\title{
Intuition, expertise and judgement in the capture and assessment of photographic images.
}

\author{
Doctor of Philosophy
}

\section{Robert James Ryan}

A thesis submitted to the University of Gloucestershire in accordance with the requirements of the degree of Doctor of Philosophy in the School of Business and the School of Art.

August 2019

82839 words 


\section{Abstract}

The aim of this thesis is to contribute to our theoretical and experiential understanding of the exercise of multivariate, short time-slice photographic judgement. This research is grounded in both the ontology and the psychology of nonconscious (intuitive) cognition and its orthogonal interaction with conscious thought at the moment of capture or assessment of a photographic image. My principal mode of empirical investigation uses a cross-sectional, correlational design employing a testing instrument, the Intuitive Mastery Photography Test (the IMP Test) originally developed to support Ryan (2017). The tests were conducted upon a mixed sample of 106 amateur and professional photographers, twenty of whom also participated in an unstructured intraspective interview. The testing and interviews establish: (i) that ten constructs satisfactorily enclose the concept of expertise for this sample of photographers in this domain, (ii) that partitioning on the basis of inter alia gender, photographic qualification and genre produce significant differences in the engagement and conjugation of the ten constructs in the intuitive moment of capture or assessment, and (iii) that 'style' or 'voice' can be explained as an emergent property derived from the complexities. of the exercise of expert, intuitive, photographic judgement. I conclude that, notwithstanding the sample size, there are grounds for strong confidence that the testing is of high external validity as a tool for individual analysis and modest confidence that it is also valid for the partitioned sub-groups. 


\section{Author's Statement}

I declare that the work in this thesis was carried out in accordance with the Regulations of the University of Gloucestershire and is original except where indicated by specific reference in the text. No part of the thesis has been submitted as part of any other academic award. The thesis has not been presented to any other education institution in the United Kingdom or overseas. Any views expressed in the thesis are those of the author and in no way represent those of the University.

Signed

Date: August 2019 


\section{Acknowledgements}

I have a debt of gratitude to my two supervisors that I can never repay: Professor Barry Davies and Dr Julia Peck. Barry's insights and Julia's forensic questioning kept me on my toes, stimulating lines of thought and development that have made a huge contribution to my research.

I have also had the benefit of the support of my youngest son Dr Joachim Ryan. Joe's deep knowledge and practice of clinical, cognitive psychology has been of enormous help in navigating the rigours of research in that area. I have also been blessed with help and support from Chris Weston who subjected himself to my research methods with outstanding good grace.

As this research progressed some very good friends, Tracy Jones, John Hobson, Peter Phillips and Rod Wainwright, have subjected my ideas to a great deal of critical scrutiny. Always friendly, but always to the point, they have helped bring this research to fruition.

I would like to thank the kindness and professionalism of Beatrice Evans who transcribed over forty hours of interviews and my friend Bob Henry whose ruthless copy-editing has exposed just how bad my punctuation can be at times.

Doctoral research can only ever come to a conclusion when three people come together, give up their time to examine the thesis and determine whether the standard for the award has been met. Accordingly, my heartfelt thanks go to the chair of my panel Dr Philippa Ward and the two external examiners Dr Branko Pecar and Dr Elizabeth Allen.

Finally, there is one person who makes everything worthwhile: my wife Alison Price. Alison's passion for photography kindled my interest twenty years ago; so to her I dedicate my love and this thesis. 
Table of Contents

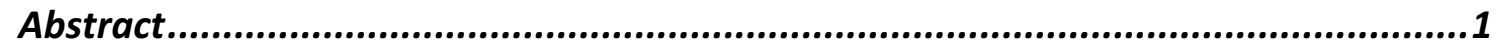

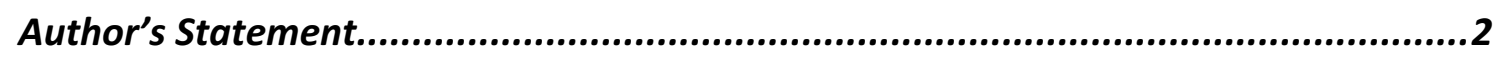

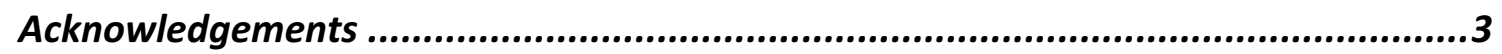

List of Exhibits........................................................................................

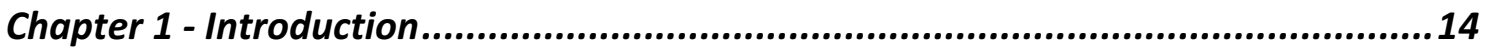

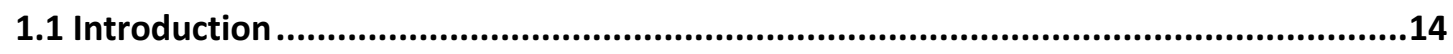

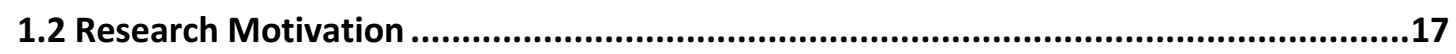

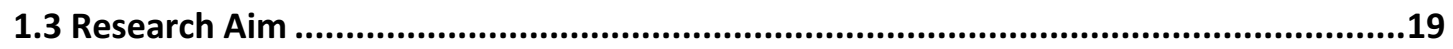

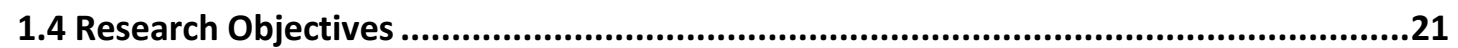

1.5 Importance and Originality of the Research.............................................................22

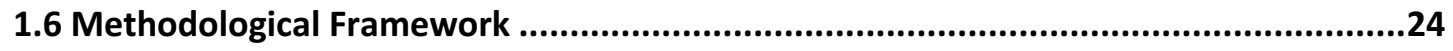

1.7 Methods, Models and Aggregate Empirical Hypotheses ................................................25

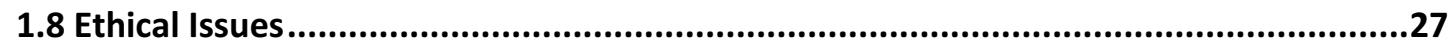

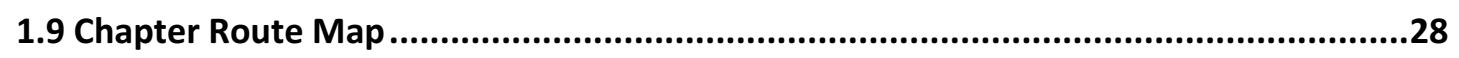

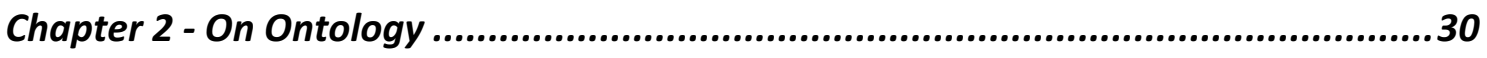

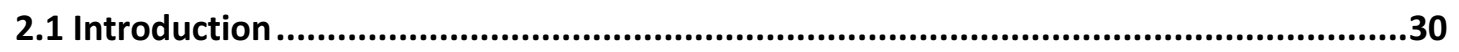

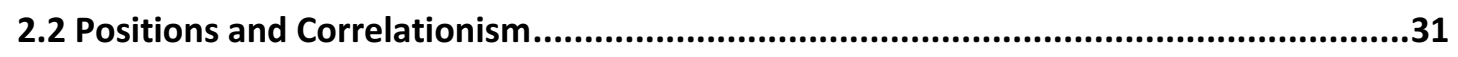

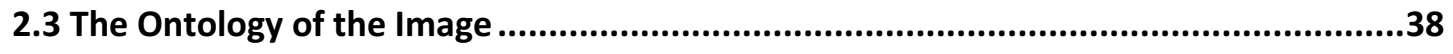

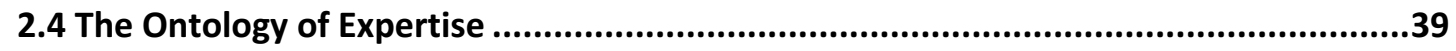

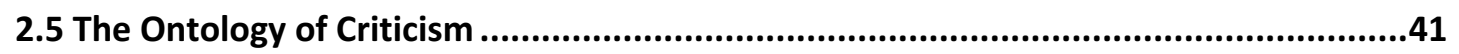

2.6 The Ontology of Meaning ..........................................................................................42

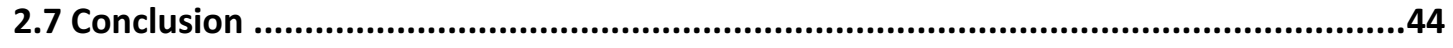

Chapter 3 - The Psychology of the Photographic Moment .....................................46

3.1 Introduction ....................................................................................................................46

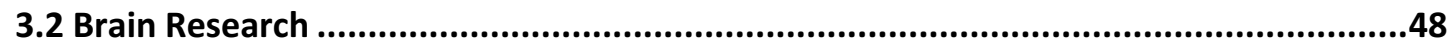

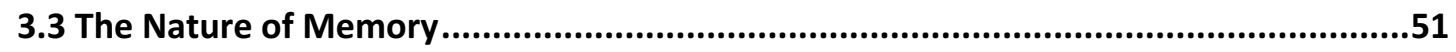

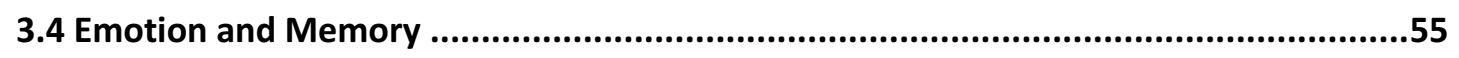

3.5 The Impact of Nonconscious Mental Activity............................................................61

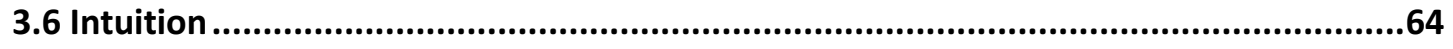

3.7 The Bayesian Brain ......................................................................................................71

3.8 Theoretical Model of the Cognitive Process in Photography ......................................75 


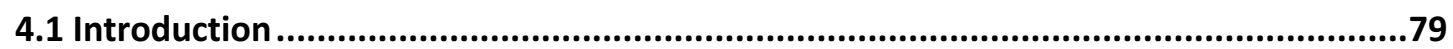

4.2 The Philosophical Origin of Cognitive Psychology ........................................................79

4.3 The Bartlett Experiments.................................................................................................81

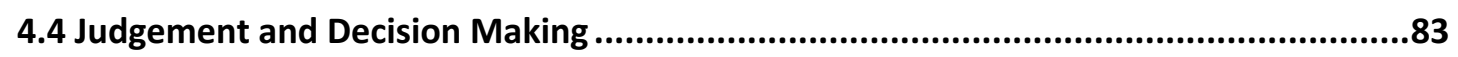

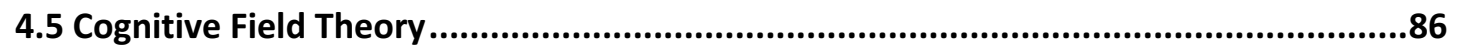

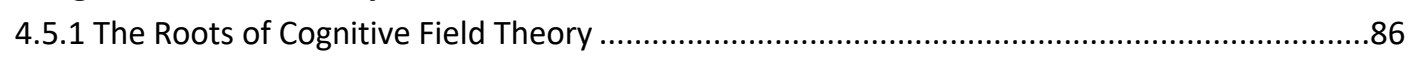

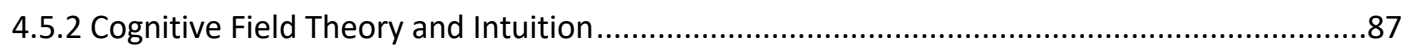

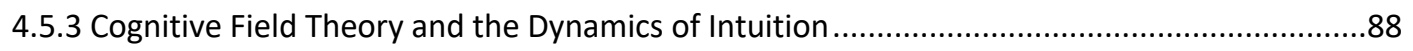

4.5.4 Cognitive Field Theory and the Development of Expertise...................................................91

4.5.5 Cognitive Field Theory and Constructs as the Units of Expertise..........................................94

4.5.6 Cognitive Field Theory and Hierarchical Processing .........................................................96

4.5.7 Cognitive Field Theory and Fuzzy Constructs...................................................................97

4.5.9 Cognitive Field Theory - the Limits of Intuitive Processing ...............................................98

4.6 The Development of Expertise ....................................................................................100

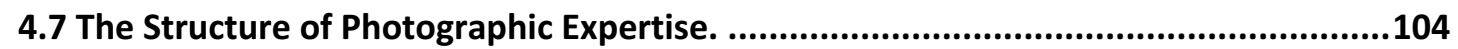

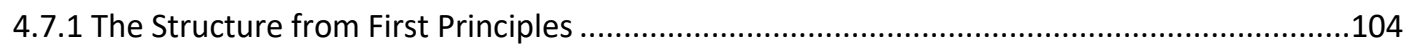

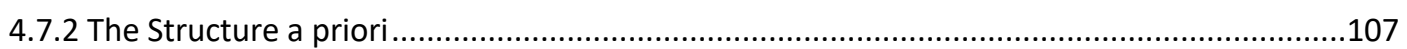

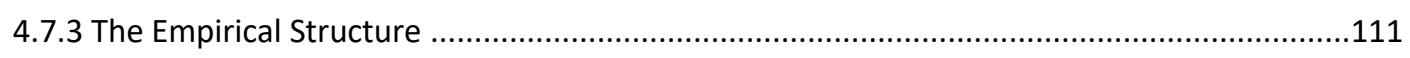

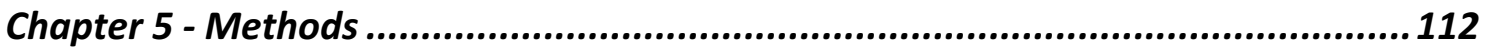

5.1 Introduction .............................................................................................112

5.2 Multi and Mixed Method Research (MMR) ................................................113

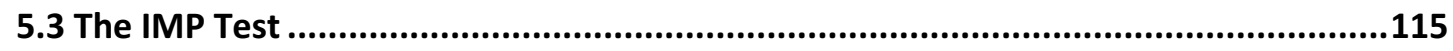

5.4 IMP Test Design and Development........................................................117

5.5 A Note on the Use of Likert Scales. .........................................................121

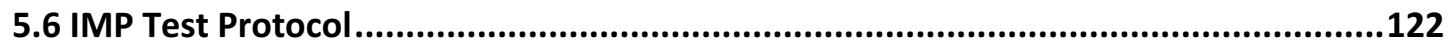

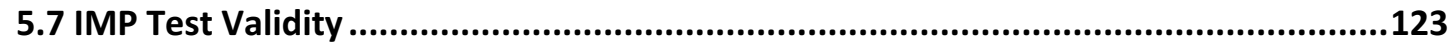

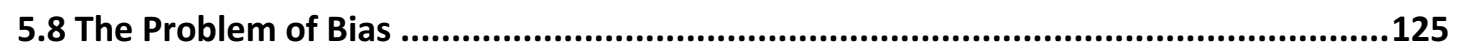

5.9 Sampling and Selection Bias ..............................................................127

5.10 The Participant Profile ...........................................................................129

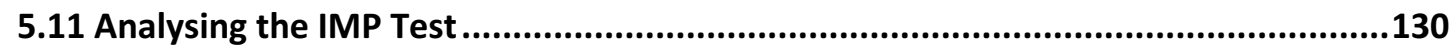

5.12 Statistical Analysis and Interpretation of the Individual's IMP Test Report .............132

5.13 Statistical Analysis and Interpretation of the Aggregate and Partitioned IMP Test Reports

5.14 The Intraspective (Cognitive) Interviews...................................................................141

5.15 Selection of the Interviewees ...................................................................145 
5.16 Confidence and Likelihood Estimation in Multi-Method Research ..........................146

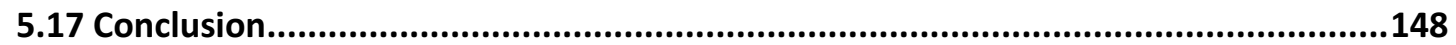

Chapter 6 - Empirical Validation of the IMP Test ........................................... 150

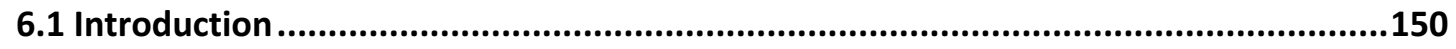

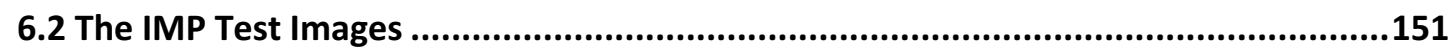

6.3 Analysis of the Construct Ranking (Aggregate Study) ...............................................152

6.4 Analysis of the Construct Ranking (Individual Study) ................................................157

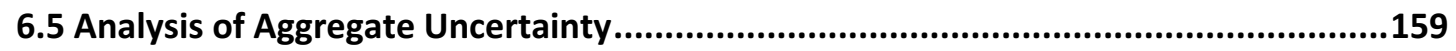

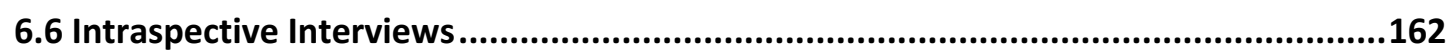

6.6.1 Intraspective Interview - Test Participant IT0084 (Simon Stafford) ....................................164

6.6.2 Intraspective Interview - Test Participant IT0079 (Neil McCoubrey) ....................................173

6.6.3 Intraspective Interview - Test Participant IT0103 (Daisy) ...................................................181

6.6.4 Intraspective Interview - Test Participant IT0106 (Simon Weir) .......................................186

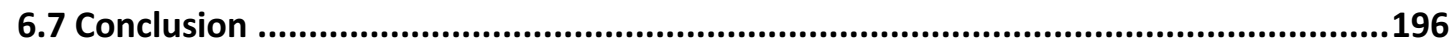

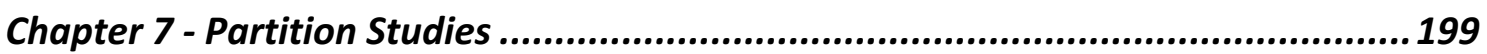

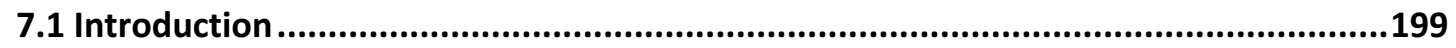

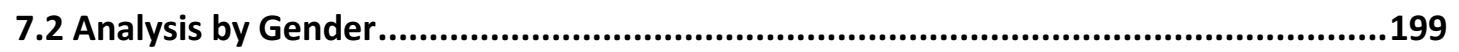

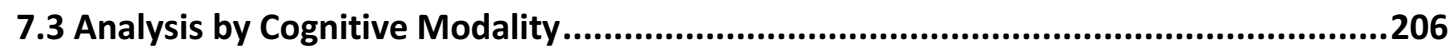

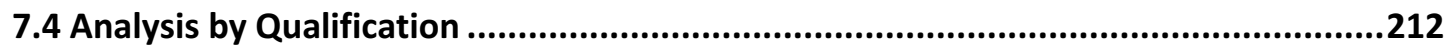

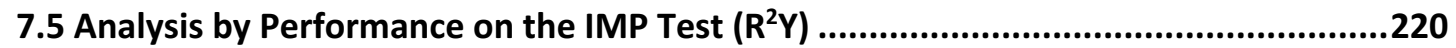

7.6 Analysis by Genre

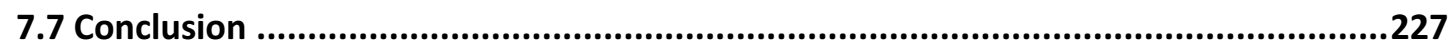

Chapter 8 - Exploring the Boundary Layer ............................................... 231

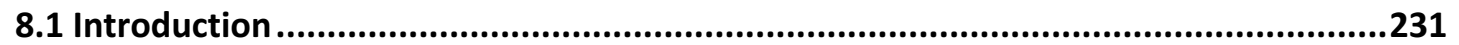

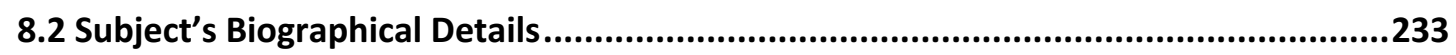

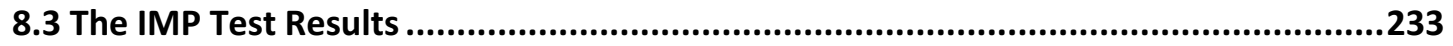

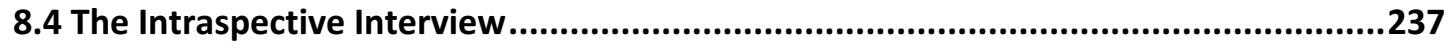

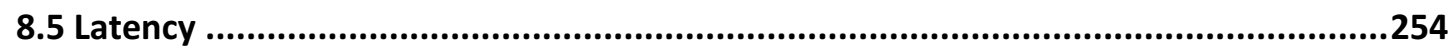

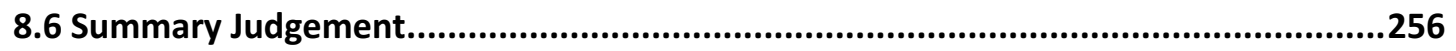

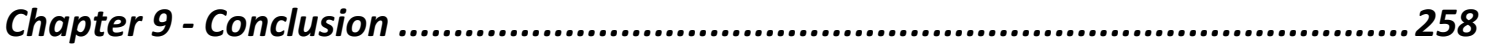

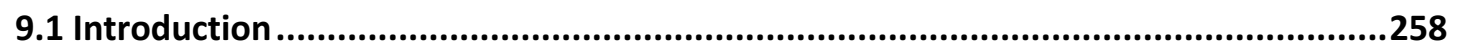

9.2 Summary Conclusions on the Research Questions .......................................................260

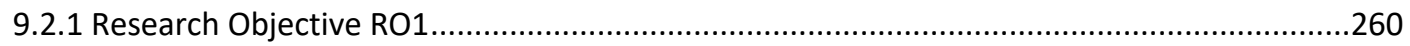

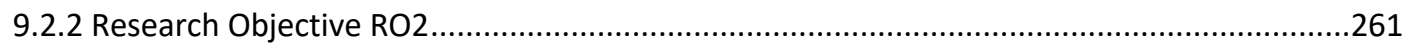

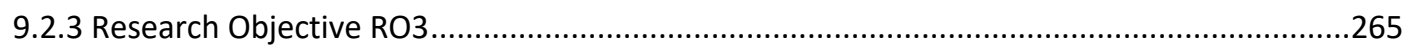




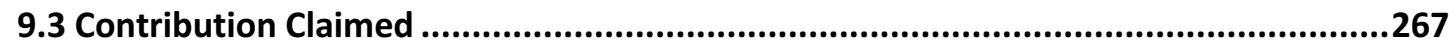

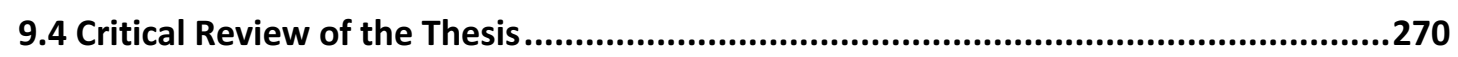

9.5 Proposals for Future Research and Development. .....................................................272

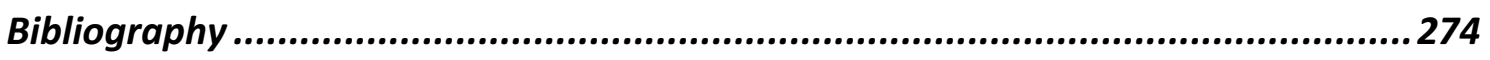

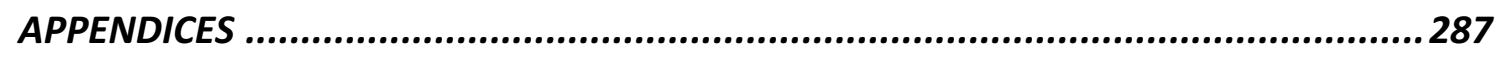

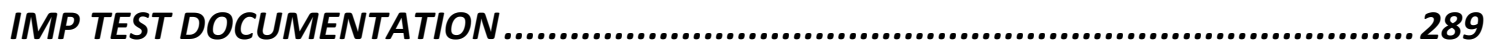

IMP Test - specimen report................................................................2295

IMP Test - instructional matter within the test ..................................................309

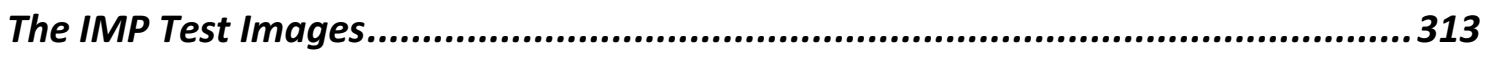

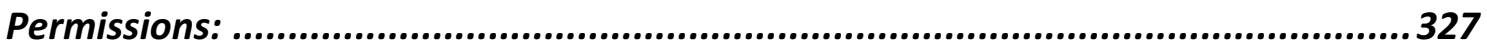

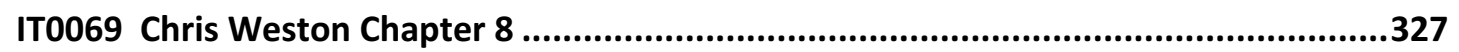

IT0079 Neil McCoubrey Chapter 6 ................................................................................328

IT0084 Simon Stafford Chapter 6...............................................................................328

IT0106 Chapter 6 Simon Weir.............................................................................................329

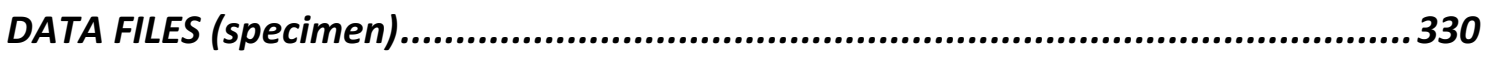

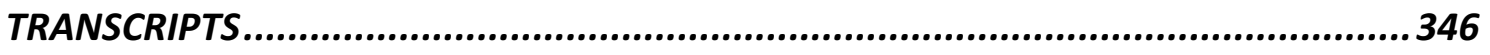

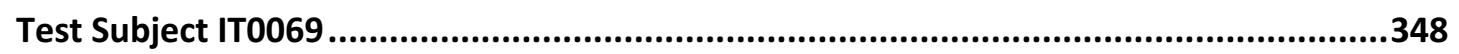

Test Subject IT0079 .....................................................................................................389

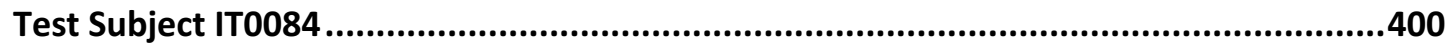

Test Subject IT0103 ......................................................................................................416

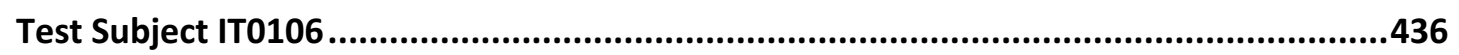

Technical note on Catastrophe Theory explanation of state changes ....................467 


\begin{tabular}{|c|c|}
\hline & $\begin{array}{l}\text { Page } \\
\text { Number }\end{array}$ \\
\hline \multicolumn{2}{|l|}{ CHAPTER 2} \\
\hline Exhibit 2.1 Strategies for approaching the essence & 40 \\
\hline \multicolumn{2}{|l|}{ CHAPTER 3} \\
\hline $\begin{array}{l}\text { Exhibit 3.1 McCullin, D., } 1968 \text { Shell Shocked Marine, The Battle of Hue, } \\
\text { held at the National Galleries of Scotland and Tate, presented by the } \\
\text { artist, 2013, viewed April } 2019 \text { and downloaded from: } \\
\text { https://www.nationalgalleries.org/art-and-artists/130204/shell- } \\
\text { shocked-us-marine-battle-hue }\end{array}$ & 47 \\
\hline $\begin{array}{l}\text { Exhibit 3.2 Axon connectivity through the multiple regions of the brain. } \\
\text { Reproduced from Bardin (2012), p.394 }\end{array}$ & 50 \\
\hline $\begin{array}{l}\text { Exhibit } 3.3 \text { Standard Model for the Formation of Long-Term Memory. } \\
\text { Reproduced from Frankland and Bontempi (2005), p.122 }\end{array}$ & 52 \\
\hline Exhibit 3.4 Ryan, R.J. (2011) - Male Lion, Kenya - author's image & 54 \\
\hline $\begin{array}{l}\text { Exhibit } 3.5 \text { Eight Affect Concepts in a Circular Order. Reproduced from } \\
\text { Russell (1980), p.1164 }\end{array}$ & 56 \\
\hline $\begin{array}{l}\text { Exhibit 3.6 Schematic representation of modulation of memory } \\
\text { consolidation. Reproduced from McGaugh (2006) }\end{array}$ & 59 \\
\hline $\begin{array}{l}\text { Exhibit 3.7 The Hierarchical Predictive Processing Model. Reproduced } \\
\text { from Clark (2016). }\end{array}$ & 73 \\
\hline $\begin{array}{l}\text { Exhibit 3.8 General Model of Photographic Image Capture and Post- } \\
\text { processing (Author's own). }\end{array}$ & 87 \\
\hline
\end{tabular}




\begin{tabular}{|c|c|}
\hline CHAPTER 4 & \\
\hline $\begin{array}{l}\text { Exhibit } 4.1 \text { Immanuel Kant }(1724-1804) \text { - artist unknown reproduced } \\
\text { under creative commons: } \\
\text { https://upload.wikimedia.org/wikipedia/commons/4/43/Immanuel Kan } \\
\text { t \%28painted portrait\%29.jpg }\end{array}$ & 80 \\
\hline $\begin{array}{l}\text { Exhibit } 4.2 \text { Sabre-tooth Tiger reproduced by kind permission of 'How it } \\
\text { Works' Magazine within the FutureNet publishing group. }\end{array}$ & 85 \\
\hline $\begin{array}{l}\text { Exhibit } 4.3 \text { Catastrophe Theory manifold showing spontaneous } \\
\text { transitions between cognitive modalities. }\end{array}$ & 89 \\
\hline $\begin{array}{l}\text { Exhibit 4.4 The hierarchy of expertise (reproduced from Ryan (2017) The } \\
\text { Master Photographer - the journey from good to great. }\end{array}$ & 105 \\
\hline $\begin{array}{l}\text { Exhibit } 4.5 \text { Royal Photographic Society - extract from guidelines for } \\
\text { distinctions. }\end{array}$ & $107-108$ \\
\hline $\begin{array}{l}\text { Exhibit } 4.6 \text { Table of constructs used in this research compared with other } \\
\text { photographic sources. }\end{array}$ & 110 \\
\hline CHAPTER 5 & \\
\hline Exhibit 5.1 The IMP Test Sequence & 118 \\
\hline Exhibit 5.2 Construct Mean & 120 \\
\hline Exhibit 5.3 Principal Quality Statistics used in the IMP Test Analytics & $124-125$ \\
\hline Exhibit 5.4 Participant Profile at a Glance & 130 \\
\hline Exhibit 5.5 Schematic of input-output arrays for the IMP Test & 132 \\
\hline Exhibit 5.6 Grand-mean Correction of Raw Scores from the IMP Test & 134 \\
\hline Exhibit 5.7 Output of a PLS Regression (sample test subject) & 136 \\
\hline
\end{tabular}




\begin{tabular}{|c|c|}
\hline $\begin{array}{l}\text { Exhibit } 5.8 \text { Multidimensional Scaling of the Scoring of the IMP Test Images } \\
\text { (sample test subject) }\end{array}$ & 137 \\
\hline Exhibit 5.9 Histogram of R2 scores from 106 IMP Test Participants & 138 \\
\hline Exhibit 5.10 Variation in R2 with increasing group size (randomly drawn) & 140 \\
\hline Exhibit 5.11 Protocol for the Intraspective Interviews & 144 \\
\hline $\begin{array}{l}\text { Exhibit } 5.12 \text { Evidential communication in multi-method research } \\
\text { (adapted from Mastrandrea et al (2011)) }\end{array}$ & 148 \\
\hline CHAPTER 6 & \\
\hline $\begin{array}{l}\text { Exhibit } 6.1 \text { Corrected average scores by image, intuitive judgement and } \\
\text { construct. }\end{array}$ & 151 \\
\hline Exhibit 6.2 Image ranking and differences & 152 \\
\hline $\begin{array}{l}\text { Exhibit } 6.3 \text { Image No. } 21 \text { - maximum disagreement intuitive rank }=18 \text {, } \\
\text { rational rank }=8\end{array}$ & 152 \\
\hline Exhibit 6.4 Variable Inflation Factors for each of the ten constructs & 153 \\
\hline $\begin{array}{l}\text { Exhibit } 6.5 \text { Partial Least Squares output for the whole sample (106 } \\
\text { participants) }\end{array}$ & 154 \\
\hline Exhibit 6.6 Output of Principal Component Regression & 155 \\
\hline Exhibit 6.7 Multidimensional Scaling data (106 participants) & 156 \\
\hline Exhibit 6.8 Number of ranked constructs & 158 \\
\hline Exhibit 6.9 Frequency of constructs that are intuitively accessible & 159 \\
\hline Exhibit 6.10 Uncertainty in judgement by group size & 160 \\
\hline Exhibit 6.11 Jarque - Bera Test for Normality of $R^{2} Y$ Distribution & 161 \\
\hline Exhibit 6.12 Participants in the Intraspective Interviews & 163 \\
\hline
\end{tabular}




\begin{tabular}{|c|c|}
\hline Exhibit 6.13 Multidimensional Scaling (IT0084) & 172 \\
\hline Exhibit 6.14 Multidimensional Scaling (IT0079) & 179 \\
\hline Exhibit 6.15 Multidimensional Scaling (IT0103) & 185 \\
\hline Exhibit 6.16 Multidimensional Scaling (IT0106) & 195 \\
\hline $\begin{array}{l}\text { Exhibit 6.17 Feedback from Simon Weir (IT0106) on the IMP test and } \\
\text { subsequent interview. }\end{array}$ & 198 \\
\hline CHAPTER 7 & \\
\hline Exhibit 7.2.1 Sample-size comparison of sub-groups by gender & 200 \\
\hline Exhibit 7.2.2 Jarque-Bera Test for normality (gender) & 200 \\
\hline Exhibit 7.2.3 Cumulative Distributions $\mathrm{R}^{2} \mathrm{Y}$ by gender & 201 \\
\hline Exhibit 7.2.4 Partial Least Squares Regression by gender & 202 \\
\hline $\begin{array}{l}\text { Exhibit 7.2.5 Multidimensional Scaling of Performance partitioned by } \\
\text { Gender }\end{array}$ & 203 \\
\hline $\begin{array}{l}\text { Exhibit 7.2.6 Frequency of constructs appearing within the intuitive } \\
\text { competency (by gender). }\end{array}$ & 205 \\
\hline Exhibit 7.2.7 Residuals from $\mathrm{Chi}^{2}$ Test - Gender & 206 \\
\hline Exhibit 7.2.8 Gender Differences & 206 \\
\hline $\begin{array}{l}\text { Exhibit 7.3.1 Sample-size comparison of sub-groups by rational versus } \\
\text { experiential modality }\end{array}$ & 207 \\
\hline Exhibit 7.3.2 Jarque-Bera Test for normality (by cognitive modality) & 207 \\
\hline Exhibit 7.3.3 Partial Least Squares by revealed bias in cognitive modality. & 208 \\
\hline $\begin{array}{l}\text { Exhibit 7.3.4 Multidimensional Scaling of Performance partitioned by } \\
\text { Revealed Cognitive Modality }\end{array}$ & 209 \\
\hline
\end{tabular}




\begin{tabular}{|c|c|}
\hline $\begin{array}{l}\text { Exhibit 7.3.5 Frequency of constructs appearing within the intuitive } \\
\text { competency (Cognitive Modality) }\end{array}$ & 210 \\
\hline Exhibit 7.3.6 Residuals from $\mathrm{Chi}^{2}$ Test - Cognitive Modality & 211 \\
\hline Exhibit 7.4.1 Sample-size comparison of sub-groups by qualification & 212 \\
\hline Exhibit 7.4.2 Jarque-Bera Test for normality by qualification & 213 \\
\hline Exhibit 7.4.3 Cumulative Distributions $\mathrm{R}^{2} \mathrm{Y}$ by qualification & 213 \\
\hline $\begin{array}{l}\text { Exhibit 7.4.4 Partial Least Squares analysis of qualified versus unqualified } \\
\text { subjects }\end{array}$ & 214 \\
\hline $\begin{array}{l}\text { Exhibit 7.4.5 Multidimensional Scaling of Performance partitioned by } \\
\text { qualified/unqualified. }\end{array}$ & 215 \\
\hline $\begin{array}{l}\text { Exhibit 7.4.6 Frequency of constructs appearing within the intuitive } \\
\text { competency (Qualified v Unqualified) }\end{array}$ & 216 \\
\hline Exhibit 7.4.7 Residuals from Chi $^{2}$ Test - Qualification Analysis & 217 \\
\hline Exhibit 7.4.8 Ranking by frequency and factorial for 'elite group'. & 218 \\
\hline $\begin{array}{l}\text { Exhibit 7.4.9 Residuals from the } \mathrm{Chi}^{2} \text { Test }- \text { qualified judges versus the } \\
\text { rest }\end{array}$ & 220 \\
\hline $\begin{array}{l}\text { Exhibit 7.5.1 Partial Least Squares analysis of subjects above and below } \\
\text { the median } R^{2} Y \text { score }\end{array}$ & 221 \\
\hline $\begin{array}{l}\text { Exhibit 7.5.2 Multidimensional Scaling of Performance partitioned by } \\
\text { subjects above and below the median } \mathrm{R}^{2} Y \text { score }\end{array}$ & 222 \\
\hline $\begin{array}{l}\text { Exhibit 7.5.3 Frequency of constructs appearing within the intuitive } \\
\text { competency (high versus low } R^{2} Y \text { scorers) }\end{array}$ & 223 \\
\hline Exhibit 7.5.4 Residuals from $\mathrm{Chi}^{2}$ Test - high versus low $\mathrm{R}^{2} Y$ scorers & 224 \\
\hline Exhibit 7.6.1 Regression Model Ordering (Landscape v other genres) & 225 \\
\hline
\end{tabular}




\begin{tabular}{|l|c|}
\hline $\begin{array}{l}\text { Exhibit 7.6.2 Analysis by frequency and factorial for landscape versus } \\
\text { other genres }\end{array}$ & 225 \\
\hline Exhibit 7.6.3 Residuals from Chi' ${ }^{2}$ est - genre analysis (landscape) & 226 \\
\hline Exhibit 7.6.4 Regression Model Ordering (Wildlife/nature v other genres) & 226 \\
\hline Exhibit 7.6.5 Residuals from Chi' Test - genre analysis (Nature/wildlife) & 227 \\
\hline CHAPTER 8 & \\
\hline Exhibit 8.1 CW - selected output from the IMP Test & 234 \\
\hline Exhibit 8.2 CW - Principal Component Analysis & 235 \\
\hline Exhibit 8.3 Wild Horses of the Camargue & 239 \\
\hline Exhibit 8.4 Dancing Cranes at Hokkaido, Japan & 247 \\
\hline Exhibit 8.5 Dali's Horses in the Camargue & \\
\hline
\end{tabular}




\section{Chapter 1 - Introduction}

\subsection{Introduction}

In the hope of achieving some personal understanding of the experiences driving my intent in preparing and writing this thesis, I ventured on a process of introspective analysis ${ }^{1}$. Looking through my personal archives of articles, books and notes, I retrieved the autobiographical note shown over page. It was composed 30 years or more ago after an evening at a Spanish club where I had been playing the flamenco guitar ${ }^{2}$. The note is important for me as it touches on a number of themes relevant to this thesis and it raises a question: how do great musicians, great painters, great photographers or, indeed, the great in any field of human endeavour achieve the sublime not once, not occasionally but time and time again? This is the question that motivates this thesis and one I presume to answer.

Some might argue that I was engaged in an act of cultural appropriation for personal gain exploiting an art form that is deeply personal to the marginalised and largely dispossessed people of Southern Spain. However, reading the note again, many years after it was composed, I am struck by the memory of the interpersonal connectedness the piece reveals. I am also struck by the clarity of that memory: I can still smell the sharp tang of the cypress wood of the guitar, the tension of the strings under my fingers, the expectation and the anxiety in the cantor's face as I started to play. I had not played for her before, she had joined us late in the evening, and she was not fooled by my fashionably long moustache and long hair. I was not Spanish. I can even remember the order and sequence of my adjustments to the tuning of the guitar. Indeed, the memories are burnt in, and it is moments like that, which led me to the insight that it was a combination of high levels of emotional intensity and equally intense and challenging practice, that allowed me, that night, to achieve my musical intent.

\footnotetext{
${ }^{1}$ The classic work on introspection as a basis for self-reflection and analysis is Ten Hoor, M. (1932). "A Critical Analysis of the Concept of Introspection." The Journal of Philosophy 29(12): 322 - 331.

${ }^{2}$ It was composed for an article I intended to publish in a local magazine. It was never published, and it is reproduced here with minor corrections from the original draft.
} 


\section{Duende}

We were into the small hours and I had been playing with little break since sun down. They had recognised me as English - a payo - but soon acknowledged that I could play, they tolerated me, I had the compas, I was a flamenco. I wiped the frets with a cloth and adjusted the capo. The tuning was perfect, but I ran down through the strings, plucking the harmonics, checking. Then I struck the first notes of the opening falseta of siguiriyas. A tiny vibrato shook the sound as it escaped from my guitar. I leaned forward, I could feel its vibration in my chest responding to the rhythm of my life, a solitary cantor clapped her hands - palmas sordas - as my right hand, in bursts of movement, shot out rasgueado after rasgueado, then a silent beat - a hole in time - a silence so loud everyone could hear it. A sigh erupted from the nearest members of the audience - a quiet ole! from someone to my right - the music was underway. From somewhere inside me the falsetas - the twelve beat bars of the rhythm came pouring out - each one a labour of love practiced a thousand times, no ten thousand times until each had passed the barrier of understanding and been burnt into my soul.

As the rhythm progressed a cantor picked up on my music and started to sing - it was poetry and it was prose, her voice was cracked but heavy with emotion, telling her tale of lost love, hardship and pain. My music pierced the room, the guitar's small brittle voice propelled by the luthier's genius, sliced through the air. Faces all around me urging me on as I, hunched over the guitar, began to lose awareness of anything but the music. Even my guitar and my hands faded from perception as the music held me and my audience in its grip. Then it was there - the lord of the dance - the moment of pure awareness of the meaning of the music - the duende - often sought, rarely found. That moment when reason goes, and artist and audience are bound in perfect communion. 
It has also led me to a personal certainty that it is by the combination of author and audience that meaning emerges that is both transcendent and transitory. That sublime moment of perfect communication, the duende, no matter what form it takes, is achieved and passes in a moment. It creates a trace of what is possible in the memories of all of those involved and a desire to seek the sublime again, in whatever form it might take. It is this trace of the sublime in the memory of those who participate in a creative act that itself forges an enduring bond of shared meaning and, indeed, mutual respect between those involved.

This autobiographical note also points to another truth that I hold as given. Beauty may be in the eye of the beholder but is also in the eye of its creator; indeed, it is inseparable from the creator. The meaning and significance of the message within the creative act is personal to the musicians involved as well as to those who are listening. Listening, like viewing, like all modes of perception, is an active act conditioned by the relationship with the author, a shared understanding of the vocabulary of the artistic form, and the variety of beliefs and expectations that define the cultural space in which the creative act occurs. The idea that the creative act can be separated from its author whose intent is quite irrelevant in deciding its meaning is, in my view, a twentieth century philosophical conceit that has impoverished critical engagement in the arts, legitimating any opinion no matter how bizarre or illconceived. Nietzsche's words are quite savage: 'imagine the inartistic natures . . against whom will they now turn their weapons? Against their arch-enemies the strong artistic spirits ... they act as though their motto were: let the dead bury the living'. (Friedrich Nietzsche's 'On the uses and disadvantages of history for life' (Nietzsche, 1874)).

The note also brings into sharp relief the personal commitment to the delivery and communication of artistic intent. In this case I, a non-Spaniard, had achieved a high level of ability in a complex musical and cultural practice through hard and systematic training and engagement. There is no objective standard against which greatness can be judged but we can recognise it in the ability of the artist to break the rules of the discipline that many years of committed practice have reinforced. In flamenco, the rules of the musical form specify a strict and regular rhythm, the twelve beat bars with repetitive accents that generate a hypnotic quality, but then comes the moment when the guitarist denies his audience what 
they are expecting - the silent beat, the hole in time. This is the moment of surprise - the phase change that has the power to convert the ordinary into the sublime ${ }^{3}$.

This willingness to break the rules that underpin any art form, to shatter an academic consensus or to make a great scientific discovery represents the pinnacle of achievement reflected in the ri of the Shu-ha-ri approach to mastery in Aikido or in the ultimate fifth stage of skill acquisition in the Dreyfus learning model (https://en.wikipedia.org/wiki/Dreyfus model of skill acquisition). The ultimate level of mastery assured by the combined wisdom of the ages only occurs, in my view, when the rational gives way and embraces the intuitive.

Thus, my working proposition some years ago when commencing this programme of research was that mastery in photography is not uniquely a product of attention to output. In music it is said that the easier an instrument is to pick up and play the harder it is to master. That is true of photography. It is an instrumental art form where the click of a button on a modern camera or mobile phone achieves an outcome that, more or less, reflects the individual's intent in taking the image. However, the ease of capture seduces the user into believing that the process of taking the image is of little consequence - all that matters is thinking up new opportunities to take images. It also ignores the point that originality in intent emerges from both engagement with the world and, through the process of achieving mastery, in its delivery. Lack of enablement in photography constrains intent and both homogenises and defers a large element of the creative process into the hands of the manufacturer and the machine. The image is no longer a creative act but a consumption product and this is, I argue, a profound and negative consequence in following an outcome-oriented approach to photography.

\subsection{Research Motivation}

Image capture and analysis is a fundamental part of contemporary culture. It drives, in large measure, modern communication and we live in what Jhally (1990) observed has become an

\footnotetext{
${ }^{3}$ The word sublime in this context I take to be the moment when reason ceases and the transcendent experience occurs (for a full discussion see Morley, S. (2010). The Sublime. London, Whitechapel Gallery).
} 
'image-saturated society' within an 'image-based culture'. Jhally's comment is doubly true in the contemporary world of 2019 where the World Wide Web hosts innumerable sites dedicated to hosting images and social media platforms that facilitate the output of the global investment by people in mobile phones. Within our modern, image-drenched society, both still and moving images have a role to play. From advertising to news media, and from science to aesthetics, the still image communicates much of our experience of the world.

The practice of photography varies from the click of a mobile phone to the MRI scanner imaging the activity of the human brain. The photography discussed in this thesis is the intentional creation and analysis of still images for aesthetic, commercial or social purposes. Specialist imaging technologies such as MRI scanners or X-ray machines are not investigated here, nor is the important field of cinematic photography.

Photography, I argue, relies upon the exercise of judgement both in the creation of the image and in the attribution of meaning to the image by the viewer. Judgement is the process by which experiential knowledge and skill is applied to sometimes inchoate though invariably complex interactions and issues on the basis of uncertain or contestable evidence. Decisionmaking is the process where we make analytically driven, risky ${ }^{4}$ choices balancing known outcomes and their associated probabilities. Skill in photographic judgement is driven by our life-world experience - realised through our 'expertise' (Harrington, 2006) which is, in part, socially shared and, in part, deeply personal. As an evidential artefact, no image offers a complete representation of the photographer's intent, or a purely veridical presentation of its subject. Rather, as I will argue in chapter 2, it reveals both.

It has been said (Patterson, 1977, p.11) that 'the camera looks both ways'. Patterson is not alone in coming to this insight - indeed, it has been made by such luminaries as Ansell Adams and Cartier Bresson. The camera - like the pen - reflects as well as illuminates, it is both a window and a mirror, and for me, the observation follows that as mastery of the camera

\footnotetext{
${ }^{4}$ The distinction between risk and uncertainty is much debated but throughout this thesis I follow Knight's (1972) definition: risk is where both outcomes and their associated probabilities of occurrence are known, uncertainty is where outcomes, their consequences and the associated probabilities are, in varying degrees, unknown.
} 
grows it illuminates the subject with a brighter light and reflects with great clarity the depth of personality and beliefs of the photographer.

Central to my research is the idea that at the heart of photography and its communicative and aesthetic imperatives lies the photographer. Intent derives from motivation that, in turn, is derived from the behaviour and ultimately from the person (in Heidegger's, 1962 terms the being behind the presence or 'Dasein'). The word 'expertise' in its common use smacks of the procedural command of public knowledge that comes from training and education in, for example, professional practice. However, in the literature on research into expertise no such limitation is imposed (Dane, Rockmann et al. 2012, Ackerman, 2014, Gobet, 2016).

Expertise is not related to any distinction between 'professional' and 'amateur' and with particular reference to photography, its early development in the Victorian era was largely an amateur pursuit. As Sieberling and Bloore (1986) comment: 'From its beginnings until the mid1850s photography in England was practiced and controlled by amateurs. Preeminent among them was William Henry Fox Talbot whose patents, and those of others, limited commercial development...Not until the mid-and late 1850 s did a variety of circumstances make photography ...less subject to direction by an elite group of amateurs' (p.1). The association of the concept of 'expertise' with the practitioner in a professional context in our public discourse is an unfortunate connotation that diminishes what I mean through the use of the word and how it is used in the literature on the topic. The evidence I will present in this thesis demonstrates the creative and artistic poverty of that interpretation of expertise - what I argue for is a much deeper understanding of the term and how it derives from and drives the impulses and intuitions of the photographer.

\subsection{Research Aim}

This research aims to explore how we judge the photographic moment both in the creation of an image and in its appreciation. From the psychological and human perspective, expert judgement, whether at the moment of capture or when exercised by the viewer, relies upon the successful integration of both conscious and nonconscious mental processes. Natural 
questions arise from this: are there underlying regularities in this judgement process? What criteria do we apply, knowingly or unknowingly, when making such judgements? What is the nature of our expertise? There are numerous dimensions to these questions and to the answers that may emerge.

This research, therefore, investigates the psychology of the photographic moment. It offers answers from neuro-psychological and other perspectives exploring the contingencies of the photographic discipline and the processes by which expertise emerges. I will argue that photographic expertise, in common with other areas of human achievement in sport, the arts and, indeed, the sciences, manifests itself through a dynamic boundary experience between our conscious (deliberative) and our nonconscious (intuitive) cognition. I will make the case that these are not diametrically opposed modes of mental activity but are interdependent of one another. The mechanics of that interdependence is explored both theoretically and empirically in this thesis.

This interdependence between the two modalities of cognition and the evolutionary necessity for the nonconscious as an enabler of conscious cognition is clearly summarised by Katherine Hayles (2017) and worth extracting in full:

'...nonconscious cognition operates at a level of neuronal processing inaccessible to the modes of awareness but nevertheless performing functions essential to consciousness... these include integrating somatic markers ${ }^{5}$ into coherent body representation (Damasio, 2000) synthesising sensory inputs so they appear consistent across time and place (Eagleman, 2011), processing information much faster than can consciousness (Dehaene, 2014), recognising patterns too complex and subtle for consciousness to discern (Kouider and Dehaene, 2007) and drawing inferences that influence behaviour and help to determine priorities (Lewicki, Hill et al. 1992). Perhaps its most important function is to keep consciousness with its slow uptake and processing ability, from being overwhelmed with the floods of interior and exterior information streaming into the brain every millisecond. (p. 10)

\footnotetext{
${ }^{5}$ Somatic markers are feelings in the body (somatic or interoceptive states) that are associated with particular emotional states: a churning stomach - fear, rapid heartbeat - anxiety being two.
} 
In developing this thesis, I recognise that photography, across its many genres, is characterised by spontaneity in judgement as the practitioner responds to the dynamics of both the external world and their internal mental states. With respect to the cognitive dynamics with the external world of perception, the evidence and arguments I present in chapters 3 and 4 lead to the conclusion that the moment of capture of a photographic image is executed in very short time slices. There may well be significant planning and previsualisation in arriving at the photographic moment; however, the point of judgement and the choice of when to press the shutter is governed by the two cognitive modalities of conscious intent and nonconscious impulse working not as opposites but together.

From the perspective of the dynamics of our internal mental states, the issue comes to this: what drives what? In chapters 3 and 4 I develop the case that our nonconscious processes with the vast well of experiential resource at their disposal and its superior pattern matching ability - is the power that drives our conscious intellect freeing it to undertake those tasks it does best namely making causal as opposed to associative connections.

Likewise, I make the case that photographic analysts also make precognitive judgements in very short time slices. However, they may subsequently reflect at great length upon their judgement and attempt to rationalise or 'unpack' the choice they have already made, convincing themselves as they do so, that their rational, conscious powers of analysis and decision making were in control all along.

The following aim is designed to capture the multiple themes that motivate and, indeed, lie at the heart of this thesis:

\begin{abstract}
Aim
The aim of this thesis is to contribute to our theoretical and experiential understanding of the exercise of multi-variate, short time-slice photographic judgement.
\end{abstract}

\title{
1.4 Research Objectives
}

In order to realise my chosen aim, I have defined four research objectives. 
R01: To develop a theoretically coherent model of photographic expertise based upon Cognitive Field Theory (CFT).

RO2: To develop an empirical protocol, based upon RO1, that enables the exploration of the exercise of intuitive expertise in photographic judgement.

RO3: To examine, using the protocol developed in RO2 whether judgement in photography is influenced by inter alia gender, cognitive processing style, photographic qualification, performance on the IMP Test or genre.

R04: To explore, using intraspective ${ }^{6}$ interviewing methods, the individual's conscious and nonconscious boundary experience in the exercise of photographic judgement.

Achieving the first (RO1) will allow us to characterise the nature of photographic expertise and how it can be framed within a cognitively feasible process of judgement. Realising the second objective (RO2) will entail the creation of an empirical instrument that will illuminate the exercise of photographic judgement both at the individual level and within groups (RO3). Finally, my purpose in achieving the final objective (RO4) is to explore the nature of the boundary experience between the spontaneous moment of intuitive judgement and the realisation of intent.

\subsection{Importance and Originality of the Research}

The importance of this research is twofold. First, it offers a novel theoretical reinterpretation and understanding of photographic expertise and judgement at both the individual and aggregate levels and, second, it demonstrates and validates a novel empirical strategy for the development of expert judgement in photography and by extension the intentionality it enables.

The case I make is that the image is an instantiation of the cognitive psychology of the socially and physically embodied photographer. Ansel Adams (1943) made the point with great clarity:

\footnotetext{
${ }^{6}$ The misspelling is deliberate and designed to reflect the process outlined below and discussed in chapter five.
} 
A great photograph is a full expression of what one feels about what is being photographed in the deepest sense, and is, thereby, a true expression of what one feels about life in its entirety.

However, reinstating the photographer does not, I argue, diminish the role of the viewer but recognises the emergent nature of meaning within a holistic perception of photography as an interpersonal social practice and act of communication. This approach whilst challenging, is a necessary precursor to my work and supports the contribution I claim for this thesis.

My intended contribution to the theory of photography is the development of an ontological framework for understanding the emergence of photographic expertise as both an individual and a socially reality. I argue that expertise - in its deepest sense - is an emergent but recursive phenomenon that both drives and is driven by intentionality. In this sense I argue that the photographer should return to the centre of our understanding of photographic art and practice - not by dispossessing the viewer but by recognising that without the one there is not the other.

This theoretical framework contributes an alternative perspective on photographic expertise by integrating the Hierarchical Predictive Processing (HPP) model of human cognition (Clark, 2013) and insights gained through my extension of Cognitive Field Theory (CFT) connecting behaviour, judgement and learning. On the basis that behaviour in general, and judgement in particular, involves intentional search for verification in the process of perception, this implies a more dynamic and active set of cognitive processes than that required in the traditional view. Here, Gobet's Template Theory (Gobet, 2013) and dynamical systems theory (Scapens, Ryan et al. 1981, Saunders, 2010) are used to model these active processes. Combining these theoretical explanations generates a number of explanatory and predictive implications including the cognitive phase-transition mechanism by which those constructs are conjugated, in the moment of hyper-attention under affective pressure. The resultant theory has generated an array of testable implications about the evolution and structure of photographic expertise.

An empirical insight into photographic expertise and its source within an individual's dynamic boundary layer is provided by my Intuitive Mastery of Photography (IMP) Test (Ryan, 2017). 
This test explores what can be described as a 'delicate empirical fabric'7 of photographic judgements where nonconscious cognitive modalities are expected to dominate. The IMP Test involves multiple variables and has been designed to investigate an individual's preferences and concerns when evaluating photographic images. This study builds on this test by linking personal characteristics with intuitive judgement and expertise.

However, what gives the IMP Test its epistemic power is that it eschews over-definition of the 'fuzzy constructs' that are constitutive of photographic expertise beyond the few words used to describe them by various international photographic bodies. At the individual level this aspect of the IMP Test protocol locates its outcomes squarely within the understanding of the person concerned. At the aggregate level, I will present evidence that idiosyncratic aspects of understanding are progressively eliminated as group size expands resolving to an irreducible core of understanding within those groups. This is a well-known phenomenon in other areas of the social sciences and, in particular, in the theory of risk and uncertainty (Beaver, 1981).

Previous studies using similar multi-variate designs (Harte and Koele 1995) to the IMP Test have been limited, not by the test method, but - as noted below - by the analytical methods employed (RO3).

\subsection{Methodological Framework}

The stance taken here reflects my long-standing engagement with research philosophy and processes. This has led me towards a 'flat ontology', which is in the Aristotelian tradition but has been reinterpreted in the phenomenology of Jose Ortega y Gassett (1968) and the anticorrelationism of Latour (2005), Meillassoux (2014), Harman (2018), Morton (2015) and others. The implication of this is that anything - following Harman (op.cit.) - can be described as an 'object' ${ }^{8}$ which is always an entity that is more than its pieces and less than its effects

\footnotetext{
${ }^{7}$ As will be discussed in this thesis the magnitude of the judgements made is irrelevant to the outcome of the test. It is how the test scores relate to one another that determines an individual's judgement 'fingerprint'.

${ }^{8}$ An object can be anything: real, abstract, aesthetic or, indeed, any category we may wish to employ. Unicorns, me, you, Hamlet, the Higgs Boson and the table in front of me fulfil this minimal definition of an object.
} 
(p53). The 'more than' is the emergent component of being that is derived from the complexity of the object and the relationships in which that object is embedded. This insight I argue provides the ontological basis for all stages of this research and accordingly forms the precursor to its various stages.

An extension of this ontology leads me to reject dualism in particular and taking positions in general both at the metaphysical and the methodological level in research. This does not mean that I do not use terms like 'conscious / nonconscious', 'subjective / objective' or 'quantitative / qualitative' - I do - but I reject the idea that they are opposites but, rather, lie in an orthogonal relationship to one another. This opposition to dualism also forms the basis of Derrida's deconstruction; however, I do not wholly accept his position that the meaning of terms is deferred solely into other terms in an infinite regress but rather are deferred into the understanding of the individual who uses them. The implication of this, supported by the results of this research, is that meaning is not unitary but plural in the sense that there is an external component that is shared and an internal component that is idiosyncratic and possessed by the individual.

\subsection{Methods, Models and Aggregate Empirical Hypotheses}

The methods employed are: (i) the empirical exploration of intuitive expertise at the nonconscious level (using the IMP Test), coupled with related personal data and (ii) intraspective interviewing methods - (Geiselman and Fisher, 2014, Matsumoto, Hwang et al. 2015) - which is designed to give insight into the conscious/nonconscious boundary experience of the individual in the exercise of their photographic judgement.

The empirical research utilised a correlational, cross sectional design (see Ryan, Scapens and Theobald, 2002). At the heart of the IMP Test is the solution of the following equation:

$$
y_{i}=\beta_{0}+\sum_{i=1}^{n} \beta_{i} X_{i}+e_{i}
$$

Where:

$y_{i}$ is the dependent variable being the short time-sliced judgement of each presented image 
$X_{i}$ are the independent variables being the unconstrained judgements of each image against each of the constructs elicited from the structure of expertise.

$\beta_{0}$ is the intercept value

$\beta_{\mathrm{i}}$ is the beta value attaching to each of the independent variables.

$e_{i}$ is the error term

The data from each individual's IMP test and related data are examined using partial least squares (PLS) regression (Abdi, 2003) and multidimensional scaling. PLS methods overcome many of the analytical issues with traditional multiple linear regression, permitting the examination of multidimensional data where the number of independent variables is high, and the number of observations permitted by any cognitively feasible testing method is relatively low. PLS regression, like all statistical methods carries with it advantages and disadvantages in any given application. It forms one part of the evidential base from which conclusions can be drawn. The use of multidimensional scaling uses a different analytical approach and provides a degree of internal (within the data) corroboration of the output from the PLS modelling.

A participant pool of 106 subjects has been used in this study of these a subset was invited to participate in the intraspective stage of the research. Those interviews were conducted using a cognitive questioning approach that is designed to reach below the levels of conscious recollection, transgress the boundary layer and enter into an exploration of the nonconscious experience of the image. This approach uses a bracketing process that draws the subject towards the moment of judgement. Sensory cues and multiple repositioning techniques are designed to elicit deep recall. This analytical method progressed until data saturation - using the criteria established in this thesis - was achieved.

Aggregate hypotheses for empirical determination (positive form) in support of each research objective:

$\mathrm{RH} 1$ : There is a set of linguistic signifiers of photographic expertise which are generally used and understood by national and international awarding bodies in photography. 
RH2: The theoretical model of expertise embedded in the IMP test effectively captures variation in photographic expertise at the conscious and nonconscious level.

RH3: There are significant differences in the structure of expertise based on (i) gender, (ii) preferred cognitive modality, (iii) holders of photographic distinctions, and (iv) favoured genre.

RH4: There is a difference in construct conjugation between conscious and nonconscious processes involved in photographic judgement.

\subsection{Ethical Issues}

This research has been conducted under the ethical principles of both the British Psychological Society and the Market Research Society.

The IMP Test is a self-report instrument where participants are offered 20 photographic images to study and to score. The test was designed as a supporting feature of Ryan's (2017) 'The Master Photographer'. This project is concerned with the analysis and interpretation of the test results at an aggregate level. Submission of the test results and the report was conducted by email, the various disclaimers and associated statement of participant rights are appended. The intraspective interviews are conducted, recorded, analysed and reported in anonymised form unless explicit written consent for the subject to be identified was obtained. Full transcripts of all interviews - anonymised and redacted where necessary - are included in the appendix. Five of the interviews were selected for detailed analysis within the thesis (see chapters 6 and 8 ).

It was not anticipated that the testing would cause harm or distress to the participants and indeed that was the case. Participants under 18 years of age were not recruited to the study. Where any participant expressed concern about the impact of the test upon their sense of self or personal wellbeing they were not tested. No deception was necessary to achieve the outcomes of this research. 


\subsection{Chapter Route Map}

Chapter 2 examines the ontological issues that frame this research. My aim in the chapter is to challenge the correlationism that influences much of the academic discourse in photography and in particular the distinctions between subjective versus objective in the conduct of research. I argue that photographic intent can only be understood as an emergent product of an individual's encounter and interaction with the world.

Chapter 3 focuses on the psychological developments that have inspired this thesis. In particular I focus on the process of memory formation, conscious and nonconscious cognitive processes, the concept of the Bayesian brain and the modern development of the Hierarchical Predictive Processing (HPP) model. These concepts represent a profound challenge for traditional ways of understanding perception in human cognition and lay the groundwork for the development of an integrated model of expertise discussed in chapter 3.

Chapter 4 integrates the HPP model and insights from cognitive field theory to generate a testable model of intuitive judgement in practice. Inter alia, this chapter explores cognition at the boundary of conscious and nonconscious processing and argues that this 'dynamic boundary experience' is the wellspring that enables, enhances and, ultimately, ennobles photographic intent.

Chapter 5 describes the development of the IMP Test and discusses the twin strands of empirical research that are employed in satisfying the research objectives established for this thesis. The two strands are complementary in design and present novel methods for investigating the boundary experience in the formation of photographic expertise. The chapter gives details and arguments in support of the research protocols used and presents a structured language for expressing confidence in multi-method research.

Chapter 6 presents and explores the results of the IMP Test at the individual level both in terms of aggregates and frequencies. Internal validity and other quality checks are presented as well as an empirical demonstration of the law of large numbers and the resolution of the degree of irreducible uncertainty in judgement evident from the aggregate data. The chapter 
concludes with external validation of the IMP Test through analysis of a series of intraspective interviews.

Chapter 7 presents the results of the empirical research with particular attention to the research objectives and associated hypotheses reflecting differences in intuitive competency across gender, genre, photographic qualification, and cognitive processing style. Both aggregate and frequency results of these partition studies are presented together with the significance of the divergence in isolating private and public expertise.

Chapter 8 This chapter presents the IMP Test results, intraspective interview and feedback interview of a successful and influential photographer. Drawing insights both from chapter 6 and this single case analysis, the chapter explores the interaction of both conscious and nonconscious cognitive modalities within the boundary layer and the emergence of latency in expertise and judgement.

Chapter 9 offers my conclusions from this research and identifies those areas where I claim a contribution to knowledge across the theoretical/empirical spectrum. There are critical issues and issues worthy of criticism in this research and I offer a brief reflective discussion of them. Finally, I believe this thesis offers many avenues for further development that I hope will be of interest to others engaged in photographic research. 


\section{Chapter 2 - On Ontology}

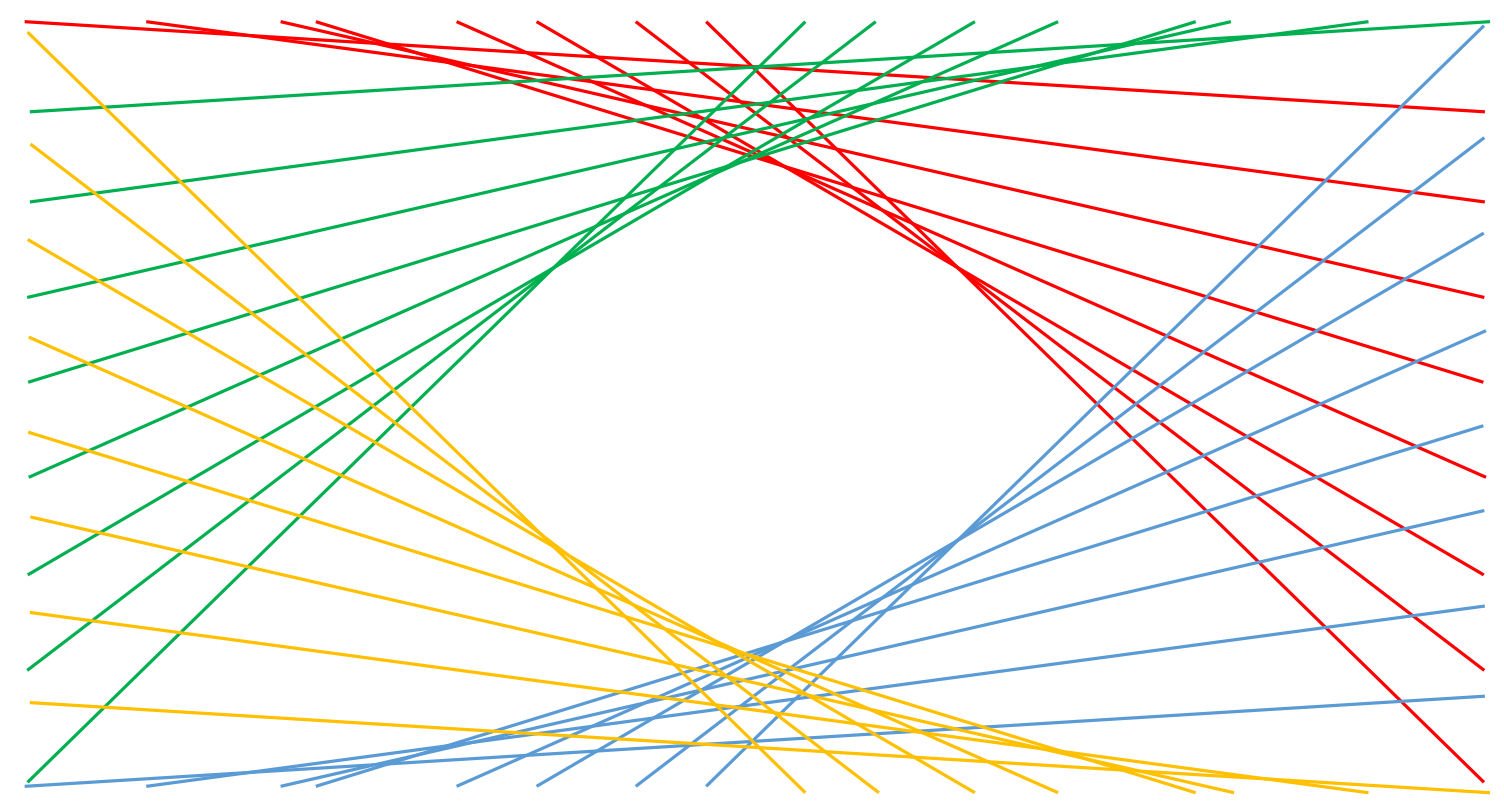

\subsection{Introduction}

In this chapter I outline my ontological position and the insights it provides in both shaping my research position and informing my approach to photography. In particular, my ontological position supports a core principle within this thesis that the camera looks both ways and the image is, in varying degrees, a representation of its subject and a reflection of the photographer going beyond his or her conscious intent. In the first part of this chapter I explore the genesis and the general implications of my ontology before turning to the problem of the photographic image. With this ontology I also contextualise my research method as an engagement, through fracturing, metaphor and the reductive process, with the essential reality of the photographer. Throughout, I address the issue of complexity, the entanglement of essential properties, the issue of emergence and how its mirror image 'withdrawal' can help us across the flimsy bridge between presence and being. 


\subsection{Positions and Correlationism}

The differing currents of western thought since the early Greeks have had two consistent themes: the first, expressed in Aristotle's Laws of Thought and the principle of excluded middle. This principle states that any truth proposition is either true or not true; it cannot be both. The second is the related issue that being, i.e., the substance of existential reality, is expressed as a relationship between that reality and our interior world of mental states and beliefs.

In natural language reasoning the logic of the excluded middle has a bias towards polarity, creating an argumentative tendency, when confronted by any propositional truth claim, to seek and assert the opposite. What may be legitimate in a formal logical system is not necessarily so in a world of natural language and complex propositions where words and meanings are deferred, and truth is an issue of coherence rather than correspondence with what is deemed to be real.

Throughout the history of western philosophy, the tyranny of the law of excluded middle has dominated much of the debate in what passes for methodology in research. It privileges differentiation over integration, disjunct over conjunct and, in logical terms, 'or' over 'and'.

To achieve personal traction on the research process I have sought to work through my own position, returning to first principles, and reviewing what I have said in the past (see Ryan, Scapens et al. 2002). It is with some gratification that my views have not changed significantly and furthermore I find myself in what has become a popular area of ontological development particularly in the area of aesthetics. I am not 'full-square' with this emerging position but it does answer many of the misgivings I have had with the post-modern and post-structural schools within continental philosophy.

My starting proposition is there is no 'ghost in the machine' and the 'subject' in the subjectobject duality is a chimera. Contrary to this, I observe that much of modern development in social theory has sought to undermine the objective and elevate the subjective. Indeed, as I will note later, the broad thrust of continental philosophy has cloven to the concept of the transcendent self. Subjectivism, interpretivism, constructionism, idealism are all of the same ilk - they are making an appeal to a knowing, conscious, psycho-centric individual who stands 
in opposition to a world of their perception or understanding. This is what Quentin Meillassoux (2014) terms 'correlationism', which he sums up in the introduction to his 'Time without Becoming': 'there can be no $X$ without a givenness of $X$, and no theory about $X$ without a positing of $X$. If you speak about something, the correlationist will say, you speak about something that is given to you, and posited by you.' (p.10)

Meillassoux goes on to make the point that correlationism appears almost impossible to refute, which of itself is good grounds for suspecting there is a very big fly in that particular ointment. However, taken as it stands, it renders impossible the idea there is any reality existing independently of the viewpoint of the mind of the person who perceives it and the consequence? Harman's (2018) pithy remark gives us half the answer '...even though we ... are of special interest to ourselves... (that does) not automatically make humans beings worthy of filling up fifty per cent of our ontology' (p.56). This search for a flat ontology represents a decisive turn against what has been taken as self-evidently true through much of Western philosophy.

From the Greeks through to the Scholastics, the idea that being lay in the co-relationship of the created to the creator was taken as a priori true and took clearest definition in Anselm's ontological argument for the existence of $\mathrm{God}^{9}$. As the necessary belief in the existence of God waned, the alternative idea that being lay in the co-relationship between creation - in the sense of the world of experience - and consciousness became firmly entrenched in western metaphysics. Indeed, the process of entrenchment began three millennia ago with Plato's argument from The Republic that the essence of being lay in ideal (Platonic) forms of which the objects of our experience were mere shadows on the cave wall. Plato's allegory of the prisoners in the cave relates how they may apply many different names to the shadow they see but they cannot see or name the real thing that casts the shadow - that is only accessible to the mind. This idea that the reality of any object of experience is accessible only

\footnotetext{
${ }^{9}$ For a thorough review of the ontological argument for God's existence see: Oppy, G. (2019). Ontological Arguments. The Stanford Encyclopedia of Philosophy (Spring 2019 Edition). E. N. Zalta.

https://plato.stanford.edu/archives/spr2019/entries/ontological-arguments
} 
to the human intellect is one of the defining aspects of Platonic philosophy; it is the basis of Cartesian dualism and, from that, post-Kantian continental philosophy.

But, is a flat ontology possible? Is correlationism so self-evidently true that it must be true? Meillassoux's answer to the problem is convincing but not, in my view, satisfying. In his After Finitude (Meillassoux 2008) he focuses on the issue of ancestrality, i.e. what sense can we make of a statement ' what is it that happened 4.56 billion years ago? Did the accretion of the earth happen, yes or no?' (p16). Taking 'objective' to mean inter-subjectively verifiable, the referent for such a statement is uncorrelated with consciousness. His argument then proceeds by demonstrating that the correlationist is stuck in the mire of "no-sense": "if ancestral statements derived their value solely from the current universality of their verification' (the most likely correlationist response) 'they would be completely devoid of interest to the scientists who take the trouble to validate them. One does not validate a measure just to demonstrate that this measure is valid for all scientists, one validates it in order to determine what is measured.' (p17)

Although confronted time and time again by the 'irrefutable and self-evident truth' of the correlationist's position (at least in their eyes) what I believed to be self-evident was the poverty of their position. Adopting an ontology where belief in the reality of reality is mocked as naïve and a sign of a lack of intellectual sophistication can be dispiriting - denial that God, consciousness, the text, power or whatever correlate happened to be the intellectual fashion of the day has always been the surest route to the heretic's stake. In the social sciences and the humanities, the apotheosis of the correlationist position has been in the anti-modernity of post-modernism where the self of self-evident belief and intellectual narcism, implicit within post-Kantian phenomenology, reached its high-water mark.

My response to the logic of correlationism was not to deny the reality of a correlate but to shift it from God, consciousness, the 'text' or 'power' and to argue for a radical objectivism. Complex things - our minds are just one example - are made up of simple things and it is emergent properties that create the 'being' but not necessarily the presence of that more complex thing ${ }^{10}$. For me the correlate shifts away from the abstraction of (say) consciousness

\footnotetext{
${ }^{10}$ This leads to the question, which I will not attempt to answer here is where does simplicity stop? Complex things are made up of less complex things and although the Greek atomists thought that the atom was the
} 
to an inherent property in all things and that property, the phenomenon of emergence, is what transforms the material stuff of sensory perception into objects and ultimately voids the distinction between the quantitative and the qualitative and the subjective and the objective.

My route to this perception arose from an anecdotal recollection of childhood. I had always been enchanted as a child with the attractive diagrams like that at the head of this chapter. With pen and ruler, and subsequently using a spirograph I once found tucked in my Christmas stocking, I was able to create wonderful shapes as the individual lines and curves criss-crossed and converged over the page. Another thing I noticed was that no matter how beautiful and colourful the shape I created, there was always a hole - a hole that emerged from the geometric convergence of line on paper. The shape was defined both by the lines and by the hole. I also became aware of the hole that was my conscious awareness as I reflected on my sins at Sunday School and in later years I experienced the meaning of the hole again as I withheld the beat, the silent note in my music. So, this was the core of my ontology: the subject is a product of the object and that the essence of what we are, 'No Thing' - the 'hole' - is an intrinsic property of the objects that constitutes, in part, our being.

This leads me to propose a simple formula:

$$
O=\sum o+e s
$$

The $O$ is any thing, or object; $O$ is a simpler little thing or object, and es is an emergent property of $O$, the 'No-thing' or the negation of its constitutive object, being an intrinsic but essential property of the sum of the $O^{\prime} \mathrm{s}^{11}$. The manifestation of $O$ to our sensory perception flows from the little ' $O$ 's, which in their turn are an aggregation of their parts and the emergent properties that generate their being as simpler beings. Our perception that an object is red, for example, is a function of the emission spectra of its component parts; however, if we want to discover the nature of 'red' we are forced to look at the emergent properties of those parts. It is at that point we appreciate that the essential property of 'redness' of those parts or indeed ' $O$ '

\footnotetext{
simplest thing, modern science has proven them wrong. Even atoms in their quantum reality are complex in relationship to the fundamental entities from which they are formed.

11 The Greek sign preceding a variable $\sum()$ is read as 'the sum of...' an array of that particular variable.
} 
is, in Heidegger's (1953) terms, withdrawn, and we can approach but never complete our understanding of the being of red nor the redness of being.

My ontology of 'No-thing', has led me to a focus on 'drawing the lines', understanding as far as I could go the structure and the organisation of the 'little things' which go to make up big things. However, mindful always that there is always a ' $+e s^{\prime}$ in the equation and that no matter how full or, indeed, parsimonious - following Ockham's Razor - we could make our understanding and definition of the sum of the little ' $O$ 's, the being of $O$ was in the 'tes'. A key point here is that +es is an essential property or conjugation of properties of the object. This essential property of the object is its essence; it is not a new object but rather a derivative quality of the object which differentiates $O$ from the sum of its o's and only emerges when those constitutive objects reach critical complexity.

The question I seek to address is how lifting the veil of complexity to approach the essence of things is possible given the essence is not just a part of an object, like the icing on the cake, it is constituted as a derivative but not the product of all the constituted essences of its parts as well as the essence that flows from their conjugation. It is the 'No-thing': the interstices or holes in the objective fabric of reality through which the immanent enters in.

As we approach the problem of emergence, we also note its mirror image withdrawal and as we approach understanding of one our reward is an approach to the understanding of the other, the trace of one always being within the other. It is important to note that emergent phenomena, being the qualitative, i.e. irreducible properties of objects, are invariably complex and that each real quality that differentiates $O$ has its own critical complexity for emergence. This takes us to the notion of withdrawal in the sense that the real object is hidden behind a veil of complexity that is just sufficient for a few, more than sufficient for some (real redundancy), and insufficient for other (real potential) effects to emerge. In this sense the essence of the object, in Kantian terms its noumena, is part of its substantial reality and unlike the phenomenon of our sensory experience, it is both withdrawn and emergent withdrawn/emergent in the mystery of objective combination (the sigma ' $o$ '), withdrawn/emergent in its objective redundancy and withdrawn/emergent in its potential.

The idea that there is no elevated ontological status to consciousness and that 'being' is just as much a fundamental property of a stone, a combined harvester or a Picasso painting form 
the basis of what has become known as 'speculative realism' (for a good overview of the state of play in 2012 see: Morelle, 2012). The recognition of a flat ontology was there in the writing of certain strands of phenomenology, but this relatively new school took shape from the writings of Bruno Latour, Quentin Meillassoux, Graham Harman, Ray Brassier, Iain Hamilton and Timothy Morton and already its intellectual vigour has led to numerous different positions. Of these, Harman's (2018) Object-Oriented Ontology (OOO) is closest to my own position, although Timothy Morton's (2015) take on what I designate +es as 'charisma' or 'weirdness' helps in penetrating the nature of essence as emergent properties. Harman acknowledges, in his development of $\mathrm{OOO}$, the influences of Kant's transcendental idealism, Husserl's phenomenology, Heidegger's radical separation of being and presence, Latour's actor network theory (ANT) and Ortega y Gassett's focus on the ' $I$ ' of being within all objects, and his insights into the role of metaphor in aesthetics. Harman's position is particularly relevant because he, like Ortega, recognises the significance of aesthetics and particularly metaphor in approaching the essence of what he terms the 'Real Object'.

An object, Harman beautifully summarises, is anything that 'is more than its pieces and less than its effects' (p. 53). In this he is not limiting himself to the world of material things, things against which we can stub our toe, but also aesthetic objects and those which are a product of our imaginations in myth and storytelling. Harman's position that an object is 'more than its pieces' places him full square within the tradition of Aristotelian ontology (Salem, 2017).

Every object, Harman argues has two aspects: the real object and the sensory object. The former being the object which is withdrawn and manifest in the +es of my formula above, and the latter which is that whose sensible effects are the correlate of our experience of the object or indeed of any other object. Given that the real object (RO) can have real qualities (RQ) (here he elides between a quality and an effect) and the sensory object (SO) can have sensory qualities (SQ), he proposes that four relationships or 'tensions' emerge from the object which, given the universal inclusivity of his ontology, is his definition of an object: RO-RQ, RO-SQ, SOSQ and SO-RQ.

Harman's (2009) and (2011) quadruple object draws extensively on Heidegger (1953) das Geviert - 'the fourfold' although resisting the correlationism in Heidegger's phenomenology. Harman argues that the fourfold structure is not two independent dualisms giving rise to four 
extremes but, rather, dualisms that intersect giving four tensions. Two of Harman's four tensions he identified with the Kantian constructs of time and space: SO-SQ through vicarious causation giving meaning to 'time' and RO-SQ giving meaning to 'space' - as the real object always lies at a distance from my sensory experience of that object (Harman 2018). He goes on to reference the phenomenon of emergence 'By showing that space and time do not just emerge arbitrarily from nowhere, but result from two types of object-quality tension, 000 is in a position to place two other terms on the same footing as space and time.' (pp.158-159)

The SO-RQ tension reflects the understanding that although there is a correlate between the sensory object of our experience of that object (being soaked by the rain which is a small ' 0 ' in the big $\mathrm{O}$ of the storm) we are also aware of the real effect, the turbulence, which is a quality of the real object which is the climate. The RO-RQ he correctly identifies as the 'essence' of the object because '(as) Leibnitz correctly noted ... that every real object is a single unit, but also that all such objects must have a multiplicity of qualities, since otherwise they would be indistinguishable from all others. The name for this tension is essence, since it concerns the real qualities that belong to a real thing.' (p.159)

However, although I am convinced by Harman's 000 in as far as it goes, there are issues he does not satisfactorily resolve. The first is that he conflates the concept of a quality and an effect whether sensory or real. In my ontology I argue that there is a distinction and a link that is important when I probe its significance in photography. The link is in the notion of 'latency': the idea that qualities are generative of effects but not all potential effects are presently emergent from a given quality, whether real or sensory. To take Morton's (op.cit.) example, the raindrops (SO) may fall on my head and the presence of 'wetness' (SQ) may make me regret forgetting my hat. However, the ability of the raindrops to quench my thirst or help the grass grow are not at that point an object of my experience. Presence in Heidegger's terms is the sensory effects revealed to our experience and the withdrawn but real effects revealed to our awareness but not our sensible perception of the object. This, for me is the second issue: awareness is not necessarily sensible experience and recognising this, which to be fair Harman does, adds a new layer of emergence and hence another source of withdrawal to the noumenal and also to the phenomenal nature of an object. It also brings into clearer focus the missing tensions in Harman's four-fold account between real qualities (RQ) and sensory qualities (SQ). 


\subsection{The Ontology of the Image}

I use the term image rather than photograph to reflect more clearly the object of interest, an instantiation of a moment and a bridge between its past and its future in the sensible perception of the viewer. Returning to a theme I have addressed before: the camera looks both ways and the image is what it has seen. It is in Szarkowski's (1984) terms a window on its subject and a mirror reflecting the mind and soul of its creator. Szarkowski's mistake was in believing that images could be sorted into one or the other. Inevitably, and no matter how literal the photographer may attempt to be in her intent, an image, in varying degrees, will be both. As Gerry Badger (2010) rightly notes in a passage from his book of essays:

'...the successful photograph by the serious quiet photographer is most likely to be a complicated amalgam of mirror and window, an ineffable struggle between subjectivity and objectivity. Like anyone else wrestling with this tricky medium, the quiet photographer is totally assured of the fact that a 'simple', 'straightforward' act of recording is anything but. The quiet photographer, however, will not draw undue attention to that process, nor, for that matter, to the process of apprehending the resultant image by the viewer.' (p.216)

So, no matter how loud a photographer shouts or no matter how many tricks they may apply in the production of the image, the same is true - the only difference is that the camera does not uncover the subtleties of the photographer's spirit but reveals instead the pretensions of their ego.

The image is from the moment of its creation an object in its own right. It has a sensible aspect but also a noumena which is the child of its parents (the photographer and her subject) but, like all children, it will be more than the sum or, indeed, the average of its parents. As we reflect on the ontology of the image, we appreciate that as an aesthetic object it is thus more than the sum of its parts - there are the choices made by the photographer and there are the elements of the subject. Indeed, there will also be the aesthetic dimension of the surface upon which the image is laid, both adding to its sensory and its real quality. However, as the image is pointing two ways it will also reveal the essential qualities of both its subject and its author, both of whom are literally withdrawn from the image. This presents us with 
two problems: how can the photographer reveal their own essential quality and the quality of the subject within the image and, in doing so, open the image to the viewer?

Harman (op. cit.) focuses upon one important strategy for approaching the essence and that is through metaphor. Here he draws upon the insight of Ortega y Gasset (1968):

'...metaphor is perhaps one of man's most fruitful potentialities. Its efficacy verges on magic, and it seems a tool for creation which God forgot inside one of His creatures when He made him. All our other faculties keep us within the realm of the real, of what is already there...The metaphor alone furnishes an escape; between the real things, it lets emerge imaginary reefs, a crop of floating islands. A strange thing, indeed, the existence in man of this mental activity which substitutes one thing for another - from an urge not so much to get at the first as to get rid of the second' (p.33)

The ability to escape from one percept to another is what gives metaphor its power and its ability to substitute the real for the sensory. However, metaphor is not the only route to the essence of the object and I would propose four others: emotional engagement of the nonconscious faculty of intuition both in and behind experience; mindfulness being the converse where the conscious mind is quietened (Thompson 2018) and the nonconscious given space to engage with the real as opposed to the sensory object; reduction where visible (sensory) effects are attenuated in order to expose the real quality of the image as object and finally, fracturing reflecting Heidegger's (1953) insight from his analysis of the hammer that when it breaks that its nature is unveiled, at least in terms of those real qualities that make it what it is sui generis. This is a theme I will return to in subsequent chapters and most specifically in chapter 8.

\subsection{The Ontology of Expertise}

At one pole of the correlationist duality sits the conscious mind but in 000 terms the mind is an object like everything else. Many of the qualities of the mind, particularly the nonconscious, I have described in metaphorical terms: the 'dark-web', information 'superhighways' and also, by analogy, the computer with its short- and its long-term memory. These metaphors are designed to give a glimpse of what is 'withdrawn' - the noumena - being that 
aspect of our cognition that we do not experience and cannot consciously recollect. The mind as a real object is deeply complex with depth upon depth of neuronal and other structures with layers upon layers of emergence giving on one side sensory awareness (the sensory object) and on the other, the 'real object'. For Husserl, Heidegger and the other phenomenologists it was the internal awareness - consciousness - that dominated their understanding of the world and gave the essence of presence. However, my argument is that it is in the conjunction of the complexity of our conscious and nonconscious mental states that the +es emerges - it is here that our being, Dasein, resides, ever present and ever withdrawn and it is in the RO-SQ and SO-RQ tensions that the motor of our creative expertise lies.

The two dimensions of the nonconscious and the conscious, the one withdrawn and the other present give a clue as to their nature in ontological terms. The process of this research is one of engagement with those tensions as a means of approaching the essence of expertise as the conscious interacts with the nonconscious in all its ineffable complexity and depth.

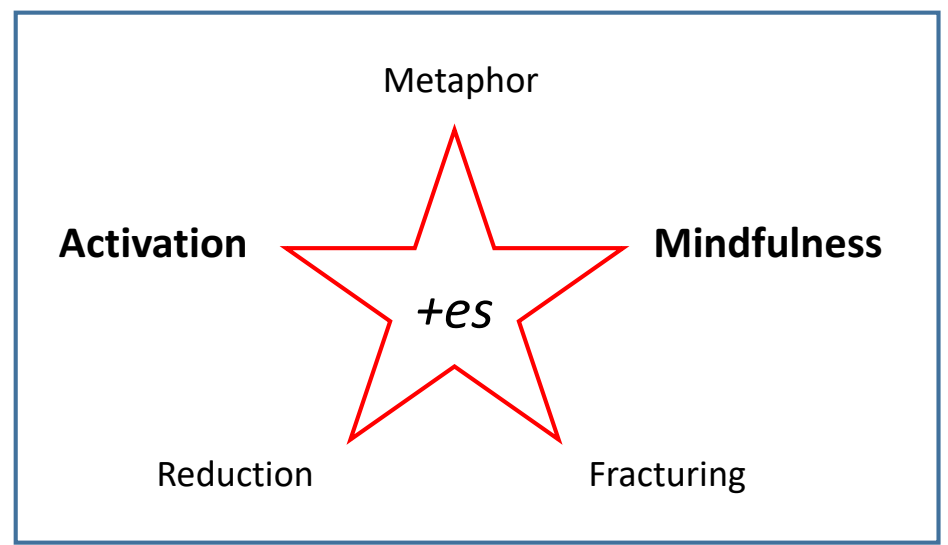

Exhibit 2.1 - Strategies for Approaching the Essence
Emotional activation is a powerful force in both opening and developing nonconscious experience and the expertise that flows from it. Meditation is an equally powerful countervailing force as we calm the 'chattering monkeys' of the conscious and

allow the nonconscious to breathe. The role of reduction and metaphor I explore in chapters 6 and 8; the role of fracturing is maybe less clear but is inherent in the idea of expertise and its conjugation developed in chapter 4 . The consequence of fracturing applies in unveiling the essence of the mind just as much as it does when it reveals the essence of a subject or indeed the essence of the image. It would be a misreading of my structure of expertise discussed in chapter 4 to assume that identifying and breaking apart its constituent elements is all that is needed to explain and ultimately to develop it.

By fracturing, the 'readiness at hand' - to use Heidegger's phrase - of the photographer's expertise is brought into sight. It is their expertise as the product of their own experience 
that is explored by the testing procedures discussed in the next chapter. If that were not the case, I would be guilty of 'undermining the object' in Harman's terms. I would be as equally guilty as the critical theorist who overmines the image as the product of that expertise and sees it purely in terms of its effects - a bundle of attributes whose merit lies only in its social or cultural significance. The essence of expertise is in the conjugation of its elements (in my terms the less complex objects or constructs that give it substance) and the latency, in terms of potential, that it generates. My argument is simple: the nonconscious is the driver of the conscious, and not vice-versa, and the essence of great photography - the RO-RQ of expertise in OOO terms - is in the former and not the latter.

\subsection{The Ontology of Criticism}

If we accept the argument that an image is an object and, like any aesthetic object, is a product of its constituent objects and the essence which creates its existent reality, then our understanding of that image has to be one of engagement as opposed to disengagement. Of course, at the moment of its creation both its subject and its author have withdrawn and, in that sense, both the author and the subject are 'dead' to the image. However, they both live on within the reality of the image and to engage with one and not the other undermines it just as effectively as if an optical scientist sought to explain the image in terms of the quality of its reflected light.

Following Ortega's insights, Harman's (2018) focus on metaphor allows the photographer to hint at the essence of their subject in front of their lens but by a process of sensible substitution where the sensory qualities of the object are replaced by the subject of the metaphor. But how do we read that metaphor and through it approach that which is withdrawn? His answer is that we are impelled to enter into the theatre of the moment, and in so doing become the RO of the metaphor. This process of 'entering in' means that we have to stand in the place of the photographer, to experience their drivers to action and through that understand the object of the image as they understood it. Hence the photographer as author is not dead, she is reincarnated in the viewer. 
Similarly, following Heidegger's analysis of the hammer (Heidegger, 1953), when we gaze on the break the essence of the object emerges into view. Similarly, the process of reduction as I discuss in chapter 8 - exposes the real object if we are prepared to commit to the task of integration. Again, we are forced to enter-in and to abandon the presumption of objectivity and to live in the photographic moment. Many critics do this of course but for others there are barriers they cannot bring themselves to cross - and as a result their commentary is not so much about the image as about themselves.

\subsection{The Ontology of Meaning}

If we accept that the camera looks both ways, does the image look both ways too? The answer in $\mathrm{OOO}$ terms is yes and no. Undoubtedly it is an emergent product of its creation, so the essence of the subject and of the author will inevitably be there. However, the image is more than the sum of two parts. When eye and heart combine in the creative act a further synergistic dimension emerges - the image reflects upon itself and, as we seek to penetrate its essence, this third, recursive level of emergence opens its meaning beyond intentionality and the representation of transitory presence.

Margaret Syverson (1999) in an exceptional book "The Wealth of Reality - the ecology of composition" explores this idea and in so doing illuminates the phenomenon of emergence and from that opens up the concept of meaning:

... meaning does not reside solely in the text (à la new criticism), nor does it reside solely in the writer (à la psychoanalytic schools of criticism), solely in the reader (à la reader-response criticism), nor even solely in the "context" (à la cultural studies and new historicism). It is not an entity that exists prior to the writing or reading of the text that instantiates it. Rather, meaning emerges as a dynamic process among these participants, a process that changes each of them even as it is unfolding. Meaning is an emergent property of the relationships among texts, writers, readers, and textual environments. (Syverson 1999)

Syverson's point here is that 'meaning' is an emergent property of both the intent with which it is created and received, and the context in which it is situated. To delve into this more deeply we bring forward the idea expressed earlier that the formation of intention is both actively conscious and also nonconscious. Meaning is also 'public' in that as soon as intent 
passes beyond thought and into action it will have consequence - those consequences may be overt, or covert, they may be bold, they may be subtle - but whatever their significance, action changes the network of the relationships in which both the actor and the audience are embedded. The significance of the act, the alteration in the network of relationships in which the act unfolds, represents the meaning of the act - whether the act is a stroke of a pen or the press of a camera's shutter.

There occurs, what theorists of complex systems term a phase transition, a qualitative global change in the system; an assortment of individuals suddenly transforms itself into a collective with distinctive global properties irreducible to its individual parts. This transition is affected through relationships and processes in densely connected networks. (Syverson, op.cit)

Syverson (op. cit) identifies ten levels of emergence of the text. Taking those ten a direct translation from the text to the image is justified:

1. Social relations in the photographer's and viewer's environments. Here Syverson gives a literary example but the image works in the same way such as in Nick Ut's classic image 'Napalm Girl' taken in the Vietnam War. The events told within the image continued to be told and retold in talk, imagery, film and texts. The image shaped the lives of those it represented, and the relationship itself emerged in the context of the image.

2. The material object or artefact; that is, the image in print or digital display generates other images in response, critical reviews, bibliographic citations, scholarly papers.

3. Local meanings, such as the emergence of stylistic signifiers within the image, its manner of composition, framing, tonality and, indeed, all the different conscious and nonconscious choices in its construction and in the way the viewer perceives those choices that those choices.

4. Global meanings, such as the emergence of genres, as well as mood, organisation, style, and other coherent structures.

5. Significance - the connection to the photographer and viewer's experience.

6. The image of the photographer in the minds of viewers that continues to emerge as viewers interact with the photographer's other work.

7. The representation of the image and of viewers in photographers' minds. 
8. Transformational effects on social structures, as when images present different ways of perceiving others, the enemy becomes seen as a hurt and terrified child.

9. Transformational effects on environmental structures, including other images as other photographers recognise that a given image has disturbed the way in which viewers are seeing and constructing their reality.

10. The justification or rationalisation of the image's existence, or its role in either reproducing or transforming social structures. This includes the critical apparatus that develops around the image as viewers respond, interpret, and analyse the image.

The understanding of the significance of any act of communication, whether via speech, text or image starts from the recognition that the act disturbs the complexity of the objective reality in which the act is embedded. That disturbance is not instantaneous but emerges through time across both the private and the public sphere. The commissioning of the act by its author was loaded with both intentional and unintentional significance but that significance is only realised through the enfolding relationships in which it subsists. The author's intent is not the whole story, but it is part of the story and the quality of the act is determined by the ability of the author to convert intention both at the conscious and the nonconscious level into action. The capture of an image has uncertain consequences, but true expertise is about opening the space for meaning to emerge and by so doing signifying the real essence within the image.

\subsection{Conclusion}

With this brief excursion into my ontology I hope to have laid the foundations for first, interpreting my focus on the mind of the photographer in the next two chapters and, second, for understanding the nature of the IMP Test in the exploration of the essence of the photographer's expertise. The IMP Test is grounded in the OOO approach to penetrating the essence of its object: the expertise of the photographer. It does not seek to impose meaning onto that expertise but instead examines its structure, its conjugation into judgement and the latent quality within that judgement that is expressed through style. In the next chapter, I commence my discussion of the cognitive architecture of the mind and how modern insights from psychology deepen our understanding of the photographic process and of photographic expertise. 


\section{Chapter 3 - The Psychology of the Photographic Moment}

\subsection{Introduction}

This chapter explores the theoretical and empirical literature that supports the research objectives and the development of the instruments used in this research (chapter 5 and 6). The first part of the chapter reviews the relevant literature concerning the operation of the brain and the formation of memory. This leads to observations about the consolidation of memory within a Hebbian framework and the role of emotion in creating persistence in nonconscious recall. The concept of nonconscious processing, research into the rationalexperiential dual processing model and finally the concept of the Bayesian Brain and the Hierarchical Processing Model is then discussed. This literature offers insights into the photographic moment both at the point of capture and in the mind of the viewer/analyst. It supports the concept that a photographic image is both a representation of its subject and a reflection of the photographers conscious and nonconscious beliefs and intent.

To motivate that story, consider for a moment one of the most iconic conflict images of the $20^{\text {th }}$ Century: Don McCullin's 'Shell-Shocked Marine', taken after the battle of Hue during the Vietnam War (see Exhibit 3.1).

My interest in this field of research was shaped by this image - it is simple, austere and painful in its intensity. Is it the soldier's face or the face of the photographer that stares at us down the decades? It goes to the essence of a man fractured ${ }^{12}$ by war, but the essence of McCullin is there too. There is something else in that the real quality of this image and its effects transcends the moment and the context of the Vietnam War. We know that the image was not staged, it was the product of a moment of insight where the camera, supported only by the hand and framed with the eye goes to the heart of the human cost of war just as surely as a bullet fired from a gun.

\footnotetext{
12 I use the term 'fractured' here reflecting the term as developed in chapter 2.
} 
Image redacted from online version of this thesis. The image can be viewed at: https://www.nationalgalleries. org/art-andartists/130204/shell-shockedus-marine-battle-hue

Exhibit3.1 Don McCullin, Shell Shocked Marine, The Battle of Hue, 1968

The psychology of the soldier and that of the photographer reveals some common lessons and they are these: both were engaged in an appalling experience under intense emotional duress (McCullin, 1992). Those experiences - learning at its most extreme - were being scorched into the dark web of their minds, emotionally tagged and ready for instant and perhaps for both, unwanted and involuntary recall. For the soldier, if he survived, what would have followed would have been a scarred psyche and possibly a life blighted by post-traumatic stress disorder (PTSD) where the nonconscious mind recalls the horrors of battle with a vividness that takes the sufferer back to the moments where bullets and steel impacted on flesh and bodies were torn apart. In his later years McCullin turned to landscape photography:

"It brings me a kind of peace," he says, "until I hear the local hunters shooting. Gunfire is a prelude to war for me. I feel I'm back there on some godforsaken road passing dying soldiers lying in culverts."

(The Observer, Shaped by War-photographs by Don McCullin, 7 February 2010).

But photographers like McCullin also learn something else beyond the horrors of war. They learn at a deep level the core skills of their art: the expertise required to capture, in the moment, their intent and, without conscious thought, make transformative judgements, exercising skills burnt in by the emotional intensity of the learning moment. 
We can learn the lessons of conflict photographers in many ways but, for me what is most pertinent is what we can learn and use to advance our understanding of the creative moment in photography and art in general. All art starts with the human experience and the construction we put upon it. Sitting at the centre of this experience is the human mind and how it works. The mind is the manifestation of the person and, based upon my ontology in chapter 2 and developed in chapter 4 , is an emergent property of the brain that as part of the nervous system interacts both within and without the individual. Central to the functioning of the brain is the process of memory formation, consolidation and retrieval.

\subsection{Brain Research}

As far as we know, the brain is the most complex structure in the natural world. It contains of the order of 86 billion specialist nerve type cells and each neuron can make multiple connections with its neighbours throughout the brain system. Interlandi (2016) estimate the neural structure has a potential of $5 \times 10^{15}$ feasible connections ${ }^{13}$ generating a memory capacity of the order of 1 petabyte (Bartol, Bromer et al. 2015), which is approximately that of the WorldWide Web. The practical storage capacity of the brain appears to be unlimited. However, even with this extraordinary computational power, the brain spends as much time disposing as it does storing what it perceives.

One aspect of this process that is important for this thesis is the way that neural structures are formed (Citri and Malenka, 2008). As particular learning activities are repeated, neural networks are formed and as they do so, myelin is progressively wrapped around the neural axons $^{14}$ increasing their speed of conduction tenfold and reinforcing the neuron's ability to fire predictably - creating what Coyle (2009) refers to as 'information super-highways within the brain'. There is evidence that myelination reinforces motor skill development, while its loss does not appear to influence recall where that learning has already developed (McKenzie, Ohayon et al. 2014). In the field of sports science, music and chess playing it is evident from

\footnotetext{
${ }^{13} 5 \times 10^{15}=5,000,000,000,000,000=5$ petabyte

${ }^{14}$ Axons are cellular conductors connecting one neural cell with another. Axons terminate in 'terminals' that link with receivers on a receiving neural cell called dendrites.
} 
fMRI studies that repetitive and challenging practice, rapid feedback and affective arousal speeds up the process of myelination and hence the predictability and speed of the brain's response to stimuli. This forms the basis of my EPF ${ }^{15}$ learning strategy for photography (see Ryan, 2017).

Across the surface of the brain, the cerebral cortex (sometimes referred to as the neo- or new cortex) forms a convoluted layer some $2-3 \mathrm{~mm}$ thick. This layer is where much of its perceptual and processing activity occurs. The cortex of 'grey matter' consists of neuron cells with 'short' axon connections from one to another. The cortical neurons also form 'long' connections passing through the interior of the brain making important connections lobe to lobe and with other parts of the brain including with three dense bundles of neurons in the limbic system: the thalamus, hippocampus and the amygdala. These three interior structures are of crucial significance in the coordination of brain activity, the formation of memory and the processing of emotions respectively.

The brain receives and interprets signals from a wide range of sense organs - the eyes, ears, taste, smell and touch being the most obvious, but also from a range of other sensory connections monitoring the interior and exterior state of the body system. However, the brain's interpretation of the inputs from the sensory system is not innate but is learnt. People who have been given their sight after a life of blindness, for example, do not suddenly see. Seeing and making sense of what is seen is learned behaviour (Kurson, 2007).

\footnotetext{
${ }^{15}$ EPF is an acronym for: emotional activation, deep practice and feedback.
} 


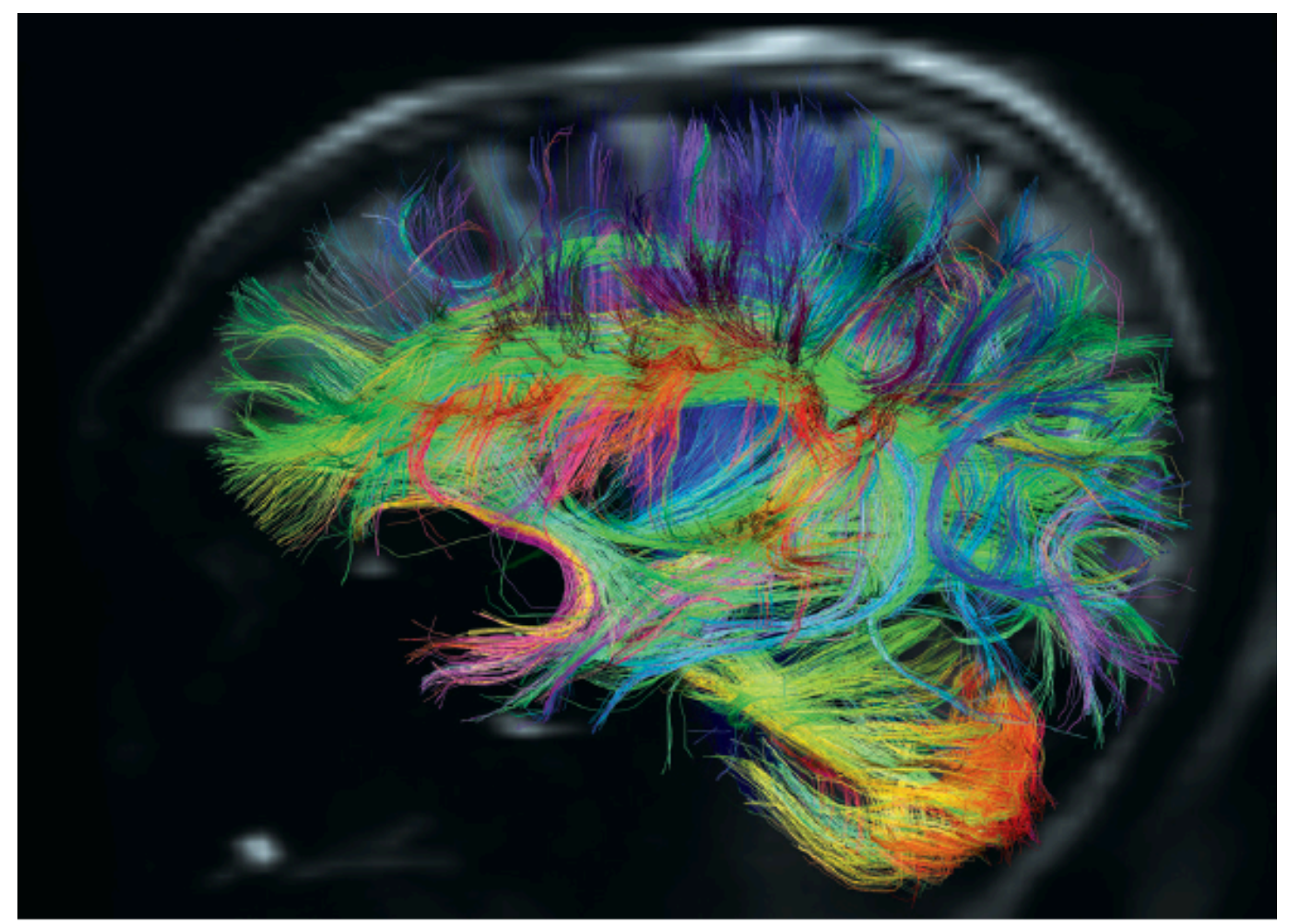

Exhibit 3.2 Axon connectivity through the multiple regions of the brain. (Bardin 2012)

The notion that the brain creates a mental model of its environment and sensory perceptions are utilised to update that model gives rise to the concept of the 'Bayesian Brain' (Doya, Ishii et al. 2006) and the Hierarchical Predictive Processing model (Clark, 2016). Rather than sense impressions continually creating a new perception of reality, those impressions revise an existing cognitive model constructed piece by piece by the individual over their lifetime. The human mind is, at one level, based upon this substrate of biochemical activity but it is more than that. The mind is enabled by the brain as part of the whole-body system and emerges as a product of countless interactions between the biochemical and the experiential in countless different ways.

In photography our principal interest is in visual processing which is conducted primarily within the occipital lobe at the rear of the brain. However, three other areas of the brain are of importance for this research. The dorsolateral prefrontal cortex where conscious executive control and rational thought is centred, the hippocampus where short-term memories are created, and the amygdala where emotions are processed. The involvement of the amygdala 
and the hippocampus in the formation of memory was believed to be well understood and established until comparatively recently. Recent work has thrown into significant doubt the processes by which long- and short-term memories are created and recalled.

\subsection{The Nature of Memory}

A photograph is the product of the process of perceiving and constraining a frame of visual perception, its capture, and transforming the result, through post-processing, into an image reflecting the potential we see in what the camera has recorded. In practice, of course, what we perceive at the moment of capture is not what we necessarily remember or, indeed, may wish for later in post-processing. The photograph is not a literal replacement for the process of remembering using the world of optics, chemistry and electronics. The brain does not remember what the camera sees - the film or the sensor does that; the brain remembers what it wants to see, and memory and intention combine to create the image.

The standard model of memory is that sense stimuli generate first a transitory memory lasting a few milliseconds. In the absence of attention these transitory memories disappear and cannot be recalled - no permanent memory trace or engram ${ }^{16}$ is created. Our ongoing engagement with everyday tasks of seeing, talking and listening all employ this 'scratchpad' memory to function effectively. Its transience is easily demonstrated if you ask someone to repeat what they have just said or engage in the childhood game of 'Telephone' (US) or Chinese Whispers (UK), where the process of attention is blocked by performance anxiety.

According to the standard model, if the individual is attentive, or the stimulus is associated with previous engrams, then a short-term memory is created by the hippocampus. Through a process of cycling through recollection and reinforcement (internal engram development) or by repetition of the external stimuli there is a process whereby long-term memory engrams are created through the hippocampus - cortical network creating engrams throughout the

\footnotetext{
${ }^{16}$ Engram: when neurons connect, they form a synaptic junction where electrochemical signals can be transferred one to another based upon their degree of activation. The connecting lines or axons can develop multiple linkages representing a given trace of memory. Linked neurons forming a permanent memory trace are referred to as an 'engram'.
} 
brain. As connections between memories in the cortex are reinforced, the connections with the hippocampus fade until a point comes where short term recall via the hippocampus disappears and recall by the prefrontal cortex for conscious processing is drawn from consolidated memory structures (long-term memory) in other areas of the brain.

The implication of the standard model is that long-term memory engrams represent consolidation of the short-term experiences of the individual and are not fundamentally different in kind but in reinforcement. This model suggests that the process of forming LT memories is derived using the original ST memory through active recollection and restore, replication or through affective pressure (Brosch, Scherer et al. 2013). There is good evidence to support the idea that emotional activation through the amygdala strongly stimulates both the creation of $L T$ engrams and in the activation of recall.

Exhibit 3.3 taken from (Frankland and Bontempi, 2005) shows the systematic process of

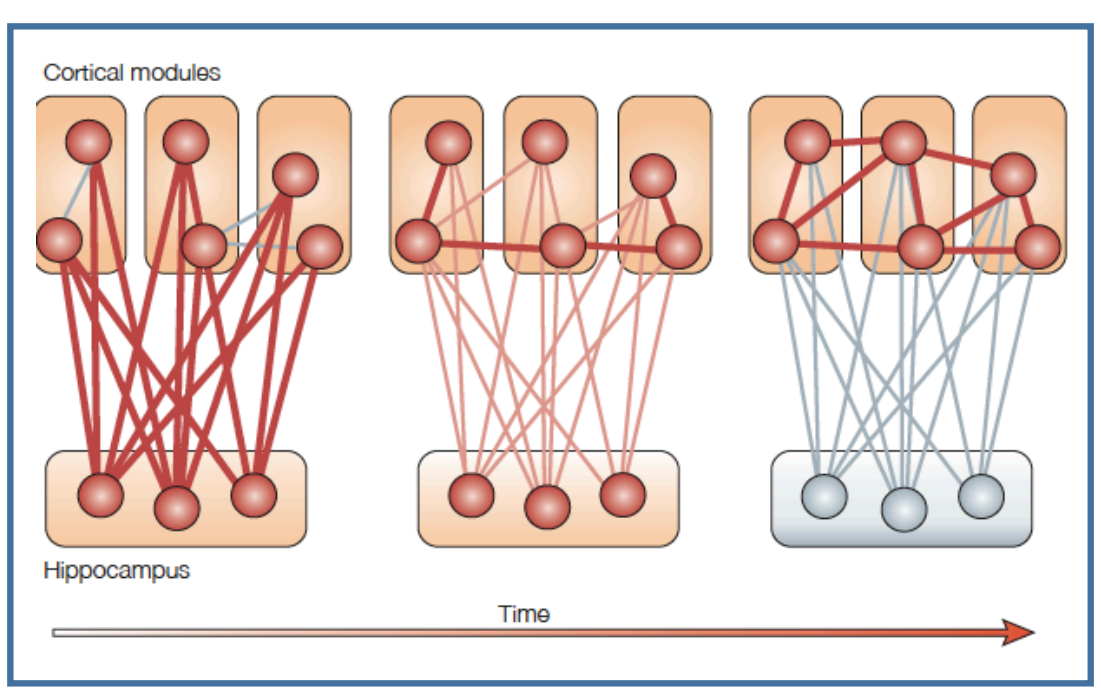

Exhibit 3.3 Standard Model for the Formation of Long-Term Memory (Frankland and Bontempi 2005) consolidation memory within the cortex and the transient role of the hippocampus.

Recent research (Kitamura, Ogawa et al. 2017) has thrown one aspect of the standard model of memory into doubt but also given strong empirical support for a multi-threaded model of memory first proposed by Nadel and Moscovitch (1997). The multi-threaded model proceeds as follows:

1. Sensory stimuli when attended to are processed within the hippocampus and synaptic consolidation occurs.

2. As memory consolidation proceeds through the hippocampus - cortex network engrams are created within different areas of the cortex upon which the prefrontal cortex can then draw through conscious recall. 
3. As connections mature within the cortex, the role of the hippocampus in coordination of memory traces fades and direct interaction between the prefrontal cortex and memories located in other parts of cortex can then occur.

This multiple trace theory where engrams are coordinated through the hippocampus has grown in significance (Frankland and Bontempi, 2005) as empirical data supporting the distributed nature of memories across the cerebral cortex were forthcoming effectively undermining the simple short-term (hippocampus) and long-term (cortex) structure of the standard model.

In their study, (Kitamura, Ogawa et al. 2017) showed that the hippocampus is involved in the creation of two independent processes of memory formation - the creation of a short-lived memory of the experience and a progressive, distributed longer term memory structure. Their study employed novel optogenetic techniques on mice where 'biochemical' markers are introduced into the brain and then traced using blue light as learning events are initiated.

If there is a strong emotional correlate involved, a memory of the emotion is created alongside in the amygdala (Hermans, Battaglia et al. 2014) (McGaugh, 2018). As these memory traces are created synaptic pathways are formed between the hippocampus/amygdala to multiple areas across the cortex (vision predominantly to the occipital lobe, sound to the primary auditory cortex, semantic content to the frontal and temporal cortex and so on).

Conscious recollection initially occurs between the prefrontal cortex and the hippocampus/amygdala system whilst the engrams forming in other parts of the cortex mature over a period of up to three days, becoming more permanent with a potential to last a lifetime. Progressively, the original memory traces, given the hippocampus's high synaptic plasticity, fade away - the short-term memory disappears to be replaced by the now maturing distributed memory across the different regions of the cortex. However, the emotional engram generated by the amygdala does not fade and hence the episodic memory and the emotional memory remain attached.

To motivate understanding of the issues involved consider the photograph of a lone male lion captured in Kenya: 


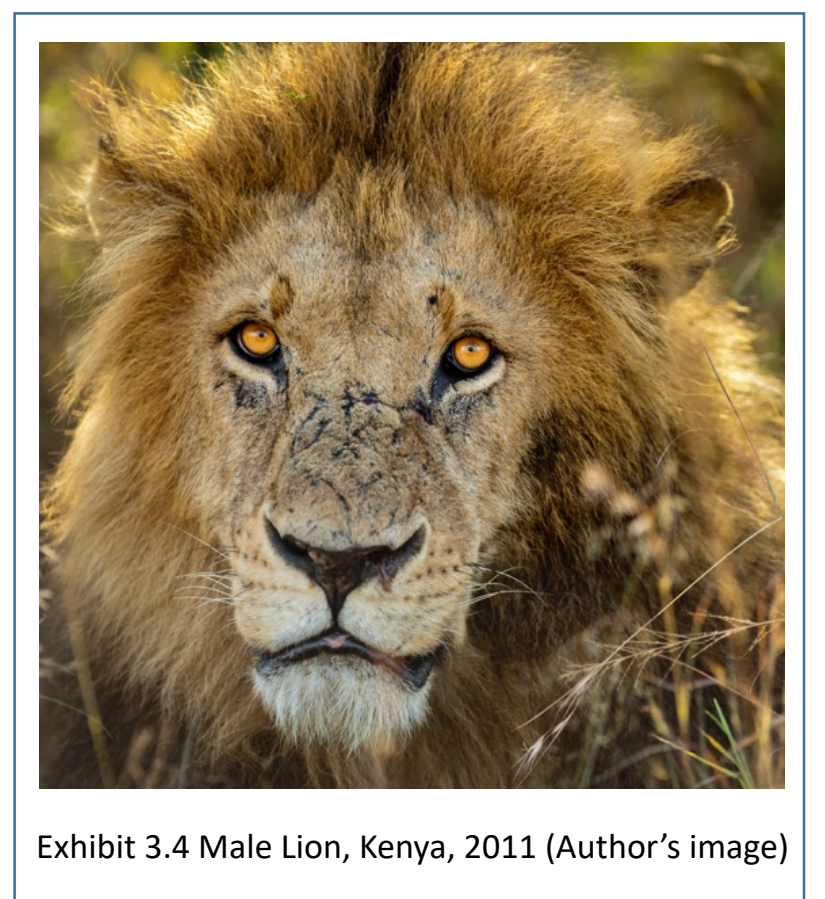

Sitting in the front of an open safari truck we had crossed open grassland and swept around an area of small trees and bush and came upon a lion sitting just in the edge of the scrub. It was early morning just after sun-up and the lion had clearly been sleeping. The driver came to a halt about 8 metres from the lion and well within its circle of fear. There is a myth that lions will not attack people in vehicles if provoked; they do, and they will. We were told by the Ranger in the front to be quiet. However, a photographer behind us, overcome with excitement, launched herself along her bench seat to get a better look pushing the long lens of her camera outside the perimeter of the truck. The lion came off its haunches with a loud, stomach churning roar. . after a moment with us frozen with fear, it settled back, and I took the shot.

The explanation of the process of memory creation in this example has significance for image making in many ways. The experience as it rapidly unfolded was transmitted into my hippocampus, stimulating the release of adrenalin from the pituitary gland creating memory of the event in the amygdala. The hippocampus, responding to the visual experience, began the slow process of forming memory traces across the cortex of the brain. Simultaneously, given my strong emotional arousal (the fear of being eaten), the prefrontal cortex was bringing forwards from my nonconscious the various memories required to handle the photographic issues to best advantage. Driving away, I could recall the event with the vivid visual and emotional load of the episode just experienced.

By the time the day's shoot was over, the hippocampus had been devoted to the task of creating the memories of a wide variety of other experiences whilst the original engrams generated through the hippocampal-cortical links were not yet mature enough to form a coherent memory of the episode, although the amygdala would contain the engrams of the emotional experience. 
Days later, when post-processing the image, what I constructed was not the original perception - that was gone - but a reconstruction drawing upon multiple engrams distributed widely across my cerebral cortex: visual episodic; visual semantic, auditory, motor, interoceptive and emotional. This has the implication that when this event was recounted around the camp-fire that evening, what is recovered is not the original short-term, veridical memory but rather a conscious and partial retrieval of an earlier version of the maturing longterm memory of the event. What is also significant is that as the story is repeated the nature of the long-term memory is transformed and accumulates semantic content in the consolidation process.

There is evidence that in this conscious retelling peripheral detail is lost and only the 'gist' is recoverable (Adolphs, Cahill et al. 1997); however, under the appropriate emotional cues the peripheral detail can also be recovered. This is an important finding because even though the capture of an image can be recalled the evidence suggests that peripheral details, such as the photographic judgements made in taking the shot, will not be available for conscious recall. The photographic implications are this: at capture, the high emotional charge will activate nonconsciously accessible skills and permit very rapid and complex judgements to be made. Later, in post-processing what is recalled is not the original veridical memory, but a reconstruction integrated, at least in part, with other associated semantic and procedural encodings but, given the attenuated emotional load, devoid of the nuanced details of the event. However, playing the sound of a lion roaring whilst post-processing might be a significantly powerful emotional cue to release those peripheral details back into conscious awareness.

\subsection{Emotion and Memory}

The research on the impact of emotion on acquisition of memory, consolidation and recall is substantial and extensive. The range and variety of emotional expression goes back to Darwin's early work (Darwin, 1872). Darwin drew finely observed parallels between the emotional responses of animals and those of humans, observing, as he did, facial expressions and other behavioural indicators of their underlying emotional states. The issue that Darwin could not deal with is the way that emotion impacts upon the process of encoding, 
consolidating and reactivating memories. Nor could he address the impact that emotion has upon the nonconscious processes of the brain and, in particular upon a mode of thinking we refer to as 'intuition', which, as we shall see in a later section, is driven by emotion. Subsequent research into emotion recognised two important dimensions to emotional activation: arousal (ranging from high to low psychosomatic disturbance) and valence (ranging from misery to pleasure) - see Russell's (1980) circumplex model (Exhibit 3.5).

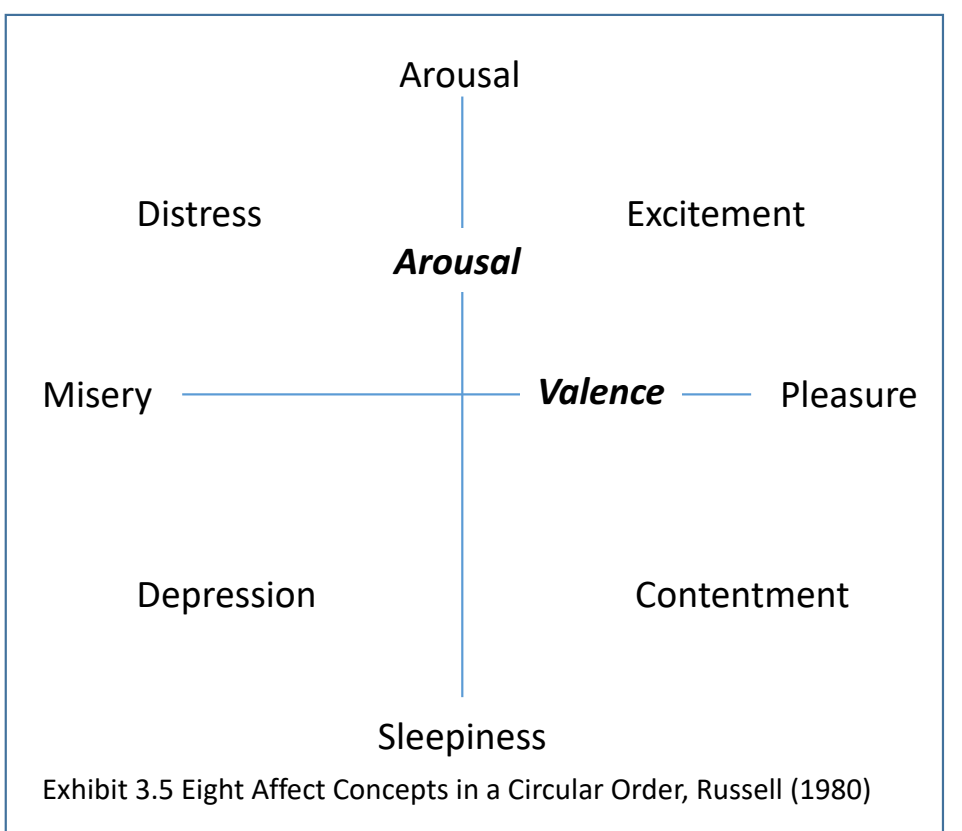

Since the $1950 \mathrm{~s}$ it has been recognised that stress leads to 'memory narrowing' where encoding and consolidation become more focused on the salient aspects of a given episode (Callaway and Dembo, 1958). Much of the research using animals has focused on fear as the driving emotion - however, other more complex emotional responses have been evoked in human studies and similar results obtained. In an early study, Cahill and McGaugh (1995) undertook laboratory studies presenting subjects with a common set of slide-based stories but attaching different degrees of emotional arousal to each. Although contestable methodologically, the results strongly indicated that the clarity and quality of recall was directly associated with arousal during the encoding stage. There is substantial evidence that arousal enhances the rate of learning and this applies to the creation of both explicit and implicit memories. The focus of research then turned to the issue of what was remembered under emotional arousal. Was it just the 'gist' of an episode, for example, that was remembered or were peripheral events remembered as well? Some studies (Adolphs, Cahill et al. 1997) concluded that it is the 'gist' that is remembered, and more peripheral detail is lost.

However, the research was highly contradictory, with one study suggesting the peripheral information was lost and others finding the opposite. Mather and Sutherland (2011) attempted to resolve this by invoking what they referred to as 'arousal-biased competition' 
or an $A B C$ theory of arousal and memory. What they suggest is that, because of the limited processing capacity of the brain, there is a 'crowding out effect' whereby certain stimuli are prioritised and others not. They identify five influencers that shape the priority with which episodes are captured and retained: 'bottom up' where highly contrasting elements are remembered and low contrasting elements discarded; 'top down', where significant goals lead to prioritising goal-relevant stimuli over others; 'surprise', where the unexpected nature of an event forces the individual's attention; 'emotional relevance', where emotional stimuli stand out against a neutral background; and finally, 'social relevance'. The evidence for the latter suggests why, for example, we tend to fixate on eyes more than would be warranted by their contrast or by their emotional relevance. Eyes carry more relevant information and we have become particularly adept at reading the signals within the eyes of a subject (Birmingham, Bischof et al. 2008).

Recent work has focused on the neurological impact of emotion and, in particular, the way that three key hormones serotonin, dopamine and adrenaline, act either singly or in combination to generate the basic eight types of emotion. What this research suggests is that under different types of sensory stimuli, the three hormones are titrated from the surrounding glands into the amygdala, creating a series of responses. Excess dopamine leads us out on an emotional vector towards fear and terror, excess serotonin towards contempt and disgust but when balanced with dopamine towards joy. The third, nor-adrenaline, leads towards distress and anguish but depending on how it is balanced with the other two can lead to anger, excitement or surprise.

These three hormones generate a 3-D model of emotion, subsuming the concept of arousal, which depends on the strength of the hormonal response and valence depending on how the three are balanced. Conventional learning and recollection tests under emotional stress suggested that in any given learning episode the 'gist' is remembered but conscious recall of peripheral detail is lost. There is evidence that emotional arousal does make the recovery of memories more vivid, although early evidence suggested that the accuracy of explicit recovery is impaired (Kensinger, Garoff-Eaton et al. 2007) and (Rimmele, Davachi et al. 2011). However, these studies must be re-examined in the light of new neuropsychological evidence. 
In high arousal situations, the process of memory formation works at two levels - vivid memories are readily recalled, although what we recall is a consolidated version of the original episode as the intensity of the moment is rehearsed, replayed and re-remembered. Peripheral detail is not processed nor recalled in that way and becomes subsumed with similar memories (Sekeres, Bonasia et al. 2016). The story of the lion above reflects that nicely: the memory of the event is vivid and encoded in long-term memory following subsequent re-rehearsal of the event as it is contextualised. We remember the surprise of the encounter, we add a semantic element to the circle of fear, we imply a motive to the photographer who produced the reaction in the animal. But what of details such as the camera settings dialled into the photographer's camera, the selection of the closest eye as the chosen point of interest, the selection of correct focus, aperture, depth of field and so on? It is these peripheral memory traces and the way in which they are consolidated that form a major theme of this research.

As we survey the literature on the impact of emotion on the vividness and persistence of memory (Ochsner, 2000), (LaBar and Cabeza, 2006), (Phelps and Sharot, 2008) the process of emotional activation has not been clear, although there has been considerable discussion on how the impact is manifest. There are a number of hypotheses suggesting how the process of emotional activation might work (McGaugh 2006):

(1) The emotional arousal focuses attention during the encoding phase;

(2) Arousal enhances rehearsal with the construction of multiple engrams;

(3) The perseverance and consolidation of memory is enhanced by the release of adrenal hormones that 'modulate' the consolidation of recent experiences (Muller and Pilzecker, 1900). 


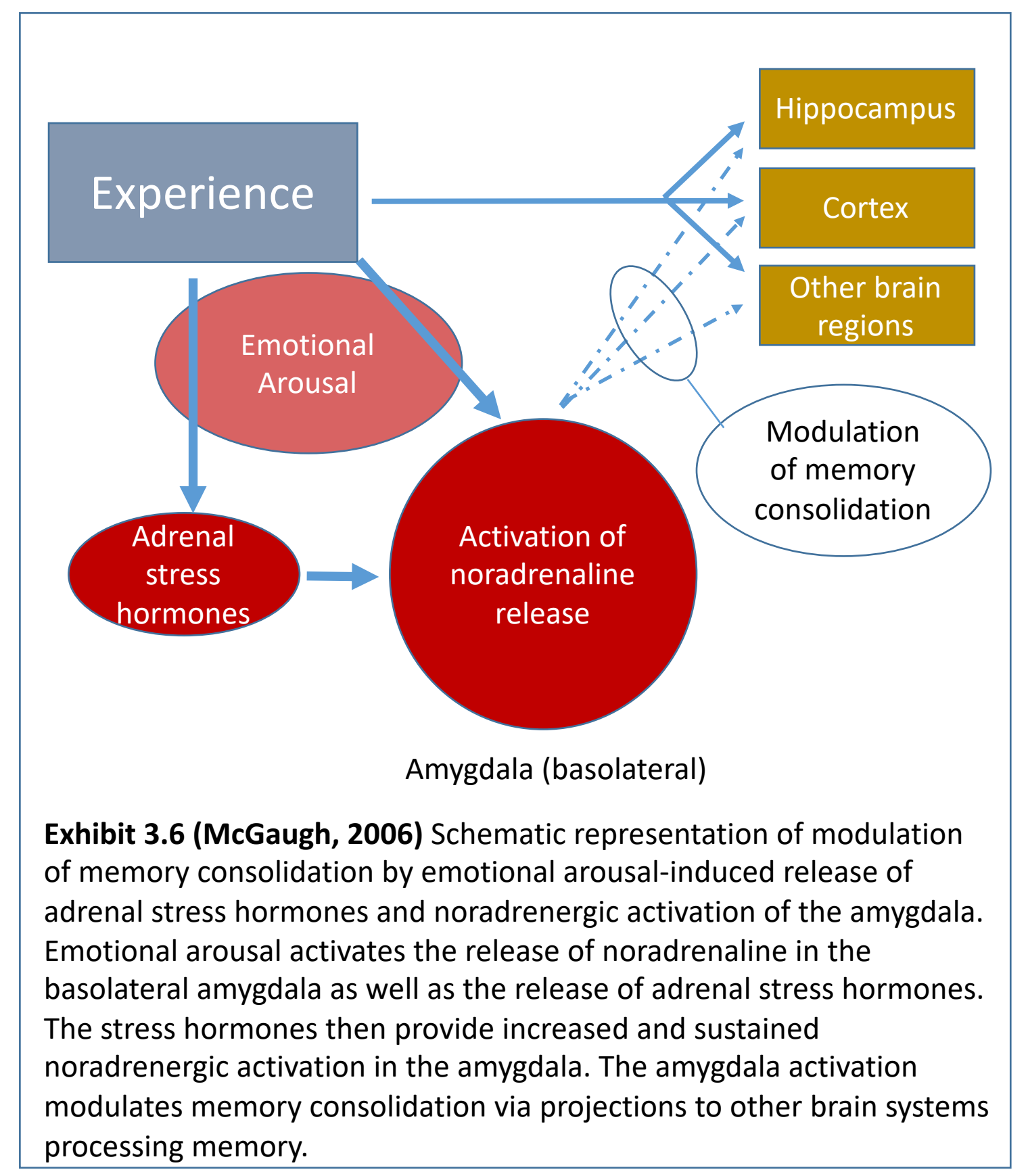

Subsequent research has provided strong evidence that the Muller and Pilzecker's conjecture is correct, and - what is of importance for this research - it is not only memory with an emotional correlate that is more vividly encoded and consolidated but also more neutral events prior to and following the event concerned. McGaugh, op. cit., describes the basic process as shown in Exhibit 3.6, much of which has been confirmed by follow-up studies using brain imaging techniques.

In one recent study Tambini, Rimmele et al. (2017) subjected individuals to a wide range of neutral and emotionally arousing images using fMRI scanning to examine arousal in the 
amygdala/hippocampus complex and then followed up with subsequent recall tests between 9 and 33 minutes later.

What is clear from this study is that both antecedent and subsequent neutral experiences are more vividly remembered than would be the case without that arousal. This gives us a clue as to how peripheral skills, necessary for the execution of the photographer's art, are retained, consolidated and subsequently reactivated. In the approach to any photographic moment, the photographer is required to make a wide variety of judgements ranging from the technical to the procedural. Where the moment of capture carries a high emotional charge, then the evidence suggests that even the more neutral judgements prior to the event will be reinforced and become 'emotionally tagged'.

The ability of the amygdala to modulate emotionally dependent information into enhanced memories as a function of their emotional significance by strengthening neuroplasticity in other brain regions is what we refer to as Emotional Tagging. (Richter-Levin and Akirav, 2003, p. 248)

Likewise, any reflective act or rehearsal following the event will also be more strongly encoded than would otherwise be the case. In a broadly-based review article, LaLumiere, McGaugh et al. (2017) review the neurological processes in memory formation and the role of emotional activation. They conclude that amygdala-based memory modulation is involved in a broad spread of learning activities, interacting across multiple brain regions and functions altering not only the encoding but also the consolidation of those memories.

From this there is support within the literature for the idea that affective tagging of otherwise neutral events will stimulate learning at the point of image capture and before and after. The final step in our discussion is the extent to which emotionally potent cues can activate spontaneous recall of events but also the peripheral learning that surrounds those events. There has been much work done in this area, particularly in the diagnosis and treatment of post-traumatic stress disorder (PTSD). Of particular interest is a review by Bergado, Lucas et al. (2011) that brings together the evidence supporting the emotional tagging hypothesis, but also discusses the variety of complicating factors that may influence the observed behavioural response: 
The literature reviewed here supports (the emotional tagging hypothesis) but points also to additional factors...such as the intensity of the emotional experience, its duration, controllability over the stressful events, age of exposure to the event and the relations between the emotional aspects of the experience and the event-to-be-remembered. These factors do not only affect the behavioural outcome of the stressful experience but also find their expression in variations in the neuronal and biochemical pathways that are activated, and in the way those will interact with memory formation mechanisms in brain regions like the hippocampus. (p.64)

\subsection{The Impact of Nonconscious Mental Activity}

Much of what we regard as conscious mental effort takes place within the dorsolateral prefrontal cortex - a relatively small area of the cortex situated above the left eye. It is here that synaptic plasticity is at its most volatile as neural connections are made and unmade across the brain. This type of mental activity is intensely effortful as different memories are tried and tested as choices are evaluated and decisions made. This area of mental functioning is richly networked to the limbic region where both short-term memories originate and the emotional and regulatory functions of the brain are concentrated in the hippocampus, amygdala and thalamus respectively.

(Eagleman 2016) in his book supporting the BBC series 'The Brain' summarises the state of current knowledge about reinforcement as follows:

Throughout our lives, our brains rewrite themselves to build dedicated circuitry for the missions we practice...It can solve the problem of complex movement using such little energy by wiring dedicated circuitry into the hardware...once etched into the circuitry of the brain these skills can be run without thinking - without conscious effort - this frees up resources allowing me consciously to attend to and absorb, other tasks. (p. 89)

To summarise the position so far, advances in the neurobiology of memory leads to the following conclusions. First, there is likely to be a significant difference between what is perceived and what is attended to and captured by the brain. Second, the initial short-term memory of, principally, a visual event is likely to be more episodic that semantic. Third, intense emotional arousal will enhance the vividness of the memory resulting in the retention of a wider range of both central and peripheral details than would be the case under 
emotionally neutral conditions. Fourth, the hippocampus, amygdala system interacts with the prefrontal cortex (PFC) to permit immediate and vivid recall. Fifth, a process of long-term memory formation will commence at the point of capture with engrams forming and maturing across the cortex. This engram formation develops out of the hippocampus and is not driven by the PFC and is not under conscious control. These engrams will consolidate as long-term memory as the immediate memory in the hippocampus fades.

One issue of importance to us now is how the vivid memories of an event with high emotional load might differ from other details that might be important in the development of expertise. Donald Hebb's work (1949) is rightly regarded as a classic and a forerunner of much of the modern work in neuropsychology. Hebb confronted the problem that much of the internal activity of the brain could not be explained in simple behavioural terms. The concept of stimulus-response (S-R) and Pavlovian conditioning was correct up to a point but could not explain the ability of the brain to intervene in the simple S-R link, nor the fact that the electrical activity within the brain was in a state of continuous flux. The brain does not respond simply under stimulus, it can create its own internal stimuli by shaping and reshaping what it knows. Hebb understood the plasticity of neural activity and how memories can be shaped and reformed through time as they are reinforced and rehearsed. His theory of Hebbian learning can be stated as follows:

'When an axon of cell $A$ is near enough to excite cell $B$ and repeatedly or persistently takes part in firing it, some growth process or metabolic change takes place in one or both cells such that A's efficiency, as one of the cells firing $B$, is increased' (p.89)

This has been paraphrased into: 'neurons that fire together, wire together' (Keysers and Gazzola 2014). This notion that as memories are built up the axons that connect them (a) become more predictable in the way they operate and (b) become consolidated into an aggregated structure, ultimately enhancing their response. As single stimuli, the elements of the consolidation process are no longer accessible to individual recall - indeed, any attempt to activate them through conscious effort will lead to haphazard and uncoordinated outcomes. Nonconsciously they are activated and fire in unison, extending our mental capabilities well beyond the capacity of rational thought.

In summary, the process of learning a new skill is enhanced by three broad mechanisms: 
(i) Reinforcement of the 'synaptic strength' between neurons firing in the engrams generated by the learning process (see Kahn, Mattar et al. 2017).

(ii) The creation of multiple synapses as the learning process is repeated enhancing the synchronicity with which they fire in response to external or internal stimuli.

(iii) The progressive myelination forming an insulating barrier around multiple axons sheaths McKenzie, Ohayon et al. (2014) and Valumian and Samoilova (2014).

The neurology of the process pointed to by Hebb is still a matter of debate, but the concept of myelination has gathered empirical support. Certainly, there is strong evidence that damage to myelin can cause severe impairment to the functioning of the brain and to the operation of memory (Baltan, Carmichael et al. 2014). There is also evidence that skill development increases the mass of white matter devoted to area of the brain implicated in that particular function (Bengtsson, Nagy et al. 2005). This conclusion has been further tested in an intriguing experiment (Kahn, Mattar et al. 2017). In their study, experimental participants were given, over a six-week training period, a simple visuo-motor task to practice and the variation in the connectivity and density of tracks within the white matter of the brain was observed through a sequence of MRI scans. They concluded:

We observed that increased white matter connectivity linking early visual (but not motor) regions was associated with a faster learning rate. Moreover, we observed that the strength of multi-edge paths between motor and visual modules was also correlated with learning rate, supporting the role of polysynaptic connections in successful skill acquisition. (p. 2.1)

Similar relationship between skill acquisition and the size and plasticity of grey matter has also been observed in the visuo-motor area using juggling and MRI scanning techniques to isolate the impact of repetitive training (Sampaio-Baptista, Scholz et al. 2014).

This research leads to the conclusion that as learning events are repeated - indeed 'overlearnt' - consolidation occurs at the neurological level with sequences of neurons forming together to create 'networked clusters'. However, no matter the exact mechanics of the process, the consolidation of skill and knowledge into expertise is a process that is enhanced by emotional activation and by repetition. Through the former, memories are made more vivid but, just as importantly, peripheral information is also absorbed both prior to, and subsequently to, the learning episode concerned. The powerful effect of emotional activation 
in learning is well established and there is a growing body of evidence that reinforcement learning may be enhanced through the reward of music that the subject finds pleasurable (Salimpoor, Benovoy et al. 2009).

The importance of much of the research looking at cerebral plasticity and learning is that the experiments examine skills that are not under conscious control. The skills used in research are necessarily simple to ensure that imaging techniques will pick up the subtle changes in the microstructure of the brain that result. However, they do reveal that repetitive and focused practice does, as Eagleman (2016) claims, 'burn-in' the knowledge and skills into the structure of the brain - altering its physical structure - and making them accessible to nonconscious activation as and when necessary. There is also sufficient evidence to suggest that Hebbian Learning is enhanced by emotional activation and that similar effects can be achieved by learning supported by music. The question that we now turn to is how such burnt-in knowledge and skills are activated where the evidence suggests that the PFC is 'switched off' by emotional activation.

\subsection{Intuition}

Intuitive processes operate autonomously and automatically, that is, they function without conscious control and cannot easily be accessed by introspection. Moreover, they can process multiple pieces of information in parallel. Analytic processes, in contrast, are performed step-by-step. The sequence and direction of these processes can be deliberatively controlled, and the actor is consciously aware of performing these processes. (Betsch and Glöckner, 2010 p.280)

The idea that intuition is a distinctive modality of thought, with its own mode of encoding, consolidating and recalling experiences, has been subject to much research. The Oxford English Dictionary defines intuition as an ability to understand or know something based upon feelings rather than facts. Betsch (2014) defines intuition as:

'... a process of thinking' where...the input to this process is mostly provided by knowledge stored in long term memory that has been primarily acquired via associative learning. The input is processed automatically and without conscious awareness. The output of the process is a feeling that can serve as a basis for judgment and decision. (p. 4) 
The idea that mental processing falls into two or more distinctive types has a long history in psychology and in philosophy since Plato, which is usefully summarised by Frankish and Evans (2009). Freud, developed the idea of the unconscious, which he regarded as a dynamic entity shaped by the traumas of childhood and ever ready to burst out, overwhelming the conscious mind, causing a wide range of mental disorders or, to use his term: 'hysterias'. His approach has been highly influential but in recent decades has been much criticised. The notion of the 'unconscious' with its connotations of subordination and subversion to the psyche has now been replaced by a dichotomy between conscious and nonconscious functioning.

Throughout all the major developments in psychology - behavioural, Neo-Freudian, Gestalt and cognitive - the idea of different levels of mental activity has been present in some shape or form, but it is only in the latter that what we now term 'dual processing theories' have emerged in testable form.

We will not review the voluminous literature supporting the development of dual processing theories as they are well covered in Frankish and Evans (op. cit.). However, it was the work of Epstein (Epstein, 1994, Epstein, 2003, Epstein, 2010) that brought together the insights of Freud and those of the developing schools of cognitive psychology to create dual processing theory in its current form. Most significantly, Epstein recognised the significance of emotion and its implication in what he referred to as the 'experiential' system.

The experiential system was largely a function of nonconscious learning (implicit), created by association and enforced by affective pressure. It stood in opposition to a 'rational system' based upon logic and conscious appraisal that is based upon 'explicit learning'. The experiential Epstein regarded as more 'passive', we are seized by our emotions, and the knowledge generated by the nonconscious is spontaneous and effortless. It is not, he proposed, as malleable and adaptable to context as rational thinking, which is both highly integrated and able to transcend contexts and make inferences in the absence of direct experience. The cost of this hugely powerful thinking tool is the effort and slowness by which it operates. In evolutionary terms, it is the thinking process that enables us to track and bring down a Sabre-tooth tiger, it is not so effective when we are confronted with one on the track in front of us. Epstein's (op. cit.) distinction between the experiential and the rational modes of thought has been styled by Stanovich (1999) as System 1 and System 2 thinking. 
There have been some attempts to place timings by which the two modes of thinking are engaged. Much depends upon the degree of consolidation of the supporting memory structures but $300 \mu$ s is one empirically supported estimate of the speed with which an intuitive judgement coheres following sensation and its encoding within the hippocampus (Bolte and Goschke 2005). This study suggests that two seconds from visual stimulus judgement is clearly intuitive in nature and the time is too short for a conscious deliberative response to occur. It is these reaction-timing differences that confer enormous evolutionary advantage

There has been a long and critical literature debating the merits of dual processing theory and extensive rebuttal (Evans and Stanovich, 2013). There appears to be little argument that there is a distinctive intuitive modality that has many defining characteristics for which individuals exhibit different abilities and preferences. However, the neurological evidence on intuition has only recently started to emerge. The two modalities, System 1 and System 2, appear to employ similarly located memory structures in different ways (Mega, Gigerenzer et al. 2015). Using face recognition studies and fMRI scanning techniques Mega, Gigerenzer et al (op. cit.) find themselves in alignment with the views of Baumeister and Bargh, (2014): 'Whatever we may have thought and seemed to say in the past, at present we both think that most human behaviour comes from a blend of conscious and unconscious processes working together to meet the person's critical needs and facilitate important goal pursuits' (p.46). They go onto conclude:

We use the term nonconscious to refer to the observation that people are not, or only partly aware of the cognitive processes underlying their judgment, in the sense that they cannot report on them. For instance, they cannot report on the cues they are using or on the way in which these cues are processed...people - when engaged in intuitive processing - act based on a strong "feeling" without being able to consciously reason about the origin of this feeling or the cognitive basis underlying their judgement...In other words, the nonconscious processing takes effect through consciously experienced signals such as "feeling of rightness" or "feeling of (processing) fluency" which are colloquially denoted as gut feelings.' (p.46)

However, although similar memory structures may be employed, there is good neurological evidence that the intuitive processing of both visual and semantic information is differently 
located. The left orbital frontal cortex shows clear activation when intuitive judgements are cued (Horr, Braun et al. 2014, Horr, Braun et al. 2015). This region of the brain is highly connected across the cortex and with the limbic system. It is also able to form and reform connections rapidly. The authors conjecture that when cued this part of the brain draws upon widely dispersed engrams and seeks to balance them until coherence is achieved. They do not provide any depth on how this coherence is achieved but they go on to suggest that there is then a process of on-going mediation with the prefrontal cortex as the conscious mind is activated by the 'feeling' generated by the intuitive or 'gut' reaction to the problem.

The neurobiological consensus appears to be converging on a series of empirical propositions that are supported by evidence:

(i) Memory is a distributed phenomenon across the brain where learning events lead to the establishment of both short-term (ST) and long-term (LT) engrams that vary in their longevity and speed of maturity.

(ii) Closely associated memories become wired together and consolidated and, as they do so, they acquire both intrinsic and semantic characteristics where the episodic nature of individual learning events are lost.

(iii) Both conscious and nonconscious recall utilise the same memory structures although highly bound LT memory structures may not be readily accessible to conscious recall but can be emotionally activated.

(iv) Nonconscious recall and 'intuitive', System 1 processing is achieved through the action of the left orbital frontal cortex which offers up a 'feeling' of coherence that can then be mediated by the more deliberative processing within the prefrontal cortex.

(v) The speed of intuitive processing is a factor order faster than deliberative processing and this processing speed is enhanced when the left orbital frontal cortex relies upon intensively consolidated memories.

Given these conclusions System 1 and System 2 thinking are not mutually exclusive but work together conferring different advantages (and dysfunctions) on the individual. Where a rapid response is required, the high speed and diverse processing of the intuitive brain offers a powerful advantage. Where time is available the deliberative, conscious brain engages, 
'rationalising' the feeling and imperatives of the intuitive brain. So, in an important sense, dual processing theory is alive and well, but System 1 and System 2 are now seen as two modalities both acting on cognition in different ways (Gobet 2016). The evidence strongly suggests the following:

(vi) Optimal learning under both differs affectively and in practice.

(vii) People will form a preferential bias between pure 'deliberative' to pure 'gut'.

(viii) The choice about the deployment and the reliance placed upon the different modes of thought will be a Bayesian combination of prior preference and contingent circumstances.

There is ample anecdotal evidence that under extreme emotional pressure expert, intuitive judgement can outperform rational analysis of a problem. Daniel Khaneman in his book 'Thinking Fast and Slow' (Khaneman 2011) cites as an example the reaction of a Fire Chief who, when facing a blazing fire, suddenly ordered his team out of the stricken building moments before the floor collapsed. However, a more potent example is given in the official and personal accounts of those involved in the landing of flight 1549 on the Hudson River in January 2009.

Following a bird strike and the ensuing complete engine failure, the Captain Chesley Sullenberger managed to land his Airbus A320 on water, where all 155 passengers and crew survived. Landing an airplane on water is extraordinarily difficult and would only be attempted when no other feasible alternative is available. The intense emotional impact of the events produced the symptoms of post-traumatic stress disorder on the part of the captain, other members of the crew and passengers. The air accident investigation (NTSB 2010, Ventura 2016) noted that the captain and co-pilot did not have time to complete the three-page checklist for the emergency they confronted. The order of the task and the time available meant that the captain brought into play an expert level of nonconscious skill and judgement. Some elements of the task employed the 'gaze heuristic' (Gigerenzer and Brighton, 2009), others involved assessing the different probabilities attaching to different outcomes, others entailed diagnosing the aircraft state from disparate sensory information and others called upon Sullenberger's deep and repetitive practice of flying in different 
simulated and real situations. In the next chapter we will explore the literature on expertise and the way the brain can adapt its mode of processing in different situations.

More formal evidence suggests that intuitive judgement can equal or outperform rational analysis in certain domains of expertise (Dane, Rockmann et al. 2012). Classic experiments have confronted subjects with video displays with 'a rolling caption' of share prices and returns across the bottom of the screen. The subjects of the study were led to believe that they would be questioned only on the content of the video advertising display (Betsch, 2008). The speed of the display was designed to induce information overload and the return distributions of share price returns was varied through the experiment. The results showed that when asked questions about the share price returns, the answers showed 'perfect rank order correlation between the mean evaluation of the shares and their actual sum of returns'. The participants on questioning said that they had no intention of thinking about the shares or their returns and that they had relied purely on their gut feeling (Betsch, op. cit. p.14). For earlier studies supporting this work see: Betsch, Plessner et al. (2004).

In photography, and taking just one of the areas of judgement concerning establishing task appropriate exposure, we can recognise different types of memory and their associated learning coming into play:

(i) Procedural: manage the camera controls in 'manual' mode without hesitation converging on chosen settings quickly and without fumbling.

(ii) Declarative and procedural: adjust the aperture, shutter speed and ISO to achieve the ideal level of exposure of the image, optimising colour, contrast and noise.

(iii) Semantic: in any given lighting conditions identify the instrumental conditions necessary to achieve optimal exposure without recourse to built-in or external light metering.

(iv) Bayesian conjunct: nuance the exposure judgement with all the other photographic judgements in order to best achieve the desired intent.

One obvious point is that (iii) is not readily accessible to rational analysis but relies upon the development of 'absolute vision', which is analogous to absolute or perfect pitch in music. There is evidence from experimental work conducted at the University of Chicago (Van Hedger, Heald et al. 2015) demonstrating that absolute pitch can be taught to adults using 
methods similar to those described in chapter 4 of this thesis. What is clear is that absolute or even relative vision (the ability to make skilled and precise exposure compensation adjustments) is not algorithmic but is acquired through intensive experience and practice.

We will discuss later in chapter 4, the conjunction of different skills and knowledge required within a comprehensive structure of photographic expertise. Our concern now is the extent to which the nonconscious might be superior to the conscious in solving the same problems. Lewicki, Hill et al. (1992), reviewing the then state of knowledge summarised it as follows:

...the nonconscious information-acquisition processes are incomparably faster and structurally more so-phisticated. They allow for the development of procedural knowledge that is "unknown" to conscious awareness not merely because it has been encoded (and entered the memory system) through channels that are independent from consciousness. This knowledge is fundamentally inaccessible to the consciousness because it involves a more advanced and structurally more complex organization than could be handled by consciously controlled thinking. (p.796)

More recent evidence has been gathered supporting the power of nonconscious processing in value integration where different attributes in a decision or judgement are weighted together by salience or affectively to achieve an outcome (Usher, Russo et al. 2011). The authors of this study created four experimental situations controlling for task fatigue and performance anxiety where they were asked to make multi-attribute assessments under both constrained and unconstrained time conditions. Three interesting conclusions arise from this study that are important for the research methods employed in this research: (i) intuitive modalities lead to more successful value integration than when the participants are induced into a rational/analytical mindset; (ii) the relative underperformance of 'rational' versus 'intuitive' modalities are not attributable to task fatigue or performance anxiety; and (iii) System 1 and System 2 modalities work together to achieve successful decision outcomes. They also suggest that when making complex judgements, 'sleeping on it' is an effective way of bringing the intuition into play.

Their results and the preponderance of the literature reinforces the view that intuition is a powerful mental capability for handling complex problems and is demonstrably reliable in its outputs (see Klein, 2015). It also leads to another important conclusion: developing high 
quality intuitive judgement requires learning methods that are different to those employed in developing rational decision-making skills. With the former, successful consolidation and emotional tagging determine the consistency of the judgement process. With the latter, recall and algorithmic intensity determine the success of the decision-making process.

\subsection{The Bayesian Brain}

Early ideas about the nature of perception maintained that experience through the senses was followed by a linear process leading to a stored recording somewhere in the brain (Fechner, 1860). However, Hermann von Helmholtz (see: Frith, 2007) noted that perception appeared to be an active and predictive process rather than a passive process of absorption and reaction. Helmholtz tried a simple experiment: if he gently poked the side of his eye the world jerked from side to side. If he moved his head from side to side the world stayed where it was. This, he reasoned was because the brain could correct, in the case of moving his head from side to side, what was seen and so maintain the stability of its current 'model' of vision.

Over the twentieth century the idea that the brain does not operate context-free gained traction and became the dominant paradigm replacing the naïve, Lockean proposition that the mind is like a blank slate upon which impressions are writ. Under this view, the brain creates a working model that it updates rather than replaces as the individual attends to a new experience. This model of brain function both predicts and interdicts the act of perception (Penny, 2012).

The search for a theoretical logic to support the emerging paradigm of the active brain directing perception and redefining itself in the light of new evidence led to the concept of the Bayesian Brain. The Bayesian Brain is an extension of the statistical theorem developed by Tomas Bayes in the $18^{\text {th }}$ Century. Bayes' theorem gives us a way of handling uncertainty by combining our pre-existing beliefs with new, but uncertain information to establish optimal posterior probabilities (Doya, Ishii et al. 2006). An 'ideal Bayesian' is someone who can assess base rate probabilities accurately and then update those 'prior probabilities' using probabilistic evidence to achieve the most efficient probability of a given outcome. 
The proposition that human decision-makers or judges are ideal Bayesians was questioned by Tversky and Kahneman (1974). They proposed that people are poor natural Bayesians and are subject to a range of biases such as 'representativeness', 'anchoring' and 'availability'. From their original study a wide range of short-cut thinking methods were discovered that appeared to circumvent the complexity of working through ideal solutions to problems. They also argue that individuals are particularly weak in assessing the significance of 'base-rate' probabilities when confronted with new evidence. However, subsequent research suggests that the problem was not with the test subjects but rather with the way in which the experimental design was constructed and how information was presented to them (Hoffrage and Gigerenzer 1998), (Gill, Sabin et al. 2005) and (Hoffrage, Krauss et al. 2015). As Frith (2007) noted, it is easy for experimenters to construct tests that will defeat the most wellqualified of statisticians, and others have noted that in the heuristics and biases field an industry has arisen where psychologists devise tests that would defeat even the most able minds.

However, Bayes theorem was soon understood to have a far wider scope than the purely statistical - it appeared to reflect the way that people naturally think. The concepts of the 'Bayesian Brain' and 'Bayesian Perception' reflect this idea that we come to any new situation with sets of prior beliefs or perceptions, we then in the light of new experience modify those beliefs or perceptions to create a new understanding of the world. So, in vision research, it is now well established (see: Frith, 2007 and Eagleman, 2016 for an overview) that sight is not a continuous recording of experience but one where we adapt a pre-existing visual model as we attend to new visual experiences. What we see is a combination of what we believe we are seeing and what we experience. The eye does not see in 3D for example, the retina is a 2D optical plane. The brain learns how to appreciate depth in objects by interpreting the input from two eyes and other visual cues.

Two challenging issues arise from the Bayesian approach: first, how does the brain search for and cope with evidence that does not match its priors and, second, how are the priors formed in the first place? The concept of the predictive brain as developed in the Hierarchical Predictive Processing Model (Clark, 2013 and 2016) suggests that based on the priors held, the individual will seek evidence supporting those priors. Where the evidence does not conform, i.e. there is prediction error, the priors will be updated, and the process of 
perceptual search repeated. This loop narrows the error on each cycle until there is no discrepancy between belief and perception and occurs so quickly (Frith, op. cit. quotes 200 milliseconds) that we are not aware this has happened. The Hierarchical Predictive Processing model has gathered strong experimental support and is now regarded as the leading contender for an overarching theory of neurocognitive development (see Euler, 2018).

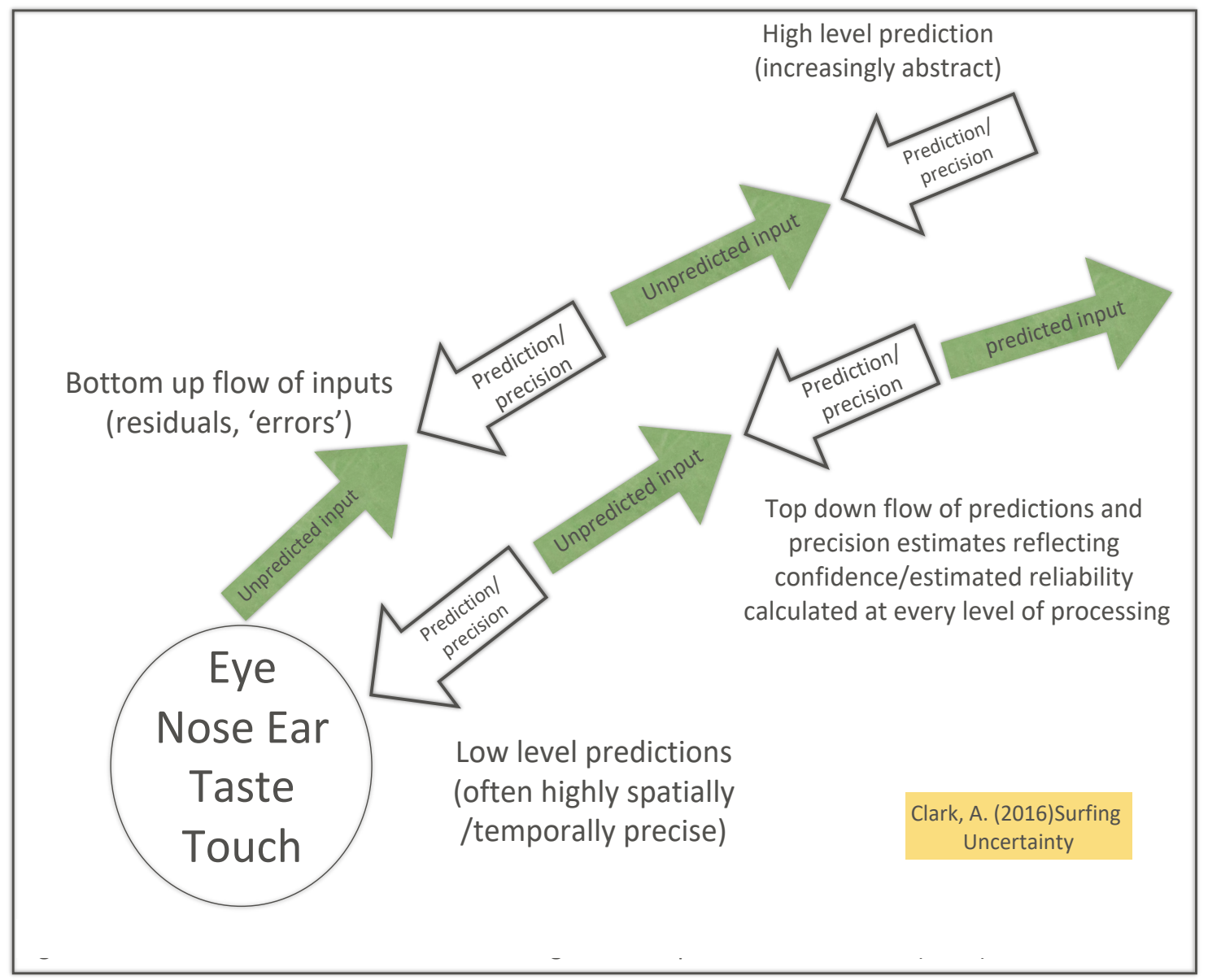

Exhibit 3.7 The Hierarchical Predictive Processing Model Reproduced from Clark (2016)

At one level, priors updated as the individual gathers the evidence of their senses leads to heavily reinforced learning, creating neural structures whose outputs, when cued, are very rapid and intense. The strength of belief in each prior will be dependent upon the strength of the reinforcement process engaged in its development. Where a complex array of expertise is called upon to deal with a given judgement task, the relative strength of reinforcement will create the weights that are nonconsciously applied in conjugating the different elements of expertise. This leads to the hypothesis that high degrees of expertise 
create highly sensitive priors in terms of their propensity to affective arousal and, secondly that those priors are likely to be more resistant to updating in the light of new evidence. This is a theme we will return to in the next chapter where we focus on the issues of expertise and its development.

Although much of what is written on the Bayesian Brain relates to perception in general the concepts have also emerged in the neural processing field with special application to human response to both static and moving visual images. In a series of studies Itti and Baldi (2009) have developed the concept of 'Bayesian Surprise', which they model as the difference between the posterior and prior distributions of the observer's beliefs. In terms of Clark's model (Exhibit 3.7), this is the 'unpredicted input' that challenges our prior beliefs.

This element of surprise works at many levels within, say, a complex image. But overall, we could also define 'surprise' as the 'impact' of an image, either as seen by the photographer or by the subsequent viewer or image analyst. The more visually literate the individual concerned, the more difficult it is to evoke surprise because, from a viewer or analyst's perspective, they have 'seen it all before'. There is evidence from saccadic and other studies that humans do tend to focus selectively on aspects of an image that (a) are of high entropy ${ }^{17}$ (Privitera and Stark, 2000), (b) strong contrast (Reinagel and Zador, 1999), (c) high personal salience (Parkhurst, Law et al. 2002), (d) novelty (Theeuwes, Kramer et al. 1998) and (e) present facial images (Guo, Mahmoodi et al. 2006). They are also attracted to motion in a moving display or as it is represented in a static image (Abrams and Christ 2003). However, Itti and Baldi (op. cit.) have taken this literature a significant step forward by providing empirical evidence that surprise 'explains best where humans look when considering all saccades, and even more so when restricting the analysis to only those saccades for which human observers tended to agree.' (p.1305)

The thrust of this research is that as the brain develops, it forms expectations about vision that it proactively seeks to predict and through prediction verify and correct, where necessary, errors in a process that has been termed the 'loopy brain' (Eagleman, 2011). As though expectations mature through the years, they become progressively consolidated into

\footnotetext{
${ }^{17}$ Entropy is a measure of the 'disorder' of a system that increases under a constant input of energy.
} 
an implicit 'model of vision' embedded within which are normative and semantic rules about what should be attended to and what not. It is the strength with which these visual 'constructs' are developed that forms the basis of the visual 'priors' in a Bayesian perceptual framework. As our discussion of the issues of memory and its consolidation indicate these priors are not readily accessible to the rational brain but drive the nonconscious processes of vision. However, as we will argue in the next chapter it is these visual priors that create the 'photographer's eye' and that, in large measure, they are beyond conscious awareness or control.

\subsection{Theoretical Model of the Cognitive Process in Photography}

Our review of the literature leads to a number of insights of research interest in photography and leads to the development of a theoretical model of the cognitive processes involved.

The conclusions from the research I would summarise as follows:

(i) Photography is a cognitive process that, just like any other cognitive process, is fundamentally Bayesian.

(ii) What the photographer or the viewer sees is a combination of perception and prior belief. Those prior beliefs will be deep and conditioned by our lifeexperiences and their degree of reinforcement.

(iii) The nature of photography lies in the 'moment' and the creative act is driven by the dynamics of the subject and the photographer's changing cognitive states. The driver is, therefore, principally intuitive, which is affective, rapid, nonconscious and constrained by the pre-existing structure of intrinsic memory which is high in semantic but low in episodic content.

(iv) Visual appreciation can be rationally driven or intuitively driven - the way they are combined is down to both conscious choices and nonconscious impulses.

(v) Nonconscious learning of photographic skill is enhanced under emotional pressure and those skills become 'tagged' and can be reactivated (cued).

(vi) Once an image is captured the memory of the scene passes through two phases: a short-term episodic phase that fades within the day and cannot be recaptured. 
A long-term phase where the episode becomes entangled with the semantic understanding of the image.

From this we can describe a simple model (see figure3.8 below) reflecting the 'bare bones' of the cognitive process in photography that commences with the formation of intent and concludes with a consolidated memory of the photographic event.

We recognise that the process commences with artistic intention. We make a choice to isolate from the world of meaning, as we understand it, a visual event. Intent precedes action and that intention will be motivated by our preferences and beliefs. The action that follows is the search for the location and the moment and when found the shutter is pressed and the image captured. Our interest is not in these stages of intention and recognition per se. Indeed, the search for the moment may be trivial or it may be traumatic or anything in between but moving towards a creative moment at any level of significance will activate both an intellectual and an emotional engagement with the problem. Indeed, the search for the moment is driven by the Bayesian processes of the brain in a conscious and nonconscious repetitive cycle of prediction, search and resolution until the point where intent and resolution converge. At the point of capture the full array of conscious choice and intuitive, nonconscious judgement is exercised and the shutter clicked. The click of the shutter works in two ways: the cameras sensor captures the image and the brain instantiates the moment. The logic of Patterson's (1977) point follows through to a secondary insight: not only does the camera look both ways, it acts two ways too.

Once the image is captured two cognitive processes commence: the formation of an episodic short-term recall that will be more or less vivid depending on the strength and nature of the emotional activation. However, the intuitive knowledge driving the photographic judgements at capture will not be accessible to recall at this stage - this short-term memory is a product of a very plastic link between hippocampus and prefrontal cortex and the channel capacity is strictly limited. It is speculative but a limited channel capacity may confer an evolutionary advantage in the very short term. Being able to think about too much when confronted with a Sabre-tooth tiger could result in getting eaten. However, be that as it may, the link is fundamentally episodic in that although memory of the event is captured across all the senses cognitive resources are not wasted in conceptualising its meaning. 
The second process commences immediately whereby the episode is progressively encoded within the neural networks across the cortex. These long-term memory networks take three days to mature in the sense that the episodic engrams are consolidated into a network of visual memories, semantic content and values along with peripheral details that did not reach surface attention but were picked up by the brain. It is during this stage that the memory of the interoceptive feelings at capture are consolidated as well as forming the cues that will enable nonconscious recovery in the future.

The final stage of this model reflects the re-engagement with the image as the photographer attempts to reconcile the inevitable differences between their artistic intent and the image through post-processing. This is the point where stylistic ability is most fully engaged.

The insights from this model support the design of the research methods developed later in chapter 5. The first examines the relationship between the intuitive and the conscious appreciation of the photographic moment and the second utilises a differential diagnostic technique examining the skills revealed by subjects processing their images at different stages in their memory cycle.

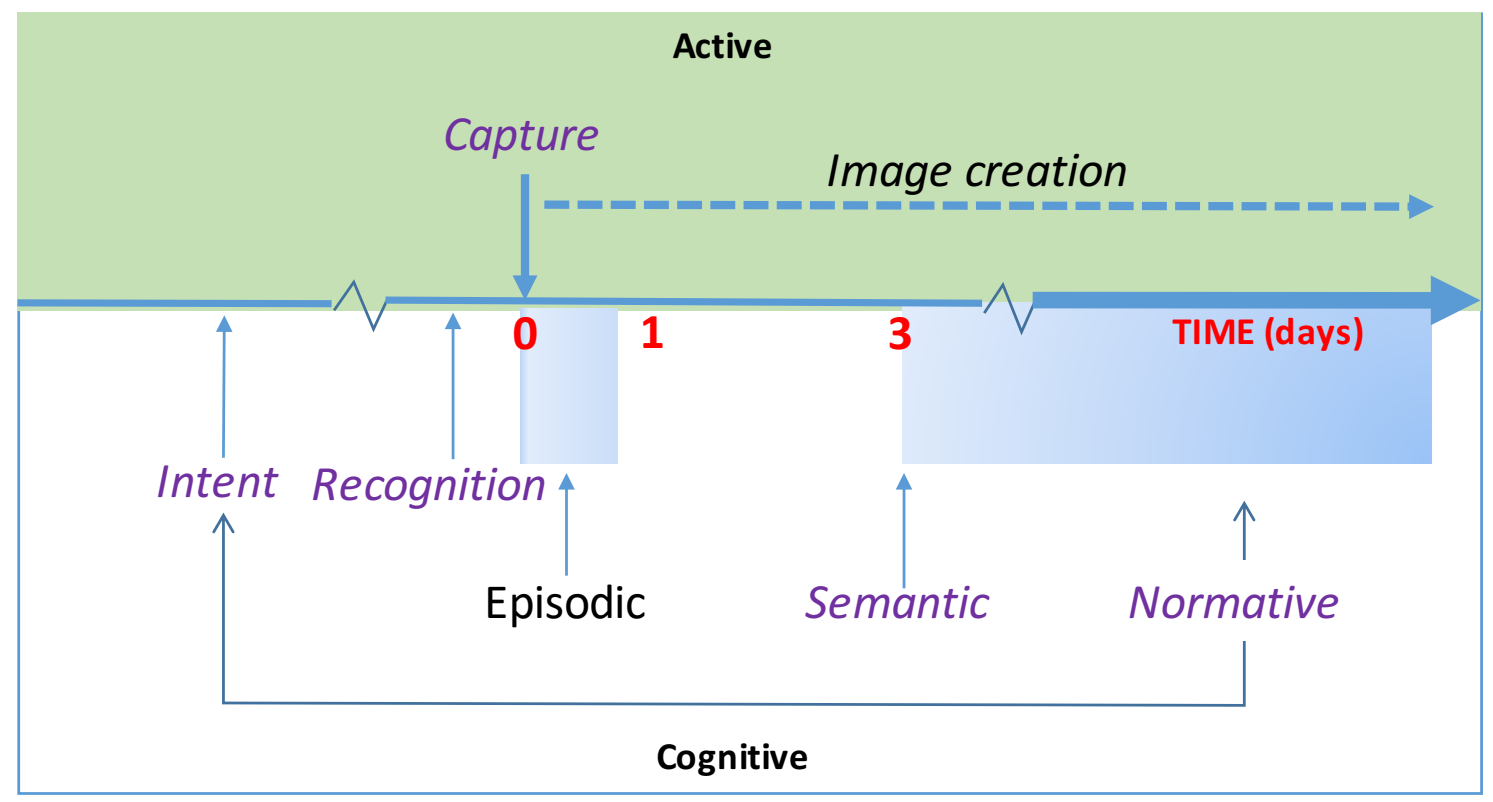

Exhibit 3.8 General Model of Photographic Image Capture and Post-processing. (Author's own).

In the next chapter, we move on from the process of the formation of memory to the problem of how it is organised. Organisation of memory is an important part of the problem of expertise and its development. By way of a taster of what is to come consider this quote by 
Gary Klein (Klein, 2015), one of the most eminent psychologists working in the area of judgement, decision making and the development of expertise:

Expertise primarily consists of tacit knowledge, rather than explicit knowledge of facts and similar forms of declarative knowledge. What differentiates the skilled decision makers....is their ability to make perceptual discriminations, to recognize patterns, to draw on rich mental models, and to judge typicality. These aspects of tacit knowledge are, by definition, tacitdifficult to articulate and often unavailable to consciousness. Judgments and decisions based on these types of tacit knowledge will, therefore, appear to be intuitive ...strengthening intuitions means building experiences that result in more accurate and comprehensive tacit knowledge. Strengthening intuition means accelerating expertise. As Kahneman and Klein (2009) stated, "a psychology of judgment and decision making that ignores intuitive skill is seriously blinkered" (p. 525) 


\section{Chapter 4 - Photographic Judgement and the Development of Expertise}

\subsection{Introduction}

In this chapter, I explore the process of memory consolidation in more depth commencing with Kant's concept of mental 'schemata'. From there I draw together the threads of the discussion to develop within a Cognitive Field framework a theoretical structure (CFT) relevant to this research. Out of CFT come several implications of direct relevance to the structure and effective acquisition of photographic expertise. This structure of expertise has broad support from the advisory output of the principal photographic bodies; its basis is Kelly's (1955) Personal Construct Theory and Chassy and Gobet's (2011) template theory, which I extend in two ways: first by proposing a cognitive capacity on the number of constructs that are meaningful to either conscious or nonconscious processing, and second, by invoking two concepts that reflect my ontology from chapter 2 and the underlying neuropsychological and cognitive-psychological processes discussed in the previous chapter: prepotency and emergence.

\subsection{The Philosophical Origin of Cognitive Psychology}

Across the sciences, practitioners in different disciplines tend to have a favourite philosopher. For psychologists and cognitive psychologists, one philosopher stands head and shoulders above the rest - Immanuel Kant (1724 - 1804).

Kant argued in his 'Critique of Pure Reason' (Kant, 1992) that there must be a link between our intuition (note here that Kant used the word 'intuition' to mean perception) and the meaning we attach to those perceptions through categorisation. 
This link between perception and categorisation was, Kant proposed, created through 'schema' or 'schemata', being a set of procedural rules or heuristics that allows us to make sense of complex perceptions and subsume those perceptions into a general category. They therefore represent a group of rules that allow us to make judgements about perceptions rapidly and below the level of consciousness. Kant's 'Critique' is obscure, to the say the least, and its meaning with respect to schema is much debated, see (Pendlebury 1955). The Bloomsbury Companion to Kant (Banham 2015) describes schema thus:

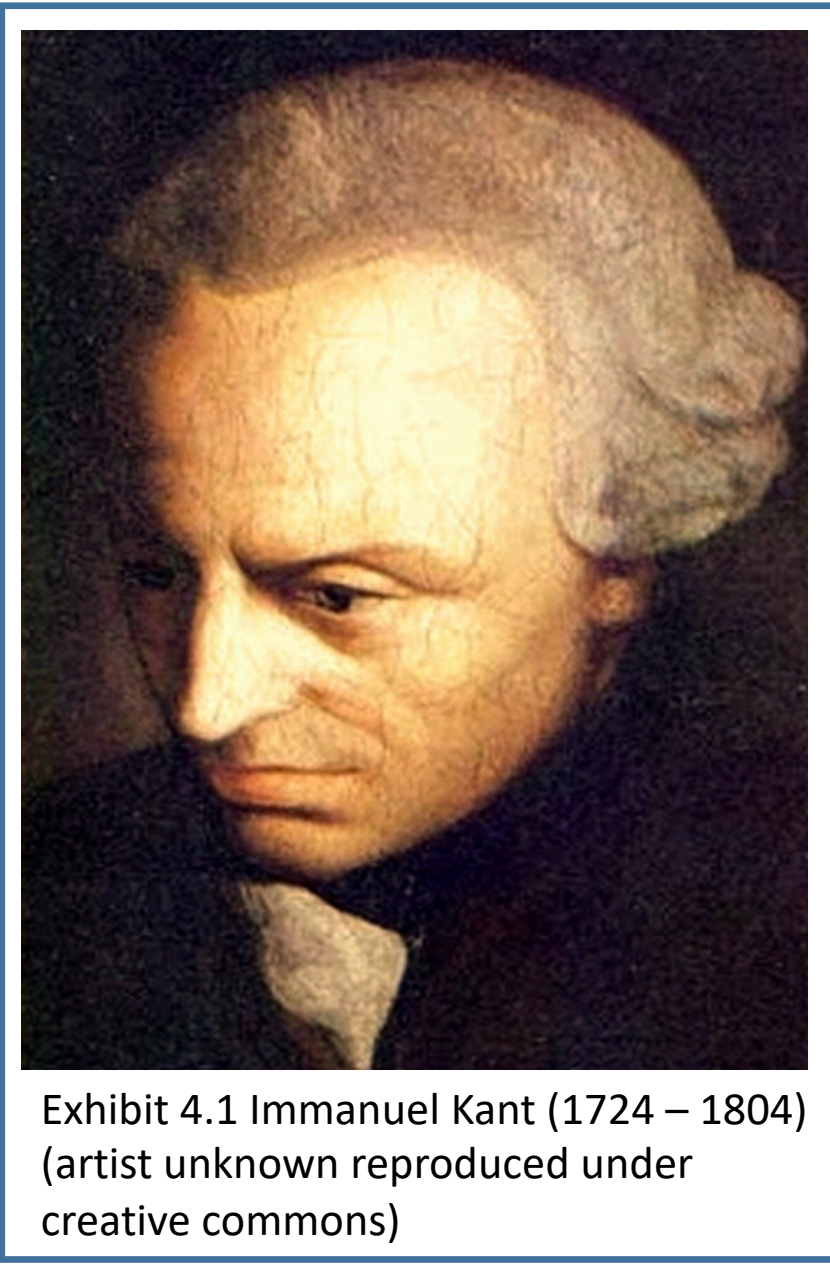

Kant's theory of concepts requires that intuitions and concepts are two different types of mental representation - that is they are heterogeneous....Kant's task....is to explain in the light of this heterogeneity, how subsumption under a concept is possible....If concepts and intuitions were ultimately a single type of mental representation as they were for his predecessors eg: Leibnitz and Hume, then there would be no apparent problem explaining how subsumption works... However when concepts and intuitions are different types of mental representation there must be some third thing that can mediate between them' (p.268)

The problem Kant left us with is that he could not describe how schema work. He says in his Critique of Pure Reason (Kant 1992) '...their mere form is a hidden art in in the depths of the human soul, whose true operations we can divine from nature and lay unveiled before our eyes only with difficulty' (B edition of CPR pp. 180-181). My conjecture here is that Kant, imbued with Christian theology in the Scholastic tradition, and in particular, the doctrine of the Trinity where God, whose love is personified by the Son must have a bridge by which that love can proceed from the Father to the Son - this being the Holy Spirit. In other words, Kant 
would have taken it as incontrovertible, that two heterogeneous concepts require a third element - a bridge - to create a relationship. This 'bridge' is, in Kant's terms, the schema that allow us to connect perception with their appropriate categories.

The other problem with Kant's understanding of the mental process of subsumption is that he believed it to be linked in a single process, whereas the evidence presented in the previous chapter is that perception involves two processes: first, its episodic nature is short term and is rapidly lost and second, it is simultaneously consolidated with a widely distributed mental construct of associated memories consisting of perceptions (original and reflected) that mature across the cortex developing semantic content as they develop. Apart from the early, largely episodic representation of perception, memory is reconstructed and a fascinating experiment by Frederic Bartlett (Wagoner, 2017) demonstrates this fact.

\subsection{The Bartlett Experiments}

In the previous chapter, we noted that initial short-term recall is highly episodic and transitory but long-term memory formation is a progressive process becoming more semantic and conceptualised as time passes. Bartlett's much replicated experiments in the 1930's (Bartlett, 1932) presaged the empirical results from neurology by 80 years.

Bartlett's interpretation of memory - based upon the Kantian concept of schemata - is often presented as opposing the Freudian view that memory represents an unalterable trace. For Freud (1900), memories leave an indelible trace on the psyche - a trace that may fade in time but is never entirely lost. However, Bartlett argued that the construction of long-term memory is a positive process whereby the original perceptions are normalised with contextual and social meaning. This process serves us well as memory takes on meaning and allows us to adapt in a rapidly changing environment. The structure by which memory becomes meaningful is through schema - a concept originating with Kant but first systematically articulated by Sir Henry Head, a clinical neurologist who met and discussed ideas with Bartlett in the 1920s (Wagoner, 2013). Bartlett's (1932) view was that memory is constructed and reconstructed through schemata, which he described as: 
'... an active organization of past reactions, or of past experiences, which must always be supposed to be operating in any well-adapted organic response. That is, whenever there is any order or regularity of behaviour, a particular response is possible only because it is related to other similar responses which have been serially organised, yet which operate, not simply as individual members coming one after another, but as a unitary mass. ... All incoming impulses of a certain kind, or mode, go together to build up an active, organised setting.' (pp. 200-201)

The role of schema in mental activity, Bartlett argued, act both at a conscious and a nonconscious level, not as passive chunks of memory but as constructions that the individual could 'turn around', separating the past from the present and self-reflectively controlling and reconstructing the schema afresh (Bartlett, 1932 p.206).

Bartlett's most famous experiment involved the telling of an obscure story drawn from the folk-tales of the middle-west of America to students at the University of Cambridge in the UK. Wagoner (2017) describes the story thus:

'... he used a Native American folk story from Boas' Kathlamet Texts (1901) called 'War of the Ghosts,' which contains many narrative disjunctions, seeming lack of logic, strange and vivid imagery, supernatural events, alongside other unfamiliar elements.' (p. 538)

His first step was to ask the participants to read the story at a normal speed twice. Fifteen minutes later he asked them to write down what they could remember of the story and then again at weekly, monthly and annual intervals. Through this process of progressive reproduction, he analysed the differences that emerged. What he observed, as the memory matured, is described by Wagoner (op. cit.) as follows:

'... (the) story ... was progressively transformed into a conventional English story: Many details dropped out, the disjunctive narrative structure was smoothed over, 'hunting seals' became the familiar activity 'fishing,' 'war cries' changed to simply 'noises,' the unfamiliar proper names ....were forgotten, and the supernatural elements were rationalized and then omitted.' (p. 539)

Bartlett's (1932) interpretation was quite specific:

Remembering is not the re-excitation of innumerable fixed, lifeless and fragmentary traces. It is an imaginative reconstruction, or construction, built out of the relation of our attitude 
towards a whole active mass of organised past reactions or experience and to a little outstanding detail which commonly appears in image or in language form. (p. 213)

Many of the early replications accepted Bartlett's interpretation and he and others developed numerous experimental variants. However, as the experimental designs became more structured, the results became more accessible to quantification and the interpretation progressively changed. Whereas the constructive process was positive for Bartlett, under the US schools of highly Positivist experimental psychology the replications only served to demonstrate the fragility and fickleness of memory. Ironically, the 'normalisation' process that Bartlett observed in the formation of long-term memory was only too apparent in the process of interpretation of the results by different researchers over time (Wagoner, 2017).

For the purposes of this research Bartlett's story is particularly relevant. The concept of schema and the active process of constructing and reconstructing memory it represents are central to the issue of expertise and its development, as we shall see later in this chapter. Second, his method of 'serial reproduction' and the analysis of differences in recall over time open the potential for the diagnosis of the involvement of schema in both photographic decision making and judgement.

\subsection{Judgement and Decision Making}

Expertise is normally associated with an ability to make 'expert decisions' and 'expert judgements. These two cognitive activities overlap to an extent, but the former relates to future choices where 'risk' dominates and the latter to circumstances characterised by 'uncertainty'. This distinction between risk and uncertainty is understood in the decision theory literature to hinge around what is 'objective' knowledge relating to the probability of future events occurring and those where only subjective assessments of future outcomes can be made. The distinction between risk and uncertainty is attributed to Frank Knight (1921) with interesting discussions by Langlois and Cosgel (1993) and Holton (2004).

Decisions and decision theory are the process and the underlying logic for making rational choices under risk and, as this suggests, is the domain of rational analysis whatever mode of rational choice is adopted. Judgement, on the other hand, is the process of making a choice 
where the underlying process is experiential and the inputs to the process are predominantly qualitative. This implies that when weighing the alternatives, the individual is driven by prior, subjective beliefs rather than objective probabilities. Given the appropriate emotional activation, the subjectivity and qualitative nature of the problem will be assessed by the intuitive brain - and where the relevant cognitive field is populated with well-formed constructs, then intuition - as noted in chapter 3 - is likely to drive a depth of insight not accessible to rational analysis. To put it this way: reason is not the driver at the wheel of creativity, that comes from the 'dark-web' of the mind or as the nineteenth experimental psychologist Wilhelm Wundt put it:

'Our mind is so fortunately equipped, that it brings us the most important bases for our thoughts without our having the least knowledge of this work of elaboration. Only the results of it become conscious. This unconscious mind is for us like an unknown being who creates and produces for us, and finally throws the ripe fruits in our lap.' (quoted from Koestler, 1964).

As we discussed in the last chapter, under emotional pressure intuition will drive the choices the individual makes. In the absence of relevant knowledge and expertise that is accessible to the intuitive mind, the individual will use whatever appears to be appropriate at the time. To bring the point into focus here is a short anecdote:

Walking down a track, if our forebears came across a Sabre-toothed tiger $^{18}$ there is no time to engage in rational analysis. The expert hunter will turn and face the animal, spread themselves as wide as possible and give a huge roar of anger. The naïve hunter will turn and run. In the former case, clusters of domain-specific expertise, developed from experience and knowledge of animal behaviour, will be activated and within 300-400 microseconds directing

\footnotetext{
${ }^{18}$ The Sabre-toothed tiger was a wide-spread species from the Eocene to the end of the Pleistocene epoch dying out about 12000 years ago.
} 


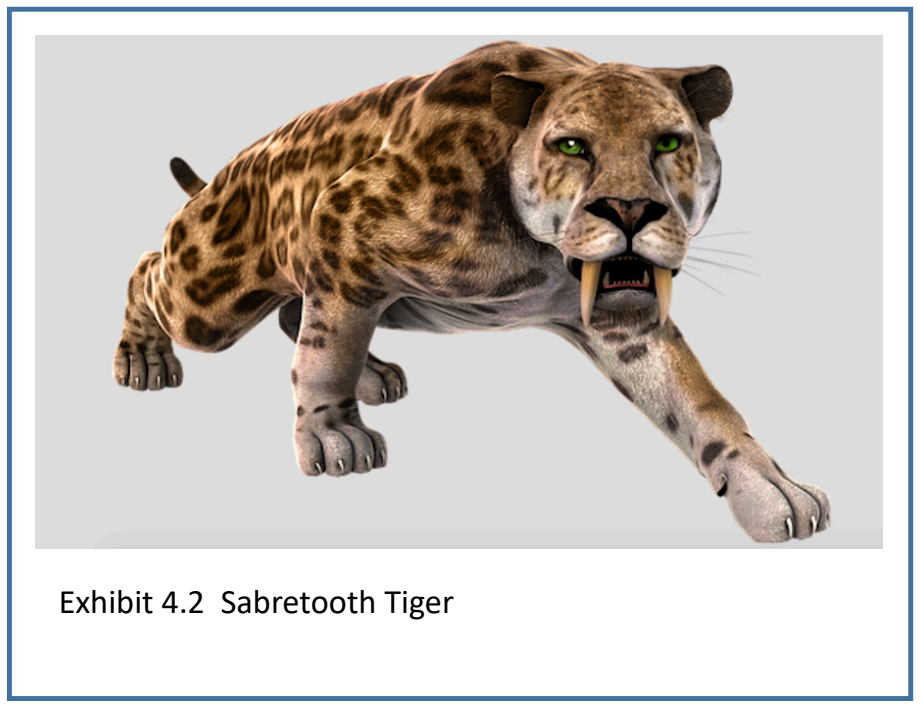

the appropriate response to the threat. The naïve hunter inexperienced in the ways of Sabretoothed tigers will find their intuition also drives their judgement. But it is intuition driven by fear and inappropriate experience. They turn and run - and in so doing become a welcome snack for the Sabre-toothed tiger.

As we reflect on this confrontation between person and animal, we can see how intuition, driven by expertise, can lead to evolutionary successful behaviour. It reacts before the conscious mind can begin to compute the risks, rationally analyse the problem, and select the best response. It may well be that the rational mind has access to tiger kill rates, probabilities of particular behaviour emerging and an assessment from the animal's condition whether it has recently fed or is actively looking for a snack. The rational mind can also make choices about the training and deployment of intuition in the future. What the rational mind does not have access to are the degrees of 'automacity' (to use Bargh and Chartrand's (1999) term), that has encoded the experience and knowledge of numerous encounters with Sabre-toothed tigers and the reflection that goes with it.

What is evident from the literature we have already discussed is that rational analysis is a scarce and effortful resource. It represents a relatively small part of human cognitive activity although its strategic functioning is very important. However, as discussed in the previous chapter, the nonconscious is the dominant motor of mental activity (see: Dresp-Langley, 2011 and Oakley and Halligan, 2017) and the driver of the choices we make and, like rational modes of thinking, it can be either trained to become function specific or not. The ability to make the type of expert judgement that allows the individual to survive an encounter with a Sabretooth tiger depends upon the successful population of the constructs with appropriate knowledge and skills to deal with the problem however it is presented. 


\subsection{Cognitive Field Theory}

"Most of what we do goes on unconsciously. It is the exception, not the rule, when thinking is conscious, but by its very nature [i.e., because we cannot experience anything else], conscious thought seems the only sort. It is not the only sort; it is the minority."

(Lachman, Lachman et al. 1979)

My first step in extending Cognitive Field Theory is to answer the question how intuition which is characterised by a nonconscious 'jump' in cognition - is a necessary outcome of the Hierarchical Bayesian Prediction (HPP) process discussed in chapter 2. From this I develop the notion of a 'construct' based upon the concepts of memory chunking, template theory and personal construct theory. I argue that an individual, when presented with a challenging perception, will utilise a hierarchically ordered 'construct field' to achieve cognitive resolution. Finally, using this theoretical and empirical architecture, I will demonstrate how a cognitive field can be constructed capturing the essence of photographic expertise. The remainder of this thesis is my attempt to demonstrate the utility and validity of that theoretical construction.

\subsubsection{The Roots of Cognitive Field Theory}

Cognitive Field Theory has its origins in the work of the Gestalt psychologist Kurt Lewin, working in the 1940s. Lewin (1939) proposed that individuals create their 'life-field', containing both their internal world of memories and thoughts, and an external environmental world of interactions and events. Behaviour, he argued, is an interaction between the cognitive and the situational environmental variables that the individual confronts. Lewin's approach placed emphasis upon the dynamic nature of the life-field. Each of the zones of cognitive expertise within the life-field are malleable and continuously changing as the individual seeks to achieve their intent by resolving internal dissonances and conflicts in their external relationships.

Lewin was highly influential in the development of Gestalt psychology and in the development of field theory within the social sciences. As the individual interacts with the environment, a 'boundary zone experience', as he referred to it, occurs. The problem with Lewin's conceptual 
structure is that it does not take us very much further forward in understanding how goalseeking behaviour can be reinforced and made more coherent and internally consistent.

Undoubtedly, Lewin had a great influence on the development of theories of learning and the thinking of David Kolb - for a recent summary see (Kolb and Kolb, 2009). Kolb, pioneered the concepts and practice of experiential learning which emphasised the importance of commitment to the process, engagement with experience, reflection on the experience and conceptualisation. Others have developed this into 'action learning' and the five-question model of (Jacobson and Ruddy, 2015). A problem with this development of learning theory is that it does not differentiate between learning targeted at the conscious mind and that which is to be embedded in the nonconscious, affective mind (what we describe as 'Hebbian learning' in the last chapter). It can be argued, that the learning methods they proposed are equally effective for both, but the evidence in the area of expertise development does not support that contention (Lachman, Lachman et al. 1979), (Gobet, Lane et al. 2015) and extensive studies by K. Anders Ericsson discussed later in this chapter.

\subsubsection{Cognitive Field Theory and Intuition}

We have already discussed in chapter 2 the attributes of System 1 thinking and how it relates to and departs from System 2 thinking. The earliest ideas in this area suggested that the two modes of thinking operated within different areas of the brain, with rational, System 2 thinking occurring with the frontal parietal cortex and System 1, intuitive thinking in the ventromedial prefrontal cortex. That idea has now been largely discarded as evidence from imaging studies suggests that both modalities are being processed within the same locations of the brain (Mega, Gigerenzer and Volz, 2015).

The consensus view is now that the strict dichotomy between the two modes articulated in the early literature is not supported by evidence. One response to this is that a continuum exists from the intuitive to the rational and that thinking can be regarded as an interplay between the two (Dunwoody, Haarbauer et al. 2000). However, this 'Cognitive Continuum Theory' (Dhami and Thomson, 2012) lacks explanatory power, particularly with respect to the speed with which intuitive processing can occur.

A more fruitful way of thinking about the relationship between intuition and conscious deliberation is that they are fundamentally the same, utilising the same neural resources but 
in different ways using different sources of energy. The rational relies upon the focused concentration of mental energy, and the intuitive is activated by emotional energy. This is an interpretation suggested by Gobet, (2016) who proposes that the rational and the intuitive modes are 'orthogonal' to one another in that there is an intuitive component involved in all thinking and this is supported by the neurological evidence as noted in chapter 3 .

Gobet's insight is, in my view, correct. The interaction of the two modes of thought, combined with the dynamics of the brain seeking cognitive resolution when prior beliefs and perception contradict one another, explains the sudden and instantaneous jumps from prior to posterior beliefs. Under 'bursts' of high emotional energy the brain is able to access much more deeply embedded resources within its neural networks - resources that are not readily accessible to the conscious mind. Under such emotional intensity the processing of new perception, and the Bayesian revision of prior beliefs is greatly enhanced both in scope and in depth.

\subsubsection{Cognitive Field Theory and the Dynamics of Intuition}

Following through the implications of Gobet's insight suggests to me that there is an explanation for the apparently instantaneous and, from the individual's perspective, incontrovertible change from one state of belief to another. Dynamical Systems Theory (DST) predicts that when any system state is under the control of two or more forces, locally irreversible state changes will occur. In the appendix, I provide a theoretical explanation for this result, although the phenomenon of sudden state change can be seen across the physical, biological and, as Zeeman (1977) would argue, the social and the psychological world. The understanding that DST brings is explored in Hooker (2011) more generally and in the work of Schmidt (2011) who uses DST to discuss the psychology of the 'Gestalt switch' and Gestalt Theory more generally (Grossberg and Pinna, 2012).

Here it is appropriate to give an intellectual caveat. Dynamical Systems Theory - and Catastrophe Theory in particular - accurately predict outcomes from physical processes under controlled conditions. However, in the application below the mathematics is not quantitatively but rather qualitatively predictive. This body of theory highlights the behaviour to be expected and forces us to think about the drivers of that behaviour. Catastrophe Theory also expresses Kantian epistemology at a deep level. Kant, in his Critique of Pure Reason, 
sought to resolve the empiricist-rationalist debate by proposing two synthetic, a priori truths about the world that are accessible through the exercise of reason alone. The first is that knowledge of spatial dimensionality is innate, the second is time. The interplay between space and time is the very stuff of dynamical systems theory and Catastrophe Theory provides a compelling representation of the mathematics of DST for any system.

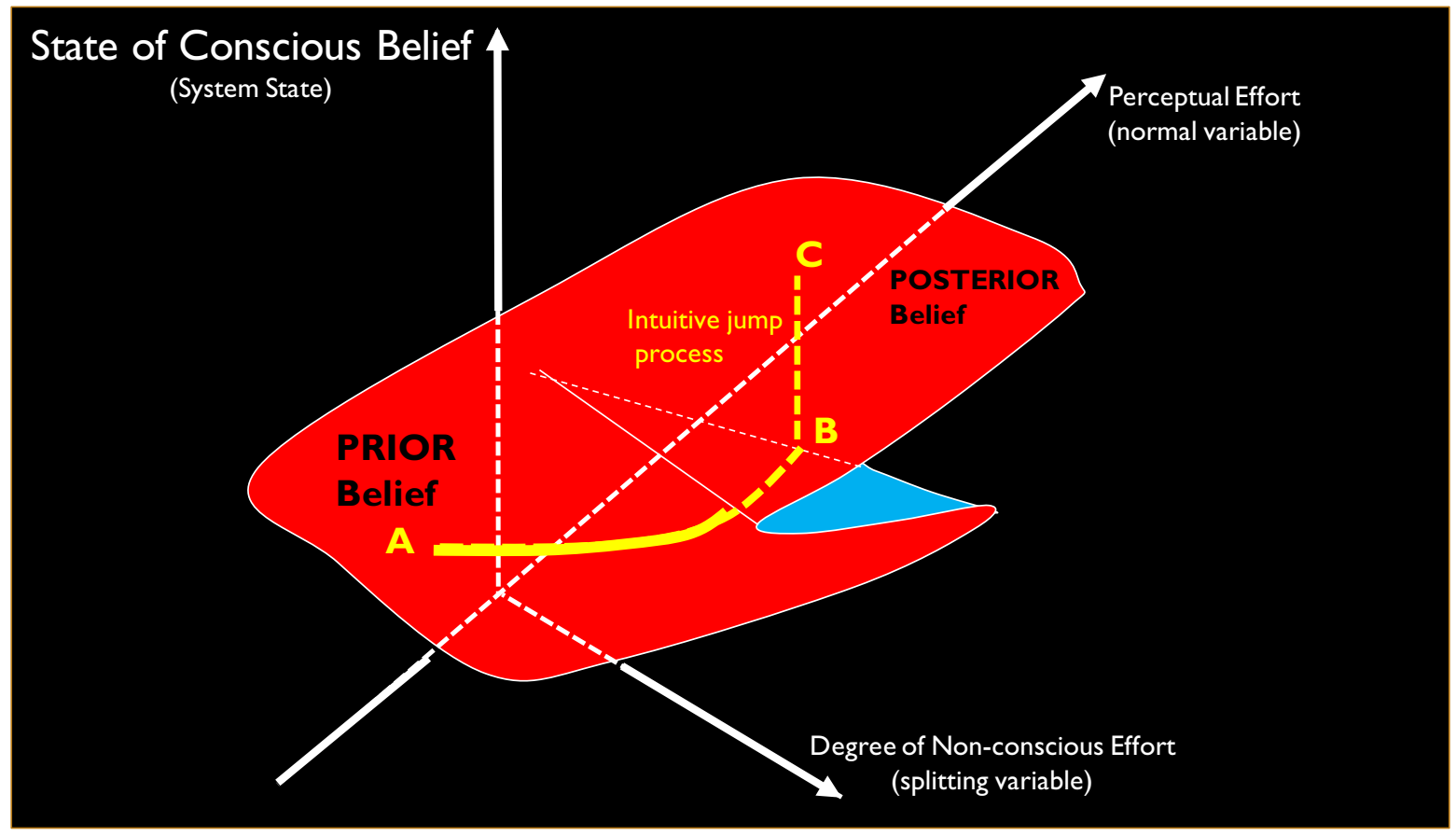

Exhibit 4.3 Catastrophe Theory Manifold showing spontaneous transitions between cognitive modalities.

Catastrophe Theory, when applied to a psychological system, relies upon a number of assumptions: first, there must be a dynamic that forces the brain system to an equilibrium state; second, the variables involved - state, normal and splitting - must change in a mathematically smooth way. The first assumption is well supported throughout the history of cognitive psychology going back to Helmholtz and before. Hohwy (2013) describes this as 'predictive error minimisation theory', which he associates with Friston's 'free energy principle' (Friston, 2009). This minimization principle lies at the heart of Hierarchical Predictive Processing Theory, and also relates to the minimisation of cognitive dissonance (see: Festinger, 1957), where ambiguity exists between what is predicted and what is 
perceived. At its simplest, these theoretical insights reflect the fact that holding dissonant beliefs, whether they be alternative perceptions or a perception that does not accord with the prior beliefs of the individual concerned, takes effort and this is the free energy to which Friston, op. cit., refers.

Mathematical smoothness, the second assumption in the Catastrophe Theory model, is required for analytical efficiency. But how realistic is it in practice? The paradox of Catastrophe Theory is that sudden state changes emerge as a consequence of smooth changes in the underlying states and variables. Leaving aside the jump changes that the theory predicts, is there any evidence that within the brain the mechanisms of belief formation or the degrees of emotional activation are not smoothly progressive? As a biophysical system, just like Rene Thom's observation of the unfolding of flowers, we have no reason to believe that nature is anything other than a smooth operator.

Given these assumptions, the red surface in Exhibit 4.3 shows the mathematical description of the equilibrium states of conscious belief as it is being driven by perceptual effort and nonconscious (emotional effort). The normal and splitting vectors are, as Gobet proposes, orthogonal and lie on the horizontal plane. As such they are not alternative forces but, in combination, drive the brain system to resolution of any discrepancy between prior belief and perception. The mathematics, as described in the appendix, predicts that, if prior belief and perception are at variance, the brain's effort to resolve the difference through conscious engagement will take it smoothly up or down the back edge of the red manifold (math speak for a topological surface). However, if the process is emotionally activated, point A will move smoothly to point $B$, at which point it reaches the inversion of a cliff edge and any small perceptual perturbation will lead to a sudden change of state to point $\mathrm{C}$.

To return to my example of an encounter with a Sabre-toothed tiger: our hunter's system state is their belief about their current state of danger. This state of belief will vary as they perceive changes in their environment as they move about. This adjustment process will be relatively smooth as they move from cave to fenced enclosure to open grassland to the rough wooded terrain where Sabre-toothed tigers are occasionally found. As the terrain becomes more dangerous, the hunter will become more and more alert as their emotional state becomes progressively more fearful. At the extreme they become 'hyper-attentive' and a 
sudden, untoward sound, a movement in the shadows, or a puff of warm air against their neck (really bad) forces a jump-change in their belief about their life expectancy. In that moment, conscious adaptation of awareness changes to nonconscious adaptation; the neural network of constructs conjugates not through a process of conscious recall but through an intuitive activation of all the relevant areas of expertise resulting in a flood of hormone through the body system. Thus, the Bayesian alteration in belief is instantaneous and the intuitive conjugation impels action in accordance with the embedded expertise of the hunter.

Further, the concept of intuition is not necessarily unimodal. Glöckner and Witteman (2010) propose four intuitive processes - associative, matching, accumulating and constructive that differ in the degree to which they are driven by affective pressure, the learning perspective employed and the way that new information is integrated into the judgment process. However, these categories, can be reinterpreted in terms of the degree of interchange between nonconscious and conscious representation of a problem and that in very short time slices, elements of all four appear depending upon the constructs and their perceived significance in the judgment process.

\subsubsection{Cognitive Field Theory and the Development of Expertise}

Much of the early work on expertise was based on the problem of chess playing (deGroot, 1965) and fascination with the idea that computers could be 'taught' to outperform humans at the highest level from opening gambit to checkmate (Simon and Chase, 1973). At one level the problem of chess playing, pattern matching, and computational logic all looked straightforward but, implicitly, it locked the mind-machine analogy into the paradigm of early cognitive psychology. The reason for this is that chess was one of the first games that attracted the attention of computer scientists and, in my view, a form of disciplinary isomorphism occurred. That is, the notion crept into psychology that, because similarities between the computational logic built into computers appeared to reflect the computational logic of humans, the brain was simply a computer and for some not a very good one at that! However, this early work did give insights that have stood the tests of replication and time.

Chess is a game that presents extraordinary complexity with millions of possibilities emerging from a single position. Much relies upon the player visually recognising the position of play and bringing from memory and selecting the best moves to achieve their objective - 
checkmate. What attracted deGroot's attention was that grandmasters could consistently find the best move although they did not appear to demonstrate any greater speed in exploring the possibilities, nor in the depth of search they undertook, than a novice. DeGroot assumed that they must employ some superior 'heuristics' in analysing the position and selecting from the choices available to them.

Indeed, much of the early work in the way experts perform followed the approach that learning occurs through a process of encoding via conscious perception proceeding through the formation of 'chunks' in short-term memory (Miller, 1956) and (Simon and Chase, 1973). These chunks are transferred to long-term memory to be recovered as necessary by a process of cues of which a maximum of seven plus or minus two (Miller, op. cit.) could be held at one time in short-term memory. They proposed that these chunks are consolidations of individual perceptions. In early research into chess playing, experts were believed to hold between 20000 and 50000 such chunks referring to specific patterns of pieces and, emerging from later studies, memories of strategic moves linking the chunks together.

The proposition that Miller, Simon, Newell, Chase and others promoted with 'chunking theory' is that experts possess more chunks and can retrieve into short-term memory those that are appropriate for dealing with a given problem (gameplay in the case of chess). Further, evidence from their studies suggested that high levels of expertise were not transferable - few experts were proficient at a very high level in more than one domain. There was, however, intriguing evidence from empirical studies of eye movement (saccades) that although eye fixing on a square on a chess board signified conscious attention, the expert was also aware, through their peripheral vision, of other pieces and could reposition them with surprising accuracy when scattered (Tikhomirov and Poznyanskaya, 1966). They were not, however, ready at that stage to acknowledge the role of nonconscious perception in either the encoding or in the reconstruction of memory although as Simon and Chase, op. cit. commented on the Russian experiments:

'.... while the saccadic eye movements themselves are serial, some parallel visual capacity appears to be operating, for, since the saccade is not random.... Visual scanning experiments show that an eye fixation does not allow enough time both to recognize a pattern in the fovea 
and to scan the visual periphery for a likely target for the next fixation unless the two processes overlap in time.'(p. 396)

The Russian studies also determined accurate timing of eye movements and the refocusing of attention at a maximum of five saccades per second (Tikhomirov and Poznyanskaya 1966). So, for example, a three-second visual input would result in a maximum of 15 perceptual captures through conscious attention and an undefined amount of peripheral, nonconscious capture of surrounding detail.

What emerged from the early studies was that expert development was a consequence of large numbers of chunks in a 'field' of expertise. However, expert ability is not just the number of chunks that are held - even though the numbers are formidable - but the processes by which those chunks are consolidated or, to use a preferable term, 'conjugated'. This is where the notion that expertise is not simply about acquiring more chunks than anyone else, especially as later evidence suggested that it is the way they are coordinated and exploited that matters. The creation of such 'super-chunks' or bundles of cognate memories that can be readily accessed is a process that has been the subject of much debate. The concept of super-chunks and the associated rules and cues by which they are accessed has been developed by Fernand Gobet (Gobet, 2013, Gobet, Lane et al. 2015, Gobet, 2016) although he prefers to call them 'templates'.

Gobet's description of the cognitive process went as follows: when perceiving a visual space an area will be selected for attention using the 'simulated eye'. From there, the information is sent to the 'mind's eye and to long-term memory where, if an appropriate memory cluster is recognised, a pointer to that cluster will be placed in short-term memory and the long-term memory unpacked into the mind's eye from which various steps can be pursued to select the next move on the chess board. The problem with this is twofold: it may represent a useful process for teaching a computer program how to recognise moves but does not reflect (i) the nature of human processing where visual perception occurs through parallel processing, and (ii) that processing occurs at both conscious and nonconscious levels. However, Gobet does see the analogy between his description of a template and what in the more traditional literature from Kant forward are described as schema. He does believe that his approach 
defines more clearly the way schema/templates works which, he argues, remedies the reason why schema had fallen into disuse in cognitive theory.

\subsubsection{Cognitive Field Theory and Constructs as the Units of Expertise}

The extension I propose to Cognitive Field Theory considers how schema or, as I prefer to call them, 'constructs' operate. I use the term construct to widen the scope of what the term signifies and to respect the terminology of the eminent, early cognitive psychologist George Kelly. Kelly's philosophical position has been the subject of debate but he clearly wished to chart a middle course between ontological realism and ontological idealism. In many respects, he was Kantian in his approach, although as far as I am aware, he never engaged with the ontological debate in any significant way. His attempt to surmount the twin horns of realism and idealism also reflects the phenomenology of Husserl (Chiari and Nuzzo, 1993) and the hermeneutics of Gadamer (2004).

In psychology, a construct has been defined as a behavioural variable, which is not directly measurable, but which is meaningful to the individual and through which different objects of experience can be discriminated. Kelly (1955) co-opted the term 'construct' in his 'personal construct theory'. For Kelly, constructs are the way that individuals model the world and form expectations about it. To use his term, they are a way that we 'psychologically channelise' our thinking about the objects of our experience and the way we understand ourselves (Markus, 1977).

What is clear is that Kelly's concept of a construct is in the tradition of Kant, Piaget and Bartlett and can be described in terms of a series of corollaries. For Kelly, constructs are predictive, they are malleable in the face of new experience, they are dichotomous, they are hierarchical and networked and, following this, their use will be appropriate for a given task depending upon their position in the hierarchy. Constructs are also idiosyncratic; that is, they are unique to the individual although our shared experiences can lead us to communicate about constructs in a meaningful way. As we review Kelly's contribution to cognitive psychology he is clearly a hierarchical 'Bayesian' in that his concept of a construct represented a tacit understanding or belief about the self or the world and that it had the potential (at least with some constructs) to be open to modification in the light of evidence. What is surprising is that Kelly's work and the location of his ideas although widely borrowed in other disciplines, 
is almost completely ignored by modern theorists such as Gobet (Gobet, Lane et al. 2015) and Clark (2016).

Kelly's concept of construct was, however, essentially bi-polar, seeking difference between objects (see Reynolds, 2013 for a discussion of his dichotomy corollary and his conception of a construct in terms of 'either-or'). Three issues arise with this: first, the bipolar concept of 'either-or' does not reflect the conditional nature of the judgement driven by each construct, so the issue of whether the focus of the image is acceptable or not depends upon the perceived intent of the photographer; second, constructs can intersect, and they may conflict with one another; third, the elicitation procedure probes the nature or the operation of different constructs as they are recalled to conscious awareness. However, constructs can operate at the nonconscious level and may not reveal their full nature through conscious recollection. This appears to me to be the most significant weakness of Kelly's conception some aspect of a construct may be amenable to elicitation that relies upon a conscious choice and a ranking of alternatives. However, other aspects of a given construct may only emerge under nonconscious activation.

My position is this: constructs are long-term memory structures consisting of accumulated neural clusters of encoded experiences, semantic interpretations of those experiences, and embedded cue dependencies and associated feelings reflecting the significance of those experiences to the individual. Those feelings can be represented heuristically as 'colours' and can be activated by given cues. The structure of a construct is infinitely variable, and it can be retrieved in part, in its mature state, via conscious recall. It can also be activated nonconsciously through emotional cues and, depending upon the strength of that cue or its resonance with the construct's embedded emotional loading, the response will be more or less vivid.

Constructs form the predictive structure of the brain. Following a Bayesian predictive processing model outlined in chapter 3 , constructs in particular cognitive fields across the brain enable a rapid process of predicting sensory perception and then adapting in response to errors between prediction and perception. This process has been demonstrated in retinal studies and has been described as dynamic predictive coding (Hosoya, Baccus et al. 2005): 
The goal of the visual system is not to construct internally a veridical reproduction of the intensity pattern on the retina. Instead, the system must reduce the onslaught of raw visual information and extract the few bits of information that are relevant to behaviour. This entails the discarding of signals that are less useful ... Thus, pattern adaptation is not merely a scheme for efficient recoding but rather serves to strip from the visual stream predictable and therefore less newsworthy signals. (p.76)

When a new experiential event occurs, the 'newsworthy' element is subsumed within the appropriate neural clusters which update and reorganise themselves as appropriate, minimising their structural entropy (or conversely optimising their coherence). This process of revising and updating follows the Bayesian process described in chapter 2 where: '...we humans are - rather astoundingly - 'Bayes' optimal' in some absolute sense.' (Clark 2016)

Simultaneously with the relatively slow process of construct revision and consolidation, a short-term, highly plastic recognition engram is activated and populated with the encoded perception. This short-term memory is highly plastic with respect to encoded experience and encoded emotional colour - an unexpected perception is encoded with high colour (vivid recollection) generating what we refer to as an episodic recollection. As noted above, this ability to create a vivid but short-term memory with minimal incoherence or interference confers significant evolutionary advantage in dealing with unexpected events of high perceived impact.

\subsubsection{Cognitive Field Theory and Hierarchical Processing}

Hierarchical Predictive Processing implies that constructs form themselves into a natural hierarchy dictated by the significance the individual attaches to them. At the first level, there are constructs that allow us to perform the basic functions of whatever behaviour we wish to pursue. These constructs depend upon high levels of repetitive encoding to become predictable in the way they operate. This hierarchy creates the framework for the 'predictive processing' of visual images either at the point of capture, during processing or when viewed subsequently.

Constructs are defined by their prepotency. Thus, the ability to select and achieve optimal focus for a given intent is a prerequisite in selecting and achieving the most appropriate depth of field for a given image - but not vice-versa. This accords with the notion that we are natural 
'hierarchical Bayesians' whose cognition is driven not passively but predictively (see chapter 3). Experimental evidence for the hierarchical nature of the Bayesian process discussed in chapter 3 has been forthcoming in the literature exploring the phenomenon of 'binocular rivalry'. Binocular rivalry was noted by Aristotle and actively investigated by the Neapolitan mathematician and polymath John Baptiste Porta (Porta, 1593) (Clark, 2013). In this experimental design, two different images are presented to each eye. What results is not a confused image but a switching process as first one side dominates then the other. The timing of this switching process and the mechanism by which it occurs strongly suggest, (Hohwy, Roepstorff et al. 2008) and Clarke, op. cit., a hierarchical process as alternative hypotheses are 'constructed' and, as a consequence, alternative visual predictions are made and the associated errors evaluated generating a 'flipping' process (Clark, 2013).

The strong evidence of hierarchical processing is not restricted to visual processing. Indeed, one of the most influential contributions to motivation theory explicitly introduced the notion of 'prepotency'. Maslow, working in the tradition of Freud and Adler, developed one of the most influential contributions to the literature of human psychology (Maslow 1943). He described the notion of prepotency and made the connection with the phenomenon of emergence, as follows:

It is quite true that man lives by bread alone - when there is no bread. But what happens to man's desires when there is plenty of bread and when his belly is chronically filled?

At once other (and "higher") needs emerge and these, rather than physiological hungers, dominate the organism. And when these in turn are satisfied, again new (and still "higher") needs emerge and so on. This is what we mean by saying that the basic human needs are organized into a hierarchy of relative prepotency.' (p. 375)

\subsubsection{Cognitive Field Theory and Fuzzy Constructs}

Constructs come in habituated clusters and a cognitive field represents a cluster of constructs for dealing with a given problem domain. The greater the cluster specialisation (its scope), its experiential depth (resource) and emotional responsiveness (vitality), the more predictable and useful it becomes - and this I argue is the basis of intuitive expertise. 
However, constructs are also 'fuzzy' in the sense that different constructs may have similar function and a degree of co-dependency. So, for example, a construct consisting of embedded skill and expertise in (say) determining ideal exposure for a given photographic intent we might label as 'exposure'. Another construct might consist of the embedded skill and expertise in determining the ideal use of light for a given intent. Being able to exercise the latter does depend to a certain degree on an ability to exercise the former; thus, they are to a degree co-dependent.

Furthermore, constructs are purposively co-dependent in that when the individual seeks to achieve a specific intent, they will be retrieved either consciously or nonconsciously and may, indeed, 'cross-cue' one another. Each construct can have an output combining with other constructs to generate a conjunct feeling with a specific neural 'colour'. This drives an intuitive response. They can also be partially recovered by the conscious mind, yielding their content at a superficial level but leaving more subtle, subjuncted memories hidden in the 'dark neural web' of the brain. This explains the early evidence that conscious recall of vivid events tends to yield the 'gist' and not peripheral detail (Adolphs, Cahill et al. 1997).

From a technical perspective, co-dependency presents both a problem and an opportunity. The problem is that standard empirical methods demand independence between measured variables. This is an issue we return to in chapter 5. However, their co-dependency does ensure their potential for synchronicity in that when they are appropriately cued there is an internal association that offers coherence in the output of the intuitive process. These codependencies between constructs are, in terms of my ontological position developed in chapter 2, a principal source of the emergent phenomenon commonly labelled 'style'. Codependency emerges as the impulse to act resulting when the nonconscious conjugation of different constructs and the individual's intent combine. This process of emergence can, potentially, be reinforced through Hebbian training methods - first by reinforcement of the individual constructs and second by varying the intensity of the emotional cue associated with each construct.

\subsubsection{Cognitive Field Theory - the Limits of Intuitive Processing}

Intuitive, or nonconscious processing, entails the activation of the most appropriate cluster of constructs to drive the nonconscious, emotional 'verdict' on the problem in hand. We have 
accepted, since the seminal work by Miller (op. cit.), that short-term conscious recall is limited to seven (plus or minus two) such constructs. In conscious processing, we rely upon a mechanism where elements embedded within long-term memory are unloaded into the prefrontal cortex and are manipulated using short-term and scratch-pad memory. This mechanism is fundamentally single threaded and is where the limit for that type of processing arises. Intuitive activation works differently, and I would argue that the limit is defined by the strength of cue dependency attaching to the individual constructs and the habituation of conjugation across the appropriate cognitive field.

Cross-culturally, cognitive fields of ten constructs appear to be a good, but not necessary, rule for defining a particular field of expertise. From the child singing 'ten green bottles' to Moses coming down the mountain with the Ten Commandments we appear to have a natural affinity - possibly physiologically based - for structuring our understanding of the world around ten elements. As a useful rule of thumb (and the remaining nine digits as well), structuring a cognitive field into ten constructs forms a justifiable but not overly parsimonious description of a given problem space. The issue of justifiability is an important - though a paradoxical aspect of any demonstration of intuitive expertise - in that it can be rationalised. More than ten and the problem of justification becomes more acute with associated constructs becoming conflated in the individual's mind.

So, we use the term 'construct' to describe these 'super-chunks' of expertise and over a lifetime of experience many countless thousands are created through the learning process. These constructs range from the most basic devoted to the problem of sense perception to recognition and differential discrimination sifting the relevant from the irrelevant. In addition, and within any specific problem space, a hierarchy of constructs ranging from the most task specific to the most general and abstract will be evident. At the moment of necessity, the individual captures, through a process of pattern recognition, the cluster of constructs they believe most appropriate for handling the cognitive task in hand.

In the intuitive moment, emotional energy drives a cognitive release of insight that emerges from the nonconscious operation of the brain to the level of conscious awareness. The crucial difference between conscious and nonconscious processing lies, I suggest, not in the neural networks and the constructs that support each modality but in the process of conjugation. In 
the intuitive moment, the conjugation is affectively driven and can access the whole resource of the required constructs. The output emerges with a surge of emotional energy that impels behaviour by releasing an emotional 'shunt' into the conscious awareness.

This simple outline of the processes of conscious and nonconscious modes of thought answers a number of observations: first, intuitive judgement appears to be spontaneous and unrehearsed; second, it does not entail conscious effort and third, it is difficult, if not impossible, for the individual to retrieve the basis of judgement. In summary, rational processing is single threaded and algorithmic and relies upon the active searching for relevant memories available for recall. Intuitive judgement is multi-threaded, drawing upon memory structures that have been packed into long-term memory and extend beyond conscious recall. It is activated by emotional intensity and is very rapid in its effect. There is good evidence that the process of conjugation of the required constructs is undertaken through a process of Bayesian averaging where a priori beliefs determine the weights that are applied to each construct.

In an important study, Nisbett and Wilson (1977) examine the related issue of the extent of correspondence between the intuitive order individuals place upon the constructs influencing their judgement and what they might believe on conscious reflection to be the most appropriate ordering. The evidence from their study and the prior literature reveals only a weak association between what experts believe they are doing and what they are actually doing (Cook, 1975), (Harte and Koele, 1995). Reviewing the more recent literature in the area of multi-attribute decision making where multiple elicitation methods have been utilised there appears to be only modest association between explicit introspection of weights and statistically implied weights (Evans, Clibbens et al. 2003).

\subsection{The Development of Expertise}

In the introduction to the first edition of the 'The Master Photographer' (Ryan, 2017), I made a provocative statement: 'talent is a concept invented by the lazy'. This reflects the view that high levels of ability in almost all spheres of human accomplishment is not predetermined by 
genetic disposition but is a product of commitment and practice ${ }^{19}$. The only qualification to that is where a physical impairment rules out a particular activity. However, although I believe the weight of evidence, discussed below, supports this view, it is not a crucial issue in developing this research. What does matter is finding the best way to enhance an individual's development of expertise to the highest level given the endowment of natural talent they possess.

The work of Anders Ericsson (Ericsson, Krampe et al. 1993) (Ericsson, Nandagopal et al. 2005, Ericsson, Nandagopal et al. 2009) led him to the conclusion that what differentiates high levels of proficiency from others, from sport to music, is practice - and lots of it. His work generated the rule of thumb that 10000 hours of deep-practice is necessary to generate expertise at the highest level. This was promoted to public awareness by Malcolm Gladwell's (2008) popular book 'Outliers: the story of success'. It also produced a professional backlash where psychologists lined up to argue otherwise culminating in a special issue of 'Intelligence' (Detterman, 2014) with a response from Ericsson (2014). The battle continues, see Macnamara, Hambrick et al. (2016) with neither side giving ground. Detterman op.cit. summarises Ericsson's position thus:

'Ericsson derived at least two general conclusions: (1) The development of expertise requires deliberate practice, a particular kind of motivated, focused practice; and, (2) with few exceptions, there is no evidence that ability is required to develop expertise.' ( $p .1)$

The observation that about 10000 hours or approximately 10 years of preparation was not unique to Ericsson. Simon and Chase (1973) and Bloom (1985) came to the same conclusion. Ericsson has been much criticised - partly because of the success of his ideas, partly because of different philosophical positions and partly because of an overly reductionist view on the part of his critics.

The two sides reflect some very old debates in the history of thought. Are human characteristics innate, or are they acquired? Is extraordinary ability the product of nature or of nurture? In most polarised debates protagonists of either position shout past one another and, in my view, this is what has happened in this case. Although this debate is of great

\footnotetext{
${ }^{19}$ Expertise, and experience take the same Latin root experiri which gives the nominative experiens- 'a trial, proof, experiment, knowledge gained by repeated trials' (http://www.etymonline.com/).
} 
interest, it focuses attention on the wrong issue. It may be that there are some innate abilities that mark out the great photographer. What matters is how we take any individual, given what endowments of talent they may have, to excellence in photography. In my view, the importance of Anderson's work is not based on the so-called 'talent myth' but in his focus upon practice as a crucial determining variable in the development of expertise. That insight flows naturally from our understanding of the psychology of nonconscious processing and the question is not whether repetition through practice is necessary but the nature of that practice and how it is conducted.

What Ericsson and his colleagues argue is that deep-practice is not the type of repetitive practice such as the endless repetition of scales in music. Deep-practice is highly focused and challenges the individual at the edge of their ability. He argues that each repetition should involve a significant chance of failure with an 80:20 ratio of success to failure being optimal. Practice must also be supported by rapid feedback on performance. Such practice is hard, it needs to be focused and implies not only strong motivation on the part of the practitioner but also strong external support and encouragement. Given this, it is easy to see that deeppractice is conceptually inclusive of many of the other factors Ericsson's critics claim are important. There is a positive feedback element in deep-practice: it enables exceptional outcomes in the individual, which motivates not only them but also those who provide support - teachers, parents, friends and, ultimately, those who are spectators or consumers of the performance in whatever field it may be.

We now turn to the specific issues of expert development in photography. Photography presents us with the problem of meeting the moment, and how we deal with it is an everpresent reality with the art. Unlike other visual arts, photography - both still and moving - is both an instantaneous and a reductive process. We hold up the canvas through our intent and nature fills it through the camera but, unlike the human eye, the camera is not selective - it is as Flusser (1983) describes it 'an apparatus'. However, it is not just the physical entity through which the photographer's expertise is channelled but rather through the intersection of the physical technology and its capability as defined by its 'firmware' and the remote choices made by its designers and software engineers. As in all other activities where tools are involved, photographic expertise lies not only in intentionality of purpose but also in the exploitation of capability as defined by others. 
Still photography depends upon the capture of the transient moment that in the majority of its sub-genres is difficult if not impossible to reliably replicate. Being able to realise and capture the moment requires a conjunction of high levels of skill at an intuitive level ${ }^{20}$. The transient nature of the external world gives us a choice - it is a choice that is existential in the sense that it represents a leap in the dark - it is a choice whether or not to press the shutter or to let the moment pass. If it is pressed, in that moment - as I discussed in chapter 2 - the camera looks two ways: first, at the object and second, at the photographer. As the shutter is pressed the photographer deploys all of the skill at her or his command, conscious and nonconscious. The quality and the depth of expertise they command are dependent upon the quality and depth of their emotional commitment to the image. It is that array of choices the individual makes that defines the image and forms the basis for the post-processing the photographer then undertakes.

Cognitive Field Theory suggests that appropriate learning strategies should be based upon identifying and enhancing a range of constructs that taken together, are most likely to enable the individual to achieve their intent at a deep as opposed to the superficial level of rational analysis. Given that, and the intuitive nature of the expertise we seek to develop, we can then establish the most appropriate ways of identifying shortfalls in knowledge and skill and the most appropriate route, through Hebbian methods, to consolidate the constructs at issue. Gobet (2005) in his exposition of the learning implications of chunking/template theory makes some very significant points:

'....an important role for teachers...is to direct learners' attention to the key features of the material to learn. One way to do this is to segment the curriculum into natural components...with perhaps an optimal ordering of these components...presenting components of the right size and difficulty... But how, then, to identify these components, their adequate level of difficulty and ordering? The answer has three parts .... The first part is to carry out a careful task analysis of the material to learn, at the level of component skills; in many domains, there exists extensive literature on which to base such an analysis (e.g. (Gagne and Briggs 1974). The second part is to combine this analysis with the study of how these skills

\footnotetext{
${ }^{20}$ At $1 / 500^{\text {th }}$ the sensor, at 10 frames per second, is only exposed for 20 micro-seconds. In many situations, the gaps are crucial.
} 
interact with broader tasks and contexts.... Another important role for teachers is to provide feedback, an obvious way to highlight the important features of a problem, and thus favour the acquisition of correct knowledge' (p.194)

The weakness in Gobet's (2005) position is that although he acknowledges the importance of the structure of expertise, he does not recognise the differences in learning for the optimisation of nonconscious encoding and intuitive recall and the optimisation of learning for conscious recollection and rational recall. He also, like much of the learning research he cites, focuses on expertise in highly rule structured and goal-oriented processes where performance can be objectively measured. He remedies that in his later work (Gobet, 2016) where he acknowledges the role of practice and, in particular, what Ericsson (Ericsson, Nandagopal et al. 2009), describes as 'deliberate practice'.

\subsection{The Structure of Photographic Expertise.}

Our strategy for determining the structure of photographic expertise is three-fold:

(i) to identify a potential structure from first principles, based upon Cognitive Field Theory,

(ii) to establish a structure a priori from bodies who have sought to define it and published their conclusions,

(iii) to identify empirically (a) the meaningfulness of that structure and (b) the degree to which it explains the variation in intuitive judgement of photographers when viewing images.

\subsubsection{The Structure from First Principles}

Following from our discussion above, Cognitive Field Theory suggests that we should seek to specify a range of 'fuzzy constructs' of no more than ten that possess a natural hierarchical ordering. The constructs should be malleable, and they will be idiosyncratic - what this means is that their content is not exclusively defined - each photographer will build their own understanding of each. It is up to the photographer to construct their own specific meaning super-setting any meaning they may share with others and constrained only by the label 
concerned. In semiotic terms, the meaning is deferred but not into an endless sequence of definitions that form the basis of Derrida's (1967) strategy of deconstruction. The deferral I argue for is into the unique and idiosyncratic understanding of the individual photographer.

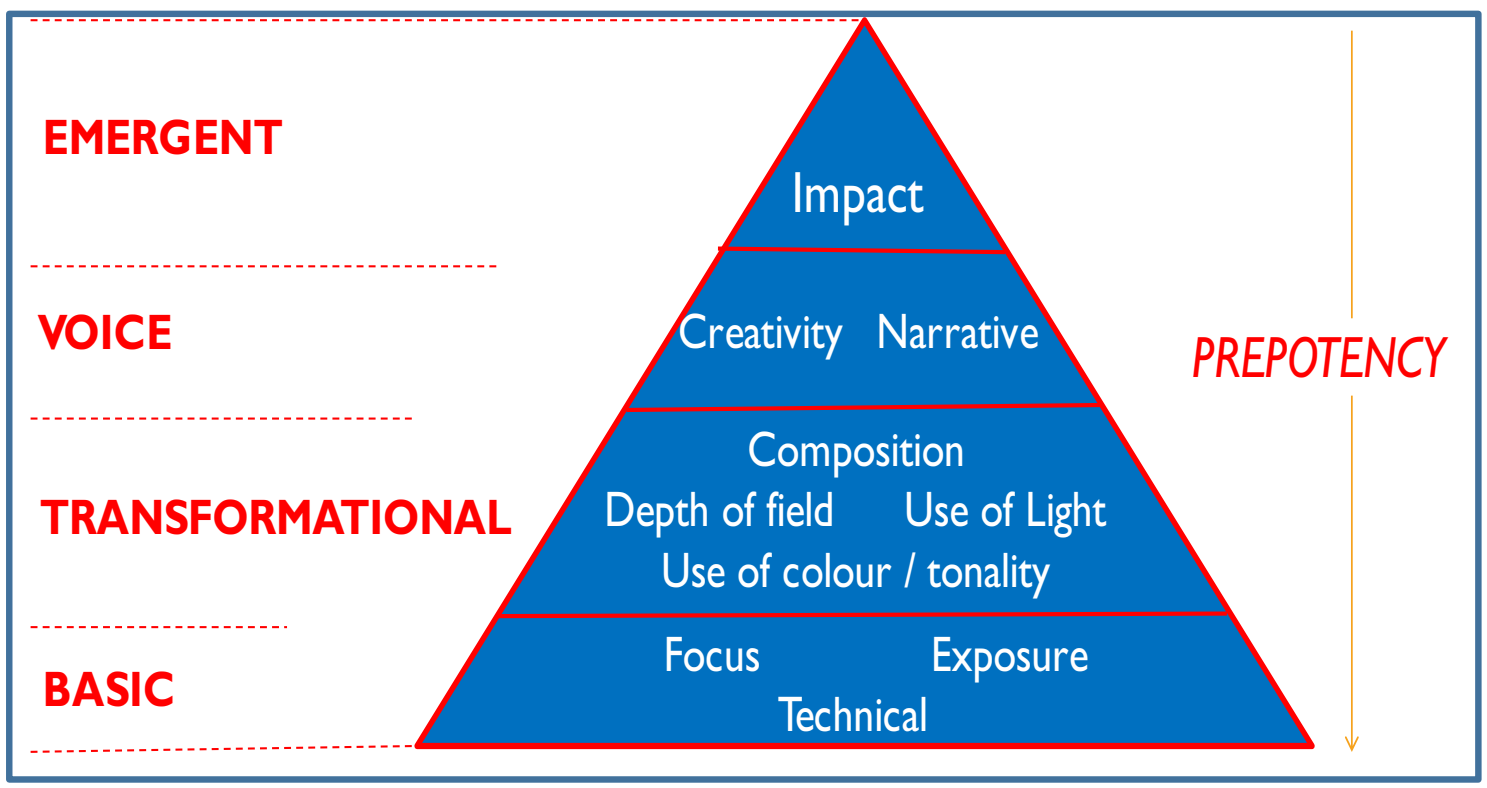

Exhibit 4.4 The hierarchy of expertise (reproduced from Ryan, 2017 The Master Photographer - the journey from good to great).

At the most basic level, the physical handling and operation of the camera - depending upon make and model - relies upon three basic skills: orientation, focus and exposure. Cameras offer three means of control - shutter speed, aperture and, with digital cameras, what is misnamed 'ISO' that controls the sensitivity of the sensor. Film cameras permitted alteration of the imaging sensitivity through different types of film. Texts have been written on two of these issues, e.g.: 'Mastering Digital Exposure and HDR Imaging: Understanding the Next Generation of Digital Cameras' (Weston, 2008), or Hagen's (2015) 'The Nikon Autofocus System'.

Rather interestingly there is paucity of literature on the problem of orientation. Most modern cameras now offer a visual horizon and vertical in the viewfinder or rear monitor - however, this is often neglected in favour of the individual's own perception of what is upright and what is level. Although superficially simple, the field of expertise required is wide: avoidance of parallax, horizontal or vertical orientation, convergence, level and uprights and so forth. For 
an overview of the literature on this topic, also see Nijhawan and Khurana (2010). The literature suggests that everyone has a personal bias in terms of the degrees they hold their camera away from true vertical and in the planar rotation of the perceived image around the vertical axis. There has been considerable recent research into the problems of postural bias in the management of stroke patients (Saj, Honore et al. 2005). The geometry of the human frame is such that bias in posture, for example, leads the eye and the brain to register the horizon as level and verticals at $90^{\circ}$ to it. However, the camera - unless corrected - will record the postural bias of the individual (Nemire and Cohen, 1993). This can be corrected in postprocessing, at the sacrifice of edge and corner content within the image ${ }^{21}$ or, more problematically, the more subtle distortions by rotational bias.

Out of the basic constructs of focus, exposure and orientation - and these we regard as the most prepotent - four others emerge. From the ability to correctly focus the camera in line with our intent we enter into the range of expertise supporting intuitive judgements controlling the depth of field in an image. These two constructs - focus and the use of depth of field - determine the 'clarity' of the image, again in line with the photographer's intent. From correct exposure two further judgements flow requiring, in their turn, the necessary expertise to exploit them to best advantage: the use of light and the use of colour/tonality. These three constructs - exposure, light and colour/tonality - largely determine the visual energy within the image. Finally, expertise in the orientation of the camera underpins appropriate composition, giving the image the level of perceptual coherence intended by the photographer. With these seven constructs the photographer can then make judgements about the delivery of their narrative and creative intent, supporting - in conjunction - the impact of the image, the tenth construct.

The structure described above goes from the lowest perceptual level to the most abstract the reverse of the way the brain works within the Hierarchical Predictive Processing model. From there the highest level is assumed to drive its subordinate level and from there down the hierarchy in turn. At each level judgements are made that dictate the judgements

\footnotetext{
${ }^{21}$ I have estimated mine to be about $12^{\circ}$ counter-clockwise, giving me a tendency to record horizons with a leftward downward tilt.
} 
immediately below with beliefs being corrected by perceptions in accordance with the Bayesian model.

\subsubsection{The Structure a priori}

The Royal Photographic Society exists, under its charter, to advance the science and art of photography. Its qualification structure - Licentiate, Associate and Fellow - are designed to give a progressive pathway from a level of all-round technical competence to outstanding ability across, at each of the three levels, a panel of images. The guidance notes describe the hierarchy in Exhibit 4.4 but give only the briefest guidance on the meaning to be attached to each of the defined areas of expertise. At the basic level its structure of expertise emphasises 'camera work' and 'technical quality'; at a more abstract level 'visual awareness' and finally 'communication'.

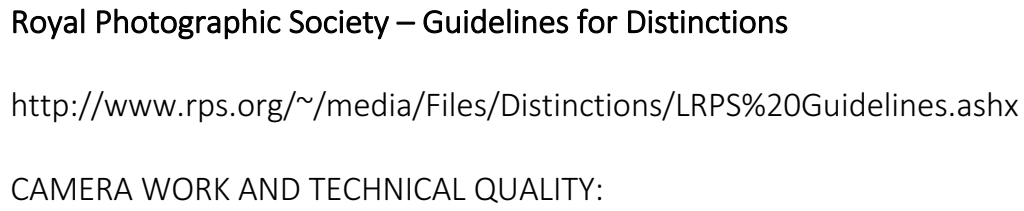

- Correct point of focus with appropriate depth of field. You should be able to demonstrate that you know how to control these elements of camera craft through your choice of shutter speed and aperture when appropriate for the subject matter.

- Suitable sharpness for the subject matter. Camera shake, over enlargement of images or a low-resolution file may result in poor technical quality.

- Correct exposure with appropriate control of highlight/shadow detail and tonal range.

- Correct colour rendition/management for the image.

- An absence of processing faults or digital defects.

VISUAL AWARENESS:

- You need to show a good understanding of how to use light in photography. The correct choice of source and direction, time of day (if natural) and intensity of light can greatly enhance an image. Correct use of light can create a mood or atmosphere and add impact to an image.

- Composition and design brings all the visual elements together. Effective composition directs the viewers' attention and prompts them to look where the creator intended. • Correct choice of viewpoint and awareness of inappropriate backgrounds and distractions. - Appropriate use of colour/monochrome medium. • Appropriate use of manipulation or post-production techniques. It is not a requirement to include manipulated images but if you choose to do so it should add value to the image and any post-production work must be technically appropriate. 
COMMUNICATION:

- Show clarity of intent with the image and where appropriate a point of interest.

- Demonstrate evidence of imagination and creativity to convey a mood, message or idea. - Show understanding and empathy with the subject matter.

- The ability to capture the decisive moment when appropriate

Exhibit 4.5 Royal Photographic Society - extract from guidelines for distinctions.

The Photographic Society of America is a little more fulsome in its description of the elements of good photography described in its online Image Analysis course, which available to members and authored by its Director of Education Jon Fishback. The elements are light (27), focus (23), composition (21), impact (18), colour (14), depth of field (8), exposure (6), story (4), creative (2) and technical (2). Each term is appended by the number of times in a text of 1700 words the specified terms are used, with the italicised terms representing significant headings. Both the RPS and the PSA do not articulate the construct 'narrative', using instead, terms such as story, or 'decisive moment'. The PSA does not use the word 'intent' except for a casual reference to intention, whereas the RPS associates it with the concept of clarity - it also makes a distinction between clarity of intent and 'point of interest'. The PSA also prefers the term 'image' to 'photograph'.

At one level, these are just labels for constructs or ideas where both societies assume there is a common understanding of their meaning and, indeed, how the quality of each might be assessed. What is noticeable is that the RPS gives little guidance on the nature of the judgement under each heading, whilst the PSA takes the view that all judgements are deeply subjective and that beneath the superficial there is little that can be taken for granted. The Image Analysis programme is deeply conditioned by the injunction to adopt language where the ownership and subjectivity of the judgement is made repeatedly clear.

The Professional Photographers Association (PPA) of America is the largest body representing the interests of professional photographers internationally with representation in 54 countries. It gives awards (it describes them as degrees) on the basis of success by photographers in its international competitions and exhibitions. It publishes and regularly updates its Twelve Elements of a Merit Image. These elements represent what it terms the 'gold standard' of a merit image: 
The Photographic Exhibitions Committee (PEC) of PPA uses the 12 elements ... as the "gold standard" to define a merit image. PEC trains judges to be mindful of these elements when judging images to the PPA merit level and to be placed in the International Print Exhibit at the annual convention. The use of these 12 elements connects the modern practice of photography and its photographers to the historical practice of photography begun nearly two centuries ago.

The twelve elements were first articulated for the PPA by Bob Hawkins (2013). The PPA's twelve elements have been utilised, copied and reinterpreted by numerous individuals and organisations as far away as the Braunton Camera Club in Devon, UK, which repeats them verbatim in its "How to Assess Photographs - a guide". In Hawkins' original drafts, he gave little guidance to assist photographers or judges in how to interpret the elements. This has been rectified, to an extent, in subsequent publications. In a useful article for Professional Photographer, Lisa Dillon (Dillon and Welsh, 2017) makes the point that 'impact' represents the combination of all the other elements. She also points out the interconnectedness of the elements and their hierarchical nature:

...rather than looking at the elements as a linear catalog or a checklist, our brains saw them as more of a constellation of interconnected elements with some in a slightly more subordinate position and others in a more prominent position, but all working together to create impact. http://ppmag.com/news/a-fresh-look-at-the-twelve-elements

As we probe the twelve elements there is one superficial point to be made, and that is two elements relate principally to the problems of presentation of a print and much of that other bodies such as the RPS would enfold within the 'technical' construct. The PPA also highlights the concept of 'style', which it associates with 'creativity. However, I argue both in chapter 2 and particularly in chapter 8 that 'style' is not a judgement construct as such but an emergent product of the subordinate constructs contributing to the impact of an image as revealed in a sequence of images or a body of work. One idiosyncrasy with the PPA elements is the concept of point of interest, which appears to me to be more a product of judgement utilising control of the depth of field underpinned by the focus judgement, along with choices in composition. However, with these caveats in mind, ten elements emerge from the PPA as described in Exhibit 4.6. 
Other organisations have produced guidelines for photography within their area of interest, and not all follow the guidelines of the RPS, PSA, SOP or PPA. It is with some delight that I discovered that the National Model Railway Association (NMRA 2017) has taken an independent view of the matter and identified the following as important judgement criteria: impact, composition, artistic approach (encompassing creativity), lighting, exposure, focus and a thorough discussion of technical issues. Control of depth of field and tonality in black and white is also discussed.

\begin{tabular}{|c|c|c|c|c|c|}
\hline $\begin{array}{l}\text { The Master } \\
\text { Photographer }\end{array}$ & RPS & PSA & PPA & SOP & NMRA \\
\hline Impact & Impact & Impact & Impact & Impact & Impact \\
\hline Narrative & $\begin{array}{l}\text { Decisive } \\
\text { Moment }\end{array}$ & Story & Story telling & Story telling & \\
\hline Creative & Creative & Creative & Creativity/Style & Creativity/Style & $\begin{array}{c}\text { Artistic } \\
\text { Approach }\end{array}$ \\
\hline Composition & Composition & Composition & Composition & Composition & Composition \\
\hline Depth of Field & Depth of Field & Depth of Field & Point of Interest & Point of Interest & Depth of Field \\
\hline $\begin{array}{c}\text { Use of } \\
\text { Colour/tonality }\end{array}$ & $\begin{array}{l}\text { Use of Colour/ } \\
\text { monochrome }\end{array}$ & Colour & Colour & Colour & Colour \\
\hline Use of Light & Use of Light & Light & Lighting & Lighting & Lighting \\
\hline Exposure & Exposure & Exposure & Exposure & Exposure & Exposure \\
\hline Focus & Focus & Focus & Sharpness & Focus & Focus \\
\hline Technical & Technical & Technical & Technical & Technique & \\
\hline
\end{tabular}

Exhibit 4.6 Table of constructs used in this research compared with other photographic sources.

As we survey the various sources, we can conclude that there is a reasonably consistent vocabulary in describing the judgements required in the capture of images and in their assessment. There appears to be a general awareness of the hierarchical nature of the judgement constructs - with impact at the top - and that the constructs overlap. All the sources reviewed limit the number of judgements to about ten, plus or minus two, and none attempt more than a brief indication of the meaning of each. There appears to be a tacit assumption amongst the professional bodies and practicing photographers that there is a common core of understanding of what each construct entails but a wide range of possible interpretation with each. It is also well understood that there is a high degree of interdependency between the constructs and it is only through their successful conjunction 
that the photographer's intent can be realised. This is in clear agreement with the development of Cognitive Field Theory discussed above.

\subsubsection{The Empirical Structure}

This leads to a series of empirical questions noted in chapter 1 :

$\mathrm{RH} 1$ : There is a set of linguistic signifiers of photographic expertise that are generally used and understood by national and international awarding bodies in photography.

$\mathrm{RH} 2$ : The theoretical model of expertise embedded in the IMP Test effectively captures variation in photographic expertise at the conscious and nonconscious level.

RH3: There are significant differences in the structure of expertise based on (i) gender, (ii) preferred cognitive modality, (iii) holders of photographic distinctions, and (iv) favoured genre.

RH4: There is a difference in construct conjugation between conscious and nonconscious processes involved in photographic judgement.

It is to the problem of answering these questions that we now turn our attention. 


\section{Chapter 5 - Methods}

\subsection{Introduction}

In 1906, a cousin of Charles Darwin, Francis Galton, an exceptional scientist, polymath and statistician, ${ }^{22}$ went to a country fair near Plymouth. Galton, the originator of eugenics, was convinced that intelligence was a hereditary trait and sought evidence to support his views. Featuring at the fair was a 'weigh the ox' competition where people could enter a raffle, putting their 'guess' of the animal's dressed weight on a ticket. Farmers, butchers, indeed everyone, including the proverbial candlestick maker, had a go at what was a very popular event. Of the 800 participants, there was a winner in that their guess was closest to the actual weight of the ox. The fact that none of the participants came close to the true weight appeared to confirm Galton's view that the common man or woman was rather stupid. However, Galton took the tickets home and analysed the results. He discovered that the average weight was just one pound different from the true weight (1198/bs). Subsequent reanalysis of the data presented in his Vox Populi (1907) ${ }^{23}$ by Wallis (2014) revealed that his original analysis contained some minor errors and that the statistical average was exactly right. The implications of this result are profound, it has been replicated on countless occasions, has formed the basis for a popular book (Surowiecki, 2005) and the insights it generates are important for the methods discussed in this chapter and my interpretation of the results presented in the following chapters.

In this chapter I will present the research methods employed, which consist of the formal statistical analysis of the data generated by the IMP Test, the testimony of a subset of photographers who had taken the IMP Test and the consilience of evidence from other sources. As such, this research can be reasonably described as 'mixed method' where the term has become synonymous with work that seeks to combine formal (quantitative) with subjective (qualitative) methods - a distinction briefly discussed in chapter 2 . I will not dwell at length on the details of the statistical methods employed which are well known, but rather

\footnotetext{
${ }^{22}$ Much of modern statistical modelling based on the concept of correlation originated with Galton's work. ' $R$ ', a universal term for correlation, was coined by Galton and is the initial letter of the word 'regression'.

${ }^{23}$ An online copy of Galton's work can be found at https://www.all-about-psychology.com/the-wisdom-ofcrowds.html
} 
focus on the following issues: the research design, the method and structure of analysis, the evaluation and communication of results. This latter area has not, in my view, received the attention it deserves in the mixed method literature.

\subsection{Multi and Mixed Method Research (MMR)}

Multi-method research is not new - the idea of transdisciplinary and interdisciplinary research is well established where disciplines as far apart as analytical chemistry and microeconomics made uneasy bedfellows. However, the more specific term 'mixed method' has been adopted where quantitative and qualitative approaches to answering specific research question are employed. A more methodological gloss has been placed upon this approach in recent years with a now well-established literature and the emergence of research based texts (see: Creswell, 2014) as a good example of the genre).

Possibly the most significant and contentious area where multi-method research has been in applied is in the evolution of the scientific and political consensus on the role of the anthropogenic drivers of climate change in the industrial era. The United Nations InterGovernmental Panel on Climate Change (IPCC) is tasked with making periodic assessments of the prevailing state of knowledge ${ }^{24}$ and presenting them in five assessment reports published between 1990 and $2014^{25}$. The early reports were mired in controversy and, as a consequence, the IPCC has developed a calibrated language for communicating the results of the research and its implications, which, in my view, represents an excellent model for assessing and communicating multi-method research and which I will discuss and, as appropriate, have modified for adoption within this thesis.

The mixed method approach has antecedents in the triangulation methods adopted in the social sciences in the 1960s as developed by Campbell and Fiske (1959), Webb, et al (1966) and Denzin (1970). Triangulation is a term that is less used now with the editorial board of the Journal of Mixed Methods Research deciding that it should no longer be used in

\footnotetext{
24 The science of climate change ranges over a number of different disciplines: computer modelling, data collection and analysis, dendrochronology, ice cores studies, as well as a range of social sciences.

25 The sixth is anticipated in 2022
} 
submissions to the journal (see Denzin, 2012, Fetters and Molina-Azorin, 2017 and Morgan, 2019).

The change of name is not, I believe, wholly positive. Triangulation as a conceptual term, and if properly applied, recognises that at all stages of the research - data collection, data analysis and conclusions - the 'legs' of the triangulation (the different methods) are recognised and respected as methodologically distinct. In my view, there is not some methodological 'blender' that can render whole the results of different approaches to a problem. The methodological blender neglects not only the links but also the divergences between the methods employed, the calibration of the weights that can be attributed to each 'leg' of the triangulation and, finally, the confidence that can be placed on the outcome. But, notwithstanding the dicta of the board of the $J M M R$, the research done in triangulation still offers some useful insights.

In an important study, Farmer, Robinson et al. (2006) utilised a six-step triangulation protocol, the first four steps - sorting, convergence coding, convergence assessment and completeness assessment - being particularly relevant for a single author study. In particular they identify a useful coding scheme for distinguishing the degree and type of convergence:

(i) Agreement where there is full agreement between the sets of results on both elements of comparison.

(ii) Partial agreement where there is agreement on one but not both components.

(iii) Silence where one set of results covers the theme whereas the other set of results is silent on the theme or example.

(iv) Dissonance where there is disagreement between the sets of results on both elements of comparison.

The principal weakness with their approach is the rather naïve process they recommend towards convergence assessment which is still there in the MMR methodology literature. Adopting a Bayesian approach in triangulation implies a search for evidence concerning the weights that should best be applied to each leg in the convergence of multiple inputs. First, and in the simplest case where there is no evidence of the appropriate balance to apply, then the law of information entropy under uncertainty indicates that equal weighting should be applied to each leg (see Shannon and Weaver, 1964). Second, where a quantitative leg generates an internal signal of its significance (such as an $\mathrm{R}^{2}$ statistic) then this can form a 
base rate probability against which the significance of the other legs of evidence can be assessed, and a statement of confidence made. My argument here is that although the quantitative leg may be of low significance from an evidential point of view, it can, in many applications, indicate a fair weighting of its significance as a useful starting point in any pursuit of rigour in the judgement process.

Formally, the principal method employed in this research is a cross-sectional, correlational design using a testing instrument (the IMP 26 Test) originally developed to support Ryan (2017). As the research has developed a second leg of the study has evolved using intraspective interviewing techniques. This second research approach has been pursued to (a) corroborate the outcome of the IMP Test, (b) gain further insights into the outputs of the IMP Test and how they can be most effectively communicated and (c) enable a mixed-method approach to the identification of latent variables in a multivariate problem. The third leg of this research, already presented in Chapter 4, seeks to establish whether there was any consensus in the definition of the signifiers of photographic expertise in practice. The development of the second and third legs employ methods differing in their approach to measurement, quantification and interpretation. Using Morgan's (2019) threefold distinction between convergent, complementary or divergent MMR, this study would be described as convergent except in so far as I place primacy on the results of the IMP Test and use the intraspective interviews as a means of increasing its external validity.

\subsection{The IMP Test}

The IMP Test was designed to identify strategies for gaining intuitive expertise in photography. Its purpose in this research has been to achieve the first three research objectives specified in chapter 1.

\footnotetext{
${ }^{26}$ IMP Test - Intuitive Mastery of Photography Test
} 
RO1: To develop a theoretically coherent model of photographic expertise based upon Cognitive Field Theory (CFT).

RO2: To develop an empirical protocol, based upon RO1, that enables the exploration of the exercise of intuitive expertise in photographic judgement.

RO3: To examine, using the protocol developed in RO2 whether judgement in photography is influenced by inter alia gender, cognitive processing style, genre or level of photographic qualification.
In constructing the IMP Test, certain design conditions were necessary: first, it should generate sufficient data inputs from the test participants to form valid inferences about their judgement processes; second, it should be simple to understand and use without support; and, third, it should not be cognitively onerous. Given those constraints the test went through a number of iterations to achieve its final form in which the subject is invited to make time constrained (three-second) judgements on twenty images scoring each on a scale of zero to 100 (stage 1), before repeating

their evaluation using the ten constructs discussed in chapter 4 (stage 2). This subsequent evaluation is not time constrained and each construct, for each image, is rated on a scale of $1-10$. The subject in stage 2 of the test is also invited to rank the ten constructs in their perceived order of importance using a slider scaling system, generating output against each in the range 0 to 1000 .

Individuals taking the IMP Test are also invited to complete Epstein's 'Rational Experiential Inventory' (REI). The REI is a well validated self-report test (for a recent review see Reyna and Ortiz, 2016) that asks 40 questions on a five-point Likert scale drawing out first, the test respondent's belief about their own aptitude for intuitive as opposed to rational thought and, second, their willingness to engage with either modality. Of these two dimensions, the second is, I suspect, most likely to be associated with genre preference in photography; however, a statistical test of this proposition is addressed both directly - statistically - and indirectly through a partitioned study on the basis of results of the IMP Test.

A number of other issues arose in the development of the IMP Test that I will discuss below. However, one issue was immediately apparent that went to the heart of the rationale for the test: is the judgement of actual images cognitively congruent with the assessment and 
selection of a potential image 'through the viewfinder'? What was clear from the prior literature, and what is addressed in chapters 3 and 4 , is that there is one important difference flowing from the difference in cognitive modality that is likely to be ascendant in either case. What is also clear from the existing literature - and what the empirical results of this study will subsequently confirm - is that the analytical mind when reviewing an image is not able to rationalise the intuitive function that impelled the photographer to press the shutter.

The question then arises: are different constructs employed in image assessment as opposed to capture, or are the prior weightings applied to those constructs different between the two activities? The answer to the latter is that they most certainly are; the answer to the former is that there is no convincing evidence to suggest there is.

At many levels, the modern understanding of the Bayesian Brain and Hierarchical Predictive Processing suggests that intent drives perception and, given the singularity of intent, there is no reason to believe that different construct signifiers are required to explain either the exante or the ex-post evaluation of a potential image space or an image outcome ${ }^{27}$. It may be that a construct (say composition) as possessed and understood by the individual is larger in terms of cognitive content than is required for either activity - but emotional activation is more likely than not to engage the whole construct in the recognition process. It is evident from the literature discussed in chapter 4 that intuition, not analysis, is the driver of cognition and that in the world of aesthetics the role of analysis is to rationalise what has already been decided. As discussed below, the IMP Test is designed to force a preponderance of intuitive judgement in the test subject's response. This is the modality that is likely to dominate at the moment of capture and unless specifically reactivated will not be present in post-processing.

\subsection{IMP Test Design and Development}

The IMP Test is based upon well-established design principles in the area of multi-attribute decision theory and practice. It has antecedents in the psychology literature (see: Nisbett and

\footnotetext{
${ }^{27}$ One trivial point is that if the skills and expertise of capture versus assessment are fundamentally different then it would be pointless reviewing images with the intent of improving image capture skills. Indeed, one might question why any teaching method apart from those advocated in Ryan (2017) could be successful.
} 
Wilson, 1977 and Schulz and Hayn-Leichsenring, 2017) and is designed to generate two judgements about each of the presented images: a judgement that is principally driven by visual activation of 'cue-dependent' memory structures, and a judgement based upon the rational evaluation of each image using a Bayesian weighting of the ten constructs. As noted in chapter 4, my argument is that emotional activation is likely to be highest at the point of image capture and that the time pressure of stage 1 of the test and the solitude of its conduct is most likely to simulate that moment. Exhibit 5.1 shows the sequencing of the test.

Research comparing intuitive visual appreciation versus rational judgement has been conducted in other areas of the arts using different experimental processes. Schulz and HaynLeichsenring op. cit. in an exceptional study of facial appreciation in painting adopted a similar approach except they alternated judgements using a Fourier mask ${ }^{28}$ to eliminate afterimage effects. My approach is somewhat more straightforward and avoids such effects.

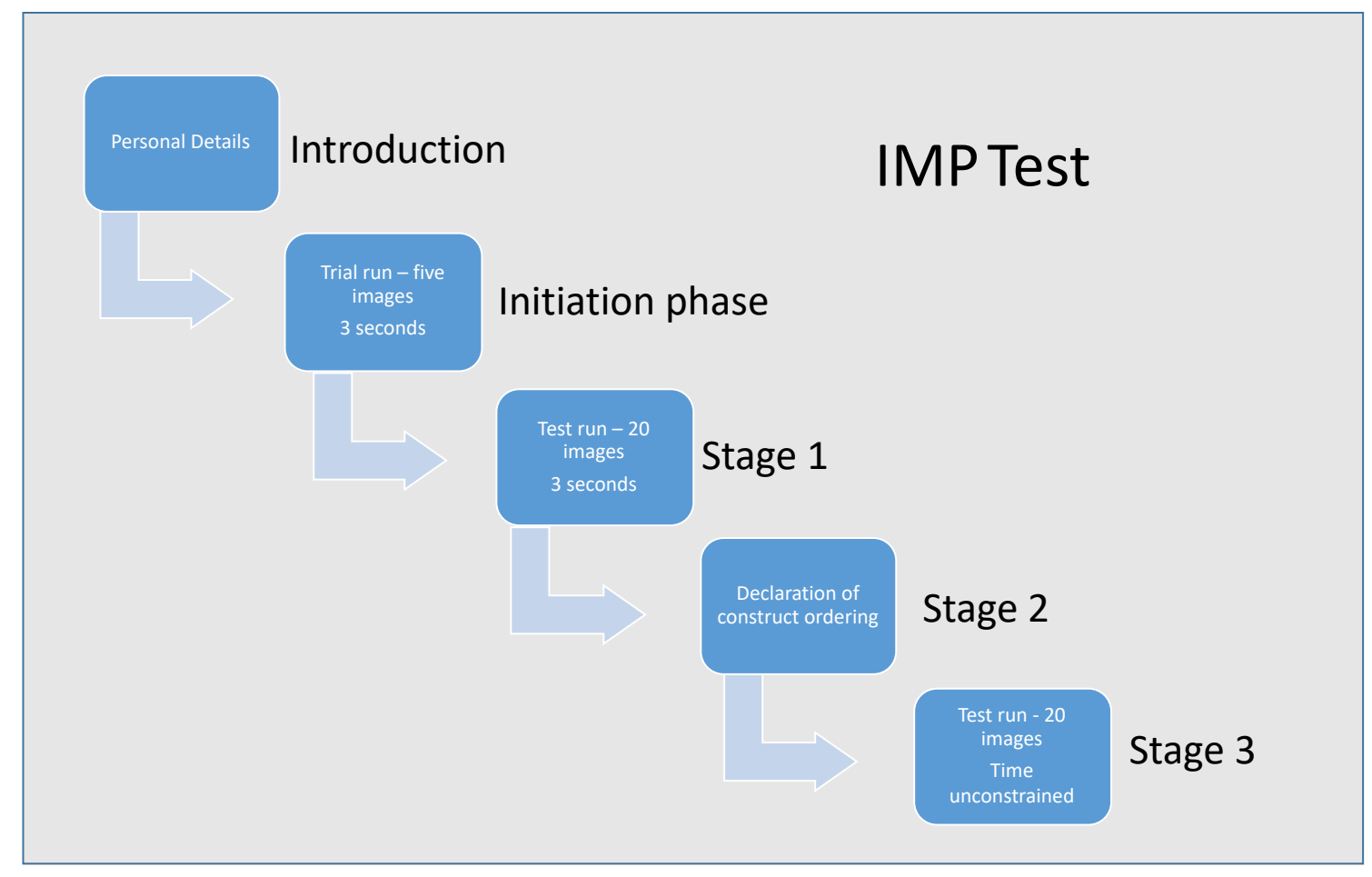

\section{Exhibit 5.1 The IMP Test Sequence}

\footnotetext{
${ }^{28}$ They do not give details of the use of the mask but, in principle, such a mask is designed to reduce noise by insertion in the diffraction plane of an optical system.
} 
The first judgement, as discussed in chapter 3 , entails a nonconscious conjugation of the ten constructs using weightings that, on the basis of previous research, are likely to be consciously inaccessible to the subject of the test. The second generates a subjective judgement of image quality using the prior stated weightings of each construct provided by the subject. The evaluation of the rational part of the judgement process is based upon the design principles of Ward Edwards' 'SMART'29 algorithm (Phillips and vonWinterfeldt, 2006).

Edwards (1954), credited as the founder of behavioural decision theory, developed SMART for its simplicity and practical utility, whilst recognising that other approaches such as Keeney and Raiffa's (1976) subjective expected utility (SEU) model might represent a more sophisticated approach to modelling complex decisions. However, in an early, in-depth, comparative study of a wide range of decision modelling techniques, Gigerenzer and Todd (2000) came to the conclusion that the simpler, less cognitively demanding models such as SMART were just as reliable than more sophisticated Bayesian network modelling methods.

It is important to note that the IMP Test is not primarily a test of image quality or standard; it represents a process for examining the (co-) relationship between the ten constructs and a forced judgement, and between the ten constructs themselves. Each individual makes 20 judgements on a 0 to 100 scale and 200 judgements on a $1-10$ scale. The possible combination of answers is such that the probability of two individuals generating the same scores purely by chance is several factors lower than winning the national lottery ${ }^{30}$. The aim of the test and the analytics that support it is to examine the nuances of variation in judgement under each of the ten construct and how those variations influence one another and most particularly the intuitively driven judgement in the first phase of the test.

\footnotetext{
${ }^{29}$ SMART - 'simple multi-attribute rating technique'

30 The odds are worked out as follows: for every construct of every image there are 10 possible outcomes. That gives $20^{10}$ variations of voting outcome or $1,024,000,000,000,000,000,000$ possible combinations. If that is combined with the range of scores on the intuitive phase of the test then, need I say, the number of possible scoring outcomes exceed the stars in the known universe. The point here is that the vast array of possible outcomes of the test mean that each individual's score sheet is effectively unique. The challenge of this research is to demonstrate that each sheet is not just unique but meaningful.
} 
In the development of the test a small panel of four photographers with a high level of formal and professional expertise advised on the images and their balance. ${ }^{31}$ The primary aim was to ensure that the images that went into beta testing of the IMP Test gave a balanced outcome of the ten constructs and were - as far as possible - stylistically neutral.

The specific choices in the development of the IMP Test were:

(i) To draw from my own portfolio, twenty images of varying quality and genre. The rationale for this was to ensure, first, that the images were novel to any potential viewer and, second, to achieve $a b$ initio equality across the ten constructs in terms of mean response (see Exhibit 5.2).

(ii) Following the arguments developed in the last chapter, only minimal pointers to the meaning attributable to the ten construct labels were given. The rationale for this was to offer ownership of what each label might signify to the test subject and through that ownership of the

\begin{tabular}{|l|c|}
\hline & Mean \\
\hline Focus & 6.73 \\
\hline Exposure & 6.90 \\
\hline Technical & 6.35 \\
\hline Col/tonality & 6.38 \\
\hline DOF & 6.70 \\
\hline Light & 6.59 \\
\hline Composition & 6.37 \\
\hline Narrative & 6.21 \\
\hline Creativity & 6.01 \\
\hline Impact & 6.50 \\
\hline Average & 6.48 \\
\hline
\end{tabular}

Exhibit 5.2 Construct means results. A primary goal of the test, as initially developed, was to optimise its salience at the individual as opposed to the aggregate level. However, aggregation served a number of purposes: first, it would provide a means of correcting one source of internal bias within the test model and, second, through the Law of Large Numbers allow us to make inferences about expertise at the group level.

(iii) To neither supervise nor set physical conditions for the conduct of the test. It is a wellknown problem across the experimental sciences (natural and social) that what you observe you change. However, it is suggested in the test instructions that subjects should use a calibrated monitor, whether using a desktop or laptop. This is to give the subject a constant viewing standard if they return to their judgement of the

\footnotetext{
31 The individuals supporting the development of the IMP Test are noted in the acknowledgements. They included two professional and two highly qualified amateur photographers.
} 
images at a later date or leave a significant gap between the different phases of the test. The risk that an individual would attempt to 'enhance' their results by manipulating their output responses was, in my view, acceptable. To be successful they would need access to the testing software or equivalent and be able to adduce the relative images scores that would achieve their desired outcome.

\subsection{A Note on the Use of Likert Scales.}

There is a voluminous literature on the use of 'Likert' scales in attitude measurement and considerable debate whether they should be treated as generating ordinal or interval data. If the former, the data generated should be treated as non-parametric with the loss of statistical power that entails. However, based upon simulation studies Carifio and Perla (2007) and Carifio and Perla (2008) concluded that Likert outcomes can be treated as interval data. Carifio and Perla (2007) present ten myths about Likert scales that deal with the majority of issues that have arisen historically in the literature.

In my view, in the IMP Test design, what underlies the scale is an individual's continuous distribution of prior beliefs. The use of the Likert scale represents a ten-point sampling of the conditional distributions of judgement as they view an image. This does not suggest to me that there is any a priori reason why the data should be regarded as non-parametric. Experiments during the development of the test with 'slider' input proved too unreliable to be useable. Although a ten-point scale does present rounding error, with twenty judgements per construct, the error distribution is insignificant.

To reduce bias towards the mean the instructions for the test specify a ten-point scoring range (1 - 10) which avoids a centre point and nudges choice one way or the other (Oppenheim, 1992). Testing the IMP Test input with various spreadsheet packages (many test subjects use open-source software) put a question mark against the use of a double conditional in each input cell. To minimise error checking during run-time, zero was left unblocked but only integer values permitted. Of the 20,000 scoring judgments made, less than 20 zeros were recorded. 


\subsection{IMP Test Protocol}

Formal analysis is not just about running a test and looking at the results. The procedure goes through a number of stages and at the individual level culminates in an extensive report that goes back to the test subject (specimen attached in the appendix). The stages are:

(i) Testing process and recovery of the data.

(ii) Checking that appropriate permissions have been received and that the test subject is within the ethical frame to proceed ${ }^{32}$.

(iii) Review and validation of the data ensuring it is complete and, if not, referring back to the test subject for additional information or clarification.

(iv) Remove all personal identifiers and contact details to an independent name file

(v) Loading and running the data through the necessary analytical software, correcting any run-time errors and checking the output.

(vi) Reviewing and interpreting the analytical output, running confirmation checks if necessary.

(vii) Preparing and submitting the analytical report to the test subject.

(viii) Consolidation of data into a database.

The formal analysis uses a range of software tools contained within XLSTAT (Addinsoft 2019), a software package that uses EXCEL as its input/output medium. Full software specifications can be obtained from the company website. Parts (vi) and (v) above incorporate the following steps:

(i) Assembly of data into matrix and arrays to run the following: Partial Least Squares Regression (PLS-R) with one dependent variable, generation of similarity/dissimilarity table using Pearson Correlation and Multidimensional Scaling. Principal Components Analysis is run as a confirmatory step if PLS-R and MDS disagree.

(ii) Using construct rankings and Variable Importance Factors generated by the PLS-R, obtain correlations of the subjects declared construct ranking with that generated by the model.

\footnotetext{
${ }^{32}$ As noted in Chapter 1 the testing was conducted under the ethical guidelines of the British Psychological Society and the University of Gloucestershire.
} 
(iii) Examine comparisons between (i) image ranking from stage 1 and stage 3 of the test noting significant discrepancies, (ii) image rankings from stage 1, stage 2 and the consensus.

(iv) Review output statistics: $Q^{2}, R^{2}(Y)$ and $R^{2}(X)$, variability in judgement across all ten constructs, cross-rank correlations between subject (intuitive), subject (rational) and consensus.

(v) Examine the output of the Rational Experiential Inventory and check for agreement with the corresponding signals from the analysis of the output from the IMP Test.

(vi) Assess quality of test outcomes against an aggregation curve reflecting confidence in judgement (see Exhibit 5.10 below).

\subsection{IMP Test Validity}

The concept of validity in experimental work was first defined by Truman Lee Kelley (1927) who forced a distinction between validity and reliability in the instrument being used: 'The problem of validity is that of whether a test measures what it purports to measure, while the question of reliability is that of how accurately a test measures the thing which it does measure' (p. 14). The IMP Test is designed to elicit the degree to which an inclusive set of cognitive constructs, as understood by the test participants, influences their nonconscious choices when assessing a potential or an actual image at the moment of capture or in subsequent assessment.

Internal validity, as we noted in (Ryan, Scapens et al. 2002) refers to the degree to which the controls in an experiment ensure that the changes in the dependent variable have been brought about, in the main, by the independent variable changes, not by other confounding factors.' (p. 122). Internal validity is determined by the sources and degree of bias induced either in the design of the experiment or in its execution. It is possible to mitigate bias either directly at the level of the individual test or through the recognition of the impact of the Law of Large Numbers with aggregate data sets. At both the individual and the aggregate level and by working within the assumptions upon which particular statistical methods are based it is possible to infer the internal validity of the underlying testing instrument using quality 
parameters appropriate to the method being used. With the IMP Test the following quality parameters are generated by the statistical methods employed:

\begin{tabular}{|c|c|c|}
\hline \multirow[t]{2}{*}{$\begin{array}{l}\text { Partial Least } \\
\text { Squares } \\
\text { Regression }\end{array}$} & $\mathrm{R}^{2}$ & $\begin{array}{l}\text { Tests the variability in the dependent variable that can be } \\
\text { explained by the independent variables. Normally an } R^{2} \text { in } \\
\text { excess of } 0.67 \text { is indicative of high predictive accuracy } \\
\text { (Henseler, Ringle et al. 2009), between } 0.3 \text { and } 0.67 \\
\text { moderate predictive accuracy and below } 0.3 \text { very little } \\
\text { confidence can be placed in the results. }\end{array}$ \\
\hline & $Q^{2}$ & $\begin{array}{l}\text { This quality measure increases by the number of } \\
\text { components extracted in the modelling process. } \\
\text { Throughout this study two components were sufficient to } \\
\text { explain the large proportion of the variance in the model. } \\
\text { Q2 is a somewhat stricter test of model quality than R2 } \\
\text { although the ranges as noted above are equally useful in } \\
\text { both metrics for interpretive purposes. }\end{array}$ \\
\hline \multirow[t]{3}{*}{$\begin{array}{l}\text { Multidimensional } \\
\text { Scaling }\end{array}$} & \multirow[t]{2}{*}{$\begin{array}{l}\text { Shepard } \\
\text { Diagram }\end{array}$} & \multirow[t]{2}{*}{$\begin{array}{l}\text { A handy visual check of the quality of the model. A reliable } \\
\text { model is indicated when the dots lie close to the line eg: }\end{array}$} \\
\hline & & \\
\hline & $\begin{array}{l}\text { Kruskal } \\
\text { Stress } \\
\text { Test }\end{array}$ & $\begin{array}{l}\text { The stress test indicates the effectiveness of the dimensions } \\
\text { in separating the data points. Normally less than } 0.1 \text { is } \\
\text { deemed excellent and between } 0.1 \text { and } 0.15 \text { is acceptable. }\end{array}$ \\
\hline Chi Squared & $P$ value & $\begin{array}{l}\text { This is a useful test of significance when comparing } \\
\text { partitioned data arrays (e.g. when considering whether the } \\
\text { responses on an array of options of one group is significantly }\end{array}$ \\
\hline
\end{tabular}




\begin{tabular}{|l|l|l|}
\hline & & $\begin{array}{l}\text { different to another). Unlike t tests and Analysis of Variance } \\
\text { (ANOVA), } \text { Chi }^{2} \text { it is tolerant of non-parametric inputs. }\end{array}$ \\
\hline Cronbach Alpha & $\begin{array}{l}\text {-infinity } \\
<\alpha<1\end{array}$ & $\begin{array}{l}\text { Alpha measures the internal consistency of a group of } \\
\text { variables in a model. An alpha greater than } 0.9 \text { with ten } \\
\text { constructs signals a high level of internal consistency. High } \\
\text { internal consistency is a necessary but not a sufficient } \\
\text { condition for internal validity of a model such as the IMP } \\
\text { Test. Values in excess of } 0.95 \text { may indicate redundancy in } \\
\text { the explanatory variables. }\end{array}$ \\
\hline
\end{tabular}

Exhibit 5.3 Principal Quality Statistics used in the IMP Test Analytics

External validity refers to the degree to which a test instrument can be generalised from the specific experimental or study setting to the relevant population as a whole. Lack of internal validity will undermine external validity to a degree but there is also a potential inverse relationship between them. For example, the conduct of a test within a laboratory setting will enable a high level of control and hence internal validity but reduce its generalisability as an analytical tool in an applied setting.

External validity can also be strengthened by corroborative experiments using the same pool of test subjects and by subsequent replication using different subjects. The first method, which is applied in this thesis, is by searching vectors of difference for evidence that any subgroup within the 106 test subjects is, as an aggregate, significantly different from the remainder. The logic of this is straightforward - if the whole group is not typical of the population as a whole, there will be a sub-group which is more typical (but not necessarily perfectly so) of the population than the remainder. If such a 'stand-out' group is not evident in the data along any of the vectors of enquiry (as measured by a general statistic of model quality), then it is reasonable to assume that the whole group is unbiased with respect to those vectors. External validity is also supported through evidence of backward validation when an experiment confirms similar experiments in other studies in different domains.

\subsection{The Problem of Bias}

loannidis (2005) gives a useful definition for bias being: '...the combination of various design, data, analysis, and presentation factors that tend to produce research findings when they 
should not be produced'. In a further study Chavalarias and loannidis (2010) identify 235 classifications of biases present in biomedical research - a veritable tour de force but which leaves one wondering if high quality, unbiased research is, indeed, possible. Pannucci and Wilkins (2010), in an interesting discussion of bias in evidence-based medicine define it as any tendency which prevents unprejudiced consideration of a question. These authors make a valuable point: bias is inevitable in any meaningful research, indeed the term itself makes some significant ontological assumptions about the nature of the reality being explored. For there to be bias, there has to be some underlying notion of what is 'unbiased', 'fair' or 'neutral' in terms of some external reference and an implicit acceptance of a correspondence theory of truth. Within the framework of Object Oriented Ontology discussed in chapter 2, the problem of bias is a real issue, and stands in opposition to the post-modern dictum that in science 'anything goes', Feyerabend (2010).

However, the inevitability of bias does not, in my view, destroy the value of an analytical method either when studying individual or group performance. In any modelling process driving an analytical outcome - whether explanation or prediction - the output error (E) can be partitioned into two components - bias and variance - and these two sources of error trade off against one another. The bias/variance trade-off is well known and can be expressed as follows:

Error $\left(f_{i}-y_{i}\right)^{2}=$ bias $^{2}+$ variance $\left(y_{i}\right)+$ variance (noise)

Where:

Error $\left.\left(f_{i}-y_{i}\right)^{2}\right\}$ is the mean square error (MSE) in judgement of an (unknown) true answer and the prediction of individual (i).

Bias is the systematic difference between prediction judgement and the true answer

Variance $\left\{y_{i}\right)$ is the variability as measured by the variance across (i) judges

Variance (noise) reflects the unattributable variability due to other factors not associated with the judgement concerned.

I have sought to reduce, mitigate or acknowledge bias throughout this research. However, one obvious bias I have not sought to eliminate is the bias in each test participant's 
understanding of the meaning of each construct. That would bias their judgement towards those definitions but decrease the variability (their uncertainty) in judgement. Working through the sources of bias is, however, valuable, although in the majority of cases reducing the bias would not have impacted upon the quality of the aggregate outcomes. In particular, I focus on bias at the following levels: sampling and selection bias, response bias, and confirmation bias.

\subsection{Sampling and Selection Bias}

The focus of this study is the process of human judgement in a particular domain. I have made the assumption that human cognition and mental processing of visual stimuli is common across our species and that there is no reason to believe that any subset of people are likely to differ from others in the way their brains function. The target population is photographers who have achieved a basic level of competency in the use of a camera. All intended participants in the test were invited to review the checklist of basic competency (see: Ryan, 2017 appendix 1).

In developing the sample of individuals for the IMP Test, I have been mindful of the issues that are likely to attenuate idiosyncrasy in judgement. Research into Galton's Ox leads to a number of required conditions for 'best judgement' to emerge (Davis-Stober, Budescu et al. 2014):

(i) The individuals involved should have an understanding of the issues involved.

(ii) Diversity of opinion is good.

(iii) The individuals should make their judgements independently with no collusion.

They then go onto say:

Our results suggest that crowd wisdom is robust to different choices of aggregation and sampling rule. That is, how one aggregates the judgments or chooses an individual judge rarely affects the qualitative conclusion that even a crowd that is a simple average of judges is wiser than the individual... First, a crowd becomes wisest when it is maximally informative, which entails that its members' judgments are as negatively correlated with each other as possible, as opposed to being independent. Thus, the best judge to add to a crowd is one 
that is maximally different from others....A second surprising conclusion is that while the absolute accuracy of the crowd depends on the direction and magnitude of members' bias, it is almost always preferable to use a weighted aggregate of judgments rather than select the single best group member, even if the crowd members are biased...the decrease in variance of predictions caused by aggregating judgments will offset the bias, a manifestation of the well-known bias/variance trade-off. (p.100)

My concern has not been, therefore, to achieve randomness in selection of the participants in this study but to maximise the 'wisdom of the collective'. The sampling process has been conducted by open invitation (volunteer sampling) with some opportunistic sampling to broaden the sample scope (see McLeod, 2014 for a useful outline and discussion of the principal sampling methods in psychological research). The sample is, therefore, largely selfselecting, so I would argue that it is safe to infer that those who choose to participate are committed to understanding the level and limitations of their expertise in photography.

Over the sampling period I made presentations on the messages within Ryan (2017) and promoted the IMP Test to in excess of 2000 photographers at camera clubs, photographic societies, trade shows and specialist conferences. I also extended an invitation to take the test through LinkedIn. At a conservative estimate the response rate to invitation was of the order of $5 \%$. On enquiry and follow-up common reasons for not submitting a test result were: (i) not interested in knowing the result, (ii) were afraid of what the test would reveal, (iii) the test and/or the instructions were too complicated, (iv) run-time problems with hardware or software, (v) lack of time, (vi) had no ambition to improve their photography. I rejected applications for the test where the candidate was underage.

The issue of test complexity was raised in feedback with one test-subject (a retired consultant forensic psychologist) responding as follows:

I'm delighted to say I found the test both absorbing and challenging - and I enjoyed the process. The instructions are perhaps a little complicated, but, given the nature of the testing process, I can't really see any simple way around that. Possibly their wording could be tweaked a tad? I'm not sure why the upload of the completed data sheet would only take place via Outlook on my desktop. I use Google Chrome and Gmail and was not computer-literate enough to get around that minor snag! (Test participant IT0100, email received and logged 26/11/2018) 
The test complexity may indicate that the sample was biased in favour of those with a high level of educational attainment. Of those who have taken the test $61 \%$ were at graduate level or above and $27 \%$ held postgraduate awards. This could potentially bias towards those who privilege conscious modalities of thought (self-selection bias) and influence the distribution of respondents (response bias). Partitioning between those who responded and submitted early following an invitation to participate and those who responded later did not show any significant differences across the test, which indicates that non-response bias was probably low and that those who did submit were a fair sub-sample of those who were invited and made aware of the test (Oppenheim 1992). However, as noted in the conclusion, any judgement of validity and generalisability of the IMP Test to a larger population would need to keep the caveats induced by the sampling method in mind.

\subsection{The Participant Profile}

Those who volunteered for the test come from a wide range of different backgrounds but with a predominance of amateur photographers, although professional photographers (i.e. individuals who make their living from photography) form a substantial minority. The sampling method restricts the external validity and generalisability of the test results (but not necessarily the validity of the IMP Test). However, the following sample constraints on generalisability are clear: the results cannot be extended to those whose interest in photography is limited to their mobile phone or simple point and shoot; and the age profile is skewed towards the older age group (mean age 61 years) because of the predominance of amateurs with sufficient surplus income to engage in what can be a high cost pursuit.

Over the time period 106 photographers submitted a test result. The test subjects were profiled by: gender, age, photographic qualification, academic qualification, judging experience, favoured genre, favoured output and cognitive profile using Epstein's Rational/Experiential Inventory. The testing was conducted unsupervised except for four participants who consented to test plus subsequent interview if they could be done together. In those cases, I withdrew during the IMP Test phase. Where test participants accepted an invitation to be interviewed, the results of the IMP Test were not divulged until the interview was completed. The participant profile is shown in the display (Exhibit 5.4). 
Participant Profile

\begin{tabular}{|llll|}
\hline Total Participants & 106 & $\begin{array}{l}\text { Testing commenced } \\
\text { Testing closed }\end{array}$ & $\begin{array}{l}\text { 01-Sep-16 } \\
\text { 20-May-19 }\end{array}$ \\
\hline
\end{tabular}

Gender Distribution

$\begin{array}{lcc} & \text { Number } & \% \\ \text { Male } & 72 & 70 \% \\ \text { Female } & 34 & 33 \% \\ \text { Not declared } & 0 & 0 \%\end{array}$

Academic Qualification

$\begin{array}{ccc} & \text { Number } & \% \\ \text { Graduate } & 36 & 34 \%\end{array}$

Postgraduate $\quad 29 \quad 27 \%$

Other $21 \quad 20 \%$

None/not decli $\quad 20 \quad 19 \%$

Age profile

$\begin{array}{cc} & \text { Years } \\ \text { Average } & 61.0\end{array}$

Standard Deviation $\quad 10.7$

$\begin{array}{lcc} & \text { Number } & \% \\ <=61 & 45 & 42 \% \\ >61 & 61 & 58 \%\end{array}$

Status

$\begin{array}{ccc} & \text { Number } & \% \\ \text { Professional } & 16 & 15 \%\end{array}$

Competition $10 \quad 10 \%$

Amateur $\quad 80 \quad 77 \%$

Judging experience

Number

Number

21

Years

Experience $\quad 9.9$
Photographic Qualification

Number \%

$\begin{array}{lll}\text { FRPS } & 2 & 2 \%\end{array}$

ARPS $\quad 10 \quad 9 \%$

LRPS $\quad 11 \quad 10 \%$

$\begin{array}{lll}\text { Other } & 6 & 6 \%\end{array}$

Unqualified $\quad 77 \quad 73 \%$

Preferred Output

$\begin{array}{lcc} & \text { Number } & \% \\ \text { Print } & 60 & 57 \% \\ \text { Photobook } & 8 & 7 \% \\ \text { DPI } & 34 & 32 \% \\ \text { Audiovisual } & 4 & 4 \%\end{array}$

Cognitive Profile

Orientation in aptitude Number $\quad$ \% $\begin{array}{lcl}\text { Rational } & 77 & 73 \%\end{array}$

Experiential $\quad 29 \quad 27 \%$

Orientation in engagement

Rational $\quad 73 \quad 69 \%$

$\begin{array}{lll}\text { Experiential } & 33 & 31 \%\end{array}$
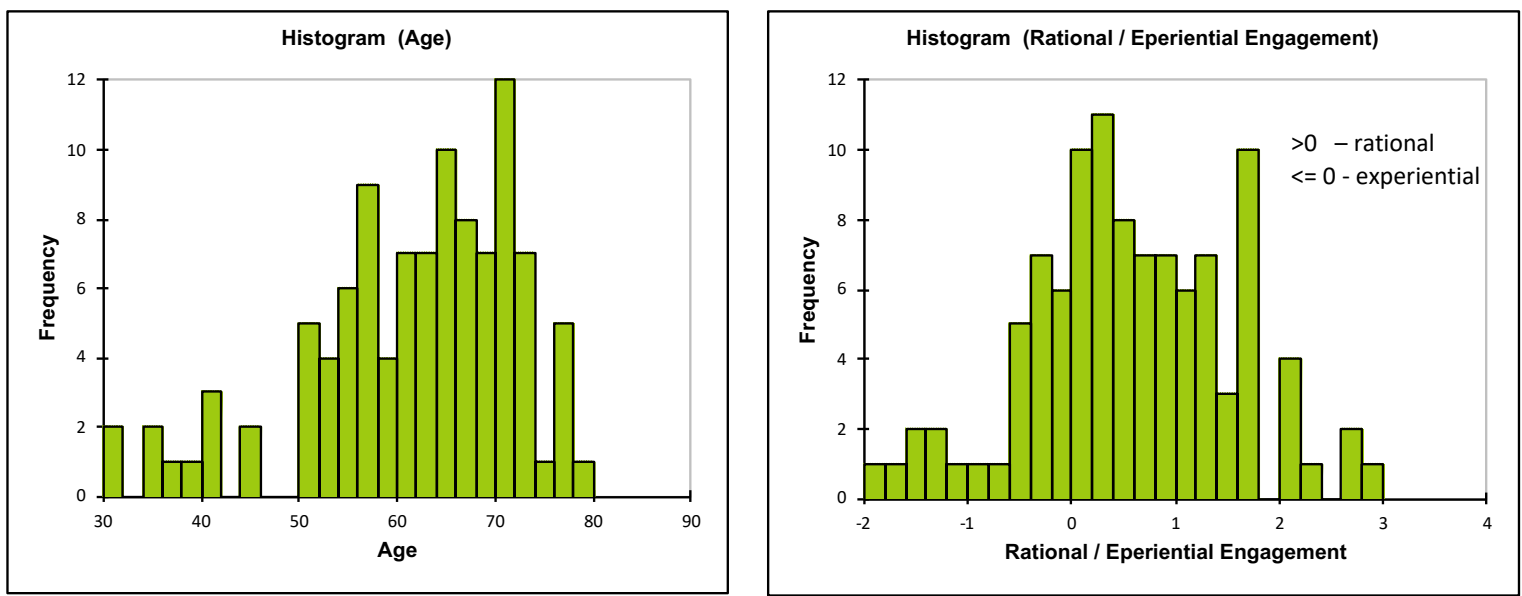

Exhibit 5.4 Participant Profile at a Glance

\subsection{Analysing the IMP Test}

In chapter 1 a series of indicative hypotheses were formulated supporting the four research objectives: 
1. $\mathrm{RH} 1$ : There is a set of linguistic signifiers of photographic expertise that are generally used and understood by national and international awarding bodies in photography.

2. $\mathrm{RH} 2$ : The theoretical model of expertise embedded in the IMP Test effectively captures variation in photographic expertise at the conscious and nonconscious levels.

3. RH3: There are significant differences in the structure of expertise based on (i) holders of photographic distinctions and those without, (ii) gender, (iii) age, (iv) favoured genre and (v) preferred cognitive modality.

4. RH4: There is a difference in construct conjugation between conscious and nonconscious processes involved in photographic judgement.

In my view, a successful experimental design is a combination of data collection and the method of analysis. Potential bias can be obvious, but in many cases a good analytical approach can eliminate the most egregious of problems. For each of these hypotheses analysis and interpretation of the IMP Test results is conducted at three levels: individual, aggregate and partitioned aggregate. A schematic of the input - output data arrays is shown in Exhibit 5.5. The data inputs and outputs generated by the IMP Test are as follows:

Inputs

[A1] The array of restricted time-slice scores from stage 1 of the test.

[A2] The array of preference weightings for the ten constructs from stage 2 of the test.

[M1] The matrix of time unconstrained scores for each of the 20 images against each of the ten constructs from stage 3 of the test.

\section{Intermediates}

[A3] An array of corrections for construct bias across the 20 images.

[M2] The matrix of time unconstrained scores corrected for construct bias. 
[A4] An ordered array of regression weights (betas) signalling the intuitive ordering of construct importance.

[A5] A rank ordering of the 20 images based upon the unconstrained judgement of the test subjects and their rated importance of the ten constructs from stage 2 of the test.

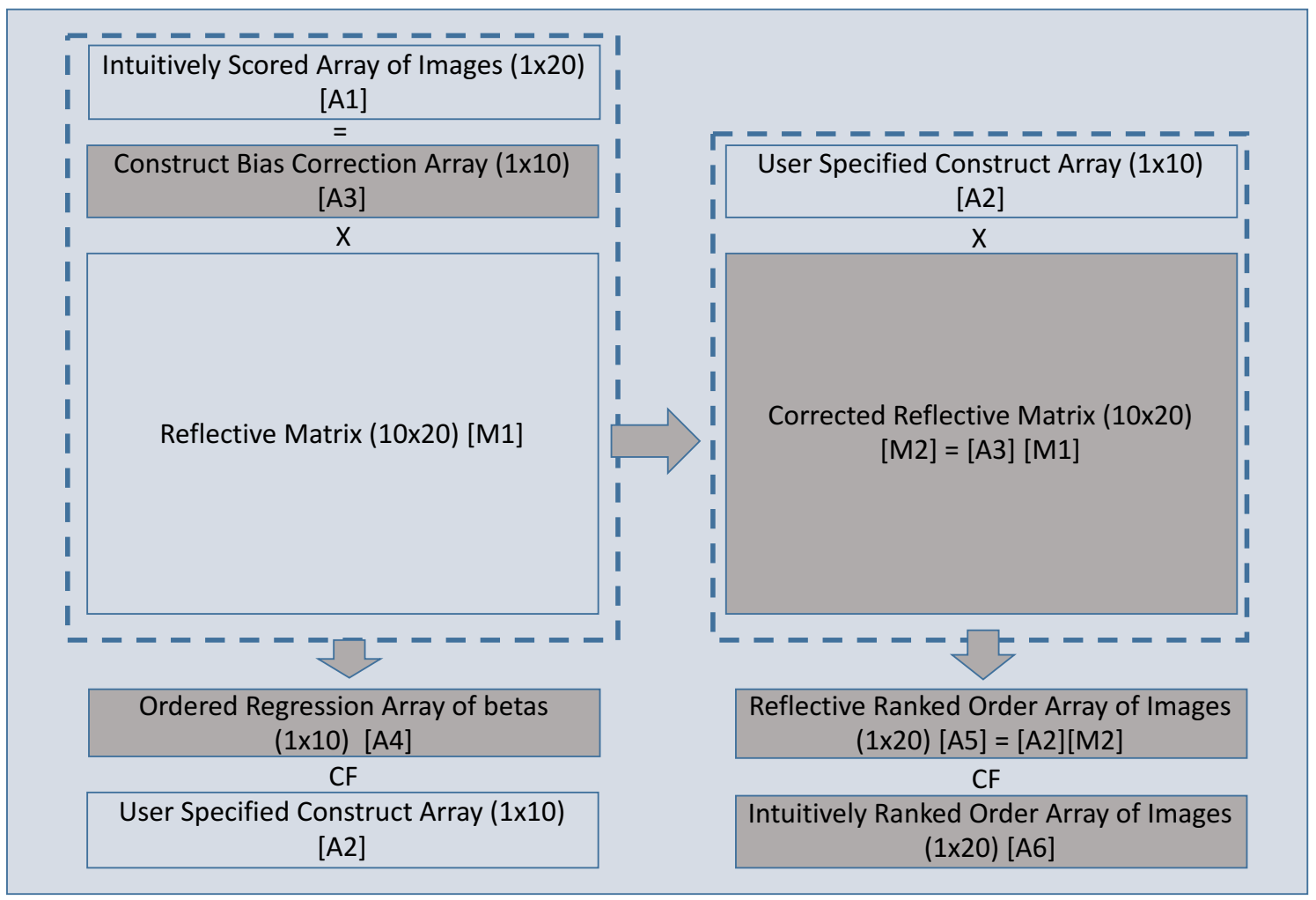

Exhibit 5.5 Schematic of input-output arrays for the IMP Test

\subsection{Statistical Analysis and Interpretation of the Individual's IMP Test Report}

The function of the IMP Test is to generate data inputs that allow inferences to be made linking short time-slice judgement of an image, which, given the time involved, is likely to be predominantly intuitively driven, to the more reflective judgement where time is unconstrained. In modelling, the intuitively driven judgement is taken to be the dependent 
variable and the more reflective judgement across the ten specified constructs of photographic expertise are the independent variables. The model is as follows:

$$
y_{i}=\beta_{0}+\sum_{i=1}^{n} \beta_{i} X_{i}+e_{i}
$$

Where:

$\mathrm{y}_{\mathrm{i}}$ is the dependent variable being the short time-slice judgement of the individual

$X_{i}$ are the independent variables being each of the ten constructs

$\beta_{0}$ is the intercept value

$\beta_{\mathrm{i}}$ is the beta value attaching to each of the ten independent variables.

$\mathrm{e}_{i}$ is the error term

At the individual level (1) of analysis the number of independent variables (10) is large relative to the number of observations (20). Such a data structure is 'fragile' for two reasons: first, the anticipated multicollinearity between the independent variables and, second, the problem of the small number of observations and the risk of 'over-fitting'. Overfitting is where any statistical model describing the relationship between the independent and dependent variables over-explains the residuals. To defeat this problem various modelling techniques were examined for appropriateness given the data structure and its limitations.

However, before analysing the data in depth the problem of 'construct' bias needs to be eliminated from the data. As noted above, during beta testing 20 images were selected which I and the reviewers believed were equivalent in revealing all ten constructs to the same level. It is also the case that some constructs may be more difficult to extract from a given image. On the basis that some constructs are more difficult to reveal than others within a given image, a low average score will be indicative of one that is more challenging to reveal photographically and a high average score one that is less so.

To a certain extent, correcting for the differences in 'construct exposure' is relatively straightforward using grand mean centring. This adjustment entails multiplying each 
individual score for a given construct by the grand mean score for all 106 participants and dividing by the grand mean for the given construct.

$$
\text { Construct Score }_{\text {corr }}=\text { Construct Score }_{\text {raw }} x \frac{\text { Grand Mean }_{\text {all }}}{\text { Grand Mean } \text { construct }_{\text {rand }}}
$$

This corrects the 'raw scores' in two ways: first it adjusts for difference in the revealed exposure of the construct in the text and, second, it changes the magnitude of the spread of an individual's scores (as measured by the standard deviation) around the construct mean. Thus, for the sample candidate whose test outcome is shown in Exhibit 5.7, their average and standard deviation for 'creativity' was 4.35 (out of 10 ) and 0.48 respectively. For all constructs the average score is 6.48 and for the creativity construct 6.01 . Multiplying any individual's raw score by $6.48 / 6.01$ will increase their reported assessment of the creativity in each image compensating for the below average revelation of that construct in the set of 20 test images. It will also tend to increase the measured standard deviation and proportionally increase the covariances used in the statistical modelling methods. The necessary adjustment for each construct was as follows:

\begin{tabular}{|c|c|c|c|c|c|c|c|c|c|c|}
\hline Raw Scores & IMPACT & CREATIVITY & NARRATIVE & DOF & LIGHT & COMP & COL/TONE & FOCUS & EXPOSURE & TECHNICAL \\
\hline Mean & 6.50 & 6.01 & 6.21 & 6.70 & 6.59 & 6.37 & 6.38 & 6.73 & 6.90 & 6.35 \\
\hline Standard Deviation & 0.17 & 0.16 & 0.21 & 0.17 & 0.16 & 0.16 & 0.14 & 0.21 & 0.16 & 0.18 \\
\hline \multicolumn{11}{|l|}{ (Grand mean $=6.48$ ) } \\
\hline Correction factor & 0.995 & 1.077 & 1.042 & 0.966 & 0.983 & 1.017 & 1.015 & 0.962 & 0.939 & 1.019 \\
\hline \multicolumn{11}{|l|}{ Corrected Scores } \\
\hline Mean & 6.48 & 6.48 & 6.48 & 6.48 & 6.48 & 6.48 & 6.48 & 6.48 & 6.48 & 6.48 \\
\hline Standard Deviation & 0.47 & 0.48 & 0.42 & 0.36 & 0.31 & 0.32 & 0.36 & 0.31 & 0.35 & 0.30 \\
\hline
\end{tabular}

\section{Exhibit 5.6 Grand-mean Correction of Raw Scores from the IMP Test}

Statistically, the problem is then devising a modelling strategy that reveals the relationships within the data. Multiple Linear Regression (MLR) is the first and most obvious technique when dealing with a single dependent variable driven by multiple independent variables. However, MLR does make a series of assumptions: first that there is a linear relationship between dependent and explanatory variables (there are solutions to non-linearity if demonstrated); second, all variables are normal with finite variances; third, the independent variables are independent of one another (no multicollinearity), the residuals are of constant variance (zero heteroscedasticity). MLR also demands a large sample size (assumed to be 20 per independent variable) to avoid overfitting. At the individual level the very small number 
of observations generated by the test rules out the parametric and other requirements of (say) traditional multiple linear regression methods.

To overcome the challenges presented by the limited data produced by each test participant, a variant of MLR, Partial Least Squares (PLS) regression, is employed. In outline, PLS modelling is an adaptation of multiple linear regression but where the latent structure of the raw $y$ and the $x$ variables are extracted. In the case of a single dependent variable the latent structure is the variable, but with multiple independent variables the latent structure will consist of bundles of the original variables (components). The first two or three such bundles should, ideally, account for better than $95 \%$ of the variability - or in simpler terms - the 'information' contained within the ten measured variables - the constructs - delivered by the testing procedure. The process is also designed such that the bundles are statistically independent of one another, i.e. have zero-correlation. Statistically, the problem then resolves to finding the regression weights of just two or three latent variables that are the new independent variables in the regression (hence reducing the minimum necessary sample size) and which possess zero correlation with one another (zero-multicollinearity). The weight that materialises for each latent variable can then be regressed against the measured variables in that 'bundle' to generate their respective beta weights.

One of the deficiencies of PLS is that although providing good predictive power provides weaker explanation of the relationships between the variables. To accommodate this multidimensional scaling is employed at all levels $(1-3)$ to give a clear visual interpretation of the data and a degree of confirmation of the relative importance of those constructs that are of significance from the PLS.

The IMP Test generates a number of outputs. Some are straightforward weighted rankings of the images using the subject's declared importance of the ten constructs [A2] - the VIPs or 'variable importance in projection' - and their vote under each [M2]. This ranking of the twenty images [A5] permits comparison with the average of all participants in the test. [A5] also permits comparison with the ranked intuitive ordering of the 20 images generated from [A1] (unranked) to give [A6] (ranked). The ranking [A5] can be modified using the construct weightings generated by the PLS regression [A4]. This is a signal of the quality of the individual's scoring of the images - superior rank correlation suggesting their nonconscious 
weighting of the constructs is a better predictor of general preference than their declared weighting.

The PLS regression generates a series of construct weights [A4] in terms of their significance in explaining the intuitive judgement of the 20 images [A1]. It also generates a number of 'quality' scores of which $\mathrm{R}^{2}$ and $\mathrm{Q}^{2}$ signal the degree of variability in the dependent variable that can be explained by the independent variables. A score of one indicates perfect covariation, zero indicates no association. The output charts from the PLS also give the degree of uncertainty associated with the judgement under each construct.

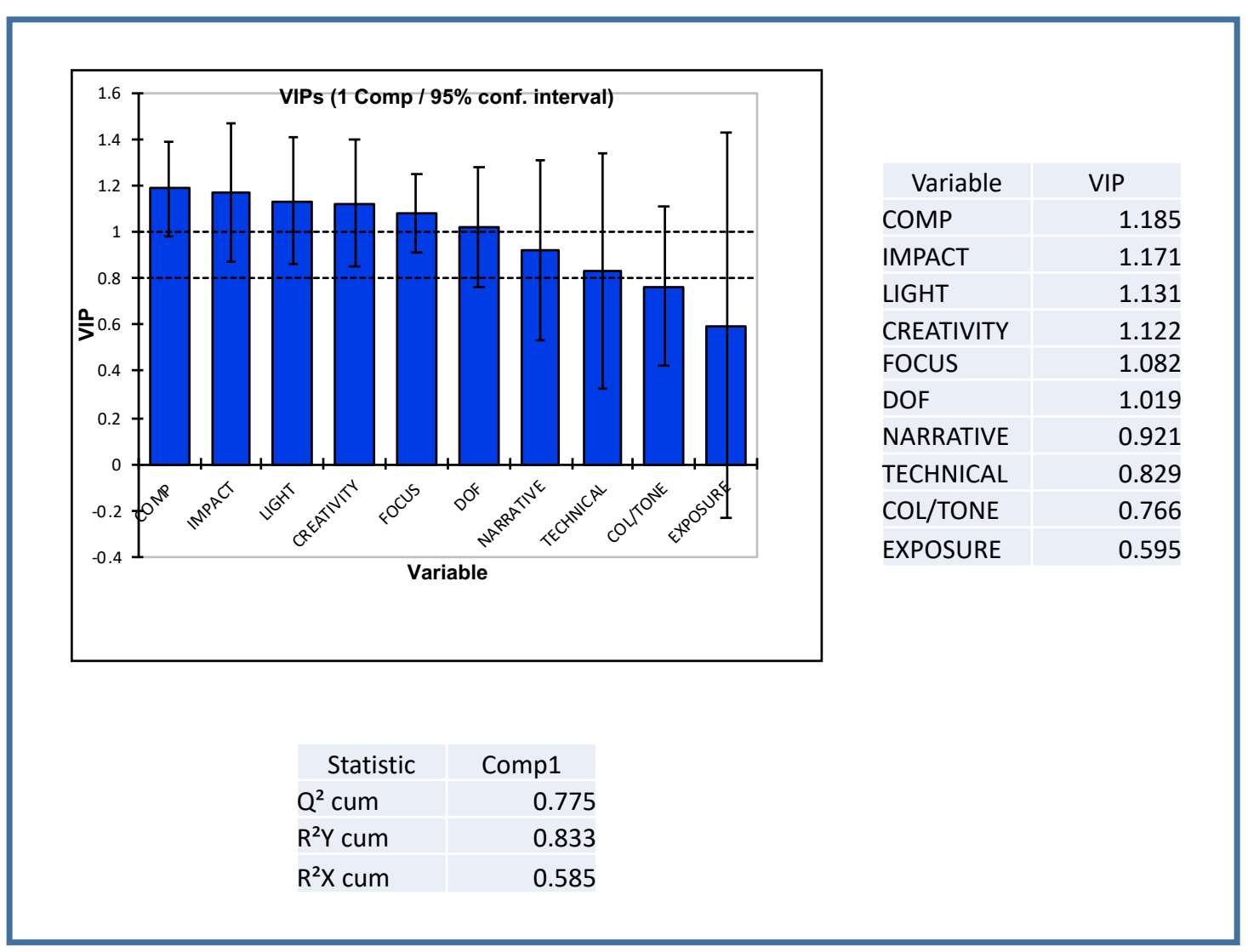

\section{Exhibit 5.7 Output of a PLS Regression (Sample Test Subject)}

Any score above 1 implies significance in prediction so, in Exhibit 5.7, composition, impact, the use of light, creativity, focus and use of depth of field are driving the individual's intuitive judgement. The whisker charts suggest a high degree of uncertainty in the individual's judgement of the four constructs outside the level signalling intuitive engagement of the construct concerned. The $R^{2} Y$, as noted, measures the predictive power of the ten constructs and at 0.833 suggests that a high level of confidence can be placed in the output of the test. 
Although statistically very effective in the analytical process, a second approach using a different method of generating the latent variables gives a much clearer visual presentation of the results. Multidimensional scaling (MDS) is a well-established technique that reveals the similarity between the constructs across two-dimensional space. Comparing the PLS ordering and the MDS display, there is a close similarity between the two outputs. The MDS confirms the PLS ordering but also reflects the correlation between the constructs. In Exhibit 5.8 we note that the photographer concerned appears to closely associate creativity with the use of light.

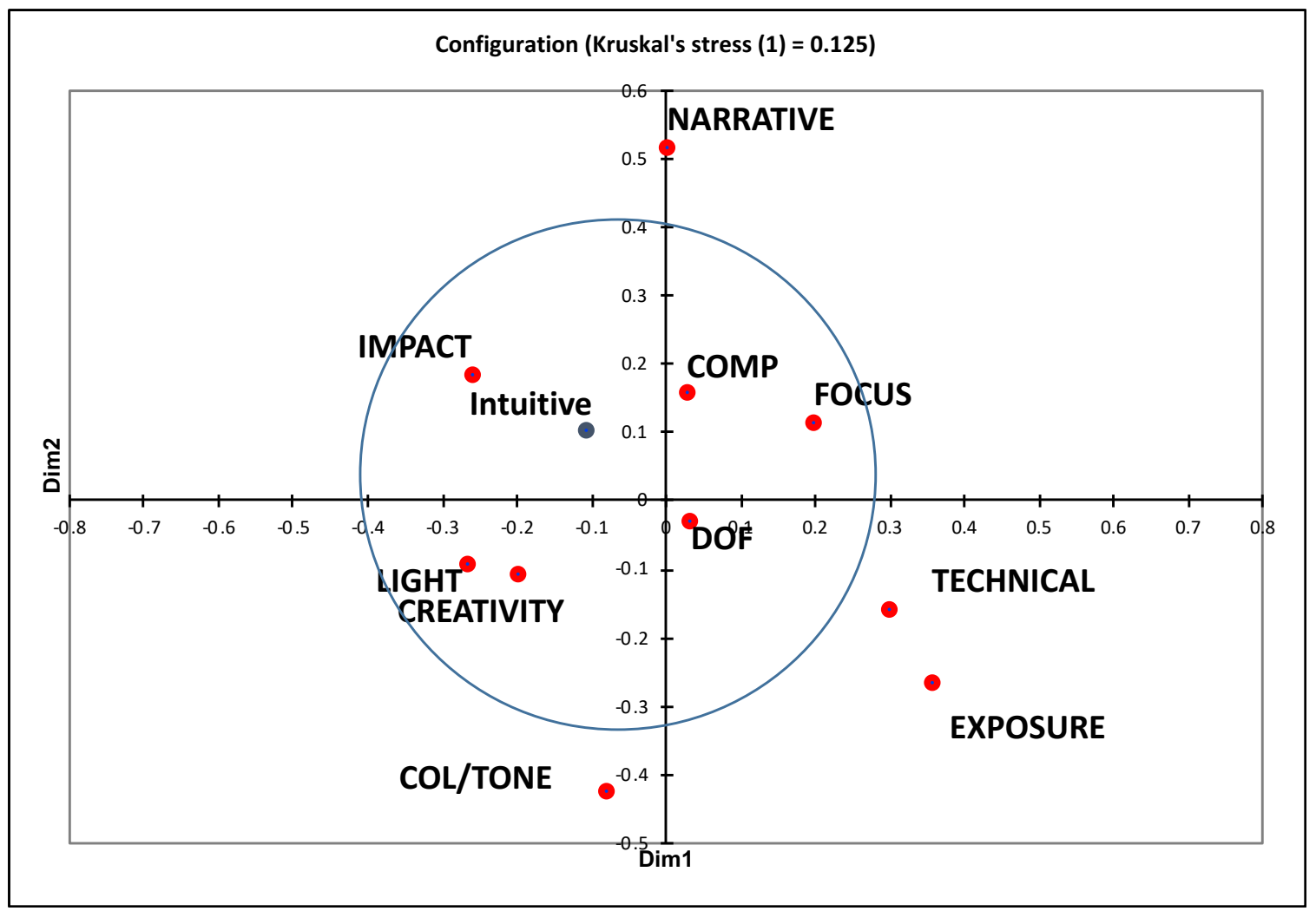

Exhibit 5.8 Multidimensional Scaling of the Scoring of the IMP Test Images (Sample Test Subject)

5.13 Statistical Analysis and Interpretation of the Aggregate and Partitioned IMP Test Reports

In aggregate studies we would normally expect the $R^{2} Y$ of the average assessments to be higher than the average of the $\mathrm{R}^{2}$ from the individual tests. Based upon 106 trials the average 
individual $R^{2} Y$ was 0.603 with a median value of 0.653 and a mode of $0.677^{33}$. The distribution of $R^{2} Y$ shows that it is skewed and part of my investigation of the aggregates will be to investigate the source of the skew and determine the extent to which the candidates scoring above the median differed in any significant way from those scoring below.

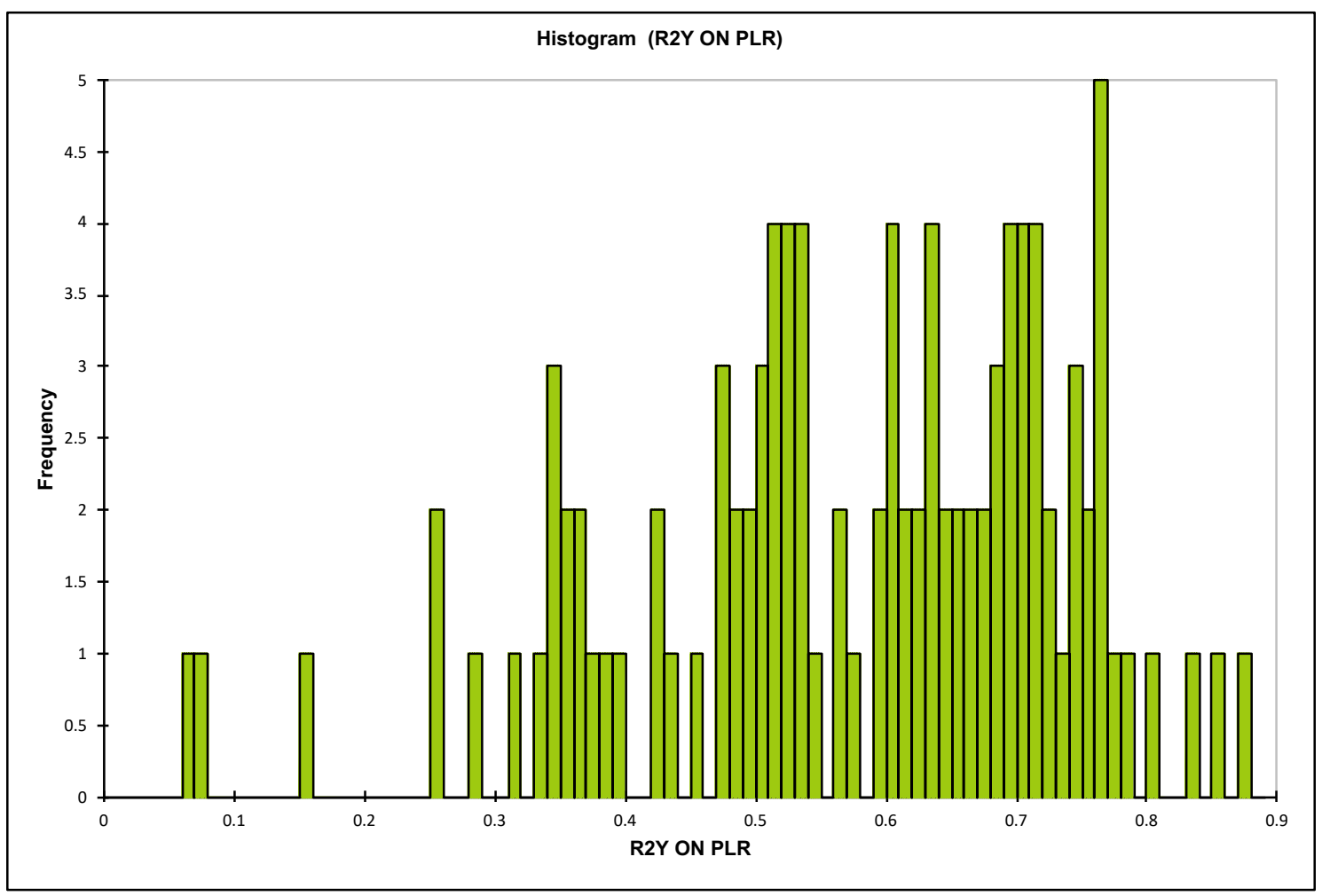

\section{Exhibit 5.9 Histogram of $R^{2} Y$ scores from 106 IMP Test Participants}

The Law of Large Numbers tells us that as we aggregate the participants in a study two things happen: first, the unexplained variability reduces and second, the variability of the dependent variable can be partitioned into two - a systematic and a non-systematic component. When assessing the variability of the returns on an equity share, for example, part will be driven by market forces and part by the drivers of return within the firm. As portfolios are built with greater numbers of shares equally weighted by value, the variability

\footnotetext{
${ }^{33}$ The median is the value that splits the distribution equally with the same number of observations above the median as there are below. The modal value is the one that appears most often and can be described as having the highest probability of being drawn from the distribution. A distribution is 'skewed' if the median, the mode and the mean are significantly different.
} 
attributable to the individual firms tends to cancel one another out (some firms do well, others badly) and eventually the variability of the portfolio converges on the variability of the market as reflected in a broadly-based market index ${ }^{34}$.

In this study we are not, of course, dealing with equity shares but we are, I argue, dealing with random individuals operating independently of one another, who are being influenced by a systematic and a non-systematic driver of variability in their judgement. However, the first study reported in chapter 6 looks at the extent to which the Law of Large Numbers is evident in the data set. A commonly seen function of the relationship between our principal measure of unexplained volatility in the dependent variable $\left(1-R^{2} Y\right)$ and the number of participants in aggregation is shown in figure 5.10. In trials with randomly selected and independent variates there is an asymptotic approach to a constant base value which defines the irreducible uncertainty. The reducible uncertainty represents the uncertainty in judgement that is (on average) idiosyncratic.

The graph in chapter 6 (exhibit 6.10) uses both corrected and uncorrected data and generates an identical result. It is also clear that increasing the number of participants in the study beyond 106 will not increase the statistical validity of inferences about the structure of expertise for the whole group. The function is drawn by randomly selecting ten sub-groups with each of 10, 20, 30 and 50 participants and estimating the $R^{2} Y$ for each. Locating a subgroup by partitioning (e.g., all females) and measuring the $R^{2} Y$ indicates the (a) the confidence that can be placed in the statistical validity of any inferences made about their structure of expertise, and (b) the difference in judgement 'skill' between that sub-group and the randomly generated function.

\footnotetext{
${ }^{34}$ Numerous studies demonstrate that the 'Law of Large Numbers' comes into effect and non-systematic influences on variability are eliminated at between $15-20$ independent and equally weighted participants.
} 


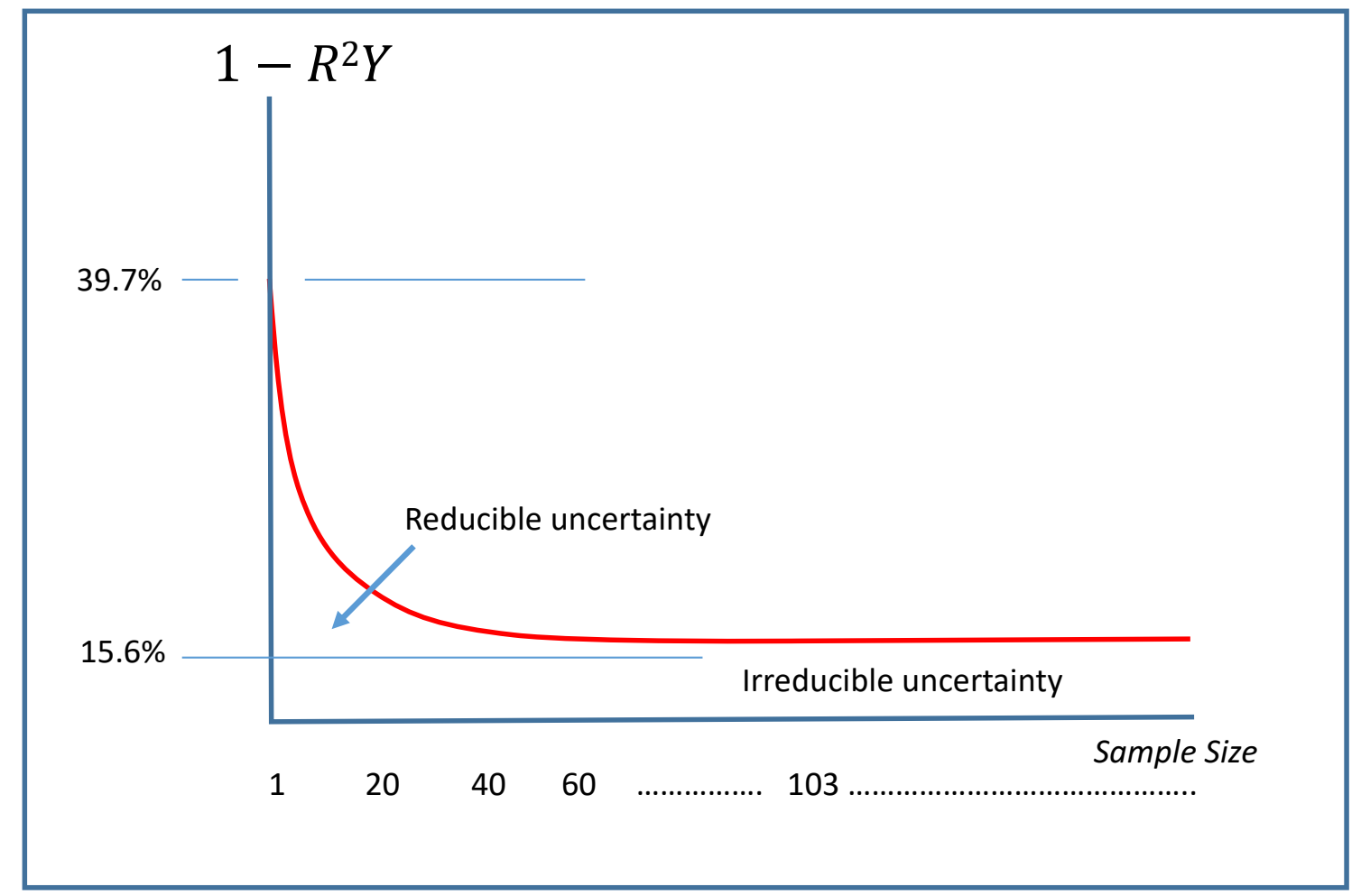

\section{Exhibit 5.10 Variation in $R^{2} Y$ with increasing group size (randomly drawn)}

Noting that the definition of the ten constructs lies with the individual, the uncorrected data suggest that just under $60 \%$ of expertise is a commonly shared 'public' good and just over $40 \%$ is idiosyncratic and largely disappears as we look at the characteristics of sub-group judgement. However, there is a small element of variability in judgement that is not explained by the model at full aggregation - this is what Hammond (1996) refers to as irreducible uncertainty. We can only speculate as to what this may be attributable to. Partly, it will be uncertainty induced by the analytical model (PLS) that seeks and maybe cannot locate latent variables that are perfectly uncorrelated. We may have a degree of uncertainty introduced because the IMP Test requires integer inputs. This should not be a problem in stage 1 or 2 of the test but may be a problem in stage 3 where an integer between 0 and 10 must be chosen. However, another explanation is that there is a missing construct that individuals are using that is not captured by the ten construct signifiers brought forward from chapter 3 and used within the IMP Test.

Given our theoretical framework centred on the Bayesian driven Hierarchical Predictive Processing model of human judgement, it is likely that the data structure will be hierarchical and that other 'latent' as opposed to 'measured' variables will be in play. One strategy for 
eliciting the underlying structure is Partial Least Squares Path Modelling (PLS-PM). Like Structural Equation Modelling, PLS-PM is designed to elicit the causal relationships that exist between defined or revealed latent variables and between those latent variables and the measured or, as they are sometimes referred to, 'manifest' variables. So, we might propose that of the ten photographic constructs scored by participants a smaller number of latent variables could be inferred and named from that data. For example: 'focus' and 'depth of field' might be related by a latent variable we might label 'clarity'; 'exposure", 'use of light' and 'use of colour' might be related by a latent variable called 'energy' and so on. Neither 'clarity' or 'energy' form part of the common language and understanding of photography but might well emerge from such a modelling exercise.

However, in my view, an extensive exercise of this nature would only be worthwhile if a latent structure is apparent from the PLS modelling, the MDS and, crucially, the intraspective interviews. For the purposes of this research, I have not pursued a formal PLS-PM route for two reasons. First, I am of the view that the credibility of the IMP Test depends upon its alignment with the experience of the individual and would not be enhanced by further recourse to 'industrial strength' statistical method. Second, there is an avenue of discovery that uses the insights from both the quantitative and qualitative method in a more convincing way. That is the approach I have taken in this thesis - particularly as much of the latency in the structure is likely to be an emergent property of particular combinations of the subordinate constructs.

\subsection{The Intraspective (Cognitive) Interviews}

The introduction of interviews into the study was intended to serve the following purposes and in so doing achieve RO4:

(i) To identify whether the constructs and their priorities as revealed by the IMP Test were reflected in the individual's judgement at the moment of capture. 


\begin{tabular}{|l|}
\hline RO4: To explore, using \\
intraspective interviewing \\
methods, the individual's \\
conscious and \\
nonconscious boundary \\
experience in the exercise of \\
photographic judgement.
\end{tabular}

(ii) To validate the relationships between the constructs as revealed by the IMP Test.

(iii) To find evidence for a latent structure behind the constructs.

It was evident that conventional interviewing or questionnaire methods would be inappropriate. Much would depend upon my interpretive skill both in situ and, subsequently, in analysing the interview transcripts.

Achieving enhanced depths of recall, cuing appropriate emotional responses and managing the flow of the discussion is a dynamic process. It was clear, that conventional methods would not achieve the ends I sought, that a variant of both funnelling and Cognitive Interviewing would be necessary.

Funnelling is a process whereby the response to a general question is used to trigger a more specific, derivative question and so on until the line of enquiry is exhausted (Matsumoto, Hwang et al. 2015). Cognitive Interviewing is a well-developed method for activating recall at greater depth than conventional interviewing methods (Geiselman, Fisher et al. 1985, Geiselman and Fisher, 2014). It has its antecedents in Interpretive Phenomenological Analysis, and in the techniques of gathering evidence from witnesses in a criminal investigation. The outline process can be described as: reinstate the context, recall everything, recall the events in different order and change perspectives. Geiselman, Fisher et al. (1985) report that the method can increase the number of elements in recall by $25 \%$ whilst maintaining the same error rate.

However, cognitive questioning has limitations in that its primary purpose is to elicit literal recall of events exposing tiny details that may allow the investigator to construct or validate a broader narrative. My task was to extend the positive elements of the funnelling and Cognitive Interviewing approach but combining both prompts for literal recall and emotional cues into a loosely structured and informal discursive interview. The advantage of this approach is that it helps the subject recall critical moments of their experience at both a conscious and a nonconscious level. In this I disagree with Ten Hoor's analysis of the concept of introspection, which he and subsequent writers limited to the conscious realm (Ten Hoor, 
1932). As such, with the assistance of the questioner, the interview operates in the boundary layer between the two modes of thought, bringing the subject, by a process of advance and retreat, progressively towards both a literal and an emotional recall of the decisive moment of capture. As I discussed in chapter 4 , this interaction between the conscious and the nonconscious is played out in the boundary layer between the two modalities by a process of recollection and resolution. This interplay gives the method its intraspective character. The method does present significant challenges for the interviewer who has to be both affectively and cognitively engaged in the process whilst probing the interviewee's experience. One interviewee commented that it felt like fruit being shaken loose from a tree as memories suddenly returned and took on a new meaning.

The process is unstructured with no specific questions set. However, I found an interview protocol of great help as an aide memoire. For this exercise, the following was developed through four pilot interviews and it helped me in the initial stages of gaining fluency in this type of interview ${ }^{35}$. One danger of a protocol is that it suggests a linear flow whereas the 'advance-retreat' mechanic is important in destabilising the process of conscious recollection and giving opportunity for the nonconscious activation of memory to work.

The analytical process is reductive and interpretive. As the interview proceeds to the heart of the moment of capture, the language used by the interviewee becomes more indicative of the structure of their judgement and hence their expertise. In this the method borrows from the reductive process in Interpretive Phenomenological Analysis where the subject is drawn, step-by-step, to the heart of their experience.

\footnotetext{
${ }^{35}$ My training in interviewing has been extensive and varied. First, as an auditor going through the investigation branch with a major London firm, second as a CNAA advisor and programme reviewer and finally as an institutional auditor for the UK Quality Assurance Agency. Funnelling was the method of choice in the training programmes at each stage of gathering experience although Cognitive Interviewing was being practiced in a primitive form in the days of my audit training.
} 


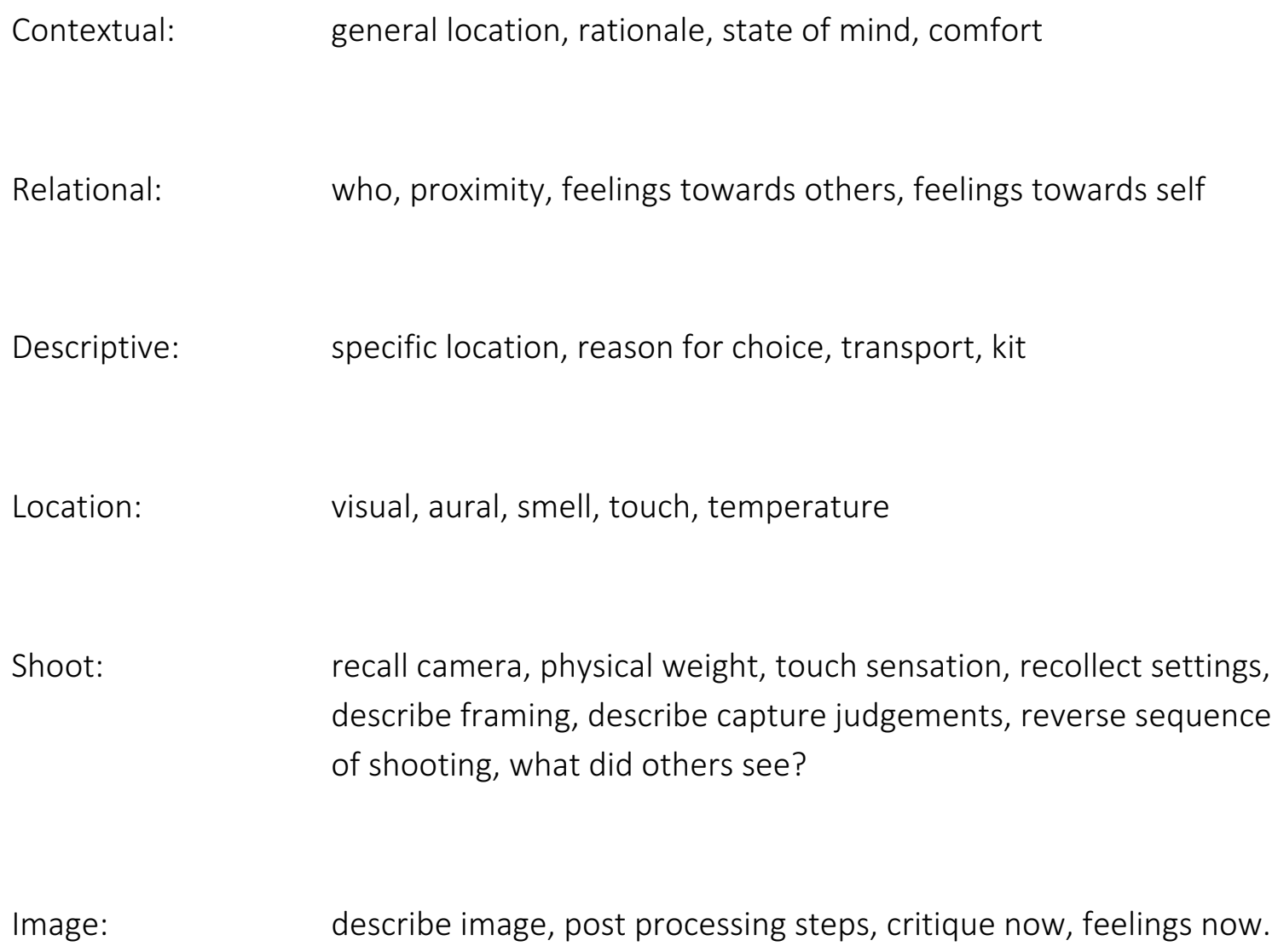

\section{Exhibit 5.11 Protocol for the Intraspective Interviews}

Candidates were selected from the last stages of the study on the basis of (i) gender, (ii) favoured genre, and (iii) the quality metrics from the regression. In selecting and inviting the sample my aim was to challenge the IMP Test across different dimensions. After 16 interviewees I formed the view that data saturation had been achieved in terms of the interview purpose outlined above.

A significant problem with this interviewing process was and is confirmation bias and whether the IMP Test is taken first or second - bias of one form or another can work either way. However, to enable sampling some of the raw output of the test was important in ensuring a fair cross-section was chosen. Therefore, invitation to participate in an interview was only given once the test had been taken and the quality metrics $R^{2} Y$ and $Q^{2} Y$ generated by the modelling obtained. However, to avoid as far as possible bias on my side, I did not examine the test data sheets before interview and avoided, as far as possible, the use of the construct terms during the interview. Where an interviewee introduced a construct term or analogue spontaneously, then follow-up questions did include the term where it was necessary to 
probe the judgement driving its use. All but four of the interviews were conducted face to face. In four cases the interview was conducted through Skype with in-system recording of the conversation. All the interviews were recorded and subsequently transcribed by an independent transcriber who is also a psychology graduate.

Each interviewee was invited to present three images that were indicative of their practice, had been taken within the previous six months, and had high emotional salience for them.

The strategy for analysis proceeds through the following steps:

1. Bracket down to experience of the image claiming words and phrases indicative of the individual's photographic intent.

2. Bracket to candidate's words and phrases about the images.

3. Examine the narrative for pauses, reasoning words and phrases, emotional words and phrases.

4. Identify explicit and implicit associations between both conscious and nonconscious reference to constructs and associated terms.

5. Word cloud and partition discourse into the ten constructs looking for linking narratives and any appeal to unifying (latent) concepts.

\subsection{Selection of the Interviewees}

The interviewees were drawn from those taking the IMP Test during the second half of the research process. Twenty-two invitations were given and two declined because of pressure of other commitments. All were given a written invitation with details of the process and the fact the interview would be recorded. Each replied confirming their willingness to proceed in writing. The appropriate number of interviews is a vexed question in qualitative research but the key issue, in my view, is not to make any appeal to the Law of Large Numbers and the rigours of selection required in quantitative research. In making my selection I followed the excellent advice in Smith, Flowers et al. (2009).

As I have noted above, the purpose of the interviews was to inform the outcome of the IMP Test and my selection process worked in three stages: four initial interviews were conducted to test the process and were discarded, twelve block selected in that all respondents to the 
IMP Test over a period of time were invited to participate, and four professionally engaged in photography were specifically invited. The only rule I imposed in the process was that interviewees could not have the results of the test prior to the interview and, apart from running the analytical software, I would not engage in the in-depth review of the results until the interview was concluded.

Data saturation is a slippery concept but is meant to imply that there comes a point in a qualitative research programme that further interviewing is not contributing to the researcher's state of knowledge. What is acceptable in any research process is debatable and, in my view, it depends upon (a) what the interview is seeking to achieve and (b) the basis upon which the selection of interviewees is made. I had become aware that I rarely received any acknowledgement of the receipt of the test results from those who took it - anecdotal evidence suggested that it was being well received but I had no hard evidence. Therefore, my primary motive was to stress test the IMP Test both in image assessment and in image capture. Furthermore, when examining the early data generated by the IMP Test patterns started to emerge that led me to believe that some latent variables were driving the results. That latency would be important in developing the conceptual basis of the IMP Test.

\subsection{Confidence and Likelihood Estimation in Multi-Method Research}

In statistical research the probability that a hypothesis is confirmed or refuted is expressed in likelihoods referred to as 'confidence levels'. If a hypothesis is accepted, or the null refuted at the $95 \%$ level, then it is regarded as 'extreme likely' to be true. In common law jurisprudence the $95 \%$ level is taken to signify 'beyond reasonable doubt' - although some place the attribution of guilt in a criminal case at a significantly lower probability (Weinstein and Dewsbury, 2007). However, in multi-method research methods using corroborative or triangulated techniques 'confidence' can be given a more general meaning and should, in my view, be distinguished from measures of statistical likelihood.

One area where significant methodological developments have occurred in the assessment of evidence is in the UN's International Panel of Climate Change (IPCC) which published its fifth assessment report (AR5) in 2014. In that report it adopted definitions from previous 
assessments and issued as guidance notes to Lead Authors (Mastrandrea, Field et al. 2010) and as brought together in Mastrandrea et al. (2011). The IPCC's mandate is to examine the evidence of anthropogenic influences on the climate and that evidence has been adduced from disciplines that are, in the main, within the conventional view of 'scientific'. Although social science disciplines such as energy economics have conditioned the judgements made, the primary problem is how to combine computer-based, ensemble modelling with measurements drawn from a wide range of incomplete data sources. Because of this, the confidence scale used by the IPCC has emerged using a different scaling when compared with the conventional ' $p$ ' scale measures of confidence intervals in statistics. Taking each line of evidence gathered across the whole field of climate and cognate research, none would be regarded as incontrovertible. However, the consilience of evidence and the agreement across the community of knowledgeable practitioners about its significance is deemed to be overwhelming.

The clear difference with a single author study such as this is in determining agreement. For this, I take agreement to mean that a reasonable interpretation of the alternative legs of the research would indicate a common result. With a given individual, if the statistical testing suggests that the independent variables 'explain' (as in the sample case above) $83.3 \%$ of the variability in their intuitive judgement then, in forming a base rate probability in the triangulation, I would attribute $83.3 \%$ weight to the output of the IMP Test PLS regression and $16.7 \%$ weight to alternative methods. Such alternatives would be (a) the multidimensional scaling, which varies the partitioning and composition of the latent variables describing distance, (b) interviews with the photographer concerned, (c) direct observation and evaluation of his or her images, and (d), any theoretical support available within the literature. In the case above, at $83.3 \%$ and given the agreement with the MDS I would place a high level of confidence that, at the point of testing, the IMP Test procedure had correctly identified the driving signifiers of the individual's expertise and that it was very unlikely that alternative signifiers would have generated a higher $\mathrm{R}^{2}$. 


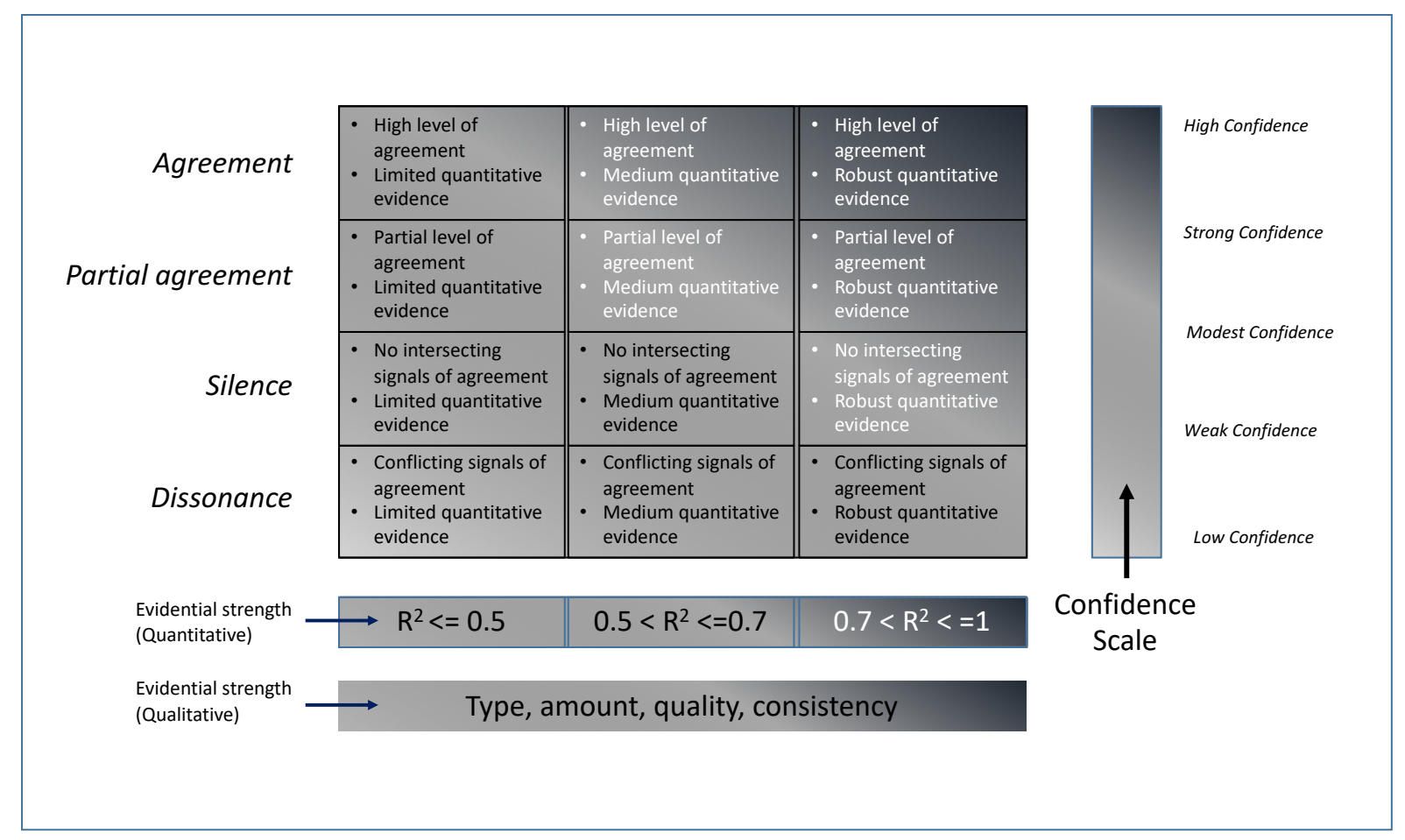

Exhibit 5.12 Evidential communication in multi-method research ladapted from Mastrandrea et al (2011))

\subsection{Conclusion}

In an ideal world research design should precede the gathering of appropriate data or the conduct of experiments to conform or refute well-formed research hypotheses. In the real world, research rarely proceeds that way. This project was initiated through a testing method developed for another purpose. Its evolution into the full-scale research exercise presented here came about as the potential of the test to explore the psychology of the photographic moment became apparent. Good research method has to be malleable particularly when engaging with a problem over a lengthy period of time and particularly when issues emerge during the research process. In this chapter I have reviewed the choices I made and pointed to their implications. Do my methods perfectly reflect my philosophical position addressed in chapter 2? In one respect they do. One outcome of this research is that individual judgement has significant differences from that of a group - this is a consequence of the Law of Large Numbers and results in the 'Wisdom of the Crowd' effect. However, it is also a consequence of my ontology in that a group is always more than the sum of its parts and less than its effects. This is a theme I will return to in subsequent chapters. 


\section{Chapter 6 - Empirical Validation of the IMP Test}

\subsection{Introduction}

In this chapter I focus on the realisation of my research objective RO2:

\section{RO2: To develop an empirical protocol, based upon RO1, that enables the exploration of the exercise of intuitive expertise in photographic judgement}

This chapter presents the results of my research into the IMP Test and the empirical protocol that generates its outcomes. As I noted in chapter 5, drawing inferences about an individual's intuitive photographic expertise depends not only on the test but the analytical procedures used in forming a confident judgement based upon its output. I will present evidence concerning the test's internal consistency and validity. Part of the problem of validity and building confidence is the degree to which the test points to the underlying psychological processes of the photographers concerned. To this end the IMP Test ${ }^{36}$ must face the challenge of experience and so the evidence presented in this chapter will be an integration of its outcomes with my analysis of the intraspective interviews.

In examining the results of the IMP Test, I will evaluate the evidence at two levels: first, in this chapter, at the aggregate level of all 106 test participants. In the next chapter I will focus on different sub-groups. At this level of analysis, we are dealing with averages: the average three-second appraisal of each image, the average ranking by test participants of the ten constructs and the average assessment of the quality of each image against each of those constructs. In the process of averaging, the individual's idiosyncrasy in judgement will disappear and what drives the overall judgement is the core of commonly shared understanding of the meaning of each of the ten constructs.

\footnotetext{
${ }^{36}$ The IMP Test can be obtained from www.G2GPhoto.com or from https://bourchierbooks.com/imp-test/ where full instructions and response forms are provided.
} 
The second level of assessment is where each individual's judgement is taken into account irrespective of their understanding of the ten constructs, whether at a conscious or a nonconscious level. In this stage of the analysis, I will examine the structure of individual as opposed to aggregate judgement using the constructs identified by the IMP Test as active in driving their three-second judgement.

This analysis does not yield any evidence about the meaning attributed to each construct by the test participants. It does reveal the degree to which they are associated, and it also suggests how the balance lies between commonality and idiosyncrasy in their meaning.

\subsection{The IMP Test Images}

Twenty Images were presented to each test subject (see the appendix) and their 'intuitive' judgement and their analytical judgement recorded. Their average judgement for each image, corrected for construct bias, was as follows:

\begin{tabular}{|c|c|c|c|c|c|c|c|c|c|c|c|}
\hline Image & INTUITIVE & IMPACT & CREATIVITY & NARRATIVE & DOF & LIGHT & COMP & COL/TONE & FOCUS & EXPOSURE & TECHNICAL \\
\hline $6^{7}$ & 53.58 & 6.69 & 6.00 & 7.63 & 5.97 & 5.71 & 5.76 & 6.01 & 6.41 & 6.58 & 6.27 \\
\hline $7^{7}$ & 54.79 & 5.42 & 5.03 & 4.75 & 6.99 & 5.67 & 5.28 & 6.32 & 7.26 & 6.93 & 6.18 \\
\hline 8 & 69.99 & 8.10 & 6.75 & 7.26 & 7.90 & 7.13 & 7.63 & 7.04 & 8.03 & 7.31 & 7.79 \\
\hline 9 & 66.59 & 6.71 & 6.46 & 5.83 & 7.07 & 7.70 & 7.11 & 7.62 & 7.06 & 7.31 & 7.01 \\
\hline $10^{2}$ & 47.92 & 4.61 & 4.92 & 3.82 & 6.16 & 6.18 & 5.04 & 6.07 & 7.01 & 6.82 & 6.22 \\
\hline $11^{7}$ & 56.00 & 6.90 & 8.23 & 5.78 & 6.13 & 6.18 & 6.40 & 6.20 & 6.50 & 5.92 & 6.80 \\
\hline 12 & 54.14 & 6.76 & 7.02 & 6.68 & 6.32 & 6.18 & 6.15 & 5.98 & 6.58 & 6.27 & 6.19 \\
\hline $13^{7}$ & 70.55 & 8.18 & 7.48 & 8.71 & 7.42 & 7.16 & 8.00 & 7.15 & 7.27 & 6.91 & 7.63 \\
\hline 14 & 59.74 & 5.71 & 6.04 & 5.30 & 6.52 & 6.70 & 6.23 & 6.70 & 7.14 & 7.03 & 7.01 \\
\hline $15^{-}$ & 73.75 & 7.93 & 7.53 & 8.03 & 7.81 & 7.75 & 7.98 & 7.82 & 7.97 & 7.71 & 8.08 \\
\hline 16 & 61.12 & 6.31 & 6.19 & 7.21 & 6.91 & 6.18 & 6.90 & 5.52 & 6.10 & 6.08 & 6.06 \\
\hline $17^{7}$ & 58.08 & 6.57 & 6.51 & 6.30 & 7.28 & 6.98 & 6.81 & 7.18 & 7.38 & 7.22 & 7.26 \\
\hline $18^{\circ}$ & 68.03 & 7.06 & 7.21 & 7.81 & 6.45 & 7.20 & 6.89 & 7.43 & 6.80 & 7.17 & 7.21 \\
\hline $19^{7}$ & 71.30 & 7.57 & 7.62 & 7.77 & 7.55 & 7.27 & 7.61 & 7.06 & 7.15 & 7.04 & 7.32 \\
\hline $20^{\circ}$ & 31.75 & 3.58 & 3.66 & 4.09 & 2.80 & 2.81 & 3.76 & 3.75 & 1.92 & 2.80 & 2.65 \\
\hline 21 & 51.72 & 6.54 & 6.46 & 7.82 & 6.98 & 6.81 & 7.00 & 7.01 & 6.93 & 6.91 & 6.93 \\
\hline 22 & 52.23 & 6.12 & 6.21 & 6.68 & 5.73 & 6.95 & 6.27 & 6.82 & 5.04 & 6.05 & 5.41 \\
\hline $23^{7}$ & 57.68 & 6.44 & 6.83 & 6.47 & 5.98 & 5.99 & 6.25 & 5.80 & 5.73 & 6.04 & 5.95 \\
\hline 24 & 53.73 & 6.35 & 6.21 & 5.41 & 6.32 & 6.77 & 6.20 & 6.10 & 6.70 & 6.14 & 6.26 \\
\hline $25^{F}$ & 53.10 & 5.94 & 7.18 & 6.17 & 5.21 & 6.19 & 6.26 & 5.94 & 4.54 & 5.29 & 5.27 \\
\hline
\end{tabular}

\section{Exhibit 6.1 corrected average scores by image, intuitive judgement and construct.}

Given the construct correction the average score under each construct was 6.48 (see Exhibit 5.2). Weighting by their declared order of importance (Rank Rational), and the implied order of importance (Rank Exp+Rat) from the IMP Test generated the rankings and differences from the consensus shown in Exhibit 6.2. 


\begin{tabular}{|ccccccc|}
\hline IMAGE & $\begin{array}{c}\text { Rank } \\
\text { Experiential }\end{array}$ & $\begin{array}{c}\text { Rank } \\
\text { Rational }\end{array}$ & $\begin{array}{c}\text { Rank } \\
\text { Exp+Rat }\end{array}$ & $\begin{array}{c}\text { Difference } \\
\text { RvE }\end{array}$ & $\begin{array}{c}\text { Difference } \\
\text { EvIR }\end{array}$ & $\begin{array}{c}\text { Difference } \\
\text { RvIR }\end{array}$ \\
$\mathbf{6}$ & 15 & 13 & 13 & 2 & 2 & 0 \\
$\mathbf{7}$ & 12 & 17 & 17 & 5 & 5 & 0 \\
$\mathbf{8}$ & 4 & 3 & 3 & 1 & 1 & 0 \\
$\mathbf{9}$ & 6 & 6 & 6 & 0 & 0 & 0 \\
$\mathbf{1 0}$ & 19 & 19 & 19 & 0 & 0 & 0 \\
$\mathbf{1 1}$ & 11 & 9 & 9 & 2 & 2 & 0 \\
$\mathbf{1 2}$ & 13 & 11 & 11 & 2 & 2 & 0 \\
$\mathbf{1 3}$ & 3 & 2 & 2 & 1 & 1 & 0 \\
$\mathbf{1 4}$ & 8 & 10 & 10 & 2 & 2 & 0 \\
$\mathbf{1 5}$ & 1 & 1 & 1 & 0 & 0 & 0 \\
$\mathbf{1 6}$ & 7 & 12 & 12 & 5 & 5 & 0 \\
$\mathbf{1 7}$ & 9 & 7 & 7 & 2 & 2 & 0 \\
$\mathbf{1 8}$ & 5 & 5 & 5 & 0 & 0 & 0 \\
$\mathbf{1 9}$ & 2 & 4 & 4 & 2 & 2 & 0 \\
$\mathbf{2 0}$ & 20 & 20 & 20 & 0 & 0 & 0 \\
$\mathbf{2 1}$ & 18 & 8 & 8 & 10 & 10 & 0 \\
$\mathbf{2 2}$ & 17 & 16 & 16 & 1 & 1 & 0 \\
$\mathbf{2 3}$ & 10 & 15 & 15 & 5 & 5 & 0 \\
$\mathbf{2 4}$ & 14 & 14 & 14 & 0 & 0 & 0 \\
$\mathbf{2 5}$ & 16 & 18 & 18 & 2 & 2 & 0 \\
\hline
\end{tabular}

Exhibit 6.2 Image Ranking and Differences

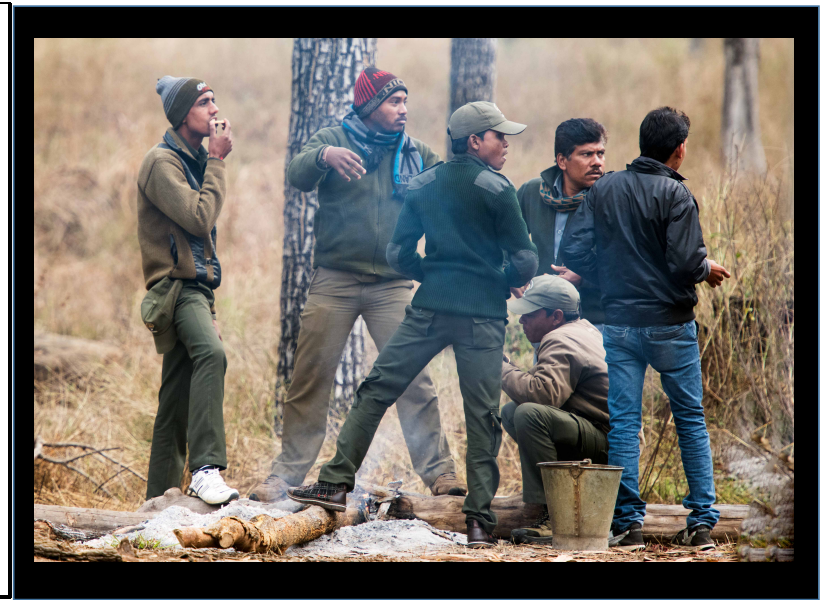

Exhibit 6.3 Image No. 21 -maximum disagreement: intuitive rank $=18$, rational rank $=8$

Exhibit 6.2 shows a reasonable degree of consistency in the scoring with some notable exceptions (images 7, 16, 21 and 23). One can speculate on why the differences have occurred but that, in my view, is counterproductive to the logic of the test where participants and anyone who views the images will possess their own understanding of the constructs and how they should be conjugated in either cognitive modality. Viewing Exhibit 6.3 the test subject will form their own view of the quality of the image or, if looking through the viewfinder, would recognise the event unfolding and decide whether or not to press the shutter. They may be of the view that it is worth more, the same, or less than the average of 7.82 for narrative power or 6.54 for impact. Nevertheless, the individual in taking the test, however they score it, will reveal the structure of their judgement in terms of how their assessment under these and the other constructs counter-varies across the 20 images. It is this counter-variation that the IMP Test explores both at the aggregate and at the individual level.

\subsection{Analysis of the Construct Ranking (Aggregate Study)}

The data at both the individual and the aggregate level is statistically fragile. First, given the nature of the constructs and their 'fuzziness' they will not pass the test of independence, i.e. in statistical terms they will, to varying extents across the ten, be collinear. The level of 'multicollinearity' in the data means that the variance attaching to the significance of each construct 
will be inflated. The VIF (Variable Inflation Factor) measures the extent of this effect as shown in Exhibit 6.4:

\begin{tabular}{|l|r|r|r|r|r|r|r|r|r|r|}
\hline \multicolumn{1}{|c|}{ Statistic } & IMPACT & CREATIVITY & NARRATIVE & DOF & LIGHT & COMP & COL/TONE & FOCUS & EXPOSURE & TECHNICAL \\
\hline$R^{2}$ & 0.957 & 0.891 & 0.914 & 0.993 & 0.983 & 0.995 & 0.955 & 0.997 & 0.990 & 0.997 \\
\hline Tolerance & 0.043 & 0.109 & 0.086 & 0.007 & 0.017 & 0.005 & 0.045 & 0.003 & 0.010 & 0.003 \\
\hline VIF & 23.331 & 9.163 & 11.613 & 137.163 & 58.662 & 202.853 & 22.456 & 298.033 & 96.849 & 298.574 \\
\hline
\end{tabular}

\section{Exhibit 6.4 Variable Inflation Factors for each of the ten constructs.}

With no multi-collinearity the VIF should be close to zero but, as theory would anticipate and this test confirms, the ten constructs are highly related.

The second problem is overfitting, where there are a limited number of observations (i.e., number of images scored). In such circumstances, multiple linear regression (MLR) tends to model the 'noise' in the data rather than the underlying relationship between intuitive judgement and the ten constructs. To surmount this problem, I have adopted a 'belt and braces' approach using three different modelling and data visualisation methods: partial least squares regression (PLS), principal components regression (PCR), and multidimensional scaling (MDS). Each method uses a different approach to data reduction and offers a different perspective on the drivers of intuitive judgement ${ }^{37}$. Clearly, the input data remains the same, but the different modelling and data visualisation methods make different assumptions about the processing of the data. A high level of agreement, between the three methods will enhance confidence that any common pattern or signal of significance is not an artefact of one method versus the other. Differences, where they occur suggest avenues of enquiry into the quality and consistency of the judgement process employed

Unlike conventional MLR, the objective of this analysis is to determine the rank order of significance of the ten constructs in the individual's intuitive judgement, rather than predict

\footnotetext{
${ }^{37}$ Partial Least Squares (PLS) regression and Principal Components Regression (PCR) work in similar ways by creating 'bundles' of the independent variables (the ten construct scorings) which have statistical properties such that the bundles can be used in a much simpler regression model. However, PLS recognises variation in the dependent variable - the three-second judgements - whereas PCR works solely with the independent variables.
} 
what their intuitive judgement would be, given their scoring of the ten constructs ${ }^{38}$. As noted in chapter 5 , partial least squares (PLS) regression generates a ranking by variable importance in the projection (VIP). This gives a strong indicator of the constructs responding in the intuitive moment. It also gives a strong pointer to the latent variables hidden behind the data (see: Chong and Jun, 2005 and Akarachantachote, Chadcham et al. 2014). The established view is that the cut-off value for the VIP is 1 (the average of the squared values of the VIP will equal 1). This is a good guide, but care needs to be taken when considering highly correlated constructs or where the range of the VIPs is low.

For the average outcomes for all 106 participants shown above, the PLS regression orders the constructs as shown in Exhibit 6.5. Four constructs are of minimal importance when determining their influence on the dependent variable at the aggregate level. They are: creativity, narrative power, focus, and exposure. The use of colour/tonality is very close to one and is counted as a minor driver of intuition. The $\mathrm{R}^{2} \mathrm{Y}$ attaching to the dependent variable is $84.3 \%$ and this is the most appropriate of the three quality measures for explaining the variability in intuitive judgement.

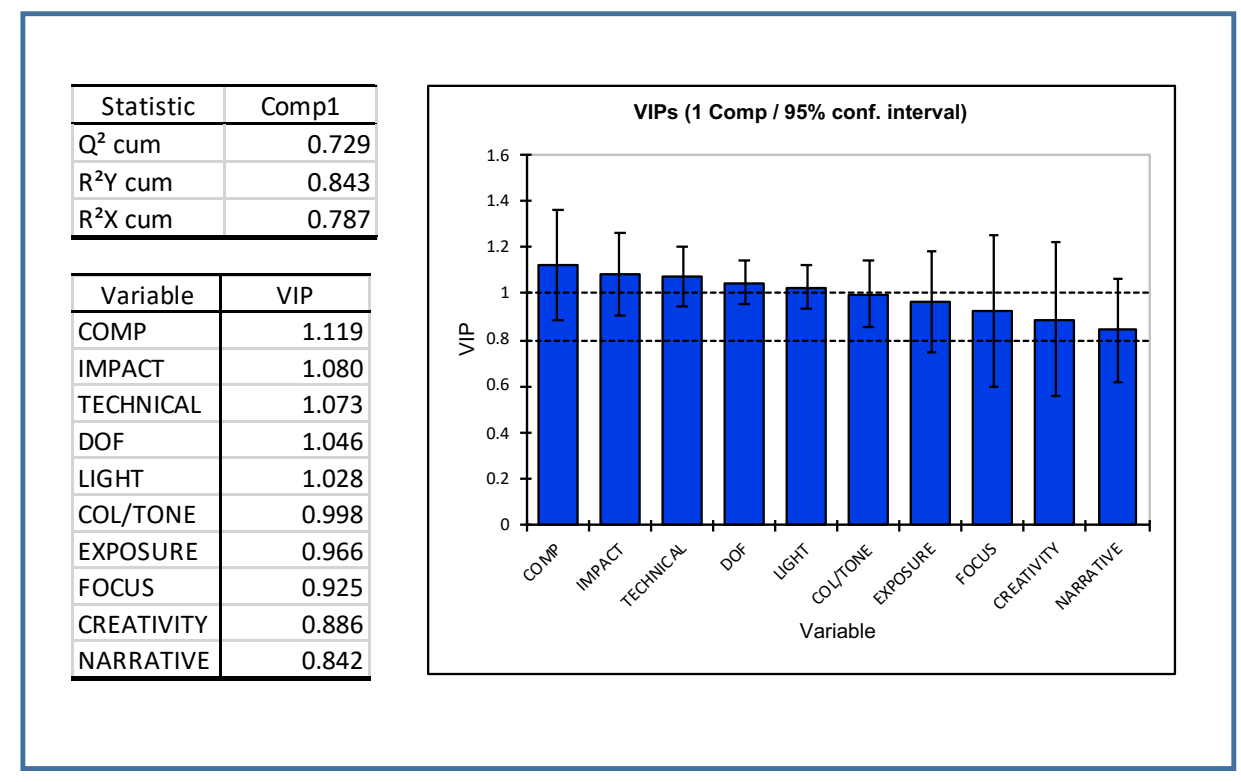

Exhibit 6.5 Partial Least Squares output for whole sample (106 participants).

\footnotetext{
38 One way of examining a model's performance is through out-of-sample testing. My use of intraspective interviews is an alternative approach and one that has the benefit of exploring the semantic basis of the model outcomes in greater depth (see chapter 5).
} 
An alternative regression strategy employs a principal components analysis as a precursor to a conventional multiple linear regression. This method reduces the 10 independent variables to two principal components (bundles of variables) that maximise their individual variance and are orthogonal to one another, i.e. have minimal correlation. Between them they account for $91.00 \%$ of the variance in the data. This method reveals details of the data structure that confirms, with minor variation, the construct ranking generated by the PLS regression (Exhibit 6.5). The intuitive judgement lies close to the F1 (horizontal) axis and the further away the constructs lie from one another, the lower their correlation.

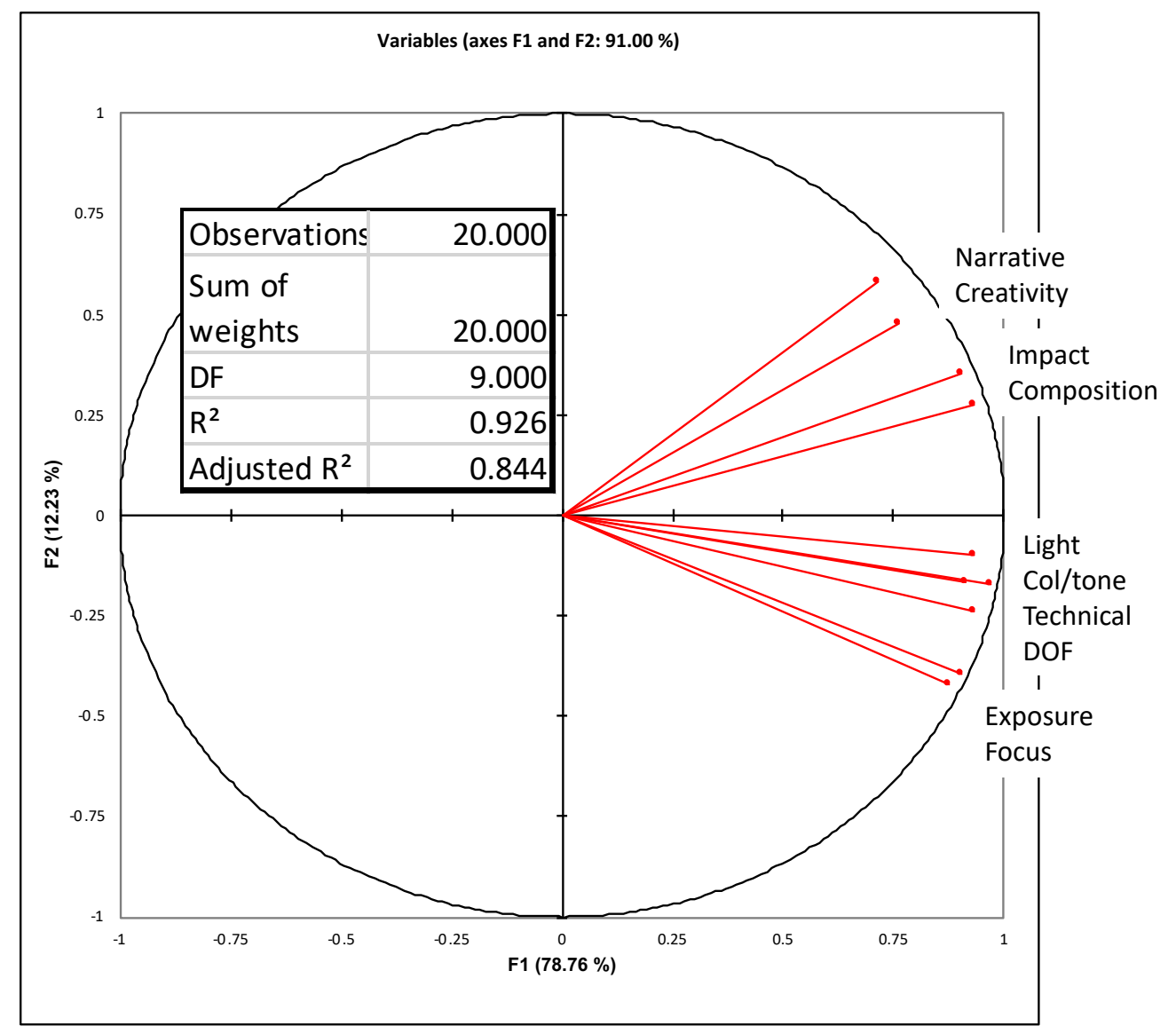

Exhibit 6.6 Output of Principal Component Regression.

What emerges from the principal component analysis (Exhibit 6.6) is that there are two groups of constructs lying close to the horizontal axis representing the first principal component: (i) Impact and composition and (ii) use of light, technical, use of colour/tonality and depth of field. Exposure and focus lie together as a pair as do narrative and creativity. This modelling method generates an $\mathrm{R}^{2}$ that represents the variability of intuitive judgement that can be explained by the ten constructs but also reports an adjusted $R^{2}$ that compensates 
for the large number of variables in the model. At 0.844 the adjusted $R^{2}$ is very close to that generated by the PLS for $R^{2} Y$.

Finally, in gaining insight into the ordering of the ten constructs in terms of their influence on the intuitive judgement of the aggregate, a multidimensional scaling was undertaken and the mapping shown in Exhibit 6.7 produced. The Shepard diagram and stress test values of 0.110 are strong inidicators of the reliability of this method. The circle encloses the constructs identified by the PLS as significant drivers of intuitive judgement at the aggregate level.

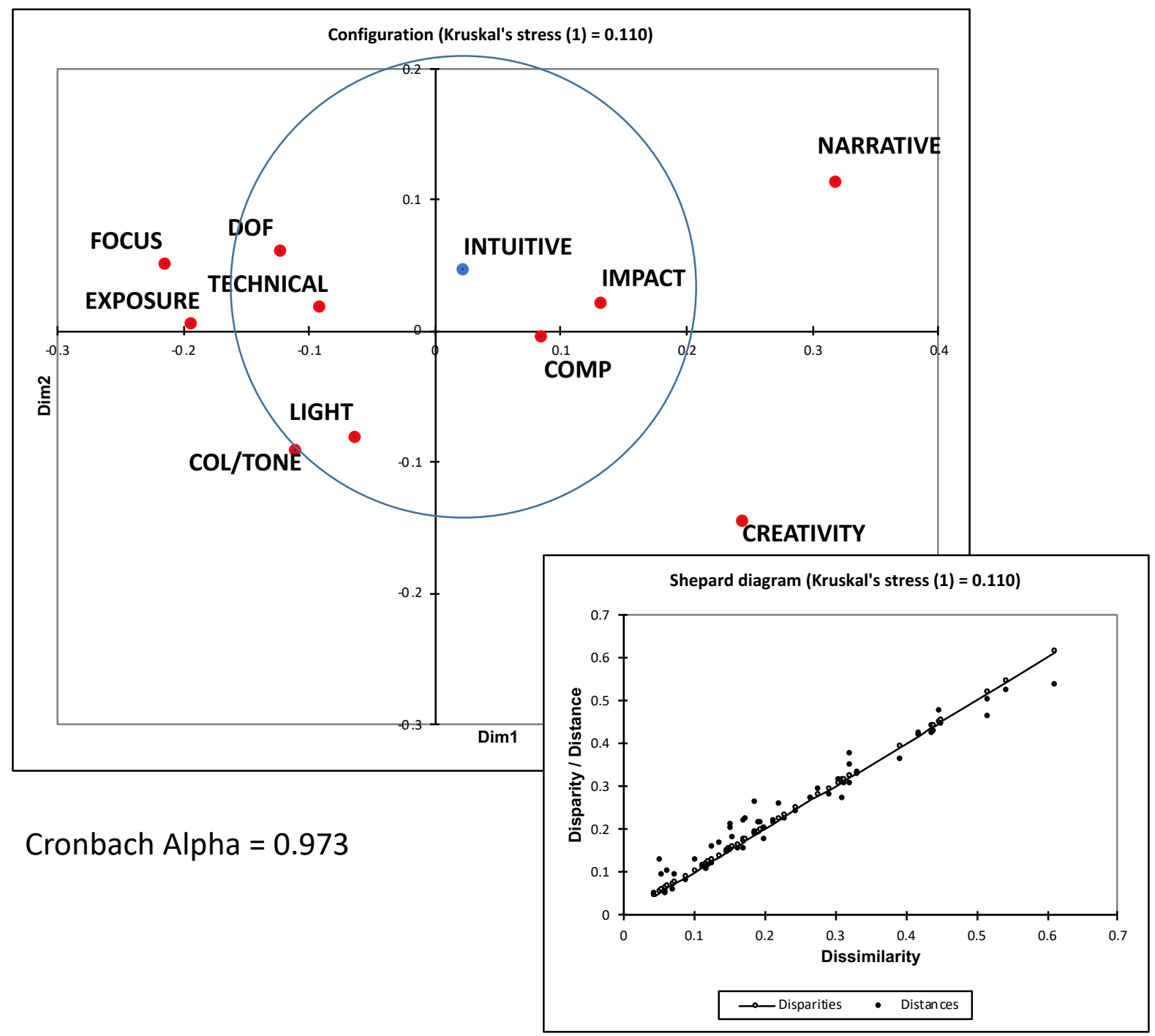

Exhibit 6.7 Multidimensional scaling of aggregate data (106 participants). Note Dim1 and Dim2 are the scales generated by the algorithm in order to reflect best the correlation between each possible pairing of constructs in two-dimensional space. 
Again, the modelling of the aggregate data is pointing to the same conclusions: focus and exposure may be within the conscious competence of the average photographer but do not punctuate their nonconscious awareness when assessing the test images and, as I will argue later in this chapter, engage at the moment of capture. The same is evident for the two highorder constructs: narrative and creativity. Again, they may be consciously accessible and can be previsualised prior to the moment of capture but they are not intuitively accessible and as such will not form part of the nonconscious conjugation process.

All three modelling methods agree, with minor exceptions, on the ordering and clustering of the naked constructs, although MDS does not show the pairing of narrative and creativity as strongly as PCR. They all show a high degree of model quality using the $\mathrm{R}^{2}$ statistic measured by the two regression methods. As noted in the last chapter, the internal consistency of the relationship between constructs and intuitive judgement can be tested using Cronbach's alpha. A score of 0.973 reflects a very high degree of consistency in that the ten constructs are all representing the same variable - intuitive judgement. However, a score at that level does signal a degree of redundancy in terms of the explanatory variables.

\subsection{Analysis of the Construct Ranking (Individual Study)}

As discussed in chapters 4 and 5 , each construct consists of two components: that which is shared and is common knowledge held in varying degrees by all test subjects and that which is their personal interpretation of the meaning of each construct. The Law of Large Numbers predicts that averaging attenuates this second, idiosyncratic element of their understanding and leaves the commonly shared 'naked construct' driving the regression at the aggregate level.

As discussed in chapters 4 and 5, the IMP Test works on the basis of what the individual understands by the terms: 'impact', 'narrative', 'creativity' and so on. Once we move to the aggregate level the averaging process strips the constructs of the variability in understanding of each held at the individual level. This is the 'wisdom of the crowd' effect and the statistical methods above work with the resulting conceptual 'core' of commonly shared meaning held 
by the whole group and the subgroups. In this section, I step back from the aggregate and return to the individual level of analysis.

A preliminary study was engaged to determine the extent to which the ten constructs form a complete set of signifiers of intuitive judgement at the moment of capture or at the moment

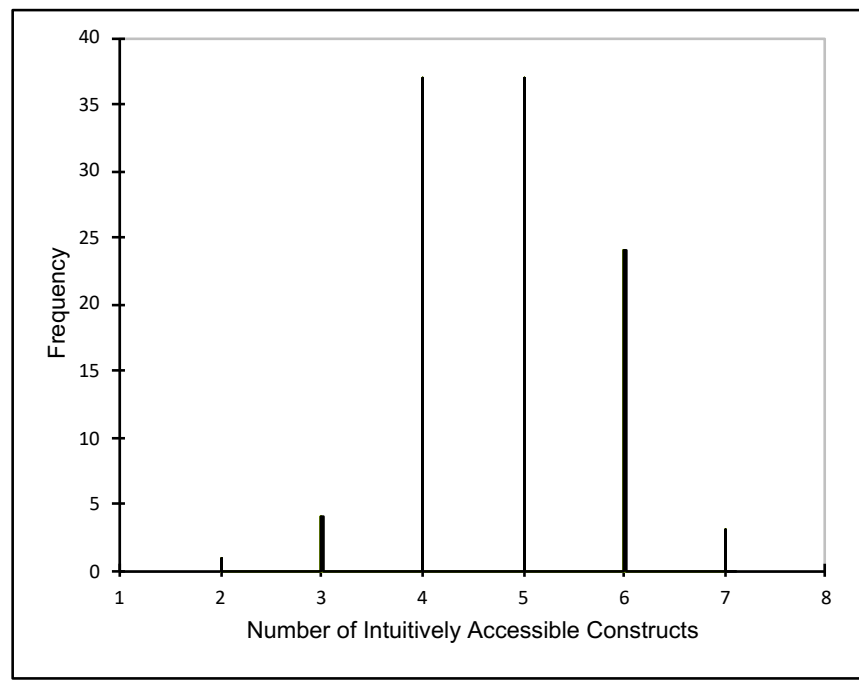

Exhibit 6.8 Number of ranked constructs of assessment. As made clear the IMP Test is not concerned with photographic intent or how that is formed, it is concerned with our response, as photographers, to the photographic moment.

The individual data reveals that of the ten constructs only three partipants drew upon more than six, the rest drew upon less. Exhibit 6.7 gives a frequency distribution of the number of constructs that are intuitively accessible. The distribution is skewed with a mean number of 4.83 constructs driving the intuitive judgement of the test subjects. Comparing with the median, this is in agreement with the three methods discussed above where 5 are clearly 'in' and one very marginal (colour/tonality).

Exhibit 6.9 below shows the frequency with which each construct appears intuitively accessible to the individual. I have also included a factorial rating that brings into account the relative importance of each construct. Impact appeared first with 41 of the 106 test subjects and is weighted 10, it appeared $2^{\text {nd }}$ with 24 and this is weighted 9 and so on to give a total number of times it appeared in the intuitive competency of the test subjects (89) and a factorial ${ }^{39}$ rating of 788 ; likewise, for the rest of the constructs.

\footnotetext{
${ }^{39}$ Factorial is a descending integer series. So factorial 10 , shown as (10!)) means the series $10,9,8,7 \ldots 2,1$.
} 


\begin{tabular}{|c|c|c|c|c|c|c|c|c|c|c|c|c|c|c|}
\hline \multicolumn{15}{|l|}{ Overall } \\
\hline & Ranked & & & & & & & & & & & & FACTORIAL & \\
\hline RANK: & 1st & 2nd & $3 r d$ & 4th & 5th & 6th & 7th & 8th & 9th & 10th & Total & Rank & (Comb.) & Rank \\
\hline Impact & 41 & 24 & 6 & 9 & 7 & 1 & 1 & 0 & 0 & 0 & 89 & 1 & 788 & 1 \\
\hline Creativity & 8 & 15 & 10 & 11 & 5 & 3 & 0 & 0 & 0 & 0 & 52 & 2 & 417 & 3 \\
\hline Narrative & 9 & 5 & 16 & 10 & 7 & 1 & 1 & 0 & 0 & 0 & 49 & 4 & 384 & 5 \\
\hline DoF & 5 & 8 & 7 & 18 & 5 & 3 & 1 & 0 & 0 & 0 & 47 & 7 & 353 & 7 \\
\hline Light & 5 & 16 & 11 & 8 & 6 & 3 & 0 & 0 & 0 & 0 & 49 & 4 & 389 & 4 \\
\hline Comp & 14 & 10 & 9 & 13 & 5 & 1 & 0 & 0 & 0 & 0 & 52 & 2 & 428 & 2 \\
\hline $\mathrm{Col} /$ tone & 6 & 8 & 11 & 12 & 6 & 2 & 0 & 0 & 0 & 0 & 45 & 8 & 350 & 8 \\
\hline Focus & 4 & 4 & 12 & 8 & 10 & 6 & 0 & 0 & 0 & 0 & 44 & 9 & 318 & 9 \\
\hline Exposure & 6 & 6 & 11 & 4 & 7 & 2 & 0 & 0 & 0 & 0 & 36 & 10 & 282 & 10 \\
\hline Technical & 8 & 10 & 12 & 8 & 6 & 5 & 0 & 0 & 0 & 0 & 49 & 4 & 383 & 6 \\
\hline Total & 106 & 106 & 105 & 101 & 64 & 27 & 3 & 0 & 0 & 0 & & & & \\
\hline
\end{tabular}

Exhibit 6.9 Frequency of constructs that are intuitively accessible.

Both the aggregate study (see Exhibit 6.5) and the count of the frequency with which they occur in the individual intuitive assessment place impact and composition as first and second (although the order is reversed). Likewise, both place a very low score on focus and exposure. This suggests that these are areas of expertise where photographers have become 'deskilled', leaving the judgement to automatic processes within camera. Unlike the aggregate study, probing the individual level reveals that creativity and narrative feature in the intuitive judgement of the individual concerned and are of relatively high importance (ranked $3^{\text {rd }}$ and $5^{\text {th }}$ respectively). The contrary results for creativity and narrative suggests that the core of commonly accepted understanding is relatively low in comparison with the level of interpretation of their meaning at the individual level.

\subsection{Analysis of Aggregate Uncertainty}

In the last chapter I showed a graphic of the impact of the Law of Large Numbers on the data set, assuming the individuals who took the test acted independently of one another. The law is stated in many forms but the simplest is as follows: given a large population of random variables, the mean of a random sample drawn from that population will, on average, draw closer to the population mean as the sample size increases. In Exhibit 6.10 the average mean $R^{2} Y$ for each individual was $0.578\left(1-R^{2} Y=0.422\right)$. As we form groups of participants drawn at random from the 106 who took the IMP Test, the mean $R^{2} Y$ at each group size increases until it approaches a constant level of 0.843. In Exhibit 6.10 thirty random drawings were made and the average $R^{2} Y$ taken at each of the following group sizes: $5,10,20,30$, and 50 . 
As noted in the previous chapter $R^{2} Y$ can be taken as the variability in intuitive judgement 'explained' by the variability of the scores assigned to the ten constructs. At the level of the individual, $57.8 \%$ of the variability in their judgement is explained by the variability in the level of the constructs that are intuitively accessible to them, $42.2 \%$ is unexplained. All that we can say with respect to this $42.2 \%$ is that it reflects variability in judgement not derived from the ten constructs - later chapters will throw some light on this. What is clear is that a large element of this judgement is idiosyncratic to the individual. As soon as we increase the sample size the idiosyncrasy across individuals in the group begins to cancel out and that cancelling effect becomes more significant as we proceed to larger group sizes. Ultimately at the full sample size of 106 test subjects we find that $84.3 \%$ of the variability in judgement is explained by the ten constructs.

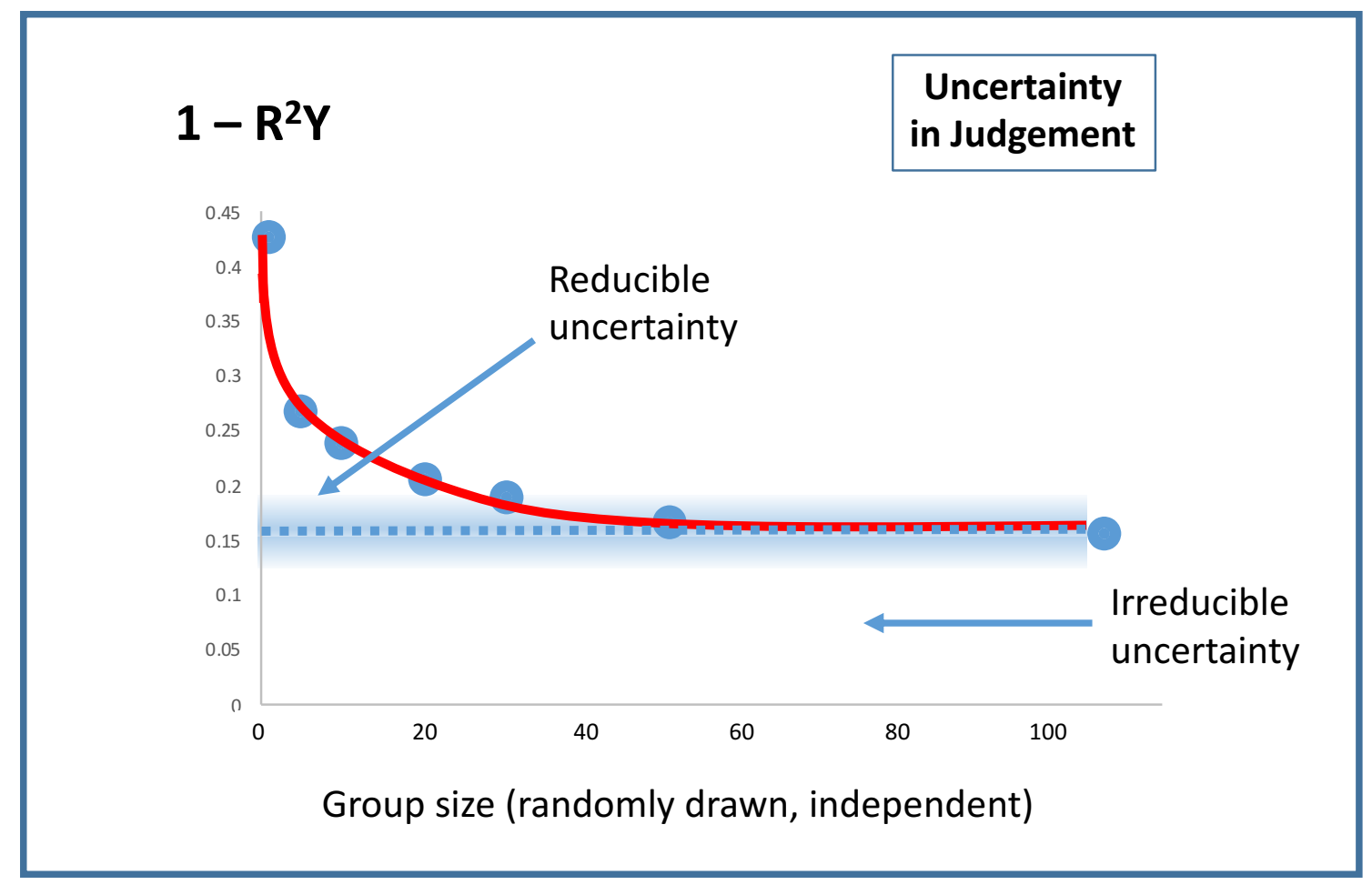

\section{Exhibit 6.10 Uncertainty in judgement by group size.}

Although at one level Exhibit 6.10 is a nice, if anticipated, demonstration of the Law of Large Numbers, it does offer some analytical dividends. The average test subject achieves a score of 0.578 with a distribution skew showing a median of just over 0.6 as shown in Exhibit 5.9. The distribution of $R^{2} Y$ fails the Jarque-Bera test for normality at the $5 \%$ level. In the next chapter I will show the results for those above and below the median. Exhibit 6.10 also gives 
us a simple way of assessing the confidence we should place in the results of any sub-group. If they are near to, or on the curve, then that is what we would expect-divergence from the curve throws into doubt any differences

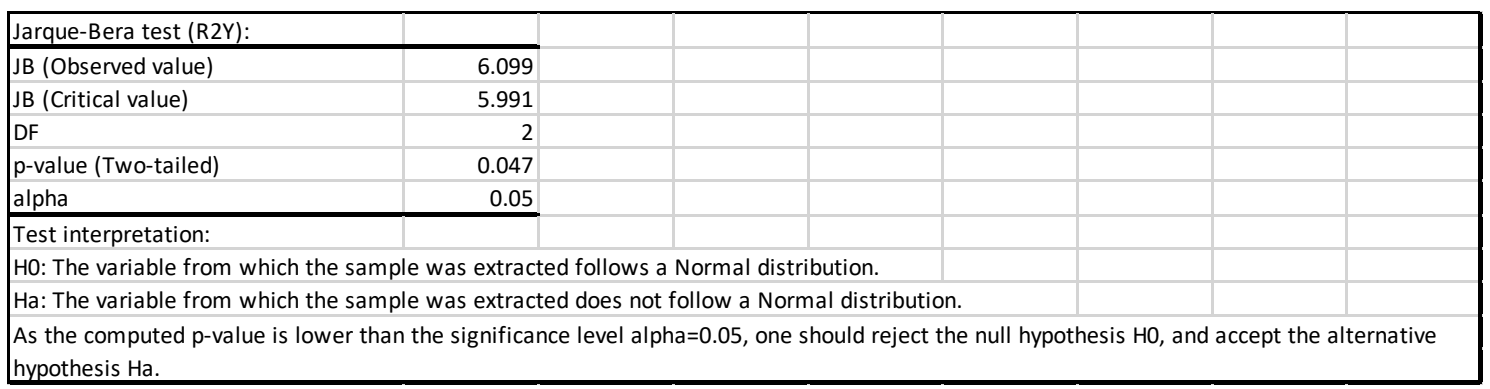

Exhibit 6.11 Jarque - Bera Test for Normality of $R^{2} Y$ Distribution.

we may note between that group's performance and any other. It also asks a question: granted the high level of internal consistency of the model why does it not approach and flatten out at zero?

Perhaps the most striking aspect of Exhibit 6.10 is the asymptotic approach to a constant level at $15.7 \%$ unexplained variability that is not due to idiosyncrasy in judgement at the individual level. Conceptually, this $15.7 \%$ is one outcome from a single study and that another group of $100+$ independent candidates, randomly drawn, would give a different outcome. Likewise, if a different set of construct signifiers representing photographic expertise were to be constructed then, again, a different result might emerge. There may, for example, be another ten constructs that are more efficient in enclosing judgement in this specific area of expertise.

In seeking an explanation of the $15.7 \%$ we need to find a common source of unavoidable error or uncertainty in judgement affecting all participants. Some explanation for the $15.7 \%$ lies in the less than perfect efficiency of the test algorithms in separating out orthogonal components in the regression. There will also be some error in any model generated by bias, excessive variance and random fluctuations. The output data suggests that the first two components account for more than $90 \%$ of the variance of the independent variables, so there will be some residual covariance in the modelling.

The use of integer scales in the assessment of the ten constructs may also induce some 'granularity' into the results. Assuming that the underlying distribution of opinion is continuous, then an integer scale likewise assumes some rounding in the mind of the test 
participant. However, as noted in the previous chapter, rounding issues are close to symmetrical around the integer values with, arguably, a slight downward 'skew', especially with an average score higher than the mid-point of 5.5. That would suggest that, with the aggregate of 106 participants, the cancelling-out effect of the Law of Large Numbers would remove most residual uncertainty from that source with perhaps a slight bias towards overscoring.

The question still remains: what is the $15.7 \%$ less any minor model misspecification? There does not appear to be any 'eleventh' term evident from the intraspective interviews, although the theory predicts, and the results confirm, a high degree of correlation between the constructs. This, I would argue, is the cause of the emergence of 'fuzzy' in the fuzzy constructs discussed in chapter 4. 'Fuzzy', given the degree of correlation between constructs, does appear to be the prime candidate for the missing percentage. It suggests that when assessing any image, whether actual or potential, the constructs are being conjugated in the photographer's perceptual system and this I argue to be the 'missing' element in judgement, and it accounts for the irreducible uncertainty in the aggregate outcome.

It points our attention to the boundary layer where theory indicates there are two systems of conjugation underway: the conjugation of the rational/analytic and the conjugation of the intuitive/experiential. What is clear from the data and very much in accord with prior studies (see chapter 4 ) is that there is very low awareness of the non-conscious ordering of constructs. The average correlation between the declared (conscious) and model (nonconscious) ordering across the 106 participants was 0.132 with a standard deviation of 0.382. Conjugation, I would argue, is the source, of 'style', which is a perceptual consistency in the conjugation of the ten constructs: and it suggests a conclusion that I will return to later in the thesis that this conjugation is of three types: that which emerges from the intuitive, emotionally driven processes of the brain (authentic style), that which emerges from its rational, analytically driven processes (synthetic style) and a third which is an idiosyncratic combination of the two approaches (dissonant style).

\subsection{Intraspective Interviews}

Twenty interviews were conducted, the first four were discarded as discussed in the previous chapter, leaving sixteen. In this section I present analysis of four and one is reserved to 
chapter 8 for a detailed summarising analysis and review covering different themes of this thesis and examining evidence of processing within the boundary layer. The remaining eleven all point to similar conclusions about the external validity of the IMP Test.

\begin{tabular}{|c|c|c|c|c|c|c|c|c|}
\hline & Int Date & Gender & Age & Genre & Status & R2Y & Qual & Judging (yrs) \\
\hline IT0069 & $10 / 05 / 2018$ & $M$ & 51 & Wildlife & Professional & 0.71 & & 10 \\
\hline IT0071 & $22 / 06 / 2018$ & $\mathrm{~F}$ & 76 & Travel & Amateur & 0.33 & & \\
\hline IT0073 & $16 / 07 / 2018$ & M & 60 & Landscape & Amateur & 0.63 & & \\
\hline IT0074 & $19 / 07 / 2018$ & M & 71 & Anything & Amateur & 0.06 & LRPS & 1 \\
\hline IT0075 & 09/07/2018 & M & 60 & Landscape & Amateur & 0.71 & & 1 \\
\hline IT0076 & $13 / 07 / 2018$ & $M$ & 69 & Creative & Amateur & 0.63 & & \\
\hline IT0077 & $12 / 07 / 2018$ & M & 78 & Landscape & Amateur & 0.63 & & \\
\hline IT0079 & $10 / 08 / 2018$ & M & 65 & Landscape & Professional & 0.66 & ARPS & 10 \\
\hline IT0081 & $23 / 10 / 2018$ & $M$ & 60 & Documentary & Professional & 0.49 & & \\
\hline IT0083 & $23 / 09 / 2018$ & $M$ & 40 & Landscape & Amateur & 0.61 & & \\
\hline IT0084 & $27 / 09 / 2018$ & M & 59 & Wildlife & Professional & 0.64 & ARPS & 20 \\
\hline IT0089 & $24 / 10 / 2019$ & $\mathrm{~F}$ & 66 & Amateur & Landscape & 0.5 & LRPS & \\
\hline IT0094 & $02 / 11 / 2018$ & $\mathrm{~F}$ & 59 & Competition & Amateur & 0.69 & & \\
\hline IT0097 & $13 / 11 / 2018$ & $\mathrm{~F}$ & 71 & Creative & Amateur & 0.43 & ARPS & \\
\hline IT0103 & $07 / 12 / 2018$ & $\mathbf{F}$ & 67 & Wildlife & Amateur & 0.83 & & \\
\hline IT0106 & $21 / 05 / 2019$ & M & 54 & Creative & Professional & 0.73 & LRPS & 10 \\
\hline
\end{tabular}

\section{Exhibit 6.12 Participants in the Intraspective Interviews (IT0069 - see chapter 8).}

The purpose of these interviews and their analysis was to provide further evidence on the cognitive processing of photographic images. My selection of the four chosen for detailed presentation in this chapter are particularly interesting because of the different stories they tell. Three are successful professional photographers, two of whom have achieved a high degree of international recognition. The first has achieved great success in international competitions; the second has decided to pursue doctoral studies in photography; the third works in theatre and music. All three have given written agreement (appended) to their story being told. The fourth interviewee is an amateur photographer presenting strong IMP Test results and who demonstrated, at interview, a profound love of photography, which she has pursued for much of her lifetime.

It soon became clear that for the purposes of corroboration, all sixteen interviews were telling a similar story, and although the interviews were very rich in insights, the incremental benefit to understanding the implications and limitations of the IMP test were, with respect to my research questions, so marginal that I formed the view that data saturation had been reached. 
The transcripts of each interview were taken from the recorded MP3 files by a specialist transcriber who is also qualified to degree level in psychology. The interviews have only been redacted where third parties or professionally sensitive information has been disclosed.

\subsubsection{Intraspective Interview - Test Participant IT0084 (Simon Stafford) ${ }^{40}$}

Simon is a professional photographer, male, 59 years of age, a graduate and has held Associateship of the Royal Photographic Society for a number of years. He is a successful wildlife photographer, author of a number of books on photography and is technical editor of Nikon User magazine. His work is regularly published, he has won the mammals section of the Wildlife Photographer of the Year (2016) and has been judging images in his professional capacity at both a national and international level for over 20 years.

He completed the IMP test prior to interview on 20/9/2018 and the results were released to him on $27 / 9 / 2018$. Neither of us were aware of the test outcomes prior to interview. His REI profile shows a marked bias towards the 'rational' in aptitude but with a more balanced score for engagement (RA:EA = 4.3, 3.7; $\mathrm{RE}: \mathrm{EE}=4.2,4.3)$.

His interview lasted 51 minutes and the full transcript is included in the appendix. Discourse focused around three images: first, a Manx Shearwater in flight taken from a boat; second, a female lion taken from a safari truck in Africa; and third, a chameleon in moult in an African encampment. On each of these occasions Simon was running a photography course and had course guests with him.

Bracketing engaged at transcript reference:

- Manx shearwater $90-138$

- Lion $148-195$

- Chameleon 206 - 226

Reducing the interview through its layers revealed a clear recollection of each stage in the process towards capture but with a tendency to abstract and conceptualise issues. This may

\footnotetext{
${ }^{40}$ Simon has kindly agreed to his name being associated with this analysis. His written agreement to disclosure is appended.
} 
have been a reflection of the situation where he was also demonstrating and teaching his guests as tour leader.

I just go into tutor mode automatically - so I'm describing what I'm seeing, what I'm using, what settings I've got.... [210]

His vocabulary, and choice of words - possibly influenced by his role as tutor - reflects a didactic thinking style, which became more evident as the process of recollection developed throughout the interview. However, as the reduction began to penetrate the experience of the subject and the moment of capture, affective and empathetic language began to emerge and became more significant.

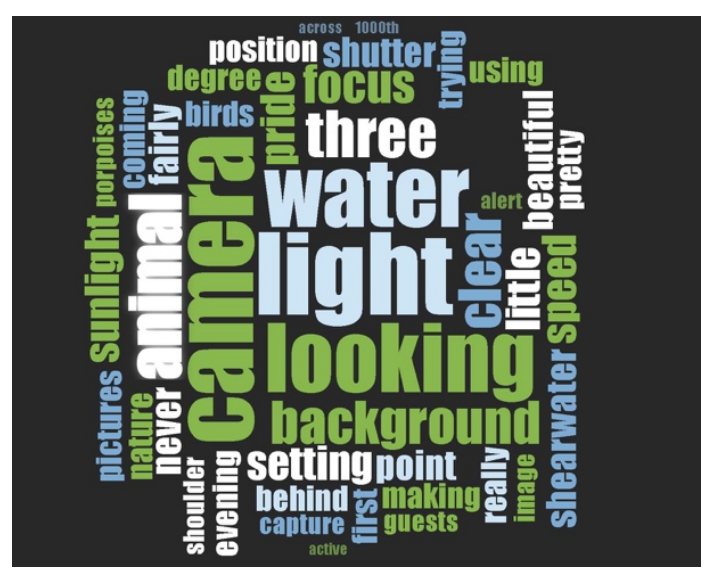

Certain themes started to emerge as soon as the reduction process got underway. Three stand out: his use of light and its relationship with the other constructs, his search for narrative power within the image, and his approach to the basic constructs and technical management of the shot. Of these the most dominant was the condition and the use of light. The word-cloud generated by his responses clearly signals 'light' and also 'camera' - in the latter case reflecting his specific professional interests. However, light was not for him simply an attribute in image-making isolated from others but was revealed as central to his understanding of creativity, narrative and composition. Each image was considered in terms of the availability and quality of the light both in enhancing the structure of the subject and in drawing the eye to the subject within the frame.

Although his style is very deliberative and well considered, from time to time emotional and empathetic responses emerged, particularly towards the animals and birds that were the subject of his images. His response to the lion reflected a concern for the continued success of the species:

.... I've been very lucky to have been on countless safaris over the years and I never, ever lose the thrill of being this close to an entirely wild animal, particularly an animal like a cat or an 
elephant, wild dog, whatever it might be, I just feel enormously privileged to actually be there and lucky to still see them in their environment and see these animals clearly, in this case, thriving.... One hears so many sad stories of declining populations - usually at the hand of man. [190]

When asked if he felt sadness:

Not sadness, anxiety, I think, if anything. Anxiety for her future and the future of her pride. [192]

The words: 'thrill', 'feel', 'sad', 'anxiety' signalled a complexity of emotional states and the presence of the images and the interview process cued his responses. These responses were within the halo of a sequence of affectively driven statements about the lion as he described both his own and his understanding of the animal's state of mind:

And it was a mixture of the peace and tranquillity and the beautiful light that was just sort of breaking across the front, sort of separating her, lifting her away from the background. [186]

\section{The use of light}

Approximately $10 \%$ of Simon's responses focused on the issue of light. In all three encounters it dominates his thinking. Sat in a safari truck approximately 20 metres from the resting lioness and three other members of her pride:

It's almost as if you're actually sitting on the ground in front of her. Clearly, we weren't but from the viewer's point of view. And the left eye is lit up - the iris of the eye is lit up with the golden light so you get that golden iris, so it sort of lures the viewer in. [188]

In these emotionally charged words we note some connections: the use of light separating the animal from her background, the association of light and colour in the animal's iris which 'lures the viewer in'. In this we can see the conjunction of light as a compositional feature as well as a signal of mood through association with the yellow of golden light. This particular hue is strongly associated with love, peacefulness and the absence of aggression. However, this understanding of light as a compositional attribute in an image was also evident in his description of the Manx Shearwater: 
We were lucky; the lateness of the day, the sun was low, there was a nice warm colour quality to the light. It was low, as well, so the underside of the bird is well-lit, as is the wing that's facing towards the sun, and the low angle of the light has also added lots of nice texture to the water and the waves. [134]

Repeatedly he talks about the beauty of the light and its ability to convert the ordinary into the sublime:

It's not unique, this image, far from it, but it's unusual to see a shearwater during daylight hours because you have to go out quite a way to see, and just to have the opportunity of being in a small boat, close to the water's surface, in some beautiful evening light... [138]

Within this discussion, the test participant's perception of composition was revealed. First, he sees it as a problem of modelling the subject in its background as opposed to framing where a 'right to left' dynamic and (unstated) bias towards centering the subject and use of rule of thirds where movement and direction of travel features within the image.

....and for me one of the first - probably the most important consideration when I'm setting up a wildlife picture - is the background... [210]

The interesting terms here are 'when I'm setting up a wildlife picture' and 'important consideration' both indicating that we are dealing with a process where dimensional depth and the use of depth of field through lens choice, focusing, ISO setting and aperture are viewed as strategic, rational as opposed to nonconscious choices. Reflecting upon the shearwaters on the water and their taking flight with the approach of the boat he waited for those who were not flying away:

...obviously from a compositional point of view there's no point photographing the bird from behind because - bum shot. [130]

... a few of them seemed to sit it out and wait and see what happened, and a couple of them as we got level with them suddenly decided, "I've had enough, I'm going to take off", which is what I was spotting for, so as soon as I certainly saw a bird starting to make those motions wings starting to flap - that's when the camera came up to the eye. Focus setting, it would have been slightly right of centre because the bird is right to left. [130] 
Here he is indicating that his preset focal point was off-centre to the right of frame. Although not explicitly stated, he had taken up a preferred starboard side as water birds tend to fly away from a boat's direction of travel when disturbed, hence right to left.

Within his deliberative process of framing and setting up the shot technical issues were considered. With both the shearwater and the chameleon he describes a sequence of deliberative choices featuring lens choice $(300 \mathrm{~mm} f 4.1$ with the shearwater, $600 \mathrm{~mm} \mathrm{f} 4$ prime with the lion and the chameleon), camera settings and angle of shot. In particular, with the moulting chameleon, he attended to a sequence of the technical problems of successfully capturing a shot under conditions of strong, overhead specular lighting and pointing upwards into a forest canopy.

It's a big lens ... supporting it on a monopod ... I'm conscious of the fact depth of field is going to be paper thin here, so again I was reminding myself and reminding the other two guests to be a little more generous with their aperture setting because although we're looking at the flank of the animal here, clearly it's a three-dimensional subject and even on that lens, although I'm focusing on the eye of the chameleon, you can see its hind quarters there - the knee is projecting forward. In fact, that kneecap is not absolutely pin-sharp; it's probably no more than a half an inch closer to the camera than the eye. But the beauty of that lens is, as you can see, the background: it's a very narrow angle of view. There were pools of sunlight coming through these trees. Obviously, you want to avoid those, so hence using a very long lens. [216]

...so I'm making sure they are looking though their viewfinders and checking for the background, any bright hotspots coming, the trees, and if they are getting that then move position, and equally I was rotating out of the position I was in so they could rotate into the position I'd been shooting from. [210]

It is interesting to note here that he has not chosen the alternative approach of shooting 'loose' with a shorter lens and cropping in post-processing. By his choice of camera, lens, support and settings he is working to avoid technical problems but also trading off technical issues (pin sharp on the eye at the expense of softness on the protruding leg, framing and avoiding irrecoverable highlights). At this level, notwithstanding his didactic concerns, he is revealing a very deliberative approach to the basics of setting up the shot. There is no 
evidence from the interview that he was making intuitively driven adjustments to settings indeed quite the reverse. In this section he describes his thinking about focus and exposure control. He has to 'remind' himself to be a 'little more generous with the aperture setting'. He is dialling in some exposure compensation as he is framing the shot:

I: So, you've got this young lady lined up, and aiming to shoot. Any adjustments at this last moment on your camera, do you remember? Anything on the focal point? [179]

$R$ : Nothing on the focus; focus is pre-set; I have a pretty standard focus configuration on the camera. Very, very rarely do I change that, so focusing is all pre-set. It was just a question of selecting the focus point - right of centre here because she's to the right side of the frame. [180]

At this point he returns to his dominant theme: the quality and control of the use of the light:

My immediate assessment was the nature of the light, as you can see it's very strong side lighting. She's in a shaft of sunlight, whereas virtually everything else in the frame is in shadow, so as I'm lifting the camera to my eye I'm already thinking about making sure I make an exposure adjustment so that I don't overexpose this area which is in the sunlight and just instinctively as the camera comes to my eye, again I have the camera set up on the rear command dial which allows me to control the shutter speed. I'm deliberately turning that away from the viewfinder because that increases the shutter speed so that it's about twothirds of a stop under the camera's recommended exposure setting because I want to make sure that I retain this brightness here without overlooking...[180]

Rather interesting here is his comment about the exposure correction. He is recalling the use of a heuristic device possibly compensating for a lack of intuitive/motor control: I'm deliberately turning that away from the viewfinder because that increases the shutter speed....

\section{Narrative power}

Simon revealed throughout the intraspective process a commitment to narrative power and its relationship to creativity. As we untangle the discourse the desire to tell a story is evident by his use of language. For the wildlife photographer, animal behaviour is a potential and potent source of narrative. 
In each case he described a process of productive dissociation and affective transference, seeing within the image potential metaphors and placing himself emotionally in the frame replacing the real subject. With the shearwater it is happily floating on the water, it suddenly becomes airborne, there is the frantic nature of the transition. The moment was clearly previsualised but the spontaneity of the moment of capture is clear as he recognises the decisive moment revealing the awkwardness of the transition and takes the shot:

...so yes, it was sort of an image l'd had in my mind's eye that sort of did come together. [136]

It was the level of animation in the bird that sort of appealed to me. It's the activity and the prospect of flight but then to some degree the awkwardness of the transition from happily floating on the waves to then suddenly being airborne, and the frantic nature that is involved in going for that transition... [136]

The interesting point here is not that Simon is just telling a story but is trying to bring the viewer into the lifestyle and life cycle of the bird:

I think it's just telling the story about the bird and the bird's behaviour, and its lifestyle and life cycle.[138]

The emphasis on flight and its conjunction with 'awkwardness' and the 'frantic nature' of its transition from peacefully floating on the water to the safety of the air is rich in metaphorical associations.

Turning to the chameleon he strongly internalises the feeling of the moult as the creature goes through the periodic and laborious process of shedding its skin. He recognised the originality in capturing such a moment, which is a rarely observed aspect of reptilian behaviour in the wild. The creative dimension here was in revealing rarely observed behaviour in a way that did justice to the moment:

I've never actually seen one mid-moult, so it was just for me something l'd not recorded before and it was just the unusual nature of the capture of the animal in that sort of stage... [218]

He then proceeds to connect what the animal is doing with the human condition and in so doing creates a metaphor for the struggle of change and rebirth - drawing the viewer in to the reality of the creature's existence: 
....from my perspective it's, what a complete palaver having to sit there rubbing yourself against this tree to get all this dead skin off. There was something running through my head about "Do real men exfoliate?" [224] ...We're meant to, of course, yes. There was almost something comical about it, not that it's comical to the chameleon, I suspect. But from my perspective there was an air of comedy about it, because as it was rocking back and forward, trying to pull the skin off, and clearly bits weren't coming off as fast or as cleanly as it wanted. You sense a degree of frustration on his part. But I couldn't help having a bit of a smile on my face. [226]

\section{Commentary on the IMP Test Result}

Simon is unusual in the very high degree of association between the way that his conscious and nonconscious choices articulate. His $\mathrm{R}^{2} \mathrm{Y}$ score of $64.5 \%$ is well above average but what is more significant is the high degree of awareness of his nonconscious judgement process with a rank correlation between declared and modelled importance of 0.845 . The test places six constructs within his intuitive competency and four without - this puts him within the upper quartile of photographers who, the test reveals, have six or more constructs under intuitive control (see Exhibit 6.13). The six are closely associated: with the use of light and the use of colour or tonality lying very close to impact. The test reports 'use of light' with its colour and compositional associates lying very close to Simon's understanding of 'creativity' and, less directly 'narrative'. What is very noticeable is the degree to which these constructs dominate Simon's recollection and understanding of the three images featuring in the interview.

As we move outside of his intuitive scope, two constructs stand out as under a high level of conscious control (focus and exposure). The IMP Test indicates that he is skilled in these two areas of photographic practice, but neither is significant in driving his intuitive judgement.

Given his interview and the strength of the themes that have arisen, I would place strong confidence in the analytical outcome. The quality of the IMP Test $\left(R^{2} Y\right)$ gives me moderate confidence that the quantitative analysis is sound. However, the high degree of association between the interview and the IMP Test increases my confidence to 'strong', particularly with respect to the status of impact, the use of light, the use of colour/tonality and narrative power. 


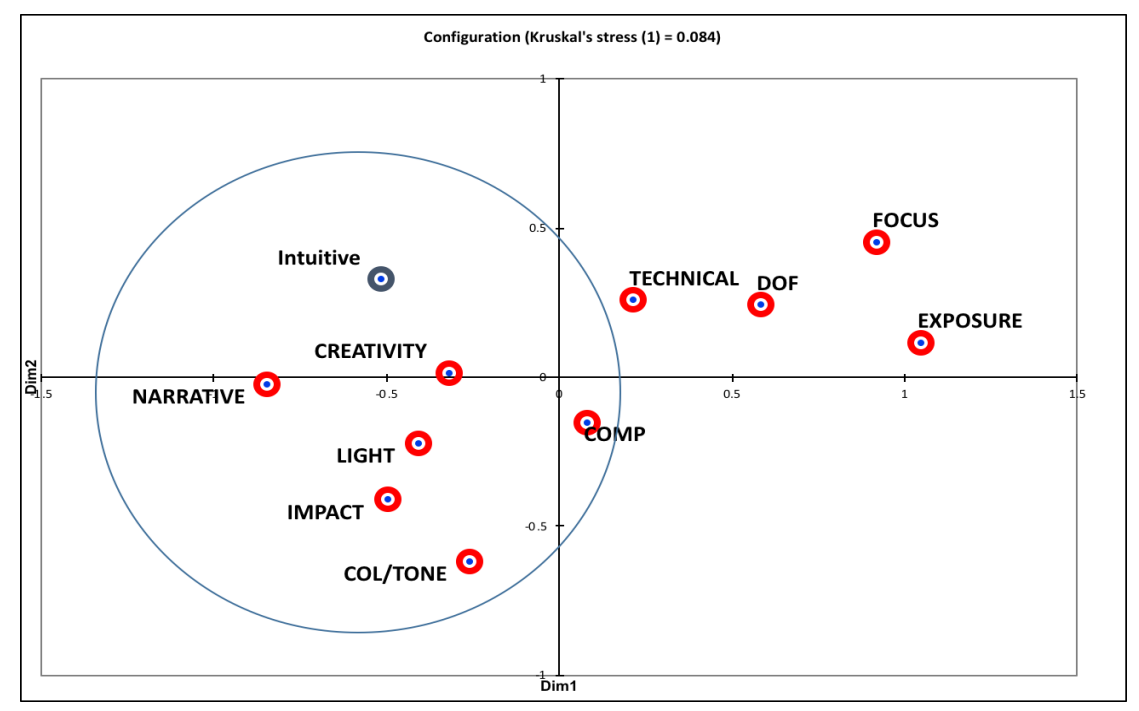

Exhibit 6.13 Multidimensional scaling (IT0084) (see exhibit 6.7 for a note on the dimensions).

The interview was silent with respect to the term 'creativity', although, in terms of Simon's understanding of the construct, and the decisive outcome of the test I have modest confidence it is within his intuitive jurisdiction. The binding of composition to the use of light and narrative suggests that it is under intuitive control, however, I am less confident about technical and the use of depth of field. The IMP Test is clear with respect to both and both appear from the interview to be a product of Simon's reflective as opposed to his intuitive judgement.

Finally, the test results with respect to the focus and exposure judgement are very clear. In this particular case they sit full square with the aggregate study and the summary of individual results. It is an open question why these two constructs do not feature as intuitively active either across the board with those tested or in any of the sub-groups.

I am most grateful for the fulsome feedback you have provided following the testing and the time you have spent discussing the results with me; it has been a very enlightening experience and one that I will certainly be drawing upon for my photography in the future. Simon Stafford, 31 March 2019 


\subsubsection{Intraspective Interview - Test Participant IT0079 (Neil McCoubrey) ${ }^{41}$}

Neil is a 65-year-old graduate with an ARPS distinction. He is predominantly interested in landscape photography with a particular interest in human-made structures in the environment. He makes a living as a professional photographer and is currently registered for a doctoral degree in photography. He is an experienced image assessor at club level (10 years) and his preferred output is via print books.

He completed the IMP Test (3/8/2018) prior to interview by Skype on 10/8/2018. The results were not revealed to him until the completion of the test. His REI profile shows a marked bias towards the 'rational' in both aptitude and engagement (RA:EA $=4.0,3.3$; RE:EE = 4.9,4.0). However, his intuitive scoring of the twenty images lies closer to the consensus than his construct-by-construct scoring. Furthermore, that deliberative scoring of the images takes him closer to the consensus when the model derived weighting is applied to his scores rather than his own declared weighting. This suggests that his experiential aptitude is higher than he believes and reports in his REI profile.

Neil submitted three images for review. Bracketing was engaged at transcript reference:

- Lerwick Harbour Building [68 - 101]

- Outside Steps [148-160]

- Tank and Field [162 - 173]

What is noticeable on first reading of the transcript is his strong interest in structures and shapes and it is through these that Neil seeks impact, creative expression and, to a degree, a narrative element in the image. As bracketing deepened his pleasure in what he was seeing became more apparent.

I can quite often see an image like this, which really excites me about its shapes...[168]

In addition, there is a clear hint of the search for creativity through the depiction of mood in the landscape he explores:

\footnotetext{
${ }^{41}$ Neil has kindly agreed to his name being associated with this analysis. His written agreement to disclosure is appended.
} 
I'd arrived at this site - because I'd seen it a year before, I had a very good idea of the mood I wanted to create and the composition. [148]

He previsualises images to a certain degree, returning to a known location, in one case a year later, looking to recreate the memory he has of a place which is essentially one of structure. As I note later, he does like to subdue the lighting and desaturate the colour in his images both at the moment of capture but, if not fully achieved in camera, later in post-processing. This appears to be a reductive process (see chapter 2 ) where aspects of the image are attenuated in order to magnify what he recognises as its essential reality.

This is an image I grew to like as I worked on it. I like the composition; I was really pleased with the placement of the big tree, the broken walls. It was a more difficult image to process than the last one because the steps themselves - the two diagonal pieces of step - did get caught up in the background. [152]

Indeed, as the interview proceeded, he became more animated by the shapes and the conjunction of elements within the frame. Spontaneously, as the emotional cues in the interview started to take effect, he recounted his childhood experiences of being driven along, noticing shapes and the balance of shapes around him. He recounts one in particular and as he spoke, I too understood its cue dependency and why it had emerged. For British people of a certain generation, any sighting of an early E-type Jaguar was a thrill and the recall of the event and its context is particularly vivid. I too can remember very clearly my first sight of an E-type and the impression it made. These vivid recollections are consciously remembered in the gist but under emotional activation the surrounding feelings and context can be reengaged as the nonconscious returns the experience to conscious awareness. In Neil's words:

...and looking at the back of the car in front and I would see its real shape as opposed to what we think it looks like, and I remember following an E-type jaguar, which is of course a beautiful car...[76]

For me it was the car's power and speed; for Neil it was his sense of the car's balance and in particular he noted the incongruity of the thinness of its wheels. 


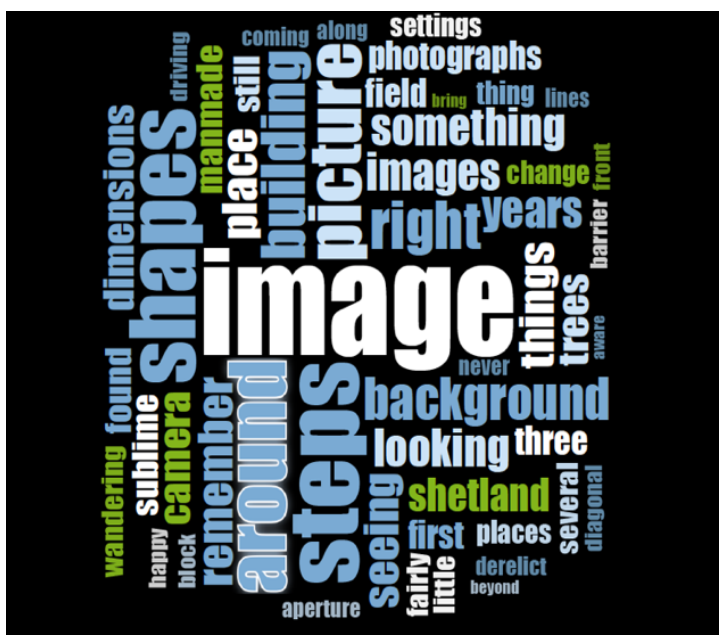

Those tyres need to be wider. It just didn't look balanced with 1960s tyres on it! And I was probably about 10 or 12 years old when I saw that, but it's things like that. I've done this all my life; I have this ability and today now it is driving possibly a quarter to a third of my image taking is seeing shapes like this. [78]

he sought spatial balance in his photographic practice along with the associated judgements about the use of light in the image.

Reflecting on his word cloud a number of words point to what he deems meaningful in terms of composition: structures, buildings, shapes, steps, and dimensions are there along with a hint of interest in age: time, dereliction and the human-made as much as natural elements within the landscape. Throughout the interview, the theme of composition - as he understands it - dominates the discourse.

\section{Composition}

As I will discuss in chapter 8, out of the archaeology of human experience particular preferred regularities emerge driving the nonconscious recognition of, amongst other things, spatial structure. In compositional terms, such nonconscious regularities reveal themselves as a preference for the strong diagonal, for others it is the use of strong 'triples', for others it is the rule of thirds. Neil associates his recognition of shape and structure as one of 'balance' that he approaches through a condition of mindfulness and 'zoning out' as he absorbs the placement of objects within the landscape. This suggests that what Neil understands as 'composition' is likely to be conditioned by his nonconscious cognition and that this will be influencing his judgements in the moment:

I do go into a state - when I intuitively see an image like this, I do go in to a state which is I'm on my own and things around me don't impact on me. [44]

Rather interestingly, he then goes onto say that the activation of this construct has both a three and a two-dimensional aspect to it: 
The reason I chose this image was it's one I saw in two dimensions, I didn't see it in three, and that also requires me to be in a certain state. [44]

Three-dimensional depth perception is not strictly dependent upon stereoscopic vision. People with one eye can still have a degree of 3D vision, the effect is created within the brain as it processes small movements, inducing changes in parallax scrolling and perspective, and assembles a 3D model of what is seen. The passive state Neil describes is, potentially, a conscious strategy to attenuate the information the brain needs to generate the $3 \mathrm{D}$ effect. This is suggestive of boundary layer processing where the intuitive recognition of a compositional opportunity combines with the conscious optimisation of a two-dimensional photographic outcome.

Mindfulness has received considerable attention in the cognitive literature as a useful therapeutic practice (Kuyken, Hayes et al. 2015). It has also been mooted as a worthwhile practice in enhancing the appreciation of creative opportunities (Thompson 2018). A plausible mechanism is that its practice does not enhance nonconscious processing as such but acts in a reductive way to suppress conscious processing in the boundary layer allowing the nonconscious to play a greater part in the creative act. Certain ambient conditions also help him in entering the 'zone':

Windless, very calm, completely quiet, as you can see from the sky it was subdued; these are all features I enjoy because that is when I become most mindful, I suppose, of the scene. [26]

On the evidence of this interview, this ability to enter into or seek the conditions to alter his mental state is a recurrent feature of his photographic experience. He finds that his enjoyment of engaging with human-made structures, even though they may not represent a sublime location, induces a feeling of the sublime in his own response:

...this is a shift in the kind of photography l'm doing ... what you're seeing is actually some shifts from what I was doing previously, which were very much more open environments, where I was feeling...the sublime. I used to feel the sublime because I was in a sublime place, whereas now, with these images I'm starting to feel the sublime but not in a sublime place. [168] 
Neil does not declare a preference for one compositional structure over another. However, his conception of composition is strongly conditioned by what he understands as 'balance' in the arrangement of elements in the frame.

I moved a couple of feet to the left so that the pole and the edge of the building were roughly the same distance each from the edge of the frame. And that was all I did, so it was a case of balancing the image; it wasn't a case of seeing the composition...[60]

His description hints at his intuitive awareness of composition and what looks right from the point of balance. Here we get a hint as to the nature of 'balance' for Neil, it is not just the mass of the elements in an image that matter but the strength of their visual presence. This is not an overtly conscious process as he adjusts his physical position until he reaches what he regards as the best angle to take the shot. In his third image:

Again it's shapes. It's the big block, rectangle, the tank, then the block on the right-hand side of the breezeblocks, the... line of trees in the background, there's a barrier similar to the line of trees in the landscape of the Lerwick picture. It's all about lines and shapes. [170]

But it is not the pristine freshness of new build that attracts him. He sees the opportunity for narrative in the sense of temporality in the image. Old and decaying structures have a past and a relationship with what was as much as with what is. But this being is mysterious - it is in Harman's terms withdrawn, but through his photography and by reduction of the sensible he believes that he can point to the essence and reveal the story behind the remains of the structure:

Again it's the placement of shapes and weights of things; there's a balance to the image. But also there's a lot of narrative in it, so this image is not just driven by shapes; it's also driven more strongly by narrative of why, who, when. [154]

It is also very noticeable that technical and basic issues do not surface significantly in his recall. He presets his camera to aperture priority setting f11 on his Nikon or f7.1 on his Fuji:

So they're the default settings and there's nothing in this image that would have caused me to change those settings. [130] 
Indeed, there is a sense that concern for the basic and the technical is a barrier to what he is trying to achieve ('let the camera deal with that'), impeding his uncovering of shape, structure and balance in the image.

Yep. It's Auto ISO, it was aperture 7.1, and I have no idea what the speed was because I let the camera deal with that...[56]

Neil's sense of light and his use of colour is the reverse of the conventional. For him it is something to be reduced to achieve the mood of the landscape he wants to convey. As I have already noted a 'subdued sky' enhances the mood and helps him achieve the state of mindfulness he values in his search for creativity. He makes a comment that this is not a deliberate act but one that lies apart from his conscious thought:

The fact that I'd seen something completely unreal. I'd just seen shapes. It's a fairly monotone type of image; l'd seen all the shapes in greys and blacks. The colours just disappeared for me. [70]

A conventional view of 'use of light' is to use it for more dramatic effect by, perhaps, stronger colour articulation or by strengthening contrast. For Neil it's quite the reverse - his use of light is to reduce the 'energy' within the image and thus allow the essence he sees in structure to emerge and dominate the viewer's perception.

Where Neil engages in post-processing, he seeks to adjust the tonal separation between elements within his image and to delete obtrusive brightness. Indeed, he does little postprocessing apart from minor cropping and working to isolate the point of interest as far as possible.

\section{Commentary on the IMP Test Result}

His quality scores on testing were well above average $\left(R^{2} Y=66.3 \%\right)$ but the primary indicator of awareness of his intuitive ordering of the constructs was negative $(-0.28)$. When adjusting the weighting in his scoring of each image from his declared to the model-defined ranking, there was a clear improvement in terms of his alignment with the consensus. However, his 
agreement with the consensus was relatively low. This, the evidence suggests, is because his non-cognitive 'latency' arises in the combination of creativity, composition and the use of light.

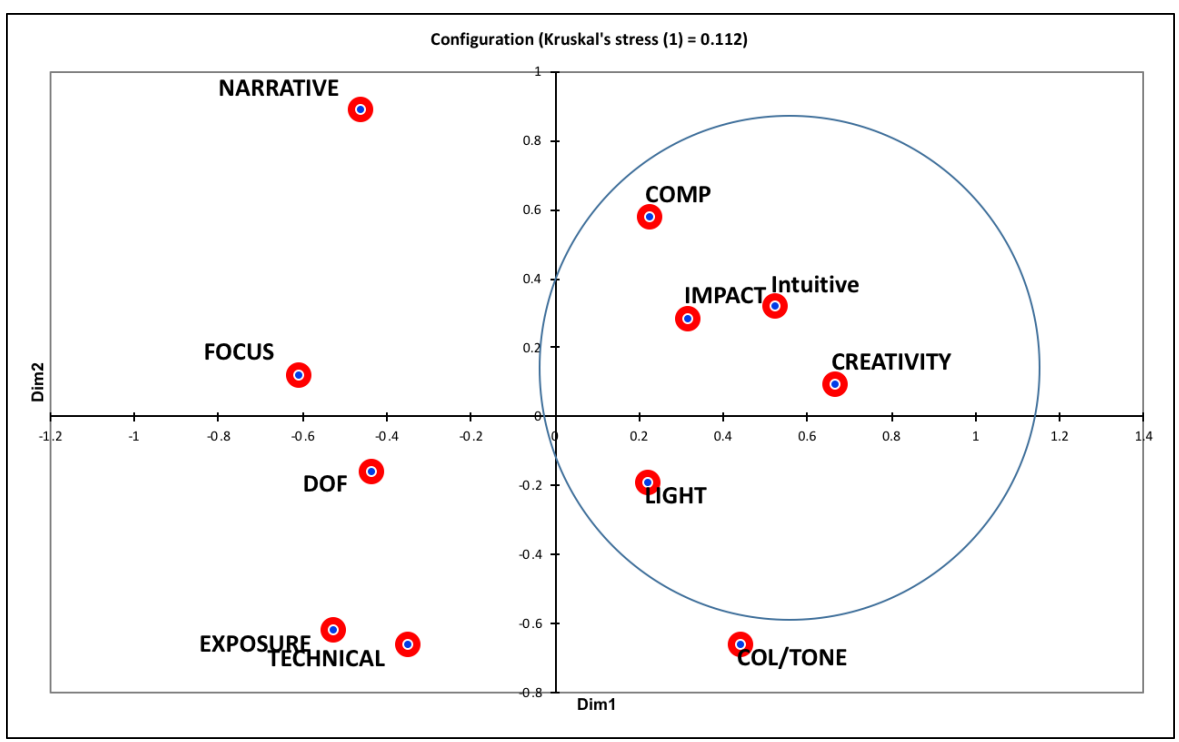

Exhibit 6.14 Multidimensional scaling (IT0079) (see Exhibit 6.7 for a note on the dimensions).

The negative correlation between the most predictively significant ordering of the ten constructs (from the IMP Test) and his declared preference ordering suggests that there may be some dissonance in his perception of the image in post-processing. That dissonance is likely to be most active around those constructs that are nonconsciously significant and, in that, composition and the use of light are where problems are likely to emerge. One comment he made is suggestive of this issue:

I was really pleased with the placement of the big tree, the broken walls. It was a more difficult image to process than the last one because the steps themselves - the two diagonal pieces of step - did get caught up in the background. The background was dark, and they were dark. In this I've actually lightened the trees in the background and the wall in the background and darkened the steps to make them stand out. So, there has been quite a significant bit of processing to make what I wanted to highlight more visible. [152]

Here his intuitive judgement in both composition and the use of light is being challenged by his more deliberative approach when post-processing his image. But he is still not content: 
(it) still works. I'd still like to separate those steps from the background a little more, especially the upper one. It's the overall arrangement that I've achieved. Again, it's placement of shapes and weights of things; there's a balance to the image. [154]

Examining the correlations, the IMP Test indicates strong correlation between his conscious judgement of focus and the use of depth of field, and between exposure and technical. He also relies upon the camera's horizon indicator when shooting and notices and corrects bright spots near the frame edge within post-processing. This provides support for the conclusion that these constructs are not part of his nonconscious processing.

Although the issue did not arise frequently in the interview, narrative was referred to once explicitly and a number of times implicitly. Working through the sections of the interview where nonconscious processing was potentially high my prior expectation was that narrative power did form part of his intuitive cognition. The IMP Test is quite categorical across all the testing algorithms that it is not. In his feedback (see below) he too registered some surprise but on reflection accepted that the outcome of the test was indicative of a more deliberative rather than intuitive process.

In feedback on the IMP Test he made the following comments first, with respect to his own photographic research, and second with respect to narrative power:

... my original ideas have been challenged, are changing and your IMP test was a cause of a significant early hiccup that has carried on delivering useful ideas. (email 31/1/2019)

He too was initially surprised that 'narrative power' was not influencing his nonconscious judgement. Initially he resisted that outcome of the test but on reflection he began to recognise the force of this test result:

I started believing that I sometimes, instantly appreciated and understood narratives in images. The IMP test suggested that all my narrative creation was actually achieved over time rather than being instant and intuitive. Thank you for that. It made me re-assess all my narrative images and I agree, my intuition does not create narrative. 
In summary, given the above average $R^{2} Y$, the low stress on the MDS but noting the lack of consistency between the test and the REI, I would place medium confidence in the quantitative element of the test. There are some significant silences in the interview around the issues of creativity, the use of light and narrative so my assessment is that the interview provides partial agreement with the IMP Test and this, in combination, gives me modest to strong confidence in its outcomes.

\subsubsection{Intraspective Interview - Test Participant IT0103 (Daisy)}

Daisy was one of the last candidates for the IMP Test before closing this empirical study. She had been reading Ryan (2017) and applying its principles for some time. Daisy took the test on $2 / 12 / 2018$ and submitted to the interview on $7 / 12 / 2018$. She is 67 years of age, is an amateur who has been taking photographs since early childhood and whose principal interest is in wildlife but who presented a portfolio of images across a wide range. Here REI scoring is very well balanced (RA:EA = 3.7, 3.6; $\mathrm{RE}: \mathrm{EE}=3.5,3.4)$.

What caught my attention with Daisy's IMP Test submission was the high-quality score $\left(R^{2} Y=\right.$ 0.83 ) and a very high level of association between her declared and the model's ranking of the ten constructs (0.93). The stress test on her MDS was acceptable at 0.125. Her average uncertainty index suggested that she was relatively more confident in her judgements across the three basic constructs and the use of colour/tonality but below average on the rest.

Daisy presented three images for review. Bracketing was engaged at reference:

- Halnaker Windmill West Sussex [74-123]

- A seascape at Rustington Beach Sussex [144 - 184]

- A pony at an animal sanctuary Storrington [211 - 241] 


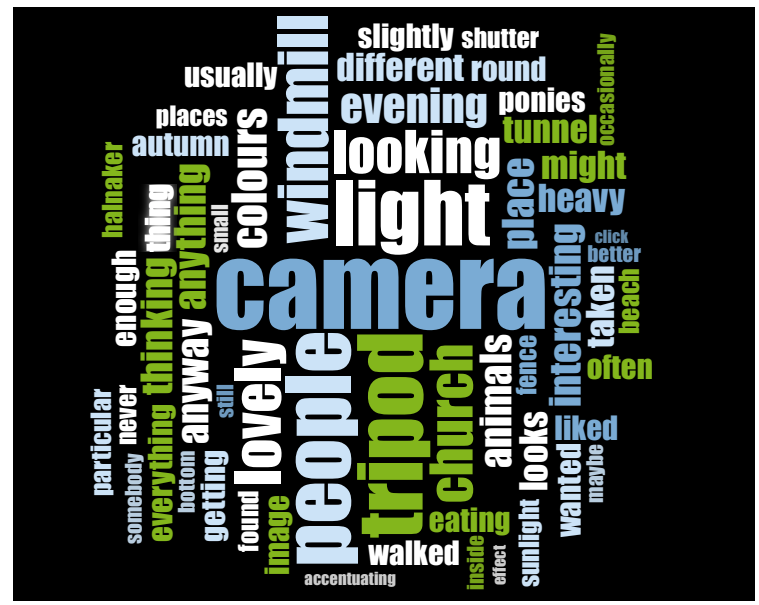

One theme started to emerge as soon as the interview and became more dominant as the reduction process commenced. She consistently focused on the quality of light in her images but in doing so made clear associations with her understanding of creativity and impact. She also became more animated in her description and detailed in her recollection of the point of capture with each image. However, what was of particular interest when analysing her interview was the relative silence with respect to focus and depth of field.

\section{The Use of Light and the Use of Colour}

In the bracketed elements of the interview light was by far and away the construct that kept being raised. In the first shot of the windmill, the view was marred by fencing and other signs of restoration. However, capturing it in backlight from a sun hidden by one of sails was foremost in her intent.

I wanted the sun; I wanted to shoot into the sun and have a silhouette, so I thought, "Well, I'll just get it as much as I can," and then when I took the pictures I noticed I was getting a lovely starburst so I thought, "Ooh, that's nice!" [8]

The "Ooh, that's nice!" is quite striking here - the lovely starburst as she calls it clearly had impact for her and was clearly reflexive and in the moment. Indeed, throughout her description, and particularly when deep into the reductive process - these small exclamatory phrases keep popping up: "Ooh, that's quite nice, I quite like that," and "Ooh, lovely! Click." [230]

Even when photographing the pony, being able to capture it in good light that enhances its face and the colour of the setting:

Well, he looks almost as if he's posing for me; I mean, he's just in a perfect position, I think. He's got the light on his face, you can see he's been eating out of this rather stylish little cart he's got next to him. I just think the whole composition was quite pleasing... [234] 
Strong lighting features in each of the three images, and it is something Daisy seeks and responds to in the moment. Colour also features in her work, particularly in the image of the sea and the pony. With the windmill she was seeking to create the strong contrasts of a silhouette, but with the other two, colour was previsualised and sought in the scene. On her way to the windmill there was an avenue of trees which she dismissed as a shooting opportunity, but she had been thinking of the autumn light and colour:

...It could be quite pretty in the right light and with the leaves the right colours...[52]

However, autumn had not advanced sufficiently, and it was too green for her taste. In a more abstract shot of the sea, Daisy is viewing the light as a means of enhancing the colour:

Well, it's clearly a photograph of the sea but accentuating all colours that evening light will give and streaming those colours in a sort of .... I suppose it's just accentuating the colours without detracting from the fact that it is the sea and you can see it's the sea. [179]...because it had the sunset light - there were sort of clouds in the sky, as you can see, sort of covering bits of the sun and bits of the sunlight, and as I swept the camera the lovely colours were in the sky and in the sea reflected so I thought, "Ooh, that's quite nice, I quite like that..." [129]

It is notable that light is the principal aesthetic driver of her experience of the sea and the strengthening of the colours was, for her, a very desirable by-product of her choice of lighting.

Asked to reflect why her image of the pony worked for her she summed it up as follows:

...I always find it difficult to explain why it's pleasing; I just kind of like looking at it and you sort of look around and it all kind of makes sense, and the light, I think, is all in the right place, and I don't mind the darkness because those bits aren't very interesting anyway, and I just think it ... highlights his gorgeous little face and lovely mane and long tail and everything, and it just sort of gives you all the information you need in a sort of nice, attractive, nicely lit way. [236]

However, one remark signalled her reflexive, in the moment, response:

...And then I turned round beautifully towards the light and I thought, "Ooh, lovely! Click." [230]

\section{Composition}


Daisy revealed a spontaneous response to composition. She noted that in her walk to the windmill that a tunnel of trees

...the tunnel was quite picturesque and there was a little curve and occasionally a person with a dog came through and that made a little bit more of a picture...[58]

However, it was not sufficiently attractive to get her camera out and take advantage of the image from a compositional point of view. There is a sense here that, of itself, composition would not have sufficient impact for her. When she reached the windmill, she was disappointed with the restoration works disturbing the view and she had to hunt for an angle to take the shot:

...it was very disappointing; it was surrounded by a fence at the bottom because they were repairing it. It's at the top of a hill, so beautiful view from there, but the windmill itself was a bit of a non-event, so I walked round and round, and I thought, "What can I make of this?" [8]

She had previsualised a shot of a beautiful view, the restoration of the fence thwarted her intent and so she begins to look for the view. Clearly, this is a deliberative process but nonconsciously she is seeking the angle and the framing that would bring strong light into the image in a creative way. We can also note the way that she frames her shot of the pony, crouching down to get a good level on the animal's head:

Because I didn't want to take a picture from the top, I wanted to take a picture more on his head height ... especially if it's a small little pony. And the bigger one, I quite like the pictures taken sort of at angle looking upwards, so I quite often crouch down...[220]

In this her framing is suggested to be both deliberative and intuitive. However, what was not forthcoming was any sense of a compositional preference - for Daisy simply sought to create an image she found aesthetically pleasing.

\section{Commentary on the IMP Test}

The interview signals the impact and the creative use of light in Daisy's image making. She gets considerable pleasure out of what has been a life-long pursuit. Turning to the IMP Test (see Exhibit 6.15), it is unequivocal in placing the use of light, creativity, impact and composition within those constructs implicated in her nonconscious processing. However, 
the interview was silent on the issue of focus and depth of field whereas the IMP Test placed them within her nonconscious set. Surprisingly, the use of colour is not within that group although the evidence of the interview is that she sees this as an outcome of her choices about lighting rather than an issue that is prominent in her intuitive judgement. The test suggests that 'technical issues' and 'exposure' are something she may deliberate upon but are not part of her nonconscious processing.

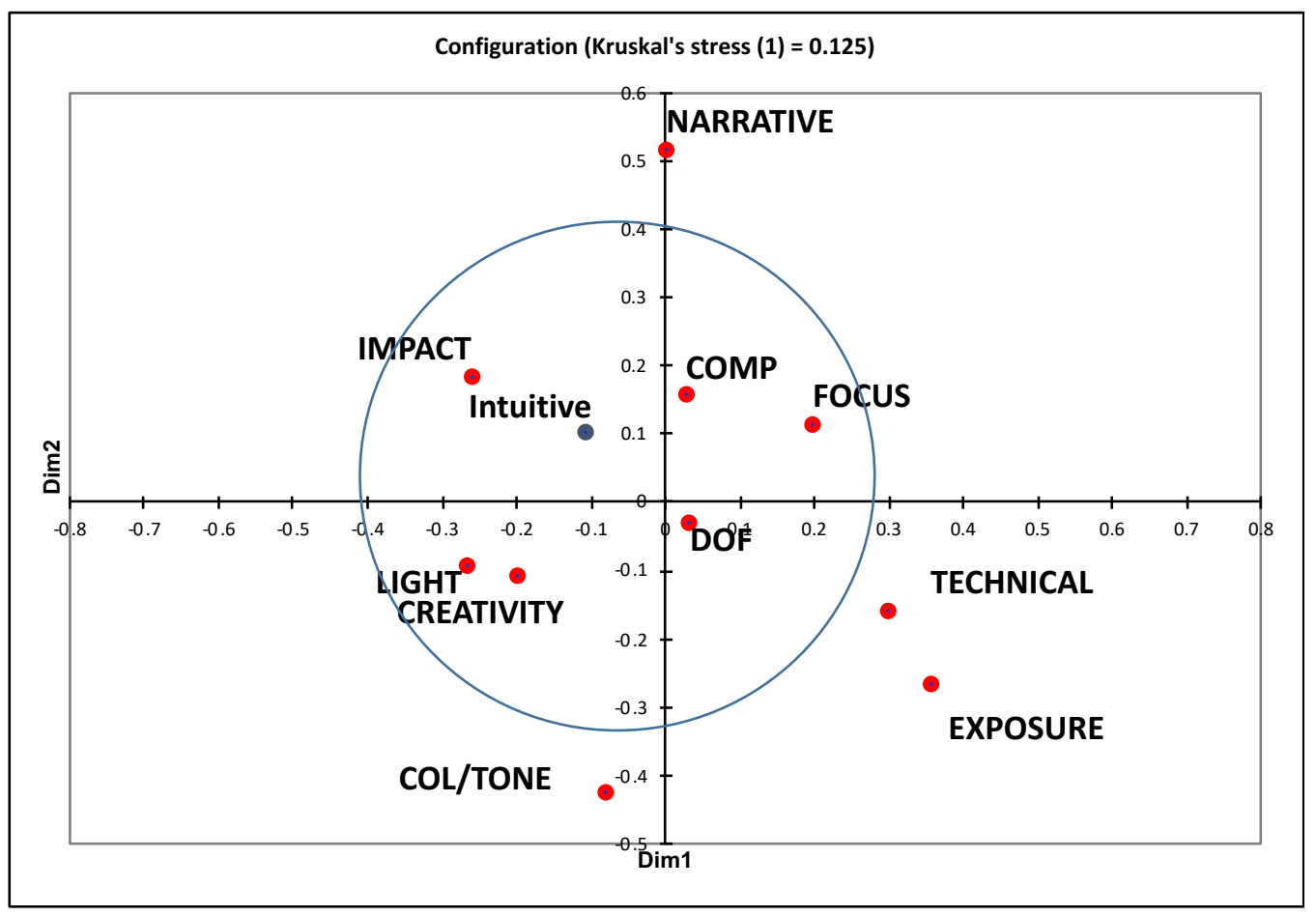

Exhibit

6.15

Multidimensional scaling (IT0103) (see exhibit 6.7 for a note on the dimensions).

Narrative power, in terms of 'story telling' does not surface within her discourse apart from some of the details of the pony's behaviour. The IMP Test places it outside of her nonconscious frame of reference and I cannot see, from the interview, any reason to disagree with that.

In summary, the IMP Test has correctly highlighted the use of light and its close association with creativity as part of Daisy's photographic make-up. There is a good association with impact, but a weaker one with composition. The absence of the exposure and the use of colour / tonality does not suggest that there is a significant latent structure in her nonconscious judgement at this point and, therefore, as a I will discuss in chapter 8 no 
emergent authentic style. Turning to her very high-quality rating on the IMP Test and the tight agreement across the three analytical methods I would place high confidence on the quantitative leg and modest confidence on the result of the interview. Given the balance that suggests strong confidence that the IMP Test has identified those constructs that form part of Daisy's nonconscious knowledge and skills and those that do not.

\subsubsection{Intraspective Interview - Test Participant IT0106 (Simon Weir) ${ }^{42}$}

Simon is a professional photographer, male, 54 years of age, a graduate and has held Licentiateship of the Royal Photographic Society for a number of years. He has extensive experience in the classical music recording business and now works as a professional photographer specialising in photographing live performance, portraiture, wildlife and nature. He has 10 years' experience in the assessment of images for publication purposes.

He completed the IMP test prior to interview on 18/5/2019 and the results were released to him on $21 / 5 / 2019$. He was not aware of the test outcomes before interview. His REI profile shows a marked bias towards the 'experiential' in both aptitude and engagement (RA:EA = 3.9, 4.7; RE: $\mathrm{EE}=4.1,4.7)$.

His interview lasted 94 minutes and the full transcript is included in the appendix. The Intraspective interview focused around three images: first, an infrared image of Venice across water with dark brooding sky above ; second an image of Venice across from St Mark's Square showing movement in gondolas in the foreground and pin-sharp images of Venice in the background and third, an image across the salt-flats in Death Valley USA with mountains in the background.

Simon presented three images for review. Bracketing engaged at transcript reference:

- Venice Image [127 - 157]

- Venice Image from St Mark's Square [197 - 236]

\footnotetext{
${ }^{42}$ Simon has kindly agreed to his name being associated with this analysis. His written agreement to disclosure is appended.
} 
- Salt-flats Death Valley, USA [283 - 303]

Simon is rather unusual in that his IMP Test results reveal a high correlation between the modelled ordering of constructs from the IMP Test and his own conscious ordering. This suggests a high level of introspective ability and represents a testing challenge both for validation of the test but also in the isolation of elements of the intraspective discourse that can be described as either consciously or nonconsciously driven.

The word cloud for his reduced bracketed discourse shows a wide range of terms indicative of his engagement with light, exposure, structure and movement. There is also a reflective element in his language with terms such as aesthetic, thought, feeling and relationships. What is also notable is the high number of constructs which the test reveals are accessible to his

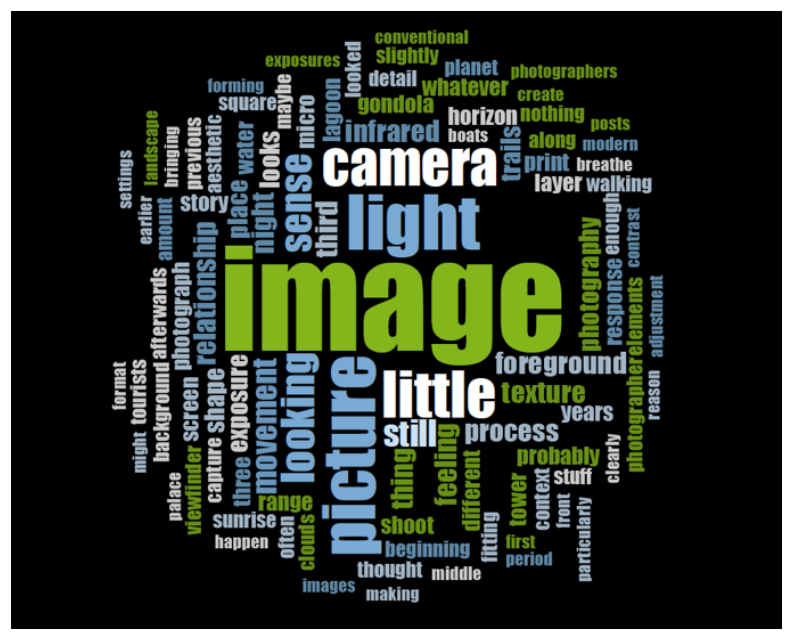
nonconscious processing. The REI shows a clear bias towards the experiential but indicates a strong willingness to use both modalities.

As the interview progressed Simon quickly moved into a reflective mode painting vivid pictures of what he had seen but also describing his motives and sense of achievement in the images he produced. There was also a strong sense that he was looking, in all three cases, for a narrative that lay behind the visual. What was also striking, particularly with images 1 and 3 was the importance of his relationship with others but also with the time and space he needed to take the images.

Throughout, his discourse revealed both conscious and nonconscious activation - there was a clear indication of both the deliberative and the emotional in his chosen words. However, the high degree of spontaneity with both modes of expression confirmed that the signal of high introspective ability in the IMP Test outcomes is justified. With Simon, the interview helped to uncover the working of the boundary layer in his cognition between the two modalities and the latency driving his image making. 
Simon's language reveals a very powerful narrative ability - his recollection of detail and feelings at the time are vivid and clear which gave me the opportunity to stand alongside him and experience the images as he had done.

When exploring the capture of the three images: three themes emerged: (i) the triple of exposure, use of light and use of colour, (ii) composition in terms of layers and relationships within his chosen field of view and (iii) creativity in the use of experimental techniques (infrared, long exposure, and night-time/marginal light shooting). He has a declared preference for wide aspect ratios in his presentation but within its constraints an eclectic compositional style.

\section{Exposure/use of light and use of colour}

Throughout his discourse on the three images exposure, and the problems associated with it appeared to be both non consciously and consciously accessible. The first image, using an infrared modified camera, presented particular challenges with obtaining the correct exposure because of its:

'...limited range of apertures you can use. So that's kind of pre-defined, and the ISO was already set because I had shot other pictures earlier on...[128]

So, to cope with the issues he presets as far as he can. However, even with that he needs to engage in fine-tuning in camera to achieve what he wants:

...the fine-tuning of the settings is done by eye through the viewfinder when I can actually see the IR image and the histogram...[128]

This suggests that with the particular challenges of this type of photography he adopts a more deliberative approach, although this also occurs when he is looking for a specific effect. With the second image he is seeking to represent movement in a night-time shot using a long exposure time. Here again, the deliberative processing comes to the fore with:

... a few test exposures to establish whether I'm in the right ballpark ... and so I made three exposures without moving the camera to find the one that was going to work-they're about a minute ... so not long. [221] 
However, with his third image he becomes more experimental and again, although working in marginal light he is now 'pointing and praying' because he is shooting in the dark. But, with another few words suggests that this shooting was working at an intuitive level:

...this is probably - I can't remember exactly - roughly 20 seconds exposure, which should give you an indication of the light level at this point... this is well pre-dawn. [251]

As they stand the words do not appear particularly significant but in the context of the clarity of his recollection elsewhere, it is striking that at this moment - in the freezing cold of Death Valley in Winter - that he was more reliant on his intuitive judgement than his deliberative and pre-considered approach. The issue becomes more intriguing as we enter into his judgement processes about the use of light and colour. He uses light as a compositional feature, and this is well described in his recollection of shooting light trails in his second image:

...When I started I wasn't particularly mindful of light trails; they weren't part of the image in my mind... until afterwards - after I'd shot a few and I'd realised actually because there's constant boat traffic going across the lagoon that these light trails have to be part of the image as well...[221]

He also refers to 'invisible light' being the light that can be captured in the darkest situations using long exposures and the sensitivity and dynamic range of his medium format camera. There is a dark aesthetic running through his images as well, and it is evident from his 'throwaway' comments that he is attracted by the deep contrasts of night and marginal light photography. The wording he uses here reflects a powerful sense of attraction to the darkness through which the light of presence breaks through - 'incredible' and 'magnificent' are the words chosen to signify his memory of the event and his mood at the time.

We spent the night before shooting by moonlight where you have incredible shadows cast across these textures from the moon, but you could just about make out with the eye, but they're magnificent. [285]

The interplay of different quality lights, light trails of different colours in the second image, and the unique light of the moon enhancing the textures of surfaces within his third image are important and their significance understood at an intuitive level. This use of light as a 
compositional feature emerged in all of his images and is connected with both colour and tonal quality:

...l knew these needed to still appear as gondolas and that's why the silver, gold coloured fin on the tail of the gondola on the left hand third was really vital to this shot. [221]

In the movement of the gondolas in the foreground of his second shot the use of colour helped distinguish one boat from another. The connectivity of foreground and background was enhanced in his mind by:

'...the colour from these light trails getting a connection between the foreground and the background.' [221]

His language, particularly when describing his second image, suggests a confluence of judgement part deliberative but part intuitive. Colour, particularly golds and blues, is important in his imagery. He notes that the pre-dawn light would give him the 'blues and creams and oranges' [285] which are important but gold is particularly significant - which is evocative of powerful emotions of love and peacefulness, for Simon serves to create a 'slightly dream-like aesthetic quality in the image'. [227]

\section{Composition}

In his discourse Simon takes a relationship as opposed to a 'placement' view in framing the image. In other words, it is not formal or classical arrangements that drive his compositional sense but rather the relationship elements have with one another, both in the plane of vision and also between foreground, middle-ground and background. He also sees composition as important in the story he is conveying through his imagery. With his images of Venice, he senses a timelessness linking the past to the present and the future - an issue I will return to in the next section. He is disturbed by modernity in the image, cranes in the extremes of the field of view or boats transgressing the field of view. Referring to his first image he brings into conjunction both the elements of composition and his narrative intent:

It's not perfect and there isn't a lot I could have done about it. I'd like to have got rid of the big boat on the left and it would be lovely if the crane wasn't there on the left, and there may be some inspiration from this image where the two cranes from the image get removed 
because they don't belong to the story, not at this point but I think the boat would be a step too far.[152]

He understands at an intellectual level the classical compositional forms - indeed he refers to the Gestalt in the grouping of buildings as one dominant structure gives coherence to the whole:

From a Gestalt point of view the tower is the dissimilar item in the buildings so we have similarities all the way across and this thing that sticks up, so immediately it's something that the eye is going to associate with.[146]

Within the three images this was one clear instance where he had recognised dissimilarity in the pattern the image presented to him. But it also occurred as he was visualising the second image and working to minimise the intrusion of unwanted light or movement. This recognition of patterns is indicative of nonconscious processing but, nevertheless, his composing is predominantly deliberative as noted in his description of his first image:

As a composition it's a deliberately slightly contrary composition because it doesn't follow the conventional rules, certainly not the rules of third - it's on the fifth rather than third - but very deliberately so because for me this image is as much about sky as it is about the landscape. [146]

Indeed, the sky is what activated his intention to take the first image - the use of infrared creating a powerful recession of cloud into the middle background. The outcomes of infrared photography are very muted in the raw file and not readily apparent through the viewfinder. An important part of the skill associated with this technique is the recognition of the potential transformation of this form of invisible light in post-processing. However, as noted in chapter 3 , one of the important characteristics of nonconscious processing is its superior pattern matching and this, combined with Simon's uncertainty about the recognition process suggests that in this instance his intuition impelled him to take the image:

I don't know whether things happen or it's more likely that I see things that come together in my mind, like the conjunction of the pattern of the sky. And that is quite a big leap because if you saw the raw file - the raw file's very neutral mid tones. Because I've done so much IR over 
the last 15 years plus, I know when I see a sky like that, I know it's going to be an easy journey to actually bring out that texture and shape in the sky. [150]

There is more than a hint here that this type of pattern matching - particularly with the use of infrared - is well 'burnt-in' to his mental firmware. So, we have an intriguing mix of cognition, at one point very deliberative and at another intuitively driven as a result of long experience. Into this cognitive mix the elements of light, colour and texture also feature in the composition of his images - all partly intuitive but also intensely deliberative. One element that does not feature is the focus judgement. In the second image he uses blur to create a sense of foreground movement but is careful to ensure pin-point clarity in his background - but this he leaves to the precision of his medium format camera.

\section{Creativity and Narrative}

With these three images Simon was seeking creative outcomes through a number of different routes. With the infrared photography he was finding drama in the sky, but the underlying narrative was the essence of Venice, its timelessness but also its vulnerability. In the bracketing processes, as we closed in on the moment of capture, he brought to the surface his feelings about the crowds and the congestion within the city as the reason for choosing a distant vantage point. He was also aware of the power of the encroaching elements - the sea and the climate. At one level the use of infrared suggests artifice, but at another the knowing reduction of visible light, the choice of the lower fifth for his sky line, and the clarity of resolution of the city itself brings into sharp relief his awareness of the real qualities that define modern Venice.

I'm not trying to create a Canaletto painting of Venice. It is Venice in 2019, but it is a sense that it is... peace and calm and tranquillity when you step back from it a bit but maybe in a context where there is a little bit of a threatening something over the city ... the city has a lot of problems and a lot of dangers, apart from sinking, but that's quite a big one. It has a constant struggle with its relationship with its visitors, so that whole thing I think in some way is built into the feeling that this is an appropriate way of conveying that environment. [156]

One aspect of the discussion that arose, particularly with respect to the $2^{\text {nd }}$ and $3^{\text {rd }}$ image was his pleasure and joy in the use of his relatively new medium format camera. In both images 
he was seeking to take the maximum advantage of its dynamic range, resolution and colour depth. In the second image he turns to a favourite location for photographers looking across the waterway with the blue covered gondolas moored in the foreground. Here he was attempting a more creative treatment registering the movement in the moored boats at night with light trails running across in the middle ground. This layering of foreground, middle and background entailed employing a challenging array of skills. Again, the use of marginal light both as a compositional and a narrative device was clear in his thinking and had been previsualised:

I got it - in the end - I kind of forced it to happen... as an image concept it was something that I had pre-visualised that I wanted to try and take and capture in some way...so it was one of those cases where I'd really gone through the structure process before pressing the button to make sure that everything was right and the only thing that was down to the random elements was, were the movements going to work? [171]

What was somewhat surprising here was his uncertainty in the outcome from what was a deliberative and consciously constructed image. Part of this may have been uncertainty about the response of his camera. However, another question was whether and to what extent he lacks confidence in his consciously accessible skill compared with the depth of his intuitive judgement, which has largely developed in a different genre. In the final image, although using the same camera his process of capture appears more spontaneous and, indeed, playful:

Oh come and look, come and look at what this is doing. Wow! God, that's so exciting! Can we change it? Can we make it better?" We're almost both kind of taking the picture together. [303]

He refers at length to his high degree of emotional activation; he is with his partner who shares his passion for photography and their relationship is important in his image making in a number of ways:

She gives me the space and the time to do this sort of photography without it being an inconvenience or an unwillingness. And she is as excited by the place and what you could convey photographically from that place as I am. I get very excited, as you've seen...[295] 
This sense of excitement comes back vividly in the bracketing process and with it the sense of the essence of this place:

...Well it's bringing back the feelings of vastness and openness and emptiness of the place. It's bringing back a whole load of feelings about the whole trip and our time together ... But beyond that I think that the feeling for me is about how amazing the planet is ... It's not tangibly planet Earth but it is, and I find that really extraordinary, that the diversity of the planet is so huge and that you can go to these places that are not even that far away from civilisation ... and nobody else is there...[289]

In this emotionally charged atmosphere he uses his camera to capture a simple image showing the weirdness of the salt-flats with their repeating patterns, the mountains and hills in the far distance and a brooding sky above horizon. The horizon line is perfectly set and the image shows technical precision from front to back but what is significant here is his search for the essence of the place. This he sees as a product of his own emotional state and the charge that had given to his photography.

\section{Commentary on the IMP Test}

As I note above, Simon's REI result and the outcome of the IMP Test point to a person with strong introspective ability. This presented an opportunity and a challenge - an opportunity to work with someone where the boundary layer between nonconscious and conscious modalities may well emerge within the interview process, but one which would provide a challenge in the interpretation of the IMP Test results. The quality indicators from the test: $R^{2} Y(0.732)$, Cronbach (0.977) and the stress test (0.161) were all high, signalling that strong to high confidence could be attached to the test results. 


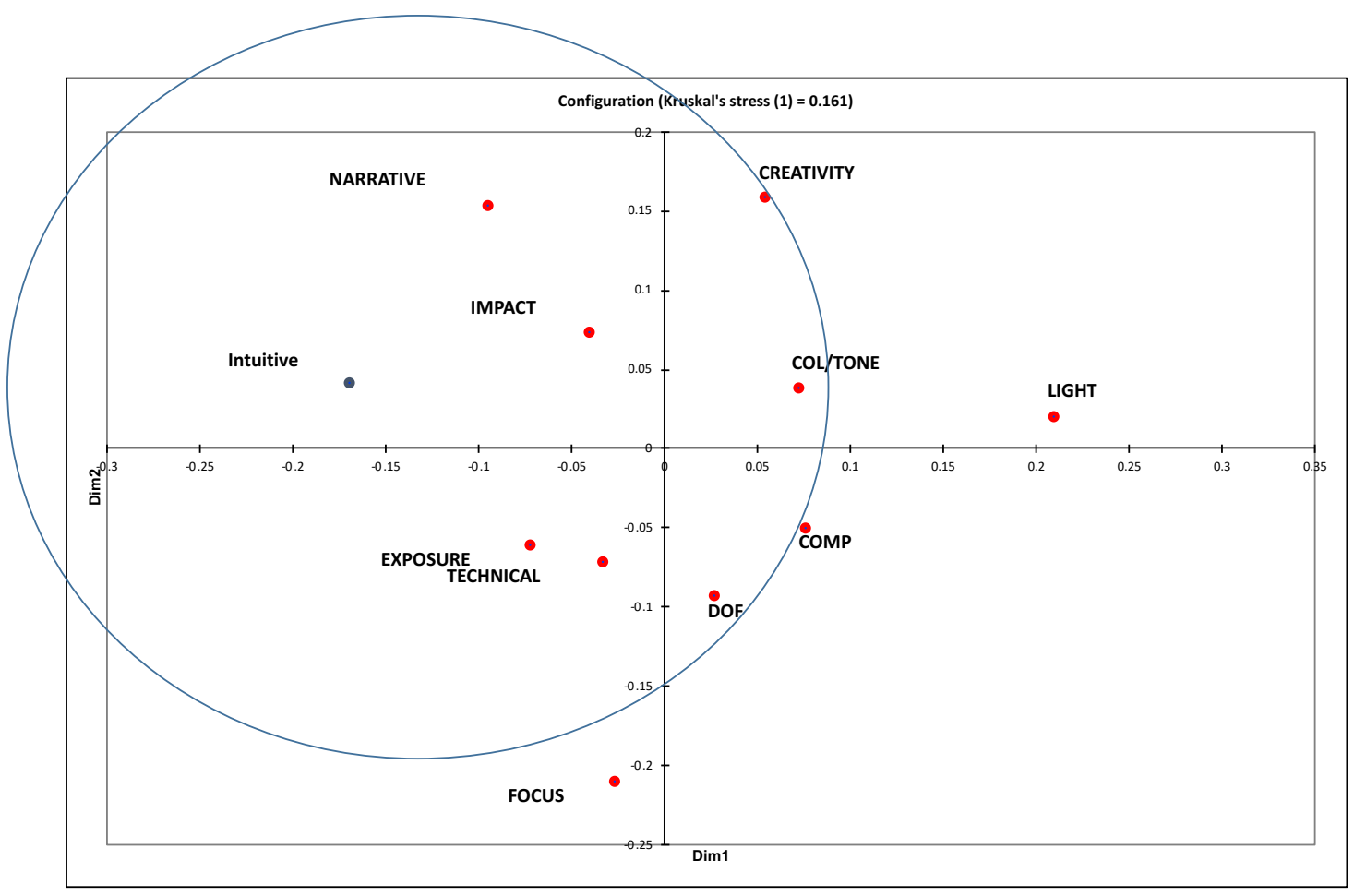

Exhibit 6.16 Multidimensional scaling (IT0106) (see Exhibit 6.7 for a note on the dimensions).

The IMP Test points to seven constructs being within his nonconscious competency. The order of importance generated by the PLS were: impact, narrative, creativity, exposure, use of colour/tonality, technical and the use of depth of field. The MDS disagrees with this ordering showing 'creativity' as less significant in terms of its correlation with his intuitive judgement (Exhibit 6.16). Composition is placed on the margin by both methods, but the focus judgement and the use of light are clearly not impacting upon his intuitive judgement to any significant degree. Generally, all of the constructs are well separated but the close correlation between technical and exposure points to a high association in Simon's mind between these two constructs. Rather interestingly there is a good association revealed by the test between his understanding to composition and the use of depth field. This accords with the observation from the interviews that he sees composition in three as opposed to two dimensional terms.

Simon does rely on the cameras autofocus mechanism to a great degree and his use of blur in the second image relied upon the use of exposure control. His work with the three images strongly suggests a very deliberative and pre-visualised approach to setting focus. The one surprise was in the use of light. Simon's extensive stage work may have preconditioned him into accepting light as largely given. The same is true with composition where the theatre or 
concert hall places significant constraints upon what is possible and where the focus of action is determined by the performance.

The interview provides strong support for the importance of the exposure judgement in Simon's nonconscious processing and, given the high quality of his work and its freedom from elementary technical errors that he is nonconsciously aware of such issues. Exploring the margins between conscious and nonconscious cognition as revealed by the IMP Test, suggests that there is latency in the way he composes, uses colour and tonality (particularly with infrared), and the three-dimensionality in his perception (both in his control of depth of field but also in his placement of elements within the frame).

My conclusion from the IMP Test and from the interview is that this latency is an emergent style that I would describe (non-pejoratively) as theatrical. The test is clear that he has a powerful emotional driver in his image making and a wide range of, and depth of nonconscious perception. In my view, on the balance of evidence there is good, but not complete, agreement between the outcome of the interview and the IMP Test. The IMP Test is of high evidential quality with in excess of $70 \%$ of his variability in judgement explained by the variability of his evaluation under each construct. In summary, I place strong confidence in the validity of the IMP Test for Simon.

\subsection{Conclusion}

At the individual level the IMP Test provides a valuable analytical tool for identifying those constructs driving nonconscious judgement and those that are not. As noted in chapter 2, the test is designed to penetrate the essence of a photographer's intuitive judgement by a process of fracturing the revealed preferences at both the nonconscious and the conscious level. The choice of testing method was designed to be simple in use, cognitively feasible, and sufficiently robust in delivering 220 judgements to permit valid inferences to be drawn. The analytical approach was chosen to complement the fragility of the data set in each case and to trade off bias and variance of outcome in the best way possible. As with all analytical methods a high degree of judgement in recognising patterns and signals in the input and output data is crucial and that takes time and commitment to achieve. The intraspective interviews come at the problem from a different perspective by employing a bracketing and reduction technique allowing the individual to retrieve what lies on the margin of their 
conscious and nonconscious processing. The last participant to take the IMP Test has given a thoughtful appraisal of the test and the interviewing process that is attached to this chapter (Exhibit 6.17 overpage). Throughout the IMP Test was well corroborated by the interviews and I now have strong confidence that the test is delivering what I set out to achieve and the second research objective (RO2) is met. 
I undertook the IMP test and Interview in May 2019...I was fascinated to see how such insight could be gleaned from what... appeared to be a relatively simple test - how hard could it be to rank a few images after all! Wow was I in for a surprise when it actually came to doing it!... I guess this is the inherent self-doubt that niggles away at so many of us working in the visual arts... Well I was in for a surprise...

The test itself was hard ...At times I found myself questioning my responses based on "not wanting to hurt the photographer's feelings" (I knew that all the images were taken by Bob...) but I ploughed on with occasionally savage scores (so sorry!) The considered ranking without time constraints was more familiar territory ... although I found the intrinsic overlap between some of the categories hard to differentiate between - to me 'Focus' and 'Exposure' are both subsets of 'Technical', and 'Impact', 'Creativity' and 'Narrative' also share much common ground. However, I was happy with the final scores and felt that my assessment was fair....

When it came to the ...Interview Bob put me immediately at ease...being taken back to the feeling, sounds and smells of a shoot is a fascinating way of re-thinking your own processes... We talked for nearly two hours and I was pretty exhausted by the end of it but then came the moment of truth - the big reveal... (At this point the result of the IMP Test was revealed)

I have a degree in Physics... and it was immediately clear that the IMP Test is based on really sound data analysis techniques. My answers were gratifyingly consistent, and I found (hopefully convincing) reasons for the outliers... the big surprise was the number of constructs under intuitive control... The results show that many of the 'process' elements are also now running under a more intuitive control ... and that together they are forming a photographic freedom that is allowing the intuitive to dominate producing images that are more connected, heartfelt and mindful not just to me as the photographer but also to the viewer. This is not to say that there is no more work to do - quite the opposite as the test revealed specific areas which... I am looking forward to exploring.

This process was hugely positive for me... and that while process has a place in structuring a photographic workflow its real strength is in giving the photographer freedom to explore and create. The word 'mindful' is over used ..., but in this context 'mindfulness' is the ability to trust your nonconscious self ... something that I strive for and that, just maybe, is beginning to happen.

Exhibit 6.17 Feedback from Simon Weir (IT0106) on the IMP test and subsequent interview. 


\section{Chapter 7 - Partition Studies}

\subsection{Introduction}

In this chapter I focus on the realisation of my research objective RO3:

RO3: To examine, using the protocol developed in RO2, whether judgement in photography is influenced by inter alia gender, cognitive processing style, genre or level of photographic qualification.

In this chapter I examine a number of data partitions to identify whether there are any significant differences between subgroups that cannot be explained by chance. Not every sub-group is sufficiently large in number to give a high level of confidence in the results but, in such cases, I have included the analysis where it represents an area of interest for future research. Each partition study consists of (i) a review of the construct ordering by each subgroup, (ii) an analysis of construct ordering and differences between sub-groups at the individual level, (iii) a commentary on the implications of the results for RO3 above and (iv) an examination of the principal vectors of difference to see, in as far as sample size permits, whether a pattern of intergroup co-variation is evident in the data.

\subsection{Analysis by Gender}

The first data partition separates men from women. Other studies in cognitive processing do reveal some gender differences (see: Proverbio, 2017), although none focus directly on the issue of photographic judgement. Some differences do touch upon spatial orientation and also differences in response to faces and aesthetic and erotic stimuli. For an excellent study and review see Shaqiri, Roinishvili et al. (2018) although they seriously question the methodological issues flowing from previous large sample studies. However, none of theses studies suggest to me that, a priori, there should be any difference between men and women in the process or structure of photographic judgement at the moment of capture or in image assessment. 
All participants in this study identified themselves as male or female and none declined to answer. The sub-group sample sizes were: Male $=72$, Female $=34$ ).

\section{Statistical Tests at a Glance (Partition by Gender)}

(i) Test of aggregate $R^{2} Y$ against the aggregation curve (Exhibit 7.2.1)

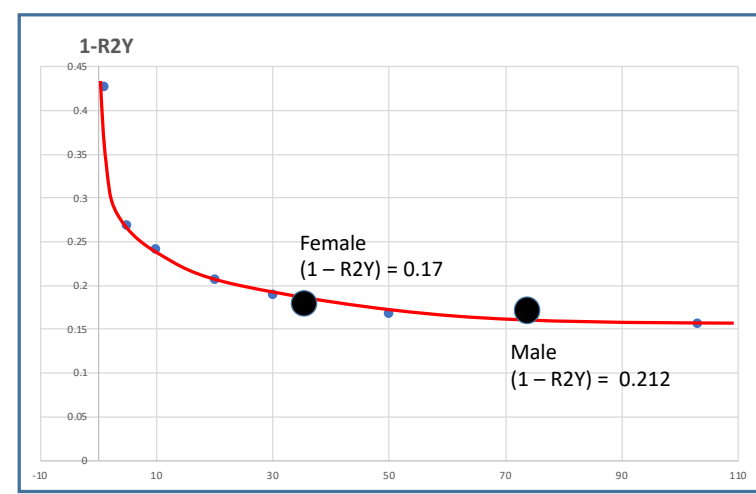

Exhibit 7.2.1 Sample size comparison of sub-groups by gender
HO The aggregate $R^{2} Y$ for each sub-sample are drawn from the aggregation curve.

H1 The aggregate $R^{2} Y$ for each sub-sample are independent of the aggregation curve.

Within the 3\% (+/- 0.03) both sub-samples are predicted by the aggregation curve and $\mathrm{HO}$ cannot be

rejected.

(ii) Test for normality of the distribution (individual test scores)

HO: The variable from which the sample extracted follows a Normal distribution.

H1: The variable from which the sample distribution extracted does not follow a Normal

\begin{tabular}{|l|c|c|}
\hline Jarque-Bera test (Gender): & & \\
\hline & Male & Female \\
\hline JB (Observed value) & 11.326 & 1.303 \\
\hline JB (Critical value) & 5.991 & 5.991 \\
\hline DF & 2 & 2 \\
\hline p-value (Two-tailed) & 0.003 & 0.521 \\
\hline alpha & 0.05 & 0.05 \\
\hline Exhibit 7.2.2 Jarque-Bera Test for Normality \\
\hline
\end{tabular}

was

was

This hypothesis was tested for both male and female $R^{2} Y$ scores using the Jarque-Bara Test. The results (Exhibit 7.2) indicate that the null must be rejected for males and cannot be rejected for females at the alpha $=0.05$ level. Given normality for the female score distribution but not for the male, a non-parametric test of the distributions is indicated.

\section{(iii) Kolmogorov-Smirnof comparison of two distributions}

HO: The two samples follow the same distribution.

H1: The distributions of the two samples are different. 


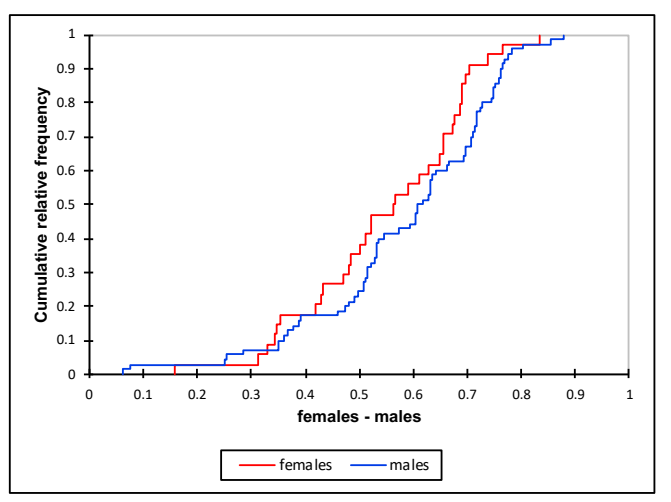

Visually the two cumulative distributions look very similar. The K-S test confirms that intuition with a $\mathrm{p}$ (two-tailed) $=0.142$, which being greater than an alpha of 0.05 means that the null cannot be rejected.

The initial statistical tests suggest, notwithstanding the disparity in the sub-group sample size, that the distributions of the quality of male and female judgements, as measured by the $R^{2} Y$ statistic, are indistinguishable from one another. That gives greater confidence in the results and conclusions we can draw both at the aggregate and at the individual level. Given the failure of the normality test for the male sub-sample, parametric comparison tests of means and variance are unreliable. To resolve this problem, I have used the Kolmogorov-Smirnof test as my method of choice. It focuses attention on both the shape and relative location of the two distributions and, just as importantly in my view, it gives a simple visual perspective on their structure (see Exhibit 7.2.3).

In Exhibit 7.2.4 I show, side by side, the output of the PLS regressions for male and female test participants. The two are strikingly similar, indeed the correlation between the two construct rankings is very high at 0.91 . With men, there are five constructs that are implicated in their intuitive judgement, four that are decisively not, and one - the use of colour/tonality - that is borderline. With women the ranking of the constructs is the same except for two points: the use of colour/tonality is within their intuitive competence and there is a different ordering to the use of light, depth of field and impact. 


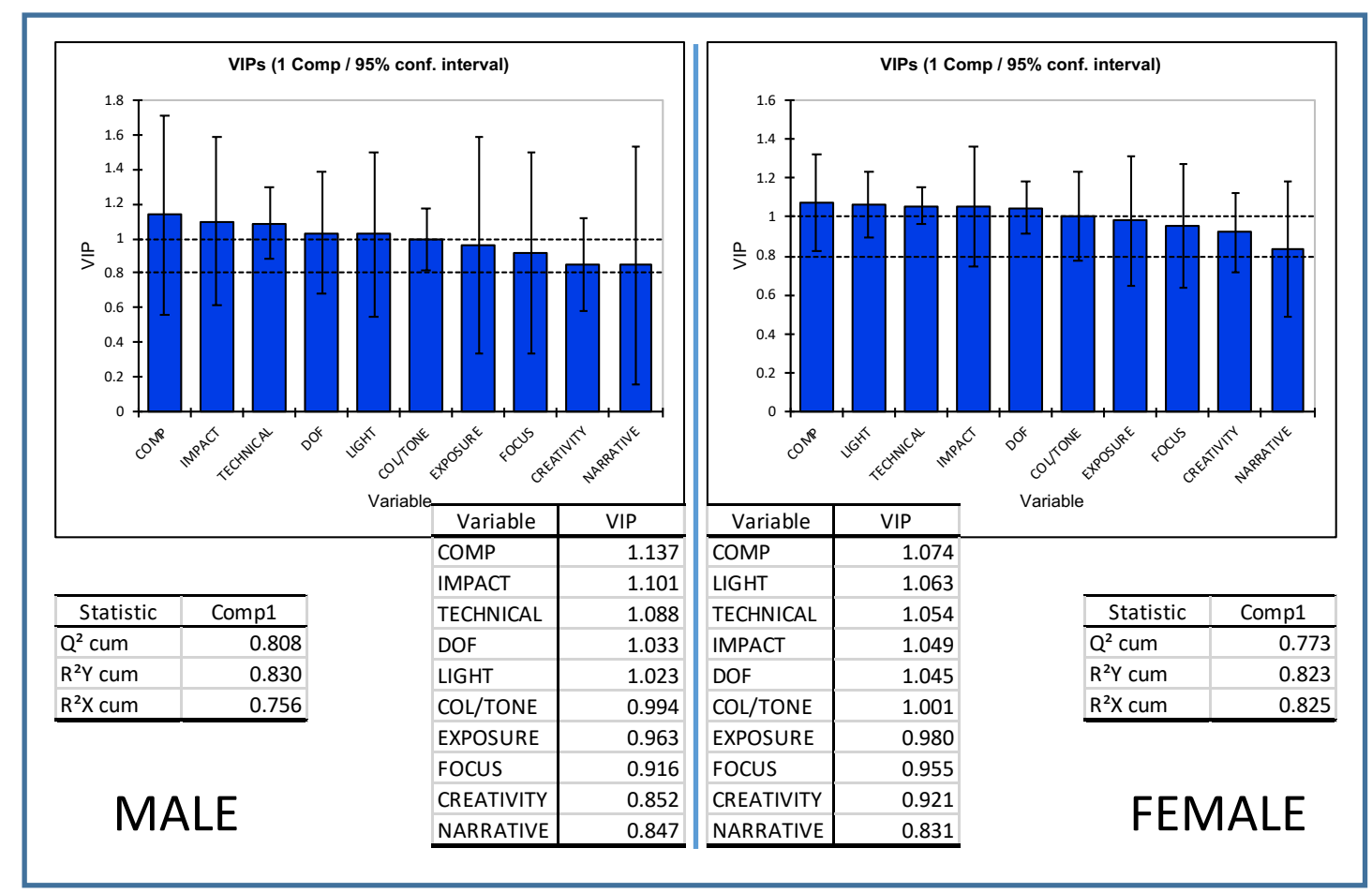

Exhibit 7.2.4 Partial Least Squares Regression by gender.

Reviewing the outcome from the multidimensional scaling (Exhibit 7.2.5), the degree of agreement between the construct ordering and their relationship is particularly marked. Note that the software orientates the constructs depending on the outcome of the data reduction process it uses to create the two dimensions of the analysis. With both groups, the three basic constructs - technical, exposure, and focus - are tightly clustered but are also closely related to the use of depth of field. In both cases, composition is closely associated with impact or, to put it another way, composition, framing and image design are the most significant drivers of both groups' assessment of impact and their intuitive response.

The use of light and the use of colour/tonality are also a closely associated pair but, strikingly, neither narrative power nor creativity form part of the intuitive response of either group at the aggregate level.

However, the picture is not as clear as it seems. When examining the frequency with which constructs are identified by the IMP Test at the individual level, a different picture emerges. As I discussed in chapter 5, at the aggregate sub-group level, idiosyncrasy in judgement is largely eliminated and the outcomes reflect the impact of the publicly 


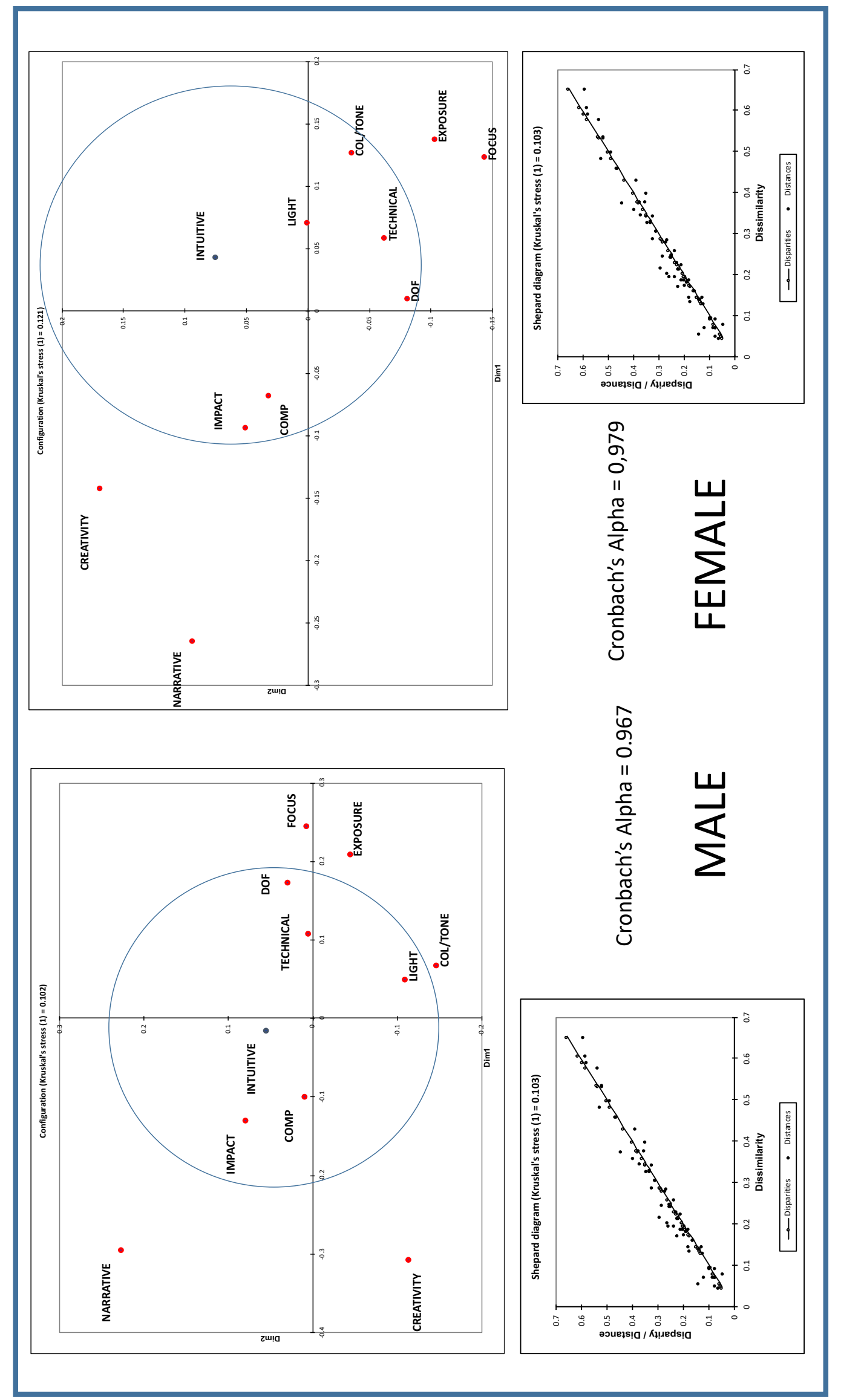

Exhibit 7.2.5 Multidimensional Scaling of performance partitioned by gender. 
shared understanding of the construct on the intuitive judgement of the average person within the sub-group. Exhibit 7.2.6 shows the frequency with which the ten constructs are identified as influencers of intuitive judgement at the individual level. So, for example, the IMP Test placed impact as the most important construct with 25 test participants, creativity was first with five, narrative with eight and so on. Both the total number of occurrences are recorded plus a factorial (sum of the digits) score and ranking.

Running a $\mathrm{Chi}^{2}$ on the total frequency of occurrence of each construct in the intuitive judgement of the individual we cannot reject the null hypothesis:

HO: The rows and the columns of the table are independent.

H1: There is a link between the rows and the columns of the table.

However, when we undertake a $\mathrm{Chi}^{2}$ by construct weighted by order of significance in the individual's nonconscious processing, a clear difference with respect to narrative power emerges. Combining both frequency and rank ordering into the factorial score gives a clearer insight into the relative importance of each construct at the individual as opposed to the aggregate level. With the factorial score, $\mathrm{HO}$ is rejected at the $5 \%$ level and four constructs now show a significant difference. Therefore, the conclusion that males place greater significance on narrative than females, and the reverse for technical, exposure, and the use of colour/tonality, cannot be rejected statistically. Indeed, the probability that the difference is purely by chance is less than $5 \%$ or, in evidential terms, is dismissed beyond reasonable doubt (see Exhibit 7.2.7).

The question is why? The first and most obvious point of note is that, within the test sample, a greater proportion of women than men hold photographic qualifications from the Royal Photographic Society and similar awarding bodies (38\% for women, $20 \%$ for men). Reviewing the data on differences (Exhibit 7.2.8), there are some genre differences worthy of note apart from the difference in the proportion holding photographic qualifications. 


\begin{tabular}{|c|c|c|c|c|c|c|c|c|c|c|c|c|c|c|}
\hline \multicolumn{15}{|l|}{ Male } \\
\hline & Ranked & & & & & & & & & & & & FACTORIAL & \\
\hline & 1st & 2nd & $3 r d$ & 4th & 5th & 6th & 7th & 8th & 9th & 10th & Total & Rank & Male & Rank \\
\hline Impact & 27 & 17 & 5 & 4 & 5 & 1 & 0 & 0 & 0 & 0 & 59 & 1 & 526 & 1 \\
\hline Creativity & 5 & 11 & 7 & 8 & 4 & 1 & 0 & 0 & 0 & 0 & 36 & 4 & 290 & 4 \\
\hline Narrative & 8 & 4 & 13 & 8 & 5 & 1 & 1 & 0 & 0 & 0 & 40 & 2 & 315 & 2 \\
\hline DoF & 3 & 7 & 6 & 11 & 4 & 1 & 1 & 0 & 0 & 0 & 33 & 5 & 251 & 6 \\
\hline Light & 4 & 9 & 7 & 7 & 4 & 2 & 0 & 0 & 0 & 0 & 33 & 5 & 260 & 5 \\
\hline Comp & 9 & 7 & 8 & 10 & 3 & 0 & 0 & 0 & 0 & 0 & 37 & 3 & 305 & 3 \\
\hline Col/tone & 3 & 4 & 6 & 10 & 4 & 2 & 0 & 0 & 0 & 0 & 29 & 8 & 218 & 8 \\
\hline Focus & 4 & 3 & 7 & 5 & 5 & 4 & 0 & 0 & 0 & 0 & 28 & 9 & 208 & 9 \\
\hline Exposure & 3 & 5 & 5 & 1 & 7 & 2 & 0 & 0 & 0 & 0 & 23 & 10 & 174 & 10 \\
\hline Technical & 6 & 5 & 7 & 4 & 4 & 4 & 0 & 0 & 0 & 0 & 30 & 7 & 233 & 7 \\
\hline Total & 72 & 72 & 71 & 68 & 45 & 18 & 2 & 0 & 0 & 0 & & & & \\
\hline \multirow{2}{*}{\multicolumn{15}{|c|}{ Female }} \\
\hline & & & & & & & & & & & & & & \\
\hline & Ranked & & & & & & & & & & & & FACTORIAL & \\
\hline & 1st & 2nd & $3 r d$ & 4th & 5th & 6th & 7th & 8th & 9th & 10th & Total & Rank & Female & Rank \\
\hline Impact & 14 & 7 & 1 & 5 & 2 & 0 & 1 & 0 & 0 & 0 & 30 & 1 & 262 & 1 \\
\hline Creativity & 3 & 4 & 3 & 3 & 1 & 2 & 0 & 0 & 0 & 0 & 16 & 3 & 127 & 5 \\
\hline Narrative & 1 & 1 & 3 & 2 & 2 & 0 & 0 & 0 & 0 & 0 & 9 & 10 & 69 & 10 \\
\hline DoF & 2 & 1 & 1 & 7 & 1 & 2 & 0 & 0 & 0 & 0 & 14 & 8 & 102 & 9 \\
\hline Light & 1 & 7 & 4 & 1 & 2 & 1 & 0 & 0 & 0 & 0 & 16 & 3 & 129 & 4 \\
\hline Comp & 5 & 3 & 1 & 3 & 2 & 1 & 0 & 0 & 0 & 0 & 15 & 7 & 123 & 6 \\
\hline Col/tone & 3 & 4 & 5 & 2 & 2 & 0 & 0 & 0 & 0 & 0 & 16 & 3 & 132 & 3 \\
\hline Focus & 0 & 1 & 5 & 3 & 5 & 2 & 0 & 0 & 0 & 0 & 16 & 3 & 110 & 7 \\
\hline Exposure & 3 & 1 & 6 & 3 & 0 & 0 & 0 & 0 & 0 & 0 & 13 & 9 & 108 & 8 \\
\hline Technical & 2 & 5 & 5 & 4 & 2 & 1 & 0 & 0 & 0 & 0 & 19 & 2 & 150 & 2 \\
\hline Total & 34 & 34 & 34 & 33 & 19 & 9 & 1 & 0 & 0 & 0 & & & & \\
\hline
\end{tabular}

Exhibit 7.2.6 Frequency of constructs appearing within the intuitive competency (by gender).

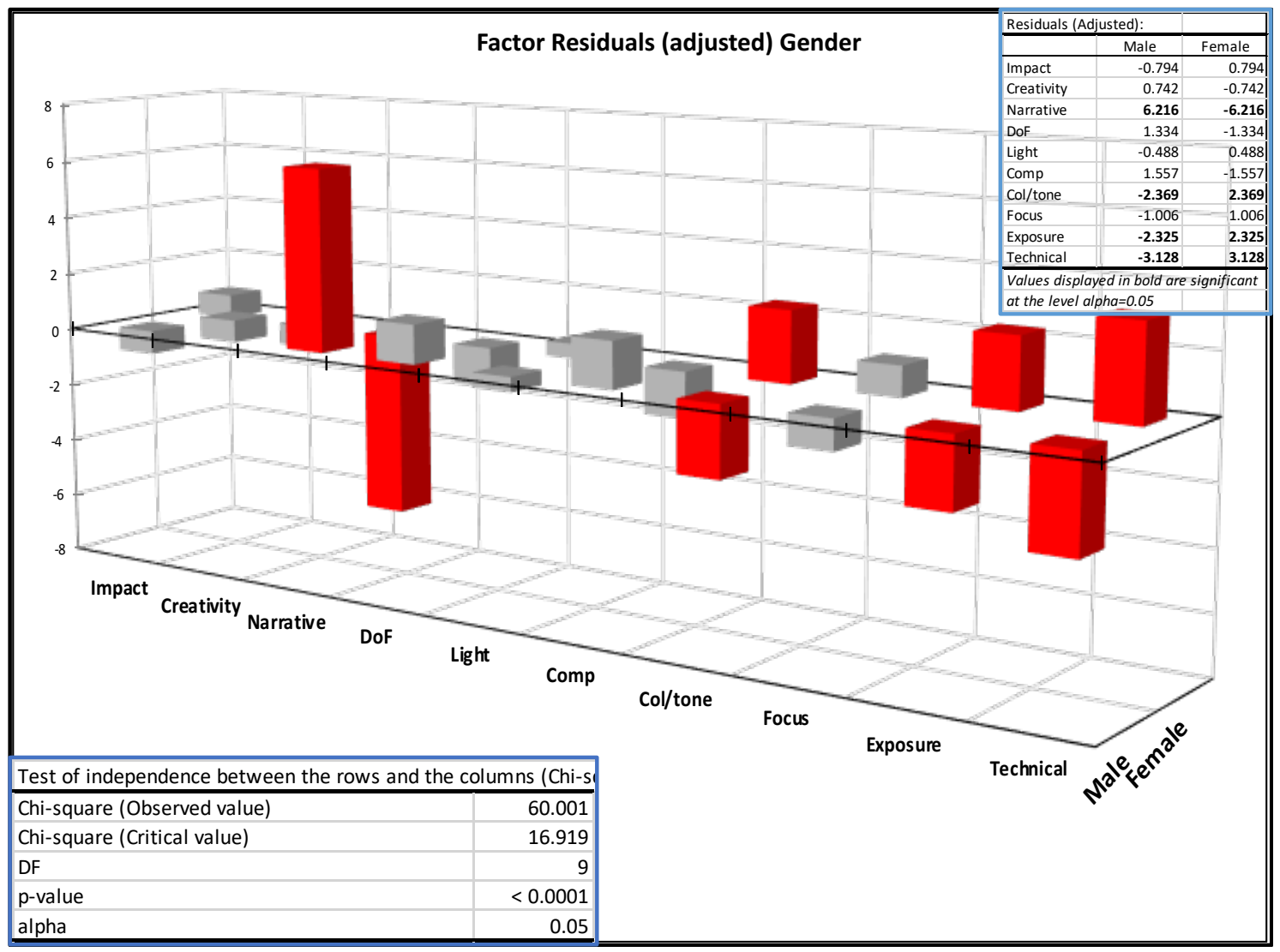

Exhibit 7.2.7 Residuals from Chi $^{2}$ Test-gender. 


\begin{tabular}{|c|c|c|c|c|c|c|c|c|c|c|c|c|c|c|}
\hline & $\begin{array}{c}\text { Sample } \\
\text { Size }\end{array}$ & $\begin{array}{c}\text { Average } \\
\text { Age }\end{array}$ & $\begin{array}{l}\text { Quals } \\
\text { (Photo) }\end{array}$ & $\begin{array}{l}\text { Quals } \\
\text { (Acad) }\end{array}$ & Professional & $\begin{array}{l}\text { Quality } \\
\text { (R2Y)> } \\
\text { median }\end{array}$ & $\begin{array}{c}\text { Intuitive } \\
\text { Bias (IMP) }\end{array}$ & $\begin{array}{l}\text { Wildlife/ } \\
\text { nature }\end{array}$ & Landscape & Creative & Documentary & Travel & $\begin{array}{c}\text { Black and } \\
\text { White }\end{array}$ & $\begin{array}{l}\text { Other } \\
\text { Genres }\end{array}$ \\
\hline Female & 34 & 60 & $38 \%$ & $82 \%$ & $3 \%$ & $44 \%$ & $50 \%$ & $29.40 \%$ & $23.50 \%$ & $5.90 \%$ & $8.30 \%$ & $20.60 \%$ & $8.80 \%$ & $12.30 \%$ \\
\hline
\end{tabular}

Exhibit 7.2.8 Gender Differences

A greater proportion of women than men pursue wildlife/nature and documentary/travel photography. Men are more likely to be engaged in other minority genres such as astrophotography, architectural, and sport photography. Males also show a greater proportion shooting in Black and White. One can speculate on why or if the gender difference is significant. The only evidence suggested by this study is that, compared with men, a greater proportion of females hold photographic qualifications and a greater proportion pursue wildlife/nature as their principal genre. Of these two, only the greater proportion of females holding photographic qualifications is negatively associated with 'narrative' power.

The results reported below (7.6) for genre, do not suggest that the difference is caused by the preponderance of wildlife/nature in the female profile. It can be argued that the problem is due to a systematic difference in the level of understanding between males and females of the concept of 'narrative'. This, however, should not significantly influence the results. As I have already noted, the IMP Test is principally sensitive to variability and co-variability in understanding between constructs and between constructs and the individual's intuitive judgement. It is well beyond the scope of this data set to support a robust conclusion about the causes of any gender difference - if such difference exists. All the IMP Test and the analysis shows is a 'might be' as opposed to 'is' at this stage, and further research would be needed on what is a complex multivariate problem.

\subsection{Analysis by Cognitive Modality}

All of the test subjects took Epstein's Rational-Experiential Inventory, which compares both aptitude and engagement with either cognitive modality. However, given the relatively small number of individuals scoring high on the experiential (intuitive) side of the REI and, given the nature of the REI as a self-report instrument, I decided to adopt an alternative approach where evidence of preferred modality is drawn from the results of the IMP Test. 
The IMP Test generates a correlation between the test subject's ranking of the test images and the overall ranking generated by the consensus. It calculates this correlation in two ways: first, using each individual's declared ranking of the significance of the ten constructs and second, the model generated ranking reflecting their nonconscious ordering. Using this as an indicator of nonconscious cognitive ability is, I would argue, a more satisfactory indicator of cognitive preference than the more general, less context specific, REI test. The sample sizes were 51:55 being those that the Imp Test identified as having a significant intuitive bias in their outcomes and those who did not.

\section{Statistical Tests at a Glance (Partition by Cognitive Modality)}

\section{(i) Test of aggregate $R^{2} Y$ against the aggregation curve (Exhibit 7.9)}

HO The aggregate $R^{2} Y$ for each subsample are drawn from the aggregation curve.

H1 The aggregate $R^{2} Y$ for each subsample are independent of the aggregation curve.

Within the $3 \%(+/-0.03)$ both subsamples are predicted by the aggregation

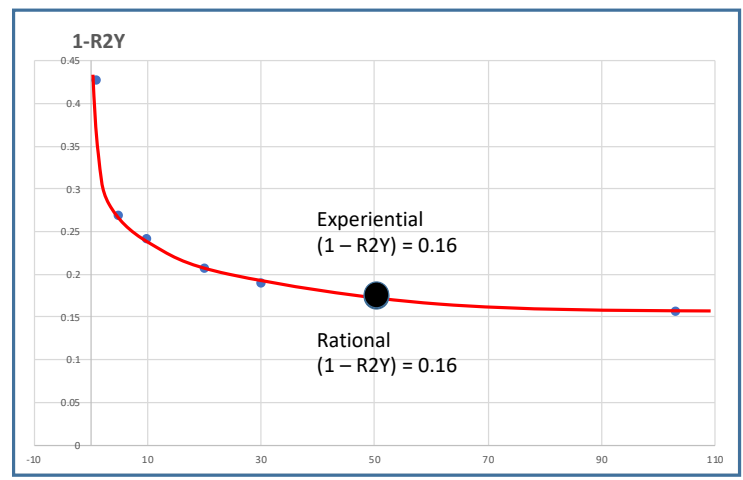

Exhibit 7.3.1 Sample size comparison of sub-groups by rational versus Experiential modality. and $\mathrm{HO}$ cannot be rejected.

\section{(ii) Test for normality of the distribution (individual test scores)}

This hypothesis was tested for rational and experiential scores using the JarqueBara Test. The results (Exhibit 7.3.2) indicate that the null is not rejected for either group at the alpha $=0.05$ level. Given this, a parametric test of the

\begin{tabular}{|l|r|r|}
\hline & Rational & Experiential \\
\hline JB (Observed value) & 4.678 & 5.182 \\
\hline JB (Critical value) & 5.991 & 5.991 \\
\hline DF & 2 & 2 \\
\hline p-value (Two-tailed) & 0.096 & 0.075 \\
\hline alpha & 0.05 & 0.05 \\
\hline
\end{tabular}

Exhibit 7.3.2 Jarque-Bera Test for normality (by cognitive modality) distributions is indicated.

\section{(iii) Parametric comparison of two distributions}


HO: The two samples follow the same distribution with respect to mean and variance.

H1: The distributions of the two samples are different with respect to mean and variance.

Both the two-sample student $\mathrm{t}$ test and Fisher $\mathrm{F}$ test confirm that both distributions are of common mean and common variance at the 0.05 level.

Given the above, there does not appear to be any significant difference between the two subgroups. As far as the distribution of $R^{2} Y$ is concerned, both appear to be drawn from the same underlying distribution. However, within that result there are nuances that the IMP Test has picked up both at the aggregate level, and at the individual level of analysis.

Running the PLS (Exhibit 7.3.3) and MDS (Exhibit 7.3.4) statistical procedures revealed a very broad similarity with both the aggregate and the individual partitions. There are minor differences of ordering within the intuitively accessible constructs, where impact emerges higher with those whose performance on the IMP Test signals a higher than average intuitive (experiential) ability.

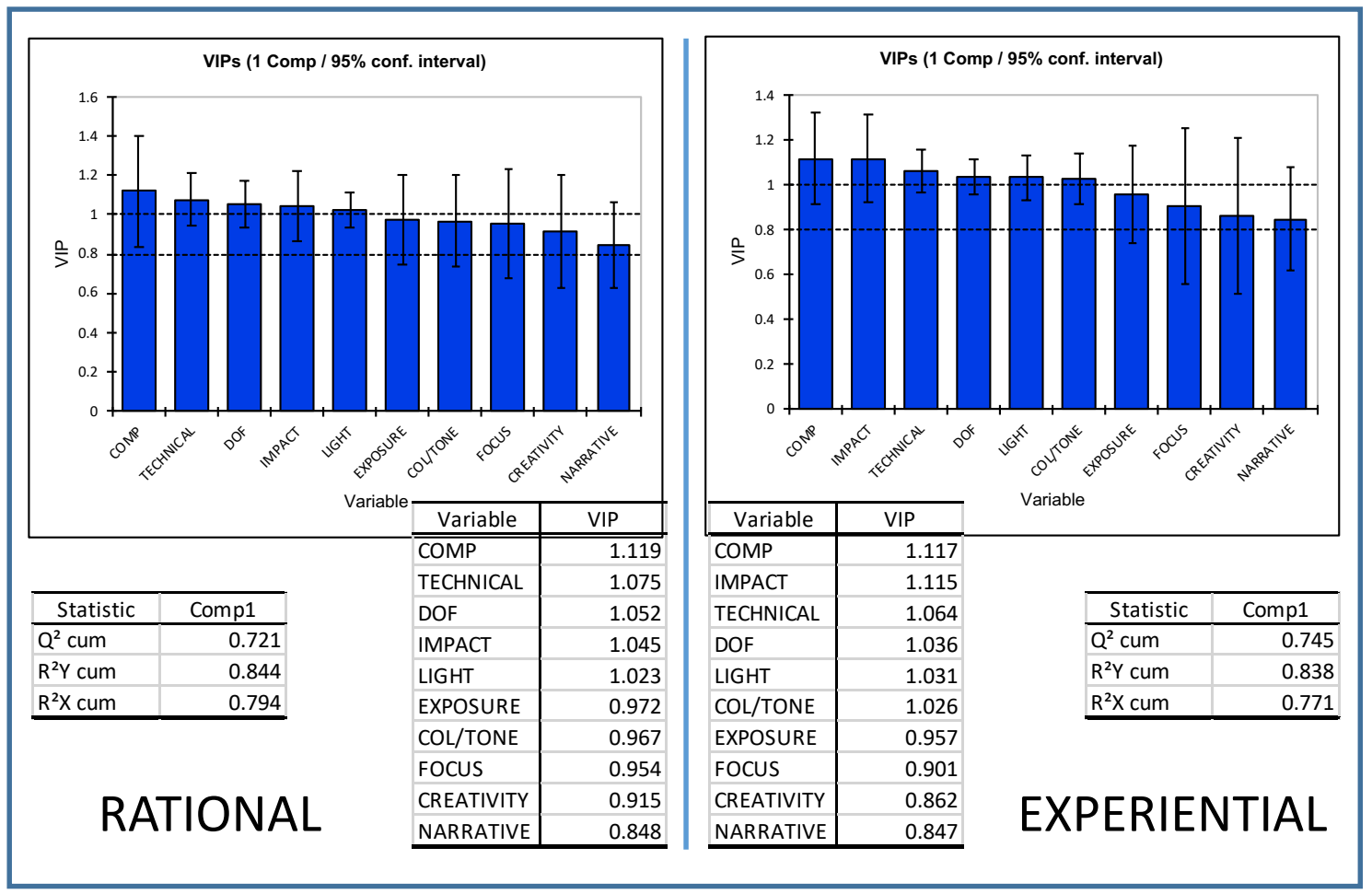

Exhibit 7.3.3 Partial Least Squares by revealed bias in cognitive modality. 


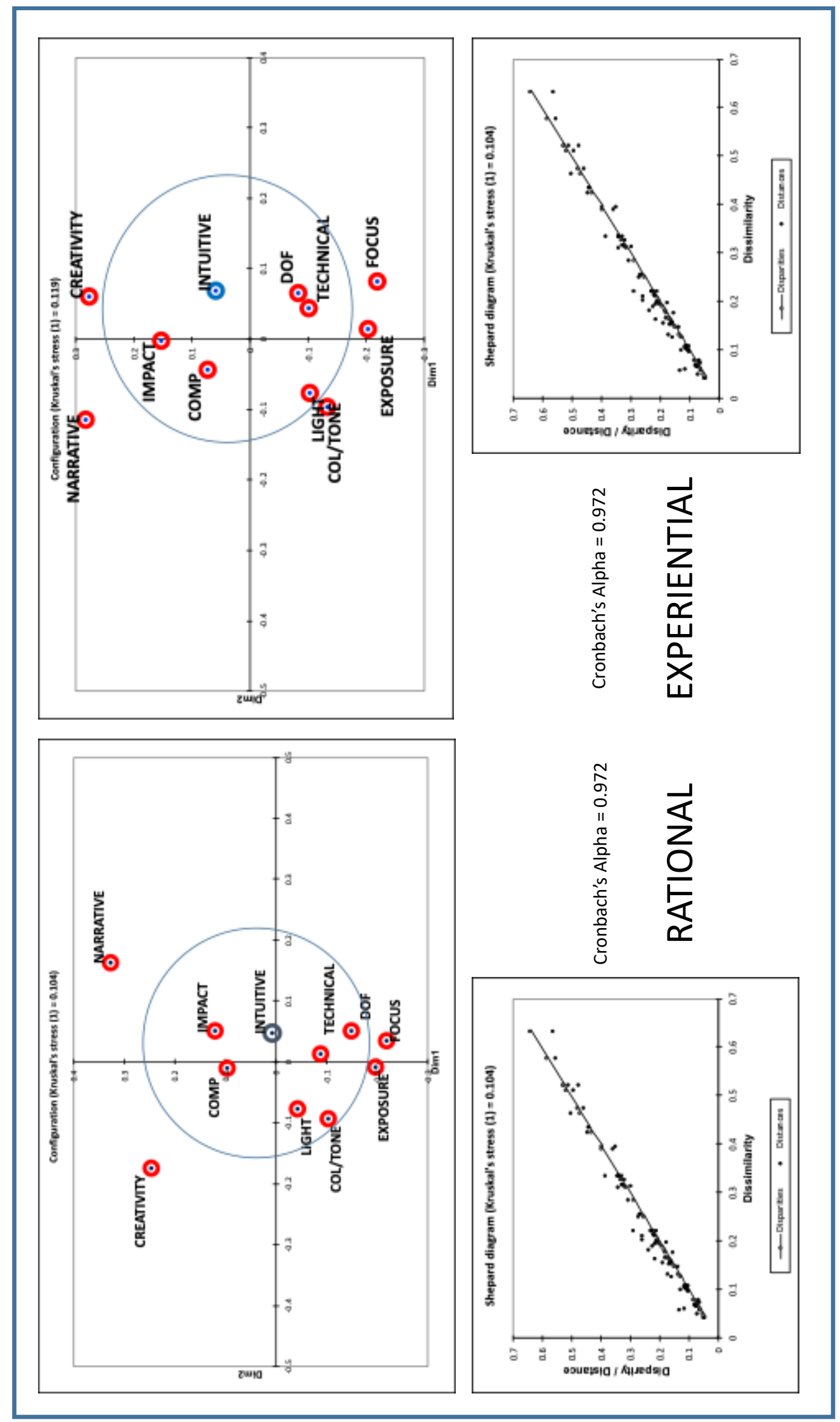

Exhibit 7.3.4 Multidimensional Scaling of performance partitioned by revealed cognitive modality. 
Again, focus, exposure, narrative and creativity are outside of the intuitive frame at the aggregate level, but the use of colour/tonality is on the margin. It is also worthy of note that the experientially declared participants recognise depth of field in their intuitive assessment of an image

The striking thing about this result is that at the aggregate level there are differences in the ordering of the ten constructs, but with the 'experiential' group six constructs lie within their intuitive competency compared with five for the 'rational' group.

However, when we probe the individual level a more nuanced picture arises (Exhibit 7.3.5). Again, we are looking at the constructs in a different way: this time in terms of their frequency of occurrence and their importance within each individual's cognitive frame of reference. Some striking observations can be made: first, exposure and focus are both low in frequency of occurrence even when they appear within the intuitive competency of the individuals concerned. This is a common theme across all the testing with aggregates and with groups.

\begin{tabular}{|c|c|c|c|c|c|c|c|c|c|c|c|c|c|c|}
\hline \multicolumn{15}{|c|}{ Intuitive Bias } \\
\hline & Ranked & & & & & & & & & & & & FACTORIAL & \\
\hline & 1st & 2nd & 3rd & 4th & 5th & 6th & 7th & 8th & 9th & 10th & Total & Rank & Intuitive bias & Rank \\
\hline Impact & 22 & 12 & 4 & 5 & 1 & 1 & 0 & 0 & 0 & 0 & 45 & 1 & 406 & 1 \\
\hline Creativity & 4 & 6 & 4 & 2 & 3 & 1 & 0 & 0 & 0 & 0 & 20 & 8 & 163 & 7 \\
\hline Narrative & 6 & 2 & 9 & 4 & 4 & 0 & 1 & 0 & 0 & 0 & 26 & 3 & 206 & 3 \\
\hline DoF & 2 & 2 & 2 & 8 & 3 & 0 & 0 & 0 & 0 & 0 & 17 & 9 & 128 & 9 \\
\hline Light & 2 & 5 & 6 & 4 & 4 & 0 & 0 & 0 & 0 & 0 & 21 & 5 & 165 & 6 \\
\hline Comp & 3 & 7 & 6 & 7 & 4 & 1 & 0 & 0 & 0 & 0 & 28 & 2 & 219 & 2 \\
\hline Col/tone & 2 & 6 & 5 & 5 & 2 & 1 & 0 & 0 & 0 & 0 & 21 & 5 & 166 & 5 \\
\hline Focus & 3 & 3 & 6 & 4 & 2 & 3 & 0 & 0 & 0 & 0 & 21 & 5 & 160 & 8 \\
\hline Exposure & 3 & 4 & 3 & 1 & 3 & 1 & 0 & 0 & 0 & 0 & 15 & 10 & 120 & 10 \\
\hline Technical & 4 & 4 & 5 & 6 & 4 & 0 & 0 & 0 & 0 & 0 & 23 & 4 & 182 & 4 \\
\hline Total & 51 & 51 & 50 & 46 & 30 & 8 & 1 & 0 & 0 & 0 & & & & \\
\hline \multirow{2}{*}{\multicolumn{15}{|c|}{ Rational Bias }} \\
\hline & & & & & & & & & & & & & & \\
\hline & Ranked & & & & & & & & & & & & FACTORIAL & \\
\hline & 1st & 2nd & $3 r d$ & 4th & 5th & 6th & 7th & 8th & 9th & 10th & Total & Rank & Rational bias & Rank \\
\hline Impact & 19 & 12 & 2 & 4 & 6 & 0 & 1 & 0 & 0 & 0 & 44 & 1 & 382 & 1 \\
\hline Creativity & 4 & 9 & 6 & 9 & 2 & 2 & 0 & 0 & 0 & 0 & 32 & 2 & 254 & 2 \\
\hline Narrative & 3 & 3 & 7 & 6 & 3 & 1 & 0 & 0 & 0 & 0 & 23 & 8 & 178 & 8 \\
\hline DoF & 3 & 6 & 5 & 10 & 2 & 3 & 1 & 0 & 0 & 0 & 30 & 3 & 225 & 3 \\
\hline Light & 3 & 11 & 5 & 4 & 2 & 3 & 0 & 0 & 0 & 0 & 28 & 4 & 224 & 4 \\
\hline Comp & 11 & 3 & 3 & 6 & 1 & 0 & 0 & 0 & 0 & 0 & 24 & 6 & 209 & 5 \\
\hline Col/tone & 4 & 2 & 6 & 7 & 4 & 1 & 0 & 0 & 0 & 0 & 24 & 6 & 184 & 7 \\
\hline Focus & 1 & 1 & 6 & 4 & 8 & 3 & 0 & 0 & 0 & 0 & 23 & 8 & 158 & 10 \\
\hline Exposure & 3 & 2 & 8 & 3 & 4 & 1 & 0 & 0 & 0 & 0 & 21 & 10 & 162 & 9 \\
\hline Technical & 4 & 6 & 7 & 2 & 2 & 5 & 0 & 0 & 0 & 0 & 26 & 5 & 201 & 6 \\
\hline Total & 55 & 55 & 55 & 55 & 34 & 19 & 2 & 0 & 0 & 0 & & & & \\
\hline
\end{tabular}

Exhibit 7.3.5 Frequency of constructs appearing within the intuitive competency (Cognitive Modality) 
Second, with the 'rational' group, 'creativity' is high both in terms of frequency of occurrence and in terms of its importance in the individuals' rankings. But narrative power is very low. The reverse is the case with the 'experiential' group. One possible explanation is that 'creative' has strong connotations in the use of post-processing techniques to create stylised images, whereas 'narrative' is more concerned with the 'reading' of an image. As discussed in chapter 3 , there is strong support within the literature for the idea that nonconscious processing is superior to conscious processing in pattern matching and recognition. This, in my view, gives some context for the higher levels of attention to narrative power, both in terms of the frequency of its appearance within the nonconscious competency of those individuals with an intuitive bias and in terms of the relative importance they attach to it. It is also worthy of note that impact, even though it is first in both rankings, is significantly more important to the 'intuitive (experiential)' as opposed to the 'rational' group. This result supports the prior belief that those who score high on the intuitive scale, are likely to be more emotionally responsive to the images they see than those who are not.

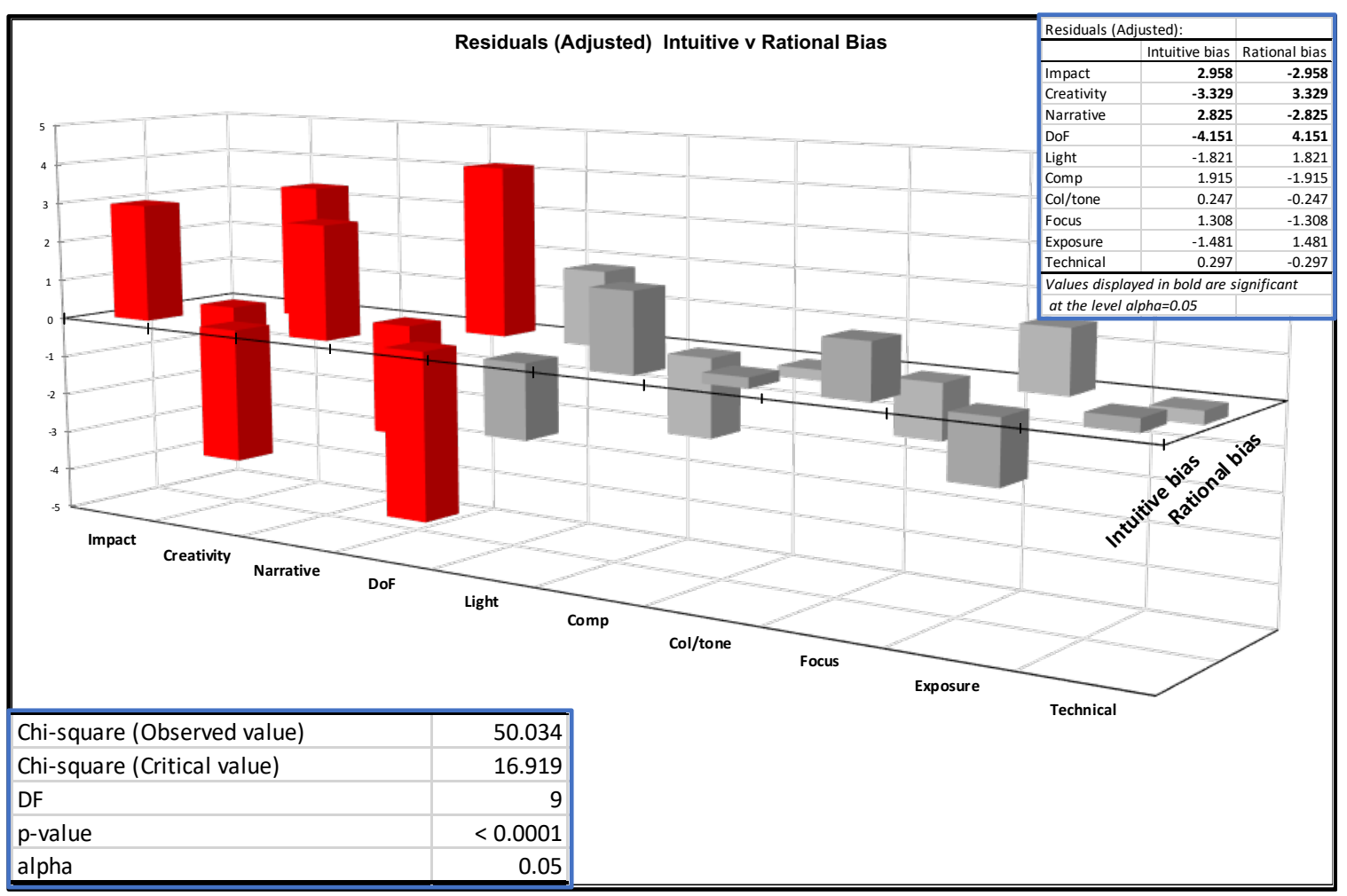

Exhibit 7.3.6 Residuals from Chi $^{2}$ Test - cognitive modality. 
One other tantalising outcome is the greater importance placed upon the use of depth of field for those in the rational as opposed to the intuitive group. I cannot see any obvious explanation for this.

Reworking the analysis with the aptitude score from the Rational Experiential Inventory (REI) does produce a markedly similar outcome with respect to creativity. This suggests that those who present a rational bias place greater significance on creativity than do those who present an intuitive bias. It does not show any significant difference between the two modalities with respect to narrative power. Reworking using the outcome from the REI for rational as opposed to intuitive 'engagement' yields the same results

\subsection{Analysis by Qualification}

For this analysis, I isolated those participants who claim a recognised photographic qualification (see the participant profile in chapter 5, Exhibit 5.4), from those who do not. Of this subsample, 23 held qualifications of the RPS and six held qualifications from other bodies. There were no declared graduates in photography at either the under- or post-graduate level.

\section{Statistical Tests at a Glance (Partition by Qualification)}

(i) Test of aggregate $R^{2} Y$ against the aggregation curve (Exhibit 7.14)

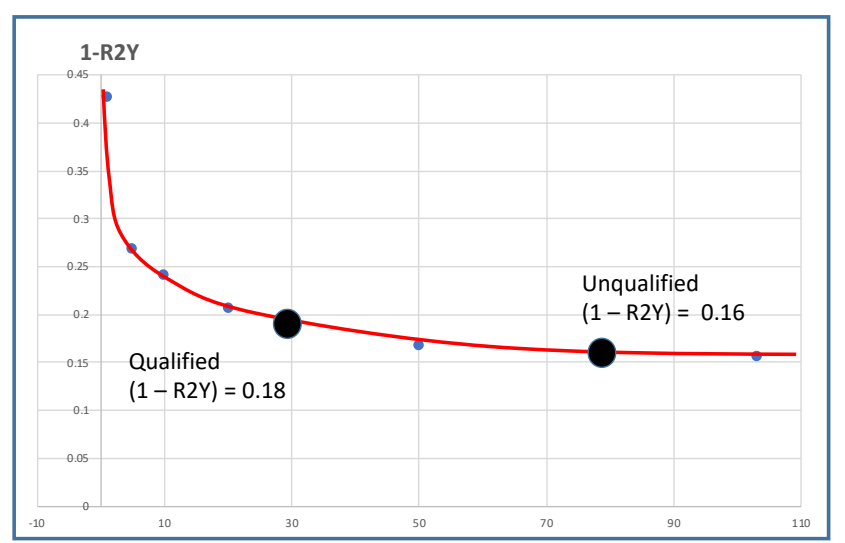

Exhibit 7.4.1 Sample size comparison of sub-groups by qualification distribution (individual test scores)
HO The aggregate $R^{2} Y$ for each sub-sample are drawn from the aggregation curve.

H1 The aggregate $R^{2} Y$ for each sub-sample are independent of the aggregation curve. Within the $3 \%(+/-0.03)$ both sub-samples are predicted by the aggregation curve and $\mathrm{HO}$ cannot be rejected.

(ii) Test for normality of the 
HO: The variable from which the sample was extracted follows a Normal distribution.

H1: The variable from which the sample was extracted does not a Normal distribution

\begin{tabular}{|l|r|r|}
\hline \multicolumn{2}{|l|}{ Jarque-Bera test (Qualified v Unqualified): } & \\
\hline JB (Observed value) & Non-qualified & Qualified \\
\hline JB (Critical value) & 10.525 & 1.554 \\
\hline DF & 5.991 & 5.991 \\
\hline p-value (Two-tailed) & 2 & 2 \\
\hline alpha & 0.005 & 0.460 \\
\hline
\end{tabular}

Exhibit 7.4.2 Jarque-Bera Test for normality by qualification

This hypothesis was tested for test subjects with and without qualification using the JarqueBara Test. The results (Exhibit 7.4.2) indicate that the null must be rejected for qualified and cannot be rejected for unqualified subjects at the alpha $=0.05$ level. Given absence of normality for the qualified group, a non-parametric test of the distributions is indicated.

\section{(iii) Kolmogorov-Smirnof comparison of two distributions}

HO: The two samples follow the same distribution.

Ha: The distributions of the two samples are different.

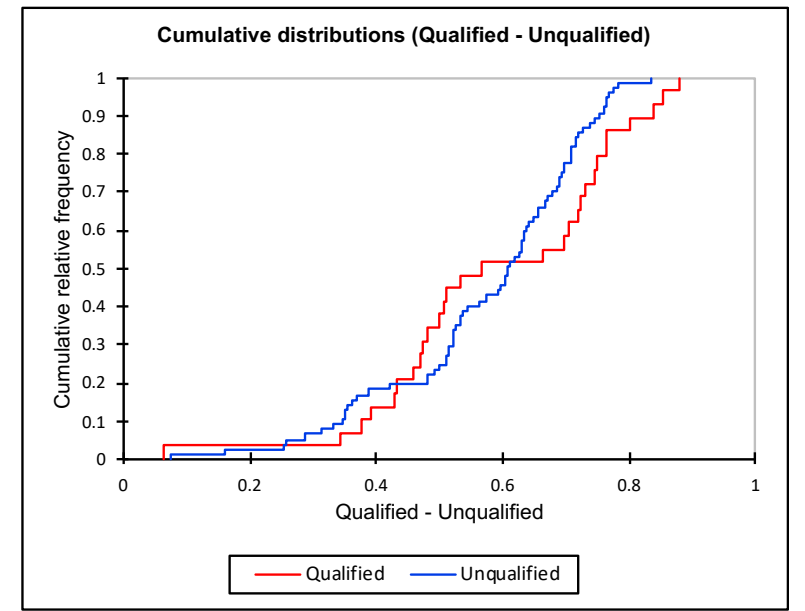

Visually the two cumulative distributions look very similar. The K-S test confirms that intuition with a $\mathrm{p}$ (two-tailed) $=0.19$, which being greater than an alpha of 0.05 means that the null cannot be rejected.

Exhibit 7.4.3 Cumulative distributions $R^{2} Y$ by

qualification

Exhibit 7.4.4 shows those with qualifications to the left and those without to the right. The PLS results show a very consistent ranking, with composition and impact at the top, and creativity and narrative at the bottom. Apart from the position of focus and exposure within the ranking, there is nothing to separate the two analyses. However, what is noticeable is that the qualified group have, on average, more constructs under their intuitive control compared with the unqualified group. That is not surprising and gives a degree of additional credibility to the analysis. Presumably those who seek qualifications have studied and practiced the subject more consistently and rigorously than others, and this is showing up in 
their intuitive access to the basic, commonly accepted elements of the subject as revealed by the aggregate analysis.

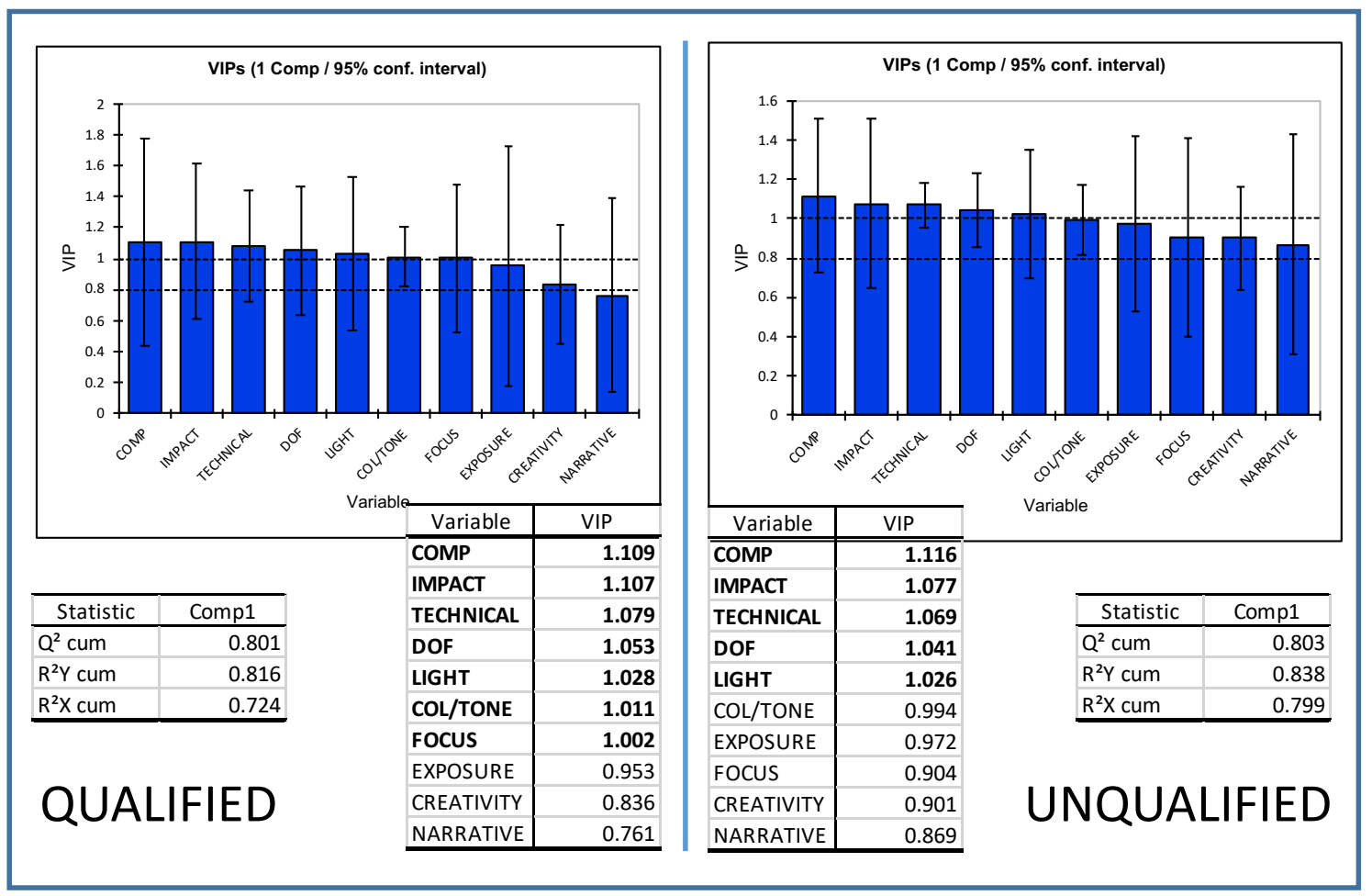

\section{Exhibit 7.4.4 Partial Least Squares analysis of qualified versus unqualified subjects.}

Again, we note what is now a common feature across both the aggregate and the individual analysis: the focus and the exposure judgement are both low in the rank ordering. Reflecting again on the nature of the aggregate analysis, and the impact of the Law of Large Numbers, it seems entirely plausible that this is attributable to the automation of camera technology. Particularly at the aggregate level, the shared understanding of what is entailed in choosing the most appropriate point of focus, or the best balance across the dynamic range of the camera, has been deferred to the manufacturers.

What is a surprise is that the qualified group do not show a greater degree of intuitive access to creativity and narrative power than others in their photographic practice. One 


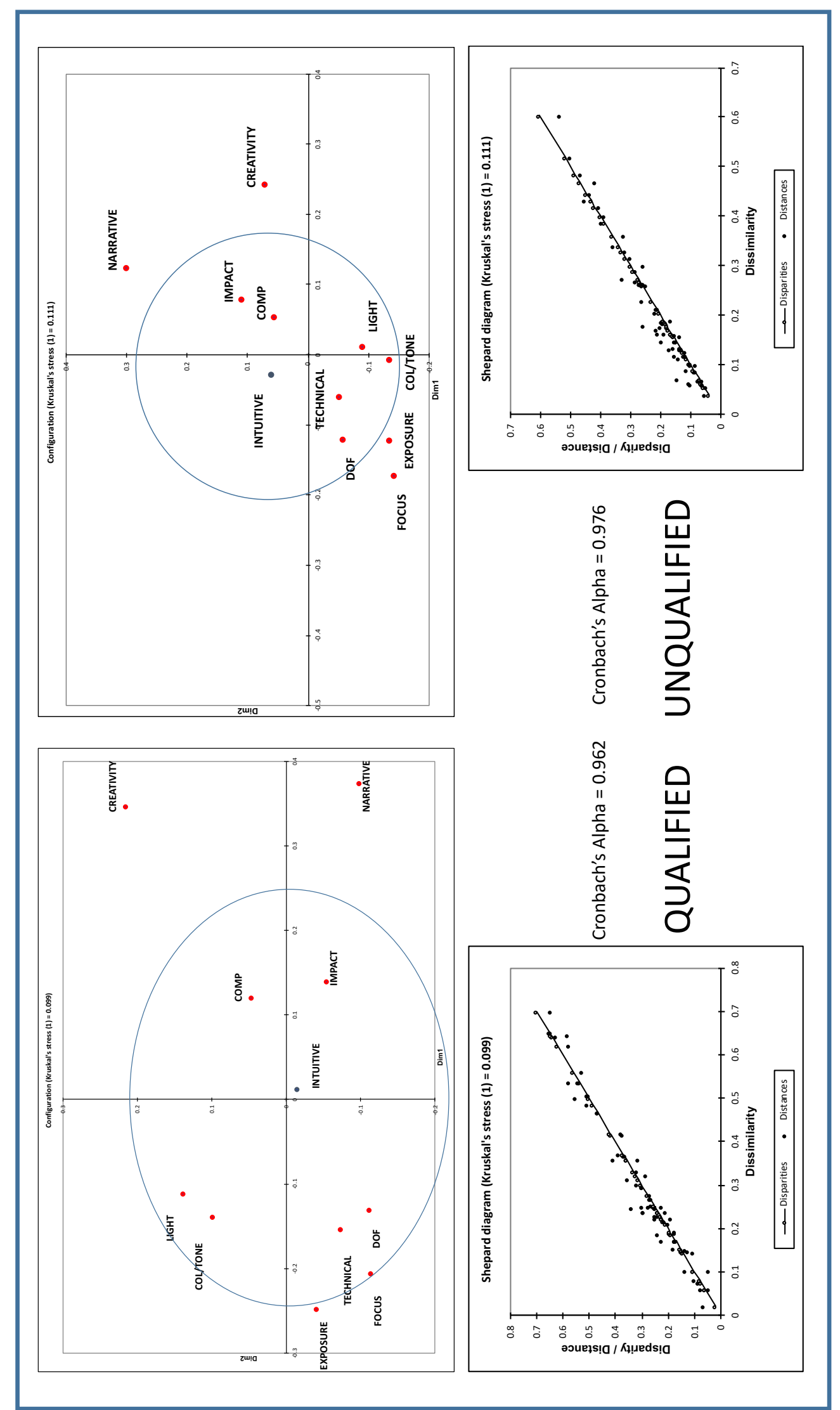

Exhibit 7.4.5 Multidimensional Scaling of performance partitioned by qualified/unqualified. 
explanation, at this level of aggregation, is that the element of shared understanding of creativity and narrative power in photography is very low or does not exist. To put it another way, the meaning of these two, albeit commonly used construct terms, is deeply deferred and withdrawn in the cognition of the individual.

The MDS analysis shown in Exhibit 7.4.5 reveals the same outcome in more visual form. It does hint at a more troubling issue: creativity and narrative power are less closely associated with the intuitive judgement of qualified subjects than those without qualifications. However, as we probe the frequency and factorial data, a more interesting picture emerges. At this level of analysis, idiosyncrasy in understanding is uncovered and is more significant in the data. The Chi ${ }^{2}$ test of the 'construct frequencies' contingency table (Exhibit 7.4.6) yields a $p$ value of 0.001 which means that there is a dependent relationship between construct score and whether the individual is qualified or not. Exhibit 7.4.7 examines the residuals across the partition and identifies the significant differences (at the $5 \%$ level) between qualified and non-qualified photographers.

\begin{tabular}{|c|c|c|c|c|c|c|c|c|c|c|c|c|c|c|}
\hline \multicolumn{15}{|l|}{ Unqualified } \\
\hline & Ranked & & & & & & & & & & & & FACTORIAL & \\
\hline & 1st & 2nd & 3rd & 4th & 5th & 6th & 7th & 8th & 9th & 10th & Total & Rank & Unqualified & Rank \\
\hline Impact & 31 & 15 & 5 & 7 & 6 & 1 & 1 & 0 & 0 & 0 & 66 & 1 & 579 & 1 \\
\hline Creativity & 6 & 11 & 7 & 10 & 4 & 3 & 0 & 0 & 0 & 0 & 41 & 2 & 324 & 2 \\
\hline Narrative & 7 & 4 & 13 & 7 & 6 & 0 & 1 & 0 & 0 & 0 & 38 & 3 & 299 & 4 \\
\hline DoF & 4 & 8 & 6 & 10 & 4 & 2 & 0 & 0 & 0 & 0 & 34 & 7 & 264 & 7 \\
\hline Light & 3 & 10 & 8 & 8 & 4 & 2 & 0 & 0 & 0 & 0 & 35 & 5 & 274 & 6 \\
\hline Comp & 11 & 9 & 7 & 6 & 4 & 1 & 0 & 0 & 0 & 0 & 38 & 3 & 318 & 3 \\
\hline Col/tone & 4 & 6 & 5 & 10 & 5 & 1 & 0 & 0 & 0 & 0 & 31 & 8 & 239 & 8 \\
\hline Focus & 3 & 2 & 8 & 6 & 6 & 6 & 0 & 0 & 0 & 0 & 31 & 8 & 220 & 9 \\
\hline Exposure & 5 & 3 & 6 & 3 & 4 & 2 & 0 & 0 & 0 & 0 & 23 & 10 & 180 & 10 \\
\hline Technical & 3 & 9 & 11 & 6 & 4 & 2 & 0 & 0 & 0 & 0 & 35 & 5 & 275 & 5 \\
\hline Total & 77 & 77 & 76 & 73 & 47 & 20 & 2 & 0 & 0 & 0 & & & & \\
\hline \multicolumn{15}{|l|}{ Qualified } \\
\hline & Ranked & & & & & & & & & & & & FACTORIAL & \\
\hline & 1st & 2nd & 3rd & 4th & 5th & 6th & 7th & 8th & 9th & 10th & Total & Rank & Qualified & Rank \\
\hline Impact & 10 & 9 & 1 & 2 & 1 & 0 & 0 & 0 & 0 & 0 & 23 & 1 & 209 & 1 \\
\hline Creativity & 2 & 4 & 3 & 1 & 1 & 0 & 0 & 0 & 0 & 0 & 11 & 9 & 93 & 8 \\
\hline Narrative & 2 & 1 & 3 & 3 & 1 & 1 & 0 & 0 & 0 & 0 & 11 & 9 & 85 & 10 \\
\hline DoF & 1 & 0 & 1 & 8 & 1 & 1 & 1 & 0 & 0 & 0 & 13 & 6 & 89 & 9 \\
\hline Light & 2 & 6 & 3 & 0 & 2 & 1 & 0 & 0 & 0 & 0 & 14 & 2 & 115 & 2 \\
\hline Comp & 3 & 1 & 2 & 7 & 1 & 0 & 0 & 0 & 0 & 0 & 14 & 2 & 110 & 4 \\
\hline $\mathrm{Col} /$ tone & 2 & 2 & 6 & 2 & 1 & 1 & 0 & 0 & 0 & 0 & 14 & 2 & 111 & 3 \\
\hline Focus & 1 & 2 & 4 & 2 & 4 & 0 & 0 & 0 & 0 & 0 & 13 & 6 & 98 & 7 \\
\hline Exposure & 1 & 3 & 5 & 1 & 3 & 0 & 0 & 0 & 0 & 0 & 13 & 6 & 102 & 6 \\
\hline Technical & 5 & 1 & 1 & 2 & 2 & 3 & 0 & 0 & 0 & 0 & 14 & 2 & 108 & 5 \\
\hline Total & 29 & 29 & 29 & 28 & 17 & 7 & 1 & 0 & 0 & 0 & & & & \\
\hline & & & & & & & & & & & & & & \\
\hline
\end{tabular}

Exhibit 7.4.6 Frequency of constructs appearing within the intuitive competency (qualified v unqualified)

The surprise is the very low ranking for creativity and narrative given by those holding qualifications in photography from the various photographic bodies. Probing the correlations 
between creativity and narrative and intuitive judgement, suggests that even at the aggregate level of analysis, where the constructs are stripped of their idiosyncratic component, they are more closely linked to the intuitive judgement of unqualified subjects compared with those with formal qualifications. At the individual level of analysis, the position is even more pronounced (see Exhibit 7.4.7). Photographers with such qualifications tend to be more concerned with problems of correct exposure than the unqualified group.

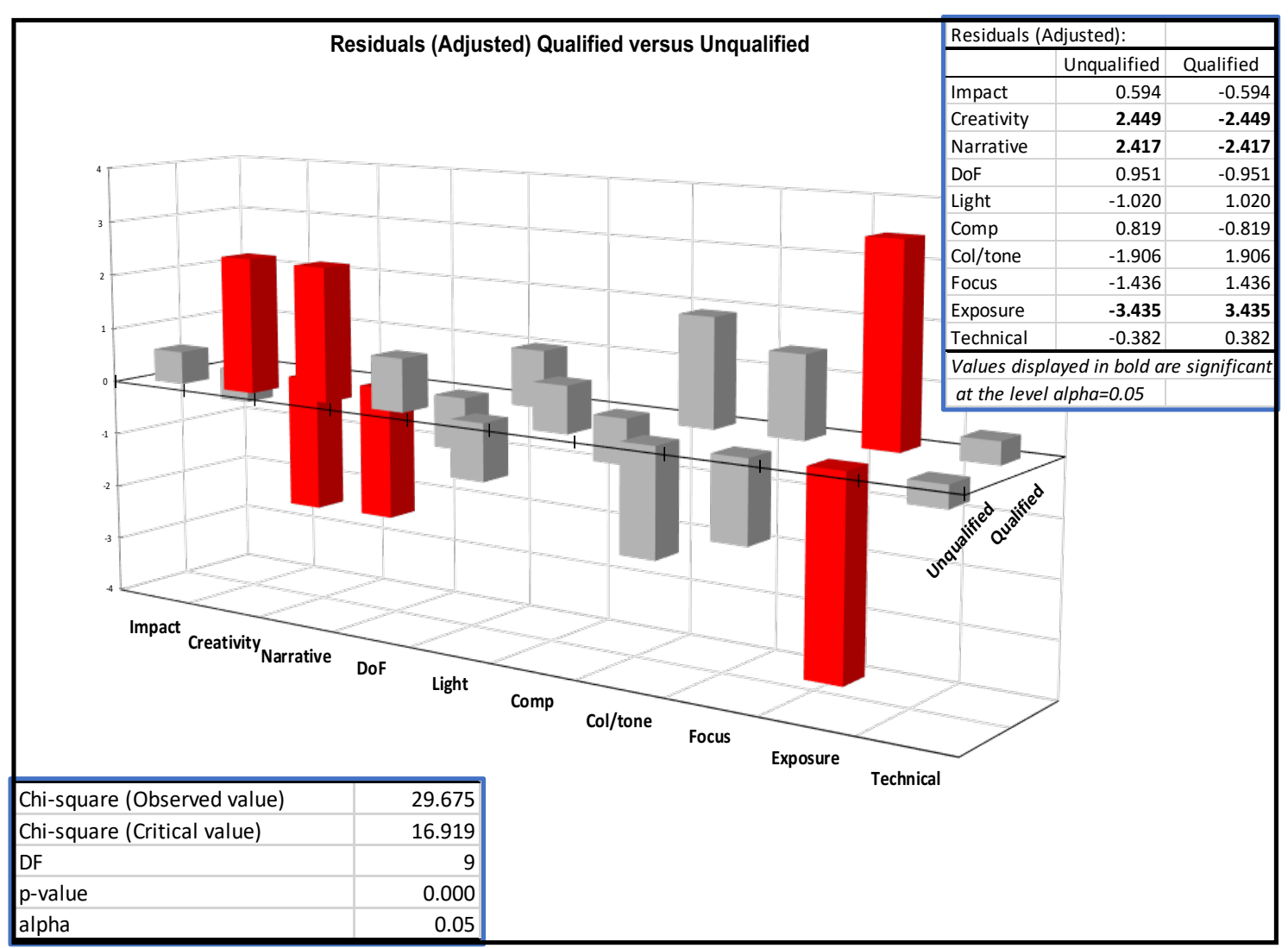

\section{Exhibit 7.4.7 Residuals from Chi $^{2}$ Test - qualification analysis}

The question is why is there such a significant disparity in intuitive competency between those with qualifications and those without, particularly with respect to narrative and creativity? The explanation may be due to a fault with the test, but other explanations are possible.

It can be argued that the test images are weaker in narrative power and creativity than the average outcomes might suggest (see chapter 5 ). This deficit is more likely to influence the intuitive judgement of those who are qualified compared with those who are not. There are two counter-arguments to this: first, the testing algorithms work on the basis of correlations rather than absolutes and the level of scoring will only have a second order effect in the 
calculation of the statistical outputs and modelling; second, filtering the data set by a small number of photographers (7) with high professional reputation, shows that their ranking is similar to the non-qualified group with respect to these high order constructs. All of these photographers have won international awards and recognition for the exhibition of their work.

\begin{tabular}{|l|c|c|c|c|}
\hline & Frequency & Rank & Factorial & Rank \\
\hline Impact & 6 & 1 & 58 & 1 \\
\hline Creativity & 6 & 1 & 44 & 2 \\
\hline Narrative & 4 & 3 & 33 & 3 \\
\hline DoF & 3 & 7 & 17 & 9 \\
\hline Light & 3 & 7 & 24 & 7 \\
\hline Comp & 4 & 3 & 32 & 4 \\
\hline Col/tone & 4 & 3 & 25 & 6 \\
\hline Focus & 3 & 7 & 20 & 8 \\
\hline Exposure & 2 & 10 & 13 & 10 \\
\hline Technical & 4 & 3 & 32 & 4 \\
\hline
\end{tabular}

\section{Exhibit 7.4.8 Ranking by frequency and factorial for 'elite group'.}

The dominant photographic body within the dataset is the Royal Photographic Society, which assesses by panel submission rather than by individual image. It can be argued that this takes the focus away from creativity and narrative power at the level of the individual image, and relocates it within the 10,15 or 20 image panels required for Licentiateship, Associateship or Fellowship respectively. The large majority of qualified test subjects were at Licentiate or Associate level and it may be the case that a different pattern would emerge at the level of Fellowship. An alternative perspective is that the educational and assessment process, particularly at Licentiate and Associate level, is over-weighted towards technical competency, or it could be that, notwithstanding their best efforts otherwise, the photographic bodies simply attract people with a mindset and orientation towards technical, high impact photography.

However, the issue may not lie with the test or the assessment methodology of the awarding bodies. A plausible explanation is that the awarding bodies are selectively attracting those who seek credentials. The theory of credentialism was first proposed by Weber (1951) as an important component of social stratification systems. Each of the bodies draw candidates for 
their awards from the amateur community, and it may be that many seek credentials either to signal status in that community or to attract commissions for their work. Credentialism has been noted as an important driving factor in other areas where individuals seek formal recognition as holders of publicly acknowledged skills and knowledge (Brown, 2001 and De Franco and Zhou, 2009).

One question the IMP Test can illuminate is whether the difference between those who are qualified, and those who are not is a problem reinforced by the judging and assessment process at club, national or international level. There is some overlap between those who are qualified and also judge but splitting them apart does raise significant issues with respect to the small sub-samples. Bearing the caveat in mind that there were 20 individuals with judging experience, 14 of whom were qualified and 6 unqualified, this is what the IMP Test has to say.

Isolating those judges who are unqualified revealed that they placed less importance on technical and exposure issues than others but placed significantly more importance on both creativity and narrative power. Examining those who are qualified and partitioning them by those who judge and those who do not suggests that those who do, place more emphasis on technical issues but less on the creativity exhibited in the images they assess (see Exhibit 7.4.9). It is also worthy of note that there is no difference in their assessment of narrative power. This is a very limited study, but the evidence suggests that there is a question mark over the relationship between the process of qualification, the amateur/club context from which many of those seeking such qualifications are drawn, and the selection and role of judges in that process. 


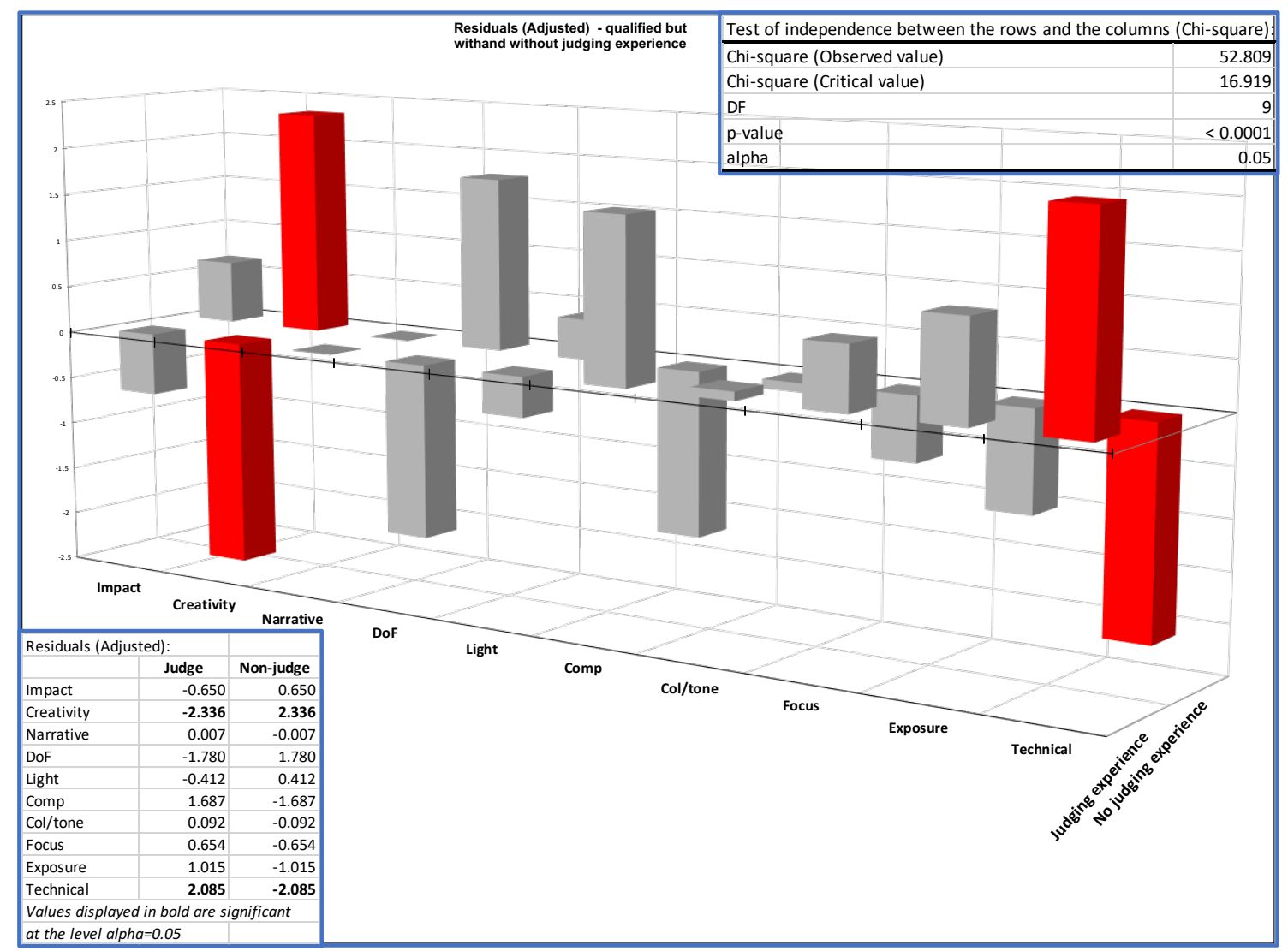

Exhibit 7.4.9 Residuals from the Chi ${ }^{2}$ test - qualified judges versus the rest.

\subsection{Analysis by Performance on the IMP Test $\left(R^{2} Y\right)$}

As noted in chapter 6 , the median score on $\mathrm{R}^{2} \mathrm{Y}$ was just above 0.6 . In this test I examine the differences between those who score above and those who score below the median ${ }^{43}$. The Jarque-Bera Test for the whole distribution is shown in Exhibit 6.11 confirms the visual inspection that normality is rejected for the distribution of $R^{2} Y$.

This test helps resolve some issues in the discriminatory power of the test between those who perform relatively well against my chosen measure of test quality, and those who do not. As expected, the high performers are high on all quality measures and the low performers are low on all quality measures. Taking the split and running a PLS regression on the aggregates for the two groups, reveals only a trivial difference in the order of the constructs with those scoring highly favouring the use of light over those with a low score, and with the reverse true

\footnotetext{
${ }^{43}$ The median was chosen over the mean simply because it gives an equal number of test subjects (53:53) above and below the actual figure of 0.603 .
} 
for the use of colour or tonality. There are three outcomes from this analysis worthy of note: first, the commonly held understanding of exposure, focus, narrative and creativity are not significant drivers of intuitive judgement for either group; second, as expected, the variability in judgement as shown by the whisker chart in Exhibit 7.5.1 is relatively low for the high $R^{2} Y$ group; and third, the low $R^{2} Y$ group have one more construct under their intuitive competency than the higher group.

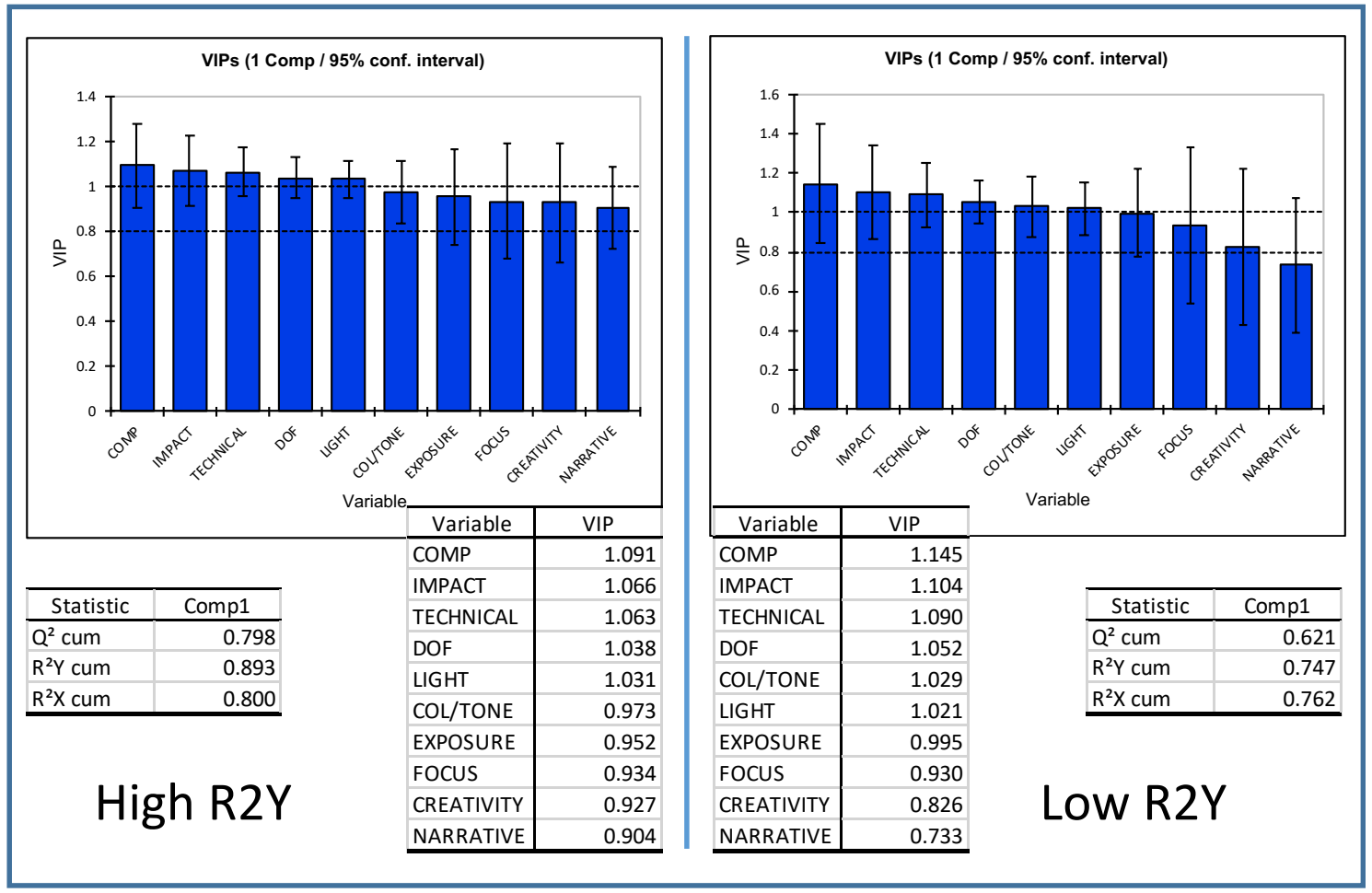

Exhibit 7.5.1 Partial Least Squares analysis of subjects above and below the median $R^{2} Y$ score.

The multidimensional scaling shown in Exhibit 7.5.2 confirms the result from the PLS regression but does suggest that the constructs may not be as well differentiated with the low scoring group. With this group, use of light and use of colour/tonality are particularly closely associated suggesting very low discrimination in understanding of their meaning. The correlations between these pair of constructs are 0.921 for the high scoring group and 0.949 for the low. 


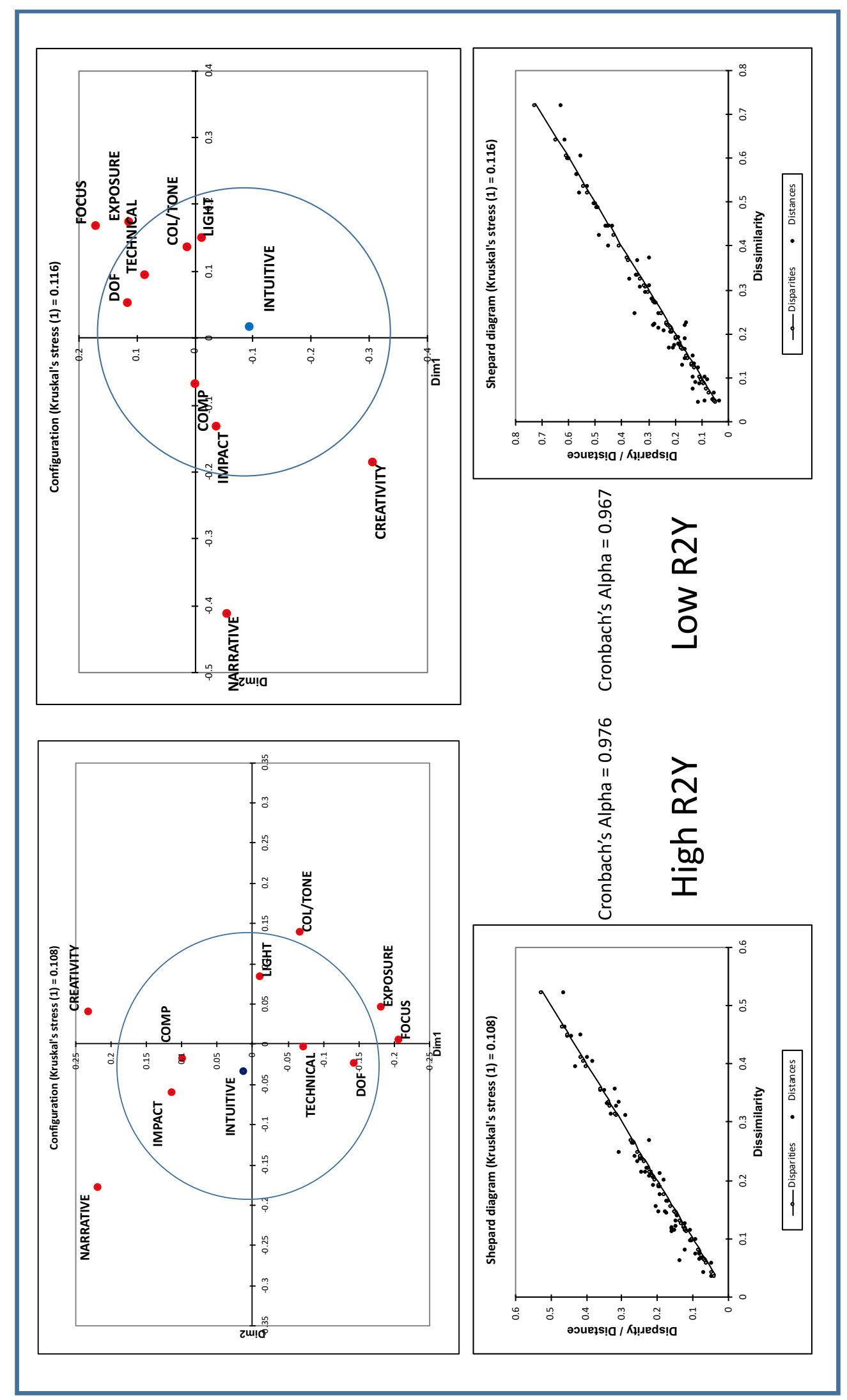

Exhibit 7.5.2 Multidimensional Scaling of Performance partitioned by subjects above and below the median $R^{2} Y$ score. 
At the individual level of analysis, a different picture emerges as shown in Exhibit 7.5.3. Focus and exposure are, as with the aggregate analysis, at the bottom of the list of constructs for the high group ( $9^{\text {th }}$ and $10^{\text {th }}$ respectively) and for the low group ( $8^{\text {th }}$ and $10^{\text {th }}$ respectively). However, narrative power is $9^{\text {th }}$ for the low group and $4^{\text {th }}$ for the high. Indeed, the high group is now showing both a higher number of constructs under their intuitive competency by frequency (5.06 compared with 4.6 per subject) and by factorial (39.91 compared with 37.30 per subject). The $\mathrm{Chi}^{2}$ analysis on the factorial contingency tables (Exhibit 7.5.4) helps unpick the difference with greater clarity. The relatively high scorers on the IMP Test place greater emphasis on composition and, particularly, narrative power than do the low scorers (at the 95\% confidence level).

\begin{tabular}{|c|c|c|c|c|c|c|c|c|c|c|c|c|c|c|}
\hline \multicolumn{15}{|l|}{ Part 1} \\
\hline & Ranked & & & & & & & & & & & & FACTORIAL & \\
\hline & 1st & 2nd & 3rd & 4th & 5th & 6th & 7th & 8th & 9th & 10th & Total & Rank & R2y > median & Rank \\
\hline Impact & 17 & 17 & 2 & 5 & 5 & 1 & 1 & 0 & 0 & 0 & 48 & 1 & 413 & 1 \\
\hline Creativity & 6 & 3 & 8 & 8 & 2 & 3 & 0 & 0 & 0 & 0 & 30 & 2 & 234 & 3 \\
\hline Narrative & 7 & 4 & 6 & 5 & 5 & 1 & 0 & 0 & 0 & 0 & 28 & 3 & 224 & 4 \\
\hline DoF & 2 & 2 & 3 & 8 & 3 & 2 & 1 & 0 & 0 & 0 & 21 & 7 & 150 & 8 \\
\hline Light & 2 & 9 & 8 & 4 & 2 & 1 & 0 & 0 & 0 & 0 & 26 & 6 & 210 & 5 \\
\hline Comp & 10 & 7 & 4 & 5 & 2 & 0 & 0 & 0 & 0 & 0 & 28 & 3 & 242 & 2 \\
\hline Col/tone & 1 & 5 & 5 & 4 & 5 & 1 & 0 & 0 & 0 & 0 & 21 & 7 & 158 & 7 \\
\hline Focus & 1 & 1 & 5 & 5 & 6 & 3 & 0 & 0 & 0 & 0 & 21 & 7 & 145 & 9 \\
\hline Exposure & 3 & 2 & 4 & 4 & 5 & 0 & 0 & 0 & 0 & 0 & 18 & 10 & 138 & 10 \\
\hline Technical & 4 & 3 & 7 & 4 & 5 & 4 & 0 & 0 & 0 & 0 & 27 & 5 & 201 & 6 \\
\hline \multirow[t]{2}{*}{ Total } & 53 & 53 & 52 & 52 & 40 & 16 & 2 & 0 & 0 & 0 & 268 & & 2115 & \\
\hline & & & & & & & & & \multicolumn{2}{|c|}{ per subject } & 5.06 & & 39.91 & \\
\hline \multicolumn{15}{|l|}{ Part 2} \\
\hline & Ranked & & & & & & & & & & & & FACTORIAL & \\
\hline & $1 s t$ & 2nd & 3rd & 4th & 5th & 6th & 7th & 8th & 9th & 10th & Total & Rank & R2y $<$ median & Rank \\
\hline Impact & 24 & 7 & 4 & 4 & 2 & 0 & 0 & 0 & 0 & 0 & 41 & 1 & 375 & 1 \\
\hline Creativity & 2 & 12 & 2 & 3 & 3 & 0 & 0 & 0 & 0 & 0 & 22 & 7 & 183 & 5 \\
\hline Narrative & 2 & 1 & 10 & 5 & 2 & 0 & 1 & 0 & 0 & 0 & 21 & 9 & 160 & 9 \\
\hline DoF & 3 & 6 & 4 & 10 & 2 & 1 & 0 & 0 & 0 & 0 & 26 & 2 & 203 & 2 \\
\hline Light & 3 & 7 & 3 & 4 & 4 & 2 & 0 & 0 & 0 & 0 & 23 & 5 & 179 & 7 \\
\hline Comp & 4 & 3 & 5 & 8 & 3 & 1 & 0 & 0 & 0 & 0 & 24 & 3 & 186 & 4 \\
\hline Col/tone & 5 & 3 & 6 & 8 & 1 & 1 & 0 & 0 & 0 & 0 & 24 & 3 & 192 & 3 \\
\hline Focus & 3 & 3 & 7 & 3 & 4 & 3 & 0 & 0 & 0 & 0 & 23 & 5 & 173 & 8 \\
\hline Exposure & 3 & 4 & 7 & 0 & 2 & 2 & 0 & 0 & 0 & 0 & 18 & 10 & 144 & 10 \\
\hline Technical & 4 & 7 & 5 & 4 & 1 & 1 & 0 & 0 & 0 & 0 & 22 & 7 & 182 & 6 \\
\hline \multirow[t]{3}{*}{ Total } & 53 & 53 & 53 & 49 & 24 & 11 & 1 & 0 & 0 & 0 & 244 & & 1977 & \\
\hline & & & & & & & & & \multicolumn{2}{|c|}{ per subject } & 4.60 & & 37.30 & \\
\hline & & & & & & & & & & & & & & \\
\hline
\end{tabular}

Exhibit 7.5.3 Frequency of constructs appearing within the intuitive competency (high versus low $R^{2} Y$ scorers). 


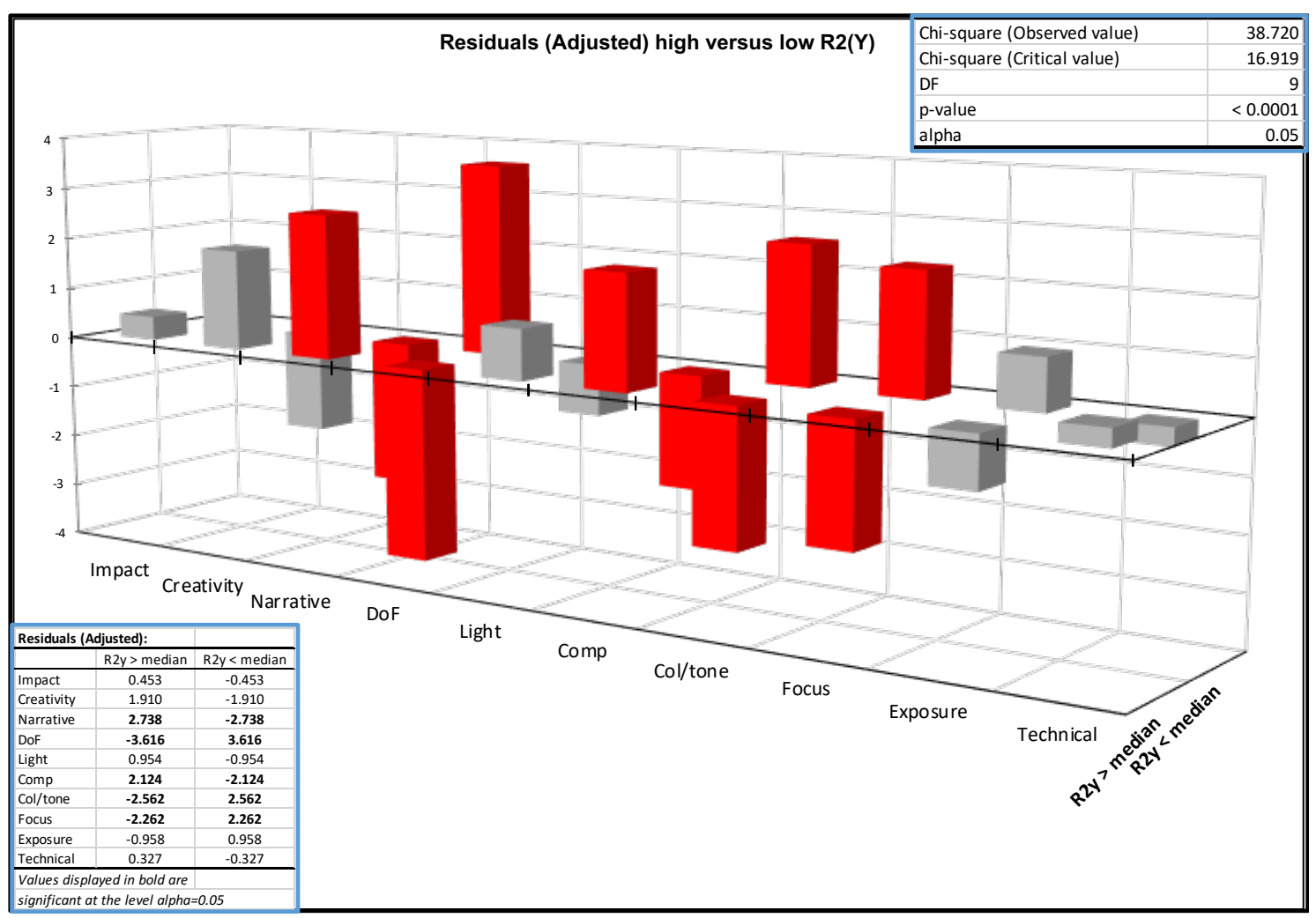

Exhibit 7.5.4 Residuals from $\mathrm{Ch}^{2}$ test - high versus low $R^{2} Y$ scorers

\subsection{Analysis by Genre}

Given the wide choice of genres from which subjects could choose, any analysis was limited by small sub-sample sizes. Given this constraint, I report here the results of two: landscape and wildlife/nature. For these small sample studies, I have not run the normality tests as above but relied upon the non-parametric tolerance of PLS regression and the chi ${ }^{2}$ test. All cell frequencies were high enough to ensure validity in the $\mathrm{Chi}^{2}$ without the need for aggregation.

Twenty-five photographers identified landscape as their preferred genre. As with all other partitions at the aggregate level, the results are very similar to what has gone before (Exhibit 7.6.1). Landscape photographers do give intuitive priority to the use of light but lower priority to the technical (as they define it) in their practice. It is important to note that low ordering does not signal inattention to the constructs concerned but rather that they are more a product of the conscious, rational modality as opposed to the nonconscious modality revealed by the test. I expect, given the research entailed in planning a shoot, and the process 
of setting up and image capture, that a more rational approach would dominate in landscape work. However, neither the Rational Experiential Inventory, nor the IMP Test, leads to the conclusion that landscape photographers have a significant cognitive bias towards that modality of thought.

\begin{tabular}{|c|c|c|c|}
\hline \multicolumn{2}{|l|}{ Landscape } & \multicolumn{2}{|c|}{ Other Genres } \\
\hline Variable & VIP & Variable & VIP \\
\hline COMP & 1.129 & COMP & 1.107 \\
\hline IMPACT & 1.111 & TECHNICAL & 1.084 \\
\hline LIGHT & 1.059 & IMPACT & 1.076 \\
\hline DOF & 1.055 & DOF & 1.044 \\
\hline TECHNICAL & 1.012 & LIGHT & 1.024 \\
\hline COL/TONE & 0.979 & COL/TONE & 1.000 \\
\hline EXPOSURE & 0.948 & EXPOSURE & 0.971 \\
\hline FOCUS & 0.929 & FOCUS & 0.929 \\
\hline CREATIVITY & 0.873 & CREATIVITY & 0.898 \\
\hline NARRATIVE & 0.865 & NARRATIVE & 0.831 \\
\hline
\end{tabular}

\section{Exhibit 7.6.1 Regression Model Ordering (landscape v other genres)}

Probing the data at the individual level gives a more illuminating insight into this genre and the priorities within the intuitive frame of the photographers concerned. Whether frequency or factorial data is used (see: Exhibit 7.6.2) impact is the principal construct for landscape photographers, as it is across all genres. Composition is next in order of importance and here we can note the similarity with the aggregate analysis. It is here that divergences start to appear: focus and control of depth of field are more important for landscape photographers than for others although they place a relatively low order of significance upon exposure and the use of light.

\begin{tabular}{|l|c|c|c|c|c|c|c|c|}
\hline & Landscape & & & & Other & & & \\
\hline & Frequency & Rank & Factorial & Rank & Frequency & Rank & Factorial & Rank \\
\hline Impact & 21 & 1 & 191 & 1 & 68 & 1 & 597 & 1 \\
\hline Creativity & 12 & 4 & 93 & 4 & 40 & 2 & 324 & 3 \\
\hline Narrative & 12 & 4 & 93 & 4 & 37 & 6 & 291 & 6 \\
\hline DoF & 13 & 2 & 96 & 3 & 34 & 8 & 257 & 8 \\
\hline Light & 11 & 7 & 85 & 7 & 38 & 5 & 304 & 4 \\
\hline Comp & 12 & 4 & 102 & 2 & 40 & 2 & 326 & 2 \\
\hline Col/tone & 8 & 9 & 62 & 10 & 37 & 6 & 288 & 7 \\
\hline Focus & 13 & 2 & 90 & 6 & 31 & 9 & 228 & 9 \\
\hline Exposure & 8 & 9 & 67 & 9 & 28 & 10 & 215 & 10 \\
\hline Technical & 10 & 8 & 84 & 8 & 39 & 4 & 299 & 5 \\
\hline
\end{tabular}

Exhibit 7.6.2 Analysis by frequency and factorial for landscape versus other genres. 
However, rank ordering can be seductive. The $\mathrm{Chi}^{2}$ test suggests that only two constructs focus and the use of colour/tonality - are significantly different from one another at the 95\% level (Exhibit 7.6.3).

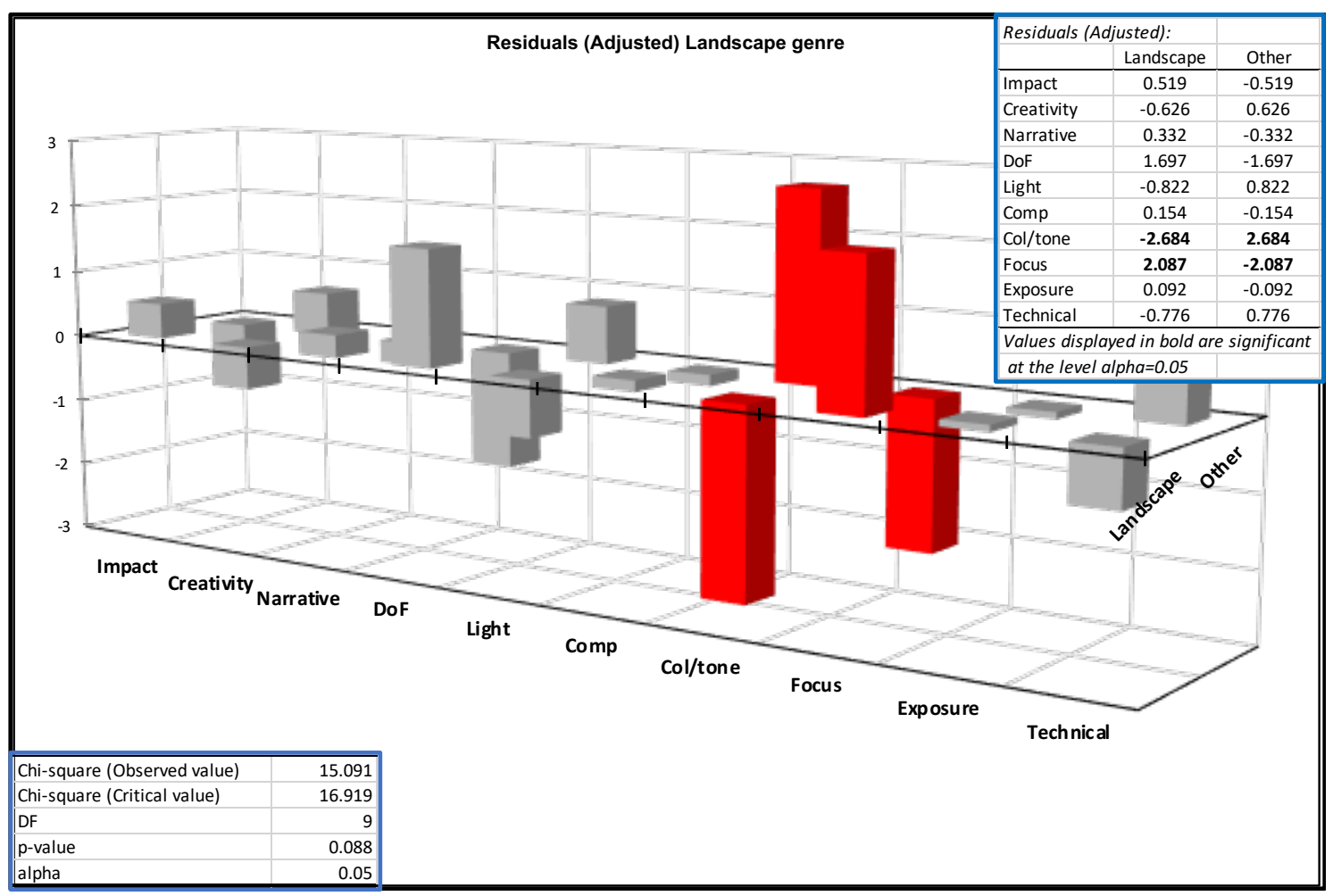

Exhibit 7.6.3 Residuals from Chi2 Test-genre analysis (Iandscape).

The next most popular genre in the data set is wildlife/nature. At the aggregate level the ordering of the constructs is again very similar (see Exhibit 7.6.4) with narrative, creativity, exposure and focus revealed as not impacting the intuitive judgement of the test subjects.

\begin{tabular}{|c|c|c|c|}
\hline \multicolumn{2}{|c|}{ Nature/wildlife } & \multirow{2}{*}{$\frac{\text { Others }}{\text { Variable }}$} & \multirow[b]{2}{*}{ VIP } \\
\hline Variable & VIP & & \\
\hline COMP & 1.140 & COMP & 1.111 \\
\hline IMPACT & 1.092 & IMPACT & 1.082 \\
\hline LIGHT & 1.046 & TECHNICAL & 1.076 \\
\hline TECHNICAL & 1.045 & DOF & 1.051 \\
\hline DOF & 1.031 & LIGHT & 1.024 \\
\hline COL/TONE & 0.987 & COL/TONE & 0.993 \\
\hline CREATIVITY & 0.935 & EXPOSURE & 0.990 \\
\hline NARRATIVE & 0.923 & FOCUS & 0.938 \\
\hline FOCUS & 0.885 & CREATIVITY & 0.871 \\
\hline EXPOSURE & 0.880 & NARRATIVE & 0.823 \\
\hline
\end{tabular}

Exhibit 7.6.4 Regression Model Ordering (wildlife/nature v other genres) 
Focusing on the significant differences at the frequencies and factorial adjusted frequencies of intuitively accessible constructs we note that wildlife/nature photographers are less concerned with impact and creativity than others. One interpretation is that practitioners of this genre are more concerned than others with the function of the image as a record of a species or behaviour.

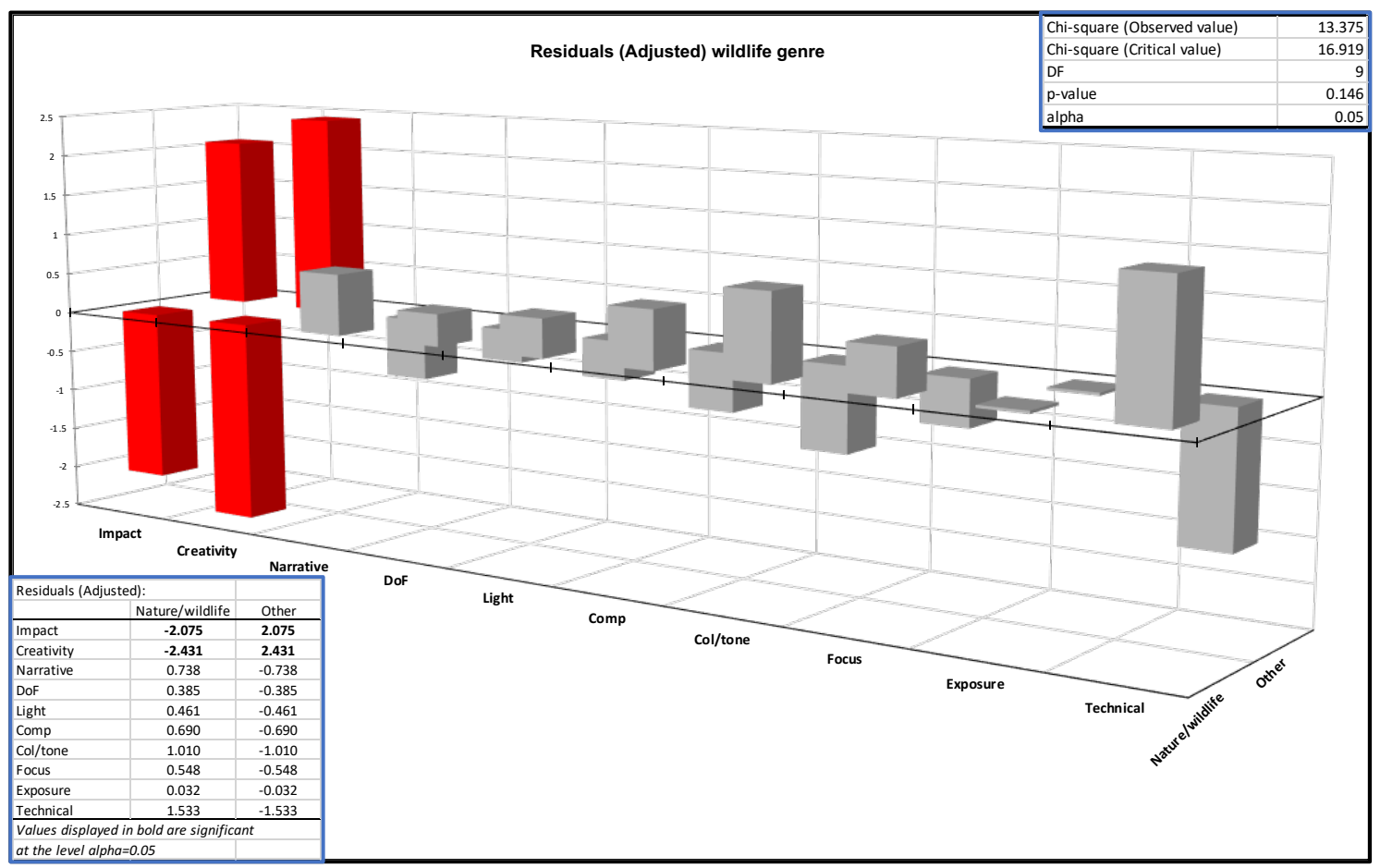

Exhibit 7.6.5 Residuals from Chi $^{2}$ Test-genre analysis (nature/wildlife)

\subsection{Conclusion}

The temptation with a research instrument such as the IMP Test is to promote it from its role as a tool for analysis at the individual level and to see whether it says anything about different groups. This, as I have noted at length, presents an immediate, analytical problem - whether to average the data for each group within a partition, and run the IMP Test, or take the individual IMP Test outcomes and aggregate using frequencies and the factorial method described above. Choosing the former approach leads to a comparison of the constructs as understood in common by the group and where individual idiosyncrasies in belief and understanding have been cancelled out. The IMP Test can, therefore, expose the 'naked constructs' but the test cannot reveal their core meaning but only their significance in the cognitive processes of the group. At this level of analysis, a common pattern is readily 
apparent. The judgement about the basic functions of focusing and exposure control has been deferred to the manufacturers of camera equipment. They both appear at the 'tail-end' of concern and rarely feature in the intuitive judgement of photographers. The other two constructs that feature at the tail-end, in the aggregate analysis, are creativity and narrative power. I would argue that with these two in particular, there is little common understanding of what the two constructs mean. If the database had drawn upon photographers who have been exposed to an undergraduate or postgraduate degree in photography - or the other visual arts - a different outcome might have emerged.

However, a more nuanced and interesting picture emerges when we focus attention on the frequencies and ranking of the constructs active in the intuitive judgement of the individual test subjects. Two results are particularly worthy of note. First, we note that women appear to be more concerned than men about the technical issues of photography but are less concerned about narrative power. Is this a gender difference - unlikely in my view - or more satisfactorily explained by the greater proportion of women in the data set who hold photographic qualifications? This is where I believe the second and real surprise of this analysis emerges. The analysis strongly suggests that those who hold photographic qualifications are more concerned with the technical aspects of photography and less concerned with creativity and narrative power than others. There are caveats here of course: a much larger data base might lead to a different conclusion and almost all the test participants had achieved only Licentiate or Associate level with the Royal Photographic Society or the equivalent with other bodies. The question remains: if this analysis is accepted, what is the reason why those who hold qualifications place low priority on creativity and narrative power? There is weak evidence of an issue surrounding where candidates for nonacademic, photographic qualification are drawn from - predominantly an amateur/club environment - and the apparently low intuitive awareness of narrative power and creativity among those who assess and advise them through the judging process.

There is one final conclusion of significance that relates back to the test and its application at the individual level. In chapter 5 and 6 , I referred to the $R^{2} Y$ statistic from the PLS regression as a good indicator of test quality. Partitioning the data set by those whose $R^{2} Y$ scores were above the median and those that were below, generated two conclusions: first, those who were above the median in their performance had marginally less constructs under their 
intuitive control than those below the median. Second, however, they were more likely to have access to both narrative power and composition as dominant, cue-dependent, nonconsciously accessible constructs. 


\section{Chapter 8 - Exploring the Boundary Layer}

\subsection{Introduction}

In this penultimate chapter I draw together the threads of my thesis with an extended intraspective interview and subsequent discussion with Chris Weston (CW) (Test Participant IT0069) ${ }^{44}$. This is in answer to the issue addressed by the fourth research objective presented in chapter 1 and discussed in detail in chapters 4,5 and 6 .

RO4: To explore, using intraspective interviewing methods, the individual's conscious and nonconscious boundary experience in the exercise of photographic judgement.

The interview also reflects many of the ontological perspectives discussed in chapter 2 and presents my conclusions about the process of conjugation of the nonconsciously accessible constructs available to him. The transcripts of the interview and the subsequent discussion (edited) are in the appendix. Elements of the intraspective interview relate to the opinions and activities of third parties and have been redacted where shown. Extracts from the subsequent discussion are highlighted below in text boxes.

This intraspective interview followed the same protocol as that presented in chapter 6 . The analysis is extended in depth and scope and verified stage by stage with the follow-up interview. As with those interviews, the reduction and bracketing led to a wealth of insights beyond verification of the results of his IMP Test. The issue of the boundary layer had surfaced and been evidenced in the intraspective interviews discussed in chapter 6 . However, In CW's case, I believed he presented an opportunity to explore, in greater depth, the issues arising from the test. I have known CW for many years, and I was acutely aware of the connection between us. Notwithstanding this, I weighed in the balance the potential error in judgement that might follow, but also recognised, as noted in chapter 5 , that such error is down to both bias and uncertainty, and that they tend to counter-vary. This presented an opportunity to observe and to control for the former and to understand the latter as he was

\footnotetext{
${ }^{44}$ Chris kindly consented to be fully identified in both the intraspective and the follow-up interview. His consent agreement is included in the appendix.
} 
keen for both the IMP Test and the interview to be done together. In my judgement, the quality of the insights to be gained outweighed, by a considerable margin, the risk of bias.

The evidence suggests that $\mathrm{CW}$ is also very reflective in his practice and has discussed at length his creative impulse in one of the most popular series of articles ever produced in 'Outdoor Photography' published in 2015 and 2016.

Steve Watkins, Editor of Outdoor Photography commenting on CW's series of 20 articles 'A Photographer's Guide to Life on Earth' (email received 5/4/2019 and appended):

What began as a possible three or four-part series ended up running for almost a couple of years and around 20 issues...the series generated more response from readers than any other I have done in my ten years at the magazine. The responses were certainly polarised and passionate. I had people seek me out at events and shows just to tell me how much Chris' article had resonated with them and how it had changed their own approaches to photography. We had numerous letters applauding his vision and courage to tackle such a deep and difficult subject.... On the other hand, we had a significant number of readers incensed by our promotion of a non-Christian view of the evolution of life and others who proclaimed it was pretty much all mumbo-jumbo. My take on it was that it was wonderful to see the readers so animated and engaged, whichever side of the fence they were on!

This reflectivity is also expressed both in the use of reduction as a strategy in his image making and in the high level of correlation between his intuitive and reflective judgement. Strikingly, even given the level of internal consistency in his judgement he does disagree with the consensus in his ranking of the images, particularly in the assessment of creativity and narrative power.

Once this chapter was substantially complete, he consented to a follow-up interview reflecting on my analysis and the conclusions I had drawn. The discussion proceeded over six hours, where the last hour summarised our discussion and is transcribed and presented in the appendix. During that discussion, I outlined my ontological position, and the conclusions I draw from that position for both practice and critical theory in photography (see chapter 2). His reflective commentary is shown in text boxes, and his email approving the text is included in the appendix along with the transcripts. 


\subsection{Subject's Biographical Details}

CW is a successful professional photographer. His biographical details are as follows:

He is 51 years of age and is resident in the UK and Switzerland. He has published numerous books and articles, lectures globally, and is the Fuji wildlife champion.

His contemporary approach to photography led Amateur Photographer to describe him as "one of the most dynamic wildlife photographers working today". A committed advocate of wildlife conservation, Chris was listed in Outdoor Photography as "one of the world's 40 mostinfluential wildlife photographers".

His work is exhibited globally, and his fine art prints are collected by private clients from around the world. He is currently represented by The Marylebone Gallery in London and, in 2018, he won the ArtHaus Gallery and Manhattan Arts International awards. He has written over 30 books on photography and his commercial credits include the BBC, ITV, The Times, The Sunday Times, Daily Telegraph, Independent and Guardian newspapers, and National Geographic. He is the presenter of The Complete Photographer - Masterclass.

(Retrieved on 15 July 2019 from https://www.photographyshow.com/theatreprogramme/speaker-list/speaker/chris-weston)

\subsection{The IMP Test Results}

As with all the other interviewees, the IMP Test generated insights and questions in equal measure. In terms of the broad brush with which the test paints CW's cognitive canvas, it gives some hints as to the vectors of difference that make his style both authentic and unique.

He consented to be interviewed and took the IMP test immediately prior on $11^{\text {th }}$ May 2018. Neither he nor I were aware of the outcomes of the test before the interview. The REI shows that he has a bias towards deliberative cognition both in aptitude and particularly in his willingness to engage that thinking modality (RA:EA $=3.9,3 ; \mathrm{RE}: \mathrm{EE}=4.9,3.8)$. 
The IMP Test provided an overall view of those constructs that were, and those that were not, active in his intuitive judgement. The $R^{2} Y$ on the test was $71.4 \%$, which is well above the average for the test. Relative to the average, his low levels of uncertainty across all but one of the ten construct areas indicated a high depth of skill in each. The only one close to the average was his assessment of 'impact'.

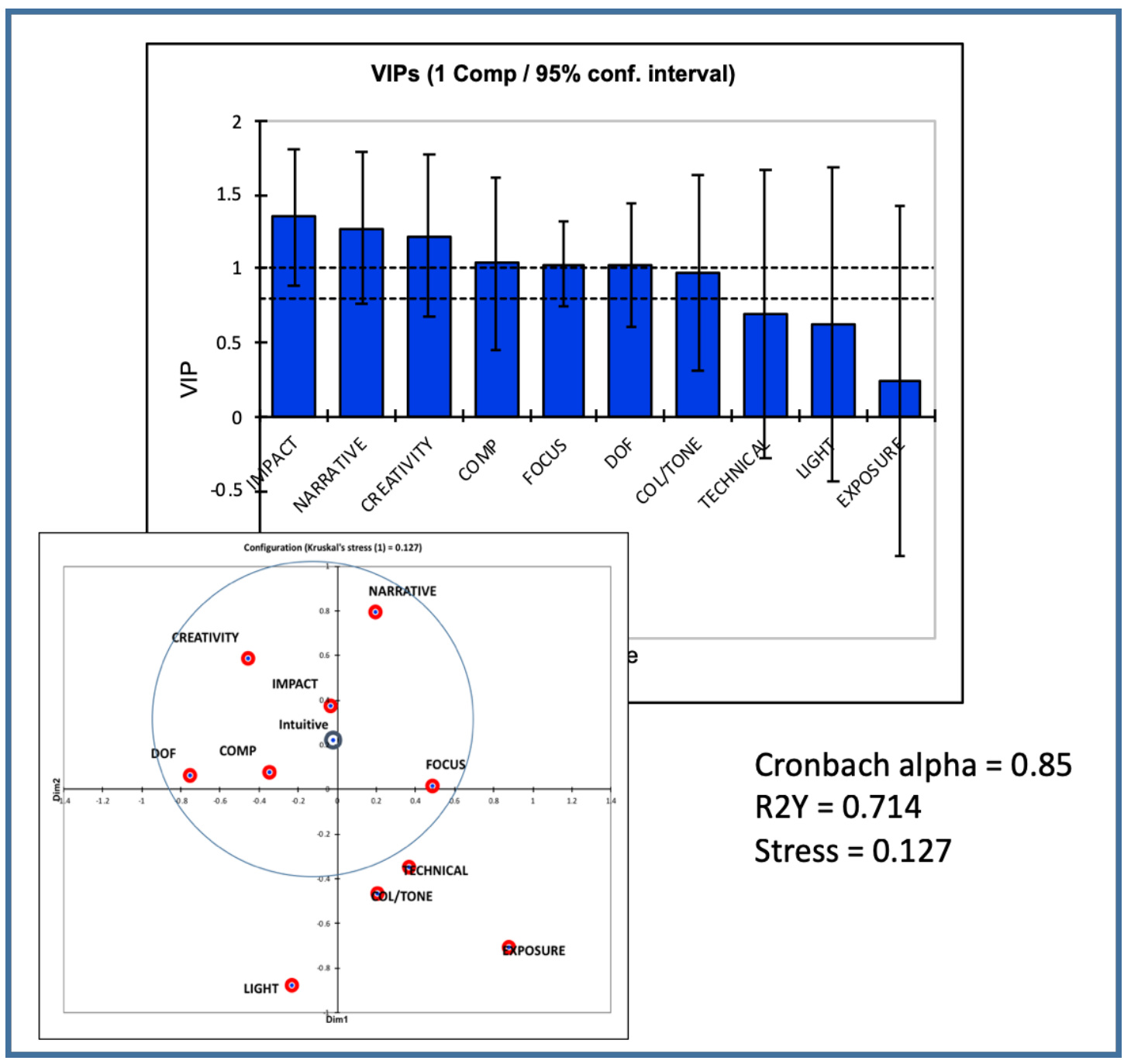

Exhibit 8.1 CW - selected output from the IMP Test.

The test results show that he scored highly in terms of the agreement between his declared and the model determined ranking of importance of the ten constructs (0.67). The major discrepancy was in the use of light - which he placed $4^{\text {th }}$ in his declared ranking while the model placed it $9^{\text {th }}$. The only other construct with more than two places of separation was exposure $\left(7^{\text {th }}\right.$ and $10^{\text {th }}$ respectively). This high degree of introspective awareness is likely to 
lead to a more phenomenologically reductive approach particularly when emotional activation and time pressures are low.

The Multidimensional Scaling (MDS) and the Partial Least Squares (PLS) regression were in agreement. What is noticeable from the MDS is the degree of correlation between the constructs. Running a principal components analysis reveals clustering and possible latency in his cognitive structuring of the ten constructs. Impact, composition, narrative and creativity form a clear cluster both with high levels of conscious skill and each highly significant within his nonconscious cognition. Both focus and the use of depth of field, are also nonconsciously accessible but are well separated. Notwithstanding their obvious relationship, they represent well separated aspects of his image

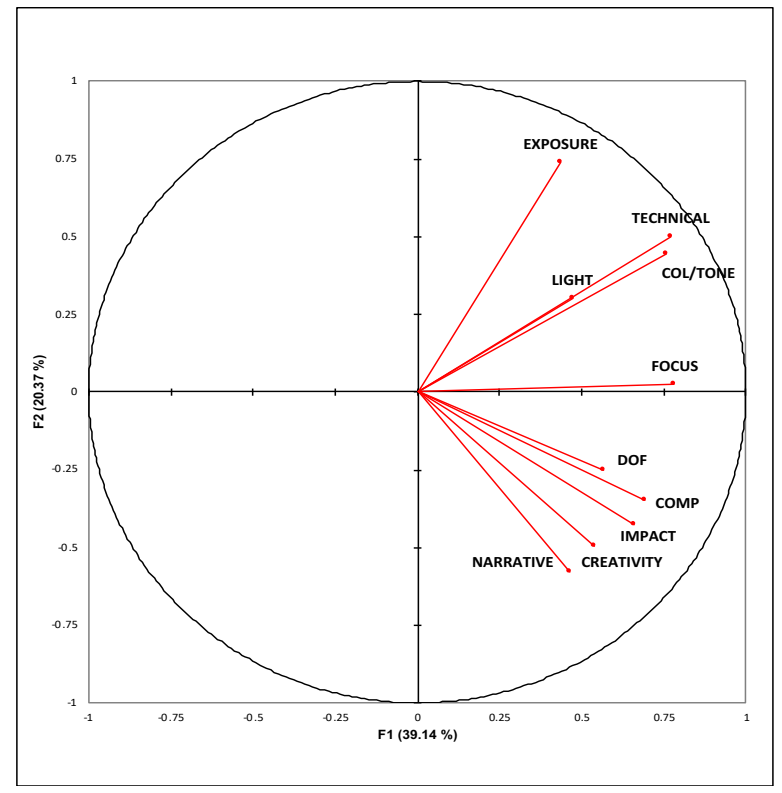

Exhibit 8.2 Chris Weston-Principal Component Analysis judgement.

The IMP Test indicates that narrative power and creativity are driving his nonconscious judgement and will be driving his previsualisation of potential images. Composition - as he understands the term - is likely to be his dominant, spontaneous response when deciding how to deliver his intent.

As we proceed down through his construct ordering I would anticipate that his choices would become more deliberative, particularly when they involve use of colour/tonality, exposure, and the use of light. If the IMP Test is correct, his use of colour will have an intuitive element to it, but he will be more deliberative and instrumental in his judgement on issues of exposure and the use of light. The reason for this may be a product of his preferred genre, which is an important part of his continuing professional practice. With wildlife one can only shoot with the light given in any situation. 
Commenting on this outcome of the analysis, CW explores his thinking about light, exposure, focus and depth of field. Discussing his approach to light he explains how he views it:

In a very conscious way. But subconsciously I don't tend to think about light, I tend to think about the narrative. Exposure again, although it's a technical requirement, is the last thing I think about. Focus and depth of field fit in there because focus and depth of field, to an extent more so than exposure, play a big part in composition because what focus and depth of field are really doing - outside of the technical reasons for something to be in focus - what depth of field and focus are doing are they are creating emphasis. They are either emphasising objects in the scene or they are deemphasising objects in the scene, and both of those things fit very closely to composition. Composition is about how do you tell the story, the narrative you're trying to tell. So, for me, focus and depth of field play a huge part in composition, and therefore they sit very closely to composition.

In summary, the IMP Test has distinguished between those constructs that are within CW's intuitive competence, and those that are without. As a result, I would make the following predictions about his photographic practice:

I. Across his practice, he seeks impact through narrative power and the creative use of subordinate constructs. With high levels of skill (as measured by the variance to average ratio within the test) and well-differentiated constructs he will make both nonconscious and conscious choices to achieve the effects he is after.

II. The test suggests that in the moment, and under strong affective pressure, his nonconscious processing will preferentially conjugate impact, narrative power, creativity, composition, focus and control of depth of field. He will seek to achieve his intent by finding strong compositions and by innovation in the control of focus and depth of field. His use of colour/tonality is predicted to be marginal to his intuitive judgement.

III. Under circumstances that permit more deliberative cognitive processing to occur, this modality will move into the ascendency and, on the basis of his high level of introspective skill, he will conjugate all ten constructs but bringing into play exposure control and innovation in the use of light. 
IV. Given his high level of introspective awareness, and his low level of dissonance, his need to make corrections in post-processing will, as a consequence, be low.

CW's commentary on the outcome of the IMP Test:

I would say it is a near perfect reflection of the way I approach photography in that, yes, the image has to have impact, so on this chart that sits at number one. The impact has to have a narrative. That narrative has to be new or interesting, which dictates it has to be creative. And it has to be unambiguous, which means it has to be composed well. So, for me the four things that drive my photography are the four things that sit at the top of the chart. Then you start to move into the technical and, interestingly, exposure and light sit right down at the bottom, which you'd think for a photographer doesn't really make sense, but to me, the way I photograph, it does make sense because... when I'm conscious of light it's because I'm trying to use light in order to create a creative narrative, so I'm using light for a purpose.

\subsection{The Intraspective Interview}

The interview lasted 73 minutes, and three images were the subject of our discussion: a team of wild horses galloping through water at the Camargue, dancing cranes at Hokkaido in Japan, and another shot entitled 'Dali's Horses' - again in the Camargue. CW emphasised that he would not regard these as his best images; however, they were recently taken and had a degree of emotional salience for him.

The bracketing process led to frequent engagements with the core of the subject matter. $\mathrm{He}$ contextualised his discussion by describing each photoshoot and his engagement with clients. Much of this has been redacted from the transcript appended. As the discussion proceeded, he revealed very quickly the ontology of his image making in a section I reproduce in full here:

The relationship doesn't have to be human-human it can be human-animal so when I'm photographing something, I want to be connected to whatever it is that I'm photographing because it is through that connection that you learn the story that is underneath the surface. So, if you think about photography as not photographing the semblance of the subject but the essence of the subject, in order to get to the essence, you have to have a connection. Now if you think about this in terms of portrait photography... In order to understand who they are 


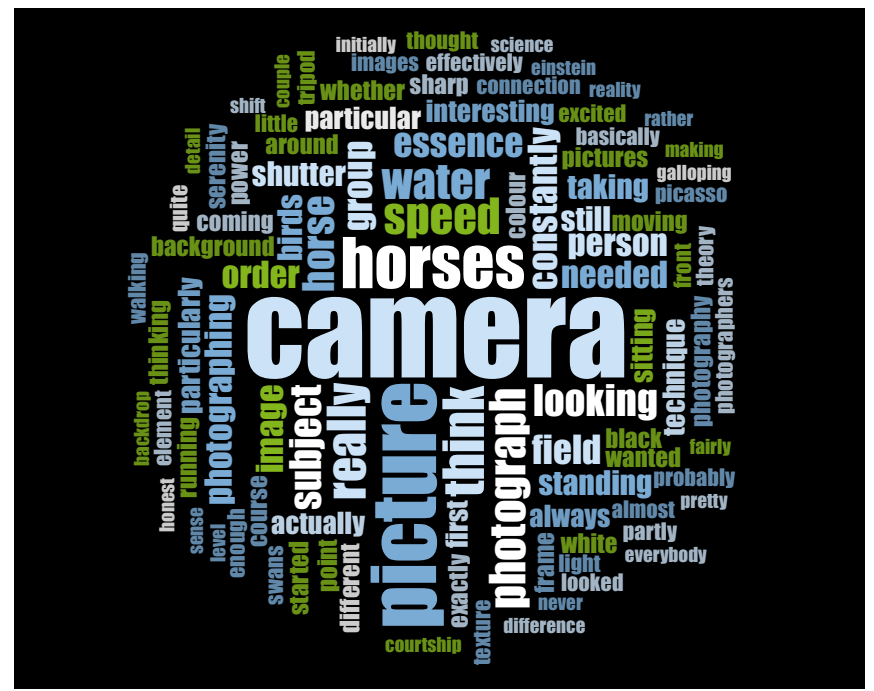

you have to make a connection with them. If you don't make that connection, you're not going to learn who they are therefore you cannot photograph... can only photograph the surface. So, for me it's applying exactly the same principles to an animal. All animals have personalities ...it's about finding the essence of the group or the herd or the pod or whatever and photographing that rather than just photographing a horse... and that comes through making a connection which allows you to communicate with the subject on an intimate level. [16]

In this lengthy section, he highlights, in $00 \mathrm{O}^{45}$ terms, his search for the 'real object' - the essence as he puts it. He does this by looking for a connection in his subject, and it is success in searching for, and achieving this connection, that gives him his 'story'. This sense of connection is both within the subject, and between himself and the subject. Clearly, the animals that are the subject of his images are not likely, at least at a distance, to form any real connection with him - however, his sense of connection can be better understood as a heightened emotional state opening his nonconscious awareness, and enhancing its ability to recognise the essence of the animals concerned.

As we scan his word cloud, 'camera', 'picture', 'photograph', 'image' all stand out, but so do 'looking', 'think', 'really', 'order', and 'essence'. Tucked away, we also note 'difference', 'different', 'connection' and 'theory', which all take on significance as his story unfolds. He was entirely comfortable with the ten signifiers used in the IMP Test, although his sense of composition also captured various elements of design. In this, he closely follows the work of his early mentor and co-author Art Wolfe.

$\mathrm{CW}$ was one of the early photographers in the digital era to explore the representation of movement in the still image through his experimental work on the use of focus, shutter speed,

\footnotetext{
${ }^{45}$ For those taking a circuitous route through this thesis: 000 means 'Object Oriented Ontology' - see chapter 2 for an in-depth discussion.
} 
and aperture control. Although technically possible with film cameras, the digital camera brought both speed and spontaneity to image capture, as well as the instant feedback of the rear screen monitor. With the first image in the sequence of three, he takes the use of focus and uses it both as a narrative device and as a vehicle of creative expression.

\section{The First Image}

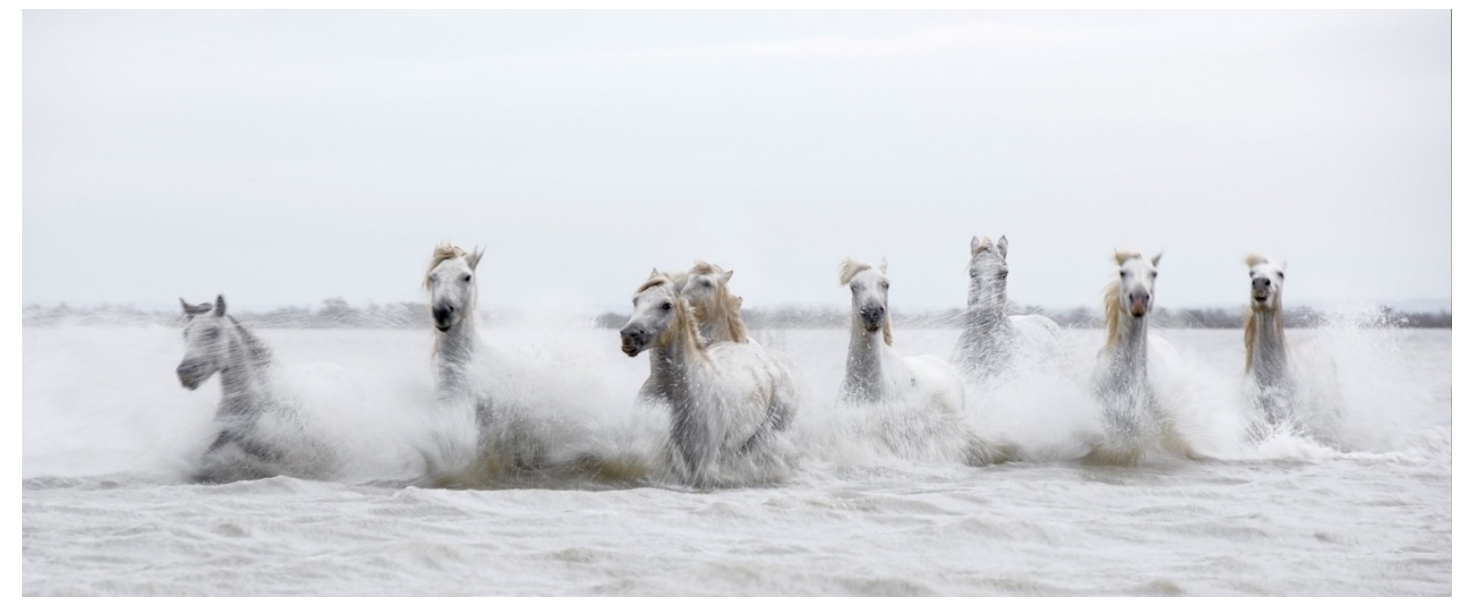

\section{Exhibit 8.3 Wild horses of the Camargue.}

CW had been leading photography courses in the Camargue for some years, and the running wild horses are one of the features of his programme. These horses have adapted to running in water and have distinctive light grey coats and yellow manes. On this occasion, he sought to explore his zoom-panning technique to capture both the power, and the movement, of the white horses of the Camargue and also the sense of serenity they conveyed to him. He does not explore his understanding of either term directly in his discourse. Their meaning is deferred, not in Derrida's sense to other signifiers but to his experience of the moment as it unfolds before him. Their meaning, and particularly their conjunct, are hidden within the complexity of his cognitive architecture and at levels of depth that go beyond the penetration of conscious introspection. In this instance, he sees them as linked, 'soft power' if you like. Not the hard power of the stampede, or the serenity of the calm ocean. There is, in his imagery, a sense of the fluidity, and drama in their movement, as perhaps depicted by the young German painter Jan Künster, or in images of the flying horse of Greek mythology.

Commenting on his intent he explains: 
Horses galloping at full pelt and yet having this softness about them and so the reason for having the horses sharp is the power and then the softer water is all about the serenity. So, the creative idea, say for the intent, was how do I bring these two essences, which are opposing, into the same picture.[55]

So, his intent is to bring two aspects of the essence together, and to focus upon their realisation within the team. He makes sure that the animals are well spread across the frame and he subsequently crops the image to give a sense of the numbers, the power and the coherence of the charge. He does this whilst recognising the animals as individuals, with their own personality. As well as the framing of the shot there are other processes underway in his image making.

First, he will not press the shutter until he feels an emotional connection with the animals:

... to understand who they are you have to make a connection with them. If you don't make that connection ... you cannot photograph...[16]

And this, he relates to the essence of what he sees:

...in order to get to the essence, you have to have a connection...it's about finding the essence of the group or the herd or the pod or whatever...[16]

Given the idea that the essence he seeks is one of connection, it is his own connection with the horses that illuminates the connection he sees between the horses, and between them and the sea. How is this done? What is $\mathrm{CW}$ invoking that reveals to the engaged viewer the real essence of these animals in their working environment? A clue to the answer emerged as the bracketing and activation process drew closer to the moment of capture, and he became more animated in his description of his image. What comes forward is the 'shadow on the cave wall', as the veil is withdrawn, and the boundary layer of his cognitive process is uncovered. Here, his creativity comes into view as, with rapid words, he paints this picture of his image:

... as you start to look at it you begin to think this chap over here on the right-hand side he has some trouble (keeping up) and has an alarmed look about him and his buddy here these two are like twins almost like they're part of the group but they are mates within the group if that 
makes sense so there is so there is this element the one on the left hand side galloping off as though needs his Weetabix so that's the thing, the longer I look at it the more I tune into the herd as opposed to the individual.[77]

Second, is he being intuitively reductive as he seeks to suppress one sensory effect to uncover the essence of his image? As I discussed in chapter 2, the reductive process is one of uncovering presence by attenuating elements within the image that pull us away from the real and towards the sensory. What do we feel if confronted with a line of charging horses? We hear the pounding hooves as they run. We see the flared nostrils and the wild eyes. We feel fear. But that is not what he wants us to feel; that is not what he is feeling. He wants us to experience what he is experiencing, and this is where the subliminal conjunct of the smoothness of the water and the dynamic of the running horses come together. The reductive approach to the sea is taking away our sensory perception of its turbulent motion and opening us to the essential reality he is trying to capture.

Here CW comments on the nature of his emotional state:

It's interesting because the reality is it wasn't fear. The one thing I never feel around wild animals is fear, which is one thing that allows me to do the job that I do...respect, for sure. Awareness, for sure. But I'm never afraid...you think, "well a wild horse is scarier than a domesticated horse." Yet for me it was the complete opposite; I wasn't at all afraid of the wild horse, but I am afraid of domesticated horses. And that was the strange thing, and I think that's what my mind picked up on, and hence that's why that became the interesting story behind the picture, these massively powerful beasts, wild, and yet are completely calm and peaceful. Underneath that, that energy, that powerful, brutal energy is being put towards good, not towards evil.

There is however, in Ortega/Harman terms, a third approach. Is CW consciously or nonconsciously recognising a metaphor, and inviting us, as the viewer, to enter into the theatre at the heart of the image? The object in 000 terms, as realised in the image, is the relationship between him and the horses, between the horses and the sea, and between them and us. Because the real objects - CW on one hand and the subject of the image on the 
other - are, in a phenomenological sense, forever withdrawn. We can only approach the reality of the image by engaging with the metaphor that he presents. We do this by stepping into the theatre of the moment and taking his place behind the camera, up to our knees in the rolling surf.

If that is his intent, what is the metaphor with image 1 that exposes the essential connection between the horses and the sea? At a simple level, we can see the flowing movement, "horses run like the wind and flow like the sea'. We can also see the allusion to the foaming white horses that appear on the wave tops as a storm comes through. Is this the allusion that he has conjured up? Or is he reflecting, in his image, the flying horses (horses that fly like a bird) of myth and legend? The image is of 'galloping horses not walking horses', and this appears to be crucial to his vision of the moment and the resulting composition.

CW's subsequent response to the use of metaphor was initially negative. Talking through the examples in my analysis, I asked him to what extent they reflected his approach to revealing the essence:

After the event. I'm not sure that they do, so I'm not sure if this picture is a metaphoric interpretation in the sense that we were talking about earlier.

However, after some reflection he returned to the metaphor of flow (white horses on breaking waves) and had this to say:

... I guess the metaphor, if we wanted to look for a metaphor in the picture... is in flow. Flow for me determines serenity. When something is flowing, then it is in harmony, and so again if I'd frozen the water you wouldn't have got flow, you would have got ice. So to make the water flow I needed to blur the water, and to reduce the detail to a point that the detail didn't exist and you had a flow because flow is movement.

It is not obvious from the interview that this particular metaphor was active in his nonconscious processing at the time of capture. It may have influenced his previsualisation of the image and his decision about placement and framing although the evidence points to reduction as his preferred strategy with this image.

At whatever level, and whatever way we wish to interpret the metaphor, the image reveals itself to us; the essence of the relationships within the image is laid bare, no matter how we read it, and his intended story is told. However, it is not just a story of horses running through water. It is a story of a relationship, and that relationship is triadic: horses, sea and CW the 
photographer. We, standing in his place, experience the essence of that relationship, and both the multiple and singular effects that it has on us as individuals, on our cultural milieu, and our collective nonconscious.

Closely related to metaphor is the issue of anthropomorphism - a term that originally applied to the transfer or carry-over of human emotions and passions to a deity. Like metaphor, it is a process of transfer, but unlike metaphor it is not the carry-over of the qualities of one object to another, but rather the carry-over of the self to another. As I have explored in depth in chapters 3 and 4, the conjugation of constructs in the intuitive moment is affectively driven. Without that emotional cue, the intuitive moment will not occur, and all that we are left with is stale literalism, and a 'record shot'. It is clear that at the moment of capture CW is emotionally charged, but is he overstepping the mark by imputing his own emotional state to the horses, and through that elevating his being as photographer above their being as animals? Has he implicitly contradicted the flat ontology discussed in chapter 2 , and reverted to a form of correlationism where everything is seen not through the lens of his conscious perception but rather through the lens of his emotional state?

Part of the issue here is the difficulty we experience in retrieving to conscious reflection the drivers of the intuitive moment. In retrieving the memories of the event, to what extent was he invoking a mode of thinking that is essentially conjugative, or an algorithmic mode of thinking that is inherently conditioned by mechanisms of cause and effect? The question then becomes whether he is being anthropomorphic in his intent, in the act of photographic capture or, is he being anthropomorphic in his rationalisation of his practice? If the first, Harman (2018) makes a powerful connection between theatre, art, and the role of metaphor where he argues that in approaching the real - to reveal 'the essence' in CW's terms - we must become the object that is portrayed:

'...strange this may sound, there is one professional realm where it is already commonplace: the craft of acting, where Konstantin Stanislavski's famous system insists that one try to become the object one portrays... This theatrical structure of metaphor strongly suggests that theatre lies at the root of the other arts.' (p. 83)

However, to what extent might anthropomorphism in intent, even if granted, influence the moment of capture? As I metaphorically 'stood beside him', and as the moment of capture 
approached in our discourse, two things became clear: first, his conscious choices and his nonconscious judgement were working together to a high degree and, second, the emotion he was feeling was driving the conjugation of an array of constructs under his intuitive command, and combining them with the deliberate employment of other technical skill and knowledge. He was experiencing calm and peacefulness in the moment and then carried that emotional state over to the horses. But is that legitimate? Jane Bennett (2010) in 'Vibrant Matter' suggests:

'We need to cultivate a bit of anthropomorphism - the idea that human agency has some echoes in non-human nature - to counter the charge of humans in charge of the world'. (p. $\mathrm{xvi})$

Indeed, for CW, it is his intuitive recognition of the emotional state of his subjects, and his understanding of their behaviour, as in human terms, that are co-dependent and which give him the ability to intuitively anticipate the moment. He also recognises that the engagement with living things, that Bennett (op. cit) calls for, cannot be one of detachment and objectivity. Given this, it is no surprise that his rationalisation reflects an awareness of the connectedness he feels and his emotional identification with their personality - even down to the Weetabix!

The IMP Test suggests that given the circumstances of the capture of image 1 , that the conceptualisation of the image is predominantly a product of his nonconscious cognition. Clearly, he had previsualised the shot he wanted to take, but the trade-off between his nonconscious, creative impulse, and his desire to tell a story, suggests that he will conjugate with composition, focus and depth of field in the mix. He then enters into a spontaneous process about how to convert his intent into outcome, and through that deliver his message. Using a slow shutter speed, but twisting the barrel of the zoom, he is able to hold precise focus on the leading animal. This is a technically complex focusing judgement that is easy to achieve with sideways-on movement, but not when it is head-on - particularly when there is a need to show movement but not radially blur the out-of-focus area. The nonconscious aspect of the capture is also revealed in his lack of recall of the settings he was using or the adjustments he was making:

...No, I don't remember adjusting them because these things are just so subconscious now that I do it without thinking. The only thought put into the camera settings for this shot is that 
I wanted a very low shutter speed so I would have set ISO and aperture to get the speed I wanted. [31]

In his view what he was doing with this focusing was both creative and telling a story. In this he reveals the conflict being played out in his boundary experience. He is trying to reveal and communicate the story - in this there is the realisation of his intuition into intent, but to achieve that intention he must solve a technical problem:

Well the aim was to capture a pin sharp image of the horses running and a blurry motion shot of the water spraying and the problem with that is that the camera needed two different shutter speeds, but of course on a camera you can only have one shutter speed ...the theory of panning being if you are moving the camera ... it is not how fast the subject is moving but how fast the subject is moving relative to the camera...But you can't pan the camera when the subject is coming straight towards you. [49]

The interesting word here is 'theory', which betrays CW's deliberative approach to the solution of the problem. It is here that we can properly appreciate the process of resolution within the boundary layer of his cognition. Composition and focus are the solution his nonconscious dictates, the effect he is after will spontaneously present itself, but there is a problem to be solved. His nonconscious has a quality and depth of resource that is not accessible to his conscious processing; but the problem is how to bring both composition and focus into equilibrium to achieve the shot. He cannot remember the camera settings, but he can remember the choice of the hand-held camera technique he needed in the few seconds available, given the speed of the animals, and their relative movement to himself.

...it's getting the zoom right to do two things, one is to get the speed of zoom correct but you are moving or changing the focal length matching the horse coming towards the camera in closing the gap between you and the camera the second thing is keeping the camera steady enough so that you are not getting camera shake. [49]

He clearly previsualises the compositional problem and how to solve it. The position he wants to adopt is in the water, so that as the horses come towards him, he does not block their gallop. In this he is making a framing judgement, positioning himself to get both the motion 
and the spread of animals across his field of view, but also putting himself in a position to achieve the differential focus he is after.

...so if you are standing on the water's edge by the time the horses get close enough to you they have stopped running as they are coming out of the water so the point where they're running at full gallop they are still in the water so I wanted to be far enough out from the edge so that when I took the picture they were still galloping rather than just walking. Because this picture is galloping horses not walking horses. [39]

Perhaps the metaphor in play here is not just the galloping horse, it is the gallop through the water - the water flowing and parting - that lies at the heart of the story. To what extent was this choice and positioning intuitive? His language does not betray strong emotion in the moment, although facing a dozen charging horses would naturally induce some feelings of fear in the hardiest of souls. However, he does say: ... actually it was anticipation based upon experience...' [39]. This ability to anticipate events and how they unfold is a familiar correlate of intuitive cognition. The language he uses is strongly suggestive of a powerful interplay between rational, previsualisation of action and consequences and experientially embedded skills and knowledge. What is also noticeable about this discussion of image 1 is the absence of two constructs: exposure and the use of light. His reference to colour is to mitigate it in the image through a consciously reductive act:

...colour is such an overpowering element of design. It gives an image context which is sometimes necessary ... this isn't about the surface reality this is not about what the horse looked like... it's about power and serenity. And for those two elements to come out you have to think about elements of design it's about texture, it's the texture of the water against the texture of the horse. So, by taking colour away from it you are allowing those other elements to breathe giving them space giving them a louder voice in the picture. [69]

This statement is highly reflective, and the judgement he makes is tied to the compositional design issues in the image. Indeed, this may have been his only significant judgement in postprocessing:

...the main adjustment I've done a little bit of tonal work on it ... I think I added a touch of yellow. Saturation into the manes and not everywhere else... [67] 
The choice of 'a touch of yellow' is significant in this context. This he says: keeps it in the realm of reality but at the same time emphasising the essence rather than the resemblance. [71]

Yellow is a powerful connecting colour. It emphasises joy, happiness, calm and indeed the love generated by the bonded relationships within the team, all of which reinforce the serenity of the scene and the sense of connectedness between the animals in the image.

\section{The Second Image}
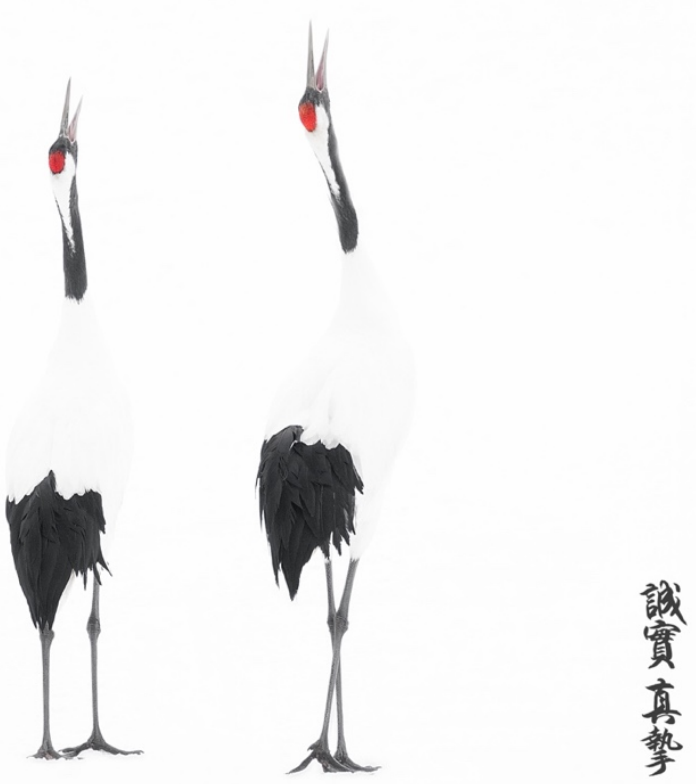

\section{Exhibit 8.4 Dancing cranes at Hokkaido, Japan}

As with the first image, the creative and compositional drive in CW's work, and the deliberative use of subordinate constructs to achieve his creative intent, is very clearly illustrated with this image of the cranes. In this image, he was seeking to emulate sumi-e 
painting through photography. As an art form, sumi-e entails years of practice not to achieve the complex, but to realise the simple.

From the Sumi-e society of America website:

Emphasis is placed on the beauty of each individual stroke of the brush. The Chinese speak of "writing a painting" and "painting a poem." A great painting was judged on three elements: the calligraphy strokes, the words of the poetry (often with double meanings and subtle puns) and the ability of the painting strokes to capture the spirit (Ch'i) of nature rather than a photographic likeness. (Extracted from http://sumiesociety.org/whatissumie.php 1 June 2019)

Sumi-e typically uses black ink on a parchment white surface, and this is the same effect CW wanted to achieve by experimentally adjusting the exposure. To do this, he positioned himself by a snow-covered field looking for a small window of opportunity to set his frame while waiting for the cranes to move into a position that would allow him to capture the structural relationship and connection he was after.

As he explains:

...this whole project was not - was very much about capturing the essence of, of the subject, and...that comes out in the, you know - this is - basically what I'm doing this is trying to recreate, the sumi-e art - Japanese art, which is all about essence. [292]

In this part of the discussion CW's normally very fluent language fractured as he struggled to find the words to summarise his recollection. With the horses we see clear previsualisation and an attempt, by exercising a high level of intuitive skill in the control and use of focus, to realise his creative and narrative intent. Here he is trying to achieve a similar intent, but this time through the intensely deliberative manipulation of exposure. With this image, he has set himself up near an upward sloping, snow covered field where a large number of cranes are moving about at the bottom end. Unlike the first image, he has less degrees of freedom in position and is using a tripod:

it's not always practical to use a tripod in wildlife photography. That's often because you're having to move the camera all over the place. I knew I was fixed on one spot... it was practical 
to use a tripod and of course it's better to use a tripod if you can, even in wildlife photography, so, for no other reason than it holds the weight of the camera, and you can walk off and leave it... [275]

The cranes have a stark tonal division between black and white in their plumage, with a red flash around the head. However, the white is not quite the white of snow, it is marginally less blue. By careful exposure control, he sought to achieve a point where the white of the plumage was just visible against the background.

I was constantly adjusting it, because there is a lot of, of technical expertise going into this picture. [220]

This, as 1 argued in chapter 2 is a characterising difference between reduction and simplification. Simplification would have been to convert to monochrome and then 'colour pop' the red. With reduction the confounding elements are attenuated to the point that they sustain the object but do not foreclose on its essence. He then proceeded to explain the method by which he reduced the image:

...the actual shutter speed was a secondary consideration, so long as it was in a range, it was fine, most of the time. So what I'm basically doing constantly is - the Fuji camera, has an exposure compensation dial on the back, and so I was constantly shifting exposure compensation, adding or taking light away in order to keep... the perfect... amount of overexposure. [226]

However, what is more striking is his search for the perfect composition, and here his normal fluency returned:

I have a fairly small window of space in which to take the photograph, so I'm waiting for the birds to come in and out of this - I mean, imagine a photo studio with a white card backdrop in a big warehouse. Well, the farmer's field is the warehouse, and my white backdrop is where I'm taking the shots, so I'm constantly waiting for the birds to pass in and out of my little window of, of shooting space.... [263]

His framing space was tightly constrained but given the rapid movement of the birds he was looking for something different. He found it with a pair who were engaged in a mating dance, 
and in capturing the moment of postural echo, he achieved his intent by revealing the connectedness between the two birds.

Connectedness is a recurrent theme in his thinking, and here, as with the horses in image 1, it emerges again:

The necks in similar positions, standing in very similar positions, it's the, it's the connectedness between the two; they're always like they're in sync with each other... which of course in a successful relationship is what you would expect; two people acting as one... and for me that's what it captures; it's two birds acting as one bird. [299]

Here we see clearly the paradox of the reflection in the window. The camera is looking both ways, the essence of the relationship between the two birds and what he values in his own relationships - two people acting as one.

In my view, much of what is being said here is a struggle to effectively rationalise a recollection of an intuitively creative event. He is framing it as about 'essence', returning to a theme in his earlier discourse on connectedness with the horses in image 1. But what is the essence he is seeking here? It is connectedness, but now the time available allows him to bring into play the lower order constructs as part of the conjugation of the whole image. The creative process was very deliberative, but in realising the opportunity multiple camera skills were entailed: composition, focus, exposure control, use of colour and tonality. But as we return to the essence of the image, and the story he is trying to communicate, we realise that the process he is pursuing is different to image 1 . There is again creative intent, but this time by trying, in-camera, to invoke the simplicity and grace of the sumi-e art form.

As discussed in chapter 2, photography can be described as the art of reduction and in this respect, it has a commonality with sumi-e, where the practitioner seeks to approach the essence but not to go beyond it. Reduction is not simplification in the sense of applying Ockham's Razor, it is bracketing the sensory experience to the point that the real experience is uncovered. Is this what he is trying to do here but by reversing the direction of travel of the sumi-e artist? His discourse is silent on this point, but his intent is clear, which is to arrive at the same point, in-camera and with the minimum of post-processing. 
By allowing the camera to reveal the structure and elegance of the dance, the viewer is brought into the image, replacing the photographer and experiencing the reality of the object. But is that all there is to it, is the story simply two birds dancing in the snow? CW observes that it is a mating dance, a dance where body echo creates a binding moment between the two birds - hinted at, but not stated. In the moment of dance, his nonconscious activates the conscious in the boundary layer of his cognition, and the communication between the two birds is revealed. However, like the horses he is revealing himself in the image - he sees the connection, he sees the communion of shape and movement, and in that lays bare his own feelings and desires within his own life-world. The poignancy of the moment is painful; there is deep ambiguity in the image, black on white, despair versus hope, and the flash of red the colour of fire and passion. But how is this realised for the viewer? A single flash of red, the gentle lines and curves of the black plumage and then, deprived of any other sensory signals, the gestalt does its work. The viewer completes the task, the sublime aesthetic, in its true meaning, is achieved - the conscious state-change that lies at the heart of the sublime occurs.

\section{The Third Image}

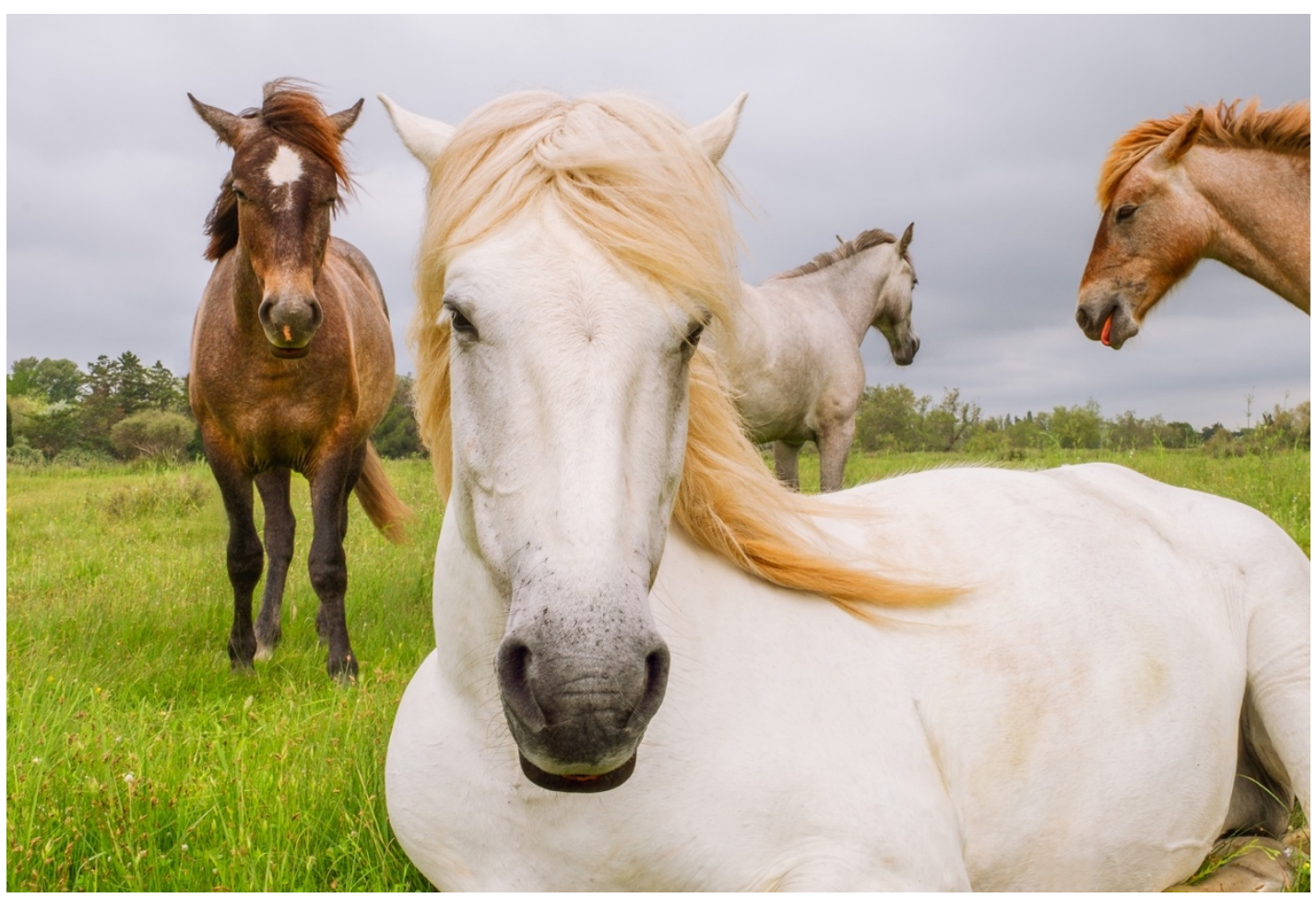

Exhibit 8.5 Dali's Horses in the Camargue 
The day after the capture of the first image discussed above, CW was invited by the farmer to take his tour group to the field where the animals were grazing. He was warned "do not approach the mare because she has the foal and she'll be very aggressive." [335] On entering the field, quite the reverse occurred when the grazing horses came towards them. Again, Chris reveals his awareness of the connectivity - this time between animal and human, and again he notices the body echo:

One of the guys who actually owns horses, he got one of them to fall asleep with him, and I've got this on video; the two of them start standing up, he sits down, she sits down, he lays down, she lays down, he puts his hand out, and she puts her nose on his hand. It's just phenomenal. So there was this beautiful energy going around the horses and the group, which was really just something to watch. [369]

Initially he did not see any worthwhile photographic opportunities:

And I became very mindful to the fact that I was completely... attached to a particular outcome... That outcome being horses running in water, charging everywhere. And because I was attached to that photograph, I couldn't see... ... what was stuck right in front of me... [383]

He recounts a process where he tries to be more meditative, exploring the creative opportunities that the scene offered. He relates how he was also preoccupied with an article he was writing, drawing inspiration and understanding from the work of Picasso and Dali. Here his focus was on the move from cubism to surrealism, drawing a parallel between the shifts and resistance to those shifts in the history of art. These are not, from his perspective, dissimilar to the shift in the sciences from Einstein's relativity to that of quantum mechanics and he conjectures that shifts in the arts follow shifts in the sciences:

... the point I was making in the article was that in order to move forward from one era in art or science, you have to have people who are prepared to accept that what we think is true now, may not be true, and there may be a different reality. And... what was also interesting at that time was that if you match the shifts in science, they are almost always followed by a shift in art movement. Picasso's Cubism came out around about the same time as Einstein's relativity theory...[389] it took Picasso and Einstein to move science and art from where it was to where it became, but interestingly, art moved on beyond Picasso and Einstein because science went from relativity theory to quantum mechanics, which is something Einstein could 
never really accept. [391] Because...cubism moved onto surrealism, which was something Picasso could never really accept... [393]

What this extract reveals is a process that he goes through in each of his images: there is previsualisation but also theorising, which, along with meditative practice, primes the creative act. He is in a mental prison of his own preconceived intent, but we see in his words a moment when the nonconscious recognises the patterns and structures of surrealism. Again, the narrative drives the inspiration, but it is in response to a compositional opportunity that opens before him.

I was sitting there, recalling this, this stuff I'd been writing about Dali, and then I looked - I didn't want to say I looked up because I was already looking at it - but I looked, and then I saw what had been sitting in front of me for the last ten minutes, which was this picture. [393]

Surrealism is the exploration through art of the nonconscious mind. Horses and the nonconscious are powerfully related in art and are a constant and recurring feature of our dreams, nightmares and desires. But what was the picture? Did he reveal the surreal? One horse sticks his head into the frame and sticks its tongue out. The mane of the central horse is curved, pointing at the intruder into the image. The combination of animals, their position and their posture, reflect the surrealism of the moment and there is no doubt about the speed of (re)cognition as Chris lifts the camera to his eye and takes the shot:

So I picked the camera up and I thought, "That's quite, that's quite an interesting shot because... it's very Dali-esque."[397]...and as I said that, that horse on the right stuck his head...[399] into the frame and stuck his tongue out and I just went, click. [401]

But what is it about the tongue sticking out? Is that an element of madness? The surreal, the unexpected, the photo-bomb that disrupts and subverts the normal? That isn't normally what horses do.

Now I don't particularly like Dali's art to be honest but I understand where it fits in the, in the history of art and its importance in the history of art, I just don't -it just doesn't speak to me. [393] 
In the discussion of the three images one construct was noticeable for its absence - technical. As noted before the 'technical' construct features in the guidance of the photographic associations. It's placing in the output of his IMP Test puts it close to the use of colour/tonality and to the use of light. Was he simply reworking these constructs in his mind as he evaluated the images in phase 3 of the test? It would appear that he has a high degree of skill in its assessment both at capture and review, but the construct is not influencing his intuitive judgement to any significant degree. It may be that it is a 'negative bin' for all those issues which detract from an image such as distractors, 'wonky' horizons and verticals, and noise indeed anything that undermines the quality of the image given its driving intent. Or, it may be that the three images discussed in this interview were free of obvious technical problems and therefore the issue did not surface.

\subsection{Latency}

In many respects, CW's interview throws a fascinating light on the interplay between conscious and nonconscious cognitive processes. The IMP Test reveals that the higher order constructs are nonconsciously accessible and are recognised in the moment of analysis or capture. The three - impact, creativity and narrative - are all under both conscious and nonconscious control, but what is most striking is the importance of design - as he understands it - to his aesthetic. His desire, conscious and nonconscious, to seek impact through creative solutions at the moment of capture, and to tell a story, drive this design aesthetic. His understanding of design is a product of both consciously and nonconsciously accessible constructs and it is their conjugation that generates his unique style.

Returning to the evidence I discuss in chapter 4 , nonconscious structures are deeper and richer in both content and problem-solving ability than conscious levels of retrieval, and that is evident from the above. With $\mathrm{CW}$, by accident or design, his conscious and nonconscious conjugation are very similar in their output and thus, what his nonconscious injects into his conscious awareness is, for him, natural and self-evident. He enfolds within design: composition, focus, use of depth of field and, when appropriate, use of colour/tonality. However, the important point to note is the close articulation between what he consciously seeks to achieve and what nonconscious access to his deepest experiences yields. 
Chris's reflections on design supports the analysis above:

Yes, because I think for me design goes beyond its perceived literal sense. When we talk about design we're typically talking about light, shape, colour, pattern, texture, the things that go to make up any object, might throw into their functionality which is another part of design. But we often talk about design in terms of composition. For me when I talk about designing an image, I talk about it in a much more 360-degree sense. Design is about the conception of the idea into which comes creativity. Where does that spark come from? So, it's the spark of the idea, that's creativity. It's taking the spark and turning it into a fire and then feeding that fire with work ethic.

Indeed, the concept of design features extensively in CW's approach to and commentary upon his work. For CW, but for no other photographers who took the IMP Test, it holds high latency. The testing shows the close cluster of the cue dependent, nonconsciously accessible constructs. However, the 'energy' triple - exposure, use of light and, to a lesser extent, use of colour/tonality - are under a high level of conscious control but are not intuitively accessible. This may be a consequence of his original genre. Wildlife photography is dependent upon scarce access to animals, where the ability to control for lighting is limited. $\mathrm{He}$, like most wildlife photographers, routinely shoots in 'aperture priority' and as a technical rule of thumb he always shoots 'to the right', i.e. with a tendency to over-expose. Providing he remains within the dynamic range of his camera, that serves to ensure that there is enough light to maintain a high signal to noise ratio and - in terms of subsequent use of the image high quality. He routinely tells his students: there is 'no such thing as bad light, just light'.

Responding to the question: How closely does the IMP Test match your perception of yourself? Chris replied:

It is almost perfect, and in that perfection, it backs up my view that the test is a very solid, reliable mechanism to determine what is going on in the mind of the photographer when they are looking at potential images or they're looking back on images they've taken and are assessing them. 


\subsection{Summary Judgement}

Following the criteria laid out in chapter 5, given the output from the IMP Test, the emergence of the constructs as uncovered in the interview, and his feedback, I have high confidence:

(i) That he was able to enclose his judgement within the ten construct signifiers and that his approach to photography reflects the ontology presented in chapter 2 and his cognitive processing reflects a high level of expertise, supporting the principles of Cognitive Field Theory discussed in Chapter 4.

(ii) That the IMP Test has correctly identified those constructs that are under his conscious and his nonconscious control, and that the correlations between them are fairly represented by the analytical methods employed.

(iii) That the test, confirmed by the interview, has uncovered the latent structure within his nonconscious that drives his style which is fairly described as authentic as opposed to synthetic or dissonant. That latency he describes as 'design' but is driven by his understanding of composition, narrative, creativity, the use of depth of field and focus. 


\section{Chapter 9 - Conclusion}

\subsection{Introduction}

Authorship - the creative act - whether the flow of the pen, the click of a shutter, or the insight of the scientist, is driven by the vast resources at the disposal of our nonconscious minds. My first working proposition is that creativity in photography comes through understanding the way the nonconscious works and how it can be developed. This is not to say our conscious ability is unimportant; it has a vital role in managing our awareness and converting the insights and intuitions of the nonconscious into intention and action. However, the route to development is not by denying the role of the nonconscious but by relearning ways it can be effectively trained to do what it does best.

Consequently, the overall summary of this research is that it is transdisciplinary - it seeks to understand the art and practice of photography through the lens of psychology. In particular, I attempt to examine the nature and development of expert, photographic judgement. One insight from psychology has become close to paradigmatic over the last quarter century. This is the understanding of the role of the nonconscious in our cognitive processes and the rather limited abilities of the conscious, rational mind in solving problems, pattern matching and influencing choices (Lewicki, Hill et al. 1992, Eagleman, 2011, 2016, Hayles, 2017). As rational, self-aware beings we are seduced by the idea that all experience both internal and external lies relative to our conscious perception. This 'relativism' - or 'correlationism' - I discuss in chapter 2 and by its rejection, I place my ontological position at the centre of this research.

The research presented, follows the ontological principles discussed in chapter 2. My ontological position - which has some commonality with Speculative Realism (Meillassoux, 2008, Meillassoux, 2014) - establishes the principles of my chosen mode of investigation, and my analysis and interpretation of the four research questions specified in chapter 1 . Speculative Realism reflects a 'flat' ontology rejecting any special ontological status for the conscious mind. I argue that we, like all aspects of our existence, are more or less complex objects who through that complexity exhibit emergent qualities and consequential effects that form the basis of our and their being in the world. In this respect, my ontology is Aristotelian (Salem 2017) but in the modern era is inspired by the work of Ortega y Gassett, 
(1968), Kant (Pendlebury, 1955, Kant, 1992, Hunter, 1999), Heidegger (Heidegger, 1953, Harman, 2009), Latour (Latour, 2005, Benzon, 2011) and others. This position leads me to reject the dualism of Plato, Descartes, and much of continental philosophy. Although opposed to the continental tradition that dominates current academic discourse in photography, I have sought to demonstrate that my position is highly productive from a research perspective. It enables me to seek coherence in both the integration of elements of cognitive psychology, aesthetics and photographic practice, allowing me to combine them into an analytical framework that transcends traditional disciplinary boundaries. This ontology also supports my experimental methods where I seek, through the IMP Test, and a process of intraspective interviews, to understand the essence of human photographic judgement both at the individual and the aggregate level.

Working with the conceptual object 'expertise', my strategy has been to avoid 'overmining' the concept, but to seek its essence through a process of fracture and reduction. 'Overmining', a term coined by Graham Harman (2018), is where the concept of experience is examined or explained simply in terms of its effects. Accordingly, I am not concerned with the issue of photographic intent or with criticising the performance of the photographer whether it is at the individual, cultural, social, or political level. My approach has been to focus attention on a set of signifiers pointing to ten cognitive constructs or 'schema' that capture the photographic expertise necessary for the effective realisation of intent: impact, narrative, creativity, composition, the use of light, colour/tonality, depth of field, control of exposure, focus and the avoidance of technical issues and distractors in the image. Through this (ontologically) reductive strategy of attenuating their 'meaning' in the analysis, I have sought to bring out the essence of photographic expertise by examining how these ten constructs are conjugated in the nonconscious processing of the individual, whether in capture or in image assessment. It is the process of conjugation that generates, out of the complexity of their combination, the emergence and evolution of what we term 'style'. 


\subsection{Summary Conclusions on the Research Questions}

\subsubsection{Research Objective RO1}

My first objective (RO1) was to develop a theoretically coherent model of photographic expertise based upon Cognitive Field Theory (CFT). Grounded within the insights of cognitive psychology and supported by recent neurological evidence, is the understanding that our experience becomes embodied within a system of distributed memories - some actively retrievable through conscious reflection and some, held as 'chunks', 'templates' or, following Kelly's term, 'constructs' that are only recoverable under affective pressure. My development of Cognitive Field Theory in chapters 3 and 4 proposes that nonconscious processing, within a domain of expertise, can be described as a conjugation of a maximum of ten such constructs. Cognitive Field Theory offers a number of insights into the operation of the nonconscious cognitive system: first, it structures the mind as an assemblage of constructs; second, those constructs form a natural hierarchy operating in a Bayesian way, where perception is driven by deep structures of belief; third, the constructs are 'fuzzy' in that they overlap, enabling conjugation under affective pressure and, fourth, the expertise of the individual is defined in terms of their ability to recover and conjugate the content of those domain specific constructs that are appropriately emotionally 'tagged'.

As I present in chapter 4 Exhibit 4.6, there is considerable agreement between the various photographic bodies about the ten construct signifiers that form a parsimonious semantic representation of photographic expertise. The meaning of these terms, as conceptual objects, is deferred or withdrawn within the understanding of the individual and it is the conjugation of these deferred terms - but not their meaning - that form the basis of the IMP Test and my exploration of the process of judgement by the individual. The empirical research presented in chapter 6 provides strong evidence that variation in the practical judgement of 106 photographers as a group is explained at the $84 \%$ level by these ten constructs. At that level we are approaching the level of irreducible uncertainty (Hammond, 1996) in any judgement process and for these photographers the empirical evidence is, in my view, overwhelming that these ten constructs fully reflect their understanding of photography and the application of their expertise. 
Individual average test subjects achieve, on average, an $\mathrm{R}^{2} \mathrm{Y}$ score of 0.578 with a distribution skew showing a median of just over 0.6 (see: Exhibit 5.9). The evidence collected from this study indicates that the mean number of constructs under intuitive control is less than five, with only a minority achieving six, and only three test participants achieving seven. This raises the question whether ten constructs are an over-ambitious basis for structuring expertise. Within conscious processes, Miller's seven plus or minus two (Miller 1956) is regarded as definitive of the maximum number of different variables that can be brought together in a given decision. There is no reason, given the parallel processing characteristic of nonconscious cognition, why this constraint might still apply. Further, there is a difference between the notion of a construct and a decision variable in terms of their specificity and computability. However, at the individual level, all ten constructs do feature in the photographers' nonconscious cognitive 'portfolio', in varying degrees of frequency and rank.

My summary judgement is that, with a high degree of confidence (see: Exhibit 5.12), RO1 is met in as far as it applies to this group of 106 test subjects. This judgement is based upon a consilience of evidence: consistency in the signifiers of expertise from the principal photographic bodies; high levels of statistical confirmation from both the individual and aggregate analyses of the results of the IMP Test; and the evidence adduced from the intraspective interviews. None of these strands of evidence would be sufficient in and of itself to justify the use of this structure of expertise in analytical work. However, taken together I believe my assessment of confidence as 'high' is justified.

\subsubsection{Research Objective RO2}

I set the development of an empirical protocol, based upon RO1, as my second research objective (RO2).

In assessing the test, two questions need to be addressed. First, is the structure of the test and the subsequent mode of analysis an efficient means of eliciting an individual's cognitive structure of expertise (the 'structural problem')? Second, does the implementation, using the structure of expertise adduced from RO1, fairly capture the domain specific expertise that is the focus of this enquiry (the 'implementation problem')? 
The empirical protocol of test and analytical method was designed to enable the exploration of the exercise of intuitive expertise in photographic judgement following the Bayesian principle of cognition. In chapter 5, I discuss at length the development of the IMP Test and its associated analytical procedures. Computationally, the principal modelling method has been chosen to (a) integrate the short time-slice assessment of each image (principally intuitively driven) with the balancing of the ten independent variables (principally consciously driven), (b) overcome the problem of overfitting, and (c) resolve the inevitable multicollinearity in the test data provided by the individual. This led to the selection of Partial Least Squares regression supported by multidimensional scaling as my analytical methods of choice. In the trade-off between internal and external validity, I have opted for the latter.

As noted in chapter 5, the IMP test is designed to be straightforward whilst yielding just sufficient data about an individual's judgement to enable formal analysis. As has been reported by numerous test subjects the test was challenging in use, took about one hour to complete, and was rewarding in its own right. The test results showed a high degree of internal consistency and all 106 candidates achieved $100 \%$ completion. As noted above, the test data revealed that in terms of judgement constructs, Miller's seven plus or minus two holds at the nonconscious level as it does for variables in a process of conscious decision making. The question the test cannot resolve is whether the construct limitation is finite around seven or can be expanded to ten through training focusing on the development of nonconscious capabilities through Hebbian learning techniques (see chapter 4). My intuitive judgement is that ten is the benchmark number of signifiers to use. There are some pointers supporting this judgement in the literature (eg Lewin's, 1939 life-field model), the upper end of Miller's rule, and our system of counting and hence enumeration on a base of ten, which is driven by the number of fingers we all possess (Changizi, 2010). There is also good evidence that ten is a significant component of our cultural enumeration (Saxe, 2016) and an important physiological driver of our cognition across a number of dimensions (Lindemann and Fischer, 2016).

The second question has a significant recursive element in that we have used the IMP Test to validate RO1, and we are using RO1 as an input into the test. This elevates RO2 to the status of a joint hypothesis, which presents a problem for cross-sectional research design. In this research, I have dealt with that in two ways: first, by a process of progressive implementation, 
examining the test's aggregate quality metrics as the sample size progressed, and (as noted in chapter 6) there was no significant difference in the temporal order of early versus late test results; second, by the use of intraspective interviews which did not signal any degree of misspecification in the specific implementation of the test.

Corroboration of the test and support of its external validity was undertaken through a series of 16 intraspective interviews that were bracketed and reduced in order to uncover the drivers of the interviewee's capture decision. All of the interviews provided a high level of corroboration; a detailed analysis of four is provided in chapter 6 and an extensive analysis, with feedback, forms the substance of chapter 8. To support the process of corroboration, I proposed and operated a structured framework of calibrated language designed to reflect my judgement about the quality of each test's outcome.

With 106 participants there is always a question of the generalisability of the results given the widespread practice of photography across the world. However, I put this question to the literature: on what grounds would the cognitive functioning and the exercise (as opposed to the structure or signification) of expertise of this sample of photographers differ in kind from the rest of humanity? One particular objection might be that the average age of the participants (61 years) is considerably higher than the mean for the population as a whole. It is a regrettable fact that brain shrinkage commences at around 40 years of age with an associated decline in cognitive abilities ${ }^{46}$. Examining the test's explanatory power above and below that mean value $\left(R^{2} Y(<61)=0.83 ; R^{2} Y(>61)=0.84\right)$ gave no red flags for an age effect. Accordingly, I was unable to conjecture a reason, theoretical or empirical, for suggesting that the sample of 106 reported in this thesis would be cognitively atypical of the rest of humanity.

The aggregation of the results at group level generated results indicating that the Law of Large Numbers emerges above a group size of between 20 and 30 individuals. The emergence and testing of the averaged data from groups, as opposed to individuals, partitioned the variability in the dependent variable into two sources: an idiosyncratic component that disappeared as

\footnotetext{
${ }^{46}$ Without opening up a new literature to explore I found support for this worrying statement of reality in a special issue of New Scientist (22 July 2019) featuring 'The Human Brain'. It appears that there are some strategies available for improving cognitive reserve, I quote: 'Continuing to Educate yourself throughout your life appears to provide one of the biggest benefits...' (p. 41) enough said!
} 
the Law of Large Numbers took effect, and a residual component reflecting a core of variability reflecting the application of meaning of the constructs held in common. For the whole group, four constructs - shorn of their idiosyncratic component - do not appear to influence the intuitive judgement of the whole group. Two of them, the judgements of focus and exposure, appear to be a likely consequence of the automation of camera technology. The two others - creativity and narrative power - are those having the lowest commonality of meaning among the group. Both go to the essence of the subject of the photographic act, but this result suggests that across the average this is difficult to discern and capture photographically and difficult to recognise intuitively in the assessment of the image.

As we turn our attention away from the analysis of the average judgements of the whole group to the results - as reflected in the frequency and the importance of the ten constructs in the intuitive judgement of each individual - a different picture emerges. At the individual level of analysis, the personal understanding of each construct is present and creativity and narrative power become more significant. By a combined measure of frequency and importance, the order revealed is as follows: impact, composition, creativity, use of light, narrative power, technical, use of depth of field, use of colour/tonality, focus, and exposure. The subordination of focus and exposure is doubly revealed. Composition or framing is revealed both at the individual and the aggregate levels as being singularly important and at the aggregate level this is undoubtedly down to a high level of common agreement on visually satisfying compositional structures.

On the basis of the degree of corroboration between the intraspective interviews and the IMP Test results, and the high recorded outcomes against a range of quality measures, I have strong confidence that the test is delivering what I set out to achieve and the second research objective (RO2) is met. Without the inevitable recursive element, my level of confidence would have been higher than 'strong'. However, at the 'strong' level I believe the IMP Test, as constructed and implemented would form a basis for further research (see below) and for guiding individuals in the systematic development of their practice. 


\subsubsection{Research Objective RO3}

This research objective sought to find any differences in the test results that could be attributed to gender, cognitive processing style, photographic qualification, performance on the IMP Test and genre. As noted with the aggregate analysis, the IMP Test showed clear differences between aggregates of performance by the whole or by sub-group, and by frequencies and rankings at the individual level. In ontological terms, we can consider the whole group, for example, as the object of study.

Partitioning the 106 test participants into sub-groups produced some surprising results. Females were shown to be less intuitively aware of issues of creativity and narrative power than men but had a greater awareness of the more basic constructs. In this, they mirrored what the test revealed for those who held photographic qualifications such as those offered by the Royal Photographic Society and other similar bodies. Holders of these qualifications have a very low level of intuitive awareness of creativity and narrative power but an above average awareness of issues concerning focus and exposure. This result raises issues about the nature of the awards concerned, the process of education and training leading to them and the types of individual attracted to apply. However, the fact that a higher proportion of women than men hold these awards is one possible reason for the noted gender difference.

Testing for differences according to intuitive modality threw up some issues with respect to the use of Epstein's $(1999,2003)$ Rational Experiential Inventory (REI). The IMP Test appears to indicate intuitive ability more satisfactorily than the REI - at least when comparing individual performance against the consensus for the whole group. Partitioning by intuitive alignment with the consensus suggest that those whose intuitive modality is more closely aligned with the consensus than others also have more constructs under their intuitive control. They also tend to be more aware of narrative in the moment, but less creative than those who have a more deliberative bias.

As noted in the conclusion to chapter 7, partitioning the data by each individual's level of explanatory performance, using the $R^{2} Y$ statistic, revealed that those above the median in their performance $(0.603)$ had marginally less constructs under their intuitive control than those below the median. However, they were more likely to have narrative and composition as dominant, cue-dependent, nonconsciously accessible constructs. This suggests that the 
$\mathrm{R}^{2} \mathrm{Y}$ score is a good indicator of the individual's alignment of their rational and intuitive judgement and would be one useful, but not exclusive, measure of their ability to make internally consistent and justifiable assessments of a given image

The problem with partition studies is that although some rationale can be found for the outcomes, the results are very fragile given the sub-sample sizes available in this research. As I note later, further research is needed to corroborate these results particularly where they may have policy implications. On this basis I have modest confidence that I have achieved my third research objective (RO3).

\subsubsection{Research Objective RO4}

In satisfying RO4, I presented the case of a photographer with an established international reputation exploring his judgement processes in great depth. Three issues were of particular importance in selecting this photographer for in-depth study: first he has a strong reputation; second, he has a high level of introspective awareness and third, he has made an issue of seeking the essence within his subjects and has written extensively on the challenges of doing so.

With this case study analysis, my objective was to pursue insights gained through the combined IMP Test/intraspective interviewing process, with the aim of discovering if, and how, they illuminate the structure of expertise and, in particular, the way that this case subject conjugates the constructs at his disposal to create a unique style or 'voice'.

Following my ontology in chapter 2 , style is an emergent property as the components of cognition combine in unique ways for the individual. It is this cognitive conjugation, as much as the depth of knowledge and skill possessed under each construct, that defines their expertise and the style that emerges. Where that style is principally driven by the conscious processes of the individual, a clear synthetic style emerges. Where it is driven by the nonconscious, a more authentic style reflecting the depth of experience and the structure of beliefs of the photographer concerned will emerge. Authenticity in style, given its genesis within the affectively driven, cue dependent structures of the nonconscious life-world of the 
individual, will be emotionally loaded at capture. Where it is synthetic, the emotional gives way to the intellectual, and the image reveals the head more than the heart.

Probing more deeply into the mind of the professional photographer, who is the case subject in chapter 8 , it became very clear that the concepts of emergence/withdrawal and the route to the essence of the image developed in chapter 2 were strongly reflected in his work and his words. What was also evident was that a small cluster of intuitively accessible constructs - including the use of narrative, composition and focus, - came together into a latent structure he described as 'design'. In this he reinforced the notion of latency as the basis of style, which was evident from the intraspective interviews presented in chapter 6 , and from many of the others whose interviews are appended as transcripts.

The important conclusion here is that the IMP Test was strongly corroborated by the intraspective interviews but more than that the whole process provides a powerful procedure for examining the parts, the sum of the parts and, more importantly, the emergent quality of photographic expertise.

\subsection{Contribution Claimed}

I claim three areas of contribution for this research: ontological, theoretical, and empirical.

(i) Ontologically: speculative realism is a new ontology with an ancient pedigree that makes a break with the correlationism and inherent dualism within continental philosophy. My variant accepts that all elements of our experience can be conceptualised as objects with both sensible and real properties, qualities, and effects - actual and potential. All objects, I argue, have emergent properties, borne of their inherent complexity, that defy definition in terms of their constituent parts. These emergent properties are recursively but not causally generative of effects. In this thesis, I have used the ontological principles discussed in chapter 2 to develop an interpretation of the cognitive literature exploring its application in the definition and investigation of photographic expertise. The principles discussed and summarised in Exhibit 2.1 lead to five strategies that enable the process of this research, support its design, and help in 
the interpretation of its outcomes. Although aspects of my ontological position are appearing in the literature (Bennett 2010, Silverman 2015, Hayles 2017), I have not found its application in experimental research in this, or any other field, thus far.

(ii) Theoretically: my theory in use is based upon a 'bottom up' understanding of cognitive processing within the framework of the Bayesian Brain and the Hierarchical Predictive Processing (HPP) model of conscious and nonconscious mental activity. The basic unit of expertise is defined as a construct being a schema or template for the acquisition and consolidation of cognate memory structures. These constructs develop experientially over time and will become, in varying degrees, associated with emotional cues that can lead to their subsequent activation and conjugation. In so doing, they can generate outputs that impel action, but lie below the level of conscious determination. They are also superficially accessible to consciousness, but the evidence suggests, and this research supports the conclusion that conscious retrieval whilst having an important strategic function in planning and developing intent, is nevertheless motivated by nonconscious imperatives.

The contribution of this research is to develop from first principles a structure of photographic expertise within the HPP framework, and to demonstrate that its cue dependence and rapid response when activated can be explained using dynamical systems theory. The theoretical exploration of the nature of construct formation leads to a four-stage process for their development: procedural, declarative, semantic, and Bayesian. At the lowest level, procedural expertise relates to the technical competence necessary for undertaking a given task. At the highest Bayesian level, the individual can make sophisticated and appropriate judgements in the realisation of their intent that, in balance with other cognate areas, produce an integrated as opposed to construct-specific optima in performance.

(iii) Empirically: I would claim this study as the first to develop and validate an efficient protocol for the examination of photographic expertise. Through careful and 
reflective use of the test, it is possible to identify those elements of knowledge and expertise that are intuitively accessible to the photographer and those that are not. With that it is possible to achieve an understanding of the way that the photographer combines them in the boundary between conscious and nonconscious cognition. There were two parts in the development of the test: first the design of a test that would be easy to undertake and have high external validity; and second, to select appropriate analytical methods that could overcome the problems of internal validity that such a test would entail. Arguably, the test as implemented can only be safely extended to the sub-population of photographers who match the participant profile presented in chapter 5 . However, I would argue that even given this constraint, the sample of 106 participants is representative of a very large underlying population of both amateur and professional photographers across a wide range of genres ${ }^{47}$.

In terms of contribution, I would argue that the construction of the test can be widely applied to anyone who possesses some degree of expertise in the capture or assessment of photographic images. Structurally the test focuses upon cognitive processes common across humanity and for which there is significant theoretical and empirical justification. The novelty of the test lies in two important rules that should be applied in its implementation: the limit applied to the number of constructs and the (ontologically) reductive process in establishing the ten signifiers of expertise. This is, I acknowledge, a significant conceptual barrier for those who feel a need to eliminate uncertainty by defining the meaning of terms in any empirical model they use. However, deferring the meaning of the signifiers has, I believe, overwhelming benefits: first, it focuses attention on how the constructs are conjugated in the cognition of the individual rather than on their meaning or content; and second, it has the effect of passing 'ownership' of the results of the test back to the test subject.

\footnotetext{
47 The Photographic Alliance of Great Britain (PAGB) notes that it has 1000 camera clubs and 40,000 members across the UK http://www.thepagb.org.uk/about/federations/ (accessed 29 July 2019)
} 
One of the challenges with this research was justifying the use of numerical and analytical techniques with what many would regard as an aesthetic and subjective process of image capture or assessment. Judgement, in my view, is characterised by uncertainty, partial and sometimes ambiguous evidence, and will be guided by experience as opposed to algorithms, probabilities, and foreseeable outcomes. In the world of judgement, numbers are a metaphor for experience rather than precise units of measurement. They can bring rigour, justification and accountability to a subjective process. Subject to appropriate replication and the development of skill in its use, the use of this testing procedure could be used to enhance the criteria for the selection of image assessors.

\subsection{Critical Review of the Thesis}

Although I undertook the supporting literature work in the early phases of the research, much of that work was done in parallel with the conduct of the test. I had made an early decision that the research would not be about the formation of photographic intent nor with the critical analysis of images above and beyond the purpose of the test. Engaging with the academic literature was a challenge in the sense that much of it had been developed within a post-modern, social/political agenda that did not sit well with my philosophical position nor, I felt, fairly reflected the decline of post-modernism as a world view in other disciplines. As a consequence, I have not engaged with the problems of meaning and intent and the debates that have followed the Wimsatt Jr and Beardsley (1946) seminal article on the intentional fallacy' reinforced by Barthes (1967) highly influential Death of the Author. Indeed, my argument has consistently been that the camera looks both ways and is as much an image of the photographer's intent as it is the meaning of the subject at which it is pointed. To what extent was my unwillingness to engage with that paradigm a mistake? I appreciate that this position might present a barrier for acceptance of the ideas and the results of this research in the academic community. Much of the photography literature I have pursued does, 
accordingly, have a practitioner bias some of which is controversial in the academic community. However, I take comfort from the fact that many photographers who have taken the test are from the 'fine art' tradition and others from successful professional practice, and they report great satisfaction with the test and the help that it has given them.

The development of the IMP Test outside the framework of this research entailed considerable planning and testing. For some years I had used a prototype of the test in the evaluation of my own images and for judging images for camera clubs and photographic societies. Indeed, the test was a crucial factor in the creation of my own panels for submission for Licentiate and 12 months later Associateship of the Royal Photographic Society. However, much of that development was not recorded and the current test was designed and implemented as an 'add-on' to the Master Photographer (Ryan, 2017). I acknowledge that this was not an ideal way to develop the test as a research instrument and subsequently accessing the 106 participant/volunteers to take the test was much harder than I anticipated, as I had already trawled the participant pool and finding 'fresh' candidates was a significant challenge. Many of the decisions were locked in, and my justification of them has been expost rather than ex-ante. Although, I am of the view that it has all worked well in the end it is not the ideal way to construct and execute a programme of research at this level.

Finally, I have one issue that has troubled me since the commencement of the test. I find the test's power in penetrating an individual's cognitive architecture profoundly fascinating and the high level of measured performance, reported in chapter 6 , very reassuring. It is a test, to reemphasise the point, that relies upon correlation as opposed to the content of the individual's expertise, although the test, through its measures of judgement uncertainty, does give some indication of their relative skill level compared with the average for all participants. Nevertheless, I have been aware all along of the 'Barnum' effect, so named after PT Barnum, the American showman, who is credited with the phrase: 'a sucker is born every minute'. My worry is that the advice given by the test is being accepted because of my perceived authority and the technical detail in the report. As a result, does the test point to real issues or is it simply demonstrating the willingness of people to believe things about themselves if told by someone they believe to be an authority figure? We know that people in general have a very weak, if non-existent awareness of their own nonconscious processes so, perhaps, any story told by the test will appear meaningful to the individual concerned. Early in the testing 
process an eminent professional with a strong science background and decades of experience in photographic practice made this comment:

Hello Bob

What a lot you seem to know about me!

The report is very detailed and complex (in places tricky to follow for a layman) so will need some serious, focused time to assimilate.

Thank you for the time and attention you have put into it.

Would it have been better for the origin of the test to have been downplayed or administered through a neutral agency?

I have sought to resolve this worry by the development of the intraspective interviews, which added to my confidence in the veracity of the test, although the ability to subliminally direct answers is a well-known skill of psychics and mind readers. I also have some reassurance that it is not possible, without access to the necessary analytical software, to cheat the test and its simplicity leads me to believe that it has the high validity and is measuring what it purports to measure. However, this leads me to my proposals for future research.

\subsection{Proposals for Future Research and Development.}

These are some of the areas suggested for further research:

I. The test in its current form should be streamlined and simplified in application. Delivery through the web, with the statistical testing and model building redeveloped in (say) R - a mathematically oriented computer language - would simplify the administration of the test and its reporting.

II. In many respects this thesis only gives evidence supporting 'proof of concept'. Like all studies, it needs replication, although by its nature it would be difficult to replicate with the current test subjects. A further trial using the same construct signifiers but 
different images would provide confirmatory evidence at the group or sub-group levels.

III. The test is very plastic within the constraints of the cognitive theory and models upon which it is built. It can be varied in terms of the images or the construct signifiers it employs.

IV. The test can be rerun at different perceived levels of photographic ability with images of higher discriminatory power for those seeking or assessing high level professional or academic awards.

V. The test can be extended to different domains of photographic expertise. Different construct labels can be developed that capture expertise in panel assessment or the assessment of a body of work. In a professional or academic setting, where assessment expertise is being developed, panels of images can be employed rather than single images.

VI. Academic teachers of photography, seeking to assess expertise in the assessment of context, narrative and creativity, might well choose ten different construct signifiers using appropriately calibrated images (see chapter 5 for the methods followed in this study).

VII. The transcript evidence and the detailed results of the IMP Test provide a substantial resource for intensive, critical review. The IMP Test results have been partitioned, and aggregate and individual results obtained for different classifications of photographer. The variety of interviews conducted cover many of the partitions discussed in chapter 7 and provide insights into the motivation for the differences found. It is beyond the scope of this research, but this body of original material could lead to and reward further investigation. 


\section{Bibliography}

Abdi, H. (2003). Partial Least Squares (PLS) Regression.

Abrams, R. S. and S. Christ, E (2003). "MOTION ONSET CAPTURES ATTENTION." PSYCHOLOGICAL SCIENCE 14(5): 427-432.

Ackerman, P. L. (2014). "Nonsense, common sense, and science of expert performance: Talent and individual differences." Intelligence 45: 6-17.

Adams, A., Ed. (1943). A Personal Credo. Photography in Print: Writings from 1816 to the Present (1988). Albuquerque, University of New Mexico Press.

Addinsoft (2019). XLSTAT statistical and data analysis solution. Long Island, NY, USA.

Adolphs, R., L. Cahill, R. Schul and R. Babinsky (1997). "Impaired declarative memory for emotional material following bilateral amygdala damage in humans." Learning \& Memory 4(291-300).

Akarachantachote, N., S. Chadcham and K. Saithanu (2014). "Cutoff Threshold of Variable Importance in Projection for Variable Selection." International Journal of Pure and Apllied Mathematics 94(3).

Badger, G. (2010). The Pleasures of Good Photographs - essays by Gerry Badger. New York, Aperture.

Baltan, S., S. T. Carmichael, C. Matute, G. Xi and J. H. Zhang, (Eds) (2014). White Matter Injury in Stroke and CNS Disease,. New York, Springer Verlag.

Banham, G., Achulting, D., Hems, N. (2015). The Bloomsbury Companion to Kant. London, Bloomsbury.

Bardin, J. (2012). "Neuroscience: Making connections." Nature 483(7390): 394-396.

Bargh, J. A. and T. L. Chartrand (1999). "The Unbearable Automaticity of Being." American Psychologist 54(7): 462479.

Barthes, R. (1967). The Death of the Author. UbuWeb Papers.

Bartlett, F. C. (1932). Remembering: A study in experimental and social psychology. Cambridge, Cambridge University Press.

Bartol, T. M., C. Bromer, J. Kinney, M. A. Chirillo, J. N. Bourne, K. M. Harris and T. J. Sejnowski (2015). "Nanoconnectomic upper bound on the variability of synaptic plasticity." Elife 4: e10778.

Baumeister, R. F. and J. A. Bargh (2014). Conscious and Unconscious - Toward an Integrative Understanding of Human Mental Life and Action. Dual-Process Theories of the Social Mind, eds (New York, NY: The Guilford Press), 35-49. J. W. Sherman, B. Gawronski and Y. Trope. New York, Guilford Press: 35-49.

Beaver, W. (1981). Financial Reporting - an accounting revolution. Englewood Cliffs, NJ Prentice Hall.

Bengtsson, S., Z. Nagy, S. Skare, L. Forsman, H. Forssberg and F. Ullen (2005). "Extensive piano practicing has regionally specific effects on white matter development." Nat. Neurosci. 8(9): 1148-1150.

Bennett, J. (2010). Vibrant Matter - a political ecology of things. Durham NC, Duke University Press. 
Benzon, W. L. (2011) "Reading Latour: Reassembling the Social." http://dx.doi.org/10.2139/ssrn.1943125

Bergado, J. A., M. Lucas and G. Richter-Levin (2011). "Emotional tagging--a simple hypothesis in a complex reality." Prog Neurobiol 94(1): 64-76.

Betsch, T. (2008). The Nature of Intuition and its Neglect in Research on Judgement and Decision Making. Intuition in Judgement and Decision Making. H. Plessner, C. Betsch and T. Betsch. New York, Lawrence Erlbaum Associates.

Betsch, T. (2014). The Nature of Intuition and its Neglect in Research in Judgement and Decision Making. Intuition in Judgement and Decision Making. H. Plessner, C. Betsch and T. Betsch. New York, Routledge.

Betsch, T. and A. Glöckner (2010). "Intuition in Judgment and Decision Making: Extensive Thinking Without Effort." Psychological Inquiry 21(4): 279-294.

Betsch, T., H. Plessner and E. Schallies (2004). The value-account model of attitude formation. Contemporary perspectives on the psychology of attitudes. M. G and H. G. Hove UK, Psychology Press: 251-273.

Birmingham, E., W. F. Bischof and A. Kingstone (2008). "Gaze selection in complex social scenes." Visual Cognition $16341-355$.

Bloom, B. S. E. (1985). Developing talent in young people. New York, Ballantine Press.

Bolte, A. and T. Goschke (2005). "On the speed of intuition: intuitive judgments of semantic coherence under different response deadlines." Mem Cognit 33(7): 1248-1255.

Brosch, T., K. R. Scherer, D. Grandjean and D. Sander (2013). "The impact of emotion on perception, attention, memory, and decision-making." Swiss Med Wkly 143: w13786.

Brown, D. K. (2001). "The Social Sources of Educational Credentialism: Status Cultures, Labor Markets, and Organizations." Sociology of Education Extra Issue 20: 19-34.

Cahill, L. and J. L. McGaugh (1995). "A Novel Demonstration of Enhanced Memory Associated with Emotional Arousal " CONSCIOUSNESS AND COGNITION 4: 410 - 421.

Callaway, E. and D. Dembo (1958). "Narrowed attention: A psychological phenomenon that accompanies a certain physiological change." Archives of Neurology and Psychiatry, 79.

Campbell, D. and D. Fiske (1959). "Convergent and Discriminant Validation by the multitrait-multimethod matrix." Psychological Bulletin 56: 81-105.

Carifio, J. and R. Perla (2008). "Resolving the 50-year debate around using and misusing Likert scales." Med Educ 42(12): 1150-1152.

Carifio, J. and R. J. Perla (2007). "Ten Common Misunderstandings, Misconceptions, Persistent Myths and Urban Legends about Likert Scales and Likert Response Formats and their Antidotes " Journal of Social Sciences 3(3): 106-116.

Changizi, M. (2010). The Brain from 20000 feet. Dordrecht, The Netherlands, Kluwer Academic Publishers.

Chassy, P. and F. Gobet (2011). "A Hypothesis about the Biological Basis of Expert Intuition." Review of General Psychology 15(3): 198-212.

Chavalarias, D. and J. P. Ioannidis (2010). "Science mapping analysis characterizes 235 biases in biomedical 
research." J Clin Epidemiol 63(11): 1205-1215.

Chiari, G. and L. Nuzzo (1993). Personal Construct Theory Within Psychological Constructivism: Precursor or Avant-Garde? Tenth International Congress of Personal Construct Psychology, James Cook University, Townsville, Australia, .

Chong, I.-G. and C. H. Jun (2005). "Performance of some variable selection methods when multicollinearity is present." Chemometrics and Intelligent Laboratory Systems 78: 103-112.

Citri, A. and R. C. Malenka (2008). "Synaptic plasticity: multiple forms, functions, and mechanisms." Neuropsychopharmacology 33(1): 18-41.

Clark, A. (2013). "Whatever next? Predictive brains, situated agents, and the future of cognitive science." Behav Brain Sci 36(3): 181-204.

Clark, A. (2016). Surfing Uncertainty - prediction, action and the embodied mind. Oxford, Oxford University Press.

Cook, R. L. a. S., T.R (1975). "Organizational Behavior and Human Performance - A comparison of seven methods for obtaining subjective descriptions of judgmental policy." Organizational Behavior and Human Performance 13(1): 31-45.

Coyle, D. (2009). The Talent Code. London, Random House Books.

Creswell, J. W. (2014). A Concise Introduction to Mixed Methods Research. Thousand Oaks, California, Sage.

Damasio, A. (2000). The Feeling Of What Happens: Body, Emotion and the Making of Consciousness. New York, Mariner Books.

Dane, E., K. W. Rockmann and M. G. Pratt (2012). "When should I trust my gut? Linking domain expertise to intuitive decision-making effectiveness." Organizational Behavior and Human Decision Processes 119(2): 187-194.

Darwin, C. R. (1872). The Expressions of the Emotions in Man and Animals 1st Edition. London, John Murray.

Davis-Stober, C. P., D. V. Budescu, J. Dana and S. Broomell (2014). "When is a Crowd Wise?" Decision 1(2): 79101.

De Franco, G. and Y. Zhou (2009). "The Performance of Analysts with a CFA ${ }^{\circledR}$ Designation: The Role of HumanCapital and Signaling Theories." The Accounting Review 84(2): 383 - 404.

deGroot, A. D. (1965). Het Denken van den Schaker. Trans. as Thought and Choice in Chess. The Hague, Mouton \& Company.

Dehaene, S. (2014). Consciousness and the Brain: Deciphering How the Brain Codes Our Thoughts. New York, Penguin, Random House.

Denzin, N. K. (1970). The Research Act: A Theoretical Introduction to Sociological Methods. Chicago, III, Aldine.

Denzin, N. K. (2012). "Triangulation 2.0." Journal of Mixed Methods Research 6(2): 80-88.

Derrida, J. (1967). Of Grammatology. Baltimore, John Jopkins University Press.

Detterman, D. K. (2014). "Introduction to the intelligence special issue on the development of expertise: is ability necessary?" Intelligence 45: 1-5. 
Dhami, M. K. and M. E. Thomson (2012). "On the relevance of Cognitive Continuum Theory and quasirationality for understanding management judgment and decision making." European Management Journal 30(4): 316-326.

Dillon, L. and B. Welsh (2017). Photo Competition Prep: A Fresh Look at the 12 Elements. Professional Photographer. Atlanta, PPA. March.

Doya, K., S. Ishii, A. Pouget and R. P. N. Rao (2006). The Bayesian Brain. Cambridge MA, The MIT Press.

Dresp-Langley, B. (2011). "Why the brain knows more than we do: nonconscious representations and their role in the construction of conscious experience." Brain Sci 2(1): 1-21.

Dunwoody, P. T., E. Haarbauer, R. P. Mahan, C. Marino and C.-C. Tang (2000). "Cognitive adaptation and its consequences." Journal of Behavioural Decision Making 13(1): 35 - 54.

Eagleman, D. (2011). Incognitio - the secret lives of the brain. Edinburgh, Cannongate

Eagleman, D. (2016). The Brain - the story of you Edinburgh, Canongate.

Edwards, W. (1954). "THE THEORY OF DECISION MAKING." PSYCHOLOGICAL BULLETIN 51(4): 380-417.

Epstein, L. G. (1999). "A Definition of Uncertainty Aversion." Review of Economic Studies 66: 579-608.

Epstein, S. (1994). "Integration of the Cognitive and Psychodynamic Unconscious." American Psychologist 49(8): 709-724.

Epstein, S. (2003). Cognitive-experiential self-theory of personality. . Comprehensive Handbook of Psychology. T. Millon and M. J. Lerner. Hoboken, NJ, Wiley \& Sons. Volume 5: Personality and Social Psychology 159-184.

Epstein, S. (2010). "Demystifying Intuition: What It Is, What It Does, and How It Does It." Psychological Inquiry 21(4): 295-312.

Ericsson, K. A. (2014). "Why expert performance is special and cannot be extrapolated from studies of performance in the general population: A response to criticisms." Intelligence 45: 81-103.

Ericsson, K. A., R. Krampe and C. Tesch-Romer (1993). "The Role of Deliberate Practice in the Acquisition of Expert Performance." Psychological Review 100(3): 363-406.

Ericsson, K. A., K. Nandagopal and R. W. Roring (2005). "Giftedness Viewed From the Expert-Performance Perspective." Journal for the Education of the Gifted 28(3/4): 287-311.

Ericsson, K. A., K. Nandagopal and R. W. Roring (2009). "Toward a science of exceptional achievement: attaining superior performance through deliberate practice." Ann N Y Acad Sci 1172: 199-217.

Euler, M. J. (2018). "Intelligence and uncertainty: Implications of hierarchical predictive processing for the neuroscience of cognitive ability." Neurosci Biobehav Rev 94: 93-112.

Evans, J., J. Clibbens, A. Cattani, A. Harris and I. Dennis (2003). "Explicit and implicit processes in multicue judgment." Memory \& Cognition 2003 31(4): 608-618.

Evans, J. S. and K. E. Stanovich (2013). "Dual-Process Theories of Higher Cognition: Advancing the Debate." Perspect Psychol Sci 8(3): 223-241.

Farmer, T., K. Robinson, S. J. Elliott and J. Eyles (2006). "Developing and implementing a triangulation protocol for 
qualitative health research." Qual Health Res 16(3): 377-394.

Fechner, G. T. (1860). Elements of psychophysics. New York, Holt, Rinehart \& Winston.

Festinger, L. (1957). A Theory of cognitive dissonance. Stanford, CA, Stanford University Press.

Fetters, M. D. and J. F. Molina-Azorin (2017). "The Journal of Mixed Methods Research Starts a New Decade." Journal of Mixed Methods Research 11(1): 3-10.

Feyerabend, P. (2010). Against Method - 4th edition. London, Verso.

Flusser, V. (1983). Towards a Philosophy of Photography. London, Reaktion Books.

Frankish, K. and J. Evans (2009). The duality of mind: an historical perspective. In two minds: Dual processes and beyond. J. Evans and K. Frankish. Oxford, Oxfoard University Press.

Frankland, P. W. and B. Bontempi (2005). "The organization of recent and remote memories." Nat Rev Neurosci 6(2): 119-130.

Freud, S. (1900). The interpretation of dreams. London, Penguin.

Friston, K. (2009). "The free-energy principle: a rough guide to the brain?" Trends Cogn Sci 13(7): 293-301.

Frith, C. (2007). Making 'up the Mind - how the brain creates our mental world. Oxford, Blackwell Publishing.

Gadamer, H.-G. (2004). Truth and Method 2nd revised edition. London, Continuum.

Gagne, R. M. and L. J. Briggs (1974). Principles of instructional design. Oxford, Holt, Rinehart \& Winston.

Galton, F. (1907). "Vox populi." Nature 75: 450-451.

Geiselman, R. E. and R. P. Fisher (2014). Interviewing Witnesses and Victims. Investigative Interviewing: Handbook of Best Practices. . M. St Yves. Toronto, Thomson Reuters Publishers.

Geiselman, R. E., R. P. Fisher, D. P. MacKinnon and H. L. Holland (1985). "Eyewitness Memory Enhancement in the Police Interview: Cognitive Retrieval Mnemonics Versus Hypnosis." Journal of Applied Psychology 70(2): 401-412.

Gigerenzer, G. and H. Brighton (2009). "Homo heuristicus: why biased minds make better inferences." Top Cogn Sci 1(1): 107-143.

Gigerenzer, G. and P. M. Todd (2000). Simple Heuristics That Make Us Smart (Evolution and Cognition). Oxford, Oxford University Press.

Gill, C. J., L. Sabin and C. H. Schmid (2005). "Why clinicians are natural bayesians." British Medical Journal $330:$ 1080-1083.

Gladwell, M. (2008). Outliers: the story of success. London, Penguin.

Glöckner, A. and C. Witteman (2010). "Beyond dual-process models: A categorisation of processes underlying intuitive judgement and decision making." Thinking \& Reasoning 16(1): 1-25.

Gobet, F. (2005). "Chunking models of expertise: implications for education." Applied Cognitive Psychology 19(2): 183-204. 
Gobet, F. (2013). Chunks and templates in semantic long term memory - the importance of specialisation. Expertise and skill acquisition - the impact of William G Chase. J. J. Staszewskil. New York, Psychology Press.

Gobet, F. (2016). Understanding Expertise - a multidisciplinary approach. London, Palgrave.

Gobet, F., P. C. Lane and M. Lloyd-Kelly (2015). "Chunks, Schemata, and Retrieval Structures: Past and Current Computational Models." Front Psychol 6: 1785.

Grossberg, S. and B. Pinna (2012). "Neural Dynamics of Gestalt Principles of Perceptual Organization: From Grouping to Shape and Meaning." Getsalt Theory 34(3/4): 399-482.

Guo, K., S. Mahmoodi, R. G. Robertson and M. P. Young (2006). "Longer fixation duration while viewing face images." Experimental Brain Research 171: 91-98.

Hagen, M. (2015). The Nikon Autofocus System - Mastering Focus for Sharp Images Every Time. San Rafael, CA, Rocky Nook.

Hammond, K. R. (1996). Human Judgment and Social Policy: Irreducible Uncertainty, Inevitable Error, Unavoidable Injustice. New York, Oxford University Press.

Harman, G. (2009). "Dwelling With the Fourfold." Space and Culture 12(3): 292-302.

Harman, G. (2011). The Quadruple Object. Alresford, UK, Zero Books.

Harman, G. (2018). Object-Oriented Ontology - a new theory of everything. London, Penguin, Random House.

Harrington, A. (2006). "Lifeworld." Theory, Culture \& Society 23(2-3) 23(2): 342-343.

Harte, J. M. and P. Koele (1995). "A comparison of different methods for the elicitation of attribute weights: Structural Modeling, process tracing, and self-reports." Organizational Behavior and Human Decision Processes 64: 49-64.

Hawkins, B. (2013). "Elements of a Merit Image." from Professional Photographers Association website www.PPA.com https://www.ppa.com/events/photo-competitions/ipc-international-photographiccompetition-overview/the-12-elements-of-a-merit-image

Hayles, N. K. (2017). Unthought: the power of the cognitve nonconscious. Chicago, Illinois, University of Chicago Press.

Hebb, D. O. (1949). The Organization of Behavior: A Neuropsychological Theory. New York, Wiley and Sons.

Heidegger, M. (1953). Being and Time. New York, State University of New York Press.

Heidegger, M. (1962). Being and Time. New York, Harper Row.

Henseler, J., C. M. Ringle and R. R. Sinkovics (2009). "THE USE OF PARTIAL LEAST SQUARES PATH MODELING IN INTERNATIONAL MARKETING." Advances in International Marketing 20 277-319.

Hermans, E. J., F. P. Battaglia, P. Atsak, L. D. de Voogd, G. Fernandez and B. Roozendaal (2014). "How the amygdala affects emotional memory by altering brain network properties." Neurobiol Learn Mem 112: 2-16.

Hoffrage, U. and G. Gigerenzer (1998). "Using Natural Frequencies to Improve Diagnostic Inferences." $\underline{\text { ACADEMIC }}$ MEDICINE 73(5): 538-540. 
Hoffrage, U., S. Krauss, L. Martignon and G. Gigerenzer (2015). "Natural frequencies improve Bayesian reasoning in simple and complex inference tasks." Front Psychol 6: 1473.

Hohwy, j. (2013). The Predictive Mind. Oxford, Oxford University Press.

Hohwy, J., A. Roepstorff and K. Friston (2008). "Predictive coding explains binocular rivalry: an epistemological review." Cognition 108(3): 687-701.

Holton, G. A. (2004). "Defining Risk." Financial Analysts Journal 60(6).

Hooker, C. A., Ed. (2011). Philosophy of Complex Systems (Handbook of the Philosophy of Science). Oxford, North Holland.

Horr, N. K., C. Braun and K. G. Volz (2014). "Feeling before knowing why: the role of the orbitofrontal cortex in intuitive judgments--an MEG study." Cogn Affect Behav Neurosci 14(4): 1271-1285.

Horr, N. K., C. Braun, T. Zander and K. G. Volz (2015). "Timing matters! The neural signature of intuitive judgments differs according to the way information is presented." Conscious Cognition 38: 71-87.

Hosoya, T., S. A. Baccus and M. Meister (2005). "Dynamic predictive coding by the retina." Nature 436(7047): 7177.

Hunter, J. L. (1999). Kant's Doctrine of Schemata. Master of Arts, Virginia Polytechnic Institute and State University.

Interlandi, J. (2016). New Estimate Boosts the Human Brain's Memory Capacity 10-Fold. Scientific American, Nature America Inc.

loannidis, J. P. (2005). "Why most published research findings are false." PLoS Med 2(8): e124.

Itti, L. and P. Baldi (2009). "Bayesian surprise attracts human attention." Vision Res 49(10): 1295-1306.

Jacobson, M. and M. Ruddy (2015). Open to Outcome: A Practical Guide for Facilitating and Teaching Experiential Reflection 2nd Ed. Bethany OK, Wood N Barnes.

Jhally, S. (1990). The Codes of Advertising - Fetishism and the Political Economy of Meaning in the Consumer Society. London, Psychology Press.

Kahn, A. E., M. G. Mattar, J. M. Vettel, N. F. Wymbs, S. T. Grafton and D. S. Bassett (2017). "Structural Pathways Supporting Swift Acquisition of New Visuomotor Skills." Cereb Cortex 27(1): 173-184.

Kahneman, D. and G. Klein (2009). "Conditions for intuitive expertise: A failure to disagree." American Psychologist 64(6): 515-526.

Kant, I. (1992). The Cambridge Edition of the Works of Immanuel Kant. Cambridge UK, Cambridge University Press.

Keeney, R. L. and H. Raiffa (1976). Decisions with multiple objectives. New York, Wiley.

Kelley, T. L. (1927). Interpretation of educational measurements New York, World Books.

Kelly, G. A. (1955). The psychology of personal constructs (vols. 1 and 2). New York, Norton.

Kensinger, E. A., R. J. Garoff-Eaton and D. L. Schacter (2007). "How negative emotion enhances the visual specificity of a memory." J Cogn Neurosci 19(11): 1872-1887. 
Keysers, C. and V. Gazzola (2014). "Hebbian learning and predictive mirror neurons for actions, sensations and emotions." Philos Trans R Soc Lond B Biol Sci 369(1644): 20130175.

Khaneman, D. (2011). Thinking Fast and Slow. London, Penguin.

Kitamura, T., S. K. Ogawa, D. S. Roy, T. Okuyama, M. D. Morrissey, L. M. Smith, R. L. Redondo and S. Tonegawa (2017). "Engrams and circuits crucial for systems consolidation of a memory." Science 356: 73-78.

Klein, G. (2015). "A naturalistic decision making perspective on studying intuitive decision making." Journal of Applied Research in Memory and Cognition 4(3): 164-168.

Knight, F. H. (1921). Risk, Uncertainty, and Profit. Boston, Houghton Mifflin.

Koestler, A. (1964). The Act of Creation. London, Hutchinson.

Kolb, A. Y. and D. A. Kolb (2009). Experiential Learning Theory: A Dynamic, Holistic Approach to Management Learning, Education and Development Handbook of Management Learning, Education and Development. S. J. Armstrong and C. Fukami. London, Sage.

Kouider, S. and S. Dehaene (2007). "Levels of processing during nonconscious perception: a critical review of visual masking." Philos Trans R Soc Lond B Biol Sci 362(1481): 857-875.

Kurson, R. (2007). Crashing Through: a true story of risk, adventure and the man who dared to see. New York, Random House.

Kuyken, W., R. Hayes, B. Barrett, R. Byng, T. Dalgleish, D. Kessler, G. Lewis, E. Watkins, C. Brejcha, J. Cardy, A. Causley, S. Cowderoy, A. Evans, F. Gradinger, S. Kaur, P. Lanham, N. Morant, J. Richards, P. Shah, H. Sutton, R. Vicary, A. Weaver, J. Wilks, M. Williams, R. S. Taylor and S. Byford (2015). "Effectiveness and costeffectiveness of mindfulness-based cognitive therapy compared with maintenance antidepressant treatment in the prevention of depressive relapse or recurrence (PREVENT): a randomised controlled trial." The Lancet 386(9988): 63-73.

LaBar, K. S. and R. Cabeza (2006). "Cognitive neuroscience of emotional memory." Nat Rev Neurosci 7(1): 54-64.

Lachman, R., J. Lachman and E. Butterfield (1979). Cognitive Psychology and Information Processing, Lawrence Erlbaum Associates. New York, Lawrence Erlbaum Associates.

LaLumiere, R. T., J. L. McGaugh, C. K. McIntyre and R. Dantzer (2017). "Emotional Modulation of Learning and Memory: Pharmacological Implications." Pharmacological Reviews 69(3): 236-255.

Langlois, R. N. and M. N. Cosgel (1993). "Frank Knight on risk, uncertainty, and the firm: A new interpretation." Economic Inquiry XXXI(July): 456-465.

Latour, B. (2005). Reassembling the Social - an introduction to actor network theory. New York, Oxford University Press.

Lewicki, P., T. Hill and M. Czyzewska (1992). "<Lewicki et al (1992).pdf>."

Lewicki, P., T. Hill and M. Czyzewska (1992). "Nonconscious Acquisition of Information." American Psychologist 47(6): 796-801.

Lewin, K. (1939). "Field Theory and Experiment in Social Psychology." American Journal of Sociology 44(6): 868896. 
Lindemann, O. and M. H. Fischer (2016). Cognitive Foundations of Human Number Representation and Mental Arithmatic. The Oxford Handbook of Numerical Cognition. R. Kadosh and A. Dowker. Oxford, Oxford University Press.

Macnamara, B. N., D. Z. Hambrick and D. Moreau (2016). "How Important Is Deliberate Practice? Reply to Ericsson (2016)." Perspect Psychol Sci 11(3): 355-358.

Markus, H. (1977). "Self-Schemata and Processing Information About the Self." Journal of Personality and Social Psychology 35(2): 63-78.

Maslow, A. H. (1943). "A Theory of Human Motivation." Psychological Review 50(4): 370-396.

Mastrandrea, M. D., C. B. Field, T. F. Stocker, O. Edenhofer, K. L. Ebi, D. J. Frame, H. Held, E. Kriegler, K. Mach, P.

Matschoss, G.-K. Plattner, G. W. Yohe and F. W. Zwiers (2010). Guidance note for lead authors of the IPCC Fifth Assessment Report on consistent treatment of uncertainties., Intergovernmental Panel on Climate Change (IPCC).

Mastrandrea, M. D., K. Mach, J, G. Plattner, O. Edenhofer, T. F. Stocker, C. B. Field, K. L. Ebi and P. R. Matschoss (2011). "The IPCC AR5 guidance note on consistent treatment of uncertainties: a common approach across the working groups." Climatic Change 108(4): 675-691.

Mather, M. and M. R. Sutherland (2011). "Arousal-Biased Competition in Perception and Memory." Perspect Psychol Sci 6(2): 114-133.

Matsumoto, D., H. C. Hwang and V. Sandoval (2015). "The Funnel Approach To Questioning And Eliciting Information." Tactics \& Preparedness

McCullin, D. (1992). Unreasonable Behaviour. London, Vintage Books.

McGaugh, J. L. (2006). "Make mild moments memorable: add a little arousal." Trends Cogn Sci 10(8): 345-347.

McGaugh, J. L. (2018). "Emotional arousal regulation of memory consolidation." Current Opinion in Behavioral Sciences 19: 55-60.

McKenzie, I. A., D. Ohayon, H. Li, J. P. de Faria, B. Emery, K. Tohyama and W. D. Richardson (2014). "Motor skill learning requires active central myelination." Science 346(6207): 318-322.

McLeod, S. A. (2014). "Sampling methods. ." from https://www.simplypsychology.org/sampling.html.

Mega, L. F., G. Gigerenzer and K. G. Volz (2015). "Do intuitive and deliberate judgments rely on two distinct neural systems? A case study in face processing." Front Hum Neurosci 9: 456.

Meillassoux, Q. (2008). After Finitude - an essay on the necessity of contingency. London, Bloomsbury.

Meillassoux, Q. (2014). Time without Becoming, Mimesis International.

Miller, G. A. (1956). "The Magical Number Seven, Plus or Minus Two: Some Limits on our Capacity for Processing Information." Psychological Review 63: 81-97.

Morelle, L. (2012). "Speculative realism : After finitude, and Beyond ?" Speculations.

Morgan, D. L. (2019). "Commentary-After Triangulation, What Next?" Journal of Mixed Methods Research 13(1): 6-14. 
Morley, S. (2010). The Sublime. London, Whitechappel Gallery.

Morton, T. (2015). "Charisma and Causality." Art Review Retrieved 22 April 2019, 2019, from https://artreview.com/features/november_2015_feature_timothy_morton_charisma_causality/.

Muller, G. E. and A. Pilzecker (1900). "Experimentalle beitrage zur lehre vom geda." chtnis. Z. Psychol 1(1-288).

Nadel, L. and M. Moscovitch (1997). "Memory consolidation, retrograde amnesia and the hippocampal complex." Current Opinion in Neurobiology 7: 217 - 227.

Nemire, K. and M. M. Cohen (1993). "Visual and somesthetic influences on postural orientation in the median plane." Perception \& Psychophysics 53 (1): 106-116.

Nietzsche, F. (1874). On the Uses and Disadvantages of History for Life, Cambridge University Press.

Nijhawan, R. and B. E. Khurana (2010). Space and Time in Perception and Action, Cambridge, UK, Cambridge University Press.

Nisbett, T., D and R. E. Wilson (1977). "Telling More Than We Can Know: Verbal Reports on Mental Processes " Psychological Review 84(3): 231 - 259.

NMRA. (2017). "National Photo Contest Judging Guidelines." from https://www.nmra.org/national-photocontest-judging-guidelines.

NTSB (2010). Loss of Thrust in Both Engines After Encountering a Flock of Birds and Subsequent Ditching on the Hudson River US Airways Flight 1549. N. T. S. Board.

Oakley, D. A. and P. W. Halligan (2017). "Chasing the Rainbow: The Nonconscious Nature of Being." Front Psychol 8: 1924

Ochsner, K. N. (2000). "Are affective events richly recollected or simply familiar? The experience and process of recognizing feelings past." J Exp Psychol Gen 129(2): 242-261.

Oppenheim, A. N. (1992). Questionnaire Design, Interviewing and Attitude Measurement 2nd Ed. New York, Continuum International Publishing.

Oppy, G. (2019). Ontological Arguments. The Stanford Encyclopedia of Philosophy (Spring 2019 Edition). E. N. Zalta.

Ortega y Gasset, J. (1968). The Dehumanization of Art and Ideas about the Novel (1925). New Jersey, Princeton University Press.

Pannucci, C. J. and E. G. Wilkins (2010). "Identifying and avoiding bias in research." Plast Reconstr Surg 126(2): 619-625.

Parkhurst, D., K. Law and E. Niebur (2002). "Modeling the role of salience in the allocation of overt visual attention." Vision Research 42: 107-123.

Patterson, F. (1977). Photography for the Joy of It Toronto, Van Nostrand Reinhold Ltd.

Pendlebury, M. (1955). "Making Sense of Kant's Schematism." Philosophy and Phenomenological Research 55(4): 777-797.

Penny, W. (2012). "Bayesian Models of Brain and Behaviour." ISRN Biomathematics 2012: 1-19. 
Phelps, E. A. and T. Sharot (2008). "How (and Why) Emotion Enhances the Subjective Sense of Recollection." Current Directions in Psychological Science 17 (2): 147-152.

Phillips, D. L. and D. vonWinterfeldt (2006). Reflections on the Contributions of Ward Edwards to Decision Analysis and Behavioural Research, London School of Economics.

Porta, J. B. (1593). De Refractione. Optices Parte Libri Novem. Naples Carlinum and Pacem.

Privitera, C. M. and L. W. Stark (2000). "Algorithms for defining visual regions-of-interest: Comparison with eye fixations. ." IEEE Transactions on Pattern Analysis and Machine Intelligence 22: 970-982.

Proverbio, A. M. (2017). "Sex differences in social cognition: The case of face processing." J Neurosci Res 95(1-2): 222-234.

Reinagel, P. and A. M. Zador (1999). "Natural scene statistics at the centre of gaze." Network 10(4): 341-350.

Reyna, C. and M. V. Ortiz (2016). "Psychometric study of the Rational Experiential Inventory among undergraduate Argentinean students." Revista de Psicología 34(2): 337-355.

Reynolds, R. (2013). Personal Construct Theory. Theory in Information Behaviour Research T. Wilson, Eiconics Ltd: 75-96.

Richter-Levin, G. and I. Akirav (2003). "Emotional tagging of memory formation-in the search for neural mechanisms." Brain Research Reviews 43(3): 247-256.

Rimmele, U., L. Davachi, R. Petrov, S. Dougal and E. A. Phelps (2011). "Emotion enhances the subjective feeling of remembering, despite lower accuracy for contextual details." Emotion 11(3): 553-562.

Russell, J. A. (1980). "A circumplex model of affect." Journal of Personality and Social Psychology 39(6): 11611178.

Ryan, R. J. (2017). The Master Photographer - the journey fro good to great. Cirencester UK, Bourchier

Ryan, R. J., R. W. Scapens and M. Theobald (2002). Research Methods and Methodology in Finance and Accounting 2nd Ed, . Andover, Hants, Cengage.

Saj, A., J. Honore, J. Davroux, Y. Coello and M. Rousseaux (2005). "Effect of posture on the perception of verticality in neglect patients." Stroke 36(10): 2203-2205.

Salem, E. (2017). Object and Oú бía: Harman and Aristotle on the Being of Things. Contemporary Encounters with Ancient Metaphysics. A. J. Greenstine and R. J. Johnson. Edinburgh, Edinburgh University Press.

Salimpoor, V. N., M. Benovoy, G. Longo, J. R. Cooperstock and R. J. Zatorre (2009). "The rewarding aspects of music listening are related to degree of emotional arousal." PLoS One 4(10): e7487.

Sampaio-Baptista, C., J. Scholz, M. Jenkinson, A. G. Thomas, N. Filippini, G. Smit, G. Douaud and H. Johansen-Berg (2014). "Gray matter volume is associated with rate of subsequent skill learning after a long term training intervention." Neuroimage 96(100): 158-166.

Saunders, P. (2010). An Introduction of Catastrophe Theory. Cambridge, Cambridge University Press.

Saxe, G. B. (2016). Culture Language and Number. Oxford Handbook of Numerical Cognition. R. Kadosh and A. Dowker. Oxford, Oxford University Press. 
Scapens, R. W., R. J. Ryan and L. Fletcher (1981). "Explaining corporate failure - a catastrophe theory approach." Journal of Business Finance and Accounting 8(1 - 26).

Schmidt, J. C. (2011). Challenged by Instability and Complexity. Philosophy of Complex Systems (Handbook of the Philosophy of Science). C. A. Hooker. Oxford, North Holland: 242.

Schulz, K. and G. U. Hayn-Leichsenring (2017). "Face Attractiveness versus Artistic Beauty in Art Portraits: A Behavioral Study." Frontiers of Psychology 8: 2254.

Sekeres, M. J., K. Bonasia, M. St-Laurent, S. Pishdadian, G. Winocur, C. Grady and M. Moscovitch (2016). "Recovering and preventing loss of detailed memory: differential rates of forgetting for detail types in episodic memory." Learn Mem 23(2): 72-82.

Shannon, C. E. and W. Weaver (1964). The Mathematical Theory of Communication Urbana, The University of Illinois Press.

Shaqiri, A., M. Roinishvili, L. Grzeczkowski, E. Chkonia, K. Pilz, C. Mohr, A. Brand, M. Kunchulia and M. H. Herzog (2018). "Sex-related differences in vision are heterogeneous." Sci Rep 8(1): 7521.

Sieberling, G. and C. Bloore (1986). Amateurs, Photography and the Mid-Victorian Imagination. London, University of Chicago Press.

Silverman, K. (2015). The Miracle of Analogy ot the History of Photography, Part 1. Stanford, California, Stanford University Press.

Simon, H. A. and W. G. Chase (1973). "Skill in Chess." Scientific American 61(4): 394-403.

Smith, J. A., P. Flowers and M. Larkin (2009). Interpretive Phenomenological Analysis - theory, method and research. London, Sage.

Stanovich, K. E. (1999). Whi is Rational - studies of individual differences in reasoning. New Jersey, Lawrence Erlbaum Associates.

Surowiecki, S. (2005). The Wisdom of the Crowds: why many are smarter than the few. London, Abacus.

Syverson, M. A. (1999). The Wealth of Reality - the ecology of composition. Carbondale, III, Southern Illinois University Press.

Szarkowski, J. (1984). Mirrors and Windows: American Photography since 1960. . New York, The Museum of Modern Art.

Tambini, A., U. Rimmele, E. A. Phelps and L. Davachi (2017). "Emotional brain states carry over and enhance future memory formation." Nat Neurosci 20(2): 271-278.

Ten Hoor, M. (1932). "A Critical Analysis of the Concept of Introspection." The Journal of Philosophy 29(12): 322 $-331$.

Theeuwes, J., A. F. Kramer, S. Hahn and D. E. Irwin (1998). "Our eyes do not always go where we want them to go: Capture of the eyes by new objects." Psychological Science 9(5): 379-385.

Thompson, G. (2018). Aesthetic Experience and Mindfulness. Compassion, Connection, and Response-Ability. Annual Human Science Institute Conferenc. CeAt: Salt Lake City, Utah.

Tikhomirov, O. K. and E. D. Poznyanskaya (1966). "An Investigation of Visual Search as a Means of Analyzing 
Heuristics." Soviet Psychology 5(2): 3-15.

Tversky, A. and D. Kahneman (1974). Judgment under uncertainty: Heuristics and biases. Judgment under uncertainty: Heuristics and biases. D. Kahneman, P. Slovic and A. Tversky. Cambridge, Cambridge University Press.

Usher, M., Z. Russo, M. Weyers, R. Brauner and D. Zakay (2011). "The Impact of the Mode of Thought in Complex Decisions: Intuitive Decisions are Better." Front Psychol 2: 37.

Valumian, A. and M. Samoilova (2014). White Matter: Basic Principles of Axonal Organization and Function. White Matter Injury in Stroke and CNS Disease. S. Baltan, S. T. Carmichael, C. Matute, G. Xi and J. H. Zhang. New York, Springer-Verlag.

Van Hedger, S. C., S. L. Heald, R. Koch and H. C. Nusbaum (2015). "Auditory working memory predicts individual differences in absolute pitch learning." Cognition 140: 95-110.

Ventura, R. (2016). "Landing on Water: The Power of Human Judgement." ConsumerGateway.org.

Wagoner, B. (2013). "Bartlett's concept of schema in reconstruction. ." Theory \& Psychology 23(5): 553-575.

Wagoner, B. (2017). Frederic Bartlett. The Routledge Handbook of Philosophy of Memory Edited by S. Bernecker and K. Michaelian, Routledge.

Wagoner, B. (2017). "What makes memory constructive? A study in the serial reproduction of Bartlett's experiments." Culture \& Psychology 23(2): 186-207.

Wallis, K. F. (2014). "Revisiting Francis Galton's Forecasting Competition." Statistical Science 29(3): 420-424.

Webb, E., D. Campbell, R. Schwartz and L. Sechrest (1966). Unobtrusive measures. NY, Guildford, New York Publishing.

Weber, M. (1951). The Religion of China. (Original work published 1916). New York, Free Press.

Weinstein, J. B. and I. Dewsbury (2007). "Comment on the meaning of 'proof beyond a reasonable doubt'." Law, Probability and Risk 5(2): 167-173.

Weston, C. (2008). Mastering Digital Exposure and HDR Imaging: Understanding the Next Generation of Digital Cameras. Brighton, UK, Rotovision.

Wimsatt Jr, W. K. and M. C. Beardsley (1946). "The Intentional Fallacy." The Sewanee Review 54(3): 468-488.

Zeeman, E. C. (1977). Catastrophe theory: Selected papers, 1972-1977. London, AddisonWesley. 
Intuition, expertise and judgement in the capture and assessment of photographic images.

Doctor of Philosophy

Robert James Ryan

APPENDICES

August 2019 


\section{Table of Contents}

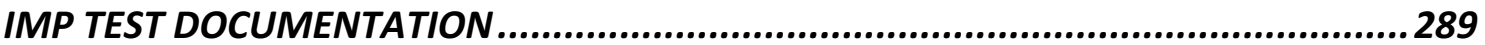

IMP Test - specimen report..................................................................................295

IMP Test - instructional matter within the test .....................................................309

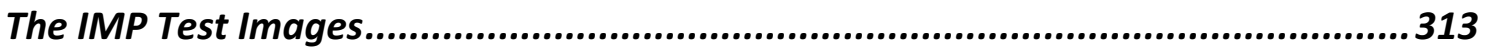

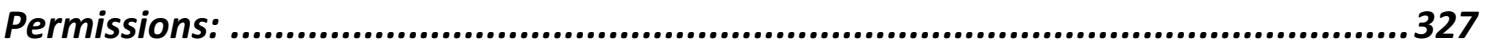

IT0069 Chris Weston Chapter 8 ......................................................................................327

IT0079 Neil McCoubrey Chapter 6 ................................................................................328

IT0084 Simon Stafford Chapter 6.............................................................................328

IT0106 Chapter 6 Simon Weir.......................................................................................329

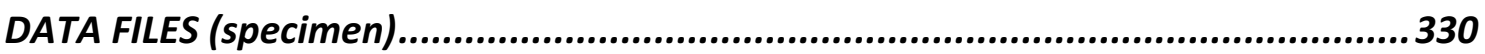

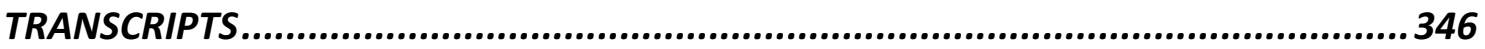

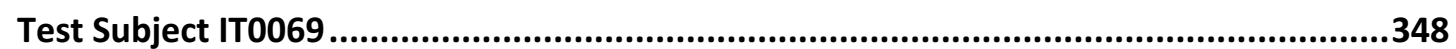

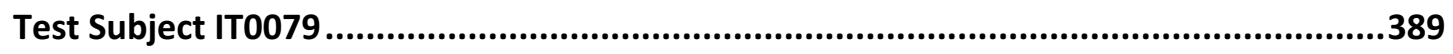

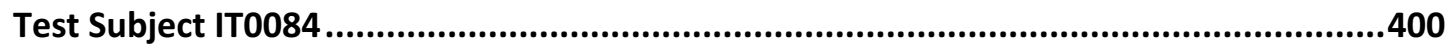

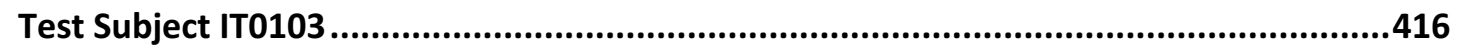

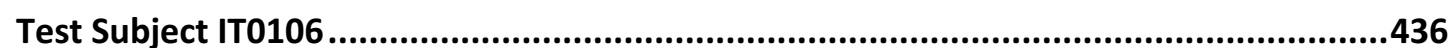

Technical note on Catastrophe Theory explanation of state changes ....................467 


\section{IMP TEST DOCUMENTATION}

Enc:

(i) Invitation to the IMP Test

(ii) IMP Test plus Experiential Interview - briefing note

(iii) IMP Test - FAQ

The Test can be downloaded from

https://bourchierbooks.com/imp-test/ 


\title{
Invitation
}

\author{
The IMP Test
}

https://bourchierbooks.com/imp-test/

Either return to me at professorbobryan@btinternet.com

Or via the publisher.

Extensive report on deficit skills

Recommendation on practice programme

Detailed report on where you are in your photographic journey. 


\section{IMP Test plus Experiential Interview - briefing note}

Thank you for volunteering to take the IMP Test and the experiential interview.

The IMP Test generates a number of outputs: it is designed to give a measure of your level of consciously accessible photographic skill and it also tells you the elements of photographic skill that are intuitively accessible to you. It points to those areas of photography where you should focus your efforts in both broadening and deepening your skill. It also helps you harmonise your intuitive and your conscious thought processes when you are both taking and assessing images.

The IMP Test has three phases: first, you will be asked to make a judgement about your perception of the quality of twenty images where you have just three seconds to form your opinion. Second, you will be asked to rank in importance to you ten areas of photographic skill and knowledge (the ten 'constructs') and third, you will be then asked to assess the twenty images again, taking as long as you like, against the same ten constructs. We will then ask you to complete a simple questionnaire which helps identify your preferred thinking processes.

The interview stage gives you an opportunity to explore the depth of your photographic expertise and develop strategies for personal development. In most cases it corroborates what the IMP Test has revealed but it can also identify more subtle issues for you to reflect upon. The way the interview proceeds is as follows:

1. Before the meeting please choose three images you have taken and processed during the preceding six months. These do not necessarily have to be what you believe to be your best images but rather ones that have high emotional salience for you - they do not need to form a triptych and can be in any genre you wish. You should be able to recall the moment of capture clearly.

2. You can present the images you choose either digitally or in print.

3. I will explain to you how we process the recording of your interview.

4. I will then invite you to talk about the events leading up to the process of capture of the images. The discussion about the images should last no more than one hour.

5. I will finish by giving you the main points from the IMP Test.

After the interview I will follow up our meeting with a more detailed assessment from both the test and the interview. 
Here are some points you need to know:

1. Both the IMP Test and the interview are free of charge.

2. Both the Test and the interview are part of a programme of research at the University of Gloucestershire.

3. Both the IMP Test the interview are confidential - your name and contact details will be kept separate from the data and information you provide.

4. Your name and contact details will not be communicated or sold to any another party.

5. Your IMP Test results and your interview form the basis for a programme of research which should be complete in 2019. Your name and contact details will be deleted once the research is complete.

6. Your interview will be recorded and transcribed for subsequent analysis and a combined report will be provided to you.

7. We will comply with any request from you to withdraw your IMP Test results and interview recording from the research.

Bob Ryan

1 May 2018 


\section{IMP Test}

\section{Frequently Asked Questions}

1. What does IMP stand for?

a. IMP is short for Intuitive Mastery of Photography

2. What does the IMP test measure?

a. It measures the speed and quality of your image taking and analysis by comparing your snap judgement of an image with your more thoughtful analysis of that image against the ten key photographic constructs.

3. How long does it take?

a. If you work through the test without a break about 40 minutes.

4. How is the test conducted?

a. There are three phases to the test: (i) you make an instant assessment of 20 images of different types and genres, (ii) you evaluate those images against the 10 key judgement constructs described in our book and (iii) you tell us how you rank those constructs in importance in your image taking or assessment.

5. What will the test tell me I don't know already?

a. It will tell you whether you can rely upon your snap judgement to capture a high quality image or to judge an image. It will also tell you those elements of photographic expertise you need to develop to bring your skills to the highest possible level.

6. How reliable is the test?

a. If you take the test conscientiously it will give you good information about where your photographic skills are currently.

7. Can I have the results straightaway?

a. When you submit your test results to us we aim to get the results back to you within 48 hours.

8. I have just started in photography. Is the test of any value to me?

a. Whatever your stage of development the IMP test will reveal those skills or areas of photographic knowledge that you cannot call upon instantly. We can then guide you on the best learning strategies for absorbing those skills at a non-conscious level.

9. Are the results confidential?

a. Yes, you will be given an anonymity number and your results will be stored against that number. Only, the lead researcher will have access to a separate, secure file linking your anonymity number to your personal details.

10. Can I take the test a second time?

a. Yes, we recommend a six month gap between testing at which time your hard work following the learning strategy in our book 'The Master Photographer - the journey from good to great' will be showing significant improvements in your non-conscious skill as a photographer. 
IMP Test-specimen report

Intuitive Mastery of Photography (IMP)

Test

RESULTS

(specimen)

Client: XXXXX

Date submitted: 11 May 2019

Date of Analysis: 18 May 2019 


\title{
THE INTUITIVE MASTERY OF PHOTOGRAPHY
}

\author{
(IMP) TEST
}

Thank you for taking the Intuitive Mastery of Photography (IMP) Test. The test is designed to reveal how your non-conscious mind operates both at the moment of capture of an image or when appraising and working on it subsequently. The test results are confidential to you.

The test will reveal the following to you:

1. The degree to which your intuitive judgment is aligned with your more deliberative appraisal of an image.

2. The extent to which your non-conscious mind is utilising the full range of photographic knowledge and skills at the moment of capture.

3. The areas where you could most profitably focus your skill development.

4. The areas of photography where your thinking style could be deployed to greatest effect.

\section{Disclaimer:}

1. Unless you specifically agree otherwise, your response to the IMP test and the REI and my analysis of your results is confidential between us.

2. My analysis of your results will be done impartially and fairly on the basis of the scores you give me. However, I cannot accept any liability resulting from the conduct of the test, downloading of files to your computer, the provision of the results or their interpretation.

3. In taking the test you agree that I may use your responses along with others for the purpose of research into the development of photographic skill and judgment. All data will be collected and analysed but individual test respondents will not be identifiable from the results.

4. If at any time you wish to have your submission removed from the study please contact me directly and I will make sure that all your data is removed from our data bases. 
5. You acknowledge that I am the sole copyright owner of the IMP test, its associated software and documentation. All rights to the IMP test, its associated software and documentation are reserved. 


\section{IMP TEST REPORT}

Well done XXX, you have answered 220 questions about the images, and your IMP Test results do give me an insight into what is going on 'in the moment' when you put your eye to your camera or look at an image for the first time.

The IMP Test has told me quite a bit about you. I have appended detailed data sheets from the analysis at the end of the report. What follows is some detailed results and my analytical summary which I hope will be useful to you.

Your results are indicative of what is driving your intuitive judgement. The test tells me which of the ten constructs are influencing your intuitive judgement and which are not. It also informs me about the depth of your knowledge of each construct as well as telling me those that are accessible to your intuitive judgement and those that are not and are a priority for development.

\section{REI Result and favoured genres}

(RA-rational aptitude, RE-rational engagement, EA-experiential (intuition) aptitude, experiential engagement).

\begin{tabular}{|c|c|c|c|c|}
\hline RA & $\mathrm{RE}$ & EA & $\mathrm{EE}$ & Comment \\
\hline 4.6 & 4.1 & 3.9 & 3.6 & $\begin{array}{l}\text { Your answers to the REI suggest that you have } \\
\text { both an aptitude and a greater willingness to } \\
\text { engage with the rational as opposed to the } \\
\text { intuitive modality of thought. Your IMP Test } \\
\text { results suggest that when the model derived } \\
\text { weightings are used in ranking the images that }\end{array}$ \\
\hline
\end{tabular}


you are closer in your assessment to the average than when you used your declared ranking preference. The same result applies when compared with your peer cluster by qualification and attainment. This indicates that the balance in your thinking (at least in terms of aptitude) is closer than you think and report.

\section{Alignment of your intuitive and rational judgement of the images}

\section{(0\% - random guessing, 100\% perfect alignment)}

\section{R2 Comment}

83.8\% All the quality stats from your analysis are high which gives me strong confidence in the results. Running a validation check using PCR gives an almost identical R2(bar) and the Cronbach Alpha is 0.923 . Confidence in judgement is taken as a primary measure of conscious skill level and is measured using normalised volatility compared with the mean of all those tested. Your results show a high level of skill across all ten constructs at $2.28 x$ the level for the whole group. You have strong conscious facility in reading colour/tonality, use of light and composition as well as the three basic constructs of exposure, focus and technical. You're slightly down on your own standard in reading narrative power and the appropriate use of depth of field. Taken overall your conventionally acquired skills are of a very high order.

Ranking of constructs (your declared ranking versus your intuitive ranking from the model) ( $0 \%$ - no relationship, $100 \%$ - perfect agreement) 
The correlation between what the model derives as your ranking in non-

$(-38.85 \%)$ conscious conjugation of constructs is negatively correlated with your declared ordering. This is full-square with all of the evidence across multiple areas of human judgement - we do not know what is going on under the 'bonnet' and driving our non-conscious as opposed to our conscious choices. This creates a problem to be aware of - particularly given your very high scoring noted above - which is dissonance in postprocessing your images. At capture, with most genres, the dominant modality is more likely to be intuitively than rationally driven under the affective pressure of the moment. When post-processing the opposite is likely to be the case and you are more likely to reject images of high emotional salience or attenuate their emotional impact. I will give you a tip later in the report that may help you deal with this not-uncommon problem.

\begin{tabular}{l|l}
\hline Intuitively Controlled Constructs & Constructs for intuitive development \\
\hline IMPACT & Composition* \\
EXPOSURE & LIGHT \\
FOCUS & CREATIVITY \\
DOF & NARRATIVE \\
\hline TECHNICAL & COL/TONE \\
\hline Composition* & \\
\hline
\end{tabular}

\section{Comment:}

As you can see the analytics tell me that 'composition' is just within your non-conscious competence whilst use of light, creativity, narrative and use of colour/tonality are not influencing your intuitive judgement. Creativity and narrative power are conventionally assigned as 'rational' or 'deliberative' processes however, I would argue that there are substantial gains to be made by making these areas of judgement cue dependent and hence retrievable in the short time-slices of non-conscious cognitive processing. I will give you a tip on this later in this report. Use of light and use of colour tonality commonly do not feature in the intuitive processing of those high on the 'rational' side of the REl scale. This may be because they, along with exposure, form a latent structure delivering energy into the image. 
Whatever the reason you may wish to start the EPF process with one of these and looking at your MDS mapping I suspect you would make the most rapid gains with the use of light.

Images to review (high difference in ranking versus the consensus)

\begin{tabular}{|l|l|l|l|l|l|l|l|l|l|}
\hline 8 & 10 & 11 & 14 & 23 & & & & & \\
\hline
\end{tabular}

Do note the consensus is not necessarily 'correct' - just ask yourself why you differ. I have appended the consensus scoring at this point (105 participants) to give you some clues as to why the differences have occurred.

\section{SUMMARY}

Your performance on the test does indicate a very high level of technical competence and it is not surprising to see you with an ' $L$ ' and indeed the higher ' $A$ " level would almost certainly be within your competency. XXXX, you have educated yourself well in the art of photography but now is a good time to starting to burn in the skills into your mental firm-ware. Your favoured genre does require high levels of spontaneity and interpretive ability in order to penetrate the visual and see the essence of whatever you choose to point your camera. Your non-conscious competency is strong across all the basics and your ability in judging appropriate depth of field and composition is evident in the data you have provided. I would recommend EPF for three areas at this point: narrative, use of light and reinforcement of your intuitive awareness of composition. Note also my remarks
$\cdot+$ ...
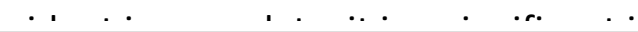


\section{Three Tips to help you on your way.}

1. Emotional activation tends to be highest at the point of capture and it is likely that your non-conscious will be pattern matching and conjugating your expertise in ways that will not be accessible to you when your conscious modality is in the ascendancy when post-processing. In order to enhance non-conscious recognition reapply any activation method you have used at the time of capture. If you seek an element of 'rawness' in the shot, then process quickly when all the peripheral details and feelings will be at their most active. A time frame of within 8 hours is best, 24 at the outside. If you want a more 'interpretive' treatment, wait at least three days before starting work on the image.

2. Use of light: move out of your favoured genre and look for a tricky lighting situation. Maybe a church interior with dramatic lighting? Work on a manual setting and spot off the most intense light and look for simple objects catching the light in unusual ways. Focus on backlighting and then use other orientations looking for dramatic effect. Also, on a dull day do the same perhaps looking for shadow contrasts. Don't worry that composition and all the rest is wrong - just work the use of light construct.

3. Narrative power is difficult to develop either at the conscious or the nonconscious level - however, emotional activation will aid the process of recognition when the extra-ordinary is about to occur. There are three basic approaches to uncovering the 'hidden' story and one I recommend commencing with is looking for fracture, no matter how it has been brought about. Take a simple example of an orange on a chopping board, photograph it in good light, and then cleave it and photograph its torn flesh. Note now how you can see much more of the hidden reality that is an orange. Find other examples and then out into a crowded street with a simple point and shoot. It is important not to worry about other issues at this point - just keep looking for the moments where order is broken, or structures fractured. Finally look for fracture in the work of others - Don McCullin is a good example. He has an uncanny knack of seeing it and 


\section{Reading the data tables (only for those who love statistics, data modelling or just want to pick through the details).}

What follows is a series of data tables and a chart. The data tables have been generated using a variety of analytical methods. For the technically minded the core of the analysis is run using a Partial Least Squares (PLS) Regression model. The PLS model surmounts the problem of the overlap between the different constructs that would undermine a conventional multiple regression technique. It also allows the analysis of data where there are a large number of explanatory variables relative to the number of observations. The cluster chart uses a method called multi-dimensional scaling. This technique uses the implicit weighting derived from PLS and your scoring under each construct. The tables are as follows:

Assessment (grey): this shows your 'intuitive' and your 'rational' ranking of the images and a 'consensus' ranking drawn from all other participants in the IMP test to date (excluding your own). Alongside the differences in ranked placement are also shown (Rvl) being between your intuitive and rational ranking of the images, (IVC) being your intuitive score compared with the consensus and $(R v C)$ being your rational score compared with the consensus. I have also recalibrated your 'rational ranking' implied by the importance of the ten constructs revealed by your scoring of the images $\left(R^{*}\right)$ rather than using the ranking you submitted.

Correlations (white): this shows the correlation between the three rankings. The range is from $-100 \%$ to $+100 \%$. Above $0 \%$ suggests that there is a positive agreement between one ranking and the other in the pair.

Differences (white): this shows the average difference between the ranking of each image under the three headings.

Placed differences (white): this shows the difference between any image ranked $6^{\text {th }}$ or better under any heading and the other rankings. 
Alignment (blue): this compares your declared ranking against your intuitive ranking. The intuitive ranking uses your votes on the ten constructs and the analytical model then works out the weighting of each construct that best explains your intuitive scoring.

Stats (green): this gives two important statistics. R2 on PLR reveals the proportion of the variability in your intuitive judgement that can be explained by the way you voted on the ten constructs. The score ranges from $0-100 \%$. A score of $100 \%$ says that nothing but the ten constructs are driving your nonconscious intuition. In practice, a score of $60 \%$ is normal for those who have not worked on the techniques in the book.

Correl on ranking looks at the ranking of the ten constructs and compares your declared ranking and the ranking implicit in your intuitive scoring. This ranges from -1 to +1 . At +1 the agreement is perfect, at -1 the two rankings of constructs are completely inverted. Scores of 0 and indeed negative are quite common until you develop your intuitive skills as described in the book.

\section{Construct Correlations}

This table compares the way you scored the 20 test images compared with the scoring of all participants. Again, beware of consensus judgements - you may see things differently to others and rightly so. However, thinking about the differences will suggest reasons why your assessment is different overall and what areas of expertise you could think about.

\section{Consensus Judgement}

This table shows how your judgement of the image (intuitive, rational and rational*) agrees with the consensus of all participants in the IMP Test. What you should look for are the differences and why they may have occurred. But, the consensus is not necessarily correct - specially if you specialise in a genre that is away from the mainstream.

\section{Construct Mapping:}

With this diagram, the software has drawn a mapping of how significant each construct is in driving your intuitive analysis of the image. Don't worry about the axes, what is important is the location of your intuitive judgement and how 
your judgement under each of the ten constructs are located relative to one another and to your intuitive judgement. The goal of the EPF technique described in the book is to bring all the ten constructs into close alignment with your intuitive score. If constructs are very close together that signals that you maybe over-associating those constructs in your mind and have not 'pulled them apart'. However, reasonable proximity is what you would expect as the constructs are 'fuzzy' and do to a certain extent overlap (use of light and exposure is a good example). 


\section{Data Sheet}

\begin{tabular}{|ccccc|cccc|}
\hline $\begin{array}{c}\text { ASSESSMENT } \\
\text { IMAGE }\end{array}$ & $\begin{array}{c}\text { Rank } \\
\text { Intuitive }\end{array}$ & $\begin{array}{c}\text { Rank } \\
\text { Rational }\end{array}$ & $\begin{array}{c}\text { Rank } \\
\text { Rational* }\end{array}$ & $\begin{array}{c}\text { Rank } \\
\text { Consensus }\end{array}$ & \multicolumn{2}{c|}{ Difference } & \multicolumn{2}{c|}{ Difference Difference Difference } \\
6 & 13 & 18 & 18 & 13 & 5 & $R^{*} v$ IVC & RvC \\
7 & 13 & 16 & 16 & 17 & 3 & 3 & 0 & 5 \\
8 & 6 & 13 & 12 & 3 & 7 & 6 & 3 & 10 \\
9 & 13 & 11 & 10 & 6 & 2 & 3 & 7 & 5 \\
10 & 6 & 10 & 11 & 19 & 4 & 5 & 13 & 9 \\
11 & 18 & 17 & 17 & 9 & 1 & 1 & 9 & 8 \\
12 & 6 & 9 & 9 & 11 & 3 & 3 & 5 & 2 \\
13 & 1 & 1 & 1 & 2 & 0 & 0 & 1 & 1 \\
14 & 1 & 8 & 8 & 10 & 7 & 7 & 9 & 2 \\
15 & 6 & 7 & 5 & 1 & 1 & 1 & 5 & 6 \\
16 & 18 & 19 & 19 & 12 & 1 & 1 & 6 & 7 \\
17 & 1 & 3 & 2 & 7 & 2 & 1 & 6 & 4 \\
18 & 1 & 6 & 6 & 5 & 5 & 5 & 4 & 1 \\
19 & 6 & 4 & 3 & 4 & 2 & 3 & 2 & 0 \\
20 & 20 & 20 & 20 & 20 & 0 & 0 & 0 & 0 \\
21 & 6 & 5 & 7 & 8 & 1 & 1 & 2 & 3 \\
22 & 13 & 15 & 15 & 16 & 2 & 2 & 3 & 1 \\
23 & 1 & 2 & 4 & 15 & 1 & 3 & 14 & 13 \\
24 & 12 & 14 & 14 & 14 & 2 & 2 & 2 & 0 \\
25 & 13 & 12 & 13 & 18 & 1 & 0 & 5 & 6 \\
\hline
\end{tabular}

Rational ${ }^{*}\left(R^{*}\right)=$ rational assessment of each image weighted by intuitive rank of importance

\begin{tabular}{|c|c|c|c|c|c|c|c|}
\hline Correlations & Intuitive & Rational & Rational* & Consensus & \multirow[t]{2}{*}{ ALIGNMENT } & \multicolumn{2}{|c|}{ INTUITIVE DECLARED } \\
\hline Intuitive & 1 & & & & & RANKING & RANKING \\
\hline Rational & $89.98 \%$ & 1 & & & IMPACT & 1 & 3 \\
\hline Rational* & $89.27 \%$ & $98.65 \%$ & 1 & & CREATIVITY & 8 & 3 \\
\hline Consensus & $48.90 \%$ & $53.23 \%$ & $62.41 \%$ & 1 & NARRATIVE & 9 & 1 \\
\hline & & & & & DEPTH OF FIELC & 4 & 9 \\
\hline Differences & Intuitive & Rational & Rational* & Consensus & USE OF LIGHT & 7 & 1 \\
\hline Intuitive & 0.0 & & & & COMPOSITION & 6 & 3 \\
\hline Rational & 2.5 & 0.0 & & & COLOUR/TONAL & 10 & 3 \\
\hline Rational* & 2.6 & 0.6 & 0.0 & & FOCUS & 3 & 8 \\
\hline Consensus & 5.0 & 4.2 & 3.8 & 0.0 & EXPOSURE & 2 & 3 \\
\hline & & & & & TECHNICAL & 5 & 10 \\
\hline Placed Diff. & Intuitive & Rational & Rational* & Consensus & & & \\
\hline Intuitive & 0.0 & & & & STATS & & \\
\hline Rational & 2.9 & 0.0 & & & R2 ON PLR & & $83.8 \%$ \\
\hline Rational* & 3.2 & 0.9 & 0.0 & & CORREL ON RAN & VKING & $-38.85 \%$ \\
\hline Consensus & 5.9 & 4.7 & 4.1 & 0.0 & & & \\
\hline
\end{tabular}

\begin{tabular}{|c|c|c|c|c|c|c|}
\hline Correlations between & Intuitive & IMPACT & CREATIVITY & NARRATIVE & DOF & LIGHT \\
\hline your intuitive and & 0.59 & 0.48 & 0.31 & 0.58 & 0.76 & 0.66 \\
\hline construct scoring and the & & COMP & COL/TONE & FOCUS & \multicolumn{2}{|c|}{ EXPOSURE TECHNICAI } \\
\hline average for all test & & 0.49 & 0.33 & 0.86 & 0.68 & 0.71 \\
\hline
\end{tabular}




\section{Consensus Judgement (Trial 3, N=105)}

\begin{tabular}{|c|c|c|c|c|c|c|c|c|c|c|c|}
\hline Image & INTUITIVE IN & IMPACT & CREATIVITY & NARRATIVE & DOF & LIGHT & COMP & COL/TONE & FOCUS & EXPOSURE & TECHNICAL \\
\hline $6^{5}$ & $53.69^{7}$ & $\quad 6.72$ & 5.58 & 7.36 & 6.21 & 5.86 & 5.70 & 5.93 & 6.68 & 7.02 & 6.15 \\
\hline 7 & 54.93 & 5.46 & 4.69 & 4.60 & 7.29 & 5.81 & 5.23 & 6.26 & 7.57 & 7.41 & 6.09 \\
\hline 8 & 69.94 & 8.15 & 6.30 & 6.98 & 8.17 & 7.27 & 7.51 & 6.95 & 8.35 & 7.78 & 7.63 \\
\hline $9^{7}$ & 66.72 & 6.76 & 6.01 & 5.62 & 7.33 & 7.85 & 7.00 & 7.53 & 7.36 & 7.80 & 6.88 \\
\hline $10^{7}$ & 47.64 & 4.58 & 4.53 & 3.62 & 6.39 & 6.29 & 4.93 & 5.98 & 7.30 & 7.27 & 6.08 \\
\hline 11 & 56.07 & 6.96 & 7.67 & 5.56 & 6.36 & 6.30 & 6.30 & 6.11 & 6.75 & 6.30 & 6.67 \\
\hline 12 & 53.93 & 6.78 & 6.53 & 6.41 & 6.54 & 6.29 & 6.06 & 5.89 & 6.84 & 6.67 & 6.06 \\
\hline $13^{\circ}$ & 70.75 & 8.23 & 6.95 & 8.38 & 7.70 & 7.30 & 7.89 & 7.06 & 7.57 & 7.37 & 7.50 \\
\hline 14 & 59.68 & 5.74 & 5.63 & 5.10 & 6.75 & 6.83 & 6.12 & 6.58 & 7.40 & 7.48 & 6.86 \\
\hline $15^{\circ}$ & 73.88 & 7.95 & 7.00 & 7.72 & 8.09 & 7.91 & 7.87 & 7.72 & 8.27 & 8.22 & 7.91 \\
\hline $16^{\circ}$ & 61.38 & 6.38 & 5.79 & 6.98 & 7.23 & 6.34 & 6.85 & 5.49 & 6.38 & 6.51 & 6.00 \\
\hline $17^{-}$ & 58.13 & 6.60 & 6.05 & 6.08 & 7.54 & 7.10 & 6.68 & 7.09 & 7.66 & 7.69 & 7.13 \\
\hline 18 & 67.94 & 7.08 & 6.70 & 7.47 & 6.66 & 7.33 & 6.79 & 7.32 & 7.05 & 7.61 & 7.06 \\
\hline $19^{\circ}$ & 71.13 & 7.58 & 7.06 & 7.44 & 7.80 & 7.38 & 7.47 & 6.92 & 7.40 & 7.47 & 7.14 \\
\hline 20 & 31.88 & 3.62 & 3.41 & 3.95 & 2.91 & 2.87 & 3.72 & 3.70 & 2.00 & 2.97 & 2.61 \\
\hline 21 & 51.70 & 6.57 & 6.03 & 7.54 & 7.23 & 6.94 & 6.89 & 6.93 & 7.20 & 7.36 & 6.81 \\
\hline $22^{\circ}$ & 52.32 & 6.16 & 5.77 & 6.43 & 5.94 & 7.07 & 6.20 & 6.74 & 5.25 & 6.44 & 5.30 \\
\hline $23^{7}$ & 57.68 & 6.48 & 6.34 & 6.23 & 6.20 & 6.12 & 6.14 & 5.71 & 5.96 & 6.42 & 5.84 \\
\hline 24 & 53.65 & 6.38 & 5.76 & 5.19 & 6.56 & 6.88 & 6.10 & 6.00 & 6.95 & 6.53 & 6.13 \\
\hline $25^{\circ}$ & 52.83 & 5.94 & 6.65 & 5.91 & 5.38 & 6.28 & 6.13 & 5.80 & 4.67 & 5.61 & 5.14 \\
\hline
\end{tabular}

\section{Construct Mapping}

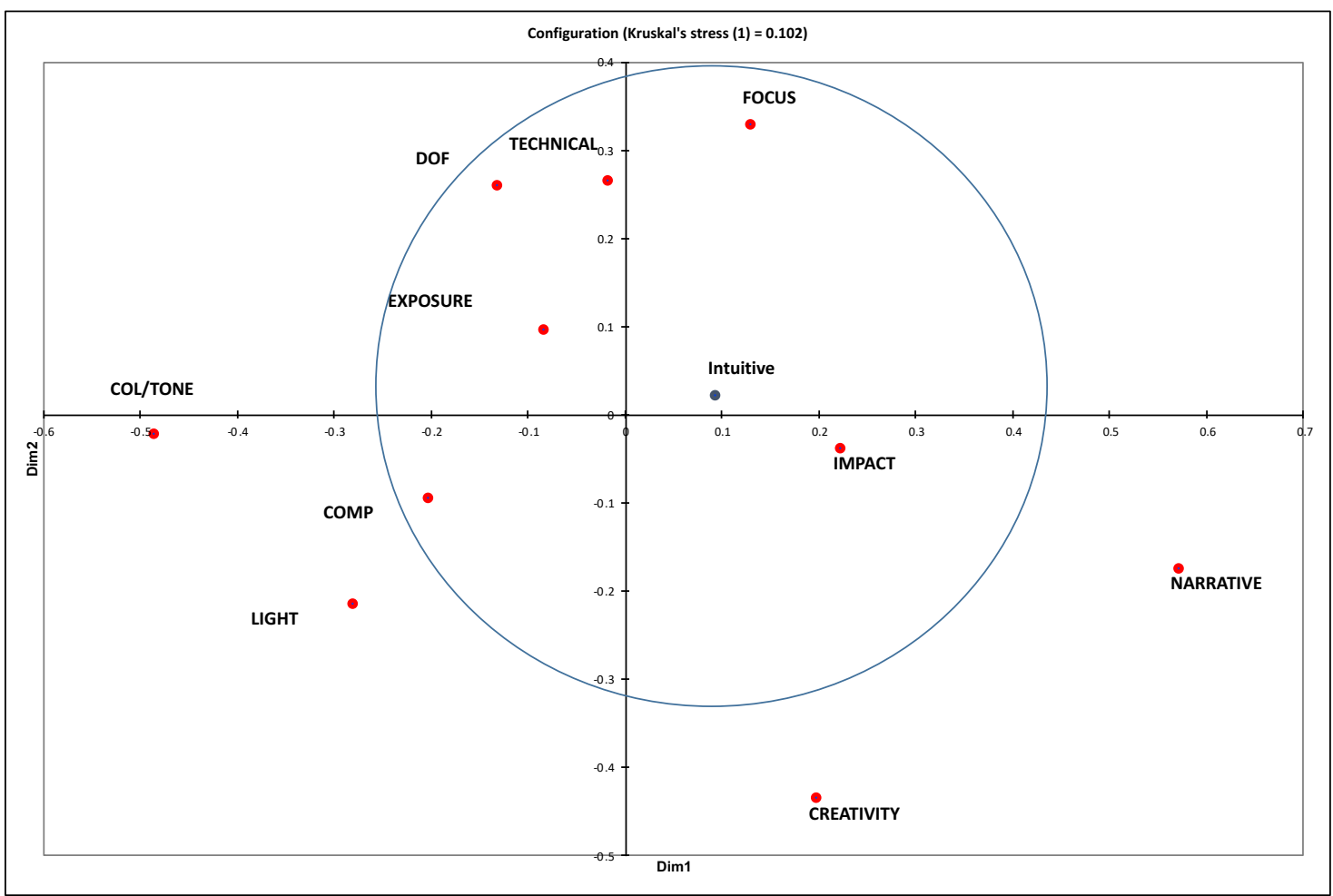




\title{
IMP Test - instructional matter within the test
}

\author{
BEFORE YOU START
}

Download the IMP Test Instructions document and if you prefer print out the scoring sheets at the end

Download and read the IMPT TEST FAQ - a WORD file

Download IMPTEST.xls - an EXCEL file

4. Select Slide Show on the top menu and then 'Play from Start'

Go to view and select 'Enter Full-Screen'

. During phase I of the test use return or enter key to move on a screen

Hit return or enter and you are on your way...

\section{IMP TEST}

๑) Bob Ryan 2016

\section{SMALL BUT IMPORTANT PRINT}

Unless you specifically agree otherwise, your response to the IMP test and the REI and $\mathrm{m}$ analysis of your results is confidential between us.

2. The REI and the IMP test are designed to help you identify your preferences for different ways of thinking and where you have strengths and weaknesses in your photographic thought you should not take the test

3. My analysis of your results will be done impartially and fairly on the basis of the scores you give me. However, I cannot accept any liability resulting from the conduct of the test, downlading a fles royour conprer, the provision of the resuls or the

4. In taking the test you agree that I may use your responses along with others for the purpose of research into the development of photographic skill and judgement. All data will be collected and analysed but individual test respondents will not be identifiable from the results.

5. You acknowledge that I am the sole copyright owner of the IMP test, its associated software and documentation. All rights to the IMP test, its associated software and documentation are reserved. 


\section{INTRODUCTION FROM}

PROF BOB

Hover pointer here for audio $\Rightarrow(j))$

WHAT YOU NEED

A quiet and comfortable place with time to concentrate (the test takes about 40 minutes to complete).

- The IMP instructions, which can be downloaded as a WORD file.

- The assessment return as an EXCEL file called IMPTEST.xls. If preferred, the IMP instructions can be printed out and the attached input forms used to fill in the spreadsheet at the end of the test.

- The final two tabs of IMPTEST.xls contain the Rational Experiential Inventory (REI) which is optional. You may find the results useful when interpreting the output from the IMP test.

\section{PHASE I}

The downloaded spreadsheet has a number of tabs - IMPTABI is for your personal details.

- IMPTAB2, is the input screen for the first phase of the test

- You are invited to assess each image on a $0-100$ scale. The first five images are there to help you start. We suggest a score of 60 for the first image you see.

- You will then go through the 20 test images scoring them as you see fit.

- Each image is visible for 3 second only. Once you have recorded your score hit return to move on to the next image.

VERY IMPORTANT: ONCEYOU HAVE COMPLETED ALL 25 IMAGES DO NOT REFER BACK TO IMPTAB2 ORTO YOUR HAND-WF

Use the return key to step through the images. 


\section{First Image and then:}

What number from 0 - 100 first came into your mind when you saw image I?

Say that number out loud, write it down on your scoring sheet or enter it directly into IMPTAB2.

Do not change that number.

Do not back-track during this phase of the test.

Use the return key

WELL DONE - THAT'S THE END OF THE FIRST PHASE OF THE TEST.

PHASE 2

Now

Don't look back at your Phase I results

On the printed form or on IMPTAB3 enter your importance score against each judgement construct using the ten sliders. You can give the same importance score to two or more constructs if you think that is appropriate.

On the next slide we show you the 10 constructs.

Bob Ryan on the 10 constructs $\square[(2))$ 


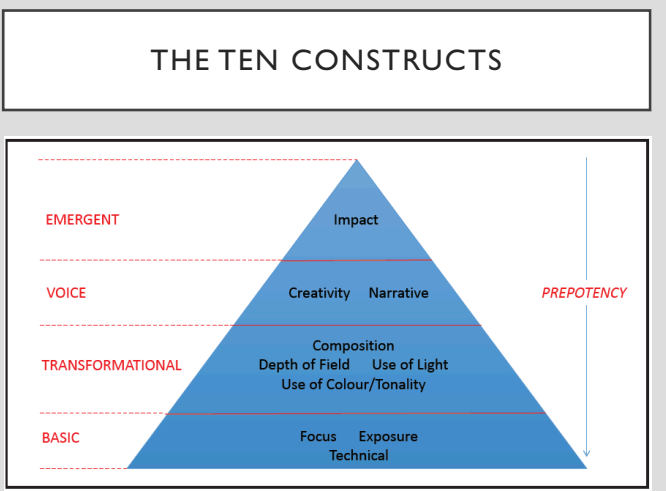

Use the return key

\section{PHASE 3}

Using IMPTAB4 or your printed form score images $6-25$ against each of the ten photographic constructs.

Don't look back at your phase I scores.

For each image score it, against each criterion, on a scale of I - 10 using whole numbers only.

Take as long as you like over this stage of the test.

THAT'S THE END OF THE IMPTEST

DON'T CHANGE ANY OFYOUR SCORES

If you have written your scores on a printed version enter them into the

respective tabs on the IMPTEST spreadsheet.

Now is the time to do the optional Rational Experiential Inventory filling in the answers on REI Input. Your scores are shown on REl Output as you complete the inventory.

Check all tabs are complete.

Save your copy of your completed test with a unique file name in the form

yournomelMPTESTreurur and upload that copy as instructed on our website or
send it to me at professorbobryan@btinternet.com. 


\section{The IMP Test Images}

All Images are Copyright Bob Ryan (2019)

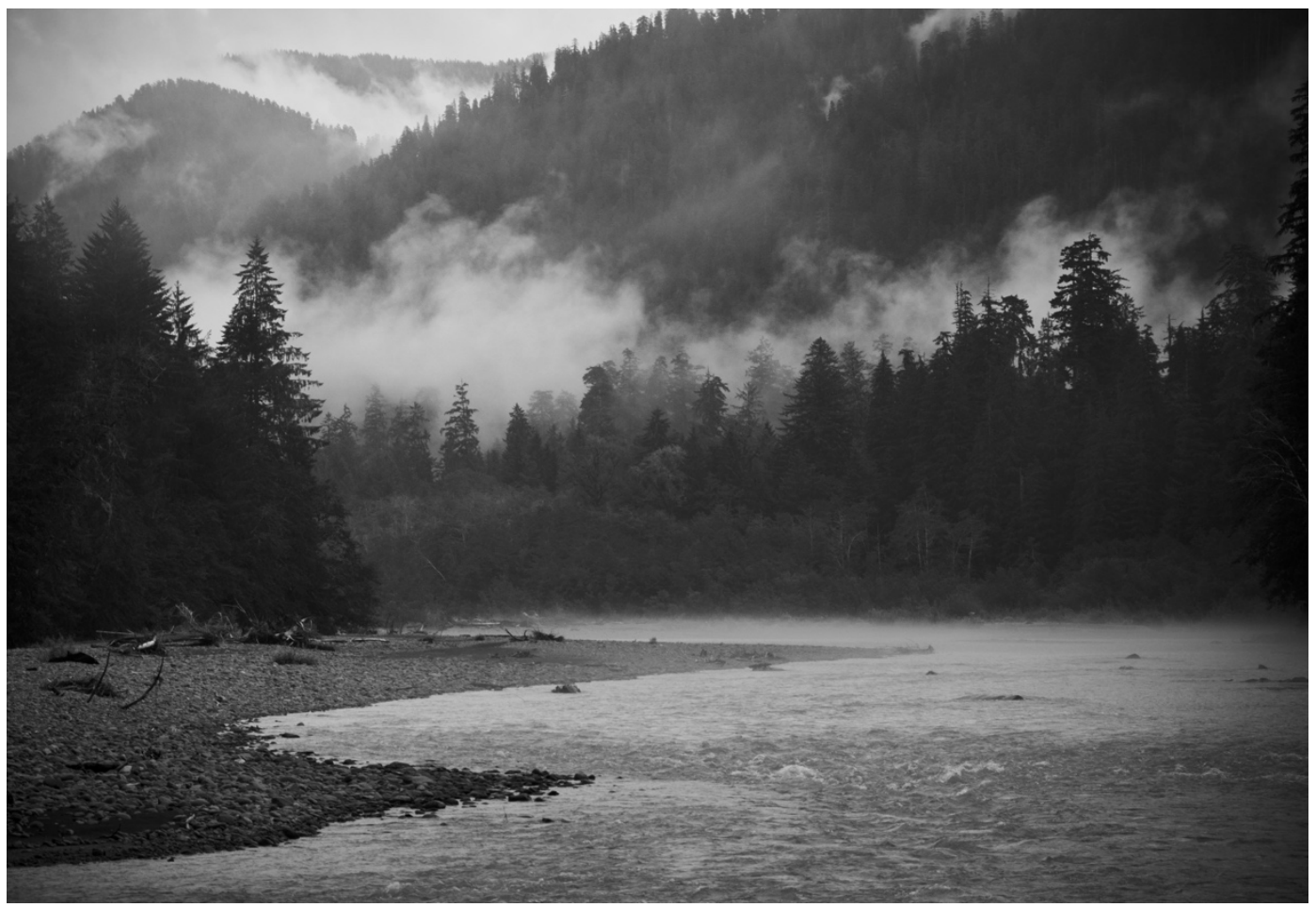

Image 1 (pretest) 


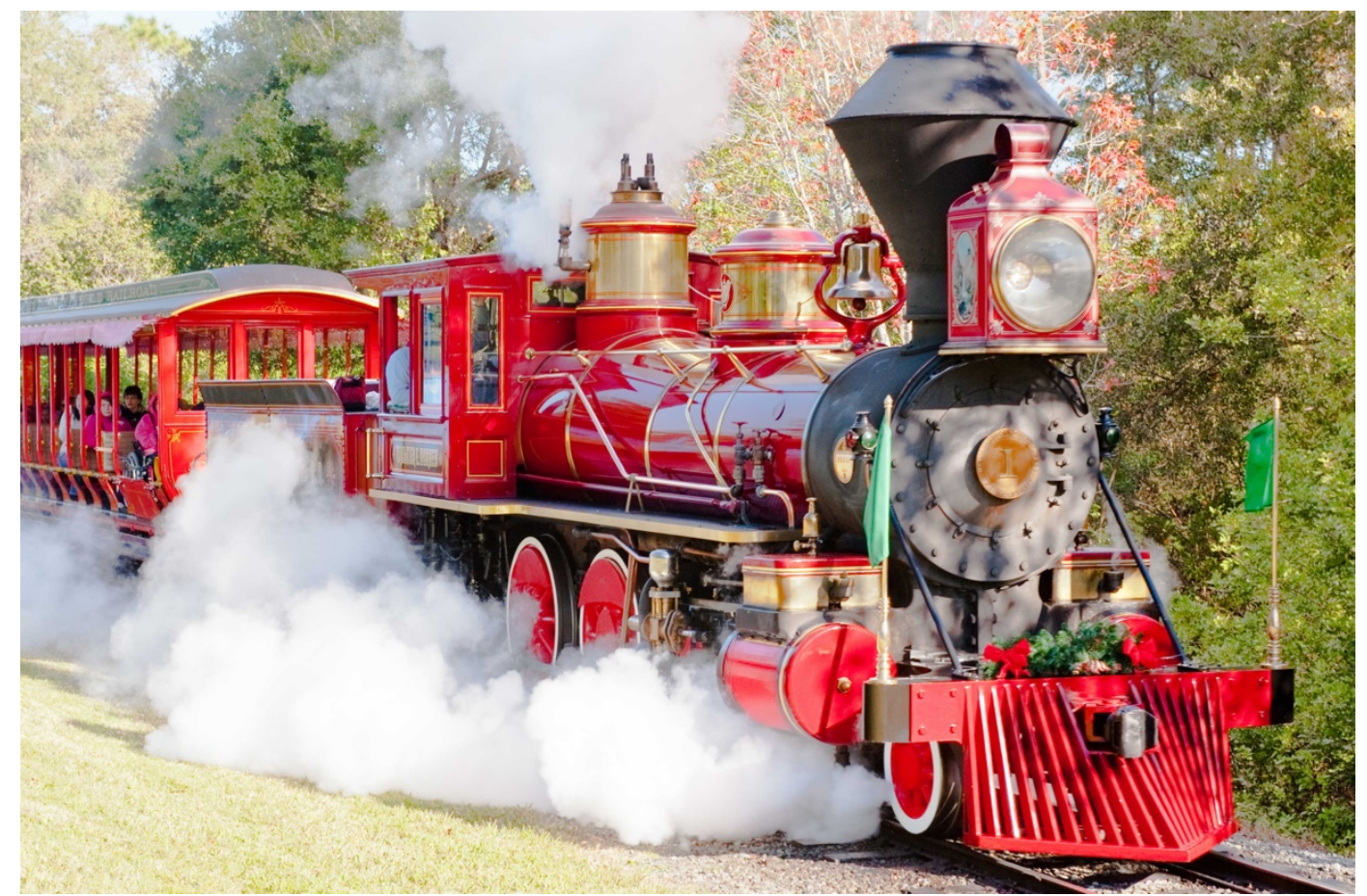

Image 2 (pretest)

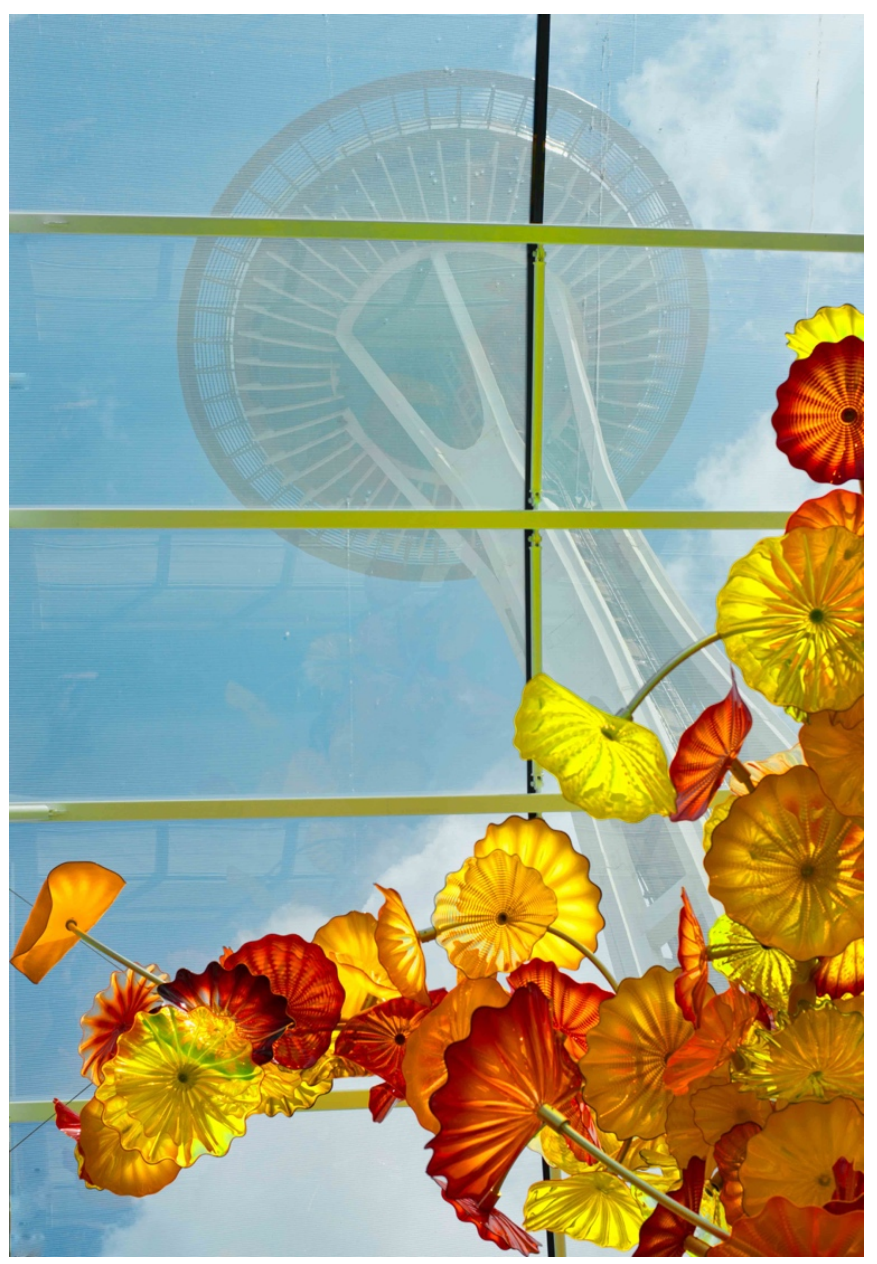

Image 3 (pretest) 


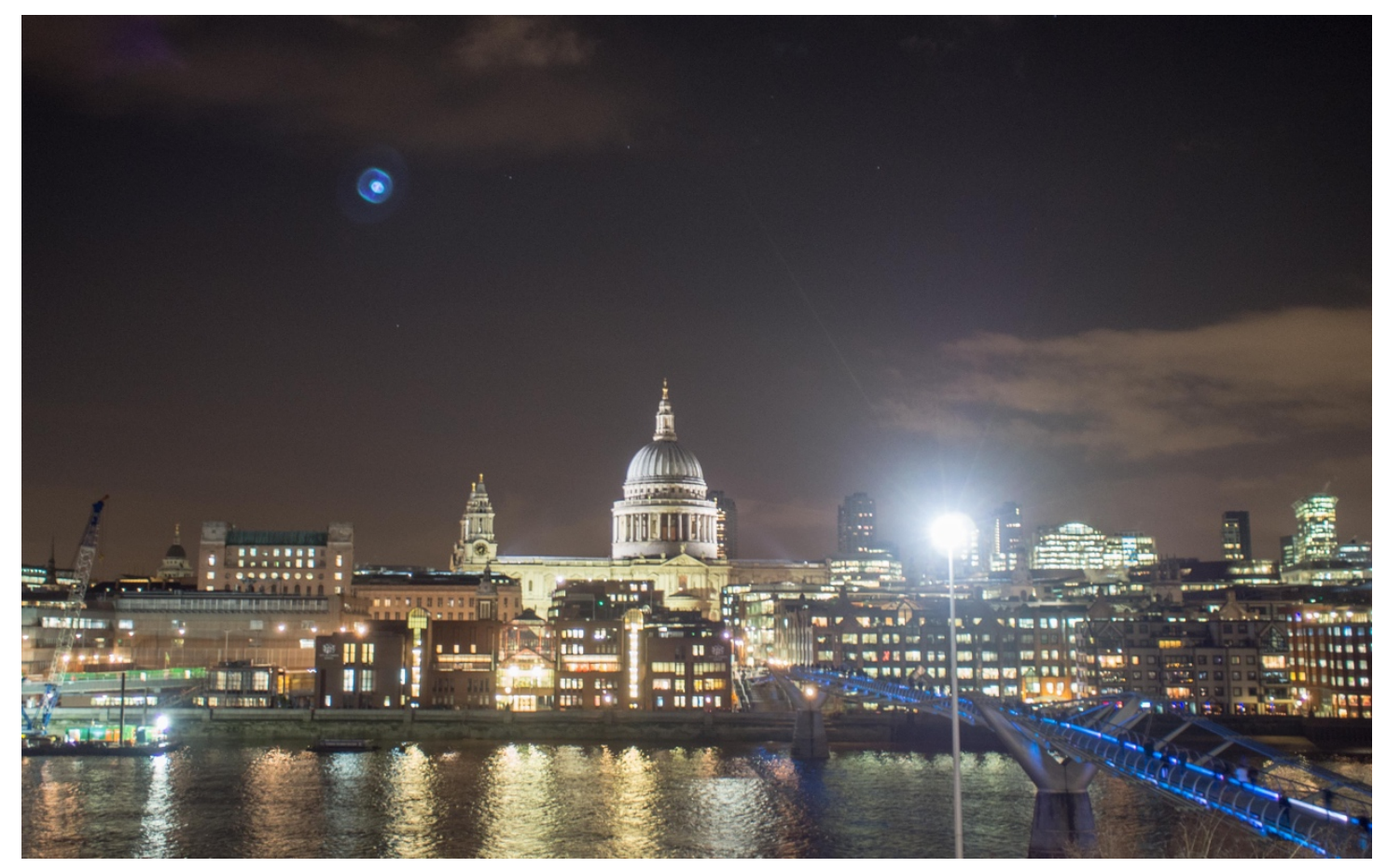

Image 4 (pretest)

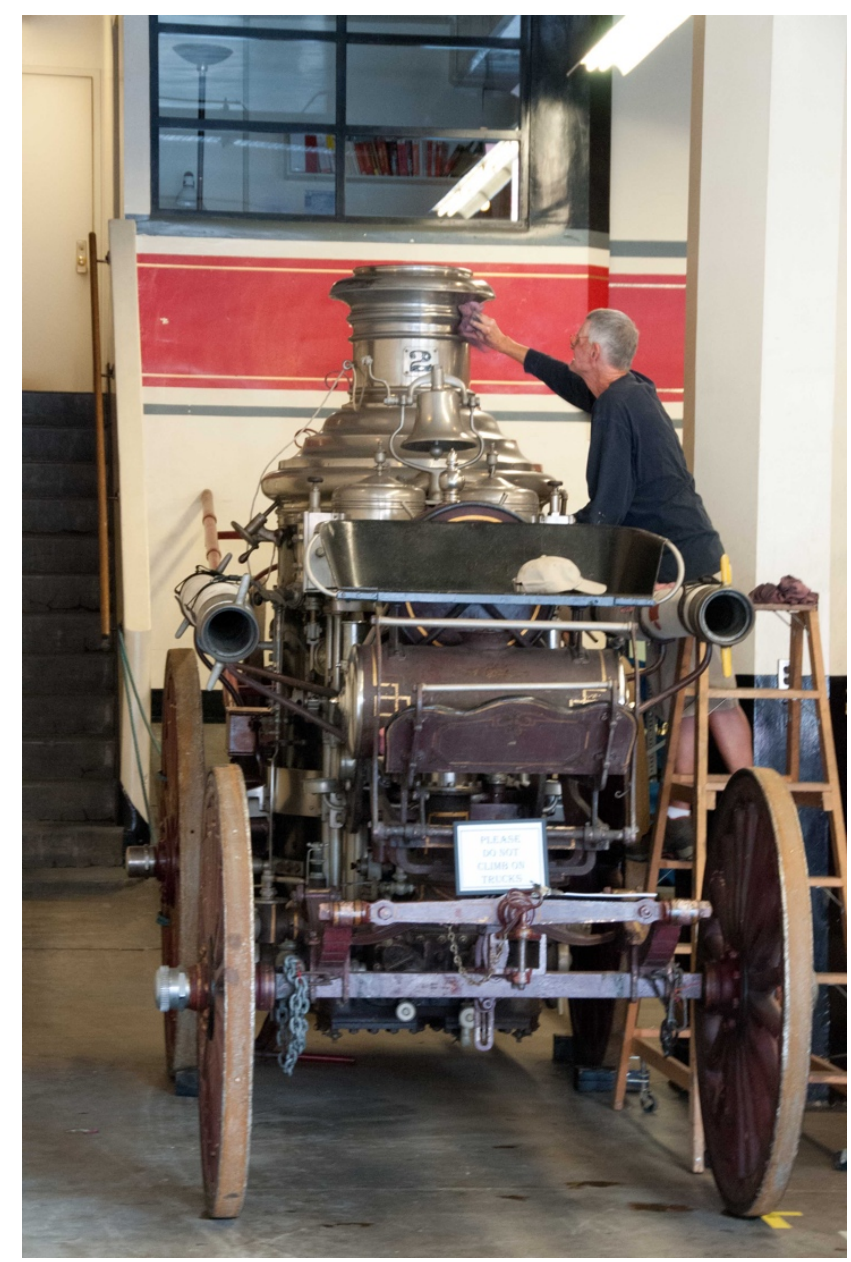

Image 5 (Pretest) 


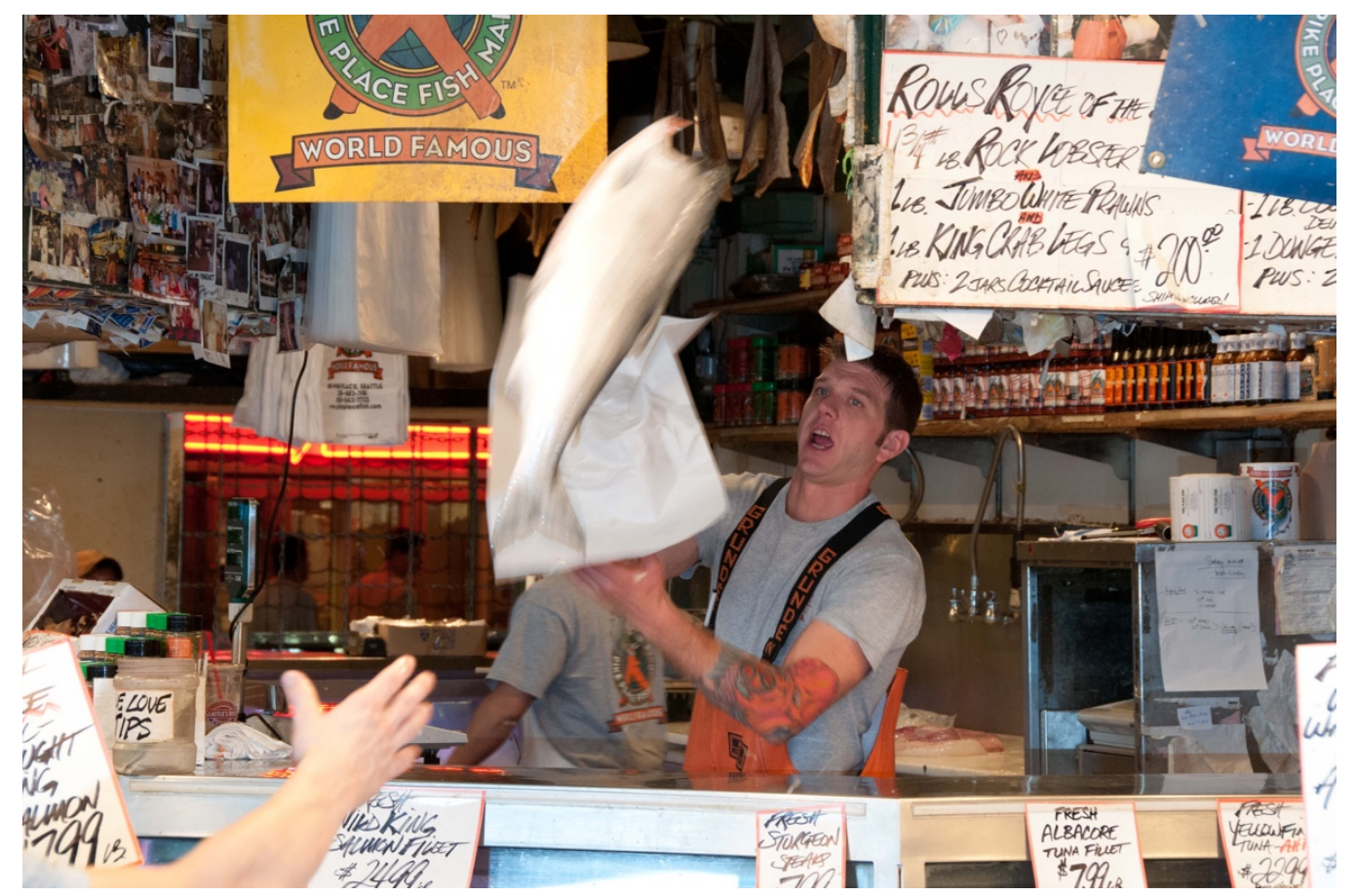

Image 6

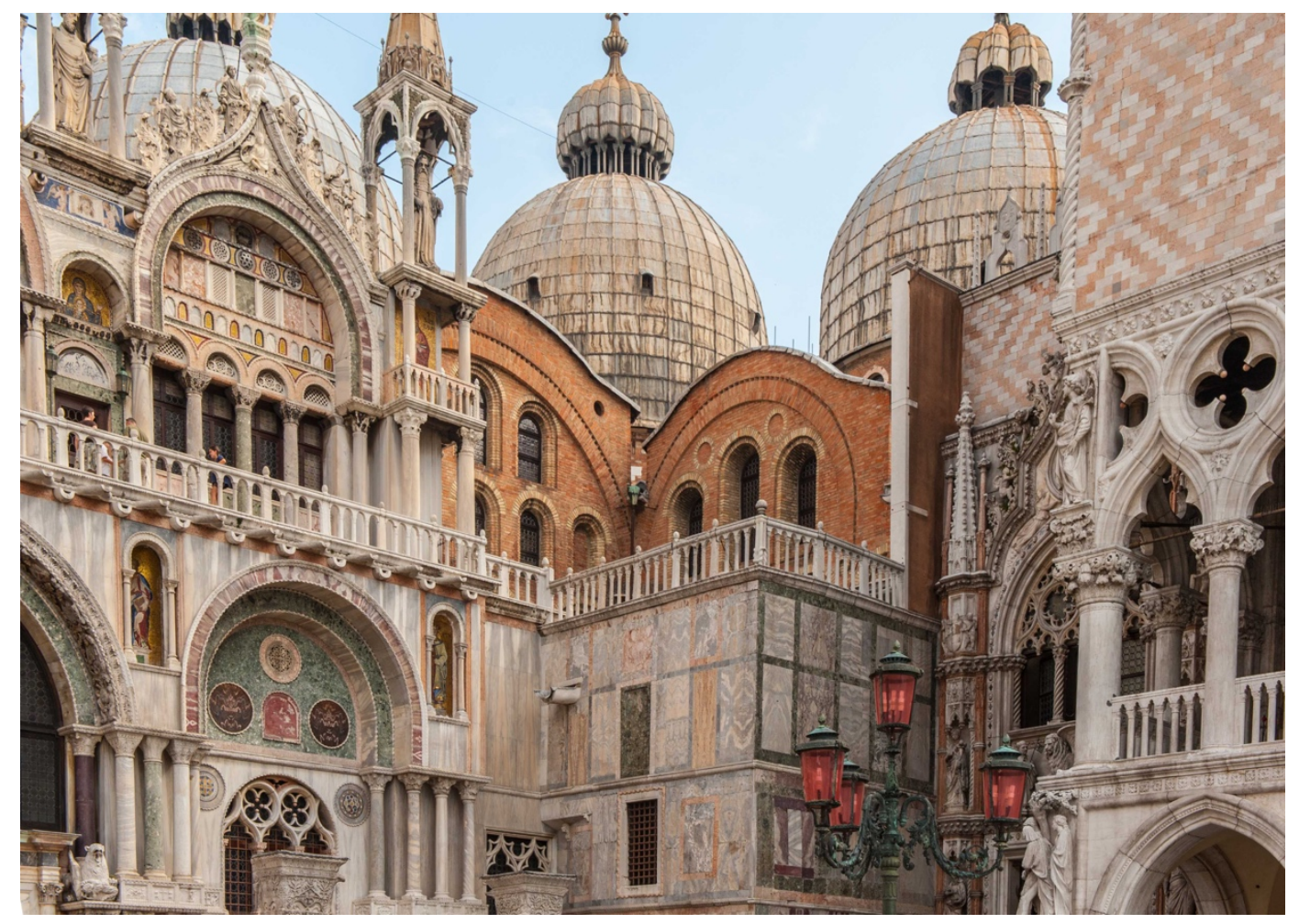

Image 7 


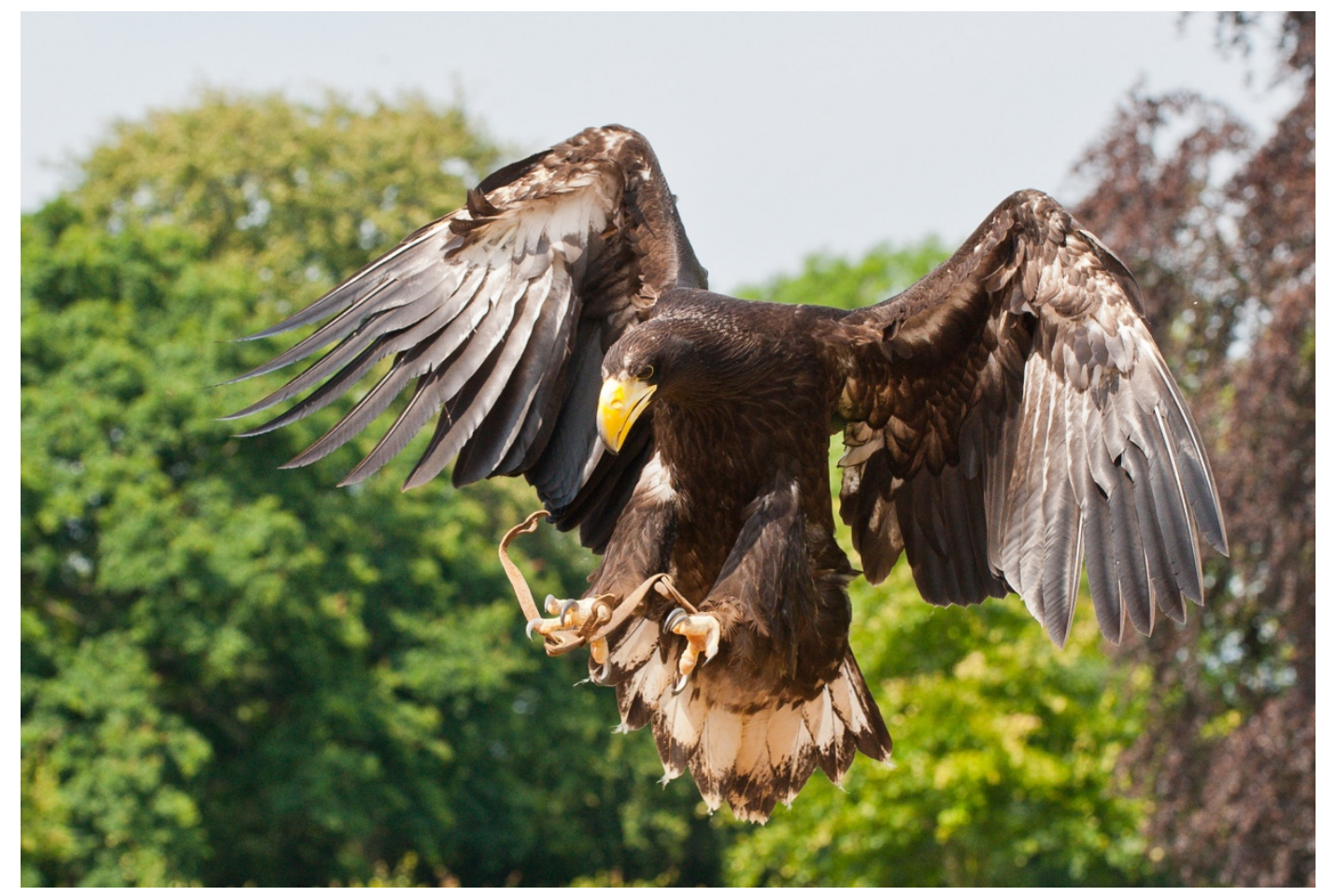

Image 8

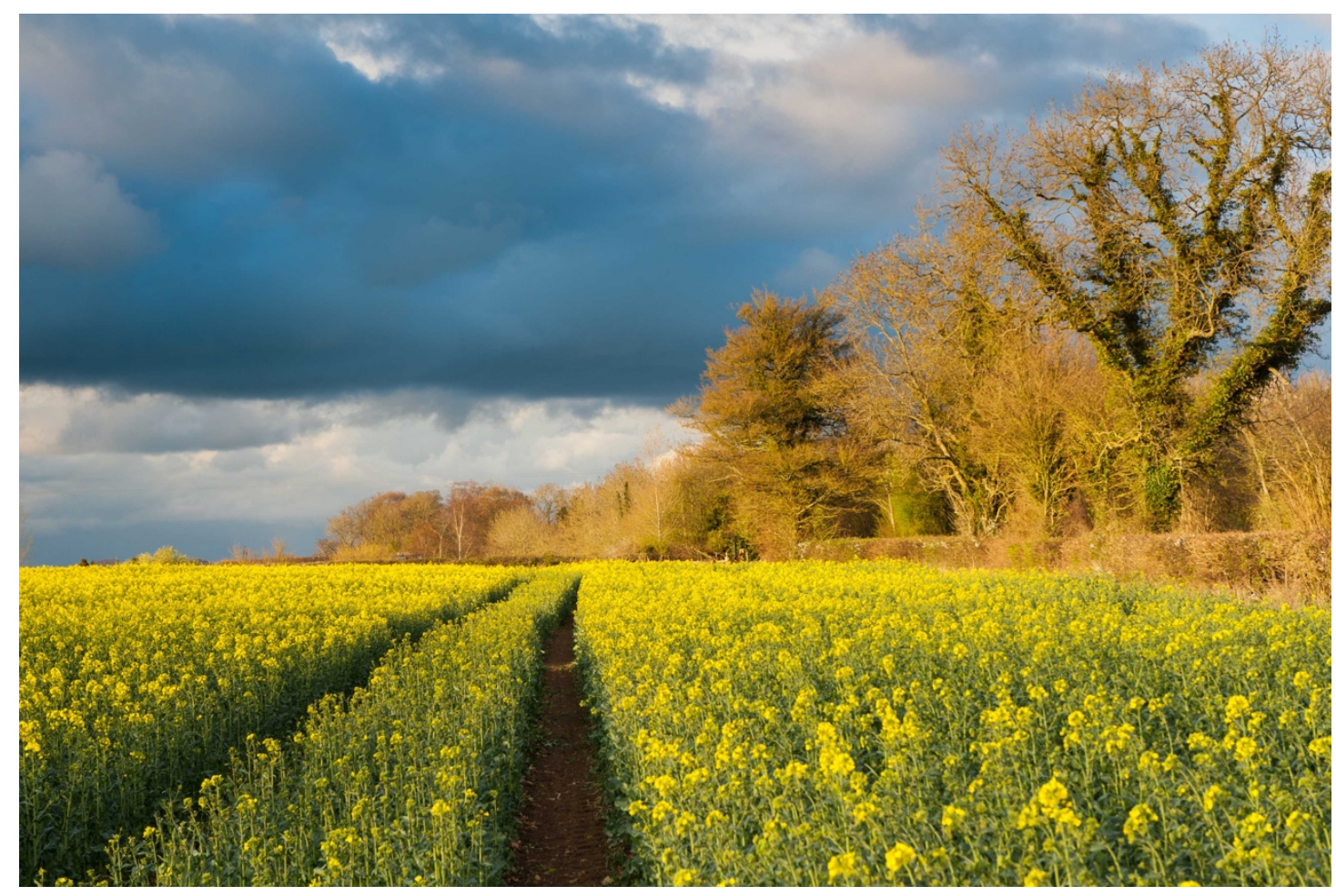

Image 9 


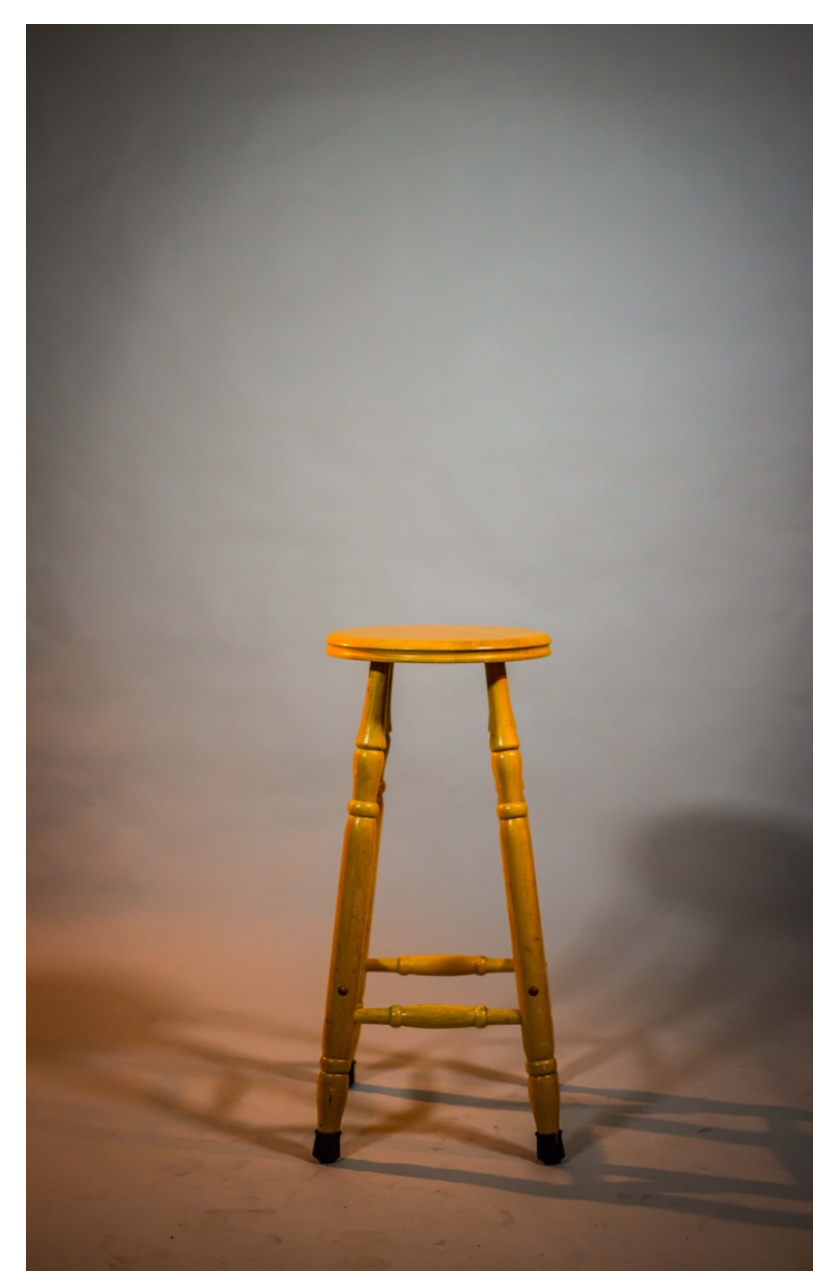

Image 10

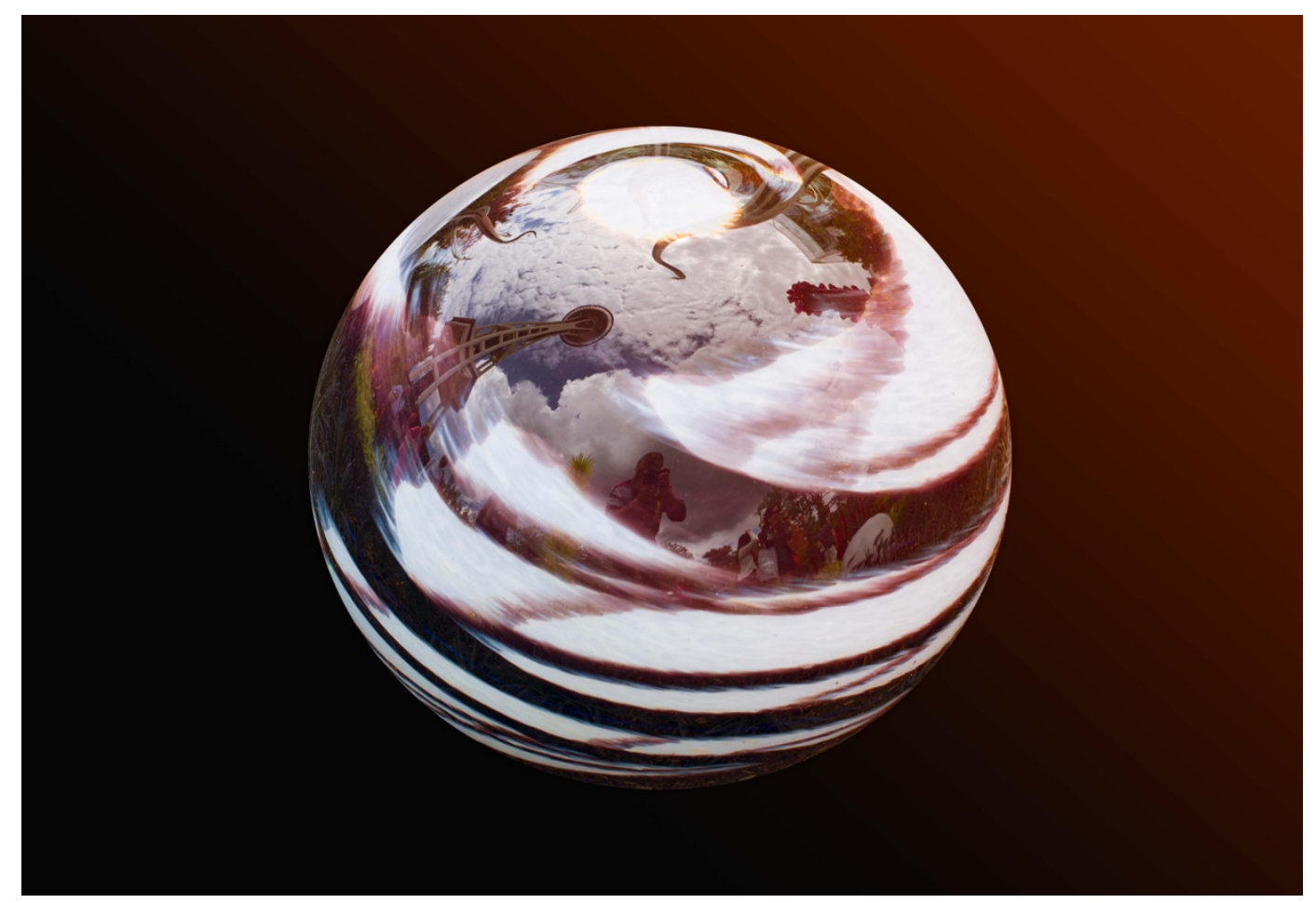

Image 11 


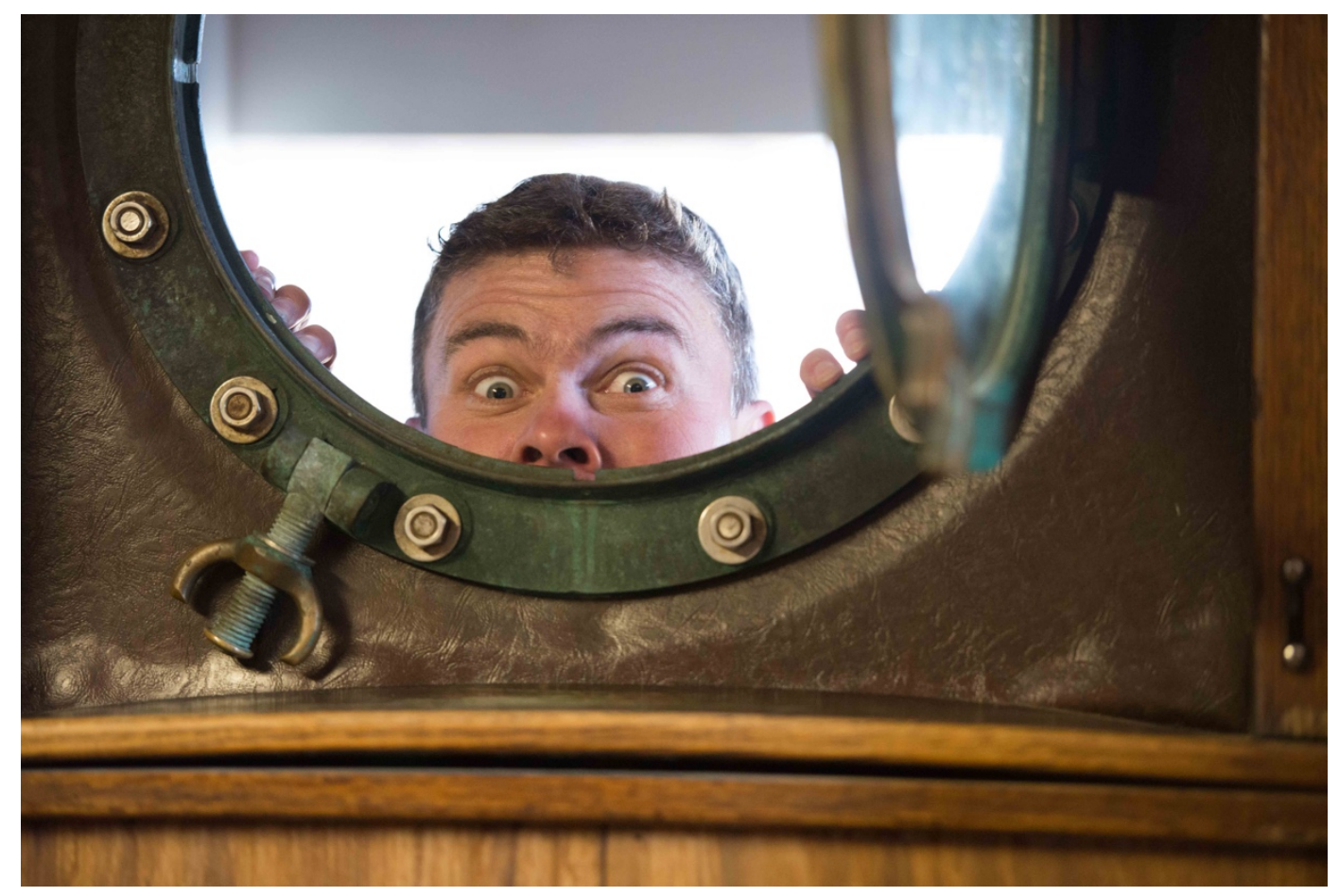

Image 12

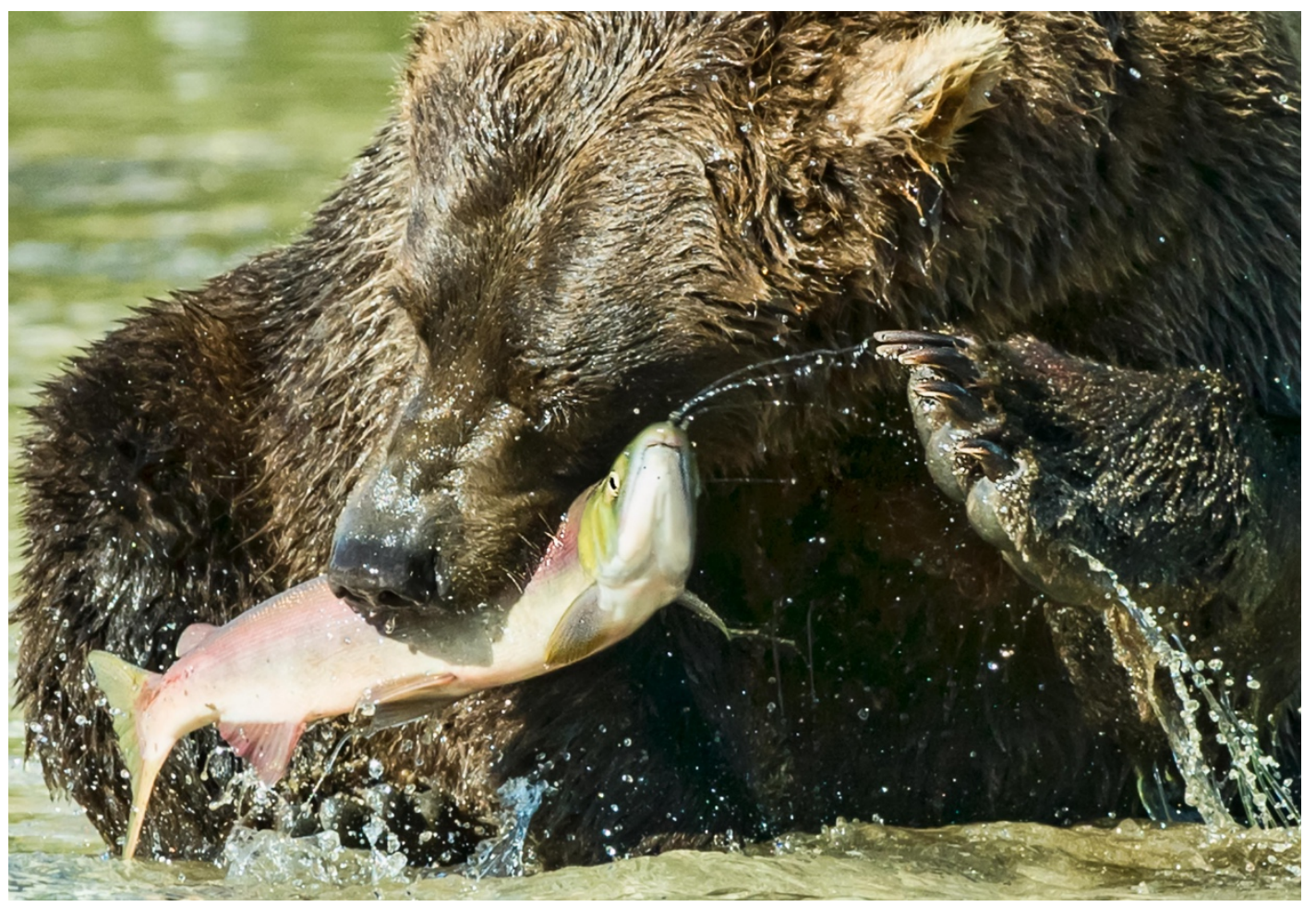

Image 13 


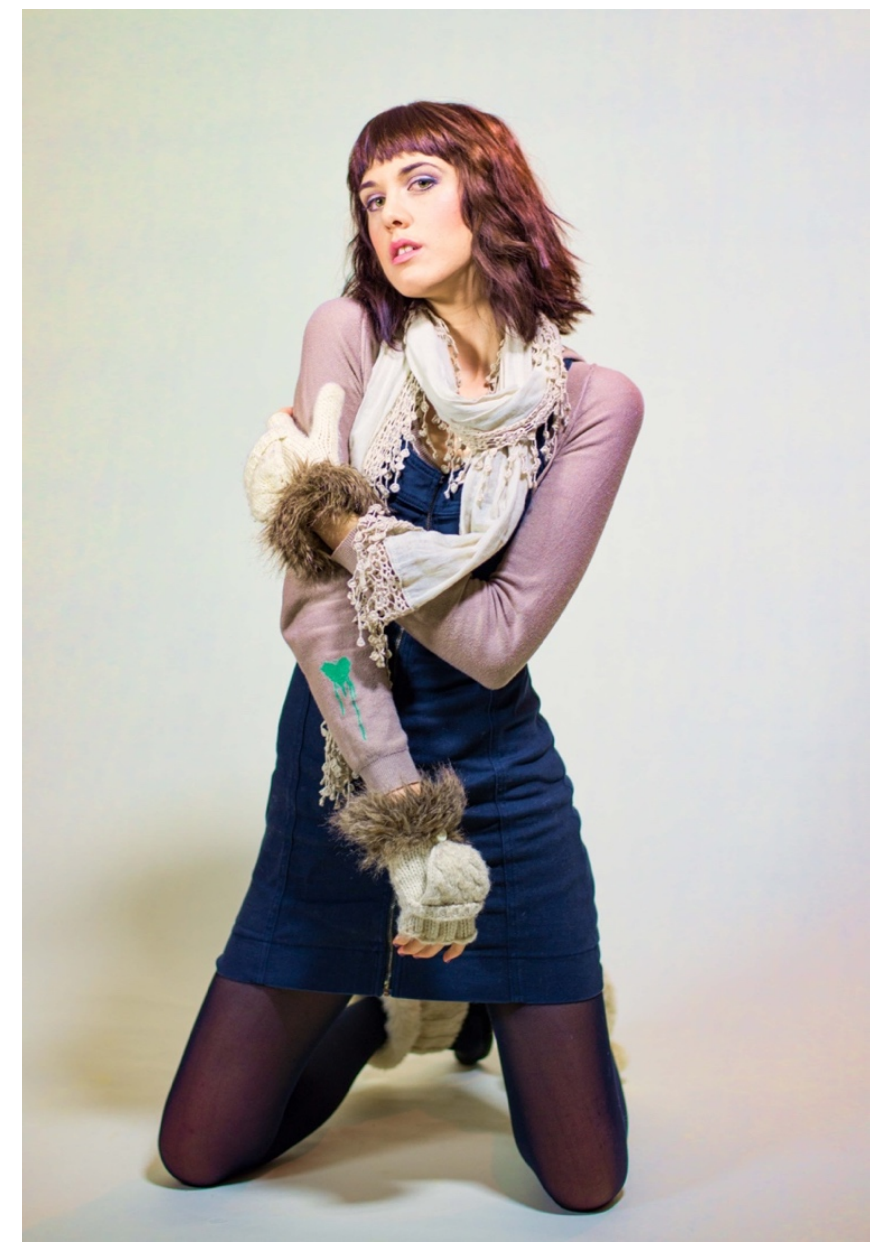

Image 14

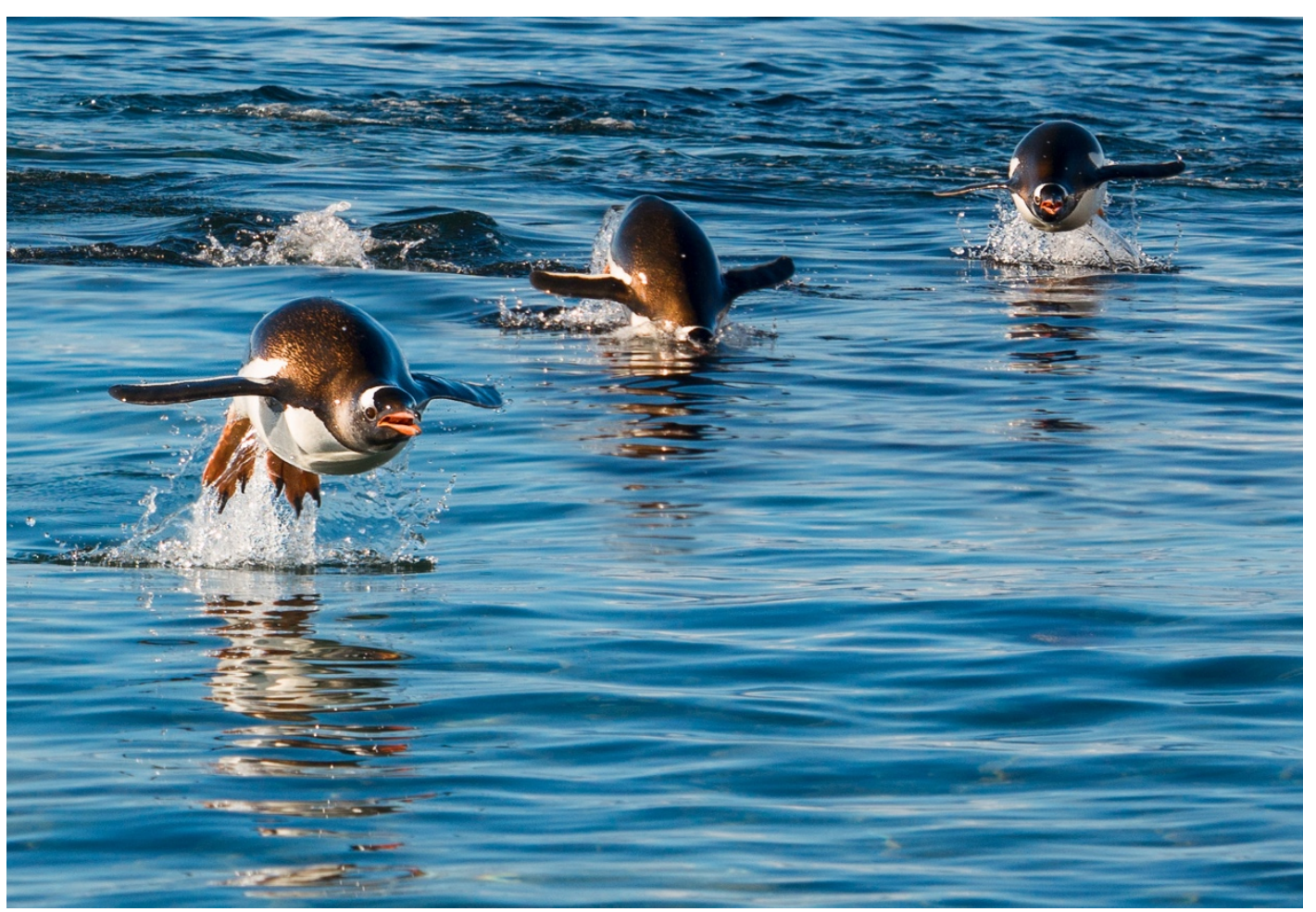

Image 15 


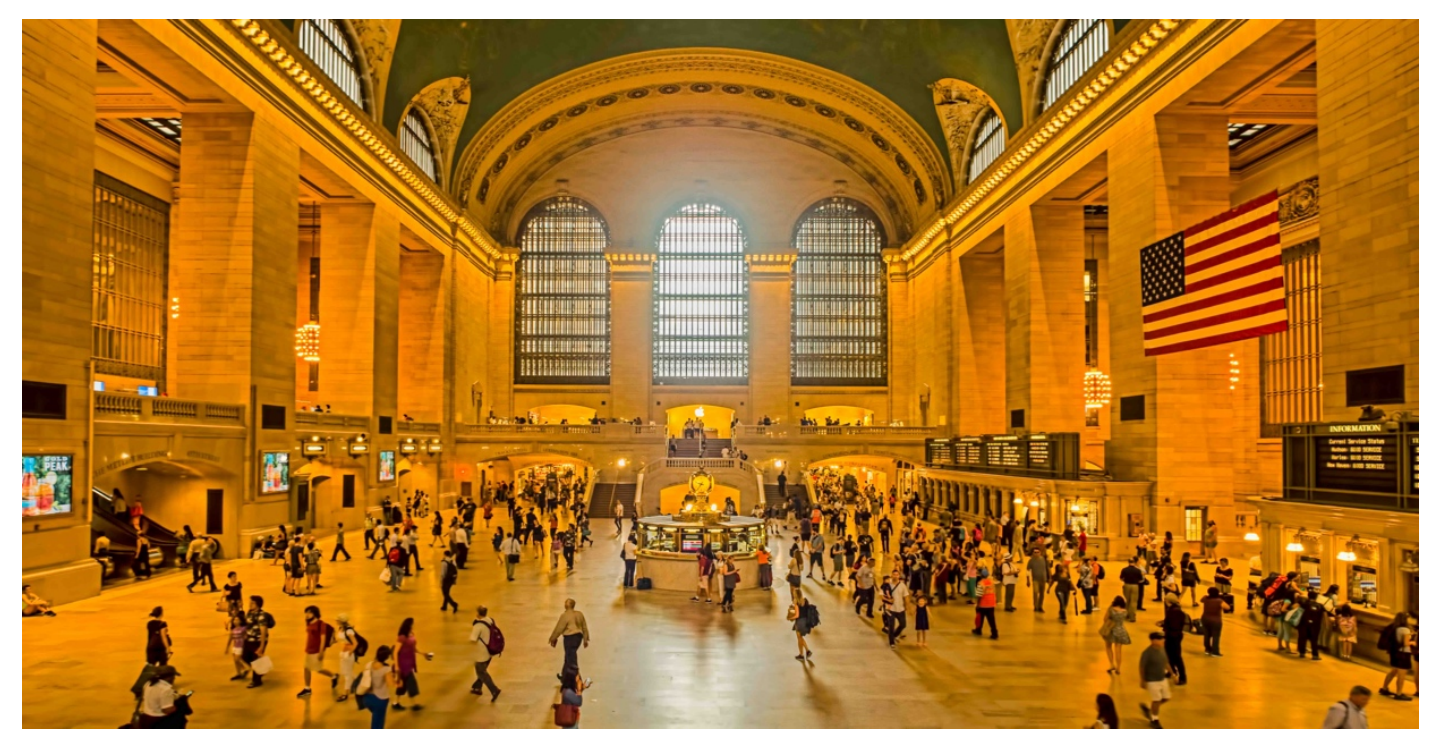

Image 16

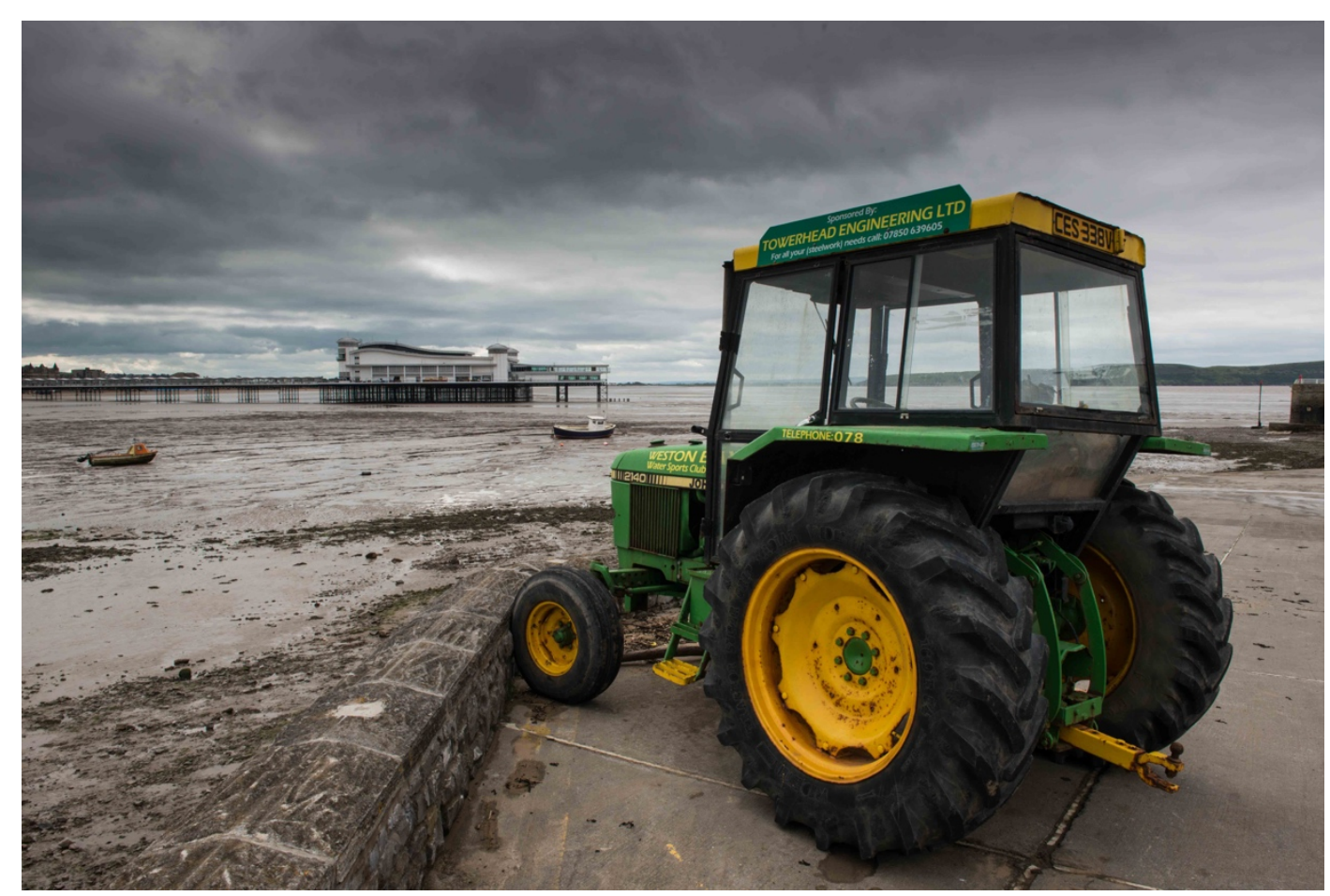

Image 17 


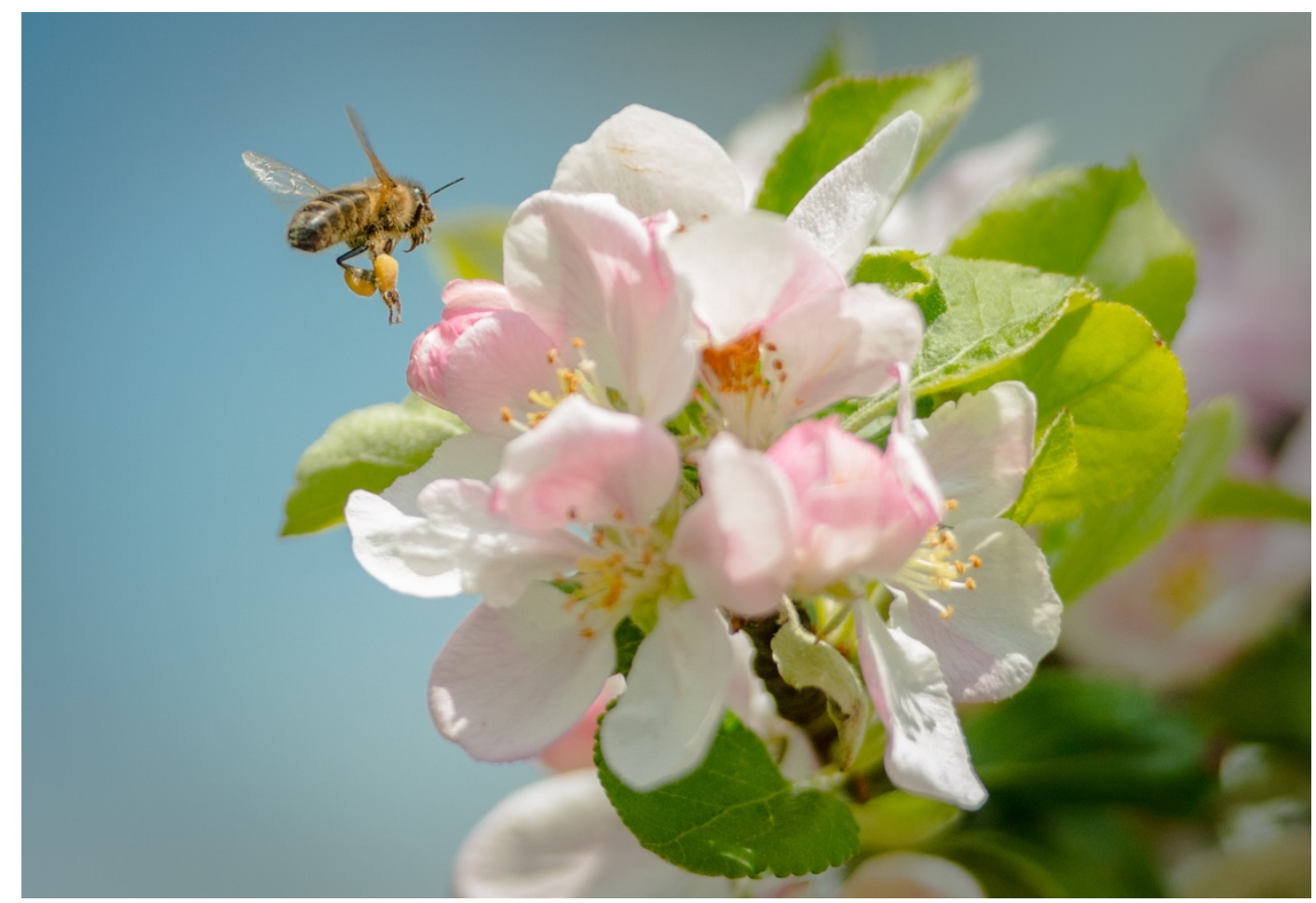

Image 18

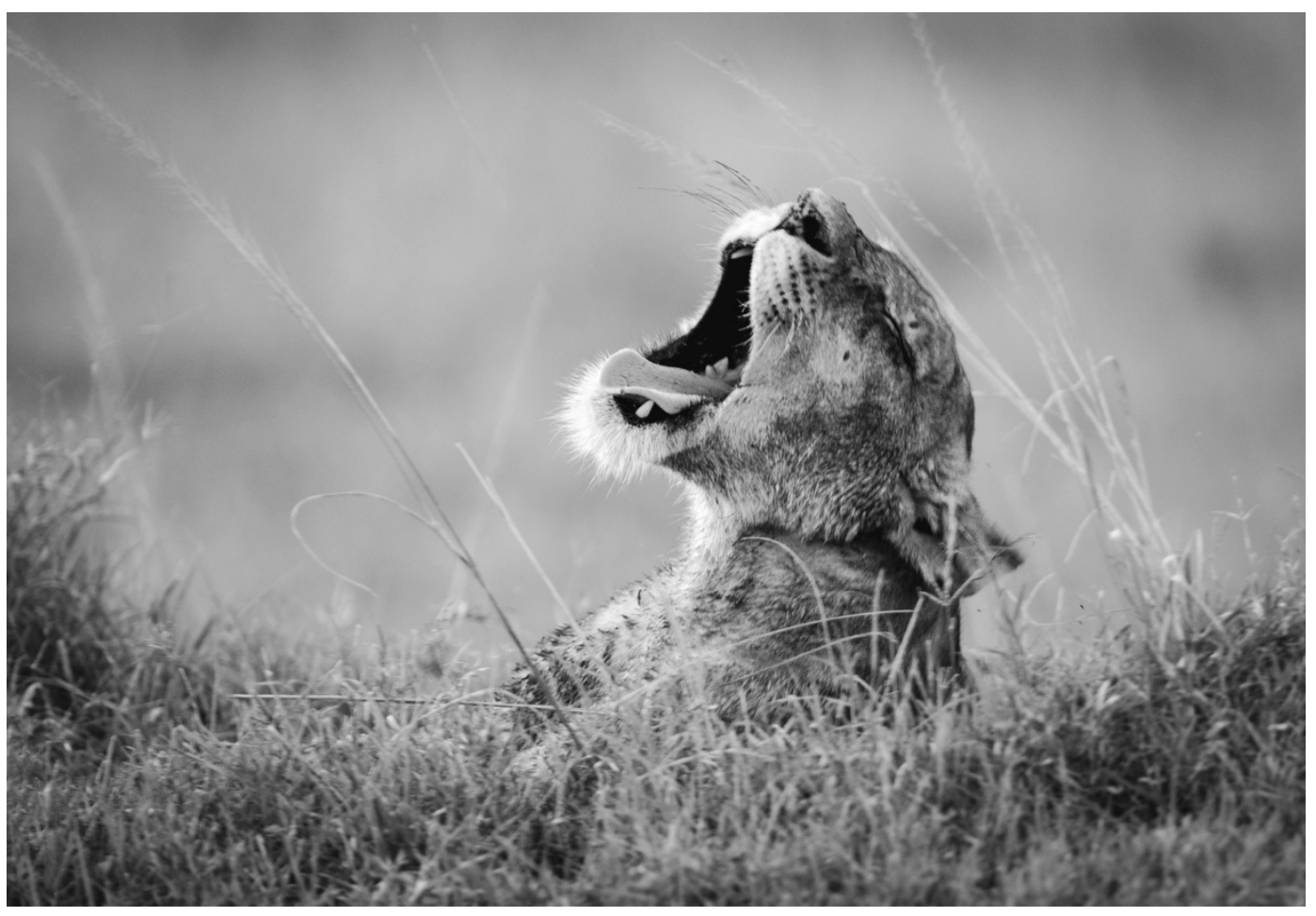

Image 19 


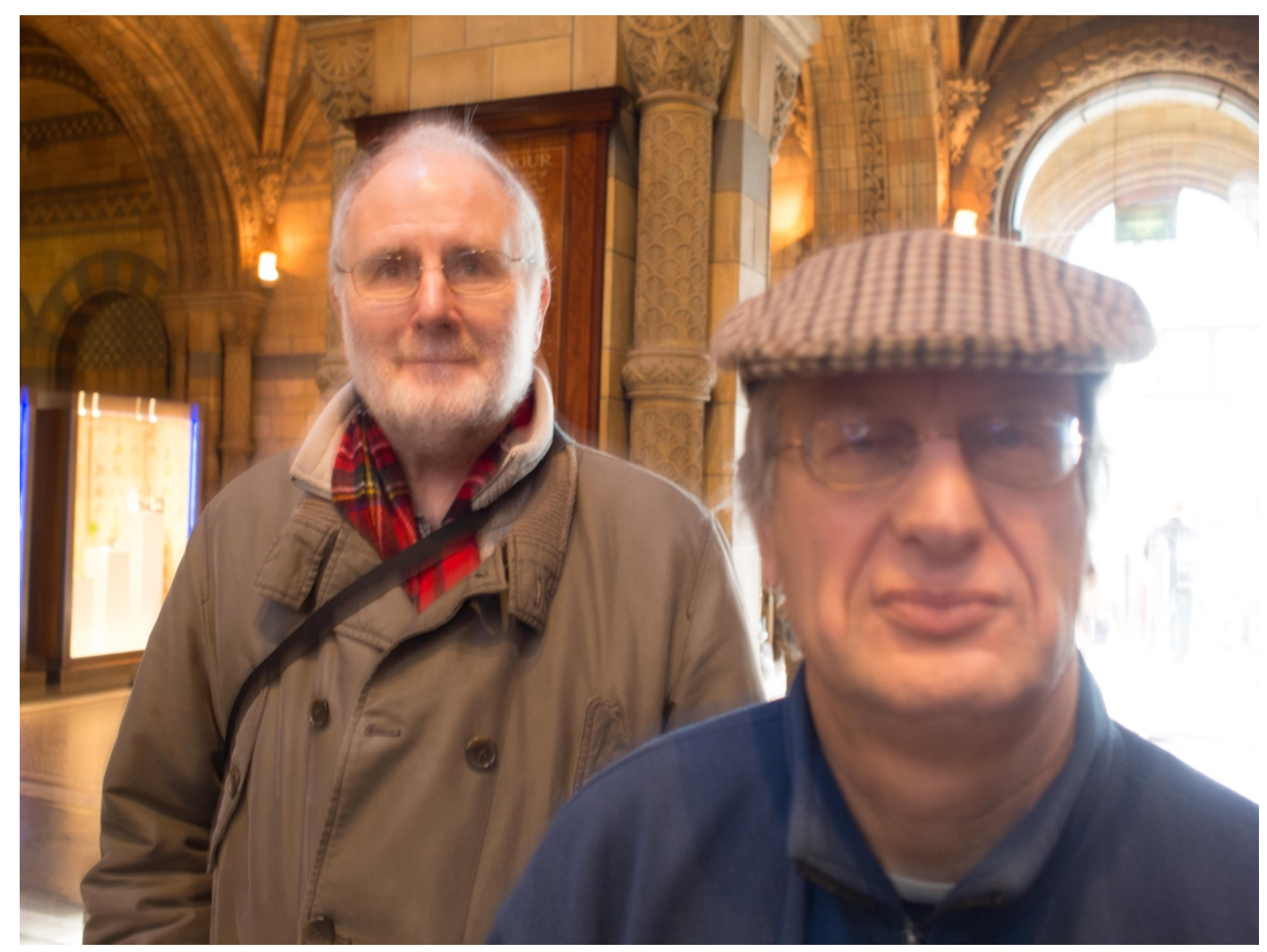

Image 20

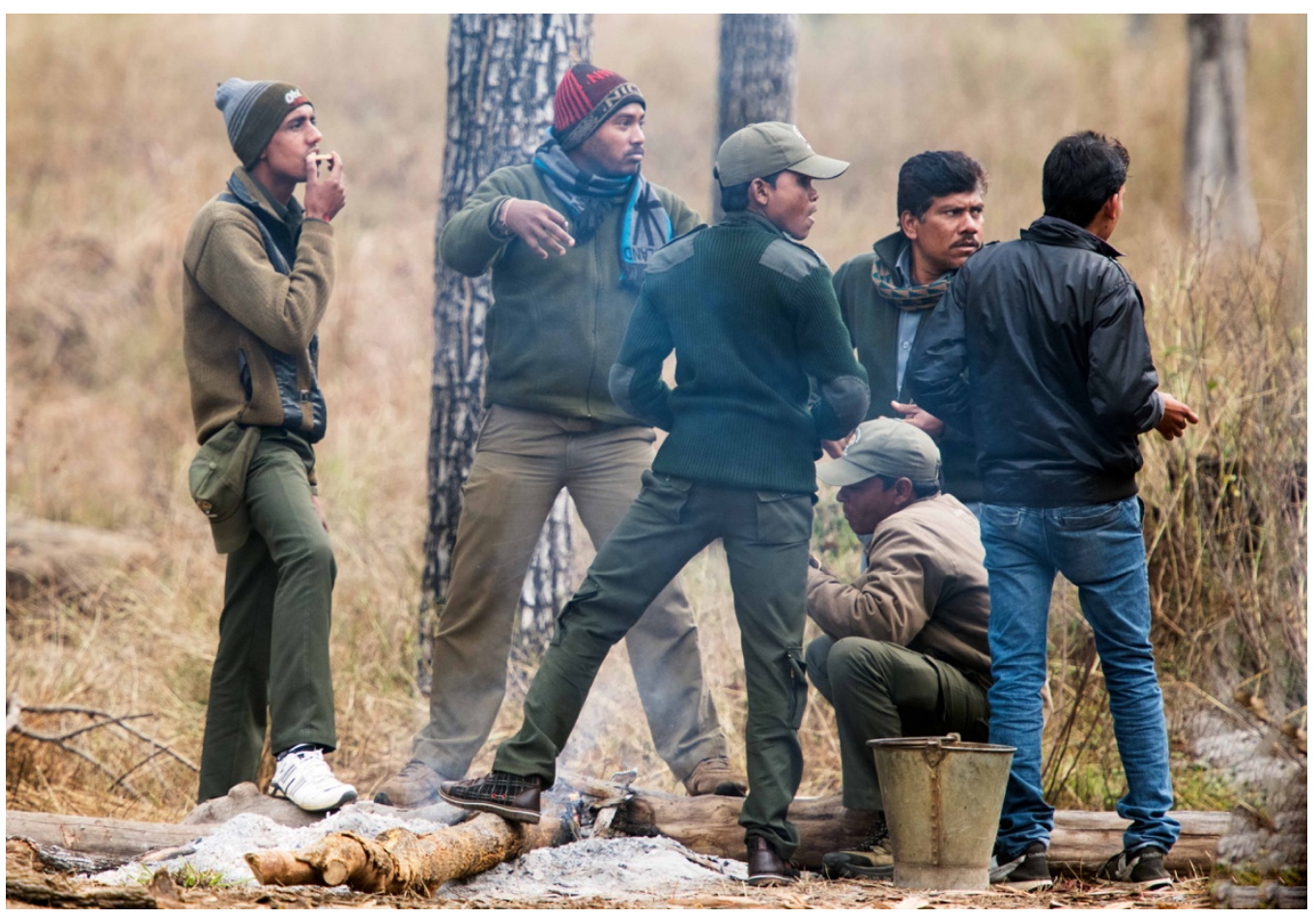

Image 21 


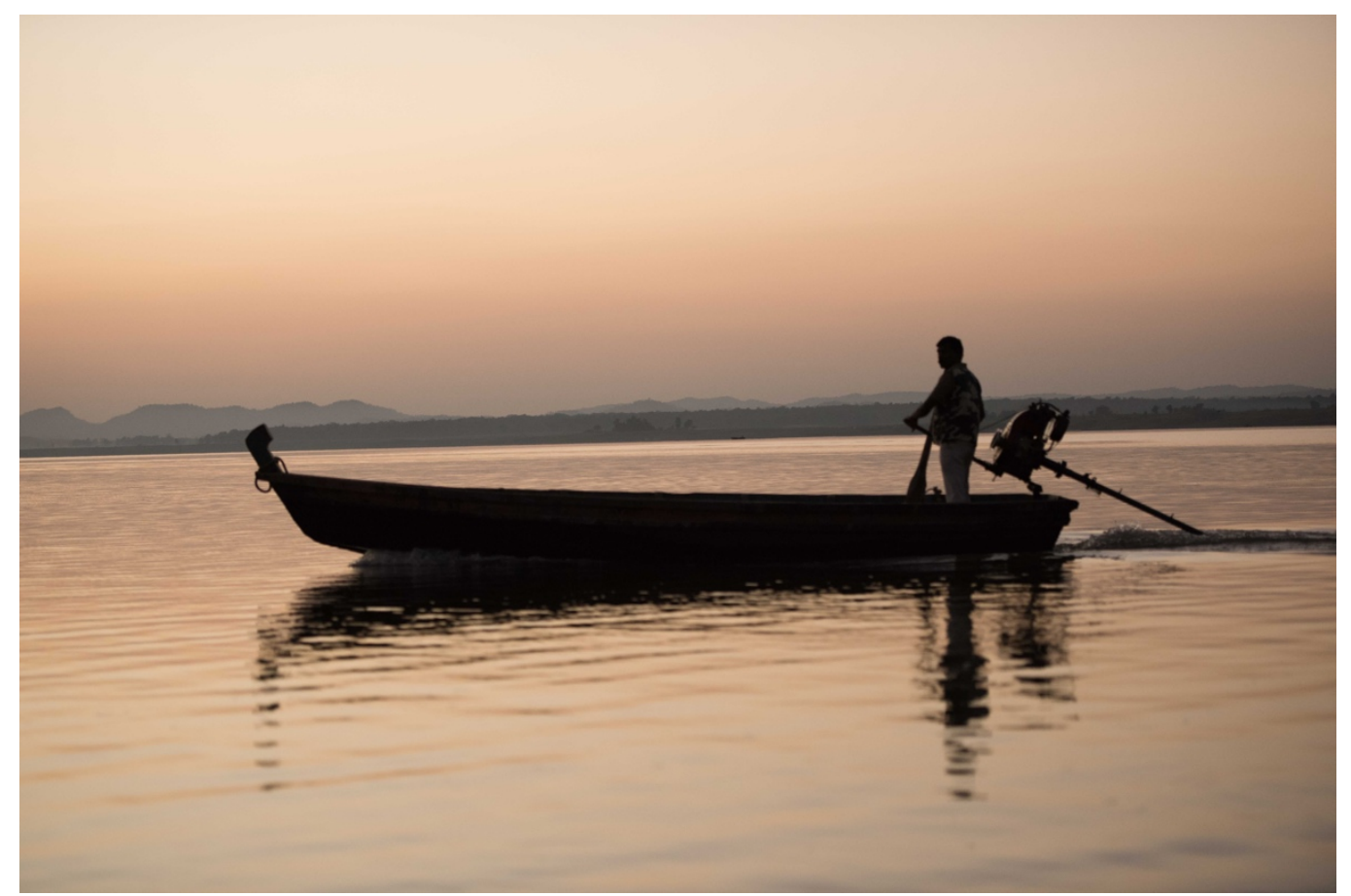

Image 22

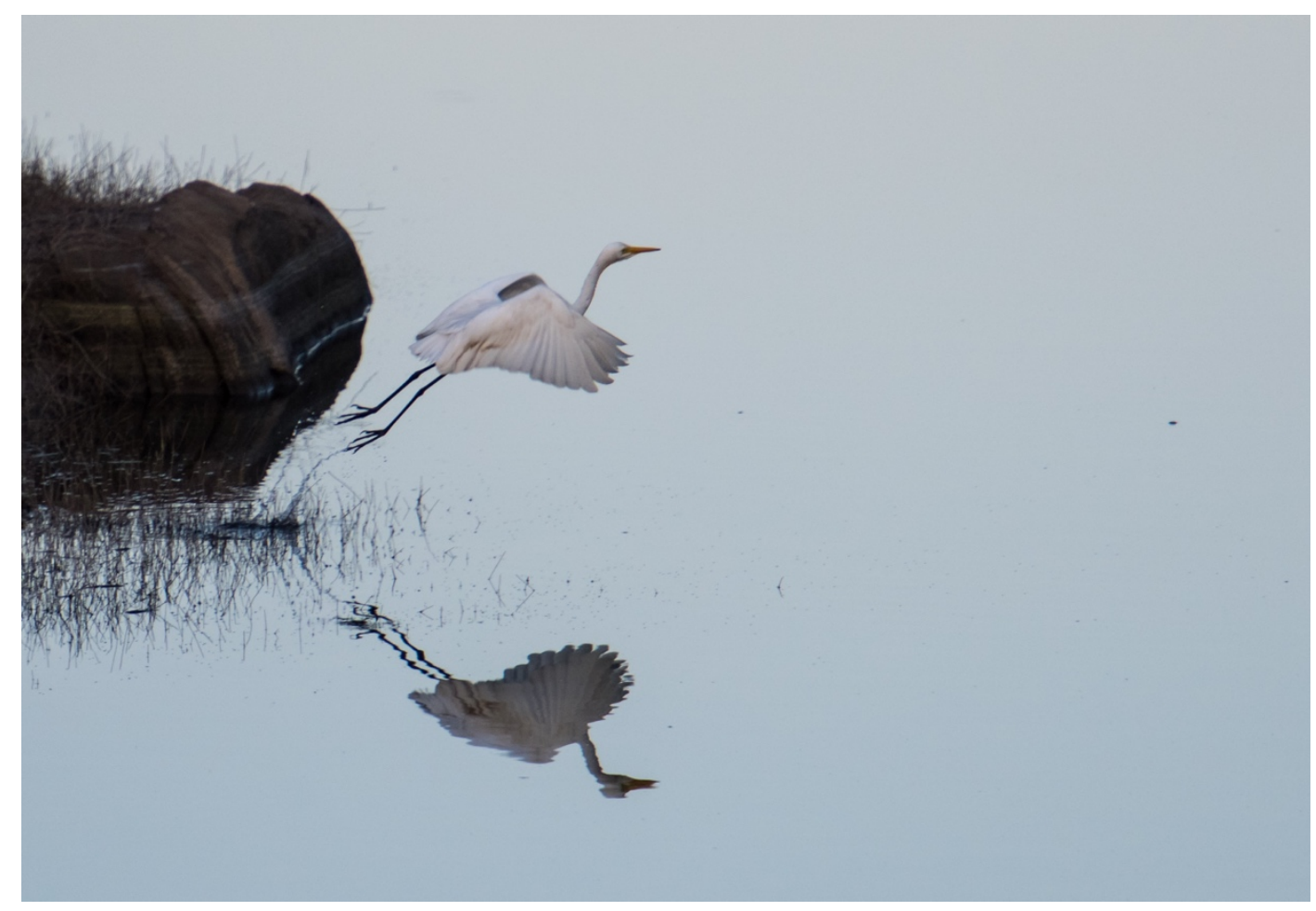

Image 23 


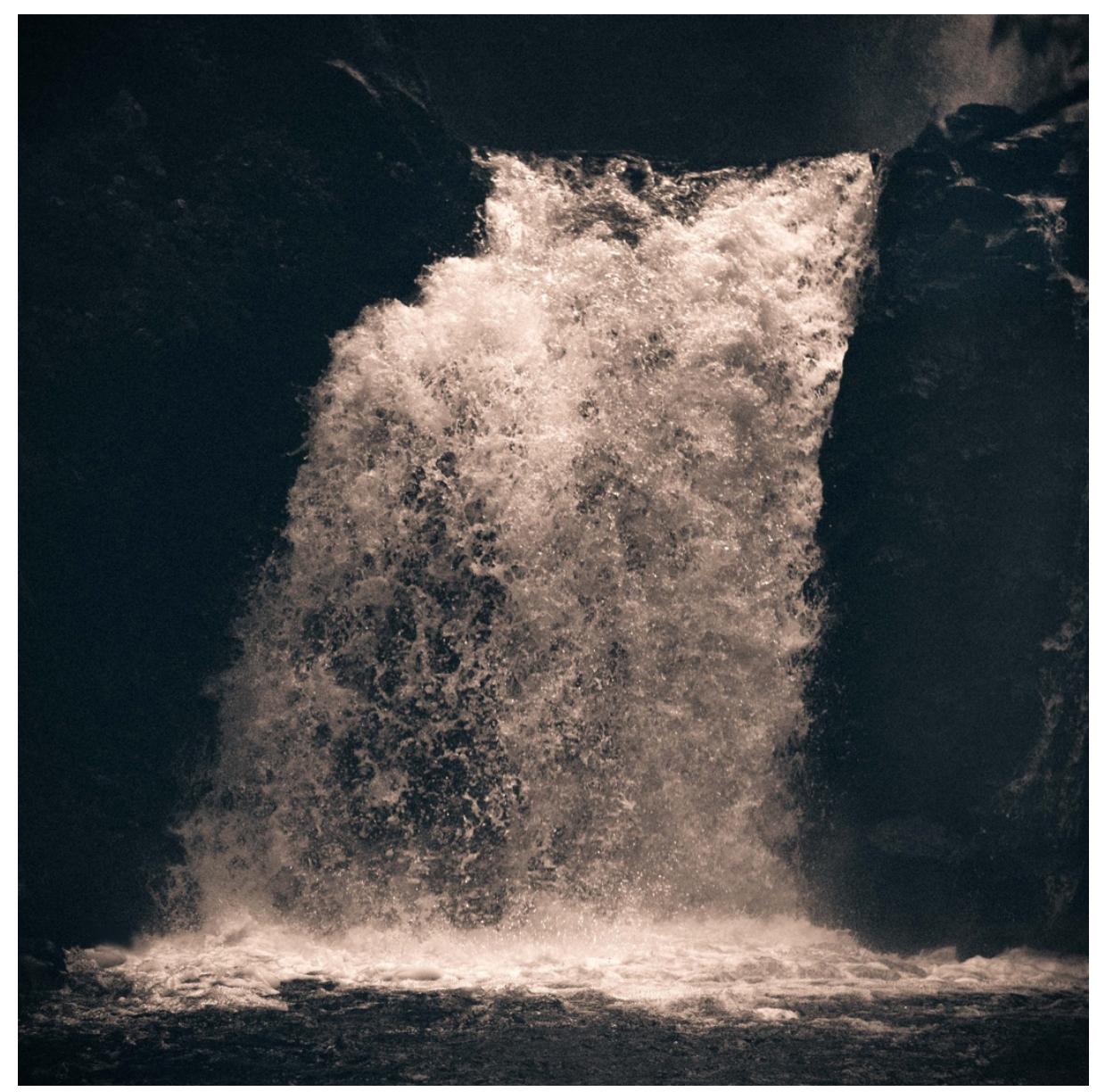

Image 24

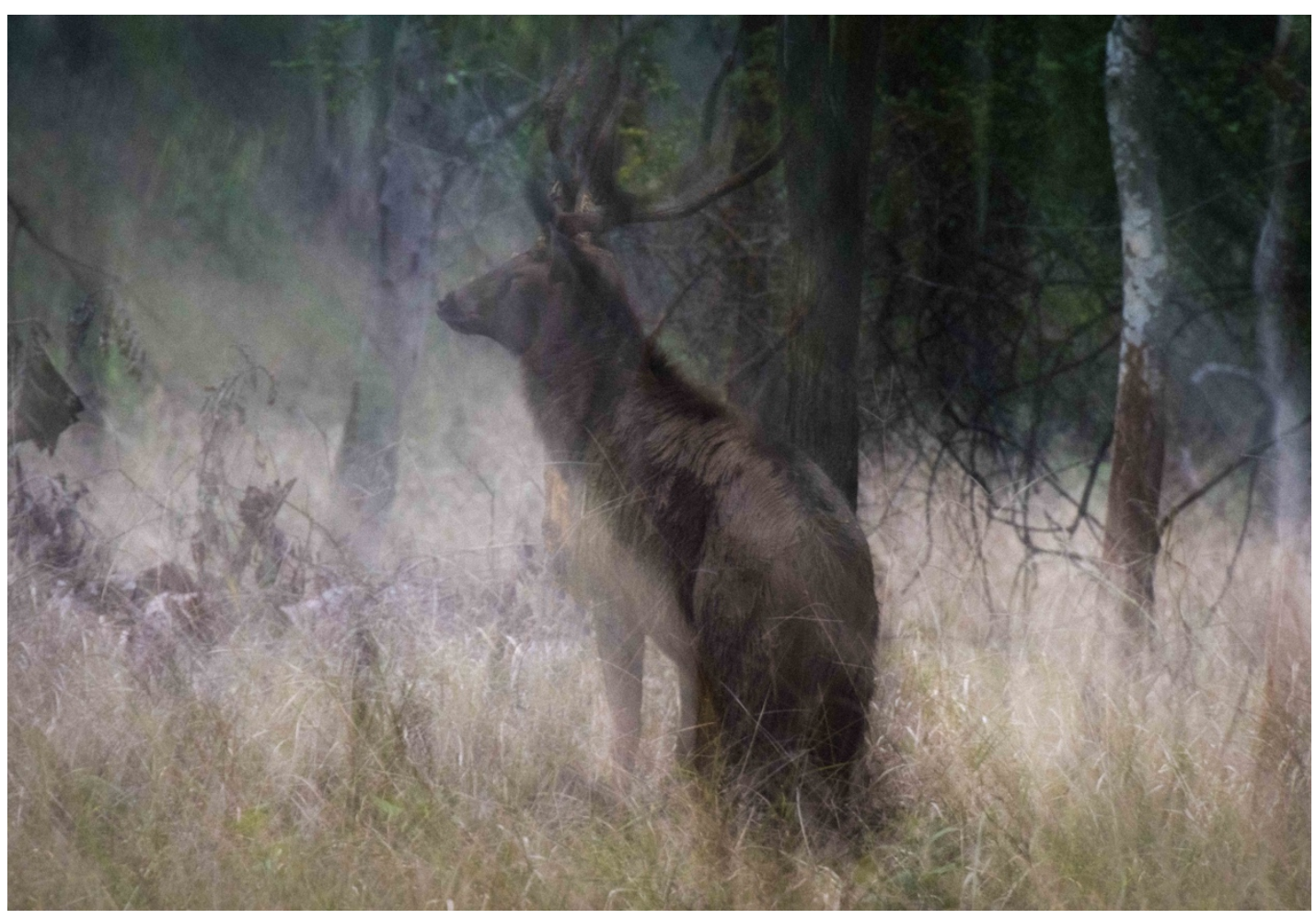

Image 25 


\section{Permissions:}

\section{IT0069 Chris Weston Chapter 8}

Re: Chapter 8 final view

Chris Weston

CW Bob Ryan

Show Details

Hi Bob

Thanks for the draft of Chapter 8, it makes fascinating reading. I'm very happy for you to include it in your thesis. Best of luck!

Chris

$>$ On 14 Jul 2019, at 23:39, Bob Ryan

wrote:

> 〈Chapter $8(\mathrm{v} 3)$.docx> 


\section{IT0079 Neil McCoubrey Chapter 6}

Re: Intraspective Interview

Neil McCoubrey
Bob Ryan
Tuesday, 9 April 2019 at $22: 22$
Show Details
Hi Bob
Thanks for sending these papers. I haven't had the chance to read them yet as I have spent the last 2 weeks in Shetland on a field trip. I go home tomorrow with
months worth of new and retested data/images. So I will read your documents when I return.
Even without reading the materials I am happy for you to use my full name in your thesis.
I will get back to you shortly.

Regards

Neil McCoubrey

www.neilmccoubrey.com

\section{IT0084 Simon Stafford Chapter 6}

\section{IMP Test \& Interview}

\section{Simon Stafford \\ SS Bob Ryan; Simon Stafford \\ Sunday, 31 March 2019 at 20:03 \\ Show Details}

You replied to this message on 01/04/2019, 10:13.

Dear Bob,

Thank you once again for the opportunity to be involved with your research and to take part in the IMP test and interview process.

I would be more than happy for you to include the analysis of my photographic skills in any published results of your research; if it is warranted you may also identify me by name.

I am most grateful for the fulsome feedback you have provided following the testing and the time you have spent discussing the results with me; it has been a very enlightening experience and one that I will certainly be drawing upon for my photography in the future.

If I can be of any further assistance please do not hesitate to ask.

Kindest regards,

Simon

\section{Simon Stafford Photography}

e:

w: www.simonstafford.co.uk

Overall Winner - Mammals - Wildlife Photographer of the Year 2016

Technical Editor to Nikon Owner magazine

Author of 'The Nikon Compendium' \& Magic Lantern Guides to the Nikon camera system published by Sterling Publishing 


\section{IT0106 Chapter 6 Simon Weir}

RQ Re: IMP Test and Interview

\section{Simon Weir}

SW

ROBERT RYAN

Monday, 15 July 2019 at 14:45

Show Details

You replied to this message on 15/07/2019, 15:19.

together sometime to share thoughts

All the best

Bob

From: Simon Weir

Date: Wednesday, 19 June 2019 at 17:28

To: ROBERT RYAN

Subject: Re: IMP Test and Interview

Hi Bob

Thanks so much for all the work you have done on the IMP test and my report - it is truly fascinating and will take me some time to fully digest. The whole process has been really positive for me and in some ways is the first proper validation of my ever-developing photographic style and technique.

I am more than happy for you to use my full name in the report - nothing there too embarrassing and I am proud of the outcome.

May I beg a little more time to send you my proper reflections on the piece and the process - I have been rushed off my feet since returning from Finland / Italy and wont really have a chance to reflect calmly until next week.

All best to you both - Simon

Simon Weir - Producer \& Photographer 
DATA FILES (specimen)

The working files are kept in this format:

\begin{tabular}{|c|c|c|c|c|}
\hline \multirow[t]{5}{*}{ Average } & stdev & 1 & 2 & \\
\hline & Cluster Flag & 1 & 1 & \\
\hline & Partition Flag & P1 & P1 & \\
\hline & Reference & IT0001 & IT0002 & \\
\hline & Gender & $\mathrm{M}$ & M & \\
\hline \multirow[t]{12}{*}{61.03} & Age & 55 & & 68 \\
\hline & Qualification flag & 1 & & 1 \\
\hline & REI Flag $(2=I, R=1)$ & 2 & & 2 \\
\hline & Qual1 & FRPS & ARPS & \\
\hline & Qual2 & 0 & & 0 \\
\hline & Qual3 & 0 & & 0 \\
\hline & Qual4 & HND & FCA & \\
\hline & ascription & Professional & Educator & \\
\hline & & Black and & & \\
\hline & Genre & White & Nature & \\
\hline & Output & Print & Print & \\
\hline & Judge & Yes & Yes & \\
\hline \multirow[t]{2}{*}{9.89} & Years & 10 & & 4 \\
\hline & Level & International & club & \\
\hline 3.88 & RA & 3.3 & & 3.8 \\
\hline 3.87 & $\mathrm{RE}$ & 3.2 & & 4.4 \\
\hline 3.44 & EA & 3.5 & & 4.2 \\
\hline 3.33 & $\mathrm{EE}$ & 4.1 & & 4.2 \\
\hline
\end{tabular}


REI differential

aptitude

Flag (female)

$29.4 \%$

\section{SUBMITTED DATA}

53.01

18.28

54.21

16.45

69.33

18.17

65.95

18.16

47.31

21.12

55.34

22.10

53.46

18.25

69.79

19.83

59.06

18.52

73.00

18.62

60.42

18.29

57.42

19.46

67.35

19.61

70.53

17.78

31.14

20.61

51.10

19.54

51.52

20.37

56.92

21.30

52.97

18.97

52.37

23.37

5.36

1.75

6.72
6 Intuitive

7 Intuitive

8 Intuitive

9 Intuitive

10 Intuitive

11 Intuitive

12 Intuitive

13 Intuitive

14 Intuitive

15 Intuitive

16 Intuitive

17 Intuitive

18 Intuitive

19 Intuitive

20 Intuitive

21 Intuitive

22 Intuitive

23 Intuitive

24 Intuitive

25 Intuitive

6 Intuitive

6 Impact
50

40

80

50

80

60

40

80

60

90

50

80

80

80

30

60

20

60

60

70

80

2

4 


\begin{tabular}{|c|c|c|c|c|c|}
\hline 5.58 & 1.99 & 6 & Creativity & 3 & 6 \\
\hline 7.32 & 1.67 & 6 & Narrative & 7 & 8 \\
\hline 6.18 & 2.05 & 6 & DoF & 7 & 4 \\
\hline 5.81 & 1.91 & 6 & Light & 4 & 3 \\
\hline 5.67 & 1.80 & 6 & Comp & 3 & 4 \\
\hline 5.92 & 1.71 & 6 & Col/tone & 2 & 7 \\
\hline 6.67 & 1.86 & 6 & Focus & 4 & 5 \\
\hline 7.01 & 1.70 & 6 & Exposure & 4 & 4 \\
\hline 6.15 & 1.94 & 6 & Technical & 5 & 2 \\
\hline 5.48 & 1.56 & 7 & Intuitive & 4 & 6 \\
\hline 5.44 & 1.61 & 7 & Impact & 4 & 6 \\
\hline 4.67 & 1.64 & 7 & Creativity & 4 & 5 \\
\hline 4.56 & 1.95 & 7 & Narrative & 3 & 4 \\
\hline 7.24 & 1.81 & 7 & DoF & 4 & 8 \\
\hline 5.76 & 2.00 & 7 & Light & 3 & 6 \\
\hline 5.19 & 2.00 & 7 & Comp & 2 & 6 \\
\hline 6.23 & 1.75 & 7 & Col/tone & 1 & 6 \\
\hline 7.55 & 1.60 & 7 & Focus & 2 & 8 \\
\hline 7.38 & 1.46 & 7 & Exposure & 5 & 7 \\
\hline 6.07 & 1.91 & 7 & Technical & 5 & 4 \\
\hline 7.00 & 1.68 & 8 & Intuitive & 8 & 4.5 \\
\hline 8.14 & 1.49 & 8 & Impact & 8 & 6 \\
\hline 6.27 & 1.96 & 8 & Creativity & 5 & 4 \\
\hline 6.96 & 1.89 & 8 & Narrative & 8 & 5 \\
\hline 8.18 & 1.14 & 8 & DoF & 7 & 7 \\
\hline 7.25 & 1.76 & 8 & Light & 6 & 5 \\
\hline 7.50 & 1.51 & 8 & Comp & 5 & 6 \\
\hline 6.93 & 1.69 & 8 & Col/tone & 7 & 3 \\
\hline 8.35 & 1.20 & 8 & Focus & 8 & 8 \\
\hline 7.78 & 1.52 & 8 & Exposure & 8 & 6 \\
\hline
\end{tabular}




\begin{tabular}{|c|c|c|c|c|c|}
\hline 7.64 & 1.64 & 8 & Technical & 5 & 6 \\
\hline 6.66 & 1.70 & 9 & Intuitive & 5 & 6 \\
\hline 6.75 & 1.62 & 9 & Impact & 4 & 6 \\
\hline 6.00 & 1.85 & 9 & Creativity & 3 & 4 \\
\hline 5.59 & 1.78 & 9 & Narrative & 2 & 4 \\
\hline 7.32 & 1.60 & 9 & DoF & 6 & 7 \\
\hline 7.83 & 1.25 & 9 & Light & 6 & 7 \\
\hline 6.99 & 1.56 & 9 & Comp & 6 & 7 \\
\hline 7.51 & 1.35 & 9 & Col/tone & 7 & 8 \\
\hline 7.34 & 1.42 & 9 & Focus & 7 & 8 \\
\hline 7.78 & 1.29 & 9 & Exposure & 6 & 7 \\
\hline 6.88 & 1.67 & 9 & Technical & 5 & 7 \\
\hline 4.79 & 2.07 & 10 & Intuitive & 8 & 5 \\
\hline 4.63 & 2.43 & 10 & Impact & 8 & 5 \\
\hline 4.57 & 2.43 & 10 & Creativity & 3 & 4 \\
\hline 3.66 & 2.28 & 10 & Narrative & 8 & 3 \\
\hline 6.38 & 1.75 & 10 & DoF & 5 & 7 \\
\hline 6.29 & 2.23 & 10 & Light & 1 & 8 \\
\hline 4.95 & 2.25 & 10 & Comp & 2 & 6 \\
\hline 5.98 & 1.87 & 10 & Col/tone & 4 & 7 \\
\hline 7.29 & 1.59 & 10 & Focus & 5 & 8 \\
\hline 7.26 & 1.50 & 10 & Exposure & 5 & 7 \\
\hline 6.10 & 2.01 & 10 & Technical & 5 & 7 \\
\hline 5.60 & 2.15 & 11 & Intuitive & 6 & 3 \\
\hline 6.93 & 2.13 & 11 & Impact & 7 & 5 \\
\hline 7.64 & 1.92 & 11 & Creativity & 8 & 8 \\
\hline 5.55 & 2.37 & 11 & Narrative & 6 & 7 \\
\hline 6.35 & 2.04 & 11 & DoF & 4 & 7 \\
\hline 6.29 & 2.00 & 11 & Light & 3 & 6 \\
\hline 6.29 & 2.20 & 11 & Comp & 4 & . \\
\hline
\end{tabular}




\begin{tabular}{|c|c|c|c|c|c|}
\hline 6.10 & 1.96 & 11 & Col/tone & 4 & 6 \\
\hline 6.75 & 1.77 & 11 & Focus & 7 & 7 \\
\hline 6.30 & 1.97 & 11 & Exposure & 7 & 7 \\
\hline 6.67 & 2.07 & 11 & Technical & 5 & 8 \\
\hline 5.41 & 1.76 & 12 & Intuitive & 4 & 5 \\
\hline 6.79 & 1.75 & 12 & Impact & 6 & 8 \\
\hline 6.52 & 1.81 & 12 & Creativity & 4 & 7 \\
\hline 6.41 & 1.69 & 12 & Narrative & 3 & 6 \\
\hline 6.54 & 1.60 & 12 & DoF & 5 & 8 \\
\hline 6.29 & 1.64 & 12 & Light & 2 & 6 \\
\hline 6.05 & 1.70 & 12 & Comp & 1 & 7 \\
\hline 5.89 & 1.64 & 12 & Col/tone & 2 & 7 \\
\hline 6.84 & 1.73 & 12 & Focus & 5 & 8 \\
\hline 6.68 & 1.53 & 12 & Exposure & 5 & 7 \\
\hline 6.08 & 1.73 & 12 & Technical & 5 & 6 \\
\hline 7.05 & 1.86 & 13 & Intuitive & 8 & 7 \\
\hline 8.22 & 1.55 & 13 & Impact & 9 & 8 \\
\hline 6.94 & 1.91 & 13 & Creativity & 6 & 7 \\
\hline 8.36 & 1.44 & 13 & Narrative & 9 & 8 \\
\hline 7.68 & 1.75 & 13 & DoF & 8 & 8 \\
\hline 7.28 & 1.90 & 13 & Light & 7 & 7 \\
\hline 7.87 & 1.69 & 13 & Comp & 9 & 9 \\
\hline 7.04 & 1.68 & 13 & Col/tone & 8 & 8 \\
\hline 7.56 & 1.93 & 13 & Focus & 9 & 9 \\
\hline 7.36 & 2.00 & 13 & Exposure & 9 & 9 \\
\hline 7.48 & 1.89 & 13 & Technical & 5 & 8 \\
\hline 5.97 & 1.76 & 14 & Intuitive & 6 & 5.5 \\
\hline 5.74 & 1.88 & 14 & Impact & 4 & 7 \\
\hline 5.61 & 2.04 & 14 & Creativity & 1 & 7 \\
\hline 5.08 & 2.04 & 14 & Narrative & 8 & 6 \\
\hline
\end{tabular}




\begin{tabular}{|c|c|c|c|c|c|}
\hline 6.75 & 1.86 & 14 & DoF & 5 & 8 \\
\hline 6.82 & 1.99 & 14 & Light & 4 & 7 \\
\hline 6.12 & 2.02 & 14 & Comp & 3 & 5 \\
\hline 6.59 & 1.96 & 14 & Col/tone & 3 & 7 \\
\hline 7.42 & 1.67 & 14 & Focus & 5 & 7 \\
\hline 7.49 & 1.53 & 14 & Exposure & 5 & 7 \\
\hline 6.88 & 1.82 & 14 & Technical & 5 & 7 \\
\hline 7.38 & 1.72 & 15 & Intuitive & 9 & 6 \\
\hline 7.96 & 1.57 & 15 & Impact & 9 & 6 \\
\hline 6.99 & 1.85 & 15 & Creativity & 6 & 5 \\
\hline 7.71 & 1.52 & 15 & Narrative & 9 & 7 \\
\hline 8.08 & 1.51 & 15 & DoF & 8 & 8 \\
\hline 7.89 & 1.37 & 15 & Light & 7 & 7 \\
\hline 7.85 & 1.28 & 15 & Comp & 8 & 6 \\
\hline 7.70 & 1.39 & 15 & Col/tone & 8 & 7 \\
\hline 8.28 & 1.45 & 15 & Focus & 9 & 8 \\
\hline 8.22 & 1.14 & 15 & Exposure & 7 & 7 \\
\hline 7.92 & 1.57 & 15 & Technical & 5 & 8 \\
\hline 6.11 & 1.74 & 16 & Intuitive & 5 & 7.5 \\
\hline 6.34 & 1.65 & 16 & Impact & 6 & 9 \\
\hline 5.75 & 1.88 & 16 & Creativity & 4 & 7 \\
\hline 6.92 & 1.96 & 16 & Narrative & 4 & 8 \\
\hline 7.15 & 1.70 & 16 & DoF & 7 & 9 \\
\hline 6.28 & 2.01 & 16 & Light & 3 & 8 \\
\hline 6.78 & 1.82 & 16 & Comp & 2 & 8 \\
\hline 5.43 & 2.21 & 16 & Col/tone & 4 & 8 \\
\hline 6.34 & 1.77 & 16 & Focus & 5 & 8 \\
\hline 6.47 & 1.85 & 16 & Exposure & 5 & 6 \\
\hline 5.94 & 1.76 & 16 & Technical & 5 & 7 \\
\hline 5.81 & 1.87 & 17 & Intuitive & 8 & 3 \\
\hline
\end{tabular}




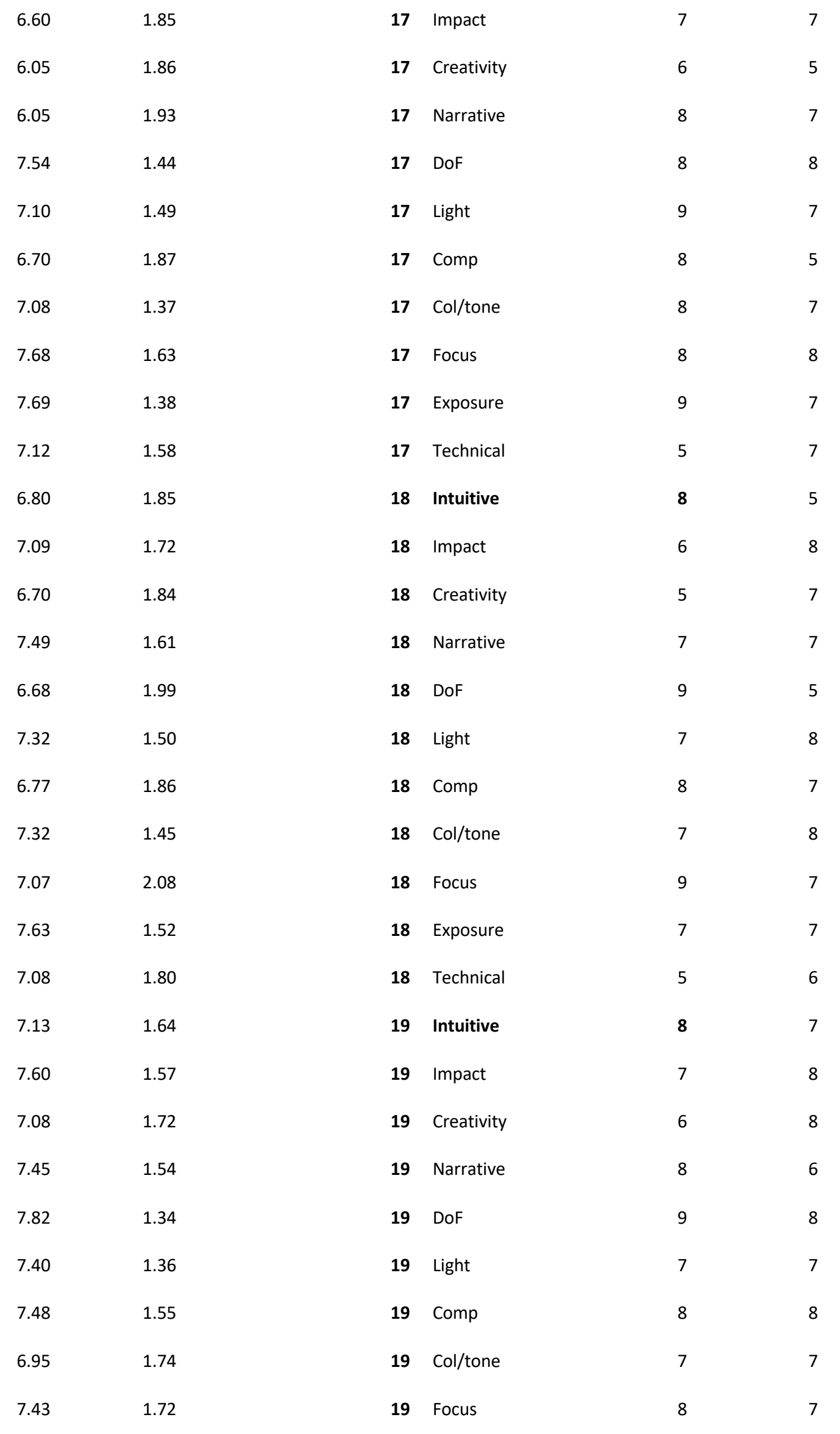




\begin{tabular}{|c|c|c|c|c|c|}
\hline 7.50 & 1.57 & 19 & Exposure & 7 & 5 \\
\hline 7.18 & 1.55 & 19 & Technical & 5 & 7 \\
\hline 3.18 & 2.06 & 20 & Intuitive & 3 & 2 \\
\hline 3.59 & 2.32 & 20 & Impact & 2 & 7 \\
\hline 3.40 & 2.39 & 20 & Creativity & 6 & 5 \\
\hline 3.92 & 2.26 & 20 & Narrative & 4 & 5 \\
\hline 2.90 & 2.05 & 20 & DoF & 3 & 3 \\
\hline 2.86 & 1.83 & 20 & Light & 1 & 2 \\
\hline 3.70 & 2.15 & 20 & Comp & 2 & 5 \\
\hline 3.69 & 1.97 & 20 & Col/tone & 3 & 6 \\
\hline 2.00 & 1.67 & 20 & Focus & 2 & 1 \\
\hline 2.98 & 1.82 & 20 & Exposure & 1 & 2 \\
\hline 2.60 & 1.73 & 20 & Technical & 5 & 2 \\
\hline 5.17 & 1.89 & 21 & Intuitive & 6 & 4.5 \\
\hline 6.57 & 1.96 & 21 & Impact & 5 & 5 \\
\hline 6.00 & 1.91 & 21 & Creativity & 4 & 4 \\
\hline 7.50 & 1.71 & 21 & Narrative & 3 & 6 \\
\hline 7.23 & 1.48 & 21 & DoF & 5 & 7 \\
\hline 6.92 & 1.39 & 21 & Light & 4 & 6 \\
\hline 6.89 & 1.65 & 21 & Comp & 7 & 5 \\
\hline 6.91 & 1.53 & 21 & Col/tone & 4 & 7 \\
\hline 7.21 & 1.43 & 21 & Focus & 7 & 8 \\
\hline 7.36 & 1.23 & 21 & Exposure & 4 & 8 \\
\hline 6.80 & 1.47 & 21 & Technical & 5 & 6 \\
\hline 5.22 & 1.99 & 22 & Intuitive & 2 & 4 \\
\hline 6.15 & 1.92 & 22 & Impact & 3 & 6 \\
\hline 5.76 & 1.96 & 22 & Creativity & 3 & 3 \\
\hline 6.41 & 1.58 & 22 & Narrative & 4 & 6 \\
\hline 5.93 & 1.97 & 22 & DoF & 5 & 8 \\
\hline 7.07 & 1.69 & 22 & Light & 8 & 7 \\
\hline
\end{tabular}




\begin{tabular}{|c|c|c|c|c|c|}
\hline 6.17 & 1.90 & 22 & Comp & 6 & 6 \\
\hline 6.72 & 1.64 & 22 & Col/tone & 7 & 7 \\
\hline 5.24 & 2.41 & 22 & Focus & 0 & 7 \\
\hline 6.44 & 1.72 & 22 & Exposure & 6 & 7 \\
\hline 5.31 & 2.02 & 22 & Technical & 1 & 3 \\
\hline 5.77 & 2.07 & 23 & Intuitive & 6 & 5.5 \\
\hline 6.47 & 2.05 & 23 & Impact & 5 & 7 \\
\hline 6.34 & 1.99 & 23 & Creativity & 4 & 7 \\
\hline 6.21 & 1.91 & 23 & Narrative & 4 & 5 \\
\hline 6.19 & 2.08 & 23 & DoF & 5 & 8 \\
\hline 6.09 & 2.03 & 23 & Light & 4 & 7 \\
\hline 6.15 & 1.98 & 23 & Comp & 3 & 7 \\
\hline 5.72 & 1.93 & 23 & Col/tone & 3 & 5 \\
\hline 5.96 & 2.16 & 23 & Focus & 3 & 8 \\
\hline 6.43 & 1.89 & 23 & Exposure & 5 & 8 \\
\hline 5.84 & 2.22 & 23 & Technical & 5 & 7 \\
\hline 5.37 & 1.84 & 24 & Intuitive & 6 & 4 \\
\hline 6.38 & 1.83 & 24 & Impact & 6 & 5 \\
\hline 5.76 & 1.89 & 24 & Creativity & 6 & 5 \\
\hline 5.19 & 2.03 & 24 & Narrative & 4 & 4 \\
\hline 6.55 & 1.49 & 24 & DoF & 5 & 7 \\
\hline 6.89 & 1.81 & 24 & Light & 3 & 7 \\
\hline 6.09 & 1.88 & 24 & Comp & 3 & 5 \\
\hline 6.01 & 1.93 & 24 & Col/tone & 4 & 6 \\
\hline 6.97 & 1.70 & 24 & Focus & 7 & 8 \\
\hline 6.54 & 1.94 & 24 & Exposure & 7 & 8 \\
\hline 6.14 & 1.99 & 24 & Technical & 5 & 6 \\
\hline 5.31 & 2.29 & 25 & Intuitive & 7 & 8 \\
\hline 5.97 & 2.18 & 25 & Impact & 5 & 9 \\
\hline 6.67 & 2.26 & 25 & Creativity & 6 & 9 \\
\hline
\end{tabular}




$\begin{array}{ll}5.92 & 2.16 \\ 5.40 & 2.09 \\ 6.30 & 2.23 \\ 6.16 & 4.37 \\ 5.85 & 2.22 \\ 4.72 & 2.32 \\ 5.63 & 2.07 \\ 5.17 & 2.40\end{array}$

25 Narrative

6

25 DoF $\quad 4 \quad 8$

25 Light $\quad 8 \quad 8$

$\begin{array}{llll}25 & \text { Comp } & 6 & 7\end{array}$

$25 \mathrm{Col} /$ tone $\quad 6 \quad 8$

25 Focus $\quad 4 \quad 9$

25 Exposure $\quad 5 \quad 8$

$\begin{array}{llll}25 & \text { Technical } & 5 & 8\end{array}$

Declared

$\begin{array}{lllll}824.92 & 179.79 & \text { IRATRANK } & 567 & 1000 \\ 738.20 & 173.27 & \text { CrRATRANK } & 805 & 679 \\ 696.24 & 203.22 & \text { NRATRANK } & 263 & 887 \\ 655.96 & 189.11 & \text { DRATRANK } & 780 & 706 \\ 798.16 & 172.25 & \text { LRATRANK } & 747 & 888 \\ 787.21 & 163.41 & \text { CORATRANK } & 851 & 477 \\ 687.70 & 166.95 & \text { CTRATRANK } & 729 & 574 \\ 738.66 & 197.22 & \text { FRATRANK } & 431 & 818 \\ 727.93 & 191.78 & \text { ERATRANK } & 797 & 944 \\ 658.96 & 204.59 & \text { TRATRANK } & 510 & 923\end{array}$

OUTPUT DATA

\begin{tabular}{ccccc} 
OUTPUT DATA & \multicolumn{2}{c}{ RANKING DATA } & Model & \\
1.20 & 0.31 & IMODRANK & 1.294 & 1.114 \\
0.97 & 0.33 & CrMODRANK & 0.570 & 0.696 \\
0.94 & 0.33 & NMODRANK & 1.160 & 0.039 \\
0.96 & 0.25 & DMODRANK & 1.031 & 1.180 \\
0.97 & 0.25 & LMODRANK & 0.652 & 1.203 \\
0.98 & 0.25 & COMODRANK & 0.927 & 1.398 \\
0.87 & 0.32 & CTMODRANK & 0.908 & 0.649
\end{tabular}




$\begin{array}{llllr}0.87 & 0.30 & \text { FMODRANK } & 1.332 & 1.113 \\ 0.84 & 0.34 & \text { EMODRANK } & 1.015 & 0.830 \\ 0.86 & 0.38 & \text { TMODRANK } & 0.824 & 1.063\end{array}$

CORRELATION (IMAGE RANKING)

0.68

0.70

0.98

0.49

0.55

0.56

3.51

3.42

0.69

4.56

4.11

4.11

3.83

3.65

0.72

5.03

4.82

4.80

0.13

0.35

0.58

0.61
INTVRAT

INTVRAT*

RATVRAT*

INTv(Consensus)

RATv(Consensus)

$\mathrm{RAT}^{*} \mathrm{v}$ (Consensus)

DIFFERENCE ON RANKING

INTVRAT

INTVRAT*

RATVRAT*

INTv(Consensus)

RATv(Consensus)

RAT*v(Consensus) $^{*}$

DIFFERENCES ON PLACED IMAGES

INTVRAT

INTVRAT*

RATVRAT*

INTv(Consensus)

RATv(Consensus)

RAT*v(Consensus) $^{*}$

Correlation (Construct Ranking)

Q2 ON PLR

R2Y ON PLR

$R 2 X O N P L R$

\begin{tabular}{ll}
$74.21 \%$ & $70.82 \%$ \\
\hline $85.58 \%$ & $80.68 \%$ \\
\hline $54.14 \%$ & $96.09 \%$ \\
\hline $59.80 \%$ & $22.29 \%$ \\
\hline $73.68 \%$ & $38.95 \%$ \\
\hline $76.84 \%$ & $26.47 \%$
\end{tabular}

3.15

3.10

2.75

2.80

1.20

1.20

3.75

5.60

3.10

4.30

2.90

5.00

3.75

3.64

3.25

2.50

1.00

1.18

4.63

6.27

2.38

5.91

2.63

6.36

$-0.50$

$-0.03$

0.52

0.59

0.75

0.72

0.52

0.47 


$\begin{array}{lr}>=.9 & 0 \\ >=.8 & 5 \\ >=.7 & 24 \\ >=.6 & 27 \\ >=.5 & 21 \\ >=.4 & 11 \\ >=.3 & 12 \\ >=.2 & 3 \\ >=.1 & 1 \\ >=0 & 2\end{array}$

24

Individual Construct Analysis

$\begin{array}{lccr}\text { IMODRANK } & 89 & 1 & 1 \\ \text { CrMODRANK } & 52 & & \\ \text { NMODRANK } & 49 & 1 & 1 \\ \text { DMODRANK } & 47 & 1 & 1 \\ \text { LMODRANK } & 49 & & 1 \\ \text { COMODRANK } & 52 & & 1 \\ \text { CTMODRANK } & 45 & & \\ \text { FMODRANK } & 44 & 1 & 1 \\ \text { EMODRANK } & 36 & 1 & \\ \text { TMODRANK } & 49 & & \end{array}$

Individual Construct Ranking

$\begin{array}{cccc}\text { IMODRANK } & 319 & 2 & 4 \\ \text { CrMODRANK } & 591 & 10 & 8 \\ \text { NMODRANK } & 619 & 3 & 10 \\ \text { DMODRANK } & 617 & 4 & 3 \\ \text { LMODRANK } & 564 & 9 & 2 \\ \text { COMODRANK } & 524 & 6 & 1\end{array}$


CTMODRANK

FMODRANK

EMODRANK

TMODRANK

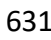

665

692

608

RANK Ist

IMODRANK

CrMODRANK

NMODRANK

DMODRANK

LMODRANK

COMODRANK

CTMODRANK

FMODRANK

EMODRANK

TMODRANK

Rank 2nd

$\begin{array}{lr}\text { IMODRANK } & 24 \\ \text { CrMODRANK } & 15 \\ \text { NMODRANK } & 5 \\ \text { DMODRANK } & 8 \\ \text { LMODRANK } & 16 \\ \text { COMODRANK } & 10 \\ \text { CTMODRANK } & 8 \\ \text { FMODRANK } & 4 \\ \text { EMODRANK } & 6 \\ \text { TMODRANK } & 10\end{array}$

Rank 3rd
41

8

9

5

5

14

1

6

4

6

8

1

16

10

10

6

10 


$\begin{array}{lr}\text { NMODRANK } & 16 \\ \text { DMODRANK } & 7 \\ \text { LMODRANK } & 11 \\ \text { COMODRANK } & 9 \\ \text { CTMODRANK } & 11 \\ \text { FMODRANK } & 12 \\ \text { EMODRANK } & 11 \\ \text { TMODRANK } & 12\end{array}$

Rank 4th

$\begin{array}{lcl}\text { IMODRANK } & 9 \\ \text { CrMODRANK } & 11 \\ \text { NMODRANK } & 10 & 1 \\ \text { DMODRANK } & 18 \\ \text { LMODRANK } & 8 \\ \text { COMODRANK } & 13 \\ \text { CTMODRANK } & 12 \\ \text { FMODRANK } & 8 \\ \text { EMODRANK } & 4 \\ \text { TMODRANK } & 8\end{array}$

Rank 5th

$\begin{array}{lcl}\text { IMODRANK } & 7 \\ \text { CrMODRANK } & 5 \\ \text { NMODRANK } & 7 \\ \text { DMODRANK } & 5 \\ \text { LMODRANK } & 6 & \\ \text { COMODRANK } & 5 & \\ \text { CTMODRANK } & 6 & \\ \text { FMODRANK } & 10 & 1 \\ \text { EMODRANK } & 7 & 1\end{array}$


Rank 6th

$\begin{array}{ll}\text { IMODRANK } & 1 \\ \text { CrMODRANK } & 3 \\ \text { NMODRANK } & 1 \\ \text { DMODRANK } & 3 \\ \text { LMODRANK } & 3 \\ \text { COMODRANK } & 1 \\ \text { CTMODRANK } & 2 \\ \text { FMODRANK } & 6 \\ \text { EMODRANK } & 2 \\ \text { TMODRANK } & 5\end{array}$

1

Rank 7th

$\begin{array}{ll}\text { IMODRANK } & 1 \\ \text { CrMODRANK } & 0 \\ \text { NMODRANK } & 1 \\ \text { DMODRANK } & 1 \\ \text { LMODRANK } & 0 \\ \text { COMODRANK } & 0 \\ \text { CTMODRANK } & 0 \\ \text { FMODRANK } & 0 \\ \text { EMODRANK } & 0 \\ \text { TMODRANK } & 0\end{array}$

Rank 8th

$\begin{array}{ll}\text { IMODRANK } & 0 \\ \text { CrMODRANK } & 0 \\ \text { NMODRANK } & 0 \\ \text { DMODRANK } & 0\end{array}$




$\begin{array}{ll}\text { LMODRANK } & 0 \\ \text { COMODRANK } & 0 \\ \text { CTMODRANK } & 0 \\ \text { FMODRANK } & 0 \\ \text { EMODRANK } & 0 \\ \text { TMODRANK } & 0\end{array}$

Candidate

1

2

Intuitively Accessible

5

6 


\section{TRANSCRIPTS}

The transcripts of the interviews analysed and presented in chapters 6 and 8 are included here. All transcripts (anonymised) and related data sheets are available in electronic form for further research by application to the author.

All requests for use of the transcripts and associated data sheets must be made in writing citing potential application and academic or professional affiliation. The voice files are not available for subsequent research.

The application must include the following wording:

1. I/We warrant that the transcripts will be used for research purposes only and that no attempt will be made to identify, name or attribute any extract to the original interviewee.

2. I/We warrant that the only data relating to the individual interviewee is that contained in the statistical data sheet accompanying the transcripts and that only that data is used as a basis for categorising or classifying their transcribed comments.

3. I/We agree not to attempt to contact any participant in this research whether named or not without the written consent of Robert James Ryan

4. I/We will keep all the downloaded transcripts and data sheets confidential and will not pass them on to any other person or organisation without the written consent of Robert James Ryan or his nominated representative.

5. I/We acknowledge the moral rights of Robert James Ryan to the transcripts and the data sheets and that he holds exclusive and non-transferable copyright to all transcripts and data originating from this research 


\section{Test Subject IT0069}

Date of Interview 11 May 2018

Transcript of Intraspective Interview

Interview commenced 14.00

\section{Image 1}

1.I: Tell me of those, which has the most impact for you

2.R: The horses,

3.I: What year did you take them?

4. R: 2017

5.I: Where were you staying?

6. R: This was photographed in the Camargue we were staying in a hotel St Marie LaMere we were staying with a group I don't remember everybody was there on it was there and then a group of around eight or 10 other photographers

7.I: What were you expecting to get from your journey?

8. R: It started off as a new job of work because it was my first visit to the Camargue I wasn't really focusing on photography as such. My main focus was on leading the group but this picture had a particular.... There was an aim to this particular photograph.

9.I: So you were there with Monique. What was her role there was she supporting you?

10. R: She was partly there as the admin. That party didn't need much. She was there photographing as much as anything else on the trip.

11. I: What was your reaction to the group?

12. R: It was at very connected group there was certainly at least two people who have experience of working with horses so the energy of the group was very relaxed so yet so it's rare to find a bad group to be honest. There was a mixture of people, some there for the experience, some there to learn how to use the camera some with a slightly higher level of technical expertise.

13. I: Tell me about his idea of connectedness

14. R: If you think about in terms of human relationships we go through life we connect with some people, we don't connect with others, those who connect with you, share with you more, you are more open with them and relax with them. You are generally more mindful with. ..

15. I: Pause 
16. R: There are probably two answers to the question. The relationship doesn't have to be human human it can be human animal so when I'm photographing something I want to be connected to whatever it is that I'm photographing because it is through that connection that you learn the story that is underneath the surface. So if you think about photography as not photographing the semblance of the subject but the essence of the subject, in order to get to the essence you have to have a connection. Now if you think about this in terms of portrait photography. If you take a picture of somebody and you don't know anything about them you will end up with a passport photo. True portrait photograph is not photographing the semblance of the person, a true is revealing the personality of the person or the mood of that person in that moment or something else it's about revealing who they are through the portrait. So in order to understand who they are you have to make a connection with them if you don't make that connection you're not going to learn who they are therefore you cannot photograph that can only photograph the surface so for me it's applying exactly the same principles to an animal. All animals have personalities, particularly obviously mammals and this may be why I specialise in photographing mammals not birds. Although I'm sure all robins are different I'm sure. No it's about finding the character of that individual or it's about finding the essence of the group or the herd or the pod or whatever, and photographing that rather than just photographing a horse and that comes through making a connection which allows you to communicate with the subject on an intimate level.

17. I: How would you describe your personal mood on the day that you took this particular image?

18. R: I would say I was probably on this particular day I'm fairly upbeat I'm excited excited more for them than from me I knew what to expect because this was their first experience I was I was excited for them and because it was the first day I had some energy. I was in a positive mood

19. R: So we drove as a group in the minibus from the hotel to the car park at the edge of the salt marshes and then we walked from the car park to the edge of this particular location about $200 \mathrm{~m}$

20. I: What did you have with you?

21. R: Camera bag and a pair of waders

22. I: Did you feel you might need them?

23. R: Of course

24. I: Talk me through this camera bag

25. R: It's comfortable, it's small. It's just small enough for a camera bag big enough to carry everything that I want. I don't give much thought to it when I'm not actually using. It's utterly irrelevant. It's a function of the job rather than anaesthetic.

26. I: When you lifted up out of the car you have any response to any feelings towards it? 
27. R: Unless its heavy. No I don't I don't get at all excited by by picking up a camera. The function of picking up a camera I have exactly the same response to it as I do when I pick up a hammer. Or a spanner or a hammer and a spanner which means I'm gonna have to do something I don't particularly want to do. I have slightly more emotion.... because I want to take pictures. On a serious note I don't get excited by cameras ... your cameras they are a tool to do a job. The camera in itself doesn't excite me. You give me a Leica or a Lumix camera compact camera it makes no difference to me, I wouldn't pick up the Leica and go wow!. It's just a tool that I need to do my job.

28. I: What settings did you have on the camera?

29. R: Approximately half a second probably probably at something like F-16 and a very low ISO.

30. I: Did you adjust the settings as you came up to the shot?

31. R: No I don't remember adjusting them because these things are just so subconscious now that I do it without thinking. The only thought put into the camera settings for this shot is that I wanted a very low shutter speed so I would have set ISO and apperture to get the speed I wanted.

32. I: Can you actually remember moving the shutter mechanisms on the camera?

33. R: No,

34. I: Did you adjust iso

35. R: No, it was already at a very low level

36. I: Where were you when you took this shot, physically?

37. R: Physically I was standing about $5 \mathrm{~m}$ into the water

38. I: Was that because you anticipated that would be the right position?

39. R: Now it's actually it was anticipation based upon experience so if you are standing on the water's edge by the time the horses get close enough to you they have stopped running as they are coming out of the water so the pint where they're running at full gallop they are still in the water so I wanted to be far enough out from the edge so that when I took the picture they were still they were still galloping rather than just walking. Because this picture is galloping horses not walking horses

40. I: When you saw these horses how far away were they from you?

41. R: When I first saw them probably about hundred metres possibly $60 \mathrm{~m}$

42. I: Where you warned they were coming?

43. R: You aren't specifically warned when they were coming but you can see when they are starting to run.

44. I: Was this the first time you had seen them on this particular trip?

45. R: Yes

46. I: Tell me how you felt at that point.

47. R: I think, if I remember rightly because we spent when they first turn up they don't go straight into the water so we had spent a little bit of time mingling with them so from that point of view I had already made an introduction with them to be honest 
when I was taking the picture because this was a technique I've never done before and I was experimenting and practising I was more focusing on what I was doing with the camera than what the horses were doing.

48. I: Tell me about this technique in what way was it different?

49. $\mathbf{R}$ : Well the aim was to capture a pin sharp image of the horses running and a blurry motion shot of the water spraying and the problem with that is that the camera needed two different shutter speeds but of course on a camera you can only have one shutter speed so my difficulty was that the tool I had to do the job couldn't do the job so I had to find another way around achieving what I wanted to achieve so what I then did I took the theory of panning so the theory of panic being if you are moving the camera ... it is not how fast the subject is moving but how fast the subject is moving relative to the camera so if I pan the camera at exactly the selfsame speed the animals are moving then I can use a slow shutter speed and still get a pin sharp image relative to the camera and the subject is effectively static now that works if the subject is running from left to right and right left across the frame. But you can't pan the camera when the subject is coming straight towards you. So, however, what I figured was that using a zoom lens, because as you zoom the lens the subject effectively gets bigger as you zoom out and smaller if you're zooming in, by zooming if I was to zoom the lens at the right speed as the horse came towards the camera the the same effect would happen the horse would stay the same size in the picture and therefore I can get away with a slow shutter speed so that's the theory - the practice it's getting the zoom right to do two things one is to get the speed of zoom correct but you are moving or changing the focal length matching the horse coming towards the camera in closing the gap between you and the camera the second thing is keeping the camera steady enough so that you are not getting camera shake. That's what I was experimenting with practising.

50. I: When did you conceive of this approach to doing it? It was this something you had already got in mind when you came to the shot or was it something you had worked out before?

51. R: The idea to experiment with it came that morning as I was kind of watching the horses and deciding what I wanted to photograph. The technique the idea for the technique came from from videography cinematography where the zoom is used or where the zoom can be used to do effectively the same thing with the size of the person who ever it is in frame exactly the same but to give the appearance of movement. The actual idea for the technique came from came from videography or cinematography the idea to use it came that morning when I was watching the horses and thinking how am I going to photograph these differently from the last eight times that I had been here!

52. I: So you were looking to photograph things differently?

53. R: The creative moment.

54. I: You thought this would give you a more creative interpretation? 
55. R: I thought it would do two things. It was a different interpretation of a of common view but also what I was really trying to do and again a you come back to this idea of the essence the thing about these horses is that they are simultaneously very powerful and very serene and now it's easy to get the concept of power into the photograph and it is easy to get the concept of serenity into a photograph but to get the concept of power and serenity into the same picture is more of a challenge so so the intent was to do that so to get the essence of power and serenity. Horses galloping at full pelt and yet having this softness about them and so the reason for having the horse sharp is the power and then the softer water is all about the serenity. So the creative idea, say for the intent, was how do I bring these two essences, which are opposing, into the same picture.

56. I: So when you got this first image then, was this the first click of your shutter?

57. R: No it was about the 100th!

58. I: Now tell me about the moment you realised you had got this shot.

59. R: Yes

60. I: Now was it at the point of capture?

61. R: No effectively you are, because by necessity the technique involves taking multiple shots whilst you're zooming you cannot take a picture then zoom, then take a picture then zoom it doesn't work. Basically, the technique was I had it on the high-speed frame advance the button was pressed and I was zooming at that time. So, it was only when I was finished that I could go back and look at the images on the screen to see what what is really happened during that two second burst of images so I didn't know I got it until after I taken it.

62. I: Was that a chimp or when you got it back to your computer?

63. R: No, it was when I was there because it was a brand-new technique and the great thing about a digital camera is that you can learn on the spot then so the playback review, revise, shoot, process is how anything like this is how it starts to develop. If I got it on the first shot then I would have been pretty amazed. So after each burst first I would look at the images I would figure out whether the zoom too fast, too slow was the right shutter speed etc. So you are doing this constant reassessment of each burst of images to see where you are sitting.....

64. I: So as you were correcting your camera can you remember the actual physical correcting of the instrument?

65. R: Well most of it, to be fair was the only thing I ever really changed was the zoom speed. I didn't ever really change the settings on the camera. I was constantly looking at how quickly do I need to turn the barrel more softly I'm only talking about four or five runs by the time I figured it out but when I saw the picture on the frame I knew I had got it.

66. I: So when you got it back what sort of adjustment did you do?

67. R: To be honest very little in that I like the fact that it is almost a monochrome image and so the sky is pretty bland but for me that works. The only thing I did which I did 
again was done on purpose it was done and when I took the images I knew that what I was going to do was to turn it into a panoramic. So I've cropped top bottom and bottom up because I wanted to accentuate the line of the horses so that was the main adjustment I've done a little bit of tonal work on it at I think and I think I added a touch of yellow. Saturation into the manes and not everywhere else

68. I: Why did you think it needed that level of tonality?

69. R: Because because, as we were talking about earlier, colour is such an overpowering element of design it's gives an image context which is sometimes necessary this isn't all about reality this isn't about the surface reality this is not about what the horse looked like it's form, it's about power and serenity. And for those two elements to come out you have to you you think about elements of design it's about texture it's the texture of the water against the texture of the horse so by taking colour away from it you are allowing those other elements two brief giving them space giving them a louder voice in the picture. At the same time I didn't want to make it purely black and white

70. I: Why no.?

71. R: I don't know as the honest answer to the question it's just having that touch of colour particularly in the mane it just it keeps it in the realm of reality but at the same time emphasising the essence Rather than the resemblance. So for me it just doesn't work as well in black and white.

72. I: As you look across this array of horses, as a scan the image does anything stand out that you find especially attractive?

73. R: I think yes, one of the reasons is that I like the picture I can keep looking at it and I can still see different aspects to it. The thing I was initially looking for for the lead horse which was, this guy, was actually sharp that was always the key they don't all have to be sharp the lead one had to be sharp and that was one of the first things but also at the same time I needed a big splash of water in order to get that element in the picture so the fact that you have got these two next to each other and this huge splash of soft water flying everywhere that's what really initially appealed to me the other thing I like about it and this is very rare with these horses that with the exception of this one they are all spread out and you can see all the faces of each one that is unusual usually they are behind one another all running in a vertical line as opposed to a horizontal line and they are all moving at pace.

74. I: So looking at this image now what is it saying to you now?

75. R: Now? Now I think it is saying very much what it did at the time and that is why I like it. It still says power and serenity.

76. I: Are there any other elements in it that connect?

77. $\mathbf{R}$ : I think over time I have started to notice if you like the personality of the other horses. So my attention initially, my attention was fixed upon this horse at the front, the leading one, but over time as you start to look at it you begin to think this chap over here on the right-hand side he has some trouble (keeping up) and has an 
alarmed look about him and his buddy here these two are like twins almost like they're part of the group but they are mates within the group if that makes sense so there is so there is this element the one on the left hand side galloping off $s$ though needs his Weetabix so that's the thing, the longer I look at it the more I tune into the herd as opposed to the individual.

78. I: So in terms of the feeling that it invokes in you?

79. R: I think it has multiple these two make me laugh this one in particular makes me laugh these two there's an element of humour in these two, there is more focus over here this one over here appearing through the mist looks perplexed so again it has one more than one level on the emotional side.

80. I: Why did you exclude that one there?

81. R: I don't know actually

82. I: Why did you leave him out?

83. R: He doesn't speak to me as much as the others

84. I: How quickly did you try to process this image in your laptop?

85. R: I did I don't tend not to tend not to process on the laptop. I did but but partly because I was doing an image review with the clients and as part of that I promised to put some of my pictures up so that they could see what I was photographing. So I did do it specifically for that. But when I got it back to the main computer I started from scratch when it came to processing.

86. I: I am still interested why that one's been left out in your narrative

87. R: I don't know.

\section{Image 2}

88. I: Now, where were you when you took this? Where were you staying?

89. R: So this was photographed in Hokkaido, in Japan.

90. I: Right. You were in one of these paper hotel rooms, were you?

91. R: No actually, no, actually for this, we were in a fairly - fairly modern... Crowne Plaza hotel.

92. I: Yeah. Impressively modern?

93. R: Like any other western-style box hotel.

94. I: Yeah.

95. R: you know, the - very little Japanese about it.

96. I: No.

97. R: I mean on a lot of the trip we were staying in traditional hotels but on this particular part we weren't.

98. I: Right. Right. So you-

99. R: And they did have a heated toilet seat.

100. I: You what, sorry?

101. R: They did have a heated toilet seat.

102. I: Oh right, a heated toilet. 
103. R: Yeah.

104. I: Excellent.

105. R: It is.

106. I: So you're in this very smart but modern hotel.

107. R: Yep.

108. I: Not particularly pleased with it, or happy with it?

109. R: No actually at that point I was exceedingly happy with it, because-

110. I: Because you've got a heated toilet seat!

111. R: Well, apart from the heated toilet seat, having spent a week of eating - of eating Japanese food, sitting on Japanese futons, er, in a traditional Japanese hotels, I was quite ready for a normal bed, and a western meal.

$112 . \quad$ I:

113. R: So I was - I was more than delighted.

114. I: OK. Without salad. OK. So you're in this hotel. Who were you with?

115. R: Again I was with a group. so this one was taken on my first group trip to Japan.

116. I: Right, OK.

117. R:

$118 . \quad$ I:

$119 . \quad \mathbf{R}:$

$120 . \quad \mathrm{I}:$

121. R:

$122 . \quad$ I:

$123 . \quad \mathbf{R}:$

$124 . \quad$ I:

125. R: ...

126.

$127 . \quad \mathbf{R}:$

$128 . \quad \mathrm{I}:$

$129 . \quad$ R:

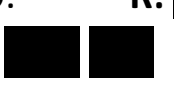

130. I: But you had been to a number of hotels before.

131. R: Yes.

132. I: So by the time you got to this place...

133. R:

$135 . \quad \mathbf{R}:$ 
136.

I:

137. R: And what was interesting about this trip, and this, this probably sums it up, is that... the day... the day before this we were photographing the swans in Lake Kussharo...

138. I: $\mathrm{Mm}$.

139. R: and I decided that we were all going to get up exceedingly early to get the sunrise...

140. I: Mm.

R: ... because that's the lake that - I mean it's mostly covered in ice but there's sort of thermal spring water that runs along the edge, which keeps at least the stream going... and, what's really nice is if you get up early you get the mist coming up from the, from, from the water - from the heat and the snow and cold, so you get this beautiful mist coming up and the swans, and then you have right light from the sunrise. It's just magical. Um. But I had no idea as to whether -no idea whether the sun was going to be visible or not so, anyway, I got everybody up in order to get the sunrise. Turns out there was no sunrise, and, er, no swans either. so we were hanging around for a while, and nothing was happening. And then, one person said... "Is there any chance we could go back for breakfast and then come back

$142 . \quad$ I:

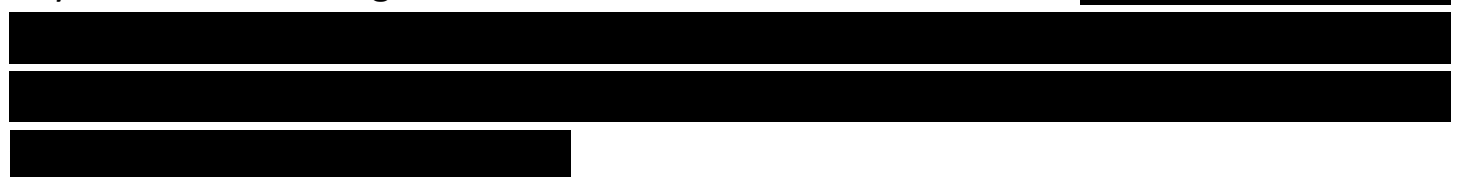

143. R:

So out of the group of 10 ,

two of them stayed... plus me. The rest of them went back to the hotel. And this is no word of a lie... as I was watching the bus just disappear...

$144 . \quad$ I:

145. R: ... out of sight; as the bus went that way and literally, literally disappeared out of sight, these enormous flock of swans...

$146 . \quad$ I:

147. R: ... came flying in through the mist, just as the sun broke, through the cloud.

I was just like, that is just poetic justice.

148. I:

149. R: So the three of us sat there for the next hour taking - I mean, my favourite photographs from that trip, this aside because this has particular, um, intents behind it, $\mathrm{w}$ - were the swans. They were just phenomenal photographs.

$150 . \quad$ I: $M m$.

151. R: While everybody else was sitting on a bus going back.

152. I: 


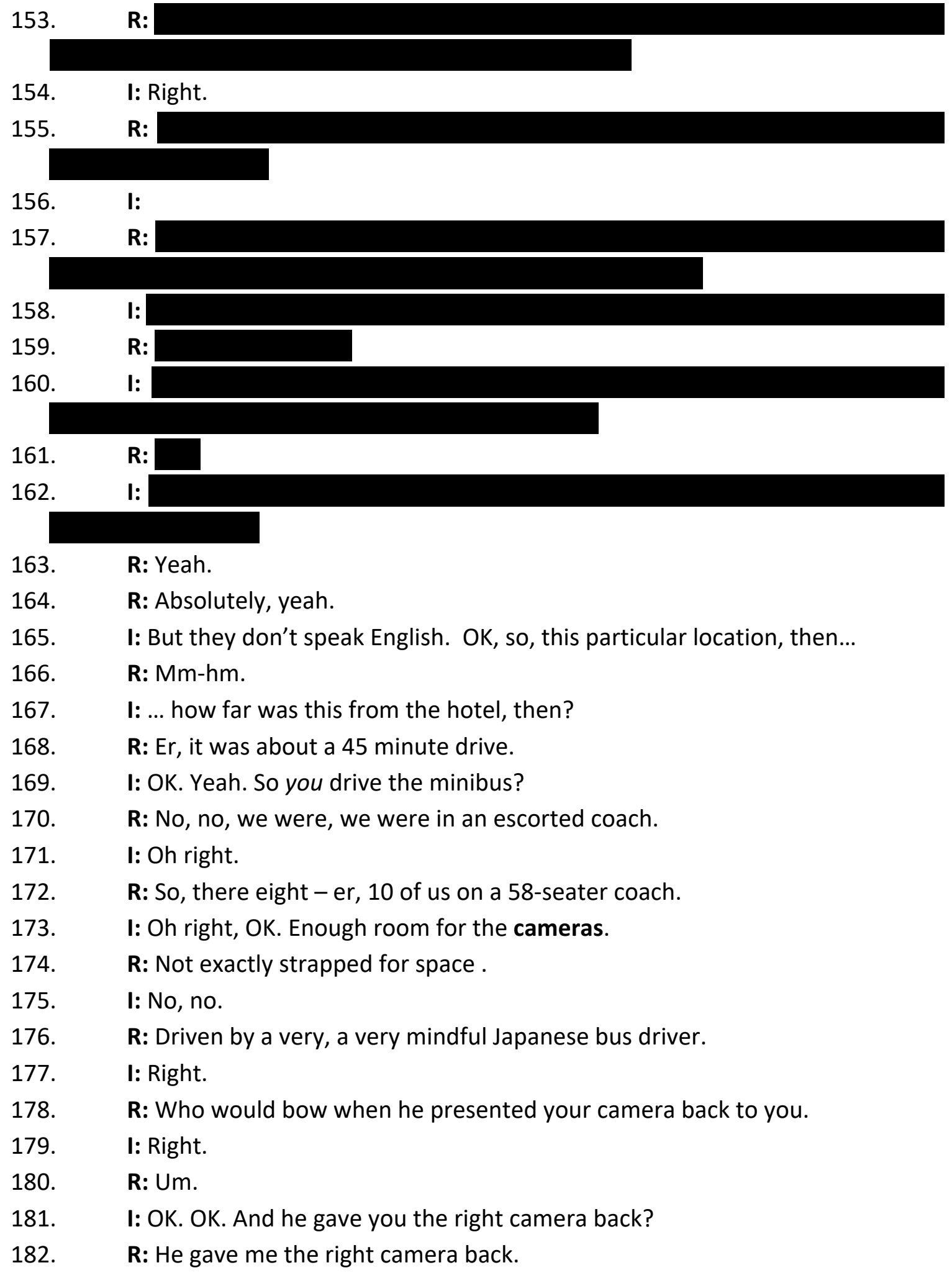

183. I: So, anyway did you know of this location particularly before you got there, or were you just...?

184. R: No, I mean I was aware of, of the very rough sense of what it would be like, but I hadn't been to any of the locations, where I photographed the cranes before so you know each, each one was - I had to think because... I needed a very, very specific spot... 
185. I: $\mathrm{Mm}$.

186. R: ... in order to do this this work, and so, when I, when I got to each location I had to figure out exactly where I needed to be in order to get the shot I needed to get. 187. I: $\mathrm{Mm}$.

188. R: Um...

189. I: What particular spot were you looking for?

190. R: Well, I needed a spot where there was snow in the background...

$191 . \quad$ I: Right.

192. R: ... and I don't mean in the background going flat, I mean almost going up...

193. I: OK.

194. R: ... because I'm using the snow as a backdrop.

195. I: OK.

196. R: Er, so that meant that - because these basically - these birds are on open fields so when there's snow on the ground there are trees and the like in the background, and so the backdrop is, is - half of it's snow but half of it is sky or tree or forest or whatever. I needed a spot where it was purely snow in the background which meant that I needed a slope where the birds were at the bottom and I could get ei- I could get either high enough or low enough in order for the, the whole slope to fill in the frame.

$197 . \quad$ I: $M$ m.

198. R: and that was very few places because they're mostly flat fields. Um. So I had a very - I had a very specific aim in mind as to where I stood.

199. I: Right.

200. R: Um, but I didn't know where that was until I got there.

201. I: OK. OK. All right. So you arrived on this spot, ok. Where were the rest of the group?

202. R: spread out, because these places are pretty busy with photographers at this time of the year, so there's at least 50

203. I: 50,000 .

204. R: $50,60,70$ photographers making...

$205 . \quad$ I: Right, OK.

206. R: er, and so the rest of the group w-was, you know, spread out amongst the... the rest of, the rest of... Japan.

207. I: OK. Excellent. Were there a lot of Japanese photographers there?

208. R: A lot of Japanese, yeah. Mo- most of the photographers there are Japanese

209. I: Right.

210. R: there are a few Americans, British, but actually, yeah, no, most of them are Japanese.

211. I: Well, you sort of think when you see a group of Japanese like that wielding cameras that have been made by Japan. 
212. R: I-I have to - I love the Japanese. And the Japanese are very different from the Chinese.

213. I: Yeah.

214. R: Very, very distinct difference in character.

215. I: Mm.

216. R: you know, the Japanese are, are very polite, and actually a lot of fun, most of the time. the photographers there who are causing problems are never the Japanese; it's always the foreigners.

217. I: Yeah.

218. R: but yeah, I mean the Japanese you know, they're just - generally just happy, laughing, making fun of each other, so, yeah.

219. I: So tell me again a bit about... you had your camera in the back. Taking this shot here, did you have to set your camera up in a specific way? Can you remember doing that?

220. R: I did and I was constantly adjusting it, because there is a lot of, of technical, erm... expertise going into this picture.

221. I: $\mathrm{Mm}$.

222. R: and even a, even the shift of a third of a stop would sometimes make the difference.

223. I: Right.

224. R: So, I had the camera set, er, I mean i- the camera's almost always set in aperture priority, I had it, I had it set at a fairly wide aperture, partly because I didn't need a lot of depth of field, and partly because I did need a relatively fast shutter speed.

225. I: Mm.

226. R: but the - the actual shutter speed was a secondary consideration, so long as it was in a, a range, it was fine, most of the time. So what I'm basically doing constantly is - the Fuji camera, has a, er, an exposure compensation dial on the back, and so I was constantly shifting exposure compensation, adding or taking light away in order to keep... the perfect... amount of overexposure, so that it's, it - because the key to this, which is what I'm saying, it's not as processed as you might imagine - the key to this image was, was overexposing it; overexposing the background snow to the point it's right on the edge of, it's [??] zero, zero in the [??] zone test. It - it's not blown out, but there's no texture, in the light, and of course any slight shift in light would take it off that. So, you know, the sun - the clouds thin a bit and the sun shines for a bit and it all brightens up, and automatically it starts - so I'm constantly doing this shifting. The beauty of doing this with a Fuji camera, as opposed to a DSLR camera, is that I'm seeing the effect of that shift in the viewfinder, because it's an electronic viewfinder. If I was doing on DSLR, I...

227. I: You wouldn't... 
228. R: ... I'd see what I always see. Yeah, so in, in that sense, the Fuji - the mirrorless Fuji camera made a big difference...

229. I: Mm.

230. R: ... in how, how accurately I could make that adjustment...

231. I: $\mathrm{Mm}$.

232. R: ... in time to take the picture, rather than doing it the other way round.

233. I: Do you think if you'd had another camera, like for example the Nikon D4 that you used to have, would you have been able to take that?

234. R: I would. it would've been a bit more hit and miss and I would've had to do a bit more processing after the event.

$235 . \quad$ I: OK.

236. R: I could still have done it.

237. I: Yeah.

238. R: but, yeah, it would have involved... more out-of-camera processing...

239. I: $\mathrm{Mm}$.

240. R: ... than I needed to do because I was able to get it more accurately in the camera.

241. I: How many birds were you seeing there at that time?

242. R: quite a lot. I mean, there must have been a couple of hundred.

243. I: Oh right, OK. OK.

244. R: Milling around, and the, the, the great difficulty actually is getting them - is isolating them.

245. I: Yes.

246. R: because they are constantly walking in front of each other and...

247. I: Yeah, yeah.

248. R:

249. I: A bit like isolating a flamingo.

250. R: Yeah. Exactly. Yeah.

251. I: Exactly.

252. R: Yeah, yeah. Yeah.

253. I: They just won't stand still. Mix up. Exactly.

254. R: Yeah, and, and, and I - and separation was, was... critical...

255. I: Mm.

256. R: ... to this style of photograph. Yeah.

257. I: So, did you spot these two? Do you remember actually spotting them as being on their own?

258. R: $\mathrm{Mm}$. because

259. R: Yeah.

260. I: Yeah.

261. R: Because that - I - that was all I was constantly looking out for. You know, you're, you, you're looking at this field which isn't particularly big; I mean, it's just a 
farmer's field, really. with a couple hundred birds on it, and I know what I may need to do, so - and I know that separation is important, so most of the time I, I have, within this fairly small field...

262. I:

263. R: ... I have a fairly small window of space in which to take the photograph, so I'm waiting for the birds to come in and out of this - I mean, imagine a, a photo studio with a white card backdrop in a big warehouse. Well, the farmer's field is the warehouse, and my, my white backdrop is where I'm taking the shots, so I'm constantly waiting for the birds to pass in and out of my little window of, of shooting space.

264. I: Right.

265. R: and then I'm constantly looking for them to walk in and out of that space, separated from themselves and...

266. I: So you weren't really moving camera much, then?

267. R: Hardly at all.

268. I: No.

269. R: No, the camera was pretty much fixed on one spot.

270. I: Is it mounted on tripod?

271. R: It was, yeah.

$272 . \quad$ I:

273. R: Yeah, it was on a tripod.

274. I: And why were you doing that? To just keep it in the space, or...?

275. R: Partly, I mean because I could, I mean, it's not always practical to use a tripod in wildlife photography. That's often because you're having to move the camera all over the place. I knew I was fixed on one spot, so I didn't know there's a slight movement left and right and sometimes up and down a little bit, but most of time, I have this relatively small window that I'm shooting into, so... it, you know, it was, it was practical to use a tripod and of course it's better to use a tripod if you can, even in wildlife photography, so, for $n$ - for no other reason than it holds the weight of the camera, and you can walk off and leave it there and not get overtaken by...

276. I:

277. R: ... 46 Japanese tourists who want your, your half, half a square foot of real estate.

278. I: Yeah.

279. R: no, I didn't - then again you're just using a tripod - just gives the camera that bit...

280. I: So you remember these two birds; they caught your attention. What was it about these two which caught your attention, apart from the fact that they wandered into your field of view?

281. R: Well, it's because they're, they're effectively mating.

282. I: Right. 
283. R: So, most of the birds there are eating; that's why they're there, because they're being fed.

284. I: $\mathrm{Mm}$.

285. R: so, most of them are eating. But occasionally, within that, you get these courtship displays, erm... and, so, and, and the courtship display, you can, you can see because it, you know, it goes on for a few minutes. Um, well at least a, you know, a few tens of seconds, so I saw them starting this courtship display and then I just, just followed them.

286. I: Did you spot them before they moved into your field of view?

287. R: $\mathrm{Mm}$.

288. R: Yes.

289. I: Yes, so you could see them moving in?

290. R: Yep.

291. I: You thought, "OK, these two characters."

292. R: Yeah. Er, I mean, that was what I was doing most of the time and, OK, I mean, sometimes they'd turn around and walk in the opposite direction, and OK, well, so be it. but you would - because again, this is about... this isn't - this whole project was not - was very much about capturing the essence of, of the subject, and not descendants and that's - that comes out in the, you know - this is - basically what I'm doing this is trying to recreate, the sumi-e art - Japanese art, which is all about essence, and so it's doing the same thing and so, you have to have the birds doing something that was interesting, otherwise it's just a bird standing in a field, and so I was constantly looking out for - and of course the courtship display is when most of that interesting activity is going on, when they're dancing or, in, in this case, you know the, the...

293. I: What catches the essence in this particular image as far as you're concerned?

294. R: falling

295. R: I think it's partly where it, it's the, the starts of the bird, the fact that they're mirroring each other.

296. I: $\mathrm{Mm}$.

297. R: you know, the beak's are both open.

298. I: Mm.

299. R: The necks in similar positions, standing in very similar positions, it's the, it's the connectedness between the two; they're always like they're in sync with each other...

$300 . \quad$ I: $\mathrm{Mm}$.

301. R: ... which of course in $a$, in a... successful relationship is what you would expect; two people acting as one.

$302 . \quad$ I: Yeah.

303. R: and for me that's what it captures; it's two birds acting as one bird.

304. I: Looking at the two of them, what would you say is the significant difference? 
305. R: the, the curve of the neck on this one...

306. I: OK.

$307 . \quad$ R:

308. I: Anything else?

309. R: ... which is what tells you that it's, um, a male.

310. I: Right.

311. R: it's got its legs crossed.

312. I:

313. R: Don't know what that tells you.

314. I: Hey?

315. R: I don't know what that tells you.

316. I: Does it tell you anything?

317. R: Er, that it's walking.

318. I: Right. Is there anything about this pose that strikes you as different to that pose? I'm not seeing it, I'm just asking you if you see anything, or saw anything different than the pose at the time.

319. R: Well... at the time I'm not sure I did. Looking at it now, there was a, there's a bit more intent in the male than there is in the female; the female seems a lot more relaxed than he does.

$320 . \quad$ I: Yeah.

321. R: Yeah.

322. I: So, looking at it, you've got these two very fine birds. You're talking about finding the essence here.

323. R: $\mathrm{Mm}$.

324. I: What sort of captures that essence for you now looking at it? Leaving this out of it.

325. R: it's, it's about... it's about brushstrokes.

326. I: $\mathrm{Mm}$.

327. R: you know, the whole point of sumi-e art is, is... texture.

$328 . \quad \mathrm{I}: \mathrm{Mm}$.

329. R: it's the - it's, it's not the thickness of the paint...

$330 . \quad$ I: $\mathrm{Mm}$.

331. $\mathbf{R}: \ldots$ it's the weight with which the paint is applied...

332. I: Yep.

333. R: ... and that is very important in sumi-e art. and I, I get the same feeling wwith these pictures, it's... you know, you look at the white of the bird.

334. I: $\mathrm{Mm}$.

335. R: It's... i-if you look at it very closely $\mathrm{i}$ - there is detail in there.

336. I: Mm.

337. R: But the detail varies, depending on where in the bird it is. You know, ththere is - the background is not overexposed. you know, if you open that, that image 
up in in Photoshop and you look at the histogram, it doesn't flash anywhere. It's right on that edge. and the whites sort of blend in and out. You know, if you look at it from over here, you start to see... because

338. I: Yeah, yeah. because

339. R: and, and there is, there is... phenomenal detail in these black feathers here, but also the, the neck there is, is a softer detail, and there's a, there's a, there's a lighter weight... to this black of the neck, as there is to the weight of the black of the tail.

340. I: Yeah.

341. R: and so it - for me it's, it, it has all to do with this, this, as I say this element of the weighting of the brushstroke. it's that essence of, you know, photography is painting with light. I-if you show the - particularly when they're in print, not so much when they're on the screen, but if you show the print of these to anybody, 99 times out of 100 they'll think it's a painting. and... and because that was the purpose - was to create a photograph that looked like a Japanese painting - that's when the essence resides.

342. I: Yeah. OK, I think you... oh good lord, yes, we've done very well.

Image 3

343. R: ... and I'll, and I'll say up front, I don't know whether I even like this picture.

344. I: Right.

345. R: However, aesthetically I don't know whether I like it, but as a piece of photographic art I love it.

346. I: $\mathrm{Mm}$.

347. R: Um. So this was taken on the following day.

348. I: Right.

349. R: After the charging horses.

350. I: OK.

351. R: And the, the, the people who run the horses said, "Look, if you want to, we have this female..."

352. I: $\mathrm{Mm}$.

353. R: ... "who is in a field with a foal and eight colts, so if you want to go and photograph them then we'll take you down there and we'll just leave you there." So the group agreed that they'd like to do that. I was personally very sceptical.

$354 . \quad$ I: Yeah.

355. R: so we got to, we got to the field and before we went in the, the, the guy who - the ranch owner - said, um, he said, "Right, this is - basically what will happen is - do not approach the mare because she has the foal and she'll be very aggressive." $356 . \quad$ I: $M m$. 
357. R: "So do not approach the mare. When you go in they will probably run away from you and you'll just have to follow them. So if they run into the next field, you just have to follow them into the next field."

358. I: Yeah.

359. R: So this is how we started, this session. We walked in, to the field - er, there was about 10 of us, as I said - and the horses were right down at the other end of the field, so we walked in and we started walking towards them... and as we started walking towards them, they started walking towards us.

$360 . \quad$ I:

361. R: And they didn't stop. The female was as relaxed as you could possibly imagine.

$362 . \quad$ I: $\mathrm{Mm}$.

363. R: And this was over a period of, of a little bit of time. But eventually, she didn't care where her foal was. she was so comfortable in our presence that she was - and this is the, this is the mare - um, she was so comfortable in our presence that she, she would just let the foal go off and do his own thing.

$364 . \quad$ I: $M m$.

365. R: We had one guy in the group who was terrified of horses.

366. I: Right.

367. R: Absolutely terrified of horses. Who by the end of this two-hour session was sitting on the ground, surrounded by a - all of the eight colts.

$368 . \quad$ I:

369. R: One of the guys who actually owns horses, he got one of them to fall asleep with him, and I've got this on video; the two of them start standing up, he sits down, she sits down, he lays down, she lays down, he puts his hand out, and she puts her nose on his hand. It's just phenomenal. So there was this beautiful energy going around the horses and the group, which was really just something to watch.

$370 . \quad$ I: $M m$.

371. R: But this picture - so I'm now standing in the field, right, with, with a group of 10 photographers who are all taking pictures of horses standing in a field, and I'm going, I have no idea what to photograph. They're just horses standing in a field.

372. I: Big field, horses, sky's pretty rubbish.

373. R: Yeah. because

$374 . \quad$ I:

375. R: They're just horses standing in a field. What, what, what? So I actually gave up at that point and just going - started walking round and talking to everybody else. You know doing what I should be doing on a photoshoot.

376. I: Yeah!

377. R: um, but we were there quite some time and then, and I saw her sitting down, she was on her own. The other horses weren't in the scene, but she was there sitting down on her own. and everybody else was off doing stuff and I saw her and I 
thought, "Mm, maybe I'll just go and sit with you" because I quite like sitting with animals. Forget the photography. I'm just going to sit with you. So I sat about this distance away, so about a metre, away from her. and we just sat there, chatting to each other. Wasn't taking pictures. but then at one point, something clicked inside my head, and so I picked the camera up and I took a couple of pictures of her, and I did a couple of real close-ups, so I thought - if you imagine a picture of just that...

$378 . \quad$ I: Yeah.

379. R: ... muzzle, square, just that. So there's a picture of there and there's another one where she turned her head that way...

$380 . \quad$ I: Yeah.

381. R: ... and so the, and so the picture is - there's no eyes in it, it's just her back, her nose and th-this part of her. Real abstracty type stuff. But there wasn't - and then I put the camera down again. But I still have this sense of - I really had no idea what photographically this is just a complete waste of time. However, this was at a time when I was writing that series of articles for 'Outdoor Photography'.

382. I: Oh yes.

383. R: A photographer's guide to life on Earth. And I started thinking about what so I became very mindful to what I was experiencing.

$384 . \quad$ I: $M m$.

385. R: And I became very mindful to the fact that I was completely... attached to a particular outcome.

386. I: Mm.

387. R: That outcome being horses running in water, charging everywhere. And because I was attached to that photograph, I couldn't see...

388. I: One that was right in front of your nose! because

389. R: ...what was stuck right in front of me. So, I started to, to lower that guard down... and then I started thinking about art, because the article I had written the month before was all about, Picasso and how he changed attitudes in art with, with Cubism, but it also then went onto surrealism and Dali. because the point I was making in, in the article was that in order to move forward from one, one era in art or science, you have to have people who are prepared to accept that what we think is to be - is true now, may not be true, and there may be a different reality. And... what was also interesting at that time was that if you, if you match the, the, the shifts in science, they are almost always followed by a shift in art movement. So, Picasso's Cubism came out around about the same time as Einstein's relativity theory.

$390 . \quad$ I: $M m$.

391. R: What shifted after that, and this is where I was kind of going with this, was that it, it took Picasso and Einstein to move science and art from where it was to where it became, but interestingly, art moved on beyond Picasso and Einstein because science went from relativity theory to quantum mechanics, which is something Einstein could never really accept. because 
393. R: And cubism moved onto surrealism, which was something Picasso could never really accept, so those two got stuck and it moved on. And so I was talking a lot about Dali in this article. Now I don't particularly like Dali's art to be honest, but I understand where it fits in the, in the history of art and its importance in the history of art, I just don't - it just doesn't speak to me. But what was really fascinating about this was I was sitting there, recalling this, this stuff I'd been writing about Dali, and then I looked - I didn't want to say I looked up because I was already looking at it but I looked, and then I saw what had been sitting in front of me for the last 10 minutes, which was this picture.

394. I:

395. R: And so I picked the camera up; now initially that horse isn't there.

396. I: Right.

397. R: OK, so I had three. So I picked the camera up and I thought, "That's quite, that's quite an interesting shot because... it's very Dali-esque."

398. I: Yeah.

399. R: And as I said that, that horse of the right stuck his head...

400. I: ... into the frame!

401. R: ... into his frame and stuck his tongue out and I just went, click.

$402 . \quad$ I:

403. R: So...

404. I: It's a good moment, actually.

405. R: It's a, it's a - as I say, I, I don't know whether I like the picture i-i-in the same way I don't particularly like Dali's art. This for me is, is photographic surrealism.

406. I: It is a bit.

407. R: It has that madness that Dali has about it. But you, you look at, you know, the, the fact that these ears and th-this mane matches these ears and mane...

408. I: Yeah.

409. R: It's in the back but it's on the same level.

410. I: Mm.

411. $\quad \mathbf{R}:$ The tail here matches the mane here.

412. I: Yeah.

413. R: You know, this guy looking off into the distance, this one just, just his head sticking in and his tongue sticking out.

414. I: [??]

415. R: And you're kind of looking at him, thinking, "What the...?"

416. I:

417. R: But what - the most fascinating thing for me is that that picture came out of something that I had opened my mind to.

$418 . \quad$ I: Yeah.

419. R: And as I opened my mind to that specific subject... 
420. I: Mm.

421. R: ... something artistically that matched that way of thinking turns up in front of the camera.

$422 . \quad$ I: $M m$.

423. R: So, I don't know where you go with that but, um...

424. I: No.

425. R: ... for me it was a really, really interesting.

426. I: Really interesting. Yeah, I see what you mean about it being Dali-esque, actually.

427. R: Really interesting. because

428. R: Mm. And this is one that has to have colour, in the same way that Dali's art is very...

429. I: Yeah.

430. R: ... colour-oriented. But...

431. I: Yeah.

432. R: ... this wouldn't work in black and white, so this is one where colour becomes very important as part of the design of the image, but yeah, it, er, it is called Dali's Horses.

433. I: $\mathrm{Mm}$.

434. R: It - it is such a strange <chuckling> picture, and interestingly I showed this one to the group as part of my image review and I said this to them, I said, "I don't know whether I like this picture and that's the reason I'm putting it in."

435. I: $\mathrm{Mm}$.

436. R: Some of them didn't express a particular opinion, some of them said that, including Monique, said that I don't like it. Monique hates this picture. The one persthe one person who loved it was - I don't know whether - do you remember Michu? 437. I: Yes. Yeah, I do.

438. R: The German baroness.

439. I: Yeah.

440. R: Michu loves it.

441. I: Right.

442. R: But Michu loves Dali's art.

443. I: $\mathrm{Mm}$.

444. R: So.

445. I: Very connected.

446. R: It obviously sits. She absolutely adored it. But she is very, as I say, she is very connected with that style of artwork. <chuckling> so it, it, it's a fascinating photograph. because of - mostly because of the story behind it.

447. I: $\mathrm{Mm}$.

448. R:

449. I: Fascinating. Really good. Well done, Chris. Well done. 
450. R: My pleasure.

451. [End of recording]

Retrospective Interview with Chris Weston

Conducted on 25 March 2019

Following an extensive review of a draft chapter 8 the following 70-minute interview was conducted and recorded.

1. Interviewer: Say that again - narrative has got to be interesting and...?

2. Respondent: Yeah, so the purpose of photography is to tell a story. In order for a story to be successful it has to achieve two things. So, in this sense a narrative is equal to the story. The two elements the story needs to achieve, one is it needs to be new or interesting. It's the newness or the interestingness of the story; it's either a completely new subject, which these days is very hard to find, or it's a new take on an old story. It is the newness or the interestingness of the narrative that is what gives it impact. Now the second point in there is it has to be unambiguous, so that you have to tell that story as clearly and precisely as possible, which comes down to your point about reduction. You have to reduce the amount of information in the scene in order that the information you want to get across is clearly definable, and that fits into what effect is fourth on my list, which is composition.

3. I: Looking at the result of the IMP test, and this may get quoted in here so care what you say, how well would you say that that sits with your understanding of yourself?

4. R: I would say it is a near perfect reflection of the way I approach photography in that, yes, the image has to have impact, so on this chart that sits at number one. The impact has to have a narrative. That narrative has to be new or interesting, which dictates it 
has to be creative. And it has to be unambiguous, which means it has to be composed well. So, for me the four things that drive my photography are the four things that sit at the top of the graph. Then you start to move into the technical and, interestingly, exposure and light sit right down at the bottom, which you'd think for a photographer doesn't really make sense, but to me, the way I photograph, it does make sense because... when I'm conscious of light it's because I'm trying to use light in order to create a creative narrative, so I'm using light for a purpose.

5. I: In a very conscious way.

6. R: In a very conscious way. But subconsciously I don't tend to think about light, I tend to think about the narrative. Exposure again, although it's a technical requirement, is the last thing I think about. Focus and depth of field fit in there because focus and depth of field, to an extent more so than exposure, play a big part in composition because what focus and depth of field are really doing - outside of the technical reasons for something to be in focus - what depth of field and focus are doing are they are creating emphasis. They are either emphasising objects in the scene or they are deemphasising objects in the scene, and both of those things fit very closely to composition. Composition is about how do you tell the story, the narrative you're trying to tell. So for me, focus and depth of field play a huge part in composition, and therefore they sit very closely to composition.

7. Colour and tone less so. Technical, which I guess includes a multiple of sins, is the last thing I'm thinking about, as is light and exposure, although there is an element that exposure also plays a part in composition. But in the technical sense of, is this image correctly exposed, I don't think either consciously or subconsciously I'm really thinking about that. And at the point we touched on earlier, although both focus and exposure are technical aspects of a photograph and in reality are the only two technical things you have to worry about on the camera, I would suggest that one reason that focus [??] exposure is that, whereas an incorrectly exposed image could be recovered in the processing stage, it is almost impossible to make an out-of-focus image in focus, and therefore focus, form a technical point of view, focus takes on - when I'm making the image itself and I'm looking through the viewfinder my attention's far more based on getting the focus right, knowing that I cannot fix it if I get it wrong, as opposed to exposure where if I get exposure slightly wrong I can actually fix it later on. 
8. I: OK.

9. R: I think it's pretty much a hundred per cent accurate.

10. I: <laughs> Do I detect a sense of rebellion there? <laughs> As we move on, on page five here, Chris, I picked out a section of your original discourse on this and this paragraph really struck me; it's when you're talking about connection and this sort of striving to find the essence within the subject. This sort of idea of essence - can you give me a bit more of a feeling of what you understand about that?

11. R: I think one of the easiest ways to show this in a way that could be understood by the layman, if you like, is imagine portrait photography. The purpose of the portrait is not to show the semblance of the person you are photographing; it is to reveal the essence of that person, it is to reveal the character of that person. When you look at a great portrait photograph it tells you what's going on behind the eyes. It tells you who that person is, what their character is. That's what I mean by the essence. Now in terms of capturing that in photography, imagine a passport photograph. A passport photograph of you would show the semblance of you, but it doesn't tell me anything about you. It doesn't tell me whether you're a terrorist or not; it just shows me what you look like. A portrait goes well beyond that of a passport photograph. A portrait needs to get beneath that semblance, and that's the difference between a great portrait shot and a passport shot. A passport shows me semblance but it tells me nothing about you, it tells me nothing about your essence. A great portrait tells me everything about you, and you may not even be visible in the picture; you could be silhouetted. That's the difference between semblance and essence.

12. I: When we get onto your images, Chris, to the image 1 which is on page 6 , we talked about the connection you felt about with these great team of horses racing towards you at breakneck speed, and obviously the sense of panic you felt, so you're very much in the moment, I image. I sort of mentioned here these two strategies of going for the essence, or exposing the essence, one through metaphor and the other one through reduction. If you just stick with metaphor for the moment, I suggest a number of metaphors which might be in play in your mind. Do any of these strike you as feasible? So if I got the metaphor of the... so, the sort of metaphor that we're talking about here, it could be, say, horses run like wind and flow like the sea. It could also be the illusion towards the foaming white horses on top of the crescent waves. It could be 
this sort of cultural reflection we have of the winged horse Pegasus flying along. Or it could be the sensual reflection that stallions - I don't know whether they were stallions; didn't do a good enough close-up - and you've got this idea of the sea parting, the sea - la mer. The mare, the female sort of parting, as well. Any of those sort of metaphors bring anything to you in the way that you think about these horses? Or have you got any other metaphor, just dealing with metaphors at the moment?

13. R: I'd love to say yes.

\section{4. $<$ Both laugh $>$}

15. R: After the event. I'm not sure that they do, so I'm not sure if this picture is a metaphoric interpretation in the sense that we were talking about earlier. For me the thing that I noticed about the Camargue horses and it was an interesting revelation as much about me personally as it was about the horses, because if there is one animal that I am terrified of, it's horses which doesn't make a lot of sense as a wildlife photographer.

16. I: I didn't know the point about fear in the air.

17. R: I'm terrified of horses - terrified is a big word, but I'm not comfortable around big horses, never have been. Now put me in front of a grizzly bear, I'm perfectly OK. Put me in front of a polar bear, I'm perfectly OK. Stick me in front of a lion, I'm perfectly OK. Stick me in front of a horse, I'm a shivering wreck. Now that doesn't seem to make a lot of sense, and this is where I'm coming to this point that in fact what I discovered around the horses in Camargue was as much about me as it was about the animals. The difference between the horses in Camargue and the horses that I had experience of is that the Camargue horses are wild and other horses are domesticated, and it's the domestication of the animal that scares me. Because a hose is an incredibly intelligent animal and in domesticating a horse and they use the term breaking in they're breaking in a horse - that's exactly what you're doing: you're breaking it. And I don't mean breaking it from a physical perspective; you're breaking it mentally. Now to me that's a little bit like taking a sane human being that you don't understand and sticking it in a lunatic asylum. Lunatic asylum in this case being a stable. Any sane human that is put into restricted confinement and mentally broken is likely at some point to rebel. And that's where I become nervous around domesticated horses; it's 
not that they're horses; it's the mentally torture that they've been put through to make them domesticated. That's the bit where I lack trust.

18. Now the thing that was interesting to me about the Camargue horses is that because they were wild they didn't have that energy about them; they had the energy of a wild animal, and a wild animal that hasn't been broken; it hasn't been mentally tortured. A wild animal behaves in a way that you expect a wild animal to behave. All wild animals are incredibly predictable because they're wild. It's the unpredictability of a domesticated horse that has a mental problem because we've given it one that makes it to me actually a little bit scary. So it was my observation of how I was responding differently emotionally, or emotionally differently, to the wild horse and the recognition of the reason behind that. And so, yes, you have this incredibly powerful animal, and the horses in the Camargue were bred to be able to run through water at speed. Now that means they have incredibly strong chest muscles because they need to be able to break that water. You know what it's like trying to run through water. So they were bred to be incredibly powerful, and they are; they're incredibly powerful all horses are incredibly powerful, but they're incredibly powerful animals that could, if they wanted to, knock you over and kill you. And, yes, they had this serene beauty about them, this peacefulness about them, which was a dichotomy compared to the domesticated horse, and that's what I was picking up on. So when I was creating the image, these were the two elements I was trying to bring together: group power and peaceful serenity all coming together in the same place. So that was the energy behind the picture; that was the narrative behind the picture.

19. I: So that was your emotional energy going into the generation of that image. I mean, fear is a very powerful emotion, even though you appreciate that they're wild horses, they're still big animals coming at you at your face, so you're not going to be emotionally neutral about that; there'll be some adrenaline pumping through the system, as it were. Do you think that was what was giving you the energy of this connection? Looking at it, then, you non-consciously, it seems to me, you've gone and you've tried to bring out this essence.

20. R: It's interesting because the reality is it wasn't fear. The one thing I never feel around wild animals is fear, which is one thing that allows me to do the job that I do.

21. I: Wariness. 
22. R: Respect, for sure. Awareness, for sure. But I'm never afraid. And, as I say, it's the thing that allows me to allow animals to come close to me is the fact that I'm not afraid when they do. And again that was the thing that struck me about the wild horses in Camargue; you think, "Well a wild horse is more scary than a domesticated horse." Yet for me it was the complete opposite; I wasn't at all afraid of the wild horse but I am afraid of <chuckling > domesticated horses. And that was the strange thing, and I think that's what my mind picked up on, and hence that's why that became the interesting story behind the picture, these massively powerful beasts, wild, and yet are completely calm and peaceful. Underneath that, that energy, that powerful, brutal energy is being put towards good, not towards evil.

23. I: To what extent do you think you did actually previsualise this sort of emotional response? Or was it something you were anticipating? You didn't know what would come out if it?

24. R: I didn't visualise the emotional response because I was in the vicinity of the horse, I didn't know what to expect. So didn't visualise the emotional response. What I did visualise was having had the emotional response, and having observed the emotional response and analysed the emotional response, I then visualised the image.

25. I: So, you talked about the image sort of reflecting you and your emotional response to the horses, the image is as much a mirror of you as it is the horses stampeding towards you. This idea of reduction, there's an explanation of how you uncover the essence here, and were talking here about reduction of the turbulence in the water. Does that ring true or do you think that's sort of putting a gloss on it that wasn't really there?

26. R: No, that's absolutely true and that, in a way, was the whole point. What I was trying to do with the picture was I was trying to capture the two different elements: power and serenity. Now in terms of how I would approach that technically, in order to create a sense of power, you have to reveal detail, so that, in the case of the horses, determined a fast shutter speed in order to free time, and in freezing time you reveal detail, revealing the detail so you can see the muscles straining in the chest of the horse. You can see the look in the horse's eye because they are sharp. So those details are what tell for me the story of power and strength. The element of serenity - now if I'd frozen the whole image, and so the water was also perfectly sharp so you could 
see every droplet, that would have added to the narrative of strength and power, but it wouldn't have given me any of the essence of serenity.

27. I: To be quite clear, this essence of serenity is not within the water, it's actually what you are perceiving within the team of horses?

28. R: Yes.

29. I: That is where the serenity, and that is a real effect of this team of horse - not just one horse, but the whole team of horses.

30. R: And also the connection within the team, the horses are under water, and so I could have blurred the horses as well as the water - that was another option - but in blurring the horse I'm reducing the detail of the horse so it becomes a picture of movement, not of strength and power and serenity. And so the trick here was to create strength and power of one element - one object in the scene - and to create serenity of another object in the scene. And so what I chose to do was to put the strength and the power into the horse, and the serenity into the water, because the water is about flow. Now serenity, for me, is about flow, when something is flowing, it's serene. When something is frozen or in discourse, then it's not serene. And so I guess the metaphor, if we wanted to look for a metaphor in the picture, the metaphor is in flow. Flow for me determines serenity. When something is flowing, then it is in harmony, and so again if I'd frozen the water you wouldn't have got flow, you would have got ice. So to make the water flow I needed to blur the water, and to reduce the detail to a point that the detail didn't exist and you had a flow because flow is movement. So by blurring the water I create movement; movement suggests flow. [??] the horse I create strength and power. You put those two things together, you get the essence of my story.

31. I: So really of those metaphorical options I gave you; that first one, the horse is flowing like the wind, is that essence, that semblance of flow, or the representation of flow is bringing out that...

32. R: Yeah. And also the horses are together. The horses are flowing as one. They are an induvial horse, but they're running as a herd. It's a single herd, not a multitude of horse, and so there is flow within the team of horses, there is flow within the water.

33. I: One of the things that really interested me about this was when you were talking about it - I'm sure it's this image here. This sense of you projecting yourself, I sort of 
thought was really quite amusing. I'm sure it was this one was talking about one horse - was it this one? - the one horse almost looks as though he's going off to get his Weetabix! In a sense you are transferring your own - I don't know if you had Weetabix for breakfast but that is you there projecting onto this horse was going, looking to go and see where I'm going to get my Weetabix, and all the horses looking sort of out of it. So even though they're a group...

34. R: ... they still have an individual personality.

35. I: Exactly, individuality there, and that, in your describing it, you're almost projecting your own personality onto these horses, which I thought was incredibly amusing.

36. R: And I think also this is where you differentiate between simplicity and reduction because the simple way to take this picture would be to remove all other horses. You have one horse to tell the story. But that's omission; it's not reduction. The thing is that, again, these horses do work as herds; in fact they don't like being on their own. To take one horse out of the herd is a punishment because that's how horses selfdiscipline; they ostracise the offending horse for a period of time, so to remove a horse from the herd, it thinks it's being punished, so it's not a good thing to do. And so these horses work as a team; they work as a herd, not as individuals. But of course within a herd, like in any team, you have individual characters and personalities, and a great team is not made up of all the same character; a great team is made of lots of different characters that come together and fill certain roles within that team that the others cannot provide. And so, yeah, that's the reason in the picture you wanted the herd, and also have them separated to a large extent. They weren't sort of overlapping each other; you could see each one individually, and you could see the character of each one individually which adds a level of complication to the image, so it's not simple; it's a complex image. But it's a complex image that has had the elements in it reduced to an extent that the only thing you see is the thing I want you to see.

37. I: Yeah. So, just sort of thinking about in the moment, then, on this image, where you are bringing together the arsenal of technical skill that you've got and the options that gives you and your storytelling. The IMP test suggests what was really driving this image was your desire to tell this story and to do it in a creative way. In your nonconscious command - that's what your conscious command; it's there in your nonconscious response - is the use of focus and [??] focus and so on. And so I'm just 
interested in your reflection there. When you got the image away and you looked at it, did you feel any sense of those constructs, the ones that were in your non-conscious [??]. Did you feel you needed to correct any of that in post-processing? You told me that you cropped a window to a...

38. R: ... Panoramic.

39. I: Made it more panoramic. So you sort of [??] a bit of sky, bit of foreground sea. Did you feel any desire to correct anything else, or did you feel that you got it right in the moment?

40. R: Yeah, I mean on the whole, yes, there was no need to really correct anything. I think I may have tweaked the exposure.

41. I: Yeah, you corrected the exposure a bit.

42. R: Fine-tuned as opposed to plagiarised.

43. I: This addition, or the enhancement, of yellow. Now as I mentioned yellow's a very powerful colour; it reflects togetherness, it reflects love. In one other dimension it reflects cowardice; we use it as a colourful symbol for cowardice, but I think you deliberately saturated the yellowness in the mane. Why did you do that? To what extent was it deliberative?

44. R: It was actually very deliberate. And the reason for that is the picture was very monotone; the light was very flat, the horses are white, the sky is light blue going on white. Everything in the picture is monotone. The water is white, there's not a lot of sky in it, the foreground doesn't have any colour, so most of this image is a image. Now, in a way I had two choices: I could have turned it into a monochrome image, but to me that didn't actually add anything to the story. Sometimes black and white does, sometimes it doesn't, and in this case it didn't add anything, and so it was a colour picture, but it was a very <laughing $>$ black colour picture. And so I wanted to put into it just a little bit of colour to enhance the observation that it was a colour image. And although when you think of the Camargue horses, we don't really call them the white horses of the Camargue; the point is that their predominant colour is white, but they're not really, and in fact their manes are - a lot of the time they're muggy grey but even the white horses, their manes aren't white; their manes are a thorny yellow, almost golden, and so, because it was a colour picture, I wanted to add some colour into it and the only place there was some colour was the manes and the tails, and so 
again just tweaking the saturation in the yellow channel just enhances that colour to tell you that it is a colour image and to accentuate a part of the horse that is the one thing in the horse that isn't white.

45. I think there's possibly a subsequent follow-on from that which is that part of the flowing of the mane is also part of the metaphoric story. It comes from this idea of flow, and so to emphasise the flowing of the mane, to emphasise the mane, I've used the colour to bring emphasis to it because one thing the colour does is in design is bring emphasis. More than any other element of design, colour is the one thing that overpowers everything else and so adding colour - if you want one of the other elements of design to stand out then you remove colour; that's why black and white works. If you want to reveal texture, you take away colour. If you want to reveal or emphasise shape, take away colour. Think about a silhouette; what does a silhouette do? A silhouette emphasises shape. It's completely black, there is no colour. With silhouette, shape becomes emphasised. In this sense because the rest of the picture is mono... mono... tonic...

46. I: Monochromatic, I think that's the word.

47. R: Because the rest of the picture is monochromatic, by adding colour I'm emphasising an element, and in this case that element has the same essence that the rest of the story has, and that essence is clear.

48. I: So really when we go through that, then, you've got out with this picture of the horses, there's sort of three strategies here for probing the essence. Metaphor; so you're not into the erotic bits so much of horses and what they do with theirs! But there is a metaphorical element there in this sort of flowing like the wind, flowing like the air. There's also a reductive process. But would I be right in saying from your point of view the big thing is your projection of your being, your emotional energy into the shot?

49. R: Yeah. Completely.

50. I: Yeah. And it's the combination of those three which would allow you to drive into the real essence to expose the real object here, in ontological terms, and to take away from sensory objects onto real objects. Let's just get your views then. I've talked about reduction versus simplification, and your two lovely little pictures, your two lovely little cranes and body echo. You describe in detail; you've written in here about this 
getting the body echo there. To what extent do you think that discovery of the essence in that image was deliberative? How much of it was coming out of your unconscious in the mixing process, as it were? An impossible question, I know, to answer but you're about the right person I could ask because you're great at understanding what you're doing under the bonnet.

51. R: Yeah, so the crane is an interesting one because in reality the crane is merely the vehicle and so I guess in that sense I'm not really applying the essence of the crane; I'm applying the essence of something else. The crane is the vehicle through which I'm applying that essence. I would have to say to a large extent it's deliberate in the sense that that idea for those photographs came many years before I actually took the photographs, and it came out over not a love of cranes because, as most people know, I don't really do birds. It came out of a love of Japanese art and, in particular, Japanese sumi-e art, and the essence of sumi-e is simplicity. It's minimalism. Not minimalism as we apply it to art today, but minimalism within the culture of Japanese society and in particular the Buddhist society.

52. I: And they approached the essence through meditative process...

53. R: Yes.

54. I: ... where they are adding to the white screen or surface a...

55. R: ... a black...

56. I: ... a black which takes them towards the essence and I guess the trick is to know when to stop adding the black.

57. R: Yes, exactly, because the whole essence of sumi-e to me is fascinating because it kind of sums up everything I feel about how we should live life. We - and I include myself in 'we' - don't. But the original sumi-e art started in China and then so the Japanese scholars visited China and saw this type of art and they took it back to Japan. It was then adopted primarily by Buddhist monks - so the scholars and the monks in Japan - and it was used as a training tool for young monks in the art of meditation. So it's kind of an interesting one because that artwork for me is a way of meditating, and yet it was used to help you meditate. So it's a sort of full-circle art, a symbiotic art, if you like. But it was used as a meditative practice, and of course a lot of the culture behind Buddhism is about simplicity, is about the lack of need of material objects and material belongings in order to amplify self. And that's what sumi-e is; it's about 
simplifying and it's about using simple brushstrokes in order to represent the essence of a being or an object. Yes, there is a huge skill; it's not just throwing paint onto a paper with a brush, but what the monks did was they visualised the brushstroke in their mind over and over. They would see that hand-drawing that brushstroke because they knew that as soon as you put the black ink onto the white paper you could not remove it. And so they would visualise until that vision became, in a sense, reality and the brush got gradually closer and closer and closer to the paper as the main brushstroke. But the strength with which the brush is applied dictates the depth of the paint or the ink that arrives on the paper and so there is an immense skill in applying just the right amount of pressure to get exactly the amount of ink to tell the story that you're trying to tell. And so in the crane picture my story is really a story about sumi-e painting. The crane becomes a metaphor. The crane is a metaphor for it. Now the crane works because it is a white and black bird with a brilliant red head, and the red of the crane really is for me symbolic of the red character which the artist used to stamp their name. So any Japanese sumi-e painting will have black and white and red. Just a little bit of red, but of course that little tip of the red head is symbolic of that.

58. I: But it actually isn't in yours a deliberate colour pop because in a sense you've not simplified the image and think, "Oh, right, now I'm going to pop red into a black and white image." It is an element within the image which you have retained within the reductive process, you've retained it, and the interesting question is, why do you retain it? Red is very symbolic of passion, fire, blood; it is a very powerful colour. A tiny amount of it reflecting- it's natural; it's there in the birds' plumage. To what extent do you think you were seeing that as a crucial element of the reductive process, if you agree that reduction is what you were doing?

59. R: Yeah, for me it was a very... deliberate act. Because it would have been very easy to turn these pictures into black and white. They're black and white birds against a white background.

60. I: That would have been simplification.

61. R: But that would have been simplification. Not reduction. And if I had turned them into black and white, I would have lost the red, but the red was, for me, important because when the artist signs his or her name they sign it in red ink. It's the only bit of 
colour in the entire picture, but they sign their name in red ink. And again for me because the crane is the metaphor for the paining, the style of painting, and the style of painting is black and white and the bit of red ink, and so it was hugely symbolic of the essence of what I was trying to do, bearing in mind that the essence is not the essence of the crane, it's the essence of the art form.

62. I: How does that connect with the relationship between the two birds? Did you see that that as part of the essence, the fact that there was this bind, connectivity between the two birds?

63. R: Yes, in the sense that the... quite often in sumi-e there is this - when they're painting the subject often they are painting the relationship between either animal and landscape, or animal and animal. In some cases there aren't two birds; in some cases there are only one bird. In the entire set of images that make up this particular artwork then, so it wasn't always the case, but the way that the original Japanese painters would paint these is they were painting behaviour and in this case I'm also representing that behaviour through including the two birds and the courtship ritual.

64. I: I want to introduce this idea of reduction versus simplification. To what extent do you feel intuitively an ownership of that distinction? Does it make more sense to you unconsciously, in the gut, as it were?

65. R: It makes complete sense, and in fact, it makes more sense to me and even enhances what I'm doing, particularly when I'm thinking about composition. We were talking before about a story has to have two elements. One of those elements is it has to be impactful. So it has to be new or interesting or relevant. You put those together and you get impact. The story also has to be unambiguous; you have to uncomplicate it. Now you have to visually tell that story in a clear and precise way so whoever looks at that photograph can read the story you're trying to tell without being distracted by lots of other things. Now, in a simplistic sense what you can say is that the easiest way to do that is to remove objects from the scene that are distracting, but that's very binary: they're either in or they're out. The idea of reduction takes that binary concept and expands it to the extent that - and this is where it gets far more... descriptive as a term, so if you take the photograph of the Japanese cranes, the real skill in the picture is - you've got a white bird against a white background. The white background has texture and the white bird has texture. In the white background I want to remove 
all sense of texture but in the white bird I want to retain that texture. So I could simplify it by removing both. Effectively what you would do is you would overexpose the white so that there was no detail, but in so doing then I would remove detail and all white and snow. That's not what I wanted to do; I wanted to reduce the level of detail in the snow to the $n$th degree, and I wanted to reduce the level of detail in the bird by $n$th minus one degree. And so it's not about removing, it's not about deleting information; it's about reducing it to a level where you are giving enough information to tell the story you want to tell.

66. And to use metaphor, when you first mentioned it the thing that sprang to mind for me was If you go cooking and you think about reducing, you put alcohol into the sauce and reduce it down, and you reduce it down in order to retain certain elements of the alcohol but to retain the flavour. You don't want to lose the flavour. The simple way of doing that is not to put the alcohol in the first place. The better way to do it is to reduce the alcohol down so you've got the flavour but you lose the sugar and the alcohol. So that for me is the metaphoric way of thinking about it. It's a much more descriptive way of assessing what I do when I'm designing a photograph.

67. I: So that idea, then, of reduction works well within your frame of thinking about how your image works. Let's just move on, then. Your Dali image - the Dali horses - I do like it; there's an element of chaos about it.

68. R: It's a Marmite picture.

69. I: The Marmite picture. And I'm very fond of Marmite.

70. <Both laugh>

71. I: I just see so much of you in it because you do have a sense of humour and you're a bit sort of whacky and off-the-wall at times.

72. R: Well I have fun with the camera.

73. I: Yeah, and it's very much in the instant, isn't it? Just pick the camera up. And you saw it - think about Steve Watson's article.

74. R: I think the important element to the photograph and perhaps the reason I... I mean, it's a really strange picture, for sure, and it's strange not as an object it's strange in the same way that Dali's artwork was strange, but it's also strange in the sense that I don't like Dali's paintings. I'm not a big fan of surrealism full-stop, to be honest. And I would not choose to put even an original Dali painting on the way. I might own it only as an 
investment. Only as an investment but I wouldn't hang it. I'm not a fan of surrealism so what's intriguing about the picture, and this is what came out in the article, is the concept of you cannot perceive what you cannot conceive, and I would hazard a guess as to say that if you'd put me in exactly that same position a year before I would not have seen that image. The reason I saw that image was because for researching the articles I'd been studying modern art. It started with Picasso and Cubism, and as part of the same article had moved onto surrealism, the idea being that Picasso moved art on where it had been stuck for a long time when he introduced Cubism, and yet Picasso was unable, because he became stuck in his own style, was unable to accept surrealism. It took another painter - or other painters, the most famous of which became Dali - to take Cubism and move it onto another level. So part of what I was doing was exploring at what point are we creative and at what point do we get blocked because Picasso was mentally creative in inventing Cubism but wasn't creative enough to get surrealism which is just one step beyond what he'd already done. So it was all linked up with that. The point was that I'd been studying Dali and surrealism as an art form for this picture. Now because I'd conceived it, when I was presented with this opportunity I was able to perceive it, and for me that's where the picture becomes interesting.

75. I: That is interesting, actually, because one of the other things I discussed certainly at the earlier part of the thesis where I'm talking about psychology and what drives us. We do not conceive what we perceive, we perceive what we conceive; what's called hierarchical predictive processing model of cognition. In other words, we have this model of the world that we create, and then we perceive things in terms of that model. You can see it in the dog; it does exactly the same thing, but if he sees a leaf being blown across the road he will chase it because it is not fitting his model of the world and therefore he's after it. What was fascinating about you discourse on that, you've got this silly-looking looking horse looking at you. I can imagine that might have tickled you, but this horse comes in from the side and sticks its tongue out! Horses do not stick their tongues out, usually.

76. R: And to be fair, actually I photographing the mare, that was my original image, and I wasn't really photographing her, I was just looking through the camera at her because I had the distraction of the horse to the background and that horse, the one 
on the right in the background, stepped into frame. Now my initial gut reaction was to recompose the image to get rid of it, and this is what I'm saying: if I had not conceived the idea of surrealism in my photography or how surrealism is applied to my photography, if I had not conceived that in the articles I had written six months before, instinctively exactly what I would have done is to recompose the image, to perceive a scene that fitted within my conception, but because I had opened my mind up to this art form that I had no interest in, I stopped judging it as an art form and I started looking at it for what it is, which is an expression of creativity, an expression itself. When this horse came in and stuck its tongue out, I instantly created a recognition of my conception and my perception, and hence took the picture.

77. I: Did you stick your tongue out at people as a child?

78. R: Not that I remem- no, actually, I remember getting told off once. No, my friend got told for sticking his tongue out.

79. I: Did you think it was funny?

80. R: I did, yeah.

81. I: What sort of triggered that?

82. R: It could be my screwed up childhood.

\section{3. <Both laugh>}

84. R: As I say, as a picture I don't even know if I like it, to be honest. It is a Marmite picture. I know some people who absolutely love it, and I know a lot of people who absolutely don't. Now interestingly the people I know who like it, they are also fans of Dali and surrealism, so obviously it touches buttons within them which Dali and surreal art touches for them, which I guess goes to prove the point that it's a very surrealistic picture. I don't know whether I like it, but I love it for the symbolism and for the story that it illustrates which, as I say, comes back down to the realisation that in photography if you are able to conceive the image in your mind - which is what [??] is called visualisation, or an element of visualisation - if you are able to conceive the images before you go out with the camera, then you are far more likely to perceive the image, you're far more likely to see that image when you're out, which for me is about opening up your mind for opportunity. So rather than going out with either an expectation that an image will appear in front of you as if by magic or what will more likely happens is if you go out with a camera because your mind's stuck in a particular 
way of thinking that doesn't appear in front of you, you go home with nothing. This idea that if you keep your mind open and you conceive ideas before you go out, you're far more likely to find them. So it's a way of approaching photography that effectively means you take more pictures. Or you find more pictures in that limited time that you have to take photographs.

85. I: Couple more points then. I think that's sort of bringing us towards the end of this particular chapter. I just want to mention this idea of latency, meta structures. An important one, it seems to me you often use this word, design. And if you look at that diagram on the imp test which is back here, would you say that this here is sort of defining for you or taking you to what you would say is design? Is that capturing this notion?

86. R: Yes, because I think for me design goes beyond its perceived literal sense. When we talk about design we're typically talking about light, shape, colour, pattern, texture, the things that go to make up any object, might throw into their functionality which is another part of design. But we often talk about design in terms of composition. For me when I talk about designing an image I talk about it in a much more 360-degree sense. Design is about the conception of the idea into which comes creativity. Where does that spark come from? So it's the spark of the idea, that's creativity. It's taking the spark and turning it into a fire and then feeding that fire with work ethic.

87. I: To what extent would you say this is spontaneous, your recognition of design?

88. R: I actually think it's mostly spontaneous, because the more you think about it the further you get from it because the problem with thinking about things is you can only think with the knowledge you have and because you're thinking with knowledge you're thinking in historical terms because everything that you know is history. And so because creativity is new it cannot come out of knowledge, because if you have the knowledge, by definition it's no longer new. So knowledge is actually the enemy of creativity. The more you know, the less creative you become, and this comes back to what we were saying about Picasso. Picasso invented Cubism and yet the more he got into Cubism, the more he knew about Cubism and what he was doing, the more closed his mind became, to the extent that when surrealism came onto the art scene, he could not accept it. It was a step beyond creativity he just couldn't take because his 
knowledge would not allow him to. It's the same with Einstein; Einstein comes up with... <chuckling $>$ the thing he came up with!

89. I: Relativity!

90. R: <chuckling> Thank you! Einstein came up with relativity theory. I mean, how much more creative can you get than relativity theory? And yet he wasn't creative enough to get his head around quantum mechanics because he knew too much at that point. And I think in a way we all get to this point and this is one of things that limits us as artists; we find a particular way of doing something and we get so involved in it that we get stuck in it and you have to find some way out of that rut, and for me the way out of that rut is to stop thinking and to start being. And this is the reason that I spent a lot of my time as a photographer without a camera because it's in those moments that you find - or if I have a camera I'm not holding it. It's nearby but I'm just not with it. And I'm just feeling. I'm being mindful - mindful is the perfect term for it. I'm being mindful in the moment. And that mindfulness is not just awareness, which is where we tend to think about mindfulness, about being aware of what's going on around us. Mindfulness is so much more than that. Mindfulness is awareness, but it's awareness of self without judgement and without attachment to an outcome. So if you become aware of you environment, you stop judging that environment, so rather than saying, “Well, I can't take a picture because it's bad weather. I can't take a photograph because the light is bad", that's a judgement. You've now just judged the light as being bad. Hence the reason I say there's no such thing as good light; there's just light. What you choose to do with it turns it into good or bad light. So you take away the judgement and you can sit there knowing that if you walk away without having taken the picture you will be equally happy as if you walked away with an award-winning competition-winning shot. It's in those moments the creativity comes.

91. I: Yeah. Yeah. Brilliant. If you think back over this process when you started nearly a year ago now, the IMP test and the first interview, which had a different method to it than this one; there you were subjected to cognitive questioning, being taken in and out of it. I mean, you didn't need much support. <laughs >

92. R: Get me going on the subject.

93. I: <laughs> But partly because in a sense you've got, as the test revealed, a very clear awareness of what's going on. The point about the cognitive interviewing technique 
is you draw the person in and out, in and out, get them to look at the situation, capture them in many different directions. What can you smell, what can you hear, and so on. And that begins to activate, as far as one can, their memories of the moment, so in a sense it's creating cues to activate their cue-dependent memory, their non-conscious memory structures. But with you, you didn't seem to need all that much cuing. Looking back over the whole process, what do you think this has brought to you? If I was putting this down, what would you say?

94. R: I would say it has brought clarification. For a very long time I was very aware that when you put a camera in my hand I became a very different person. What I wasn't able to tell you at that time was why, and in teaching photography I'm eminently capable of telling you how to use a camera. I'm eminently able to tell you what the buttons and controls in the camera will do to you photographic experience. I can tell you how to compose an image. I can tell you the psychology behind pretty much all of the elements of design that you are likely to include in a photograph, what it will and won't do to the person looking at it. What I was never able to do was to tell you what that X-factor was that allowed me to see images that other people couldn't see, even though we may have been standing in exactly the same place. I seem to have this innate ability to see how that image should look so that I could then fly the camera in order to capture it.

95. I: To what extent do you think these IMP test process and the interviews have pushed you on?

96. R: I think what then happened was I... somehow - long story, so I won't bore you with it - I became very aware of why I was able to what I was able to do. I became aware as to why I was a different person when you put a camera in my hand. And because I became aware of the reason for that, I then started to study it, and I started to study neurology and I started to understand creativity. I started to study... how we conceive and perceive things, and everything that was attached to it, and so I guess that for a lot of people the work that you are doing, with the test in particular, would have been either completely unaware to that and certainly completely new to that. In a sense with me l'd already done some of that work myself, which led to the outdoor photography articles, in fact. What the test did for me was to take what for me was a very subjective opinion. This was the way I saw myself and these were the reasons - I 
thought were the reasons - I was doing what I was doing. What the test does is actually comes along and show me a way of making that more of an objective opinion.

97. As you said right at the beginning, you said, "How closely does it match your perception of yourself?" And I said, "Actually, it's almost perfect." It is almost perfect, and in that perfection, it backs up my view that the test is a very solid, reliable mechanism to determine what is going on in the mind of the photographer when they are looking at potential images or they're looking back on images they've taken and are assessing them. So, as I say, in some ways it's not an eye opener...

98. I: ... for you.

99. R: ... for me, because I kind of was already there. It was a very reliable, a very solid confirmation that what I believe is true.

100. I: Yes. Yes. A real object has been exposed by Chris Weston <chuckles>

101. [End of interview] 


\section{Test Subject IT0079}

Date of Interview 10 August 2018

[Interview starts at 00:09]

1. I: You've given me three [images]; one of outside steps, another Lerwick Harbour, and the third one a tank and field. All came through nicely, JPEGs coming out quite nicely on my display. This is the first time l've done one of these interviews over Skype, which is a very interesting experiment for me.

2. R: This is the first time in eight years I've used Skype.

3. I: Oh, right, OK. I've produced your report, I've got it on file here, and at the end of this phase of our conversation I will indicate to you some very interesting results it's generated for you, so you're going to have to wait.

4. R: I was going to say that you're saying it in the way that a doctor might say it. Makes me wonder what's coming! <laughs>

5. I: Don't worry, it's not terminal! <laughs> You're safe! They are interesting and ones that gave me great food for thought but I don't want to discuss the specifics of them until we've had a good look at these images. Which of these three would you like to start with? Which one has got the, let's say, the most salience for you as you're looking at it now?

6. R: Well, let's start with the Lerwick Harbour one. Can you bring it up on my screen or shall I get another one to look at?

7. I: You get another one to look at.

8. R: OK. <pause>

9. I: Skype doesn't allow others to see your screen; you have to use specialist software for that.

10. <pause>

11. R: Right, l'll just have to bring it up on my phone instead.

12. <pause>

13. I: If it's on your laptop you can bring it up there.

14. R: Then I'll lose the picture I'm seeing of you.

15. I: No, you will appear as a little window in the corner. I will, rather. So if you bring it up, what should happen is my face will go off to your top left, so if you're using preview, for example. You've got a Mac, haven't you?

16. R: No, no.

17. I: You've got a Windows machine?

18. R: I've been PC for 25 years.

19. I: <laughs> So you've been politically correct. 
20. R: No, not quite that long.

21. I: And it shoulder come up, and as soon as you...

22. R: Yeah. I've got it and you've gone into a small square.

23. I: Yeah, that's it. Should be top left. So, I'm looking at your image now. Now, the questions I ask you may seem a bit odd in the way they work. There is, as perhaps we can talk about later or some other day, some rationale in this. First of all, Lerwick Harbour, that's the location. What took you to that location, Neil? Why were you there?

24. R: Well, this was a field trip I did earlier this year, where I was going for the first time ever to Shetland, looking for remote landscapes with manmade structures, and I planned my trip around a lot of World War 2 structures, around Unst and places like that, and I was staying in Lerwick, and what I found and the reason this picture's significant for this year is that what I found with this picture and some others, I didn't see it in three dimensions. I was wandering around the harbour with my camera and not looking for photographs - this wasn't part of my actively look for photographs - I was just wandering around and I saw this picture in two dimensions and what I saw was that pole on the right side and an imaginary line up the steps to the top of the pole, and then the block of the building on the left, and the land in the background I just saw as a parallel rail. Line.

25. I: Before we go down that, I'd just like to ask you - the technique I'm using here is I'm going to draw you into the image because what I'm going to try and do is draw out some deeper memories of what you were doing at the point that you took your image. The way I'm going to do this is I'm going to draw you in. What time of day was this?

26. $\mathbf{R}$ : Windless, very calm, completely quiet, as you can see form the sky it was subdued; these are all features I enjoy because that is when I become most mindful, I suppose, of the scene.

27. I: That's an important point. Did you have anyone with you?

28. R: Yeah, my wife was with me but she was wandering off, looking at other things.

29. I: I just want you to go back to this moment, then. Where was she wandering with respect to you? Were you aware of that?

30. R: No, I wasn't aware of her, at the instant or the moment of taking the photograph, but I know from memory she was somewhere maybe 50 metres off to the left.

31. I: What was she wearing?

32. R: I have no idea. It wouldn't be an anorak because it was damn cold. <laughs> No, I could work it out because I know what colour her anoraks are, but that is not in my mind.

33. I: I know you're very good at working things out! I have discovered about you, you know how to work things out very well! You can't remember anything about her at the moment. I want you just sort of stay with your presence there, then, at this point where you got your camera, you're looking at this image. Can you sort of reflect a little 
but on your state of mind? This was associated with a search for location, et cetera. How would you describe your state of mind at this point?

34. R: Very peaceful. Very calm, enjoying that moment of...

35. I: You used the term mindfulness, didn't you?

36. R: It's mindful and that is very much to do with the weather conditions at the time, and I didn't have any stresses; I'd been on the island for about 10 days at this point. The wind had died down, which made a big difference.

37. I: You mentioned it was very quiet and peaceful. Can you remember anything else? You're near water; could you smell anything?

38. R: I don't remember. I can imagine there was probably seaweed, seaside smells, but that did not impinge on my psyche at the time, as I'm aware.

39. I: OK, you don't have any recollection of smell. Were there any specific sounds that you can remember? You're saying that it was calm and quite, but there's quiet and quiet, isn't there? Were there any specific sounds?

40. R: No, not that I can remember.

41. I: Any sounds of birds flying? Sea gulls?

42. R: Possibly but l'd zoned out. <chuckles>

43. I: You'd zoned out, OK.

44. R: No, I do go into a state - when I intuitively see an image like this I do go in to a state which is - I'm on my own and things around me don't impact on me. The reason I chose this image was it's one I saw in two dimensions, I didn't see it in three, and that also requires me to be in a certain state. <stutters>

45. I: That's good. You can probably see, I'm here, drawing you closer to the image. I'm just getting you to try and remember as much detail as you can. How'd you actually got to this location?

46. R: Just walking. We'd been walking around the town for about an hour, an hour and a half. We just ended up at this place.

47. I: Yeah, doing a bit of window shopping, that sort of stuff.

48. R: No, it was just wandering. My wife had never been to Shetland before; this was her first time looking around the city - town, really. We walked past the places where the film series Shetland is filmed. Do you know, the house?

49. I: What kit did you have with you?

50. R: When I'm not on my own, when I'm with other people, I carry a Fuji CSC camera and no tripod.

51. I: That's the CSC - is that a medium format camera?

52. R: No, no. It's an APSC. It's the XT1 I've got and I just carry a single zoom lens with that.

53. I: Do you carry a tripod with you?

54. R: If I'm going out on my own, I will go with my Nikon D800, the tripod and probably several lenses. I'm now specifically using a 24 , a 35 and a 50, and I've found those three lenses cover $95 \%$ of what I want to take. 
55. I: So this shot you had just the camera and this [??] purpose lens. Now, can you remember the settings you had on your camera?

56. R: Yep. It's Auto ISO, it was aperture 7.1, and I have no idea what the speed was because I let the camera deal with that.

57. I: So you were aperture priority, were you?

58. R: Aperture priority and for landscape my defaults on my Nikon is F/11 and on my Fuji's F/7.1. Those I find are the settings I use most often because they give me the depth of field I'm looking for. I will change the aperture usually down the way, down to $F / 2.8$ or something like that if I want a shallow depth of field. With this kind of landscape photography I do, that's not a common occurrence.

59. I: So can you remember actually making any changes at the point of shoot here, when you were approaching the moment of taking this shot? You recognise the location, you recognise the possibility. Can you remember actually handling, changing the camera settings?

60. R: I didn't change the camera settings - oh, by the way, I always have it on [??] + or 1 , so I just trust I'm going to get the image. The only change I made from seeing the image to pressing the shutter was I moved a couple of feet to the left so that the pole and the edge of the building were roughly the same distance each from the edge of the frame. And that was all I did, so it was a case of balancing the image; it wasn't a case of seeing the composition.

61. I: What was behind you at this point of shooting?

62. R: It's the Shetland Museum. I was on a pavement, or on the dockside which is a pavement next to a carpark for the Shetland Museum, that's it.

63. I: So, you were aware of what was behind you but you're looking out into this image. Your wife had gone away; there was no one else looking at this scene with you.

64. R: No.

65. I: Is that correct?

66. R: Yep.

67. I: You were totally on your own. Now, I want you to - you've got the image there, you might want to close your eyes now - could you describe to me, as fully as you can, what you were seeing there?

68. R: OK, so, what I saw was a flat plain, that pole and this imaginary diagonal line, plus the edge of the building. And I saw these as flat plains and I also saw the land in the distance as a thin ribbon and it was the lines, it was the shapes, it's a geometric image for me. I had had this thing that I've done all my life where I can see things and initially if I quickly go I can see the two dimensions first and it catches up and I see them in three dimensions, but if I can capture that moment of the two dimensions, I can quite often see an image like this which really excites me about its shapes.

69. I: Let's just talk for a moment about that excitement because that's actually a feeling. What was exciting you about this? 
70. R: The fact that I'd seen something completely unreal. I'd just seen shapes. It's a fairly monotone type of image; l'd seen all the shapes in greys and blacks. The colours just disappeared for me.

71. I: Why did that excite you?

72. R: Just seeing this conjunction of shapes, especially that line of the steps going up to the top of the pole. And the pole is pretty well halved by the land behind and then when I looked to the left then I had the pole about a further way in, I had the front of the building further way in. That beam coming out of the top of the building seemed to nicely balance the bollard going up in the middle of the picture.

73. I: Have you ever experienced that excitement before an image taking, or was this something you felt was quite unique in your experience of photography?

74. R: Sorry, when you say have I experienced before myself?

75. I: Yes, yourself. Have you appreciated a geometry like this?

76. R: Many times through my life. It started when I was a young child, and I can remember sitting in the back of the car, going along motorways and looking at the back of the car in front and I would see its real shape as opposed to what we think it looks like, and I remember following an E-type jaguar, which is of course a beautiful car...

77. I: A wonderful car.

78. R: ... and my brain thinking, "Those tyres need to be wider." It just didn't look balanced with 1960s tyres on it! And I was probably about 10 or 12 years old when I saw that, but it's things like that. I've done this all my life, I have this ability and today now it is driving possibly a quarter to a third of my image taking is seeing shapes like this.

79. I: Well, it's interesting, isn't it? So, I'm getting a sense from you almost of a tangible excitement.

80. R: Oh yeah. Yeah. The one photographer who's very influential for me is Lewis Baltz. Do you know him?

81. I: Yep, yep.

82. R: And his industrial park type images, photographs of warehouses and things like that; that aesthetic really appeals to me and I'm seeing that aesthetic in this image here as well.

83. I: But for you this is almost becoming from what I'm hearing here almost sublime; you're seeing great impact in the recognition of this structural form here, which is one of many possible structural forms you could encounter, but you've recognised it in the landscape in front of you.

84. R: Yeah, it's found still life in my mind - that's a term I've been using in photography for many years, and that's how I see it: it's an arrangement that I find is accidentally beautiful, because nobody intended to do it.

85. I: That's good. Now, when you got this image home and first popped it up on your computer, what was your feeling about it then? 
86. R: Still exciting. And now, three or four months later, I'm still really happy with this image, it's one of my best images that I took in Shetland. I did take quite a few others that I'm really pleased with as well, but this, of its type, was the best because I did take others of this genre. This is the one that I enjoy the most because it's the most complicated but it achieves my...

87. I: Did you do anything in post-processing - I expect you may have done a little crop and that sort of thing but was there anything else you felt necessary to do to this? Or, I should ask you, sorry Neil, how long after taking it did you actually do the postprocessing?

88. R: Week, ten days.

89. I: Week to ten days. Sorry to interrupt you there. Just take me through what adjustments you would have made or might have made here?

90. R: I don't recall cropping it. If I did it would have been trivial cropping. I don't think I have to level the horizon because l've got an artificial horizon in the camera. In terms of the tonal qualities, I often slightly desaturate my images. Certainly take down blue content because I find both the Fuji and the Nikon seem to emphasise the blues more.

91. I: Definitely do. Yeah, absolutely.

92. R: I probably desaturated that orange thing in the buoy in the foreground. I didn't clone anything out. It's fairly minimal.

93. I: Just take me to the desaturation point again. When you originally took the image, what was your perception of it tonally, can you remember?

94. R: Well, as I said earlier, I saw it in shades of grey. I didn't see all that yellow on the roof and in the stones.

95. I: And you took it into post-processing and did you find you had to do much desaturation to get the effect you wanted, or was it a fairly minor amount of tweaking of the colours and so on?

96. R: It's usually fairly minor. Unless I'm going for a specific colour to desaturate, what I would tend to do is make a simple black and white layer over the top and reduce its capacity, usually down to between 10 and 30 per cent, something like that, until I get the effect I like.

97. I: You're doing that in Photoshop?

98. R: Yep. I can't remember whether I did that on this image but that's typical what I would do.

99. I: Can you remember anything else specifically about this image that you might have done in correction?

100. R: No, just thinking about the cropping. If there's any cropping at all on this it's probably in the foreground. I can just see a little highlight coming in on the bottom edge. I probably cropped in to get rid of highlights in the base, but that would've been it.

101. I: OK. OK. Lovely image.

102. R: Thank you. 
103.

I: I like it. <laughs>

104. R: I just see it as a very calming image.

105. I: Very calm, yes, it is very calm. I'm going to move you onto your second image now, and that was very useful. Which one should we do second? You've got two images which have got some superficial similarity about them but you choose which one you would prefer to go with.

106. R: I'd like to do the outside steps.

107. I: OK, and l've got that one in front of me now.

108. R: There's a bit of history in this one.

109. I: Where are we now? Outside steps. Where are you and why are you there?

110. R: Right, right on the tip of the heel of Italy, [??] Apulia. My wife and I have been to this place on holiday twice, last year and then again this year. Last year I saw this location, this derelict house. It is a building project that was started and never finished, and that happened many years ago, and there's quite a few of these in Italy around that part, where people - I understand they would get grants from the EU to start building something and then run out of money and never finish, or then just put them on hold until they got money. And this was an example that I guess had been derelict or unfished maybe five, 10 years.

111. I: So who were you with this one?

112. R: I was on my own. So I saw the scene 12 months ago, and then when we went back to the same place this year I decided to find it because I wasn't able to take the photograph before but I was so intrigued by these steps, so I went back, found the location, and was able to go in and take lots of images around the area. This particular composition appealed because of the shapes of the steps, the obvious unfinished nature of them, and space around where nature is taking over.

113. I: What time of day were you here?

114. R: This would have been about four o'clock in the afternoon.

115. I: OK, so it was an afternoon shot.

116. R: Yep.

117. I: And where were you immediately before this? Where had you come from?

118. R: I'd left my wife at the hotel, she was swimming and I'd just got in the car and I was hunting for sites like this. It took me an hour to find it, but l'd been around several other places taking similar kinds of photographs.

119. I: So, middle of the afternoon, still got a couple of hours to go until evening drink and food, so are you feeling pretty comfortable?

120. R: Yeah, I'm very relaxed because we're on holiday, we're in a place we know having been there once before. Although driving in Italy's not that easy, this part of the country's quite sparsely populated.

121. I: So you're driving around, you've got your kit with you. Are you fully tooled up this time? 
122. R: No. Same kit, whenever I go away with my wife travelling, I only take the Fuji handheld, one lens. My wife is very tolerant but I don't find I get into the right mood to seriously take photographs.

123. I: <laughs> Your wife isn't a photographer, I gather by what you're saying.

124. R: She's not. She's not. This is a case where l'd left her swimming off the rocks next to the hotel and she was quite happy to do that all afternoon while I went off and did my thing. And the Fuji gives really good results; I can blow this picture up to a two without any issues.

125. I: I've got a very close friend who's the Fuji wildlife champion and he's being trying to persuade me to get one for a long time.

126. R: I've got the XT1. It's beautiful. The size of the camera is about the same as my old Olympus OM10 and I like its size.

127. I: And when you're hefting it, it feels quite light in your hands, does it? Certainly compared with the D800.

128. R: D800's a monster and 98\% of the time I have it on the tripod.

129. I: Yeah, simply through the physical weight of it. So you had your Fuji camera, the [??] lens. I'm going to ask you the same question again: when you were preparing to take this shot, you're standing there, you're holding the camera; what were the settings on the camera?

130. R: Same as before. So they're the default settings and there's nothing in this image that would have caused me to change those settings.

131. I: So at the moment of capture, then, or just prior to that moment of capture, can you actually remember making any changes to the adjustments on the camera?

132. R: No. No.

133. I: What could you smell when you were here?

134. R: <inhales, then exhales> I don't think the sense of smell impinges on me that much. I can't remember. I'm sure there's lots of Mediterranean smells of olives and all sorts, but no, I can't remember.

135. I: Sounds. Let's just probe the sounds, then.

136. R: Sounds... probably birds, [??].

137. I: How far away were you from the road?

138. R: Well, the road is about 50 metres behind me. But I'm hidden from the road by fences and hedges.

139. I: Looking at this, was there much in the way of wind or anything else?

140. R: No, there's no wind.

141. I: Dead still?

142. R: It was hot and quite humid.

143. I: Yeah. Were you feeling uncomfortable at that point?

144. R: No.

145. I: With the humidity? It wasn't that hot? 
146. R: No, the only uncomfortable feelings I had were on approaching this site because it's not very remote there are houses all around it, I was conscious of, is somebody going to come along and say, "What are you doing on this property?" The mafia are quite strong down there.

147. I: Did you have a look behind you at the point of shoot? Just prior to the point of shoot?

148. R: No. No. l'd arrived at this site - because l'd seen it a year before, I had a very good idea of the mood I wanted to create and the composition. I'd arrived at this site, I'd taken a few pictures off to my right here, I then walked right round the building and back to the front, so I'd been on the site for 15, 20 minutes already when I finally took this shot. Previous shots had focused on trying to make the steps really big and important, and then finally this where I thought, not just the steps but the space and that tree seemed to be nicely positioned. This was an image which I would describe as a 'belief' image; it was intuitive that that there would be a good picture there, I believed that there'd be a good picture there; it didn't just jump out at me.

149. I: I'm now going to get you to do what you did a few moments back with the previous image. You don't have to shut your eyes but really l'd like you to describe this image to me as it's coming up in your perception now and you memory, what were you looking at? Can you describe it?

150. R: I'm looking at the juxtaposition of different shapes: the triangles of the steps, both the big triangle of the two sets of steps but also the little steps; that concrete pillar on the left side, sort of the solidity of that; the kind of fragility of the steps but they're all pointing towards the tree and the broken wall beyond; and you've got this scrubby grassland where they're pointing to.

151. I: So, describing that, when you brought this back home a week later or whenever it is you post-processed it, what was its impact upon you then? What were your thoughts towards it then?

152. R: This is an image I grew to like as I worked on it. I like the composition; I was really pleased with the placement of the big tree, the broken walls. It was a more difficult image to process than the last one because the steps themselves - the two diagonal pieces of step - did get caught up in the background. The background was dark and they were dark. In this I've actually lightened the trees in the background and the wall in the background, and darkened the steps to make them stand out. So there has been quite a significant bit of processing to make what I wanted to highlight more visible.

153. I: Well, that's fair enough, and as you're looking at it now, what do you feel about the image now? Has it worked for you?

154. R: Yeah. Still works. I'd still like to separate those steps from the background a little more, especially the upper one. It's the overall arrangement that I've achieved. Again is placement of shapes and weights of things; there's a balance to the image. 
But also there's a lot of narrative in it, so this image is not just driven by shapes; it's also driven more strongly by narrative of why, who, when.

155. I: Yes, yes. You think a lot of money and ambition went into this but... <laughs>

156. R: It's been abandoned.

157. I: ... It's not a complete job, isn't it?

158. R: Yeah. And you can't see it from this angle, this is a big house. It's got a big basement underneath with garages for several cars, things like that.

159. I: And obviously a fair estate around it as well.

160. R: Oh, it was in about, l'd say half an acre of ground.

161. I: OK. Fascinating. Let's go to your last image, then. You're doing very well, Neil. Thank you very much for your time here. But the last one you've got is a tank and field. Where did you take this shot? What location am I looking at here?

162. R: It's only about a kilometre from the previous one. It's taken about half an hour later. I was driving along this lane and I saw this, about a hundred metres off the road, and I thought, "There's got to be a photograph there." Again, my big thing is the juxtaposition of the manmade against the natural, and as I walked up to it, I took initially a photograph to the left where that wall you can see and the tank were more centrally placed, so that was the first image, and I wasn't quite as happy with that and then I moved over, and that's when I saw the old milk bottle which I presume is full of fuel or something like that. Certainly wouldn't want to drink it. And this, with the tank sort of dominant in the foreground on the left but kind of balanced by the breezeblocks on the right wall separating you from the field which has got all those lovely diagonal lines from the straw. So it's manmade structures, it's shapes, there's also a sense of barriers; there's a barrier from where I am, very manmade into the famer's field and then the trees beyond like a barrier where you don't know whether there's a cliff over there or something else.

163. I: And you're on your own again with this one, were you?

164. R: Yeah.

165. I: With the Fuji? The same settings?

166. R: Yeah.

167. I: So, looking at this image, and I think we can move straight to it then, you're recollecting strongly now. Can I just ask you then to describe this image to me.

168. R: It's got all the features in it that I've been doing for several years: it's manmade against a [??] natural background, but these last two images were fairly new for me in that they were in relatively built-up and busy areas. I know the Lerwick one as well, that's a small town, but the vast majority of my images up to that point were taken in places where I was the only person, there's very little, really open spaces and no trees, so there's no people, no trees, old buildings that are now derelict. Whereas this is more like a newer building, maybe it's in progress, this is a functional place, obviously work has been done here recently. So this is a shift in the kind of photography I'm doing, so when you asked for something I've done in the last six 
months, what you're seeing is actually some shifts from what I was doing previously, which were very much more open environments, where I was feeling the, as you described, the sublime. I used to feel the sublime because I was in a sublime place, whereas now, with these images I'm starting to feel the sublime but not in a sublime place.

169. I: For you, what is the sublime in this? What is it really catching your attention in the image? You've given me a lot of context here but in the image what is it that is really working for you again?

170. R: Again it's shapes. It's the big block, rectangle, the tank, then the block on the right hand side of the breezeblocks, the... line of trees in the background, there's a barrier similar to the line of trees in the landscape of the Lerwick picture. It's all about lines and shapes.

171. I: It is about lines and shapes.

172. R: But it's also more narrative. The Lerwick picture for me had no narrative. The last picture we looked at, the steps, had a lot of narrative. This is somewhere in between. It's got narrative but it's also about the shapes.

173. I: That is interesting and coming back to it, I think I'm discerning a similarity in the structures of these three images, around the triangle again. And you've got it here, quite dominant in my eyes, possibly even more so than the shape of the tank which is very dominant but it does form a very strong diagonal here in my eyes and I've seen that in both of your previous images as a structural form which it has emerged here; it would be interesting if it was in your other images as well. I think with composition, we do tend to have a dominant structural form. You're in good company with this. If you look at Don McCullin's photographs, images, the triangle is there very much in his image-making.

174. R: I don't know his work, so I'll write the name down.

175. I: Yeah, it is fascinating. Well, that is the end of that phase of the interview. 
Test Subject IT0084

Date of Interview 20 September 2018

Transcript

[Interview starts at 11:18]

Image 1

1. Interviewer: This is your first image and it's an image of a Manx shearwater?

2. Respondent: It's a Manx shearwater.

3. I: First thing I want you to tell me: can you remember the day you took this?

4. R: Specifically the date?

5. I: Do you just remember the day?

6. R: I remember the day. It was the end of a very long day so I'm actually on boat, off shore, obviously, by a couple of miles from the Skomer Island area. Pembrokeshire coast.

7. I: You were away from home?

8. R: Yeah, I'm actually on a workshop here, so I'm with a group of people throughout the day. We spent the day on Skomer Island photographing seabird colonies principally but other flora and fauna as well.

9. I: When you start your day, what did you have for breakfast?

10. R: That day? I think probably a couple of poached eggs on toast, I should think.

11. I: Can you remember the poached eggs on toast?

12. R: Probably, because we stayed at a guesthouse in Broad Haven and I've been using this location now for the last three years, and Helen Parry, the proprietor there who knows me quite well - because I generally visit at least two if not three times a year. Breakfast that morning and then we were off to Skomer. Probably onto Skomer by took the first boat so we were on the island by about quarter to eleven.

13. I: Can you remember sitting down for breakfast?

14. R: Oh yes.

15. I: Can you?

16. R: Breakfast I had with the guests on the workshop. 
17. I: Just think: what did those eggs smell like?

18. R: Eggs, just mainly eggs. <laughs>

19. I: Were they tasty?

20. R: <pause> Oh yes, because Helen's a very good cook and as is her kitchen assistant,

21. I: Did you sprinkle anything on them?

22. R: Knowing me, probably a very light sprinkling of some black pepper and some salt.

23. I: And you're in the room with all your other guests?

24. $\mathbf{R}$ : Yes, we'd all have breakfast together.

25. I: Can you remember what you were wearing?

26. R: I can, and the reason I can remember what I'm wearing because we were down there at the beginning of July in that middle part of that heatwave we had and I can remember remarking to the guests that in 15 years of running workshops on Skomer, it was the first ever time I'd chosen to wear shorts. For the day. So I was wearing canvas cotton shorts and a short-sleeved shirt, because the forecast was it was going to be pretty warm on the island.

27. I: What did you feel about wearing shorts? Is this exposing your legs too much?

28. R: <laughter> Well, apart from scaring the horses, and there aren't any of those on Skomer! Well, no, it was just practical, because, as I say, I do remember having a conversation with people about their welfare in terms of making sure they got plenty of fluid with them because there are virtually no services on Skomer, or facilities. Sunblock, appropriate headwear and everything else. Roll through the usual pre-trip briefing.

29. I: And your camera kit - was that up in the room? Did you have to go back and get it at the end of breakfast?

30. R: Typically yes, I would've left the kit. Pre-packed the bag before coming down to breakfast. Breakfast is a social event for me on those trips, so the kit's up in the room.

31. I: So you remember going back up and retrieving that?

32. R: I presume I would have done. I don't for sure know. I might have - it's unusual - I might've left the gear in the Land Rover, but probably left it in the room.

33. I: And it was a Land Rover you went to the location in, was it?

34. R: Absolutely, yes.

35. I: Was this one of these long wheel-base Land Rovers?

36. R: It is indeed. 110 Defender.

37. I: So you had these other people with you. How many?

38. R: We were full that workshop, so it was eight.

39. I: Any one of them you felt more attractive and affiliation to, responsive to the other?

40. R: No, no, I mean there were two, three who'd been in workshops with me to that location previously. One chap I do remember specifically because he'd been a previous year and for a couple of reasons, he'd been unsuccessful in getting a, what he thought, and I would agree with him, successful photograph of a puffin in flight. So, he'd come 
back with the sole purpose, the entire day on Skomer to get a puffin in flight. I'm pleased to say he did achieve his objectives.

41. I: Are you saying there, then, that with this individual that he had a particular objective that you were very sympathetic towards?

42. R: Oh, completely, yes. As I say, he'd taken the time and the trouble - and the expense - to come back for a second year running to have another crack at doing it. l'd known him or a number of years; he's been on several workshops around about with me so, yes, he was up for the challenge, as it were, and was completely satisfied with the previous year's attempts - or failed attempts - were down to principally operator error on his part, and to some extent equipment, sort of, not failings but misunderstanding or mis-setting of the camera.

43. I: So when you were in your Land Rover, the, how far was the Land Rover from the place you were going to disembark Land Rover?

44. R: As the crow flies, it's probably no more than about five or six miles but we are talking deepest, darkest Pembrokeshire where the lanes are about six inches wide, so you allow plenty of time to meet Farmer Giles in his tractor and a few others. Probably took us about 40 minutes to drive over in a two or three vehicle convoy.

45. I: So you were there all day round the island, then, were you?

46. R: Well, you're limited - I don't know if you've been to Skomer before?

47. I: No, l've never been to Skomer.

48. R: Skomer is part of the South West Wales Wildlife Trust and they control visitors in terms of number and duration of visit quite strictly, so long story short, they run a number of boats, weather permitting, each day. It's 50 people per boat to a maximum of four sailings and it's first boat out, you're on the first boat back, so if you take the 10 o' clock boat you are obliged by whatever colour-coded ticketing system to be on the first boat back, and they make it very clear to you, if you miss that boat, there won't be any room on subsequent boats because they're already fully booked, so it's down to you to charter the boat to turn around and pick you up.

49. I: And that will cost you some money.

50. R: $£ 250$.

51. I: Oh, fair enough. That's a good fine, isn't it?

52. R: It tends to concentrate people's minds about being back in time for the boat.

53. I: so, roughly what time did you embark on the boat, then?

54. R: The boat's pretty punctual so generally at the landing stage about ten minutes before, so the boat would have gone at about ten o' clock.

55. I: And that was the boat you were on?

56. R: Yeah.

57. I: So you're on this landing stage, you've got all of your pilgrims with you. All ready and kitted up?

58. R: Absolutely.

59. I: And you had your kit back? 
60. R: Yes.

61. I: You carrying it?

62. R: Not at that stage, it's parked on the landing stage.

63. I: It's parked on the landing stage.

64. R: By my feet.

65. I: So do you pick it up to take it on the boat?

66. R: Yep. It's a stipulation of the workshop. I put it out in the initial information - the booking information - that goes out: each guest is responsible for carrying their own equipment.

67. I: So you lift up your bag. How heavy is it? Does it feel heavy?

68. R: It's reasonably heavy. I've obviously over the years managed to fine tune what I take in terms of anticipating what I will need, therefore I take just that what I would consider to be essential, but it's probably still weighing in at about 10 kilograms.

69. I: Can you remember the act of lifting it?

70. R: Not specifically that day but I know that particular bag and it is configured for this particular workshop, I know how much it weighs.

71. I: Would you know just by feel of the weight that that was your bag you had picked up?

72. R: Some people don't necessarily heed all the advice I give them and bring the kitchen sink, regret it halfway round the island. Other people, of course, come - I'm lucky, privileged enough to own a fairly wide range of equipment; some people bring what they've got, it might only be one camera, one lens. It doesn't mean that it diminishes their enjoyment of the day, or indeed their ability to take pictures. Skomer is one of those where even a very modest focal length of lens still permits you to take close-up pictures of birds because unlike many wildlife subjects, you can actually get very close. I've actually had birds sitting on the toecaps of my boots before. Puffins, that is.

73. I: So you're on the island now and are you dispersed from your group?

74. R: No. the first thing you're obliged to do is listen to a five-minute briefing from one of the wardens or deputy wardens about your behaviour on the island. Bear in mind that environmentally it is a critically important site for a number of species and there are huge numbers, so the Manx shearwater, there are 320,000 pairs on the island. It's the largest population in the world. The puffin - currently it's the only puffin population in the UK increasing in numbers; it's now about 27,000 individual birds, whereas all the other puffin populations around the UK coast are in decline, principally those around the north east coast of England, Scotland, and that's a direct consequence of the over-fishing of sand eels in the North Sea. But the island-because puffins obviously live in burrows, Manx shearwater live in burrows - it's a fairly small land mass and it is literally peppered with burrows; you can't move for them. So the purpose of the briefing is to impress upon people the importance of strictly staying on the paths. 
75. I: Can you remember who actually gave the briefing that time? Can you visualise that person?

76. R: I can visualise her, yes, she's actually not a UK national, she's actually a Swedish lady, and she volunteer to work there each summer.

77. I: What was she dressed in?

78. R: Shorts, T-shirt, sunhat. <laughs>

79. I: Large sunhat.

80. R: Yeah, large sunhat, and a pair of binoculars.

81. I: Well smothered up with suntan lotion, I would imagine.

82. R: Yes, absolutely, and again she rolled through all of the advice that I had given over breakfast about making sure you keep your head covered, lots of sunblock, lots of fluid, and so on and so forth.

83. I: You had this briefing, you were moving around. How far were you from the location you took this shot?

84. R: Well, that picture was taken a couple of miles offshore.

85. I: Right, on the boat.

86. R: Yeah, on the boat much later on in the day. But the actual bird population you tend to gravitate towards because of their accessibility on the island are probably a 25,30 minute walk.

87. I: So you're back on the boat.

88. R: To take this picture?

89. I: Yeah, to take this picture.

90. R: Yeah, this picture - the day on the island has finished, and we've then gone back to the guesthouse, had some refreshments, and then myself and three others had separately arranged to go out on the boat this evening - this is purely optional, this boat trip - so three of them wanted to go on the boat trip so I obviously booked to go with them. The boat trip starts 7pm. It's generally an hour and a half's duration, bear in mind again obviously the days are quite long and we're talking early July, and the boatman [??] take where you go because obviously around that particular part of the world, there are all sorts of very important considerations: tides, wind direction and strength, so he will be restricted to where he can go based on the prevailing weather conditions and the sea conditions. It just so happened that this particular evening we were blessed with fairly calm conditions, beautiful light and we actually picked up a pod of passing porpoises. We'd gone out much further into the Irish sea than we would normally have gone on that boat trip, tracking the porpoises, and this is actually on the return towards Skomer and so we'd gone probably a couple of miles offshore from the mainland. Normally he'd probably only go half, three-quarters of a mile at most, but specifically to follow the porpoises.

91. I: How high was the sun in the sky as you approached this?

92. R: This is pushing on towards last light, so it's probably the last half an hour of light.

93. I: So the sun's getting pretty low. 
94. R: Pretty low, yeah. We're out in open water here so there's no obstructions in terms of any landmass.

95. I: What about low lying cloud on the horizon?

96. R: No, it was pretty much clear; there was a little bit of wispy cloud above but it was a beautiful, beautiful evening.

97. I: How many were on the boat with you?

98. R: Probably about 35 people in total.

99. I: On the boat? So it's quite a crowded boat, then?

100. R: It's not too bad. I mean, it'll take 60. It's an open deck at the back there's just a small wheelhouse. It'll take 60 , so it's probably just over $50 \%$ capacity and the reason for that is obviously people like to move around; it's quite popular with photographers and birdwatchers so people like to have flexibility of moving around.

101. I: So you moved around. Presumably you got quite close to one of the rails.

102. R: Oh yes, you're on the edge of the boat here. The boat is motoring; we're not stationary but as you can see from the waves there's a little bit of a swell, so yes, you're bracing yourself against the rail, trying to hold onto something with your knees.

103. I: Getting any travel sickness at this point?

104. R: Touch wood. I tend not to suffer, even on small boats in sort of <laughing> relatively choppy seas. I think probably the concentration on the subject and trying to concentrate on the camera tends to take your mind off it. The fact that you're actually out on an open deck I think also helps because subliminally you've got the horizon there. What I'd be like below decks, I'm not sure. In this instance, I've never had any problems.

105. I: Did you have your camera out of the bag?

106. R: Oh, absolutely.

107. I: Yeah, you had it out of the bag. Describe your kit, then. What have you got? You've got your Nikon, I presume.

108. R: Yeah, both Nikon cameras; I'm working with a pair of D850s and each camera's got a different lens on. This was taken on a $\mathbf{3 0 0}$ millimetre lens, the other lens is a shorter 70-200 zoom.

109. I: Who's standing next to you?

110. R: Standing next to me as I was shooting this sequence was my son.

111. I: Oh right.

112. R: He'd come on this trip specifically to come on the boat trip with me. So he'd joined me on this workshop, and then I had the three guests who were aligned on the rail the other side.

113. I: What was your son dressed in?

114. R: Pretty much the same sort of kit that I was wearing, so, shorts, walking shoes or boots - I can't remember which - and then a couple of layered tops because it tends to get quite breezy.

115. I: What was he looking at? 
116. R: Well, it was the first time he'd ever actually been on a wildlife shoot - in this circumstance. He'd come on a safari a few years ago to South Africa with me, but in terms of being out on open water photographing wildlife, that was a first for him. And I think it was the first time he'd really encountered large numbers - and I'm talking hundreds, if not thousands - of birds, both on the water and in the air, so there was a degree of spectacle for him in terms of, he was sort of more taken in by what he was expecting visually than he was actually with the camera.

117. I: You're a man with a mission.

118. R: <laughing > I'm a man with a mission! Specifically to photograph Manx shearwater.

119. I: What were your settings on your camera?

120. R: They would have all been pre-set based on the fact that the light was very consistent: clear sky, sun low in the sky but still at a consistent level of light, so I'm prioritising initially on shutter speed, and then aperture and ISO were selected accordingly. Typically the aperture is quite wide, and then the priority here is shutter speed.

121. I: So can you remember the ISO setting you had?

122. R: Probably for this it was around about 400 because I'd have kept the lens pretty much wide open. Shutter speed would have been up around about $1000^{\text {th }}$, $2000^{\text {th }}$ of a second.

123. I: OK, so you'd have been on F5 point-

124. R: F4 probably.

125. I: F4, OK. As you're lifting the camera to your eye you caught the sight of the bird, did you make any changes to the setting of the camera when you took this shot?

126. R: No. Because I know that the light is consistent, I'm actually not paying any attention to the camera settings in terms of the exposure whatsoever because it's already pre-set. All I'm interested in is the focusing and the composition.

127. I: So what about the focal point? Did you hold it centre frame or did you correct your focal point?

128. R: No, because the background to the shot is that as we were returning to the island of the mainland on our post-hour porpoise tracking we saw a large number of Manx shearwater on the water. I don't know if you're aware but essentially they are a nocturnal species so if you spend a day on the water, they will only go to their burrows on Skomer after dark because they are predated by the black backed gulls, and they won't risk going at even twilight. So they wait until complete darkness before they go into land. You don't see them during the day at all unless you're out on the boat and to capture them in this lovely light and, as I say, being fairly active is a fairly rare opportunity.

129. I: So you picked this bird up, then, in your viewfinder. How far away would you have said it was coming towards you? Was it coming across your frame or sweeping in? 
130. R: The boatman was being very obliging because he could see probably two, maybe three hundred shearwater on the surface of the water just bobbing up and down because they're little dots at this stage as we're approaching, and he's forewarned everybody on the boat that what he's intending to do is get round behind the birds - because they're all generally facing towards the island and the mainland and then he'll sort of open the throttle slightly on the boat - not excessively so but get up a little bit of speed and the whole idea is to drive the birds forward and induce some of them to take flight, which is exactly what happened so there was a degree of preparation. It's not causing the birds any distress; we've got to go past them or through them anyway to get back to mainland, so it's a question of just using the circumstance to our advantage. If it was question then of trying to pick up on one or two birds and hope one of them was going to react, but obviously from a compositional point of view there's no point photographing the bird from behind because - bum shot. So we had to get alongside or ahead of the bird, so it was a question of most of them did start to fly or head off on the water, paddling furiously away from the boat. But a few of them seemed to sit it out and wait and see what happened, and a couple of them as we got level with them suddenly decided, "I've had enough, I'm going to take off", which is what I was spotting for, so as soon as I certainly saw a bird starting to make those motions - wings starting to flap - that's when the camera came up to the eye. Focus setting, it would have been slightly right of centre because the bird is right to left.

131. I: I want you to have a look at that image now and I'd like you to describe it to me.

132. R: So it's a Manx shearwater with both wings fully outstretched above its head. It's clear of the water but only just. Both of its feet are visible and its left foot is behind its body. The Manx shearwater is designed for flying and swimming; it's not designed for walking. The legs are set so far back on the body that if it is on land it can't actually walk; it literally sits on its belly and propels itself along in a sort of sliding action. But to capture the bird actually on the water with just one foot in touch with the water the idea of the image was in my head and of course little bit of luck comes into it because you can't time with absolute precision the exposure, but as you can see from the image I've got actually two splashes; the previous splash with the droplets of the water still in the air, and then the splash he's making currently with the left foot actually still in contact with the water. And then otherwise just clear waves behind as a fairly clear, uninterrupted background.

133. I: Is there anything else notable about the image?

134. R: Principally for me it was the light. We were lucky; the lateness of the day, the sun was low, there was a nice warm colour quality to the light. It was low, as well, so the underside of the bird is well-lit, as is the wing that's facing towards the sun, and the low angle of the light has also added lots of nice texture to the water and the 
waves. And of course the proximity of the bird to the water surface has then added the reflection, which has added some nice interest in the foreground.

135. I: Looking at the image, what do you feel about it? What is your response to it as an image?

136. R: It was the level of animation in the bird that sort of appealed to me. It's the activity and the prospect of flight but then to some degree the awkwardness of the transition from happily floating on the waves to then suddenly being airborne, and the frantic nature that is involved in going for that transition, so yes, it was sort of an image I'd had in my mind's eye that sort of did come together.

137. I: You talk about this 'frantic'. Why do you feel that was important to you for the image?

138. R: Because I think it's just telling the story about the bird and the bird's behaviour, and its lifestyle and life cycle. It's not unique, this image, far from it, but it's unusual to see a shearwater during daylight hours because you have to go out quite a way to see, and just to have the opportunity of being in a small boat, close to the water's surface, in some beautiful evening light, and capturing it in a sort of state of high animation.

Image 2

139. I: Excellent. Well done. Let's move onto another image. Now, I'm looking a picture of a lion here, and presumably not taken in the United Kingdom.

140. R: Most definitely not taken in the United Kingdom!

141. I: She looks like a wild lion!

142. R: Very much a wild lion. This is a young female lion.

143. I: OK. Let's just open up your day a moment, though I think your memory's now well activated. Where are you?

144. R: this is South Luangwa Valley in Zambia. This is back in late May this year and a trip there I was leading with six other guests. We're staying in a camp, actually quite a way from where this picture was taken, and this, believe it or not, was the very first $<$ chuckling > day of the trip and we were just blessed. We'd been out on an afternoon game drive having arrived. It had been a very, very long 24 hours because I travel with the guests from London the previous day, we'd had an overnight flight into Nairobi, and then Nairobi on down to Lusaka, and then Lusaka into the bush. We'd arrived at camp at lunchtime. We'd had a quick refresh and our lunch and then three-thirty that afternoon we were out on a game drive. And this is about six o' clock in the evening. I can tell that because Zambia and that particular part of the park, the sun sets at about six-thirty and at that time of the year six-thirty, six-forty-ish because you're only a few degrees south of the equator, so there's not much variance through the year.

145. I: What time, then, were you taking this shot?

146. R: This was around $6 \mathrm{pm}$. So we'd been out on the vehicle for about three hours - two and a half, pushing on three hours by then. 
148. R: I had one-myself and two other guests, and the other three - three guests with me and three guests in the other vehicle and on this particular occasion - because we only had two guests that afternoon, that's right - I was actually sitting in the front passenger seat beside the driver.

149. I: Right. So you're quite low down then?

150. R: I'm actually quite low down relative to - for the purpose of the recording I should explain: the vehicles have tiered seating; three benches, so the bench immediately behind the driver's position is the lowest, and then each one behind successively higher. The rear seat is the highest possession. But I'm actually right at the front of the vehicle in the passenger's seat, therefore at the sort of height of a typical $4 \times 4$ type vehicle, a little bit higher than a saloon type road-going vehicle.

151. I: So you've been out all day. You're just getting near to the sundowner and so on. Are you feeling hungry at this stage?

152. R: No, no thoughts whatsoever of sundowners or food. We had just had - I mean, it was the most incredible game drive - we had - bear in mind, as I said, everyone was fairly fatigued. We had a long period of travel, and it is a long journey, several flights involved and so on and so forth. It was very hot, probably about 26, 27 degrees centigrade when we set off from camp that afternoon. But all thoughts of any sort of food or drink were well out of people's minds because we'd just spent the previous hour photographing a young male leopard which was a fantastic sighting for the first game drive; bearing in mind of the six guests I had, four had never been to Africa before, let alone been on a safari, so they were absolutely cock-a-hoop, as I was for them that they'd had that fantastic sighting of the leopard. And as we were turning our attentions to the hour of the day and starting to head back to camp, not that there's any real timetable to these sorts of things, we rounded the corner, only to find this young lady and three other members of her pride, just lounging in the evening sunlight.

153. I: So you've just come round the corner. You've got your camera in your bag.

154. R: Oh no, camera's on my neck. Literally at the ready to go.

155. I: What lens did you have on here?

156. R: This was taken on a $600 \mathrm{~F} 4$.

157. I: So $600 \mathrm{~mm}$ lens you've got on here?

158. R: Yes, 600 prime lens.

159. I: How were you supporting your camera?

160. R: I actually reclined across the passenger seat; my legs were hanging out the door - there is no door, I should say; the passenger and driver door don't exist; they're taken off for access to the vehicle. So my feet are actually outside the vehicle, sort of resting on the running board and then the lens is actually propped up on my knee.

161. I: And that's how you intended to shoot? 
162. R: Absolutely, because I was trying to get the lens as low as I could, relative to the subject, and then we were fortuitous as much as the track that we were in was in a bit of a gully, so it brought the lens almost but not quite down to the same level as the subject.

163. I: Feel the weight of the camera. Can you feel it?

164. R: Yes I can, weight and size. It's quite cumbersome, but it's my favourite lens of all. And I wouldn't go on a trip like this without it.

165. I: Give me your settings. What had you got on it?

166. R: It would have been wide open at F4, ISO was probably no more than 400 , and shutter speed would have probably been around about $1000^{\text {th }}$ of a second. I'm not looking at any depth of field issues here at all. I'm using the lens at its maximum aperture because I want to really isolate the subject. I'm not even thinking about and because of her posture and the nature of the light, all I'm looking for here is just her nice pin sharp eyes.

167. I: So what do you feel when you get this close to a lion? How far physically were you from it? You shot with a 600.

168. R: She's probably about 25 metres away.

169. I: So not in a life-threatening position for you?

170. R: No. No. Looking at the other, she was reclined as you see her in the photograph now. The other three cats I think we saw all had quite distended bellies, so they were well fed; they weren't looking in the least bit hungry, they weren't looking ill or distressed in any way; they were all very relaxed in my presence, so the initial assessment was they were all quite placid. They're still wild lions, you know, you're not going to do anything stupid, but you can sort of make an assessment as to what condition the cats are in in this situation, and obviously the driver guide is extremely knowledgeable - far more knowledgeable of these things than I am. He too will make an assessment and if he doesn't think it's safe he won't stop. Simple as that.

171. I: So your stomach's not active, you're not getting hungry yet, but what can you smell? Remember the smell?

172. R: Oh the smell? <pause> Sort of earthy smell, I suppose, because you're not far from the Luangwa River and in May the water's still relatively high, so there was sort of dampy, sort of earthy smell.

173. I: Can you smell your guests?

174. R: Certainly can't smell guests. No. I just remember it being - just sensing the air cooling and, as I say, the gully behind the cat that you see in the photograph here still actually had running water. Not much, but it was still damp.

175. I: What's on the dashboard?

176. R: Nothing. There's no windscreen; there's nothing.

177. I: No gun laid across or anything?

178. R: No, no weapons carried. They're not authorised to carry weapons in the park. No weapons, there's nothing on the dashboard immediately in front on me. 
Wedged in the foot well is my small camera bag and wedged round the side pocket behind the gearstick is my binoculars.

179. I: So, you've got this young lady lined up, and aiming to shoot. Any adjustments at this last moment on your camera, do you remember? Anything on the focal point?

180. R: Nothing on the focus; focus is pre-set; I have a pretty standard focus configuration on the camera. Very, very rarely do I change that, so focusing is all preset. It was just a question of selecting the focus point - right of centre here because she's to the right side of the frame. My immediate assessment was the nature of the light, as you can see it's very strong side lighting. She's in a shaft of sunlight, whereas virtually everything else in the frame is in shadow, so as I'm lifting the camera to my eye I'm already thinking about making sure I make an exposure adjustment so that I don't overexposure this area which is in the sunlight and just instinctively as the camera comes to my eye, again I have the camera set up on the rear command aisle which allows me to control the shutter speed. I'm deliberately turning that away from the viewfinder because that increases the shutter speed so that it's about two-thirds of a stop under the camera's recommended exposure setting because I want to make sure that I retain this brightness here without overlooking...

181. I: Burn out...

182. R: ... The highlights, yeah.

183. I: You're now looking at that image. I'd like you to describe it as fully as you can to me.

184. R: So it's a young female lion, identifiable because she's still got a pink skin on her nose. She's reclined, head up, shoulders up, two front paws outstretched in front of her, both ears are up, she's alert, eyes open. She's not looking directly at the camera; she's looking slightly off to the left of the camera and her left side - left side of her head, left shoulder and her chest, as you can see it - are in a shaft of yellowywarm sunlight. The remainder of the frame is lit but it's in shade and it's basically soft earth and some stumps of scrub grass.

185. I: Looking at your lion there, then, what are you feeling now about your image?

186. R: What struck me about her when we saw her - we drove very slowly around the bend in the track and we saw her, she just reclined - she just looked very peaceful, very tranquil, very self-assured, didn't really react to our presence at all. We got a cursory glance and she went back to just watching the other three or four members of her pride who were around about; they weren't really doing anything, there was no point of interest, there was no kill or cubs or anything like that; they were all adult cats. And it was a mixture of the peace and tranquillity and the beautiful light that was just sort of breaking across the front, sort of separating her, lifting her away from the background. As I say, fortuitously we were in a gully so the vehicle was low relative to her and therefore the camera position was as well.

187. I: You get that. 
188. R: It's almost as if you're actually sitting on the ground in front of her. Clearly we weren't but from the viewer's point of view. And the left eye is lit up - the iris of the eye is lit up with the golden light so you get that golden iris, so it sort of lures the viewer in.

189. I: What do you feel when you get close to a lion?

190. R: Well, I've been very lucky to have been on countless safaris over the years and I never, ever lose the thrill of being this close to an entirely wild animal, particularly an animal like a cat or an elephant, wild dog, whatever it might be, I just feel enormously privileged to actually be there and lucky to still see them in their environment and see these animals clearly, in this case, thriving. One hears so many sad stories of declining populations - usually at the hand of man.

191. I: So would you say there's an element of sadness in your feeling there?

192. R: $<$ pause $>$ Not sadness, anxiety, I think, if anything. Anxiety for her future and the future of her pride.

193. I: How many little ones did she have?

194. R: No cubs present. We never actually found the cubs. The guides were convinced that not this particular cat but at least two of the cats in the pride had got cubs or had cubs at that time.

$195 . \quad$ I: But were somewhere else.

196. R: But we couldn't find them. The next couple of days we did go looking - when we were in this particular park the pride didn't move, which is another indication the cubs are nearby but the cubs are hidden away. The lionesses can go hunting. And we actually never saw the male lion where we found him. We saw another male lion with the entire pride, including cubs, but this was a different pride, not associated, and that was almost at the end of the trip, the other end of the tip, so it was six days later.

Image 3

197. I: Let's move onto your next image. Just describe this image to me, if you would, in terms of what we're looking at.

198. R: This is a chameleon. It's a native species, quite a common species. Again this in Zambia; in fact it was taken on the same trip as the shot of the lion but not the same day.

199. I: Can you remember which day it was, this one? Was it towards the end?

200. R: I think it was more the middle part of the week and this was just one of those happy accidents. This is actually taken in the camp. The camp, again for the purpose of the recording, comprises - it's a small camp, privately owned and it's ten chalets around a central lodge building. The lodge building is completely open to the elements; one side there's no walls, doors, windows, or anything, and it just looks out over the floodplain by the river in the South Luangwa Valley. And as part and parcel of the camp structure, they actually have an elevated platform - an open platform built on a very large tree where you can have lunch and you can overlook this glorious 
scene. So we've had lunch and I'm walking back down the central path through the camp back towards where I'm billeted. As the tutor, I have the privilege of staying in what's known as the pilot's hut, which is a little less luxurious than the chalets but still. So I'm making my way, which is at the very far end of the camp.

201. I: Were you accompanied?

202. R: No, no, I was on my own.

203. I: So you could have been attacked by one of those?

204. R: No.

205. <Both laugh>

206. R: And as I'm walking aback through the pathway of the camp, one of the staff, who was diligently sweeping up the leaves on the path, looked up to me and as he looked up to me he sort of raised his eyebrows and pointed, and I looked and there's this chameleon halfway up a tree and the interest here is - the chameleon in itself is not unusual, as I say, they're not particularly rare species - but this one is actually about halfway through its moult so it's actually shedding its skin, and that is something that is relatively unusual to see, particularly as it's actually out in the open, although it's clinging to a very smell stem of the branch of a tree, and fortuitously by the time I went back to the pilot's hut, grabbed my camera, ran back up the path, it was still there, making very slow progress going up the tree and I was able to position myself choosing the background to get a nice clean, uncluttered background, again using the same $600 \mathrm{~mm}$ F4 lens.

207. I: So when you actually took this shot were you on your own now? This follow actually pointed it out?

208. R: No. Time was of the essence because obviously this animal was on the move. I was able to alert two other of the guests who were on my trip - we weren't alone in camp; there were other guests staying in camp - I managed to alert them and they too went back to their chalets, got cameras, so there was the three of us, stood shoulder to shoulder, photographing this chap.

209. I: Describe their perspective on this, then. One, presumably to your left, might have been a bit distracted with it?

210. R: Well, no, I was making sure - <chuckles> I just go into tutor mode automatically - so I'm describing what I'm seeing, what I'm using, what settings I've got, and for me one of the first - probably the most important consideration when I'm setting up a wildlife picture - is the background, so I'm making sure they are looking though their viewfinders and checking for the background, any bright hotspots coming, the trees, and if they are getting that then move position, and equally I was rotating out of the position I was in so they could rotate into the position I'd been shooting from. So we all had a variety of pictures and I took pictures side-on, head-on, from behind to give options.

211. I: So what settings were you carrying on your camera?

212. R: Here I think I was shooting at around F5, 6 . 
214. R: The $600 \mathrm{~F} 4$. It may have even been F8 because I was very close, very close indeed. I was just on around about, no more than about sort of... 20 feet away.

215. I: That's a $600 \mathrm{~mm}$ pride. It's a heavy lens.

216. R: It's a big lens. I'm working on a monopod, supporting it on a monopod, but I'm conscious of the fact depth of field is going to be paper thin here, so again I was reminding myself and reminding the other two guests to be a little more generous with their aperture setting because although we're looking at the flank of the animal here, clearly it's a three-dimensional subject and even on that lens, although I'm focusing on the eye of the chameleon, you can see its hind quarters there - the knee is projecting forward. In fact that kneecap is not absolutely pin-sharp; it's probably no more than a half an inch closer to the camera than the eye. But the beauty of that lens is, as you can see, the background: it's a very narrow angle of view. There were pools of sunlight coming through these trees. Obviously you want to avoid those, so hence using a very long lens.

217. I: Looking at that image, then, describe it.

218. R: It was just something I'd never witnessed before, despite many, many trips to Africa and I've seen chameleons in many different countries, I've never actually seen one mid-moult, so it was just for me something I'd not recorded before and it was just the unusual nature of the capture of the animal in that sort of stage. And even some of the members of staff of the camp later remarked when they saw the pictures, despite living there, they too had not seen it before, particularly out in the open because obviously because it can only move quite slowly as it's trying to shed the skin and it's in part rubbing itself against a tree to try and get the old skin away, it does make it rather more vulnerable. So, yes, it's not usually done in such an open setting as this.

219. I: Anything else about the image?

220. R: The lighting was fairly flat and harsh, I mean obviously I had no <laughing> choice in that. It's middle of the day African sunlight. It was tempered slightly by some very thin clouds that just took the edge off the really harsh nature of it. It's still, as you can see, some quite well-defined shadows. Again I took the conscious decision, relative to the background, to move round, so it was a little bit of side lighting but it's still quite high; it still gives a little bit of texture to the subject. Ideally, of course, it would have been early morning or later afternoon warm sunlight but that wasn't going to be the case. So one shoots as one finds, but just trying to turn the prevailing conditions, make the most of them.

221. I: What do you feel about chameleons? This little chap. Or chapess.

222. R: <laughs> Chapess, I couldn't sex the animal for you from here. What do I feel about it? I found it absolutely fascinating. I was there for probably 40 minutes because he wasn't moving very quickly and 40 minutes watching this animal progress probably no more than three feet up this tree in that time and I just thought, "What a 
complete palaver to have to go through periodically, having to shed this skin." But it was just one of these interesting facts of the lifecycle of the animal itself.

223. I: But this poor little creature. Are you feeling some sympathy for it at this point?

224. R: It just looks horribly uncomfortable. I can't imagine that he's sitting there thinking, "Oh God, here we go again." But from my perspective it's, what a complete palaver having to sit there rubbing yourself against this tree to get all this dead skin off. There was something running through my head about "Do real men exfoliate?"

225. I: Well, we're meant to.

226. R: We're meant to, of course, yes. There was almost something comical about it, not that its comical to the chameleon, I suspect, but from my perspective there was an air of comedy about it, because as it was rocking back and forward, trying to pull the skin off, and clearly bits weren't coming off as fast or as cleanly as it wanted. You sense a degree of frustration on his part. But I couldn't help having a bit of a smile on my face.

\section{[End of interview]}




\section{Test Subject IT0103}

Date of Interview 7 December 2018

[Interview starts at 00:18]

1. I: Daisy, thank you very much for your three images, which I've got on the screen in front of me now, and l'd like you to choose for me one of these three images to start our discussion. Have you got any particular one that you would like to start with?

2. R: No, no, any would be fine by me. If you have any particular one you say.

3. I: No, I think what I'Il do is then I'm going to start with I think it's the first one that popped up actually, which is the windmill in the - is that sunlight or moonlight?

4. R: Sunlight. Sunlight.

5. I: Yes, we've got your windmill in the moonlight, we've got your horse and the car in the background, and you've also got a seascape. So I think we'll start then with the image of the windmill... Right, let's go back to the day that you took this shot. Where was it? Where did you take the photograph?

6. R: Well, we went for a walk with the camera club to I think it's called the tunnel of trees or something in Halnaker, here in Sussex.

7. I: Oh right, OK, so quite close to home then.

8. R: Yeah, fairly close to home. We were going to basically take pictures of the tunnel of trees in autumn but of course autumn was very late and this was just before anything turned brown or yellow or red or anything, so anyway we walked through and took a few pictures and then some of us - well, actually all of us in the end - walked up to Halnaker Windmill. And Halnaker Windmill, I thought it was going to be a lovely photo opportunity and actually it was very disappointing; it was surrounded by a fence at the bottom because they were repairing it. It's at the top of a hill, so beautiful view from there, but the windmill itself was a bit of a non-event, so I walked round and round, and I thought, "What can I make of this?" and so then the sun was in the sky, just behind it, not exactly in the right position because you'll notice in the picture one of the arms of the windmill can't be in the shot and the sun at the same time. I wanted the sun; I wanted to shoot into the sun and have a silhouette, so I thought, "Well, I'll just get it as much as I can," and then when I took the pictures I noticed I was getting a lovely starburst so I thought, "Ooh, that's nice!" I took lots of pictures of that and I know it looks like it might be the moon because it's so dark, but the only way I could get the fence out of the picture without cloning it all which was a huge trouble to clone and it was all messy down below anyway. I thought, "Oh well, I'll just vignette it and make it dark and make the whole thing rather dark and just have the silhouette and have the lovely starburst," so that was the thinking behind that.

9. I: OK, let's just back up a bit then. About what time of day was this, Daisy?

10. R: Well, it was, I suppose, I don't know, about midday probably. Probably got the time on the picture if you look at the... I could look it up if you want to know. 
11. I: No, no, that's fine. It's what you can remember. So you left home and met up with the camera club. Were you on your or did you go with someone personally, partner or...?

12. R: No, no, I drove there on my own. Met up with about, I suppose we must have been about eight or 10 of us from the camera club.

13. I: Right, OK, and where did you meet them?

14. R: We met at <pause> we met at a church, actually, because we went first to... can't remember the name of the church in Halnaker also, and then we went to this place, so we met and took some interior pictures of this church, which I'm not very keen on, interior pictures of churches; I just took a few, but it doesn't really excite me very much. So we took a few pictures there and then we moved on to this place.

15. I: Now let's get you recalling things. Can you remember exactly what you were wearing on this day?

16. R: <laughs> No, not really, but I would have been wearing probably trousers and a shirt and a coat. It was sort of cool-ish, I think.

17. I: So it was a cool-ish day. So when did you note it was cool? When you left the house or did you just look out the window, see frost on the ground, thinking...

18. R: Oh no, not that cool, just cool-ish enough to wear a coat over a bottom layer but no, not cold. It was sunny, as you can see by the sun in the sky, so it was a nice day.

19. I: It was a nice day. What did you have on your feet?

20. R: Well, I had some walking shoes because we were going to be walking in, sort of, rough terrain. I might have made some cut-off wellies, it might have been. I can't remember actually.

21. I: No, that's fine. So you climbed into your motor car, you get under way. How much fuel did you have in your car? Can you remember looking?

22. R: No, absolutely not. <laughs>

23. I: That's all right, don't worry. I'm just sort of drawing you back.

24. R: I'm not running on empty so if it's getting low I fill up but I'm not always conscious exactly how much fuel is in the car. Enough to get me there and back!

25. I: What car were you driving?

26. R: Oh, I was driving my Volvo V70.

27. I: Oh right, OK. A big vehicle. Where did you put your camera kit?

28. R: On the seat beside me.

29. I: Oh right, OK, so you put it on the seat beside you. How long did it take you to get to the point where you met your camera club friends?

30. R: Oh, about 20 minutes.

31. I: OK, so a reasonably quick drive. Was the traffic busy?

32. R: I think it was, sort of... oh, it was slightly busy because I had to go through Arundel and things like that so it was slightly busy through Arundel and things like that but nothing out of the ordinary.

33. I: Right, OK. Can you describe what sort of state of mind you were in? Were you really looking forward to it or was it a little bit of "Oh dear, I wish I hadn't agreed to go out today"?

34. R: No, no, no, I'm always looking forward to going out to new places because I hadn't been there, so I thought, "Oh, this might be a nice photo opportunity" and I'm always interested to go to somewhere where it might be a nice place and good place to take some good pictures so no, I was looking forward to it. 
35. I: And you didn't know which eight that you were going to meet, I guess. Any particular friend there that you do know particularly well who you do like to go shooting together with?

36. R: Well, funnily enough my closest friend at camera club wasn't there that day, but we're all quite close-ish friends - photogaphically, anyway - because we're a very small camera club. We all know each other and it's usually sort of the same group that go on the photographic outing. Some people never come, some people very occasionally come. A bit of [??] of us who are game for few places.

37. I: <laughs> Yeah, the hardy group! So where did you park the car? Did you drive on with a group of them, or did you park the car and then walk on? What was the plan of getting to this location?

38. R: Well, there wasn't a very fixed plan; in fact when I got there, I thought, "Oh gosh, nobody's here" because somebody told me on the wrong side of the church; they had parked on the other side and so I thought, "Oh, there's no one else here, a single car, but I'll still park and look around," and then I found them inside the church. Actually, I do remember now, it was quite cold because they were all inside rather than taking pictures outside because it was quite cold.

39. I: Oh right, OK. So you went into the church. How long were you in the church, then, with the group?

40. $\mathbf{R}$ : I suppose we must have been there about 20 minutes, half an hour. Not more. Then I went out into the church grounds because there's an abbey there - there's a ruin of an abbey - so I went out to see if there any good pictures to be taken of that and it wasn't terribly interesting. I took pictures of some autumn leaves more than the abbey, I think.

41. I: So when you were actually in the church, you're looking around now, did you find anything that you particularly wanted to photograph or was it just not interesting for you?

42. R: Well, very unusually for me, because I don't use my tripod very much; I take it on all sorts of trips and it sits in the car or its round my shoulder and I never take it out because I'm a bit more of a snapper than a sort of 'sit down and take out the tripod and take ages and ages taking a picture,' so I had brought it, so I went to the car and I thought, "This is the opportunity to use a tripod and get a decent picture of the inside of the church, get the lighting right and everything." So I did set up the tripod and just took pictures of the... it's got quite a [??] sort of feeling and sort of autumn. It's quite a pretty church inside, small church. And so I took a few pictures there, but when I got back and looked at them they didn't really talk to me very much, so I've got them and they're not bad exposure and everything but just not very exciting!

43. I: Right, was it bad echoey in the church with a lot of noise rattling around? People putting up tripods and dropping their cameras?

44. R: Small little country church. No, it wasn't echoey.

45. I: OK, can you remember the smell?

46. R: Well, all churches kind of smell the same, don't they?

47. I: <laughs> Can you remember the smell of that one?

48. R: Well, no, not particularly. No, not particularly, I don't think.

49. I: All right, let's move on then. You left the church and you moved onto this location where you took this shot here - to the windmill. How far away was that from the church? 
50. R: Not very far at all. We all went in convoy because I didn't know how to get there.

51. I: OK, but it was a drive, yeah?

52. R: It was a drive; we all got back in our cars and went and parked and then walked up a path which is a sort of tunnel of trees; it's a sort of cavern of trees; they sort of grow all around the path. It could be quite pretty in the right light and with the leaves the right colours. As it was, it was a bit disappointing because it was all still green and it wasn't very exciting at all.

53. I: Autumn hadn't turned up! Very irritating!

54. R: Autumn hadn't turned up yet; no, it hadn't.

55. I: So anyway, Daisy, did you drive alone or did you take somebody else in the car?

56. R: No, I was alone because my camera club is in Storrington and I'm in Rustington so most of the people who go to Storrington camera club are more local to there, so they were sort of together in cars; they bunched up together, and I come from a different place so I was on my own.

57. I: So you really didn't have too much awareness of what it was you were going to see from what you said earlier, but you drove there. This tunnel of trees - did you take any photographs there of that tunnel of trees, or did you move on fairly quickly up to this windmill?

58. R: No, no, we did, we took quite a few of the tunnel of trees. It was quite interesting but not hugely interesting, I didn't find. I tried to do something with the pictures afterwards. It was anything terribly special because although there was a bit of dappled light coming in and the tunnel was quite picturesque and there was a little curve and occasionally a person with a dog came through and that made a little bit more of a picture, but it wasn't - in my view anyway - it wasn't very exciting, and I haven't seen anyone else from the camera club put up their pictures or anything else, so I didn't think anybody else thought it worthy of too much publicity afterwards, either!

59. I: Anyway, let's move you on a little bit. Was it a walk up to the windmill? You didn't climb?

60. R: Yeah, on a hill, because it's on the top of a hill. It was quite a hilly walk up, yeah.

61. I: How were your feet feeling as you walked up this hill?

62. R: Oh, the feet were fine, but you sort of feel that you're walking up a hill. <laughs>

63. I: How heavy was your camera bag?

64. R: Oh, not very heavy because I had taken just the bare minimum for what I thought I would need... what had I taken? I took my wide-angle lens because I knew we were going to be in a church, and I think I just took my 24-105 and the camera, so not too heavy. And my tripod. I did take the tripod up the hill.

65. I: You did take the tripod up the hill?

66. R: I did, because I had it with me because of the dark tunnel and I did use it a little bit in the tunnel. I was determined to use the tripod a bit and see if I could become accustomed to using it, so I did have the tripod with me also, but I put it in a bag over my shoulder so it wasn't too lumpy.

67. I: So you had your camera bag, and you had your tripod; you're carrying all this lot up the hill. When you got to the shooting location, were you feeling quite tired physically?

68. R: Well, you sort of start on the way up feeling a bit tired and you stop. No, it was all right. I mean, it was a hill but it wasn't anything; it wasn't a mountain. 
69. I: It wasn't quite Everest. You've got to your shooting location then, and you're now looking at this windmill and everything around it. Were all eight of you still together?

70. R: Yes, we all went up the hill.

71. I: Right. Can you just describe to me, who was closest to you as you came up this particular shot? Can you remember?

72. R: Well, we sort of changed around as we went up because some of us would stop on the way up and see if there was a good view of the windmill as we were approaching it, or the windmill and the fence; there's a fence that sort of goes up towards it. So if somebody stops to take a picture and you weren't going to take a picture, then you suddenly moved off to somewhere else, so there wasn't anybody... I didn't walk up the whole way with one person. We were sort of talking to each other and sort of taking different pictures, so it wasn't one person in particular.

73. I: You're coming up to the windmill and I want you to now think about looking at this windmill, and everything else that's around it. How long did it take you to select the location that you took this shot from?

74. R: Well, when I got up there I thought, "Ooh, OK, this is a windmill," but a pity there isn't much context because it was just windmill and sky and the sun on it, and it was being repaired so there was this fence around the bottom of it and it was just sort of sitting there in bright sunlight and I thought, "This is not very good", and you couldn't get a good picture because it was right on the top of a sort of pimple hill. You couldn't get a right picture with the views - the views were lovely, although photographically I don't know. They were lovely to look at but I don't think they were so beautiful photographically because there weren't many features in the view; it was just a long, lovely view. So I sort of walked around and thought, "Where can I find a picture here?" I sort of zoomed in on some detail and that didn't really inspire me. I love taking pictures into light; I take lots of flower pictures and I love pictures with the backlit flowers and things like that, so I thought, "Ooh, good, there's a bit of a possibility of backlighting here!" so as I walked round I saw that, and that's when I took these shots, and I took several of them and those were the ones in the end that I liked best from the whole day.

75. I: OK, lovely, so you've now found this shot and you're weighing up its possibilities. Did you put your camera on the tripod for this shot, or did you handhold it?

76. R: No, all handheld I'm afraid here.

77. I: All handheld, right. That's great. So you handheld the shot. I want you just to stop for a moment, and I want you to remember the weight of the camera in your hand and the lens that you got. Can you feel how heavy the camera was?

78. R: Well, the camera is pretty heavy, but it's a Canon 7D II and the 24-105 is a heavy lens too, so it's always heavy and I'm used to that because I actually also have a 28300 lens, which I quite often take because it's so versatile; it's got such a lovely length, scope. So I often take that and I haven't yet succumbed to mutating like a lot of my friends have to the light cameras because they're so heavy to carry. To me it's just worth carrying the weight because the quality of picture is, I think, superior. I have a little Sony as well, a little light one, and I'm sort of thinking, "Well, the day this camera becomes too heavy I'll go to the lighter one" but at the moment I hardly feel the weight. I only feel the weight when I get back and suddenly I've been carrying the camera for a long time or whatever and I sort of feel it on my shoulders and I think, 
"Ooh, I've been carrying it all day!" But at the time I don't feel the weight of the camera at all.

79. I: OK, so you're there at the moment of shoot, you've got the camera in your hands. Take me through the settings that you put into the camera to take this shot.

80. R: Ah, well that's interesting. Because I was taking right into the sun, I was sort of experimenting with all sorts of settings, so just try it and see. I was sort of using one setting and thinking, "No, that's not enough, a little bit more, a little bit more." So I was moving it backwards and forwards until I got a setting that didn't blow out the sun too much. Still got the starburst, still got the contrast. I probably didn't even achieve the right setting and I'm not saying that's a top-notch photograph but I was sort of pleased with it and reading your book I realised that one should do more trial and error and then go back and do it again and all that sort of thing, which I never do. That's why your book was so interesting to me; because it sort of will prompt me to go back and do it again and do it better, rather than just saying, "Oh, this was quite good, this wasn't quite good, full-stop, never do that again." I'm sort of inclined to do that: not go back and sort of learn from it and get right what I didn't get right that time; I sort of move on and go somewhere else, instead of going there, getting it right, and then you probably get it right in more places next time!

81. I: You're at the point of taking the shot, you've been experimenting with the various settings. What aperture mode were you in?

82. R: I was... What was I in? I'm usually in shutter mode. I find that more sort of useful for everything. I was probably in shutter mode, or did I go into manual a bit? I can't remember. I was just sort of trying to experiment with everything to see what looked best.

83. I: Can you feel the intensity of the sunlight on your eyes as you're looking at it?

84. R: Yes. <laughs>

85. I: Was it very bright?

86. R: Very bright, yes.

87. I: OK. And I want you to take a deep breath now and can you remember any smells around you?

88. R: No, I don't think so; it was sort of fairly windy up there, so I don't think there were any distinct smells of any kind.

89. I: Was the wind disturbing you? Did you feel it pressing against you?

90. R: No, no.

91. I: No? So you're quite calm, you're not having the camera blown out of your hands by a roaring gale?

92. R: No, no.

93. I: <laughs> Excellent. What I'd like you to do now - you've got the image in front of you - I've got it here as well. What I want you to do is just have a look at that image now for a moment.

94. R: I'll just put it in front of me; I haven't got it in front of me at the moment.

95. I: OK, pop your image up in front of you.

96. <pause>

97. R: I'm trying to find the email I sent them to you in, because I sent them in very low res, didn't I?

98. I: That's fine, yeah. Don't worry, take your time, it's not a problem.

99. <pause> 
100.

R: When did I send you the pictures, Bob?

101. I: It was about the day before yesterday, I think. I'll just check that for you.

102. R: Ah, found it, I've found it. OK, I've got the picture up now.

103. I: It's probably raised your tension level quite a bit! <laughs> Well done, Daisy. Save me a lot of work! Look at the image now and l'd like you to describe it to me, please.

104. R: OK, well, it's a sort of dark moody contrast-y picture of Halnaker Windmill with a starburst sun behind it.

105. I: OK, OK. Anything else that strikes you about the image as you're looking at it?

106. R: I like the cloud formation; that sorts of made it. It if hasn't got the clouds there it would have been a different type of image, I guess. But I quite like the clouds.

107. I: Anything else about the image that you think is worthy of description? So if you were describing this image and somebody didn't have it, what else would you use to describe it?

108. R: OK, well, I suppose I haven't said that the windmill is in silhouette, and it's a picture vignetted top and bottom to sort of draw your eye basically to the windmill in the sun, that was the idea, anyway.

109. I: Right, OK, and why is that idea important in the image?

110. R: Well, because they're the two things in the image, really. Two subjects of the image.

111. I: So now you're looking at it and you're back there in the moment. Other people are around but you're looking at this image in the viewfinder at this image, and I want you to describe what it is you are feeling as you look at this image. What feeling is it evoking for you?

112. R: Well, what I'm thinking at the time is trying to produce an image that's different, that has some artistic value, rather than just record value, so that was my thought, and in fact you were saying people around me - there wasn't anybody actually around me; everybody else was somewhere else. Actually one member of the camera club came out with me and took a picture of the same kind of thing I was taking. Everyone else was sort of looking round other places and taking very different pictures. In fact I don't know what pictures they took because I haven't seen them yet. I haven't discussed it with them.

113. I: So if you look at this, what would be your emotional response to this scene? What sort of emotion does it evoke in you?

114. R: Well, I suppose it's a sort of a bit forlorn and a bit lonely, but other than that! <laughs>

115. I: Why's it a bit lonely?

116. R: Well, because it's got no people in it and it's got no activity in it; it's just a windmill and the sun. As far as it looks like, there are no people and no buildings and no signs of life at all, really.

117. I: Do you find that as a negative or a positive when you look an image like this? There are no people, as you say. How do you respond to that feeling of, you used the word loneliness?

118. R: No, no, I don't say lonely, but I was reluctant to mention the word loneliness because I don't see it as a negative at all; I rather like isolating and just getting one or 
two things in a picture, even though there are lots of people around, I quite like isolating something and simplifying a picture rather than cluttering a picture.

119. I: Right, why do you want to do that?

120. R: Well, it's an artistic form that I would quite like to pursue.

121. I: Can you describe that artistic form to me?

122. <pause>

123. R: Well, I suppose it's looking for shapes and forms and light and dark. It's quite difficult to express actually; I found it difficult in your test, even, with your 10 constructs, and I thought, "Well, I probably, on certain days, I would give all of these 10." Because they're all important; it depends what mood you're in and what you want to catch on that particular day, and other times if you're taking pictures of a busy railway station you probably like the clutter and all the stuff. On this occasion and for this subject, I thought the uncluttered bit was an interesting way to go but that's on that day. Other days if there's stuff happening around that windmill, if there was a, I don't know... a balloon taking off or something, I would probably have liked to have that in it. Yeah, I suppose it's just on that day at that time, that's what I felt I would like to pursue.

124. I: Good. OK. Well done, Daisy, I think you did very well there! Let's move on to another of your three images now. I've got the picture of your horse and I've also got this little seascape that you've also got. Which of the two would you like to talk about now?

125. R: Well, let's go for the seascape, shall we?

126. I: OK, yes. Now, seascape. This will proceed now a little quicker because you probably got the idea of the way my questioning will go, but I want to take you back to the day of taking this seascape. Was this a seascape near home or further away?

127. R: Very near home, yes, it's on Rustington beach.

128. I: Oh right, OK, so you're on the beach. What took you down to the beach then? Come on.

129. R: Well, I quite often go down to the beach looking for pictures or for a walk or whatever, but this day I went because we have a Flickr monthly challenge with the camera club, and this one was on intentional camera movements, and I love the sea anyway; I love pictures of the sea, anything about the sea, so I thought, "Ooh, I'll go down at sunset" - there was rather a nice sunset that evening - "and see if I can get some nice sort of sweeping long exposure pictures." So I took quite a few as you can imagine, and that black blob that you can see in the picture is actually Little Hampton buildings and skylines, getting dark, and it sort of, I thought, worked quite well, this particular series, because it had the sunset light - there were sort of clouds in the sky, as you can see, sort of covering bits of the sun and bits of the sunlight, and as I swept the camera the lovely colours were in the sky and in the sea reflected so I thought, "Ooh, that's quite nice, I quite like that," and there were all other bits of shadow and whatever to cause these dark shadows to come, so I thought all of that made rather nice colours. So that's a different style of picture completely based mostly on colour.

130. I: So did you go down here on your own? Were you on your own here?

131. R: Yes, on my own.

132. I: How far is this from your house?

133. R: Oh, about 500 yards.

134. I: Oh right, OK, so you walked? <laughs> 
136. I: Did you just take your camera or was it in your bag?

137. R: I took my tripod as well! <laughs>

138. I: Not that tripod! <laughs>

139. $\mathbf{R}:$ I took the tripod just in case there was something to take with the tripod but obviously these, because they were moving l'll not take them with the tripod. Yes, I had my camera and my tripod over my shoulder.

140. I: Tripods don't work well on sand, do they?

141. R: Well, no, it's fine actually. It did work. I took a few with the tripod but I wasn't very pleased with what I took because it was quite low light by then, so I thought, "Well, maybe there's a picture to be had with the tripod", but I took a few and I didn't them; I preferred all these sweeping ones much better than the tripod ones!

142. I: Was there anybody on the beach with you?

143. R: Oh yes, lots of people. People walk their dogs there and there's a path along the seafront. Yes, there's quite a few people.

144. I: Right, when you're getting ready to take this shot, so you've got your camera, tripod planted to one side, you pick up your camera. Can you recollect anybody else around you, looking at the sea in the way you were?

145. R: Well some people do, they sort of look at you slightly funny and think, "What are you taking a picture of?" <laughs>

146. I: Boring old sea!

147. R: People just walk by and they sort of give you an understanding nod and sort of think, "Oh yeah, nice sunset."

148. I: OK, so tell me about your camera now. Can you remember what settings you had in your camera?

149. R: Well, again, it's another case of experimenting with all sorts of settings, so I sort of go backwards and forwards until I think I've got it more or less right, and then of course you can always tweak it slightly, especially in this kind of shot, you can always tweak it slightly afterwards, so I wasn't too worried as long as I didn't get things burnt out or too dark. Because the picture was too dark, because it was getting quite dark.

150. I: It's getting quite dark, you're getting on towards sunset. I want to draw you to the moment now. You're lifting the camera up to your eye. Can you smell the sea?

151. R: Yes. I mean usually there's a sort of strong-ish smell of seaweed on our beach; it's a sort of stony and seaweed-y, it's not a sort of Riviera beach of any kind. So yes, there are sort of maritime smells. <laughs>

152. I: OK, so how would you describe that smell? Is it smelling quite pungent?

153. R: Well, no, not overly. Not overly. There are some days that it is, but I don't recall that day being particularly. It was just a sort of nice, nice smell of the sea really.

154. I: Yes, so that smell then, that sort of evokes in your mind the sea, and does that add to your feeling of pleasure at what you're looking at?

155. R: Oh yes, yes, I love the sea.

156. I: Right. Was there anybody talking around you?

157. R: No, not really, it was mainly people with their dogs. I mean, occasionally, if it was two people with a dog they may have been talking amongst themselves but...

158. I: Can you hear them?

159. R: ... it wasn't the noise of talking, no. 
160. I: What about gulls? Were there any gulls about?

161. R: Oh yes, always gulls about.

162. I: Right, did you see any gulls flying through your field of view?

163. R: Yes, occasionally, but they never flew in quite the right place to be included in the picture.

164. I: Oh, right, OK, so they weren't being cooperative? Annoying, but yes! $<$ laughs>

165. R: Well, also I was sweeping, so any gulls that there were would have been swept out of the picture.

166. I: So you're sweeping and you're sweeping from right to left or left to right?

167. R: Well, because of the place I was going, it had to be right to left.

168. I: Right to left, OK. So you're sweeping right to left, OK. What are you wearing?

169. R: Well, I can't tell you exactly but it would have been probably some wellies

because I was on the beach and sort of near the edge, and the tide had just gone out, so it was quite wet down at your ankles, sort of amongst the stones, and I would have been wearing trousers and a sweater and maybe a coat. I can't remember how hot it was; I don't think it was very hot because it was evening, so it would have been coolish. It's always cool-ish on the seafront.

170. I: Yeah, quite. Did you feel a sense of restriction as you were swinging your camera.

171. R: No. No, no.

172. I: OK, so nice easy movements going from right to left. Can you remember doing that now?

173. R: Yeah, yeah, yeah. Trying to keep it from wobbling! <chuckles> And trying to keep it straight too because some of them you look up and think, "Ooh, that horizon's a bit wonky!"

174. I: Bit wonky, yeah, OK, so you wanted to keep a nice straight horizon. So you're sweeping from right to left. Did you have a slow or a fast shutter speed?

175. R: Slow shutter speed.

176. I: Can you remember consciously setting that shutter speed?

177. R: Well, yes, I was setting it all the time to try and get the optimum shutter speed because the optimum shutter speed combined with the optimum speed and the sweeping, so it was a lot of trial and error. <chuckles>

178. I: OK, you're looking at this image now, Daisy, and you're there on the beach and the tide is receding, you can feel yourself sinking down into the slime and you're swinging your camera from right to left, taking shots as you go, and you've got that image in front of you now, in movement as you're swinging it. l'd like you to describe the photograph to me, so I want you to say what is here. What am I looking at?

179. R: Well, it's clearly a photograph of the sea but accentuating all colours that evening light will give and streaming those colours in a sort of <pause> I suppose it's just accentuating the colours without detracting from the fact that it is the sea and you can see it's the sea. You can't see its little Hampton but you can see there's something there in the black, so you might assume it's the land, which it is. And just accentuating evening colours, really.

180. I: Is there anything else that you would use here to describe this? Anything else that strikes you as you're looking at it?

181. <pause> 
182. R: Well, not really, I think it's quite simple, really: lights and darks and colours and brightness and varying tones, which it mainly is for me!

183. I: That's great. So looking at it now, I'm taking you back to the moment of capture, what were you feeling? What was your feeling as you saw this?

184. R: Well, I was thinking I could possibly produce a picture that I would like to hang up on my wall and would sort of stand the test of time; I wouldn't get tired of looking at it. So that had to have a subject I liked and it had to have colours I liked and it had to have a sort of pleasing overall image, really. So that's what I was thinking, and then I was thinking, of course, "Oh good, I might have an image amongst this lot to put on the Flickr challenge this month." <laughs>

185. I: OK, so you got an image, OK. I'm going to take you onto your final photograph now that you sent which is a photograph of a horse, or was it pony? Could you tell me when you took this?

186. R: OK, this one has a little bit of a story. I took this at an animal sanctuary. We were asked by a local animal sanctuary in Storrington whether several of us from the camera club would come and take some pictures of the animals because they wanted to do a new brochure and they wanted to publicise it and they wanted to get these animals to have a home and so on, so we went and we went twice, actually, and we took the pictures, and they have horses and cats and pigs and a huge variety of animals there. I particularly love horses myself and so I took lots of pictures of all the animals and we gave them all the pictures and they were very pleased with it and all that, and then fairly recently I was looking at my pictures - I hadn't sort of done anything with those pictures other than prepare them for the sanctuary - and I saw this picture and I thought, "Oh, I rather like that picture." I rather like the lighting, I rather like the sort of old-fashioned hay stack cart beside the little pony. So <chuckles> I thought, "Maybe I can do something with those?" so I sort of planned an effect which is very contrast$y$ and slightly blurry. In one of my programmes it's sort of known as the Orton Effect and it blurs part and it contrasts part and it sorts of highlights part, and then I sort of filled it in a bit more, and I quite liked the lighting and, of course, the cute little pony, and that's what I love about it. I love the fact that it's a pony, I love horses, and I just liked the colouring. This picture wasn't taken in the evening although it looks like it was taken in late evening. It wasn't; it was taken with low sort of with wintery autumny sunlight, but because of the effect I put on it, it looks a bit like evening light. Evening light is always lovely so that's fine if it looks like evening light!

187. I: You can remember the day that you took this, then? This particular shot?

188. R: Yes. Well it was one of the two days that we went to take pictures of all the animals.

189. I: And you can remember which of the two days it was that you took this shot?

190. R: I think it was the first day! <chuckles>

191. I: That's fine. Did you have anybody there with you?

192. R: Yes, three of us went. Three or four of us went.

193. I: OK. As you took this shot, who was the nearest person to you?

194. R: Oh no, nobody was near me when I took this shot. We were all just roaming around doing our own thing. Taking different animals, and there were people looking after the animals, sort of feeding them and cleaning them out and doing this. There were people around, but there was nobody sort of near me when I took this shot. 
195. I: So you're there in the moment with this animal. How actually physically close to it were you?

196. R: Oh quite close. I mean, I was in the enclosure with it and most of the time we were stroking the donkeys and the ponies and everything.

197. I: And had you stroked this one?

198. R: Yes.

199. I: OK. Where? Where did you stroke it? Physically. On its head? Side?

200. R: On its head. <laughs>

201. I: On its head. <laughs> Yeah, OK. So you stoked this pony, is it, on its head, and how was it responding to you?

202. R: Oh. Fine. I mean, they're very tame little ponies.

203. I: And what was your feeling towards this little pony?

204. R: Well, I was sort of wondering where it had come from, where it would be going to. I mean, a lot of these animals sort of in the end kind of spend their lives in these sanctuaries because people don't - I don't know how long it takes before somebody adopts them or takes them. They've all got a history of why they had to be got rid of; the child grew up and wasn't riding any more, I don't know. I was sort of thinking, "I wonder what will become of you."

205. I: Yes. So you're looking at this pony. What would describe this pony for you? If you had to find some words which describe it.

206. R: Describe the pony or the picture?

207. I: The pony for the moment.

208. R: Well, it's a little Shetland pony who's probably had a stint in a field belonging to someone with several children who rode them and then they maybe sold the house and this pony was left to go to the sanctuary.

209. I: How would you describe that feeling? Were you feeling a little sad for it?

210. R: Well, actually, there were several of them; this sanctuary had at least a dozen of these little ponies. They were all together, they were in a field and they were well fed so, as you can see, it's a very healthy little pony so they're not at all badly off, I don't suppose they're ridden very much, being in the sanctuary; I suppose they just eat and sleep, but they're perfectly happy and well looked after, so you don't really feel sorry for them, you just sort of think, "Well it'd be nice to have a home where somebody really loves you particularly, rather than loves the $12 . "$

211. I: Take me to the moment then that you got the - you were handholding, you didn't have a tripod I guess in there?

212. R: Hand holding. I didn't even take the tripod! <laughs>

213. I: Sensible move! So you're there, you're hand holding. Which camera were you using?

214. R: I was using my Canon.

215. I: Were you standing right upright or did you crouch down?

216. R: I was crouched down, yeah. Because tiny little pony! <laughs>

217. I: <laughs> It's only a little chap! How far physically did you have to crouch down? Did you have a knee on the ground? Can feel your knee on the ground?

218. R: I don't usually put my knee on the ground because the ground there is not very knee-friendly, so no, I just crouched down.

219. I: Why were you couching down? 
220. R: Because I didn't want to take a picture from the top, I wanted to take a picture more on his head height. And usually from lower down - I could often crouch down for pictures; I mean, depending what they are, but especially if it's a small little pony. And the bigger one, I quite like the pictures taken sort of at angle looking upwards, so I quite often crouch down anyway.

221. I: Was he a smelly little beast or was she quite wholesome?

222. R: No, he was fine. There was a general smell of stables all around.

223. I: Can you remember that smell?

224. R: Yes, yes.

225. I: Can be quite strong. Was he sort of munching away on stuff?

226. R: Yes, he was, he was, and in fact I considered when I saw this picture taking the bits out of his mouth and I thought, "No, I quite like them there!" <chuckles>

227. I: Can you hear him munching?

228. R: Yes.

229. I: So you can hear him munching, you're looking through the camera viewfinder at him. Describe to me what you're seeing. Describe this to me.

230. R: Well, he was busy eating, so I went up to him and stroked him and sort of thought, "Well, he's busy eating." I think I took some pictures of him actually sort of eating out of the thing as well. And then I turned round beautifully towards the light and I thought, "Ooh, lovely! Click."

231. I: Right. Why was that important then?

232. R: Oh, because he's looking into the light and it's all sort of a nicer view, really, with him turning his head and just sort of eating over the edge of the cart - actually of three little ponies eating over the edge of the cart, that's quite sweet, but I just prefer this view, really.

233. I: And what is nice about this view? What is it particularly that's catching your attention here? You said he's looking into the light.

234. R: Well, he looks almost as if he's posing for me; I mean, he's just in a perfect position, I think. He's got the light on his face, you can see he's been eating out of this rather stylish little cart he's got next to him. I just think the whole composition was quite pleasing.

235. I: Right. What was pleasing about the composition?

236. R: Well, I always find it difficult to explain why it's pleasing; I just kind of like looking at it and you sort of look around and it all kind of makes sense, and the light, I think, is all in the right place, and I don't mind the darkness because those bits aren't very interesting anyway, and I just think it sort of highlights his gorgeous little face and lovely mane and long tail and everything, and it just sort of gives you all the information you need in a sort of nice, attractive, nicely lit way.

237. I: OK, good. That's smashing. Look back at it now. What are you feeling as you look at him? It? He? She?

238. R: I don't know, I honestly don't know if it's a he or a she. <laughs> Well, it's again gorgeous little pony that I would love to have if I had ponies! <laughs>

239. I: Why would you like to have him?

240. R: Oh, because he's gorgeous! He's absolutely lovely. And visiting children could ride him. I just have a particular affection for horses and ponies.

241. I: Brilliant. Excellent. I think we'll leave him there chomping away at his food!

242. <Both laugh> 
244. R: Well, I hope whatever I've shown you and said has been useful.

245. I: Well, the question is, as well, will it be useful for you?

246. R: I'm sure it will. I'm sure it will. You know, it makes you think of things. I often don't think enough about my photography, which is why I found your book interesting because, as I think I mentioned, I'm more of a snapper; I see something and I go click. I usually have my camera with me; these days I either have my phone or a camera and I sort of just click things as I see them because I think, "Ooh, that's nice." But I would like my photography to come a bit more thoughtful and as one of the things you say, which I think is a very valuable thing, is try imitating photographers or pictures that you like and try and go and take something similar and work on it, and if it doesn't work the first time go back, see what didn't work. Because you can't really tell on the back of your camera if it's worked or not; I can't tell until I go back to a computer and see it bigger what has worked and what hasn't. On the back of the camera sometimes it looks great and then you get home and it's not worth anything. I want to try and make myself somehow come back to it, go back again and take a better one, and perfect the art because it's not that I feel I can't do it; I don't sort of take enough time doing it, and even when I go out and take pictures of places, I sort of take a picture and move on instead of staying there and waiting for the light to be better or for something to come into the picture, so I need to be a bit more thoughtful about my photography, let's say.

247. I: OK. Before I debrief you on your IMPTest and take you through what the results are and what it's telling me and what I hope will be very helpful for you, can you just tell me a little bit about taking the IMPTest? How long did it take you to do the test?

248. R: Well, I sort of got the impression by the instructions that you wanted me to do it kind of as quickly as possible so that first impressions count, rather than think about it too much, which would probably ruin the spontaneity of it, so I did it sort of fairly quickly, trying to follow that sort of idea and afterwards I thought, "Well, I wonder if I did it a bit too quickly and maybe I didn't think about it enough." The instructions seemed to indicate that you wanted first impressions and you wanted that kind of reaction, rather than to think about it too much.

249. I: OK. When you went through the very fast, just the three seconds, did you find that reasonably easy to do, to think of a number that expressed your thoughts? You'd only seen the image for three seconds, did you find that reasonably easy to surface a number?

250. R: Well, it's not easy, actually. I mean, you do your bets. We've done at camera club several attempts at judging each other's pictures and that sort of thing and you sort of get a picture up and you have to sort of say something constructive - think of something constructive, think of why you like it, think of why you don't, so it is quite difficult. I think you probably can through practice train yourself to be better at it. I think also in the case of your pictures you didn't know what was coming next or what you were comparing it to either, so yeah, I don't think it's easy one sort of tries to rise to the occasion! <laughs>

251. I: OK, as you see the image for three seconds it goes blank. How long do you think it took for a number to come up in your mind to express what it was that you saw? 
252. R: Well, I tried to make that as quick as possible, so that it was a bit more of an instinctive reaction, so I did it as quick as I could, sort of thing.

253. I: The first number that came into your head was what went down. Right, OK.

254. R: Yes, mostly yes. Occasionally a little bit more, occasionally a little bit less.

255. I: That's fine. Right, let me just say what the IMPTest said about you. What I've done here with your report because I've put the technical answers down but I'm taking this interview here as an opportunity to help shape up perhaps more helpfully what would be good steps on for you. Let me go through what it says about you. First of all, you also very kindly did what's called the Rational-Experiential Inventory. Can you remember those 40 questions asking you about whether you like to think intuitively or rationally? You may well have looked at the output page on that. You've come out with a very even balance between the two, very even. With no real distinction here, your mind is able to think deliberatively, rationally - I don't ever like using the word rational because being not rational seems to be a negative. You can think very deliberatively, you can also think intuitively; you do respond to your intuitive judgement and the test, as I say, is a very even balance in the way that you make your judgements, and you demonstrate quite a good level of willingness to use either way of thinking to rely on your intuition sometimes, to rely on thinking things through on others. Now the implication of that is that you're going to be open to a huge range of genres; I don't think there's any genre here that you wouldn't be able to cope with. Some genres require very rapid intuitive judgement of what's going to happen, as I say, wildlife shot and photographing your pony. You've got to anticipate what is going on and take the shot. Contrariwise, you would be comfortable with a genre of photography that requires a high degree of pre-visualisation, landscapes. This part of the test - well, it's not part of the IMPTest - suggests to me that you would be open to almost any genre that you felt you wanted to pursue.

256. Now what I found very interesting about your results was two things: first one, your intuitive judgement your rational judgement is highly aligned. Those 10 constructs, to varying degrees, do explain your intuitive judgement. There's a very high degree of association with them and the sort of association that normally I would be expecting to see with very highly skilled photographers, actually, in the top cluster. What that means is that there's not a missing variable the test hasn't accounted for. Those 10 labels as you defined them - this is important because it's your definition of those 10 labels really: what you mean by composition, use of light, use of the colour - those 10 labels are doing it for you; in other words they are capturing your expertise at a high level, there's not a missing label at number 11 which should be there. So that was what caught my eye, but what really struck me and why I was pretty keen to talk to you: do you remember I gave you an ordering of those 10 constructs, their importance to you? Do you remember that?

$257 . \quad$ R: Yes.

258. I: Well, the IMPTest, what it does is it analyses, using the scoring that you gave to the images and your intuitive judgement, what it does is it works out how important those 10 constructs are to your non-conscious, your intuitive judgement, and you came out with the highest score anybody's ever recorded on the IMPTest!

259. R: Oh really?

260. I: The average is close to zero relationship. You came out with very close to $100 \%$. 
261.

R: Really?

262. I: Now what that means is that you've got a very high degree of alignment between your deliberative thinking and what is happening in that fleeting moment when you decide to press the shutter, so in that fraction of a second where you see it and you press it, you've you a very high degree of alignment with what is important to you in that moment and what is important to you when you actually think about it all in a more deliberative way. Now that does have a lot of significance for you; there's not a lot of conflict going on between what you're feeling and what you are thinking. They're in a high degree of alignment, very surprising. I tested it and ran some more tests on your data and that is what the IMPTest is suggesting. Now, the IMPTest could work out your skill level - how far you've sort of progressed in your study of photography - and it's showing you at around about the average for all participants in the test, so your actual kind of knowledge of photography is around about the average, but that is something as you do get into it more and more, deeper and deeper, that scoring will come up higher and higher, but it was this very high degree of self-awareness, of what is important to you in the intuitive moment, that caught my attention.

263. The IMPTest tells me, of those 10 constructs, which of those 10 constructs are driving or really crucial when you press the shutter. In that three seconds that you saw the image, and I only can go on what you've said to me here and I will have to analyse your discussion in more detail, but I get the sense that the way you've describes it does describe what it is you are feeling at that fraction of a second when you press the shutter, and these are the constructs which are describing your intuitive judgement. This is your non-conscious brain working; it's sort of going on under the bonnet, as it were. These are a composition - you have a sense of the structure of what you are looking at. Impact is also important; you're looking for something striking within the image. Light, use of light, the test tells me unequivocally that your use of light, the light in the image, the way that it's playing within the image is very important. Creativity - the IMPTest is telling me that you can sense what it is for you in the way you understand the word creativity, what is creative in the moment, so you are looking for that little spark of difference, as it were. And focus and depth of field, that you are aware of the issue of gaining the focus that you want and the appropriate depth of field to capture what it is you want. Now there are four constructs which are not driving your intuition, and your conscious skill level is OK and good with them, but they're not yet burnt into the firmware of your brain, such that three-second moment that you are using them. The narrative power - the telling a story - what you mean by technical, which is probably different from what I mean by technical, is not driving your intuitive judgement. Colour and tonality is not driving your intuitive judgement; it's there in your deliberative thinking but it's not influencing the moment that you press the shutter, and ditto exposure; you know how to expose your camera; you experiment with exposure so you know how to exposure your camera, but the exposure judgement is not there in the intuitive moment. Looking at that, those six that are driving your intuitive judgement are very important constructs and I think that you are in a very strong position here to really push your photography onto another level, and I'm going to suggest to you first of all your knowledge of exposure you know how to expose your camera properly, I have no doubt about that, but what 
I want to try and do is to burn that into the firmware of your brain - we're doing to firmware update here...

264. <Both laugh>

265. I: ...to burn that into your brain so that your brain will respond to it in that intuitively moment. That'll be really enhance and strengthen this use of light which is so important to you in that intuitive moment, so I'm going to recommend to you that you focus on exposure and I'm also going to recommend for a starting point that you focus on the narrative power, and I'm going to give you a couple of tips in your report, and focusing on those two to start off with. If you got those two into your firmware such that if you did the IMPTest again in a year's time there wouldn't be six there, there'd be eight there, you would find that your photography would leap up a level.

266. Now I think, as you remember reading the book, I suggest to you strongly that you seek a high level of emotional activation when you are practicing these constructs; one, for example, on exposure. Exposure's an interesting one. How long have you been taking photographs, Daisy? How long have you had your camera?

267. R: Well, I've been taking photographs since I was a child; I think my mother used to take photographs. And we've always been a very photographic family. I mean, in a totally amateur way - no professional photographers, but just as a hobby.

268. I: I think it's important that you become the best that you can be. Now on exposure, when I finalise your report today I'm going to put in a little game that you can play to develop your intuitive control of exposure and it's one that is very good fun to play with friends but loosely it goes like this: I'm going to suggest a way that you can acquire perfect exposure sight. In other words what I want you to do is to look at anything - it could be a shot indoors or outdoors somewhere - and I want you to in manual mode set up an ISO perhaps of 400 or 200, if it's a nice bright day, or perhaps a little bit higher if doing an internal shot, and I want you, given that you're shooting, let's say it's an indoor shot, you're shooting at F11. Before you check the exposure through the camera is to say what shutter speed you would need to get that exposure exactly right, so just by looking at it - not with your camera or light meter; just by looking at it. And then you look through the camera and you check to see whether you've visually got it correct or not. I only give this particular tip to people who've been - this is why I asked you how long you've been doing your photography - people who are well used to their photography, because its trying to acquire in photographic terms the equivalent in music terms perfect pitch; in other words, your eye becomes attuned to the light that's out there in terms of the exposure that your camera will need to give you that perfect dynamic range across the image.

269. When you play this game, see if you can get yourself geed up a little bit. Several ways of doing it: you could have a row with somebody; that might get you geed up. Or you could have some music playing or some other way in order to get you going. And I think very quickly with this one - with exposure - you will update your firmware very quickly! Narrative, I'll give you some tips on how to develop your narrative power and in other words to expand the levels of meaning which are within the shot, to expand the story, as it were, in order to add strength of the story to the image. And I think very quickly you would be able to bring both of these into your cognitive processing and I think as a result, following the EPF method that I describe in the book that you will find quite a rapid transformation in what it is that you're seeking to do. What the IMPTest also tells me is these four constructs that are not in at the moment 
- narrative, technical, exposure, colour and tonality - they're not that far away, and my suspicion is if you do pursue what I suggest eventually you'll be able to work on all four. In practice, it's always worth practising from time to time all 10. I do, for example - haven't been down there for a few weeks but I usually go down to Slimbridge or somewhere like that in order to do a bit more burning in on focus and depth of field and so on. Other days I play the exposure game and so on. You've got to work on all 10 to sort of build in regular practice with all 10 but I think the four that the test has highlighted are not part of your intuitive capture that they soon will be if you do some really focused work on each of them.

270. Lights and creativity are very closely associated in your mind and this came out in the discussion that we had about your images. As I'm looking at the result of the IMPTest - and when you get the document through there's a diagram on the final page which shows how these various things are linked, how close they are to one another - light and creativity are very closely aligned in your intuitive judgement, very closely aligned. Depth of field, focus are reasonably well-aligned, and composition. So they're all in sort of positioning that I would expect. Looking at this - I mean I think that you could make some really significant jumps in your photographic skill and satisfaction that you get from it very short order actually with some of that practice that is described in the book. But I'll send you three tips; I'll send one on exposure, I'll send one on narrative as part of the report, and I think it said that you were interested in Andy's photography, as well, didn't you?

271. R: Yes.

272. I: Do you know the work of Sebastião Salgado?

273. R: Yes, I do. I mean not intimately but I do know him, yeah.

274. I: Yeah, I'm going to suggest looking at Sebastião Salgado's image-making as well, which I think will help you, to really enhance this narrative power, as well. I think your judgement about the 20 images was pretty close to the consensus; I'm just having a quick check on that. There's five images here where you were in significant disagreement with the consensus; that doesn't matter. One thing I would say to you, as well, is as you develop your intuitive scope, as it were, your personal style will emerge much more clearly, such that people will look at an image and say, "Oh, that's a Daisy image!"

275. R: $<$ laughs $>$

276. I: Style is an emergent phenomena; it's something that comes from deep within you. As you take images it sort of comes almost spontaneously. Well, we all copy styles of other people, like Andy's work, like Salgado's work, if you like that sort of moody black and white type photography, that's wonderful. But you will evolve your own style; it will emerge the more and more you pursue. The development of these constructs, rest assured that your non-conscious brain is putting them together in a way which the test tells me is the way you want to put them together, and that was what's so striking about yours is that your intuitive brain is putting those 10 constructs together in a way that you think is the correct way to do it, and I think that is very interesting.

277. There, Daisy. That's what the IMPTest tells you.

278. R: Gosh, well, Bob, thank you so much for taking all the trouble to analyse all of this and to impart all your advice! I do think you're absolutely right; what I feel with my photography is don't have a style, really. You asked in one of your questions, what 
is my favourite type of photography. I like all types of photography. That's my problem; I like too many types of photography, so I haven't sort of homed in [sic? should this be 'honed in'?] on one to sort of pursue because I just like anything that sort of inspires me and looks good, and I find a lot of things inspire me and look good, so I haven't homed in on one in particular. I tell you I like Andy's picture and I love it because it's something I've never done before and it's a new thing and I think it's wonderful. But then I love other styles of photography, as well, so I haven't sort of quite decided, and I don't think I ever will decide on one style - I'll probably always have more than one style - but I would like to pursue at least some styles in greater depth.

279. I: As I say, style is something to be authentic to yourself, to represent yourself. The camera's got this uncanny ability of looking both ways; it's as much a photograph of what's going on in your head as it is a photograph of a pony or a windmill with the sunshine. It's what's going on in your head, and as you deepen your intuitive grasp of these 10 constructs, you will start to emerge a distinctive style, and it doesn't matter whether it's landscape or if it's a wildlife shot, or whatever it is that you're taking, that style will emerge. Adopting a black and white moody style or way of presenting the images, that in a sense is an imposed style, but I think that over time, probably quite rapidly, actually, if you do what the book suggests, I think what you will find is that Daisy will be emerging very powerfully in her images, such that people when they look at them will say, "Ah. We know who this is!" It'll just be you talking and when your images make the jump from being good images to being great images that they'll become a very powerful, personal expression of what you photograph. So I think, yeah, you've got a lot to play for here, Daisy, and I'm not suggesting taking awards or licenceships or associateships or anything like that, but whatever direction you decide to take your photography I think you're well on your way, as it were.

280. R: Well, thank you for saying that. I've always loved photography and I just love images. I love looking at things, I love shapes of things, colours of things; it's just sort of part of me and I really enjoy it. I was very interested in your book and in you approach because I think it opens lots of avenues.

281. I: Well, I hope it does work for you. I'll get this report - I've got some writing to do in it now; I've done all the analysis but I'm going to make my selection of your tips and so one. Hopefully get it finished today and over to you later today or possibly tomorrow. Do let me know if you've got any feedback or anything about the books or any comments on it; delighted to get them. I just can't say thank you enough for taking part in this exercise.

282. R: Well, I can't thank you enough for taking the trouble to do all this. It's been a huge eye-opener and really enjoyed it and will read the rest of the book - I've nearly finished the book, actually; I think I'm sort of three-quarters of the way through - but I think I will not just read it once; I think I'll read it again, because another thing I've got is very bad memory, so I think, "Oh gosh, I've got to go back on this! What was it you said about this?" So I will be reading it more than once.

283. I: Oh good! Well, thank you very much, Daisy. You have a lovely weekend. Thanks, it's a privilege really for me and I hope you have great success with your photography.

284. R: Well, I hope you have a great success with your future research, and thank you so much for all your help; really, really appreciate it. 
285. I: OK, Daisy, we're drawing to a close there. Thanks very much and I'll get this over to you later today or tomorrow. Is that fine?

286. R: Thank you, Bob. Thanks so much.

287. I: OK, Daisy. Bye-bye now. Bye-bye. 
Test Subject IT0106

Date of Interview 21 May 2016

Transcript

\section{Interview Commenced 10.00am}

1. Interviewer: What we're going to do, then, is I'm going to take you through the interview process. I've got your IMP Test results and at the end of the interview I will send them over to you on the email, so you should get the within seconds, they're not a big file; there's about 10 pages in all. You can have that. And I'll then take you through your IMP Test results. So for the moment, in a sense, you forget about the IMP Test; we're just going to have a look at your images.

2. Respondent: You're looking at them from the context of knowing the IMP Test results?

3. I: Yes, I do know the IMP Test results, but I'm blanking my mind, so to speak.

4. R: OK.

5. I: <laughs> There's no way I can get round this problem, I do know, but my danger is that as this interview proceeds I will be avoiding any language which might take you into the IMP Test.

6. R: Yeah, yeah. Yeah.

7. I: ... which reflects the IMP Test. If you want to talk about elements within the IMP Test, that's absolutely fine but I won't be cueing you, as it were, as that... oh, what's his name? Brown. Do you know the magician?

8. R: Derren Brown.

9. I: Darren Brown.

10. R: No, Derren, isn't it?

11. I: Yeah, Derren. I watch him and I think, "You've cued them! Of course they're going to come up with number seven! You've given them cues!" <laughs>

12. R: Yep. Yeah. And he doesn't deny it! The way he [??] and you see it going past them.

13. I: Anyway, so, we're going to go through them, and the process will seem a little bit strange. I won't get you describing the images to start with; I'm going draw you into 
the image. I'm recording this, so I've got an online recording which as you're speaking is recording every word. What will happen at the end is I will get all of this transcribed and I will then prepare, on top of the IMP Test, I'Il call it a vignette but it's going to be about 2,500 words of commentary on what the implications of this are as far as I see them. I will send you a copy of that for your reflections, and of course you can have the full transcript as well if you might find that useful in any way. Some people do; they like the transcript. Once or twice they said, “Oh, I didn't mean to say that." Well, I said, "Tough, you've said it!"

14. <Both laugh>

15. I: Everything's totally confidential. The only people who know about this conversation now is Fred and Henry!

16. R: Excellent!

17. I: They are totally schtum.

18. R: It's going to be around the retriever world in a moment.

19. I: Yeah, that's right; they're off out of the door. Even Alison doesn't hear what really goes on here. It's between you and me. She may come steaming through the door; if she does she will transcend rapidly to the other room, disappear. Right, let's get on with the images, then. I've got them here on the screen. I can still see you in a tiny window up to the left. The image I suggest we start with first - up to you; if you want another one, fine - is the one that's most recent for you.

20. R: So that's the Venice...?

21. I: You've got two of Venice here, haven't you?

22. R: OK, so, yes, that's right, so those are days apart.

23. I: It's not horrifically important.

24. R: The black and white one, so the one that's number three of the three was one of the last images that I shot in Venice. And it's significant in that context so I think it'll be a good one to start with.

25. I: OK.

26. R: So I've got it up here as well.

27. I: OK, you've got that in front of you. Right. You're in Venice then. I want to take you to the day now, when you took this shot. You've woken up in the morning. What are you doing in Venice? You're running a course, if I remember rightly. 
28. R: I was prepping for a course we're going to run in the autumn. So it was kind of a full-day preview, photographic preview, meeting people preview.

29. I: OK. So, about what time in the morning did you get up?

30. R: About eight o' clock in the morning. Not massively early. Quite late night the night before.

31. I: OK. Where were you staying?

32. R: I was staying in a little tiny Airbnb apartment in one of the north west quarters of Venice city. Literally a one bedroom bijou flat, it was very sweet. Turned out to be a really good location.

33. I: What woke you up?

34. R: I woke up at the same time as I always wake up, which is between quarter past seven and quarter to eight. Don't need an alarm clock, just always wake up at that time.

35. I: Can you remember anything about the room?

36. R: It was very dark. Very long and thin; it was just a weird shape. It is literally a oneperson apartment with a single bed, that's all.

37. I: For a tall man, then. Nice long room.

38. R: Exactly. Exactly. And the window that looks out onto a courtyard, so hasn't got any sort of a view, but does look out onto a courtyard. It wasn't massively comfortable as an apartment but it was completely functional. The main thing was the location; it was all about where it was; it was in the heart of the right bit of Venice for me, what I wanted to go and see, people I wanted to be with.

39. I: You were alone in this property. As I say, it's all confidential! So, you were alone?

40. R: Yes.

41. I: So you wake up. What did you have for breakfast?

42. R: I had a coffee first thing in the morning, even before I had my shower. The only things I bought to feed myself in the apartment were coffee and a few biscuits and some green things because it seemed really hard to find green food in Venice; a lot of red food but not much green food. So I had a coffee and then I went down to a café in the square just two minutes away from the apartment.

43. I: So you go down to this café then, Simon. What did you have there?

44. R: So I had a cappuccino and an almond croissant. 
45. I: And where did you sit? Outside or inside?

46. R: Yeah, I sat outside.

47. I: And can you remember what anyone sitting near you?

48. R: There were people around me, nobody in particular I interacted with. I did use that time to have a quick Skype with Meredith in New Zealand because that's the end her day, beginning of my day, so that's the sort of pattern that we have every day.

49. I: So you had a conversation with Meredith. What was she wearing?

50. R: Golly! <pause> I honestly couldn't tell you because I can remember things but I don't know whether I'm remembering it from that day or from other days in Venice or many other Skype conversations.

51. I: That's absolutely fine.

52. R: Would have been work clothes probably.

53. I: What were your feelings speaking to her? Were you sort of excited or not terribly convenient to be speaking to her at this moment? What sort of feelings were going through your mind when you were speaking to her?

54. R: Yeah, no, quite excited because this was the morning of the penultimate day in Venice. I'd had some really good encounters with people on the previous days and I had got lined up later in that morning to meet an artist that I was really excited about meeting up with because I think her work's quite extraordinary and we chatted on the phone and on email so she just seemed like a very interesting person, so I was meeting her that morning. Yeah, so I was kind of pretty full of that, probably slightly too full of everything I was doing and probably not quite as attentive to what she'd been doing and that she'd had a fairly shitty day at the hospital but that's often the way.

55. I: Often the way. Often the way. Yeah, that's interesting. If you had to judge her mood before and after you'd had this conversation with Meredith was it sort of more up at the beginning and down at the end because she'd sort of unloaded a bit onto you, passed one or two monkeys onto your shoulder?

56. R: No, not really, because that's what we do. As you realise it's a slightly unusual relationship with all of that distance involved in it and people find it quite hard to get their heads around it. Actually we tend to just condense it to the morning or the evening conversation and all those other bits of conversation that people have across the day, so it is about being excited about what you're doing and what you've done 
the previous day, and it's about having a good whinge about a colleague at work who screwed up or whatever it is and so you have to act as a sounding board for the other person as well. So I think on that morning for me I was very excited about what I'd been doing, so that was very positive, I was looking forward to what was going to come that day and so I was pretty upbeat. I had a pretty good time in Venice and it was quite an unusual time for me which is why two of these pictures are from Venice, because they have very strong resonances for me.

57. I: Brilliant. That's good. So anyway, you've had this conversation with Meredith. What time did you meet this other person?

58. R: Half-past ten in the morning.

59. I: Half-past ten. And that was before you took this image or after?

60. R: Yes. Yeah, yeah, this image is taken towards the end of the day but it exists because of a sequence of events that then followed on from that meeting.

61. I: Let me just take you into this encounter very briefly with this woman. Where did you actually meet her physically? Where were you?

62. R: So she has a little gallery that was in a side street just off the square just a few hundred yards from the apartment, so it was a road I'd been up and down many times coming in and out of the square. And the gallery had been closed. Every time I'd walked past it it'd been closed. And she's a watercolour artist primarily and I really liked some of the work and some of the etchings that she'd got in the gallery and I didn't know anything about her particularly. But she had an iPad scrolling through a slideshow of things in the window and it said on there - in English - it said, If my gallery's closed and you want to find out more, then here's my mobile number and my email address. And so do reach out. And I thought, "OK, if you're actually asking people to reach out to you, I won't be shy about doing that." And so a couple of days previous I'd emailed her and said, "Look, this is what I'm doing here. I'd be really interested to meet with you and I'm very interested in seeing some more of your work." And so she came back very quickly and we had a chat via email and then WhatsApp and then arranged to meet, said she'd be in the gallery this day. And so she was. 
63. I: OK. So you actually go into the gallery - I want you to now get yourself into that moment - you're walking into the gallery, you now meet this person for the first time. Try and answer this question: describe the similarities between her and Meredith.

64. R: Gosh. <pause> Passionate about what they do.

65. I: Any physical similarity?

66. R: Sorry?

67. I: Any physical similarity?

68. R: Not especially. Same height, I guess. Similar age; Meredith's late forties, her early fifties, mid-fifties.

69. I: Attractive?

70. R: Oh, without a doubt, but very much in that Italian slightly swarthy, dark-haired attractive. Attractive woman, very beautiful lady, very passionate about what she does. And I find passion attractive in anybody - male or female - to be honest.

71. I: Wat was she wearing?

72. R: <pause> Don't know. Didn't really register it.

73. I: It's all right. I'm just drawing memories up.

74. R: I can see where you're going. And I think the reason I didn't register is because I was in a place surrounded by other visual stimuli, Because it's a smallish, a very small one room gallery with a little office off to the side of it and art on every surface, and on tables and in racks and literally practically to the ceiling height, paintings and etchings and drawings. And in fact when I first went in there she was with a customer, so I lurked and looked a bit. So I had a moment to sort of assess the drawings and so on before she decided to acknowledge and realised that I was the Englishman who'd been in contact before, so she knew who I was and why I was there, but I also knew that I was taking her time and this was her living and her customers were more important than me at that point, so she was dealing with them.

75. I: What were you dressed in at the moment?

76. R: It wasn't that warm, so I was in trousers and a polo shirt and a jumper.

77. I: OK. So you say it wasn't that warm. Were you feeling distinctly chilly? Can you remember the feeling? What was your body feeling?

78. R: No, no, it felt like shoulder season, when you never know what to wear. I thought it was going to be warmer and I'd taken clothes for it being slightly warmer with me 
and in the end it wasn't warm enough to wear shorts and so I was kind of layered a bit.

79. I: Did you have your camera kit with you at this point?

80. R: Yes.

81. I: You did?

82. R: Yes. In a backpack. Not a camera backpack. Just a day bag.

83. I: So you've got this big bag...

84. R: Actually pretty small bag.

85. I: Pretty small bag. Can you feel the weight of it on your shoulders at this moment?

86. R: Oh yeah, I know exactly what it feels like.

87. I: OK, good. When you spoke to her what did you do with the bag?

88. R: I'd actually taken it off my back and I'd got it casually slung on one shoulder.

89. I: Cas, yeah. <laughs>

90. R: Cas, yeah.

91. I: Trying to look impressive! <laughs>

92. R: <laughs>

93. I: OK, so you're sitting there.

94. R: Standing.

95. I: Standing, trying to engage conversation. You've got the camera. OK, so let me just move you on now through time. About what time did you decide you were going to go looking for an image to take? Was this very quickly afterwards or some hours afterwards? At what point did you decide?

96. R: An image or this image specifically?

97. I: Well, just say generally an image at the moment.

98. R: Well, I was there in Venice in part to photograph so I was looking for photographs the whole time and because this was the third full day l'd been in Venice and the next day was the day I was travelling down to Naples, then there were some images that I had pre-visualised and knew I was still looking for and didn't have what I wanted to get. And then others that I'd known that I wanted but I knew l'd bagged and didn't have to think about any more. So for part of the morning I was very specifically chasing around trying to get a particular, particular image. Not terribly constructively actually, to be honest. I got it - in the end - I kind of forced it to happen. It wasn't a particularly 
fluid moment, but it was an image I really wanted to try and get because l'd got a very clear mind, this sort of visualisation of what it was.

99. I: OK. So let's draw you on towards this image then. What time of day were you at now?

100. R: So can I sort of flesh out the context of the rest of the day to [??] here? The meeting with Monica was fantastic; we got on incredibly well and she was very interested in what we were doing on the course in Naples and following on, and said her studio is down on Giudecca which is the island along the southern part of Venice, so off the mainland there's a long scoop that goes along the bottom, and her workshop is one of about eight artisans' workshop in a monastery that's been restored and turned into... so she said, "Do you want to come down there and meet some people there?" and so I thought, "Yeah, hell yes, absolutely!" So we arranged to meet - the night we go down there - to meet at about half-past five that afternoon so Giudecca wasn't part of where I was expecting to go in Venice. It's an odd bit of the city it's a mixture of new build and old build, but it's a very thriving community but as it turns out it has a lot to offer a photographer. And not least the gallery and the [??] where she works from is incredibly beautiful and other artisans that are there are amazing. What it led to was an exploration of her other work, beyond her saleable commercial work for tourists, her sort of projects. So we spent a couple of hours just talking through that and seeing what role she could play in a travel light workshop and so it's very exciting. So I was on Giudecca and I went over to the island - so you had to take a waterbus over from the main part of Venice - so I had quite a weedy walk through to the [??], took the waterbus across and I was on Giudecca for a couple of hours before we'd agreed to meet just to explore and walk around. And it has this extraordinary view back across Venice which, although Giudecca's very long, actually the view doesn't really much as you go along it. Actually it's all similar and you could actually take this picture from any point that view but you walk as far as you can, you get this very abrupt stop at the end because there's a naval base. You walk the last bit of it.

101. I: I think I remember it vaguely, I do. Yeah.

102. R: And so I got to the end and there is a photographic gallery called the Tre Occhi - The Three Eyes- which was an old palace that's been restored and is now a 
foundation and a teaching space for photography and they had an exhibition of an Italian photojournalist - female photojournalist's work - so I went around the exhibition and very much enjoyed that and came out from that and was walking aback along but now with a little bit more of a mission of where I was going because I had to find where Monica's place was. And earlier in the day it had been very sunny and it was very boring photographically the light was very, very flat and even but by now so were now talking about maybe half past four in the afternoon - these clouds had rolled in and I was beginning to see an image forming in this infrared. So this is an infrared picture so that's actually probably quite significant to point out that none of the light in this picture can be seen by the human eye. This is pure infrared light. Because I've done so much now of it now over the years I kind of know when the sky's beginning to do something really interesting that's going to take a nice shape in IR and so now my antennae was twitching a bit as the potential for an image that would now come together.

103. I: OK, let me just pull you there for a moment, then, because I'm walking alongside you here; you're giving a very vivid description of where you are and what you're doing. I just want to sort of get you to begin to recollect your physical feelings at this moment. You've got your camera bag with you. What camera did you have?

104. R: Well, this is taken on my modified IR camera so it's a little Fuji X-T1, very little small range-finding camera that only sees infrared so I looked through the viewfinder and I see the world in infrared.

105. I: Was that in your bag?

106. R: That was in my hand.

107. I: Yeah. Oh that was in your hand?

108. R: And medium format camera was in my bag so those were the two things I had with me.

109. I: So you've got the block of concrete in your bag?

110. R: Block of concrete's in the bag, yeah.

111. I: <laughing > It's in the bag. And you've got this IR modifying camera in your hand. Hefting it, can you feel the weight of it in your hand now?

112. R: Yeah, it's very, very light, it's a tiny camera. It's like the size of your pen.

113. I: Yeah, my little Olympus. 
114. R: Yeah, so it actually has very little heft. It's very immediate.

115. I: Did you have it connected round your neck with the strap?

116. R: No, I have the strap wrapped around my wrist, so like a way that I always carry cameras with the strap wrapped around and tucked under my little finger. So it doesn't get in the way.

117. I: No, got you. Draw a deep breath. You're coming up to recognising this scene. What can you smell?

118. R: Seaweed.

119. I: Can you remember the smell of the seaweed?

120. R: Yeah. Yeah. Because you're right on the waterfront.

121. I: Was it pungent? Describe that smell.

122. R: Salty. Salty, irony. Salty, rusty smell.

123. I: Any other smells invading you?

124. R: <pause > Coffee. There's a bakery behind me, people having coffee outside, that's around. Trees; there was some blossom trees. There was quite a wide pavement area that's been nicely landscaped and there was some orange blossom or something, but it was quite a gentle smell, but there was definitely a smell from that.

125. I: Good, good. And what noise was going on here? I'm getting the sense that you can smell but the noise? Could you hear the chatter of the people?

126. R: Yeah, the chatter of people. The sound the water. The sound of the water lapping on pontoons and occasional seagulls - no pigeons this time; they're all over in St Mark's Square. A little bit of wind, the odd riverboat or river taxi going along. Very occasional bells, as you find anywhere in Venice.

127. I: And your camera, then, in your hand, you're feeling down - bringing you to the moment - you're holding the camera. How do you set the camera up? Was it set up in terms of basic aperture and ISO and all that? Was that already set? You had it on a pre-setting? Or had you set about adjusting it at this point?

128. R: So it was to some degree pre-set. The range of adjustment on an infrared camera is rather narrower. You have a limited range of apertures you can use. So that's kind of pre-defined, and the ISO was already set because I had shot other pictures earlier on, walking along, walking to that point, and now I'm walking the other way, so I shot a few others, sort of knew what the settings were, but the fine-tuning 
of the settings is done by eye through the viewfinder when I can actually see the IR image and the histogram through the viewfinder.

129. I: Did you physically switch the camera on from an off mode? Can you remember that movement?

130. R: I would have done but I don't remember doing it because it's part of the picking up to my eye action.

131. I: So you're drawing it up to your eye. Now I want you to try and remember did you make any last minute adjustments?

132. R: $<$ pause $>$ Yeah, well, I would have certainly dropped the active AF point down to the buildings line in the photograph, so from that point of view of adjustment, I would have checked that nothing had slipped but particularly aperture's easy to knock, and I would have checked that it's giving me a shutter speed that I'm going to be able to hold all right because these cameras are slightly less sensitive to sensitive to visible light so you have to be a lot more mindful. It's a prime [??] that's what I always shoot on on that camera so there's no focal length adjustment to do, so from a settings point of view it's more about checking that things are right and that the focus is going to fit the right spot.

133. I: So you're looking through the viewfinder now. How much framing did you have to do to get this shot the way you wanted it? Can you remember moving the camera physically?

134. R: Yeah. So I shoot quite a lot in this proportion. This big sky, little foreground proportion when I'm shooting in IR because one of the effects that I find fascinating in infrared is the modelling of clouds, the texturing that clouds get and how that responds to whatever is in the foreground and the mid-ground. And that's why I love shooting with such a wide angle lens - $21 \mathrm{~mm}$ equivalent so it's wide by the [??] standard.

135. I: What's attracting you? What are you seeing here? What is it about this particular image now as you're looking at it through the viewfinder which is getting the juices flowing? What is it?

136. R: So it's many layers all at once. The one layer is a response to the whole reaction I had to Venice over the previous days on the main island of Venice which to one degree was on of shock at the number of people that were there. I haven't been 
to Venice since I was a teenager; I went with my parents when I was 18 when, sure, it was busy, but it wasn't like this and... so the first day l'd been there went to St Mark's Square and kind of reminded myself of the geography and could barely move for people. And then a lot of the other times I'd been walking around in very narrow streets over the little bridges it's been very much in the thick of Venice with the buildings around you and the crowds and the hustle and bustle and the tourists and the more tourists and the yet more tourists kind of layering in on top of that, making it very difficult to engage with the place. And now suddenly I find myself across the water looking back at it, feeling that I've got a sense here of the bigger place that you can't tell from this vista whether it's busy or it's not busy. There's one boat in the water and I would have liked it even more if the one little boat wasn't in the water. There's no sense of bustle, of modern tourism, there's a couple of cranes which is really the only thing in the image that tells you that this picture's contemporary and one largish passenger boat on the left hand end which tells you it's contemporary, but it could in a sense have been taken 300,400 years ago. So it's a response to that feeling of slightly overwhelmed congestion that actually this is a place of incredible beauty that sits within a sea-sky landscape.

137. I: Right, OK, so we're both looking intently at this image now. Have you got it up on your screen?

138. R: Yep.

139. I: Full screen?

140. R: Yep.

141. I: Big enough, it's dominating your visual field as it were.

142. R: Absolutely.

143. I: I would like you before we go back to the emotional response to this is if you would just describe it to me please as thoroughly as you can and just give me a good description.

144. R: From an aesthetic?

145. I: I think you can choose whichever way you wish to describe it.

146. R: So there is a very big difference between a raw capture of an infrared picture and the finished picture. Possibly a whole order of magnitude than more than most conventional photographs would have. Whilst it looks black and white through the 
camera's viewfinder, it doesn't have the range of contrast or detail that you can extract from the picture in post-production. And one of the things that intrigues me about infrared is the micro detail, so there's always something I'm looking for. As a composition it's a deliberately slightly contrary composition because it doesn't follow the conventional rules, certainly not the rules of third - it's on the fifth rather than third - but very deliberately so because for me this image is as much about sky as it is about the landscape. So the visual journey that I saw through the viewfinder and that I've tried to reinforce in the print is one where you quite quickly go from the buildings in the centre of the image and then you scan around the sky but that the sky's all pointing back in towards the tower. From a Gestalt point of view the tower is the dissimilar item in the buildings so we have similarities all the way across and this [??] thing that sticks up, so immediately it's something that the eye is going to associate with. So the first point of contact for most viewers of the image is they're going to look at the tower; many people will recognise where it is geographically because it is iconic as a building as is the palace to the right of it and then clearly it's been placed in the context of water which is intrinsic to Venice's existence, Venice's history, and then at the same time it's been placed in the context of this big, big dramatic skyscape that is giving a sort of natural power to the image and bringing you back in to the buildings in the centre.

147. I: Just now let's pick up on that then, Simon. What is the sense of power that we've got here?

148. R: Because we don't see sky like this with our eyes. It never looks as contrast$y$ as that. This is not a particularly threatening sky but it is a very sort of geometrically structured sky. It's a really usual cloud pattern and it's unusual in the way that it's forming this pan shape that, at the lower tip of which, is the tower and the palace. But it's not just a fan shape it goes out and takes you out of the picture because it also resolves itself in the middle top and kind of stops you bouncing out the edges. So this is definitely humourless photographers. This is clouds working for the photographer to enhance the texture in the landscape - for me.

149. I: OK, so let me just draw you back to your feelings about what you were photographing here. You were talking about the very crowded feeling you got in Venice of being, personally I would guess, of feeling very crowded, lots and lots of 
people around and that was not what you said you remembered as a child. Now you're looking through it, what is the feeling you've got now looking through the viewfinder at this image now? What is that feeling? Is it - you described it as that hustle of bustle has gone - you're now looking back at the image as you remember Venice?

150. R: Yes, in a sense it's a little bit like looking back in time. I'd not seen this view of Venice when I was a child; we never went over to this island, but we did go over to other islands and had had views of Venice. I suppose in one sense any monochrome image has a little feeling of period to it - it can have feeling of period to it - so there is an element there of it being sort of looking back through the retrospective scope. But I think my emotional response to the image is that I can breathe when I see it and I think that it partly because there's been a lot of time when I'm in the crowded part of the city when it's been all like this. So I don't know if you're looking at me or the image but all very tensed up and tight, and now suddenly it's a feeling that it can breathe and that the sort of creative muse is flowing a little bit more and that when I relax and when I'm in the zone, so to speak, of taking pictures but then other things happen - I don't know whether things happen or it's more likely that I see things that come together in my mind, like the conjunction of the pattern of the sky. And that is quite a big leap because if you saw the raw file - the raw file's very neutral mid tones. Because I've done so much IR over the last 15 years plus, I know when I see a sky like that, I know it's going to be an easy journey to actually bring out that texture and shape in the sky.

151. I: What are your feelings about this image now looking at it?

152. R: $<$ pause > I still really like it. It's not perfect and there isn't a lot I could have done about it. I'd like to have got rid of the big boat on the left and it would be lovely if the crane wasn't there on the left, and there may be some inspiration from this image where the two cranes from the image get removed because they don't belong to the story, not at this point but I think the boat would be a step too far.

153. I: <laughs > Yeah. <laughs >

154. R: So I have another image taken a few minutes later when one of the commuter boats crosses in front so it's in the middle - in the water in front of it. And I also really like that image. It's a portrait format image, it's a different story because that is a story about here is historic Venice in all its context but it's still living, it's still 
people doing what they do, but I don't like it as much as this image; the sky's not as successful in that one. So I would love to have had a tilt shift when I shot it. It's not something I've ever been able to use in IR but it is something I want to be able to use in IR when I get my converted medium format camera back because as capture this had hideous verticals as you can imagine on a 44 lens with the horizon set that high, so has been vertically perspective. So it would have been nice not to have had to do that in post-production, to have been able to have that correction there in camera.

155. I: Are you looking at it now and feeling that the image has accomplished what it is you wanted to represent at that time, and I notice what you say about the big boat and the crane and all that sort of stuff.

156. R: But I don't think any of those really detach from the power of the image. I'm not trying to create a Canaletto painting of Venice. It is Venice in 2019, but it is a sense that it is... peace and calm and tranquillity when you step back from it a bit but maybe in a context where there is a little bit of a threatening something over the city. Which there is a big threatening something over the city; the city has a lot of problems and a lot of dangers, apart from sinking, but that's quite a big one. It has a constant struggle with its relationship with its visitors, so that whole thing I think in some way is built into the feeling that this is an appropriate way of conveying that environment.

157. <pause>

158. I: Excellent. Right, Simon, we're going to move onto your next image now.

159. R: Shall we stay in Venice?

160. I: Up to you. If you'd like to stay in Venice. I've always found it a very attractive city myself. If you fancy staying in Venice.

161. R: Let's stay in Venice, then. I tell you, picking three images to send to you was really, really hard. But you'd said once that you had some degree of emotional connection.

162. I: Salience.

163. R: Yes, salience, that's right.

164. I: I need then to activate a strong response for you and you could now sort of see the way the questions are flowing, usually the next two images, the whole pace of the interview picks up because I think I detected that your brain is now working the way I want it to work. 
165.

R: Good. Hope so.

166. I: <laughs> Let's take you to this image. Was this taken on the same day?

167. R: This was taken the previous night.

168. I: The previous night? OK. And had you arrived into Venice that night or was it...?

169.

R: No. No, that was the end of my second full day.

170. I: Right. OK, end of your second full day. So at what time in the evening was this then? Must have been quite late.

171. R: Very late. Very late indeed. I'd been out shooting and walking the whole day from sort of eight o' clock in the morning. This was probably around $11 \mathrm{o}^{\prime}$ clock at night and <pause > as an image concept it was something that I had pre-visualised that I wanted to try and take and capture in some way. Blurred gondolas is not exactly reinventing the wheel in Venice, let's face it! Plenty of people have done it.

172. I: I've seen a few in my time. <laughs>

173. R: Exactly. But I wanted to do it in a different way and I think what I found very attractive was that this image has three very distinct layers in it.

174. I: Right. Before we get onto that I just want to make absolutely sure that I'm with you. You've had your dinner?

175. R: Yeah, yeah. Yeah, yeah, yeah.

176. I: What had you had? Was it pizza again?

177. R: Probably. What did I have? No, I didn't have pizza, I had spaghetti carbonara in a little bistro just round the corner from the apartment.

178. I: With a glass of wine attached?

179. R: Sorry?

180. I: With a glass of wine attached?

181. R: Definitely.

182. I: Oh definitely? <laughs> OK, so you're feeling pretty relaxed...?

183. R: Or two.

184. I: <laughs> So you're feeling very relaxed and comfortable?

185. R: Very relaxed and comfortable, then I went back to the apartment and I picked up a tripod because I knew I was going to stay out and do some lowlight stuff 
and some light stuff. And I didn't have the IR camera, just had the medium format camera and the tripod.

186. I: So medium format and tripod. Medium format in your bag, over your shoulders, tripod in your hand.

187. R: Tripod in a bag over my shoulder actually.

188. I: Oh was it?

189. R: Yeah.

190. I: So you're well laden down.

191. R: Yeah.

192. I: OK. All right. You knew where you were going to? Was there anybody else with you at the time?

193. R: No, I was on my own. Yeah, knew where I was going to. Had reccied it as a location earlier in the day, so knew that there was some interesting things going on around here that could potentially combine together to make an image.

194. I: Right. OK. So you've approached this place. You've got an idea in your mind of where you're going to be choosing, so you're approaching this place. Can you remember exactly where you were standing?

195. R: Yeah. Yeah, I could take you to this exact spot very easily.

196. I: Describe the spot. What's behind you as you're looking out here?

197. R: So, St Mark's Square exits onto the main lagoon and it's one of the areas where many, many, many thousands of tourists pick up gondolas for their trips. So there are these pontoons go on for several hundred yards where these gondolas sit. I photographed earlier on; there's a bronze brass glass lantern that's very distinctive that's like a little sort of lighthouse for the gondolas that's been there for probably a couple of hundred years and I think it got a Madonna inside it, so that's just off to the right hand side and that's level with the opening from St Mark's Square into the lagoon, so this is just a little bit further along and affords that view across to the other palace and the other tower that is the mirror of the two in St Mark's Square and on the other side of the lagoon. So the picture we just looked at in fact, if you scan along the horizon of this to the right, hit the last pole on the right and come left one building, that is where I was standing photographing back in the opposite direction to the previous picture, so I can in fact locate both of those two places very, very accurately. 
198. I: So you get your tripod out?

199. R: Yep.

200. I: What did you do with the bag?

201. R: The tripod bag I stuff into the backpack, I just leave that on my back which now weighs nothing because it's not got a camera in it.

202. I: Did you have any trouble getting the tripod up?

203. R: No. No. Second nature.

204. I: Yeah, I know. The ground - was it levelled up?

205. R: Yeah, the ground's level. I'm down on some steps so there's the pavement level and then there are three steps down and then the water lapping, so the front two legs of the tripod are longer than the back leg because I needed to get down and forward quite a lot to get the camera position, so in fact what l've done is I've figured out by hand where I need the tripod to be before I even take it off my shoulder and realised, "OK, this is where I need to be" so when I open the tripod up I already knew that it needed to be two legs longer and one leg short in that way to sit across the steps.

206. I: Take a deep breath. Was it very smelly?

207. R: $<$ pause $>$ No. No. No, not really.

208. I: Any rotting vegetation floating past?

209. R: No. Well, yeah, there's seaweed flapping around. There's seaweed in the water, but it's not particularly smelly. It's busy behind me. Well busy. Lots of people around. A few other photographers.

210. I: Irritated by them?

211. R: No. No. There weren't people jockeying to take the same picture or anything like that; it wasn't that sort of a situation. I've been in that situation before, but it wasn't like that. So although it was quite busy it was quite solitary as well. And I was very, very much in the zone.

212. I: Good.

213. R: Yes.

214. I: So, Simon, you're in the zone. What settings did you put not the camera at this point? 
R: So <pause> I knew that I wanted an exposure that was long enough to achieve blur so I did a few test shots to see what sort of exposure time was going to give the right amount of blur in the gondolas and it's surprisingly hit and miss because the amount the gondolas move depends on how much other traffic is going past in the lagoon, and so you just find one that was right while everything was bobbing gently. And then a waterbus would go across and there' $d$ be a big wake and they' $d$ be heaving up and down and then it would be completely wrong. So it was not quite as straightforward as I had perhaps originally thought it might be to get just the right amount of movement and just the right amount of light trail. The exposure is defined by the background.

216. I: So you've got this set up on the tripod. Was it height that you could look directly through the viewfinder? You don't have [??] monitor through this, do you?

217. R: Yeah, I do. It's got a flip screen, so that screen tipped.

218. I: That was how you were setting it up.

219. R: Yeah, I was looking at it on the screen.

220. I: So you're looking down at it as opposed to through it? OK, so you're looking at this scene visually unimpeded by camera, you're then looking down. I want you to describe what you're looking at now. You've got this image here to remind you now. What are you actually looking at?

221. R: <pause > Well clearly you're not looking at what the finished picture looks like because you're looking at effectively a video on the screen, so what I'm looking at then is about am I in the right position? I've established the height is right - am I in exactly the right position left and right? Do I have enough width to get what I want width-wise or do I need to come back to widen the angle of view in terms of that? A few test exposures to establish whether I'm in the right ballpark and then beginning to think a little bit more about the post-production in the image, so, OK, what's going to resolve in the movement because with any picture that's conveying a sense of movement in a still image you've got to hit that sweet spot where the object still clearly resolves as what it is. So I knew these needed to still appear as gondola and that's why the silver, gold coloured fin on the tail of the gondola on the left hand third was really vital to this shot. So l'd moved around a little bit to make sure there was light glinting in that, so that's actually light glinting from the street lamp. Because I 
knew that I needed that to become a really strong highlight because it's going to be a blurred image but it still had to have some semblance of that iconic shape which, in fact, that shape represents the islands of Venice. So I'm kind of thinking about how that's going to work in the long exposure and how I'm going to process the image afterwards to work with that. When I started I wasn't particularly mindful of light trails; they weren't part of the image in my mind... until afterwards - after I'd sot a few and I'd realised actually because there's constant boat traffic going across the lagoon that these light trails have to be part of the image as well, so that's what I meant to add the third layer. So that the gondola are $a$ layer, the buildings at the back are $a$ layer, but the light trails that divide them are yet another layer and then perhaps the most abstract one, because it's not immediately clear as to what's going on there, but because we're conscious of what light trails look like these days because there's a lot of imagery of them around, because I'd done projects with my daughter for her Alevel photography that involved light trails, I was suddenly switched on to think, "Actually, the colour from these light trails is getting a connection between the foreground and the background." So where some people maybe would have looked at this and gone, "I don't want those there, I'm just going to wait until nothing's going past", I couldn't do that because if I waited until nothing's going past, the gondolas aren't going to move. The whole thing is that sort of interconnected process.

222. I: You're coming across to me and I'm looking at it as a very sort of deliberate process that you're going through there with these light trails. There's a very thought through - you talk about your daughter's been doing it in her photography course. Would you describe this as very deliberative?

223. R: Yeah, because it is a very consciously constructed image because there's a lot of very detailed micro placement of elements in the image for it to work, so for example the bright white light on the left hand side fitting just between the two posts, that's like one centimetre left or right and that's gone, matched with the two lights on the right hand third fitting between their posts. So those little highlight moments are very constructed as well as fitting the steeple and the church in between its framing posts and then trying to find movement on the far left end and far right end to frame it that way with substance going on in the middle, so it's a very conscious, deliberate, iterative process of where the camera's going to go, and then it's a subsequent 
iterative process of when I'm actually going to file the shutter and for how long to get the right movement and so I made three exposures without moving the camera to find the one that was going to work - they're about a minute, the exposures so not long.

224. I: I was guessing something of that sort of order. Looking at the image now, Simon, you've got it in front of you - what sort of feeling does it evoke in your now looking at it? Can you describe those feelings to me?

225. R: $<$ pause $>$ There's a slight feel of smug satisfaction that it was worth spending the time to get those bits right because there are no bits in the image that annoy me in the way that the boat in the previous one, you know, wouldn't it be nicer if...? There isn't any element of that in it and so it was one of those cases where I'd really gone through the structure process before pressing the button to make sure that everything was right and the only thing that was down to the random elements was, were the movements going to work? But I knew I was in the right ballpark of shutter speeds and that I would get the right sequence of movements eventually if I persevered at it. Funnily enough this wasn't the third of the three; this is the second of the three. I thought this was a keeper; I thought, "Well, I'll do one more. I'm just not sure." I was just kind of banking it a bit. But this was a head and shoulders above the other two. So yeah, a little bit of smug satisfaction that l'd worked the problem and had resolved it and had been able to create it.

226. I: Why did you feel a need to do that?

227. R: Because I think that $<$ pause $>$ too much of our photography has corners cut for whatever reasons and it might be because you're there with your partner or your children and they want to go off and they don't want to spend hours doing this. Or it might be because you're hungry or you're tried or whatever, or get disturbed or somebody else is waiting to stand there to take their picture or whatever it was. So there's so often, for externally perceived reasons, a compromise introduced into a picture and this is not one of those times. This was a time where I had no temporal constraints on me at all; there was no pressure of somebody saying, "Oh, you've got to be here, you've got to be there." I could have stayed out all night if I wanted to; it was my call. And that l'd gone out there with a clear desire to use a technique that's been used a lot but to try and do something unusual and new with it and all of those 
elements to me came together in the image - in the print. And the other thing I should have put in there, as you probably sensed when we were in Naples, that I was blown away by what this camera could do, as well, because I don't think I could have got this image in a single capture from my X-T2; it didn't have the dynamic range. Although it's a night shot, the dynamic range is enormous in this picture. Absolutely enormous. And I actually did a rough development of this picture when I got home that night because I wanted to know that I'd got it. And I was sort of squeaking at that point because the camera has translatable eyesore in my eye into the image. It hasn't captured that sort of sense of disappointment that you so often get when you look at the raw file afterwards. So there's a lot of that technical stuff going into it at the same time as it is telling a story to me. It's telling the story in the way that I want to tell it of this fusion of the old city, the piles, the lagoons as a means of transport, but the modern transport going in between the two all the time. So you've got this mixture of old and new and historic and sailing boats in the back but gondolas in the front at a golden slightly dream-like aesthetic quality in the image, but still got these blocks of colour, these blocks of blue from the tarpaulins that are used to cover the gondolas. And the way the ropes sway, that wasn't expected at all. But I think it's incredibly beautiful. And it's a little bit like the wings of a seagull - maybe I'm reading too much into it, and now l'm doing a deconstruction.

228. I: <laughs $>$ Go for it, Simon!

229. R: I feel that there's waves and there's birds wings coming, these little things. I love the fact that there's movement in these vertical poles as well because the boats knock against them.

230. I: There is a little bit, isn't there?

231. R: So they're sort of solid and yet not solid. The picture is framed on the left and right edges with movement from those gondola but the two gondola are really striking and really resolve for what they are in the central place but fitting with the church. I've got the foreground and the background framing in darkness, which kind of forces you into this thin strip, and I shoot a lot in this sort of [??]. I like as a format. It's kind of wide yet narrow, which I think is how how we see things very often; we scan horizontally way more than we scan vertically, so whilst I love big skies, I also like the horizontal too so... 
232. I: If I said to you this was an image for Simon, in the sense this is for you a personal accomplishment, this image is perhaps not so much for another viewer but is a sense for you the fact that you have solved so many problems as you would perceive them in getting an image like this and producing it, would that be fair, do you think?

233. R: It's certainly not unfair. It is certainly $a$ way of viewing it, but I know because quite a lot of people have seen this image now that it has a very strong impact with views, as well. it's an image that people have looked at and dwelt on and thought about, so it is something going on in the aesthetic and the construct in this image that does make people stop and look a little but deeper and that's something that I'm very passionate about in all of my photography, and it certainly sits in all three of the images were talking about today because <pause > my biggest bugbear in imagery is the sort of transitory flick and swipe, or the nine-second judging in a camera club or anything like that. You know, what I love to do, as you well know and you were part of this process with me, is I love to actually sit and breathe and think about an image, and look at all the stuff that's going on subconsciously. We don't analyse and I find that that whole... any image that kind of draws you in, sucks you and keeps you there for a little bit where the more you look the more you see. I talked about micro detail with the other one and there is the same level of micro detail in here and this - I want to print this big, I want to print this two metres wide and see what it looks like because this is some silly number of pixels across this image; this will print huge. So I want to be able to a walk into it and I want to be able to walk along it physically and have a physical relationship with the buildings in the background.

234. I: What does it tell me about Venice that I would not get from one of the many images one sees across these gondolas over to the square? What is this giving me, do you think? Take me into that aesthetic. What do you think this is giving me that...?

235. R: I think, so <pause> I think it's a process, so there's certainly a "Wow"; there's an impact. Because you look at it, you go, "Wow, how did you take that?" Which <pause $>$ which hopefully morphs into the individual elements in this picture are as components really beautiful, be they sharp or they blurred. But putting them together in a single capture <pause $>$ I hope creates a relationship between those components that is both a temporal relationship and a spatial relationship and a 
contextual relationship. You can't separate out Venice as a city from either its history or its modern day. And you shouldn't separate them out, so you have to acknowledge that the city exists both in the past and in the present and in the future at the same time. That is what I'm trying to create some sense of in this picture - that there's stillness and there's beauty and there's busyness and there's movement and there's life going on, and there's past and present and future if you wish to extrapolate to it, going on all in one image, so <pause $>$ yes, at one level it is a technical achievement a bit like any long exposure or astro photograph or whatever, but I hope that that is the bit that's for me, the technical achievement, but that the aesthetic response to the image works on a whole other level and there's a non-photographer - because let's face it: we're hopefully not making images for photographers to view, we're making images for non-photographers to view - that the non-photographer's response, who has no concept of what you have to go through to take an image that does this. I want them to go, "Wow! Look!" and to travel through the image and to dwell, to spend time with it. Does that answer your question at all?

236. I: Yes, it does. You've done very well there, Simon. What's your sate of mental exhaustion like? Do you think you could manage another one?

237. R: Oh God, yes, I could go on all day. I'm very happy.

238. I: <laughs>

239. R: Sip of water. I was well caffeinated at the beginning.

240. I: Let me find... Oh, right, Simon, we've got you in the mood now.

241. R: Oh yes.

242. I: Oh he's in the groove!

243. R: Oh yes!

244. I: Now, this is the final one of the three and where have you taken this?

245. R: So this was taken in Death Valley.

246. I: OK. When?

247. R: About... well, it was February. Middle end of February that we were there. So, I'm with Meredith and we're towards the end of two weeks of travelling around California, starting off in LA, going out to Park Springs, and then picking up an RV, and so we're camping in this RV. We went up to the Grand Canyon; spent three day up at the Grand Canyon in the snow. Really close call as to whether it would be this picture 
or one from the Grand Canyon. Then very traumatic through foul weather drive across to Death Valley, involving driving three-quarters up a mountain and having to turn back because we couldn't go past because the weather was so bad, but anyway eventually getting into Death Valley and being completely mind blown by the vistas in Death Valley. So this is taken on the salt flats.

248. I: Time of day?

249. R: Before dawn.

250. I: Before dawn? Right.

251. R: Before dawn. So this again feeds into my invisible light aesthetic, to some extent. I mean, it's a conventional regular image, it's a GFX image again. It's not an invisible light but it's an enhanced light in that it was pretty damn dark when I took it. So again this is probably - I can't remember exactly - roughly 20 seconds exposure, which should give you an indication of the light level at this point. So this is well predawn. We shot there the evening before and during the night so I'd done some astro photography during the night there. And then we went back to the campervan and slept and got back up in the dark, walked out, so this is about 45 minutes' walk out to get to the road at this point.

252. I: So you're away from your RV. You've walked away.

253. R: Yep.

254. I: Yep, yep. OK. Meredith with you?

255. R: Yes. Yep. We were both shooting in different directions. There was one other person out on the flats at dawn at that time, another photographer.

256. I: What were you wearing? Were you both in your jim-jams? <laughs>

257. R: No, it was cold. It was quite cold so we were wrapped up quite warm.

258. I: Were you topped up?

259. R: Yeah, topped up. And it's incredibly quiet there. I mean just unbelievably quiet. There's not even any wind because you're sheltered down in the valley. And it's vast.

260. I: And you say it's cold. How cold? Can you feel the cold?

261. R: Yeah, gloves cold.

262. I: Gloves cold.

263. R: Gloves cold. Woolly hat cold. 
264. I: Woolly hat cold. And what was Meredith wearing? Just bringing the memory back.

265. R: Yeah, same, thick quilted blanket coat, and fleecy trousers, very attractive. $<$ laughs>

266. I: <laughs> It's all right, Simon, I know it's being recorded! Very attractive, Meredith, alongside in her black kit. OK, and she stands typically to the right or to the left of you?

267. R: Actually she was standing behind me because she was photographing in the other direction up the valley.

268. I: What was she looking for up there then?

269. R: Well, we hoped for a sunrise, that the sun was going to rise from, as my picture shows, the right hand side. And the forecast from the night before was not great but we figured it was going to be good enough to get up early and see whether we could get a sunrise, so we were hoping for sun striking the hills that are to my left in this picture, but I realised pretty quickly that with this level of cloud cover nothing was going to happen. And in fact in Death Valley the sun has to get really high - as had been discovered later - before it gets over the mountains to either side to illuminate the mountains to the opposing side, at which you're out of the sunrise time anyway, but we didn't quite work that one out properly.

270. I: What were you shooting with here?

271. R: This is the GFX and the $23 \mathrm{~mm}$ prime.

272. I: On tripod or handheld?

273. R: Yep. Tripod.

274. I: Tripod. OK. So you've set yourself up, you're looking out at these flats. Was the tripod set up on this sort of ground or are you on some sort of edge now?

275. R: No. No, I was absolutely in the middle of this extraordinary salt texture.

276. I: OK. And is it the sort of salt texture that really crunches under your feet as you walk?

277. R: Yeah, very tactile.

278. I: So you could actually hear it crunching?

279. R: Absolutely. It's pretty strong. I mean you can stand on the ridge-y bits and they don't crumble underneath you. And there is a sort of diminishing path that comes 
out from where you can park - you can't see it; it's off to the right hand side - that starts out being there's no salt on it at all, it's just mud, it's all been crumbled away, and as you further out it gets crumblier and crumblier, and then the path disappears because that's as far as most people will go - about a quarter of an hour out probably, so we're now about 45 minutes out into the middle. So at this distance you can look all around you and you can't even see that path anymore; it's disappeared off. So you are sort of marooned in the salt to some degree. It's an extraordinary alien environment.

280. I: Do you feel a sense of isolation looking at this?

281. R: $<$ pause $>$ Yeah, good isolation though. Not bad isolation.

282. I: So you're not feeling hostile or?

283. R: Not at all. No, no, not hostile. More dramatic isolation. But it is an otherworldly place. I've never been to anything like this. I've been out to salt flats before but they've always been flat flats. I've never been anywhere with this geometry, this regular repeating, hexagonal geometry around you. That stretches for as far as the eye can see. In all directions. It's just bizarre. So, yeah.

284. I: So, you've got your camera on the tripod. Let's get to the practicalities. How have you set your camera up? Did you make any last minute adjustments to it?

285. R: This is point and pray because I'm shooting in the dark. I mean obviously the light levels at this point are just beginning to activate the eyes, so this is, in a luminosity sense, magnified many times from what we as humans can see. And I find this whole thing is one of the miracles of the camera to me: that it sees in a totally different way to how we see, whether it's blur, as in the previous photograph, or whether it's light that we can't see as in the wavelengths that we can't see as in the first photograph, or whether it's light that is simply so dim that we cannot collect it in our photo receptors. But the camera can because the camera can make a single moment from a longer temporal period and so can reveal things that are not receivable to us and can reveal colours and textures that are gone. Because once the light gets up any more all these blues and creams and oranges disappear totally. We spent the night before shooting by moonlight where you have incredibly shadows cast across these textures from the moon, but you could just about make out with the eye, but they're magnificent. 
286. I: To what extent, as you were sort of approaching, taking this shot, Simon, were you aware, couldn't physically see them, but were you aware of the textures and colours that potentially might be there that the camera could see?

287. R: Very aware because I'd started shooting test shots so I'd done a lot of photography at this time of day and we'd done a lot on this trip as well, up at the Grand Canyon and we'd done some stuff at the sand dunes in Death Valley before dawn as well, so I was kind of in that zeitgeist a little bit and so I just started shooting. We walked all the way out there, we weren't going to get a sunrise, doesn't mean to say we weren't going to get an image that we like, so l'd done some test shots and looked at them on the back of the screen and gone, "Ooh, wow, OK. This is dramatic." This is a texture that is the same but is different to the foreground texture. The foreground texture's an absolute; I know what that looks like, but what I'm seeing and what I'm hoping that this image gives the viewer is the contrast between the texture and the sky in some sense forming quasi-geometric patterns that relate to the texture in the foreground. This slither of mountain as the dividing line and as is so often the way for me, I start tipping the camera up more and more and dropping the horizon down more and more, but this picture could probably have worked in a mid-line horizon or an upper-third horizon. And I took it in all three. But this is a complete uncropped frame out of the camera. This was a composed in camera but as a result of shooting, looking, tweaking, shooting, looking, because I couldn't see it with the clarity that the camera could see it.

288. I: OK. So looking at it now, I'd like you to describe to me what sort of feelings this is evoking in you as you look at it now?

289. R: Well it's bringing back the feelings of vastness and openness and emptiness of the place. It's bringing back a whole load of feelings about the whole trip and our time together and our time together in the RV and using the amazon base to explore the photograph from. <pause $>$ But beyond that I think that the feeling for me is about how amazing the planet is, even though this looks like it may have been taken on a different planet almost. It's not tangibly planet Earth but it is, and I find that really extraordinary, that the diversity of the planet is so huge and that you can go to these places that are not even that far away from civilisation and see these sights on your own or with your chosen partner and nobody else is there. Like I said one other 
photographer somewhere on a different bit of the salt flats, but still it's possible in this world to have these individual isolated experiences so...

290. I: I just want to test that briefly then with you. You've got Meredith to the side of you. In order to communicate, how important was it having her alongside?

291. R: I think we motivate each other to go out and do this. To go and take pictures and to go to these places.

292. I: Could you have taken this shot, do you think, without her there?

293. R: I probably wouldn't have gone to Death Valley - at the moment - on my own.

294. I: Was there some element of the relationship that you have with her that was important for you? Do you see that reflected in this image?

295. R: She gives me the space and the time to do this sort of photography without it being an inconvenience or an unwillingness. And she is as excited by the place and what you could convey photographically from that place as I am. I get very excited, as you've seen. <laughs>

296. I: I've noticed that, Simon.

297. R: Funny that, isn't it? I know.

298. I: You're looking at very much a sense of isolation, grandeur; the planet is looking quite unlike anything you've seen before. You describe that very eloquently. Is there a degree of comfort in having a relationship there with her which allows you to experience and communicate this isolation?

299. R: Yes. Yeah, absolutely. It's on a macro and a micro level. So at the macro level it's having someone you want to travel with and go and do this stuff with that sort of motivates you to go and do it so l'm not <pause $>$ I'm not a sort of <pause $>$ what's the word I'm looking for? My travelling and my photography works best when I have a reason for being there, some sort of a purpose. I can't be a sort of mindless traveller just wandering around and letting things chance upon me; I don't function terribly well in that situation. So I like to have a reason for being in a place. Now that reason could simply be because it's somewhere two people want to go to and they go, "Well, OK, let's go and do it." "All right, why not? Let's get rid of any obstacles to go and do it and let's go and do it." And so she is the person in my life and has been the person in my life for some years now that I can go and do that with. It's not a relationship I've 
had with anybody else in the same way. So, you know, Chris and I don't go and shoot together. We'll go somewhere and we'll both go and shoot and we'll meet up afterwards and we'll talk about it and bounce ideas around and we'll then go off and go and shoot. Whereas...

300. I: But not a man to hold hands with!

301. R: So that's kind of the macro level.

302. I: Yeah. And the micro level?

303. R: And the micro level. It's bouncing ideas off each other on the ground at the time. It's playing with techniques to see what you can get out of it. We'd been doing light trails the night before with laser pointers. We'd been light painting in the middle of this landscape with a starscape behind us. I've seen that done - I've never done it. I've watched Meredith - she's a consultant, plastic surgeon. She's at one level a very cerebral person and at the other level she was playing like a five-year-old with a torch in the dark. It was one of the most beautiful things I've ever seen somebody do. She completely let go of all inhibitions. Was just having fun and that was so lovely. So lovely to see someone unlock themselves like that. So there's that going on. So, yeah. So at the micro level it's about that "Oh come and look, come and look at what this is doing. Wow! God, that's so exciting! Can we change it? Can we make it better?" We're almost both kind of taking the picture together.

304. I: As I look at this with you it's not just a straight landscape. I guess a strong emotional response to it; I'm looking at the source of that emotional energy that's coming from it and I'm just wanting to explore with you there how you've managed to collect that emotional energy to delivery it in the image. The degree to which Meredith being there alongside you was, in a sense, giving you that...

$305 . \quad \mathbf{R}: \ldots$ the space and the freedom.

306. I: ... to deliver that shot.

307. R: To deliver it, yeah.

308. I: Fascinating. Simon, you must be exhausted!

309. R: I'm all right! I'm on home turf, you see!

310. I: You're on home turf! Good for you! 
311. R: It's very good because you've not made any subjective assessment yourself of the images so am I allowed to ask you whether you detached from the process? Are you allowed to form a subjective opinion yourself on them?

312. I: No, in fact it's not a subjective opinion. You took me into that shot with you. I'll just make a note here on the recorder for my transcriber that the interview as such is at an end, but I think it's absolutely crucial when you look at an image to attempt to enter into the image with the photographer, to stand alongside them. In a one sense, I mean if you take the meme of the death of the author; once the image is taken the author and the subject is then eternally withdrawn, then you've got an image. But, no, the author remains reincarnated in the viewer. I have to come into that image to experience it with you. So I'm not going to look at it and make technical criticisms of it; I'm sort of getting in there with you alongside you to try and explore with you what it is that you were experiencing at the moment that you shot that image.

313. [End of interview] 


\section{Technical note on Catastrophe Theory explanation of state changes}

This Extract is taken from Scapens, Ryan and Fletcher (1981)

Catastrophe Theory emerged in the 1970s' following the work of Rene Thom (1989) and in the UK of Carl Zeeman (1977), Ian Stewart and others (Poston and Stewart, 1998). It offers an elegant mathematical approach to the problem of sudden state change in any field. Wherever there is an equilibrating force in place any state variable (performance in the case of sport science or belief in the case of judgement theory) under the influence of more than one control variable will exhibit points of disequilibria where instantaneous state changes will occur. The operative word here is 'will' and the mathematical logic centres around the concept of structural stability. If we take a simple function of the form:

$f(x)=x^{2}$

perturbation of the function by the addition of another term in $\mathrm{x}$ does not disturb the basic geometry of a quadratic function.

However, when we reach functions of the form:

$f(x)=x^{4}$

perturbation of the function can lead to structural instability. 


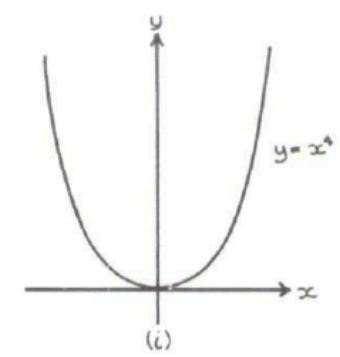

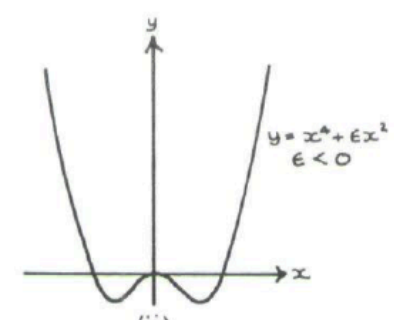

(ii)

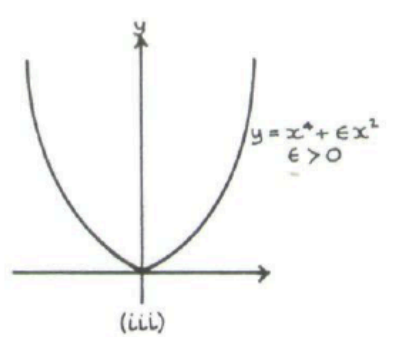

(iii)

A function to the fourth power is the lowest functional order in $\mathrm{x}$ that will allow us to examine the resulting equilibrium states in three dimensional space (one order is lost on differentiation). For example, the functional relationship which expresses a state variable $(x)$ in terms of two, perturbation or control variables (a) and (b):

$f\left(x_{a, b}\right)=x^{4}+2 a x^{2}+4 b x$

gives on differentiation:

$f^{\prime}\left(x_{a, b}\right)=4 x^{3}+4 a x+4 b$

If we set this to zero, we generate a family of functions representing the three dimensional surface of equilibrium states in ( $x$ ) under the control of (a) and (b):

$f^{\prime}\left(x_{a, b}\right)=4 x^{3}+4 a x+4 b=0$

therefore:

$x^{3}+a x+b=0$

Letting $y=-b$

$y=x^{3}+a x$ 
Experimenting with different values of (a) generates a series of functions which vary from the smooth to the folded in terms of (x) (Scapens, Ryan and Fletcher, 1981).

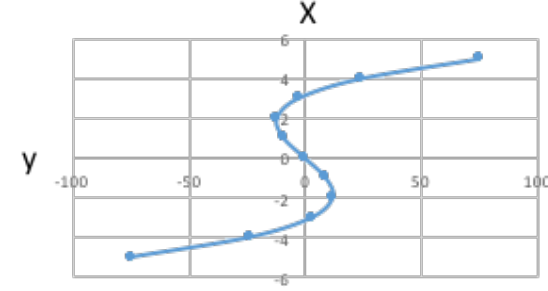

$y=a x^{3}+a x$ where $a<0$

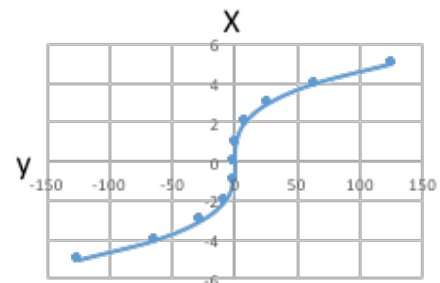

$y=a x^{3}+a x$ where $a=0$

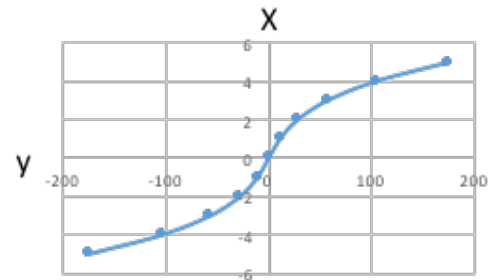

$y=a x^{3}+a x$ where $a>0$

I have rotated the $x, y$ axis to enable a clearer geometric understanding of the different curves that arise under different values of (a). Under different values of (a) the resulting function moves from smooth $(a>0)$ to inflected $(a=0)$ to folded $(a<0)$. By varying the values of the splitting variable (a) Looking at the geometry of the family of functions representing the critical points of the function in $x^{4}$ gives the three-dimensional surface as follows:

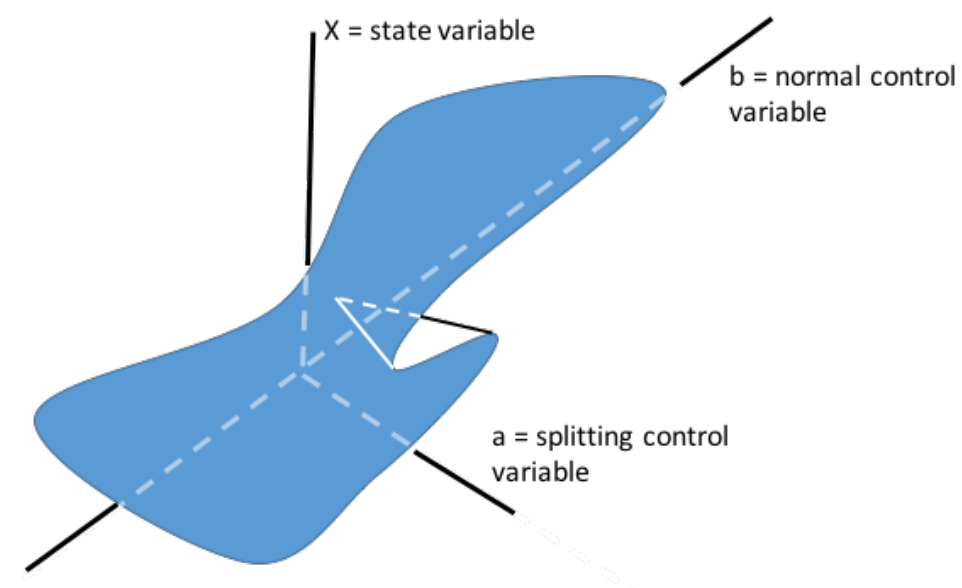

The shaded surface - or manifold - represents the critical points defined by the solution of the first derivative when set to zero sketched in three-dimensional space. Holding (a) equal to zero gives a smooth transition of the state variable under the influence of (a). However, setting any value for (a) greater than zero, results in a discontinuity through the fold and creates the possibility of a 'jump' process. 


\section{Interpreting the variables in an intuitive process.}

Catastrophe Theory as an explanation of sudden changes of state relies upon the definition of the state variable $(x)$, the splitting variable (a) and the normal control variable (b). It also relies upon the identification of the attractor or force which drives a system to the critical point of $(x)$ in terms of $(a)$ and (b). Intuition and deliberative rationality are processes by which individuals change their state of belief in the search for knowledge. Using Plato's definition of knowledge as 'justified true belief', epistemic state is a state of belief and we would define its range as varying from complete uncertainty to complete certainty (i.e., a subjective probability of zero to one).

The normal state variable we argue is deliberative, system 2 cognitive processing where the individual accesses conscious modes of analytical reasoning. As discussed in the thesis, this process in effortful and relatively slow and the process of state change from uncertainty to certainty is gradual and piecemeal as memories are selected, retrieved and processed through whatever algorithms are driving the thinking process. Movement of belief is easily reversible as conflicting cognitions are processed and evaluated. The rear edge of the manifold reflects cognitive activity resulting in epistemic state change in the absence of the splitting variable.

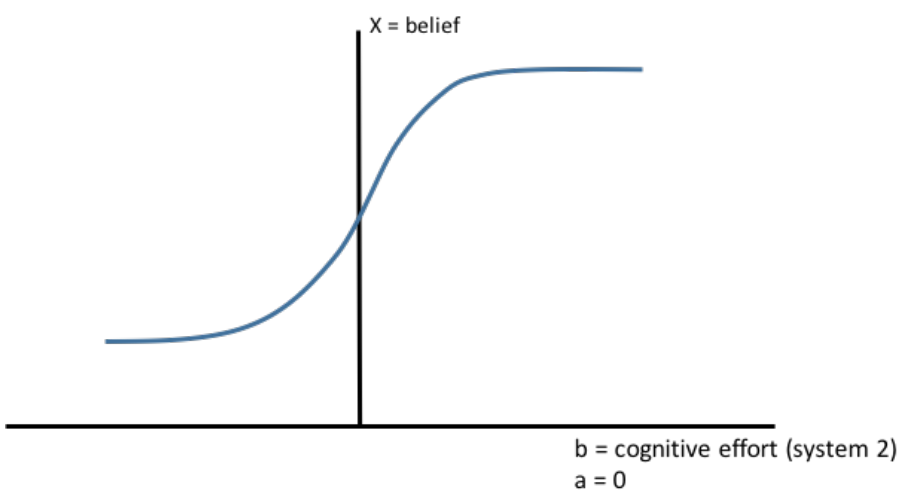

The shape of the function when $a=0$ reflects the degree of effort required to initiate the process of changing belief state and, ultimately achieving certainty of belief. Movement can be in either direction in the face of evidence; confirmatory evidence taking the individual closer 
to certainty, refuting evidence taking them further away. The rate of change in one direction or another is complex and will be conditioned both by prior belief and the reliability which is attached to the evidence acquired (see section $\mathrm{xx}$ on Bayesian inferencing). In a situation of zero affective arousal, the tolerance for ambiguity and uncertainty will be high and the resistance to reconsidering prior beliefs will be low but effortful.

However, when a is greater than zero the manifold of states begins to unfold creating an area of unstable equilibria.

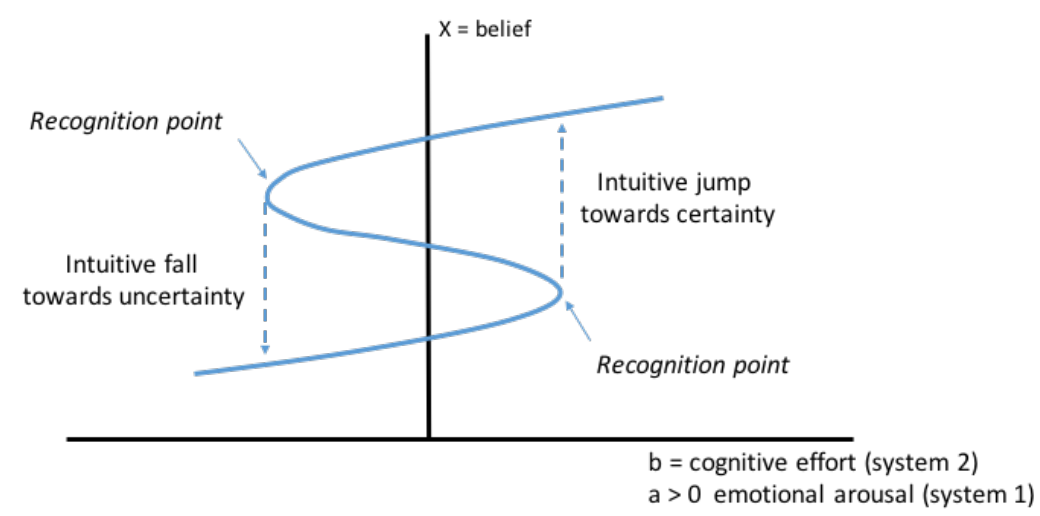

The splitting variable (a) I propose is emotional or affective arousal. The more powerful the emotional state the greater the commitment to a given belief system (as reflected in their 'priors' in a Bayesian sense) and the greater the resistance towards new evidence disconfirming the certainty with which it is held. Further, the higher the arousal state the higher the desire to resolve the uncertainty of belief and hence the stronger the attractor force pulling the mental state to equilibria.

Under uncertainty, the individual's prior beliefs, I would argue, are likely to be strengthened under emotional arousal and there will be resistance to revision even in the presence of strong counterbalancing evidence. However, Catastrophe Theory implies that a certain point will be reached (the recognition point) where the smallest evidential cue will lead to a sudden and locally irreversible state change from uncertainty to high certainty (or vice versa). 
Therefore, Catastrophe Theory provides a necessary explanation for what we commonly observe that under high emotional arousal individuals become strongly committed to their priors and we get the 'anchoring' and 'recency' heuristics dominating their judgement. However, the theory also suggests that the over-commitment effect binds the individual to their prior belief system but that a point will be achieved where even an insignificant item of evidence will act as a cue that activates the jump process. We should also note that intermediate positions can occur where neither deliberative or intuitive modalities are dominant. In this situation, the theory predicts that a hysteresis effect is likely to occur where the individual vacillates between certainty and uncertainty.

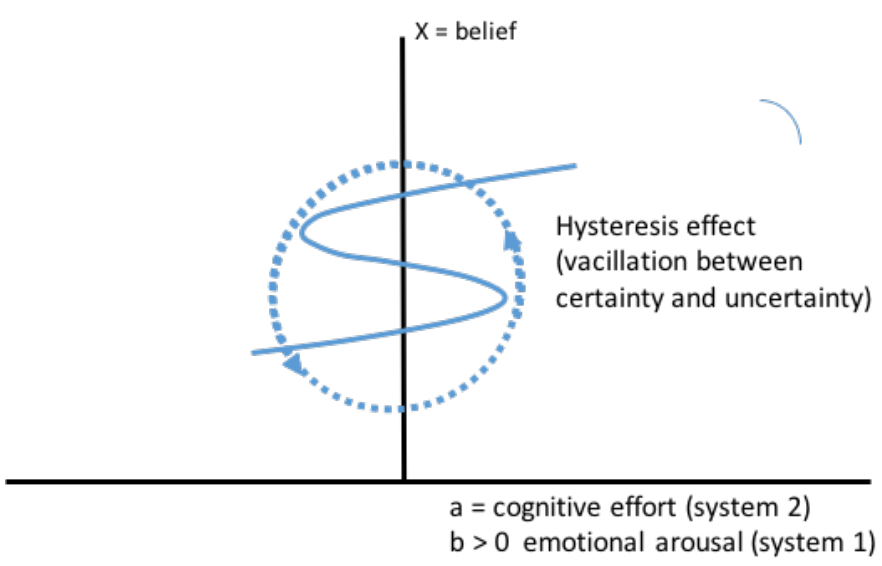

In summary, our discussion of the psychology involved and Catastrophe Theory reinforces the two state model and supports the contention that there is a difference in both the kind and the speed of the cognitive processing under varying states of emotional arousal. Catastrophe Theory also supports the idea that there may be intervening states where emotional engagement is low and the attractor force is relatively weak (see Mega et al, 2015) leading to a more unified theory where pure reasoning and pure intuition sit at two ends of a spectrum of modalities of thought. In these intervening states we expect to see hysteresis effects where individuals vacillate between certainty and uncertainty as their cognitive states operate on the borderline between deliberative and intuitive modalities. However, two important questions remain unanswered: what is the speed of the intuitive jump and what does the answer to that 
question predicate for the minimum time slice during which we would expect intuition to dominate?

The geometry of Catastrophe Theory also focuses attention another aspect of the phenomenon of intuition and the role of expertise. Let us assume we have a complex multivariate problem with multiple interactions and nuances. Developing formal expertise in the solution of such problems, as a class, is likely to involve considerable sophistication in subject expertise, personal skill and in problem and pattern recognition. 